\section{UCDNN}

LIBRARY
University of Connecticut OpenCommons@UConn

$1-4-2019$

\title{
Electric Form Factor of the Neutron from Asymmetry Measurements
}

Richard Frederick Obrecht Jr

richard.obrecht@uconn.edu

Follow this and additional works at: https://opencommons.uconn.edu/dissertations

\section{Recommended Citation}

Obrecht, Richard Frederick Jr, "Electric Form Factor of the Neutron from Asymmetry Measurements" (2019). Doctoral Dissertations. 2045.

https://opencommons.uconn.edu/dissertations/2045 


\title{
Electric Form Factor of the Neutron from Asymmetry Measurements
}

\author{
Richard Frederick Obrecht Jr., Ph.D. \\ University of Connecticut, 2019
}

The overwhelming majority of visible mass in the universe is composed of protons and neutrons, collectively known as nucleons, which are arguably the most important strongly interacting systems to study. A nucleon may naively be thought of as a composite object built out of three non-interacting sub-objects, known as quarks, in the large energy limit; however, the structure of the nucleon is much more complicated, and consequently far richer, than a simple valencequark picture. There are sea quarks in addition to valence quarks that interact strongly via the exchange of gluons resulting in a complex vacuum structure, and the collective system must give rise to the observed properties of the nucleon, e.g. the radius and mass. The nucleon is the most well-studied hadron and yet there are still unresolved complexities in the calculation of properties from first principles of QCD; this represents a central problem in nuclear physics. The theoretical difficulty with the nucleon requires experimentation, which is steadily increasing the knowledge of nucleon structure and the strong interaction.

The subject of this thesis pertains to nucleon electromagnetic form factors which are fundamental quantities containing information on the spatial and momentum distributions of charge and current within the nucleon. Nucleon form factors may be accessed through well-understood 
Richard Frederick Obrecht Jr. - University of Connecticut, 2019

leading order electromagnetic processes with a leptonic probe, and provide strong constraints on testing non-perturbative QCD and nuclear structure models. The form factor ratio of the neutron has been extracted at a negative momentum transfer squared of $Q^{2}=1.16 \mathrm{GeV}^{2}$ via a beamtarget helicity asymmetry measurement using the semi-exclusive reaction ${ }^{3} \mathrm{He}\left(\vec{e}, e^{\prime} n\right) p p$. The Jefferson Lab Hall A experiment E02-013 ran in 2006 utilizing the $6 \mathrm{GeV}$ CEBAF for its highduty, longitudinally polarized electron beam. The double-arm coincidence experiment detected the quasielastically scattered electrons in a large angular and momentum acceptance spectrometer. The recoiling nucleons were detected and momentum analyzed in a large scintillator-iron based neutron detector. The analysis of a new extraction at $Q^{2}=1.16 \mathrm{GeV}^{2}$ will be presented, and concludes with the result of $G_{E}^{n} / G_{M}^{n}=-0.1247 \pm 0.0088 \pm 0.0121$. 


\title{
Electric Form Factor of the Neutron from
}

\section{Asymmetry Measurements}

Richard Frederick Obrecht Jr.

B.A., Boston University, 2011

M.S., University of Connecticut, 2014

\author{
A Dissertation \\ Submitted in Partial Fulfillment of the \\ Requirements for the Degree of \\ Doctor of Philosophy \\ at the \\ University of Connecticut \\ 2019
}


Copyright by

Richard Frederick Obrecht Jr. 


\title{
APPROVAL PAGE
}

Doctor of Philosophy Dissertation

\section{Electric Form Factor of the Neutron from}

\section{Asymmetry Measurements}

\author{
Presented by
}

Richard Frederick Obrecht Jr.

Major Advisor

Andrew Puckett

Associate Advisor

Thomas Blum

Associate Advisor

Peter Schweitzer

University of Connecticut

2019 
To my mother

Susan B. Obrecht 


\section{ACKNOWLEDGEMENTS}

There is a cliché quote from a movie* that I am embarrassed to say has stuck with me for many years: "I guess that's what happens in the end, you start thinking about the beginning." The completion of this document partially fulfills my Ph.D. requirements, but on a more personal level it represents the end of my years as a graduate student which is especially bitter-sweet. As the six year long marathon comes to a close, I cannot help but to reminisce about the people who have made this wonderful journey possible, starting from the beginning. My Ph.D. experience was somewhat different than most in the sense that I analyzed data that already existed; therefore, I did not spend long hours in the counting house during production anxiously awaiting for data. However, I did spend significant time and effort trying to resurrect old code and relevant details, which included harassing many people to test their memory of E02-013. With this being said, my list of people to thank is much smaller than one would typically see, but I'd like to give thanks to the entire E02-013 collaboration despite not being here in 2006 . Note that I will be reminiscing and expressing my gratitude in chronological order.

There are a handful a people that come to mind from my high school and college days. From Boys' Latin, I'd like to thank Molly Mullally and Dr. Guilo Venezian. I am particularly grateful to Molly for her patience, and for finding ways to make calculus interesting to a room full of immature boys, otherwise I probably would have moved on to a less technical subject. Dr. V taught a double-length physics class with only 5 students, thank you Oliver, Jack, Alex, and Juicy for taking it with me. When I first arrived at Boston University, I thought I was taking courses to become an engineer...or an earth scientist...or a geophysicist. It took me until the Fall of my junior year (2009) to return to physics, which I discovered was in fact my true passion.

\footnotetext{
* Mr. and Mrs. Smith
} 
I would like to thank Raj Mohanty for allowing me to work in his nanoelectronics laboratory; additionally, thank you Colleen Dalton for giving me a chance in seismology even though it turned out not to be for me. I had a great physics crew at BU who are now friends for life, thank you Francine and Sol.

My graduate school days were split between UConn and Jefferson Lab, roughly $60 \%$ of my time at the former. From UConn, I'd like to thank Philip Mannheim and Gerald Dunne for the difficult, yet stimulating classes, among many other things. I am thankful to have Kyungseon Joo and Tom Blum as my associate advisors. Tom, thank you for humoring the quantum field theory questions and being the guide during the P\&S days; also, thanks for just being there when needed. UConn is located in the middle-of-nowhere and consequently there is little to do; therefore, I am thankful for the friends that I have acquired to keep me busy outside of physics: Brad C., John M., Brandon C., Kegan M., Odin and Lucy, Tian the Titan, Anees A., David R., Vincent T., Shankar, and the entire Willi-family. Most importantly, though, I am forever appreciative of the time and effort given by my Ph.D. advisor Andrew Puckett. You accepted me as your student even though I had just about zero experience in programming or nuclear physics, and your patience and mentoring will never be forgotten. The amount of genuine support and concern you have given me (while simultaneously allowing me to be independent) truly made the journey great, and I owe the vast majority of my scientific/programming growth to you. I am so thankful for the opportunity to present research at the many conferences, and in general I am 100\% satisfied with the Ph.D. experience mainly because of you, so thank you!

I moved to JLab in the summer of 2016 in order to start my dissertation work on GEn (note that I originally was working on the Super BigBite family of experiments, but due to accelerator scheduling concerns I had to change my topic in the interest of graduation time), where I was fortunate enough to acquire two more mentors: Bogdan Wojtsekhowski and Seamus Riordan. Bogdan and Seamus were not assigned to me formally, and they were by no means forced to 
assist me, yet they put in significant time to help complete my research in addition to genuinely being interested in the project and my well-being. Both of you are busy, and I understand that the thesis, the Phys. Rev. $C$ paper, and the $G_{E}^{n}$ meetings only added to your busyness. Bogdan, thank you so much for humoring my questions any day of the week (I still remember going into your office on a Sunday) and for the advice you have given me. Your relentless pursuit of perfection is contagious. And Seamus, I cannot begin to thank you enough for your help, guidance, and support. We have spent countless hours discussing analysis-related topics over Slack, and you helped save me during one of the most frustrating times of my Ph.D. (when I could not retrieve helicity information from the 2006 analyzer because of an old $\mathrm{C}++$ type issue - an insidious bug to track down, indeed). Thank you for reading this document and supplying me with suggestions as well. Thank you Albert Shahinya for your generosity and help digging up old photographs; lastly, I want to thank Kalyan Allada for machine learning and career advice.

I have many thanks to give to my family. Thank you Dad and Gail for the wonderful support, and supplying me with the perfect get-away location during my JLab years. I want to thank my sister Bethany and her family (Sean, Charlie, and Jack) for the support in addition to the times we shared in NY/Brooklyn. A huge thank you to my mother and step-dad John for always being there for me; the difficult decisions and sacrifices that were made early on are directly related to the completion of my Ph.D., and I am forever grateful. Lastly, I want to thank my beautiful Kristin who without a doubt suffered the most while seeing this project to the end. Long distance can be difficult and tiresome, but you stuck with me; I am forever appreciative of your love as there is truly 'nothing better.'

Freddy Obrecht

Newport News, VA

August 2018 


\section{TABLE OF CONTENTS}

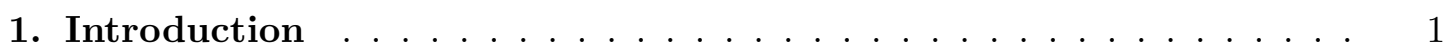

2. Elastic $e N$ Scattering Formalism ................ 14

2.1 Fundamentals . . . . . . . . . . . . . . . . . . . 14

2.2 Unpolarized Scattering . . . . . . . . . . . . . . . 18

2.2 .1 Lab Frame Kinematics . . . . . . . . . . . . . . . . 21

2.2.2 Differential Cross Section ..................... 23

2.2.3 Rosenbluth Cross Section .................... 25

2.2.4 Rosenbluth Separation Technique ................ 30

2.2.5 Initial Form Factor Discussion . . . . . . . . . . . . . 31

2.3 Polarized Scattering . . . . . . . . . . . . . . . 34

2.3.1 Breit Frame Kinematics . . . . . . . . . . . . . 36

2.3.2 Beam-Target Asymmetry . . . . . . . . . . . . . 43

2.3.3 Recoil Polarimetry ...................... 54

2.3.4 Closing Remarks on Double Polarization ............ 56

3. Nucleon Form Factors . . . . . . . . . . . . . . . . . 57

3.1 Previous Experiments ........................ 57

3.1.1 Unpolarized Proton Results . . . . . . . . . . . . . . . 59

3.1 .2 Unpolarized Neutron Results . . . . . . . . . . . . . . . . . 60

3.1 .3 Polarized Results . . . . . . . . . . . . . . . . . 69

3.1.4 Radiative Corrections and Proton Data . . . . . . . . . . . 76 
3.1 .5 Nucleon Charge Radii . . . . . . . . . . . . . . . . . 82

3.2 Parametrizations . . . . . . . . . . . . . . . 86

3.2 .1 Dipole Form Factor $\ldots \ldots \ldots \ldots$

3.2 .2 Galster Fit $\ldots \ldots \ldots \ldots \ldots \ldots$

3.2 .3 Kelly and Galster-like Fits . . . . . . . . . . . . . . 88

3.2 .4 Diehl and Kroll . . . . . . . . . . . . . . . . . 89

3.3 Theoretical Predictions . . . . . . . . . . . . . . . . . 90

3.3.1 Vector Meson Dominance . . . . . . . . . . . . . . . . . . 91

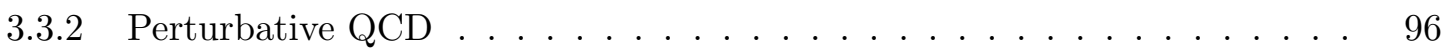

3.3.3 Generalized Parton Distributions . . . . . . . . . . . . . . . 101

3.3 .4 Summary . . . . . . . . . . . . . . . . . . . 104

4. Experimental Description . . . . . . . . . . . . . 107

4.1 Introduction to E02-013 . . . . . . . . . . . . . . . . . 108

4.2 Coordinate Systems . . . . . . . . . . . . . . . . . . . 114

4.3 CEBAF: The Electron Beam . . . . . . . . . . . . . . . 116

4.4 Hall A Beam Monitoring and Diagnostics . . . . . . . . . . . . 121

4.4 .1 Beam Position . . . . . . . . . . . . . . . . . . 122

4.4 .2 Beam Current . . . . . . . . . . . . . . . . . . 123

4.4 .3 Rastering the Beam . . . . . . . . . . . . . . . . . . 124

4.4.4 Absolute Energy Measurements . . . . . . . . . . . . . . . . 125

4.4 .5 Polarimetry ......................... 127

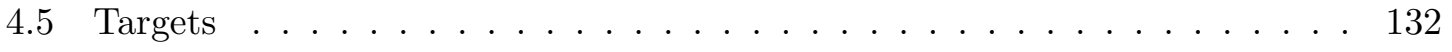


4.5.1 Helium-3 as a Neutron Target . . . . . . . . . . . . . 133

4.5.2 Principles of Polarizing a ${ }^{3} \mathrm{He}$ Target . . . . . . . . . . . . . . 134

$4.5 .3{ }^{3} \mathrm{He}$ Target Cells . . . . . . . . . . . . . . . . . . . . . 140

4.5.4 Monitoring the Polarization: NMR and EPR . . . . . . . . . . 142

4.5.5 Measuring the Polarization Direction . . . . . . . . . . . . . 146

4.5.6 Description of the Target Box and Magnetic Field . . . . . . . . . . 151

4.5.7 Carbon Foil Target . . . . . . . . . . . . . . . 154

4.5.8 Reference Cell . . . . . . . . . . . . . . . . 155

4.6 Electron Arm and the BigBite Spectrometer . . . . . . . . . . . 155

4.6.1 Multi-Wire Drift Chambers . . . . . . . . . . . . . . . 160

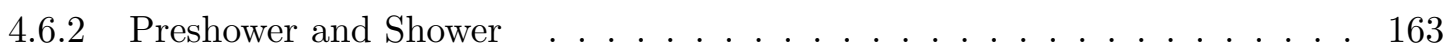

4.6 .3 Timing Hodoscope . . . . . . . . . . . . . . . . . 167

4.7 Neutron Arm and the Neutron Detector . . . . . . . . . . . . 168

4.8 Trigger and Data Acquisition . . . . . . . . . . . . 175

4.8.1 BigBite Trigger and Read-out Electronics . . . . . . . . . . . 177

4.8.2 Neutron Arm Trigger and Read-out Electronics . . . . . . . . . . . . 181

4.8.3 Triggering on a Coincidence Event . . . . . . . . . . . 183

4.8 .4 Scalers . . . . . . . . . . . . . . . . . . . 185

4.8.5 Beam Helicity Determination _.............. 187

4.8 .6 Summary of Triggers . . . . . . . . . . . . . . . . 188

4.9 Analysis Software: AGen and the Hall A Analyzer . . . . . . . . . . . . 189

4.10 Monte Carlo Simulation: G4SBS . . . . . . . . . . . . . . . . 190 
5. Data Analysis . . . . . . . . . . . . . . . . . . 192

5.1 CODA Raw Data . . . . . . . . . . . . . . . . . 193

5.2 Available Data . . . . . . . . . . . . . . . . . . 194

5.3 BigBite: Raw Analysis and Calibration Procedures . . . . . . . . . 195

5.3 .1 Drift Chambers . . . . . . . . . . . . . . . 196

5.3.2 Description of BigBite Track Reconstruction . . . . . . . . . . . 200

5.3.3 BigBite Optics Model: Vertex and Momentum Reconstruction . . . . . 203

5.3 .4 Invariant Mass . . . . . . . . . . . . . . . . . . . . . . . . 214

5.3.5 Preshower and Shower .................. 216

5.3.6 Simulating the BigBite Calorimeter . . . . . . . . . . . 228

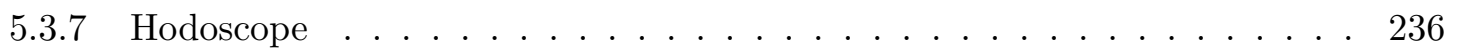

5.3.8 Hodoscope Time Delay of Neighboring Paddles . . . . . . . . . . . . . 240

5.4 Neutron Detector: Raw Analysis and Calibration Procedures . . . . . . . 244

5.4.1 Raw Time of Flight Determination . . . . . . . . . . . . . . . 246

5.4 .2 RF Correction to $\mathrm{ToF} \ldots \ldots \ldots \ldots \ldots$

5.4 .3 Calibration to an Expected Time of Flight . . . . . . . . . . 251

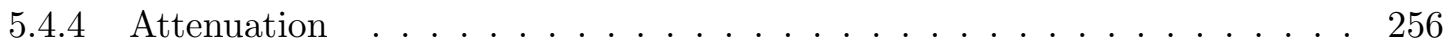

$5.4 .5 \quad$ Nucleon Cluster . . . . . . . . . . . . . . . . 258

5.4.6 Matching Veto Hits to a Cluster and Charge Identification . . . . . . . 263

5.4.7 Simulating the Neutron Detector and a Coincidence Event . . . . . . . 271

5.5 Polarized ${ }^{3}$ He Run Selection . . . . . . . . . . . . . . 285

5.6 Quasielastic Event Selection _. . . . . . . . . . . . . . 289 
5.7 Experimental Asymmetry Formalism . . . . . . . . . . . . . 302

5.7 .1 Corrections to the Raw Asymmetry . . . . . . . . . . . . 307

5.7.2 Finite Acceptance Corrections to the Physical Asymmetry . . . . . . . 314

5.7 .3 Accidental Background . . . . . . . . . . . . . . 318

5.7 .4 Nitrogen in the ${ }^{3} \mathrm{He}$ Cell . . . . . . . . . . . . . . . 324

5.7.5 Misidentified Protons . . . . . . . . . . . . . . . 325

5.7 .6 BigBite Pions . . . . . . . . . . . . . . . . . 331

5.7 .7 Inelastic Events . . . . . . . . . . . . . . . . 335

5.7.8 Nuclear Corrections and Final State Interactions . . . . . . . . . . 337

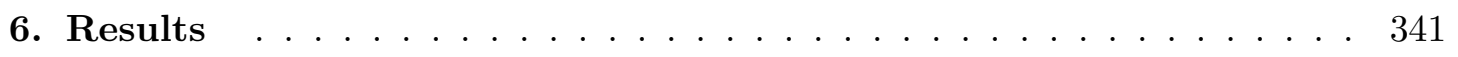

6.1 Calculations for $G_{E}^{n} / G_{M}^{n} \ldots \ldots \ldots \ldots \ldots \ldots \ldots \ldots$

6.1 .1 Uncertainties . . . . . . . . . . . . . . . . . . 342

6.1 .2 Polarizations . . . . . . . . . . . . . . . . . 343

6.1.3 Background .................................. 345

6.1.4 Nitrogen in the ${ }^{3} \mathrm{He}$ Cell . . . . . . . . . . . . . . . . 347

6.1.5 Charge Misidentification . . . . . . . . . . . . . 353

6.1.6 BigBite Preshower Pions . . . . . . . . . . . . . . 360

6.1.7 Inelastic Contamination . . . . . . . . . . . . . . 366

6.1 .8 Nuclear Corrections . . . . . . . . . . . . . . . . . 369

6.1 .9 Physical Asymmetry from Data . . . . . . . . . . . 376

6.2 Neutron Form Factor Ratio . . . . . . . . . . . . . . . 378

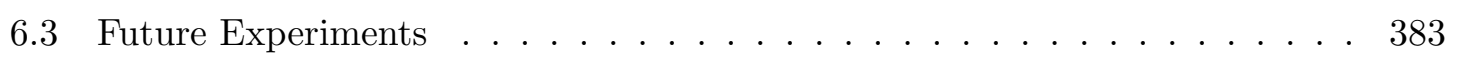


A. Useful Results from the Dirac Equation . . . . . . . . . . . . 385

A.1 Dirac Representation . . . . . . . . . . . . . . . . . 3855

A.2 Dirac Equation . . . . . . . . . . . . . . . . 386

B. Useful Trace Identities $\ldots \ldots \ldots \ldots$

C. Results for the Remaining Configurations . . . . . . . . . . . 388

C.1 Kinematic $4: Q^{2}=1.72 \mathrm{GeV}^{2} \ldots \ldots \ldots \ldots \ldots$

C.2 Kinematic 2: $Q^{2}=2.48 \mathrm{GeV}^{2} \ldots \ldots \ldots \ldots \ldots$. . . . . . . . . . . . . . .

C.3 Kinematic $3: Q^{2}=3.41 \mathrm{GeV}^{2} \ldots \ldots \ldots \ldots \ldots \ldots$

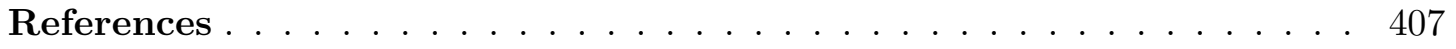




\section{LIST OF FIGURES}

2.1 Tree level elastic $e N$ scattering $\ldots \ldots \ldots \ldots \ldots \ldots$

2.2 Breit frame displaying the chosen coordinate system . . . . . . . 37

2.3 Beam-target asymmetry diagram . . . . . . . . . . . 50

2.4 Polarization transfer diagram $\ldots \ldots \ldots \ldots \ldots \ldots$

3.1 Selected proton form factor data extracted via Rosenbluth separation . . . 61

3.2 Selected $G_{E}^{p} / G_{M}^{p}$ data extracted via Rosenbluth separation . . . . . . . 62

3.3 Selected $G_{E}^{n}$ extractions from unpolarized elastic ed . . . . . . 67

3.4 Selected $G_{M}^{n}$ extractions from unpolarized quasielastic ed . . . . . . 68

3.5 Post-1999 double polarization extractions of $G_{E}^{n} \quad \ldots \ldots \ldots$

3.6 Double polarization extractions of the ratio $\mu_{n} G_{E}^{n} / G_{M}^{n} \ldots \ldots \ldots$

3.7 Selected $\mu_{p} G_{E}^{p} / G_{M}^{p}$ data displaying the difference in technique . . . . . 74

3.8 Selected data of $\mu_{p} G_{E}^{p} / G_{M}^{p}$ focusing on low $Q^{2} \ldots \ldots \ldots$

3.9 One loop radiative corrections to elastic $e N$ scattering $\ldots \ldots \ldots 77$

3.10 VMD fits to selected data of the ratio $\mu_{p} G_{E}^{p} / G_{M}^{p} \ldots \ldots \ldots$

3.11 VMD fits to selected data of the ratio $\mu_{n} G_{E}^{n} / G_{M}^{n} \ldots \ldots \ldots$

3.12 Perturbative QCD predictions and proton form factors . . . . . . . . 100

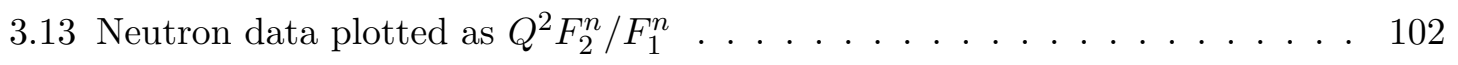

3.14 DVCS "handbag" diagram . . . . . . . . . . . . . . . . . 103

3.15 Selected models and $G_{E}^{n} / G_{M}^{n} \ldots \ldots \ldots \ldots$ 106 . . . . . . . . 
4.1 E02-013 detector layout $\ldots \ldots \ldots \ldots$. . . . . . . . . . . . . . . . . .

4.2 Aerial view of Jefferson Lab . . . . . . . . . . . . . . . 112

4.3 Experimental layout as seen in Monte Carlo . . . . . . . . . . . . . 113

4.4 Summary of the coordinate systems . . . . . . . . . . . . 115

4.5 Diagram of CEBAF .................... 117

4.6 Hall A beamline diagnostic instrumentation . . . . . . . . . . . . . 121

4.7 Diagram of a BPM antenna array . . . . . . . . . . . . . . 122

4.8 Hall A Compton polarimetry schematic . . . . . . . . . . . . . 130

4.9 Compton polarimetry results . . . . . . . . . . . . 131

4.10 Dominating spin states of the ${ }^{3} \mathrm{He}$ nucleus . . . . . . . . . . . . 134

4.11 Optical pumping of $\mathrm{Rb} \ldots \ldots \ldots \ldots \ldots \ldots \ldots$. . . . . . . . . . . .

4.12 Helium-3 target cell . . . . . . . . . . . . . . . . . 141

4.13 Components of the AFP NMR technique . . . . . . . . . . 144

4.14 Compass to measure the magnetic holding field direction . . . . . . . . . 148

4.15 The compass absolute measurement . . . . . . . . . . . . . 149

4.16 The compass relative measurement . . . . . . . . . . . . . 149

4.17 Magnetic holding field angle across the target cell . . . . . . . . . . 150

4.18 Top down schematic of the target magnet box . . . . . . . . . 152

4.19 Major components within target box . . . . . . . . . . 153

4.20 Electron traveling through BigBite in simulation . . . . . . . . 157

4.21 BigBite Spectrometer . . . . . . . . . . . . . . . 158

4.22 BigBite in Monte Carlo . . . . . . . . . . . . . . . 159 
4.23 Wire plane diagram $\ldots \ldots \ldots \ldots \ldots$

4.24 Orientations of XVU wires relative to $y_{\text {det }} \ldots \ldots \ldots \ldots 16 \ldots$

4.25 Electron energy deposition as a function of longitudinal depth in lead-glass 165

4.26 Dimensions and types of ND scintillating bars . . . . . . . . . . 169

4.27 Cross section of the neutron detector . . . . . . . . . . . . 170

4.28 Cassettes and shielding of the $\mathrm{ND} \ldots \ldots \ldots \ldots$. . . . . . . . . . . . .

4.29 Charge identification concept . . . . . . . . . . . 173

4.30 BigBite trigger sum logic . . . . . . . . . . . . 178

4.31 BigBite trigger logic . . . . . . . . . . . . . . . . 179

4.32 Neutron detector trigger sum logic . . . . . . . . . . . . 182

4.33 The trigger diagram of $G_{E}^{n} \ldots \ldots \ldots \ldots \ldots$. . . . . . . . . . . . .

4.34 Determination of L1A . . . . . . . . . . . . . . 186

4.35 Helicity structure and corresponding signals . . . . . . . . . . . . 187

5.1 Method to obtain drift chamber $t_{0, i}$ time offsets $\ldots \ldots \ldots \ldots$

5.2 Drift time to drift distance conversion . . . . . . . . . . . . 200

5.3 Description of the BigBite magnetic bend plane model . . . . . . . . . 203

5.4 Vertex z position prior to calibration . . . . . . . . 206

5.5 Vertex z position post calibration . . . . . . . . . . 207

5.6 Reconstructed vertex $z$ position and the foil data . . . . . . . 208

5.7 Calibrated reconstructed momentum using $\mathrm{H}_{2}$ data . . . . . . . . . 211

5.8 Calibrated momentum resolution versus tracking variables . . . . . . . 213

5.9 The sharp peak at $M_{p}$ within the $\mathrm{H}_{2}$ invariant mass spectrum . . . . . 215 
5.10 Clustering algorithm example . . . . . . . . . . . . . 217

5.11 Calibration coefficients for the preshower and shower . . . . . . . 220

5.12 Calorimeter pedestal and gain distributions . . . . . . . . . . 220

$5.13{ }^{3}$ He preshower energy spectrum before and after calibrations . . . . . . 222

5.14 Preshower energy as a function of $\frac{E_{\text {cluster }}}{E_{\text {optics }}} \ldots \ldots \ldots \ldots \ldots \ldots$

5.15 BigBite energy resolution . . . . . . . . . . . . . . . 224

$5.16 \frac{\delta E}{E}$ as a function of calorimeter $x$ and $y$ coordinates . . . . . . . . 225

5.17 BigBite $x$ and $y$ coordinate resolutions $\ldots \ldots \ldots \ldots \ldots$

5.18 Maximum ADC energy deposition sum for a given TSUM . . . . . . . . 229

5.19 Maximum ADC energy deposition for TSUM 10 through TSUM 18 . . . 230

5.20 Average value of the ADC trigger threshold . . . . . . . . . . . 230

5.21 Comparing the simulated preshower spectrum to $\mathrm{H}_{2}$ data . . . . . . 232

5.22 The simulated BigBite resolution using G4SBS and ESEPP . . . . . . 233

5.23 Comparing the elastic ADC signal between simulation and data . . . . . 234

5.24 Comparing the maximum ADC sums between data and simulation . . . 235

5.25 Description of the hodoscope timing analysis . . . . . . . . . 238

5.26 Hodoscope neighbor paddle vs. $t_{\text {primary }}-t_{\text {neigh }} \ldots \ldots \ldots \ldots \ldots 24$

$5.27 t_{\text {primary }}-t_{\text {neigh }}$ before and after corrections have been applied $\ldots \ldots . .243$

5.28 Conceptual diagram of ToF determination . . . . . . . . . . 247

$5.29 \mathrm{RF}$ correction applied to the nucleon time of flight $\ldots \ldots \ldots . . \ldots 250$

5.30 Results of calibrating the neutron detector . . . . . . . . 253

5.31 Residual times for planes N1-N6 prior to calibration . . . . . . . 254 
5.32 Residual times for planes N1-N6 post calibration . . . . . . . . 255

5.33 Extracting attenuation coefficients ................. 257

5.34 Attenuation factors organized by bar type . . . . . . . . 257

$5.35 \mathrm{ToF}$ and inverse beta distributions . . . . . . . . . . . 259

5.36 Concept of missing momentum . . . . . . . . . . . . . 261

$5.37 p_{\text {miss }, \|}$ and $p_{\text {miss }, \perp}$ distributions for $\mathrm{H}_{2}$ elastic data $\ldots \ldots \ldots . \ldots 262$

5.38 Defining the veto-cluster space window for charge identification . . . . 264

5.39 Defining the veto-cluster time window for charge identification . . . . . 266

5.40 Veto-cluster time difference before and after corrections . . . . . . . 267

5.41 Example of a veto channel deadtime . . . . . . . . . . . 269

5.42 Distribution of $\mathrm{V} 1$ and $\mathrm{V} 2$ deadtimes . . . . . . . . . . . 269

$5.43 \mathrm{~V} 1$ and V2 deadtimes for all channels . . . . . . . . . . . . . 270

5.44 Cross section of the neutron detector displaying trigger logic $\ldots \ldots$. . . 272

5.45 Left and right maximum ADC sums of 32 blocks used to generate a T1 . . 274

5.46 Neutron detector ADC sum32 thresholds . . . . . . . . . . 275

5.47 Simulating the neutron detector response . . . . . . . . . . 278

5.48 Simulating the neutron detector left and right sum32s . . . . . . 279

5.49 Full simulation machinery compared to hydrogen coincidence data . . . . 281

5.50 Beam current data over the duration of the entire data set . . . . 286

5.51 BigBite helicity gated T2 asymmetry as a function of ${ }^{3} \mathrm{He}$ run number . . 287

5.52 Beam current as a function of time for an arbitrary ${ }^{3} \mathrm{He}$ run . . . . . 289

5.53 Missing momentum and Fermi motion . . . . . . . . . . . 292 
5.54 Missing momenta components for the polarized ${ }^{3} \mathrm{He}$ data . . . . . . . 293

5.55 Missing mass spectrum for the ${ }^{3} \mathrm{He}$ data set . . . . . . . . . . . . . . 294

5.56 Chosen cuts to select the quasielastic region . . . . . . . . . . 296

5.57 Invariant mass spectrum before and after QE cuts . . . . . . . . . . 297

5.58 Kinematic distributions at each stage of the quasielastic cut selection . . . 298

5.59 Quasielastic count over the accumulated beam charge . . . . . . . 300

5.60 Quasielastic uncharged to charged ratio . . . . . . . . . . 301

5.61 Factors that determine the sign of $A_{\text {meas }} \ldots \ldots \ldots \ldots$. . . . . . . . . .

5.62 Raw asymmetry for the QE charged and uncharged samples. . . . . . . 312

5.63 Raw asymmetry binned for important kinematic variables . . . . . . . 313

5.64 Method to estimate the random background contribution . . . . . . . 319

5.65 Overlap of QE data and the random background for various kinematics . . 320

5.66 Background uncharged to charged ratio for the ${ }^{3}$ He data set . . . . . . . 322

5.67 Uncharged-to-charged ratio for events and background . . . . . . . . . 323

$5.68{ }^{3}$ He nucleon density distribution $\ldots \ldots \ldots \ldots . \ldots . \ldots . \ldots 328$

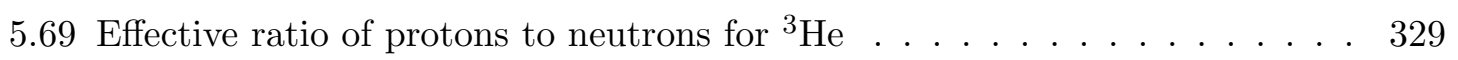

5.70 Dominant contributions in semi-exclusive electro-nuclear reactions . . . . 339

6.1 Polarization of target cell Barbara . . . . . . . . . . . . . . 344

6.2 Carbon foils in the context of the $\mathrm{N}_{2}$ dilution calculation . . . . . 348

6.3 Vertex cut selection and $D_{\mathrm{N}_{2}} \ldots \ldots \ldots \ldots \ldots \ldots \ldots \ldots$

6.4 Uncharged-to-charged ratio $R$ and fit result for the three targets . . . . . 354

6.5 Calculating $D_{\pi}$ by matching simulation to the quasielastic ${ }^{3} \mathrm{He}$ data $\ldots . .364$ 
6.6 Distributions used in the calculation of $D_{\pi}$ and $A_{\pi} \ldots \ldots \ldots$

6.7 Simulating the invariant mass with a loose cut selection . . . . . . . 367

6.8 QE $W$ spectra with and without proper charge identification . . . . . 369

6.9 Six $W$ spectra required for the inelastic contamination estimation . . . . . . 370

6.10 Log scale $W$ spectra required for the inelastic contamination estimation . . 371

6.11 Estimating the error in $A \ldots \ldots \ldots \ldots \ldots \ldots$

6.12 Mapping between $A$ and $A_{\text {free }} \ldots \ldots \ldots \ldots \ldots$

$6.13 A_{\mathrm{phys}, i}$ and the error contributions $\ldots \ldots \ldots \ldots \ldots \ldots$

6.14 New result of the neutron form factor . . . . . . . . . . . 382 


\section{LIST OF TABLES}

1.1 Elementary particles of the Standard Model as of $2018 \ldots \ldots$. . . . . . .

3.1 Summary of neutron form factor extractions via double polarization . . . 71

4.1 Experimental parameters of the four measurements . . . . . . . . . . . 110

4.2 Dates of production .................... 111

4.3 Møller polarimetry results . . . . . . . . . . . . . . . . . . 129

4.4 Mott polarimetry results . . . . . . . . . . . . . . 131

4.5 Selected parameters of ${ }^{3} \mathrm{He}$ production cells . . . . . . . . . . . . . . 140

4.6 Summary of target cell wall thicknesses . . . . . . . . . . . . . . . . 142

$4.7 \mathrm{C}$ and $\mathrm{BeO}$ foil specifications $\ldots \ldots \ldots \ldots \ldots$

4.8 MWDC geometry specifications . . . . . . . . . . . . . 162

4.9 Trigger types $\ldots \ldots \ldots \ldots \ldots \ldots$

5.1 Time cuts to associate veto hits to a nucleon cluster . . . . . . . 268

5.2 Results of the simulated charge identification study . . . . . . . . . . . 284

5.3 Quasielastic cut selection . . . . . . . . . . . . . . 299

5.4 Summary of the analysis notation $\ldots \ldots \ldots \ldots \ldots \ldots$

6.1 Polarization values used in calculations . . . . . . . . . . . . . 344

6.2 Results of the background correction . . . . . . . . . . . . 346

6.3 Parameters used in the calculation of $D_{\mathrm{N}_{2}} \ldots \ldots \ldots \ldots \ldots \ldots$

6.4 Results of the nitrogen correction . . . . . . . . . . . . 352 
6.5 Results of the charge misidentification dilution factors . . . . . . . . 357

6.6 Charge identification comparison between data and MC . . . . . . . 358

6.7 Results of the proton asymmetry correction to $A_{\text {raw }} \ldots \ldots$. . . . . 359

6.8 Results of the BigBite preshower pion dilution factor correction . . . . . 363

6.9 Results of the BigBite preshower pion asymmetry correction . . . . . . . 363

6.10 Results of the nuclear corrections . . . . . . . . . . . . . . 374

6.11 All parameters used in the calculation of $A_{\text {phys }} \ldots \ldots \ldots$. . . . . . 379

6.12 Neutron form factor ratio . . . . . . . . . . . . . . . 381

6.13 Systematic error budget . . . . . . . . . . . . . . . 383

6.14 Upcoming JLab $12 \mathrm{GeV}$ approved experiments . . . . . . . . . . 384

C.1 $Q^{2}=1.72 \mathrm{GeV}^{2}$ : Background corrections . . . . . . . . . . 388

C.2 Parameters used in the calculation of $D_{\mathrm{N}_{2}} \ldots \ldots \ldots \ldots$

C.3 Results of the nitrogen correction . . . . . . . . . . . . . . . . 389

C.4 Results of the charge misidentification dilution factors . . . . . . . . 390

C.5 Results of the proton asymmetry correction . . . . . . . . . . 391

C.6 Results of the pion dilution factors . . . . . . . . . . . . . . 392

C.7 Results of the pion asymmetry corrections . . . . . . . . . . . 392

C.8 Results of the inelastic contamination . . . . . . . . . . 393

C.9 Results of the nuclear corrections . . . . . . . . . . . . . 393

C.10 $Q^{2}=2.48 \mathrm{GeV}^{2}$ : Background corrections . . . . . . . . . . . 394

C.11 Parameters used in the calculation of $D_{\mathrm{N}_{2}} \ldots \ldots \ldots \ldots \ldots \ldots$

C.12 Results of the nitrogen correction . . . . . . . . . . . . 395 
C.13 Results of the charge misidentification dilution factors $\ldots \ldots \ldots$

C.14 Results of the proton asymmetry correction . . . . . . . . . . . 397

C.15 Results of the pion dilution factors . . . . . . . . . . . . . . 398

C.16 Results of the pion asymmetry corrections . . . . . . . . . . . 398

C.17 Results of the inelastic contamination . . . . . . . . . . . . . 399

C.18 Results of the nuclear corrections . . . . . . . . . . . . . 399

C.19 $Q^{2}=3.41 \mathrm{GeV}^{2}$ : Background corrections . . . . . . . . . 400

C.20 Parameters used in the calculation of $D_{\mathrm{N}_{2}} \ldots \ldots \ldots \ldots \ldots$. . . . . . 401

C.21 Results of the nitrogen correction . . . . . . . . . . . . 401

C.22 Results of the charge misidentification dilution factors $\ldots \ldots \ldots$. . . . 402

C.23 Results of the proton asymmetry correction . . . . . . . . . . . . 403

C.24 Results of the pion dilution factors . . . . . . . . . . . . . 404

C.25 Results of the pion asymmetry corrections . . . . . . . . . . . . 404

C.26 Results of the inelastic contamination . . . . . . . . . . 405

C.27 Results of the nuclear corrections . . . . . . . . . . . . 405

C.28 Summary of the reanalysis . . . . . . . . . . . . . . . . 406 


\section{Chapter 1}

\section{Introduction}

Humans have been seeking an answer to "What is the world made of?" for millennia. A seemingly simple question often asked by children and yet the answer is still not fully understood. This work attempts to contribute towards the understanding of matter by providing an experimental extraction of the electric form factor of the neutron, a fundamental quantity containing information on the spatial and momentum distributions of charge and current within one of Nature's most prominent building blocks. Protons and neutrons, collectively known as nucleons, are formed by strongly interacting quarks bound together through the exchange of gluons, the mediators of the strong-sector of the Standard Model. A complete description of nucleons in terms of fundamental degrees of freedom is currently a central problem of nuclear physics, and requires steady feedback between experiment and theory. Nucleon form factors, which may be accessed through well-understood electromagnetic processes, contain information regarding the quark and gluon dynamics that may be used to constrain the computational complexities associated with the strong interaction. In order to put a neutron form factor measurement into context within the big picture, namely the Standard Model, a brief historical interlude highlighting the unique significance of the nucleon with respect to 
the basic constituents of matter will be presented, eventually coming full circle back to the importance of form factors.

The investigation towards understanding matter arguably began in ancient times with the philosophical concept of an atom, or an uncuttable unit, as an elementary building block of nature. Thousands of years were needed to prove the existence of atoms, culminating in a categorical description known as the periodic table. The story quickly becomes more complicated, though, when J.J. Thompson discovered the electron in 1897 , and proposed that this particle exists within the atom. In order to keep the overall neutrality of the atom intact, the negatively charged particles, or corpuscles as he called them, must occupy a positively charged region. While no evidence of the positively charged "cloud" existed, the plum pudding model served as a useful guide for his student Ernest Rutherford. In the early 1900s, Rutherford had been working on some of the first scattering experiments in which alpha particles were emitted from a radium source and collimated towards a gold foil. The beam of alpha particles deflected slightly when passing nearby gold atoms, and consequently a fluorescent spread was observed on a zinc sulfide sheet. The number of fluorescent "flashes" rapidly decreased with increasing scattering angle, and a negligible amount of particles deflected more than a few degrees [1]. Two years later in 1909, Rutherford, Geiger and Marsden fortuitously checked to see if alpha particles were observed to scatter at large angles, or perhaps even backwards. To their surprise, alpha particles were found to scatter backwards, a truly unexpected behavior. Their findings led to the discovery of the atomic nucleus and a revision of the understanding of the atom in which electrons were thought to 
orbit about a dense positively charged center composed of protons, often known as the Rutherford-Bohr model. It took approximately 20 more years for James Chadwick to discover the other particle occupying the nucleus, a neutral particle similar in mass to the proton suitably called the neutron. As the neutron and proton mass difference is small, around $1.3 \mathrm{MeV}$, it is fruitful to consider them as different states of the same particle called the nucleon, an argument first made by Heisenberg in the early 1930s. If the nucleon is thought of as a particle with two different states, then due to the mass degeneracy an $\mathrm{SU}(2)$ symmetry arises from which the proton and neutron form a doublet. In this picture, a new quantum number is introduced called isospin in which the proton (neutron) has isospin up (down), respectively; the details are completely analogous to a spin- $\frac{1}{2}$ particle with two spin states allowed, $\pm \frac{1}{2}$. While nucleon isospin is not an exact symmetry of nature, the idea of interpreting particles of similar mass as states of a single particle will be a powerful tool towards understanding how particles interact.

Shortly after the discovery of the neutron, Otto Stern in 1933 measured the magnetic moment of the proton* and reported a value that is roughly 2.5 times larger than the Dirac theory prediction for a structureless, spin- $\frac{1}{2}$ particle [2]. This was the first indirect experimental evidence that subatomic particles, for example nucleons, could possibly be made up of smaller, more fundamental objects; while this statement is easy to make in hindsight, it would take many more decades for the physics community to come to this conclusion. An accurate and absolute measurement of the magnetic moment of the neutron was performed by Alvarez and Bloch, publishing a value of

\footnotetext{
* He also measured the magnetic moment of the deuteron, indirectly obtaining a value for the neutron.
} 
$\mu_{n}=-1.93 \pm 0.02$ in units of nuclear magnetons in 1940 [3]. Since the neutron does not have an overall charge, it was expected to have a magnetic moment of zero but the measurements, like the measurements of Stern, point to a reality that is perhaps more complicated. In the early days of quantum physics, physicists were reluctant to introduce new particles into the picture in an attempt to explain the discrepancies between the observations and the expectations; more particles destroy the simplicity and elegance of the Bohr atom. However, throughout the next several decades (1930s1960s) the number of observed and/or theoretically introduced particles proliferated, and physicists attempted to categorize them in analogy to the periodic table. Pre-1960s discoveries included the muon, the pion, the kaon and the $\Lambda^{0}$. Furthermore, there were also discoveries in which a new particle exhibited identical properties to an already known particle except with the opposite electric charge (antiparticles). An example would be the positron, or the antielectron, which was predicted by Dirac in the late 1920s, i.e. the famous negative-energy solutions to the free particle Dirac equation, and discovered soon after by Anderson in 1932. Particles with different fundamental properties continued to be discovered, and there was no theoretical framework that could consistently explain the experimental observations; the confusion and shear number of particles led to the informal comparison to the number of species within a zoo, or the particle zoo.

A deeper understanding of matter began with the pioneering experiments of Robert Hofstadter and collaborators in the 1950s at Stanford. Similar in concept to the early experiments of Rutherford, a beam of high energy electrons were scattered 
from the assumed point-like nucleon.* The experiments were able to see scattering deviations from a Dirac spin- $\frac{1}{2}$, structureless particle, a consequence of the electron ${ }^{\dagger}$ scattering off of structure within the proton. The direct experimental evidence of a proton substructure presented a key which may be used to unlock the mysteries of matter, and as a result initiated a world-wide effort in an attempt to understand the nucleon structure at the most fundamental level. With advances in accelerator and detector technology, facilities were able to produce more energetic beams with increasingly better quality to the point where the electron probe could resolve the structures within the nucleon. For example, the deep inelastic scattering (DIS) experiments that took place at SLAC throughout the 1960s provided the first evidence of "lumps" within the nucleon. By the mid-1970s, it was realized that the lump structure could be partially explained by Feynman's partons or the quarks of Gell-Mann/Zweig.

The time has come to re-evaluate what exactly is considered a fundamental building block of nature, and how can all these units be related theoretically in order to explain observed phenomena. While the theoretical side of particle physics has a rich history, it has culminated with the Standard Model ${ }^{\ddagger}$ (SM), humanity’s best and perhaps most ambitious attempt towards understanding the interactions of the most basic units of matter, thus explaining the world around us. The SM attempts to explain three out of the four fundamental forces of nature through renormalizable quantum field theories called quantum chromodynamics (QCD) and the electroweak interaction, or the uni-

\footnotetext{
* At the time, an electron beam of a few hundred MeV was considered high energy.

$\dagger$ The leptons are assumed to be point-like [4].

$\ddagger$ There are exciting topics beyond the SM, but they will not be discussed here.
} 


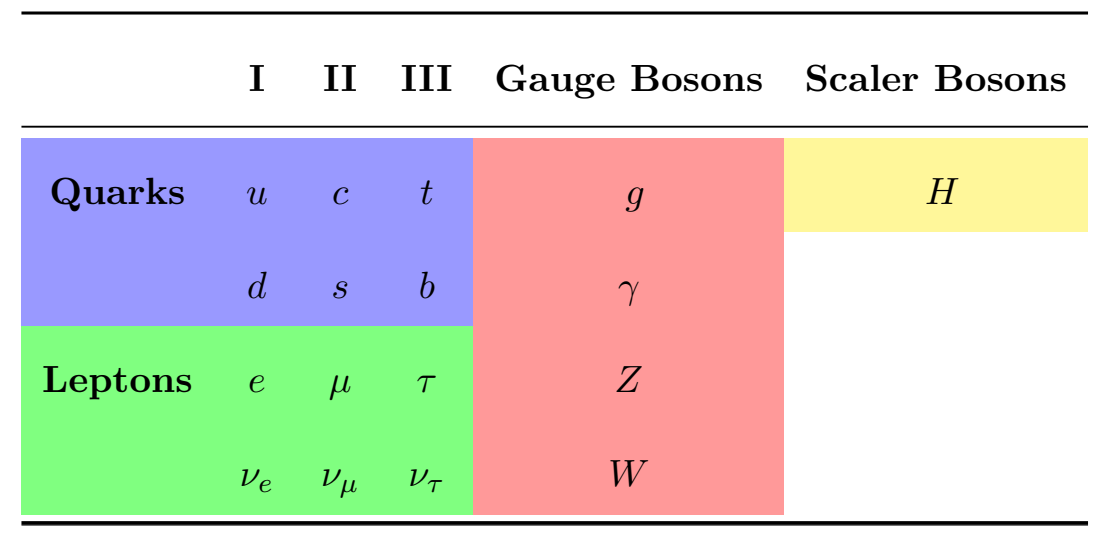

Table 1.1: The elementary particles of the Standard Model as of 2018.

fication of quantum electrodynamics (QED) and the weak interaction.* Incorporating the remaining force, or gravitation, into the framework is still an open problem, but fortunately due to its weak strength gravity is not a factor for short distance particle physics. The fundamental particles of the Standard Model, see Table 1.1 for the current list, "feel" forces through the exchange of the gauge bosons unique to that particular force. For example, a charged lepton like a muon may interact electromagnetically with another charged lepton by exchanging virtual photons with each other. The virtual photon mediates the electromagnetic interaction or in other words carries the exchanged energy and momentum between the two charged leptons. The particle interpretation of exchanged four momenta is physically more intuitive when compared to its abstract "action at a distance" conceptual predecessor. QED was firmly established as the correct renormalizable field theory of the electromagnetic interaction by the mid 1960s. ${ }^{\dagger}$ Similarly, there is a particle interpretation for the weak and strong force, but the details are more complicated.

\footnotetext{
* Electromagnetism and the weak interaction were unified by Glashow, Salam and Weinberg.

${ }^{\dagger}$ Feynman, Schwinger, and Tomonaga shared the Nobel prize in 1965 for this work.
} 
The strong sector of the SM inherited beautiful symmetry ideas from the early quark model, which was quite successful in explaining static properties of observed particles. The quark model was introduced prior to the discovery of most of the particles listed in Table 1.1. nevertheless the theory attempted to explain experimental observations by introducing the strongly interacting spin- $\frac{1}{2}$ quark and exploiting the power of symmetry arguments similar to isospin and the nucleon. The quarks are differentiated by flavor (up, down, charm, strange, top or bottom) and color charge (red, blue or green), a quantum number originally introduced to avoid violating the Pauli exclusion principle. Quarks only appear in nature in colorless configurations, a concept later explained by a phenomenon called confinement, or the idea that a color charged particle cannot be isolated. The need for colorless configurations reflects the fact that no quark has been directly observed in nature, but its existence and flavor are inferred by detecting the products of a particular reaction. Subsequently, according to the quark model it is possible to form two classes* of bound-state colorless systems collectively known as hadrons:

1. quark-antiquark bound-systems called mesons like the $\pi$,

2. triquark bound-systems called baryons, for example protons and neutrons.

Protons and neutrons can be built out of three valence quarks in the colorless triquark configurations uud and $u d d$, respectively. In order to preserve the overall charge of the nucleon, quarks must have fractional electric charge; therefore, the sum of the

\footnotetext{
* There will be no discussions of exotic hadrons, e.g. the pentaquark.
} 
constituent quark charges must equate to zero for the neutron,

$$
e_{u}+2 e_{d}=\frac{2}{3} e-2 \times \frac{1}{3} e=0
$$

and similarly the sum must equate to $e$ for the proton,

$$
2 e_{u}+e_{d}=2 \times \frac{2}{3} e-\frac{1}{3} e=e .
$$

Summarizing the charge of the quarks: $+\frac{2}{3}$ for $u, c, t$ and $-\frac{1}{3}$ for $d, s, b$. The initial success of the quark model was the ability to predict gross features observed in nature; a few examples include spectra of various hadrons, the prediction of hadrons leading to the discovery, and the anomalous magnetic moment of the nucleons. As Otto Stern's magnetic moment measurements are perhaps the first indication of nucleon internal structure, it is instructive to briefly discuss what the quark model can predict. By considering the spin/flavor wavefunctions of the proton and neutron, the magnetic moment ratio may be calculated by considering only the valence quarks and assuming that $m_{u}=m_{d}$ (see chapter 5 of Ref. [5]); the quark model result is

$$
\frac{\mu_{n}}{\mu_{p}}=-\frac{2}{3}
$$

Experimentally, the magnetic moments of the proton and neutron in units of nuclear magnetons have measurements of $\mu_{p}=2.793$ and $\mu_{n}=-1.913$, respectively; this corresponds to a ratio of -0.6849 which is in good agreement with the quark model.

Historically, the lighter quarks $u$ and $d$ were indirectly observed first. In complete analogy to nucleon isospin, the $u$ and $d$ quarks can be treated as the fundamental representation of an $\mathrm{SU}(2)$ group, thereby constructing a doublet from which the $u$ quark has isospin $u p$ and the $d$ quark has isospin down. This means that there exists a 
unitary transformation of $u$ and $d$ states that is a symmetry of the strong interaction. Once the strange quark was "observed," one can carry the symmetry arguments a step further as the quarks $u, d$ and $s$ form the fundamental representation of an approximate* flavor SU(3) symmetry. To make this more explicit, the combination of a three quark representation decomposes to $3 \otimes 3 \otimes 3=10 \oplus 8 \oplus 8 \oplus 1$ where there is a flavor symmetric decuplet, two mixed-symmetric octets, and an antisymmetric singlet. This SU(3) flavor symmetry explains aspects of Gell-Man's Eightfold Way, meaning that an initial baryon state within the octet representation, e.g the neutron $u d d$, can be flavor rotated into various tri-combinations of $u, d$ and $s$ quarks within the 8-dimensional representation. The 8 states of the baryon octet correspond to different particles that have $u, d$ and $s$ tricombinations of quarks. A similar decomposition for mesons exists, or $3 \otimes \overline{3}=8 \oplus 1$, with a similar interpretation. As the remaining quarks continued to be observed, ${ }^{\dagger}$ the degree of flavor symmetry was enumerated to $\mathrm{SU}(6)$, but the symmetry becomes increasingly "broken" as the mass difference between $u$ and $t$ is roughly $170 \mathrm{GeV}$. While the $\mathrm{SU}(3)$ flavor symmetry breaks due to the large mass of the strange quark relative to the up and down quarks, the $\mathrm{SU}(3)$ color symmetry is believed to be an exact symmetry of nature. As particles observed in nature must be colorless, this forces the antisymmetric color singlet to be chosen. In order to generate a singlet within $\mathrm{SU}(3)$, a $q \bar{q}(3 \otimes \overline{3}=8 \oplus 1)$ or a $q q q(3 \otimes 3 \otimes 3=10 \oplus 8 \oplus 8 \oplus 1)$ is necessary, thereby defining the required amount of valence quarks for mesons and baryons, respectively.

The success of the quark model solidified the importance of the valence quarks;

\footnotetext{
* It is approximate as the quark masses are treated as equal, but $s$ is much more massive than $u$ or $d$.

$\dagger$ The heaviest quark, the top, was discovered in 1995 at Fermilab.
} 
however, it became clear that a theory describing the strong interaction should be modeled after the already successful QED. The briefly discussed DIS experiments at SLAC observed a behavior known as Bjorken scaling, a critical clue as to how the new quantum field theory should be constructed. Within the context of SLAC's deep inelastic experiments of the 1960s, Bjorken scaling essentially implies that over short time intervals, or equivalently large energy transfers between the beam electrons and target protons, the "lumps" within the proton (Feynman's partons or the quarks of Gell-Mann/Zweig) can be approximated as free particles. In other words, the strongly interacting quarks within the proton behave as if they are not in the presence of other strongly interacting particles. In the language of quantum field theory, the running coupling constant of the strong interaction decreases with increasing momentum, resulting in a system where the quarks may be well approximated as non-interacting. This behavior is referred to as asymptotic freedom, and constrains the class of field theories to non-Abelian gauge theories, a generalization to the Abelian QED template. By combining the key symmetry ideas of the quark model, namely identifying the color SU(3) symmetry with the gauge group, with a renormalizable non-Abelian four-dimensional field theory results in quantum chromodynamics, the theory of the strong interaction. The quanta, or vector gauge bosons, are called gluons which mediate the strong interaction between quarks, and unlike the QED photon the gluon carries a (color) charge. The generalization of QED to QCD introduces an extra term within the field strength tensor, and consequently an additional technical difficulty arises: gluons may interact with themselves. QCD also exhibits color confinement, or for large strong coupling (large distances, small 
energy) a color singlet state cannot be separated. Therefore, the ad hoc introduction of the color quantum number within the quark model is justified by the non-Abelian properties of the field theory; the concept of only three colors has been experimentally observed via the reaction $\sigma\left(e^{-} e^{+} \rightarrow\right.$ hadrons $)$. The behavior of the strong interaction may be explicitly seen by the running of the strong coupling constant,

$$
\alpha_{s}\left(\mu_{R}^{2}\right) \approx \frac{1}{b_{0} \ln \left(\frac{\mu_{R}^{2}}{\Lambda^{2}}\right)},
$$

where $\Lambda$ is the non-perturbative QCD scale, $b_{0}$ is a $\beta$-function coefficient, and $\mu_{R}^{2}$ is the momentum scale [6]. Recall that the coupling constant is a measure of the strength of the interaction. At large momentum transfer $\alpha_{s}$ becomes small and the theory is asymptotically free where perturbative QCD (pQCD) is applicable; however, as momentum transfer decreases the coupling strength increases to the point where perturbation theory is no longer a good approximation. The strong interaction becomes appreciable corresponding to the QCD scale $\Lambda \approx 200 \mathrm{MeV}$, or a distance larger than $\sim \frac{1}{\Lambda} \sim 1 \mathrm{fm}$ which is roughly the size of the proton.* As the scale of the interaction becomes order 1 fermi, then the coupling between the quark and gluon fields becomes strong. In this regime, other computational methods are required and has been an intensely active area within the nuclear physics community for decades. There are many promising methods and phenomenological models, but bridging the gap between the asymptotically free and the strongly coupled regime is currently unsolved.

The nucleon is the most abundant visible hadron found in nature, and therefore is an excellent strongly interacting system to study. Even though the nucleon was the

\footnotetext{
* In natural units, $1 \mathrm{fm}^{-1} \approx 197 \mathrm{MeV}$.
} 
first hadron discovered, a surprisingly small amount of physical information is fully understood. Difficulties associated with the prediction of fundamental properties, e.g. the radius and mass, from first principles still persist. Intuitively, the particles making up the nucleon, namely the valence and sea quarks all strongly interacting via the exchange of gluons, are required to collectively give rise to the measured properties of the proton. In order to study nucleon structure, the nucleon may be probed using well-understood electromagnetic processes with a lepton, or an assumed point-like particle that does not participate in the strong interaction. Assuming that the energy-scale is much smaller than the mass of the $Z$ boson, then the unpolarized elastic electron-nucleon scattering process $e(k)+N(p) \rightarrow e\left(k^{\prime}\right)+N\left(k^{\prime}\right)$ is precisely calculable within the well-established framework of QED; the calculation introduces functions known as nucleon electromagnetic form factors which encode information of the electric and magnetic structure of the nucleon. While the form factors may be extracted via purely electromagnetic processes, information about the strongly interacting constituents of the nucleon may be inferred providing an observable that may be used as a powerful constraint towards non-perturbative QCD efforts. The subject of this thesis is an extraction of the electric to magnetic form factor ratio of the neutron from the data of Jefferson Lab's Hall A experiment E02-013. The experimental goal was to measure the so-called beam-target asymmetry at four values of $Q^{2}$ and to extract the form factor ratio $G_{E}^{n} / G_{M}^{n}$. The three higher $Q^{2}$ extractions have been published by Riordan et al. in 2010 [7]; the purpose of this work is to analyze the unpublished data at $Q^{2}=1.16 \mathrm{GeV}^{2}$.

The remainder of this work has been organized as follows. Chapter 2 presents the 
physics formalism of E02-013, namely a QED calculation of the polarized elastic scattering process $\vec{e}(k)+\vec{N}(p) \rightarrow e\left(k^{\prime}\right)+N\left(k^{\prime}\right)$ resulting in an expression for the beam-target asymmetry, or the theoretical formalism of E02-013. A connection between an experimentally measured asymmetry and the derived asymmetry will be made. Chapter 3 surveys the experimental results of previous nucleon form factor measurements, and attempts to put nucleon form factors into context amongst current theoretical models striving to handle the non-perturbative regime. The experimental apparatus of $G_{E}^{n}$ will be presented within Chapter 4 , which includes a description of the detector equipment, the triggering schemes and the software. The details of the calibration procedures and the data analysis will be presented in Chapter 5 in addition to the formalism to correct the measured asymmetry such that the result may be compared to the theoretical free neutron asymmetry. Lastly, all calculations required to extract the neutron form factor ratio will be presented in Chapter 6. While the thesis focuses on the $Q^{2}=1.16 \mathrm{GeV}^{2}$ data, the results for the reanalysis of the remaining $Q^{2}$ points may be found in Appendix C, specifically Table C.28. 


\section{Chapter 2}

\section{Elastic $e N$ Scattering Formalism}

The purpose of this chapter is to present the scattering formalism of E02-013, starting with an analysis of unpolarized elastic $e N$ scattering. The result is the well-known Rosenbluth formula which has served as a theoretical tool towards understanding the structure of the nucleon for over half of a century. The calculation will be redone but for polarized elastic $e N$ scattering and concludes with an alternative and perhaps superior method of studying the nucleon. The polarized calculation will result in an expression for the so-called beam-target asymmetry, which is the theoretical basis of $G_{E}^{n}$.

\subsection{Fundamentals}

The leading order elastic scattering process $e(k)+N(p) \rightarrow e\left(k^{\prime}\right)+N\left(p^{\prime}\right)$ as depicted by Figure 2.1 is analyzed in detail. An incoming electron of four momentum $k$ interacts with a nucleon of initial four momentum $p$ via the exchange of one virtual photon, ${ }^{\dagger}$ resulting in the same electron and nucleon but with different outgoing four momenta, $k^{\prime}$ and $p^{\prime}$, respectively, constrained by four momentum conservation. The virtual photon, denoted by four momentum $q$, characterizes the amount of energy and momentum that

\footnotetext{
${ }^{\dagger}$ Commonly referred to as the one-photon exchange approximation (OPEX) or the Born approximation.
} 


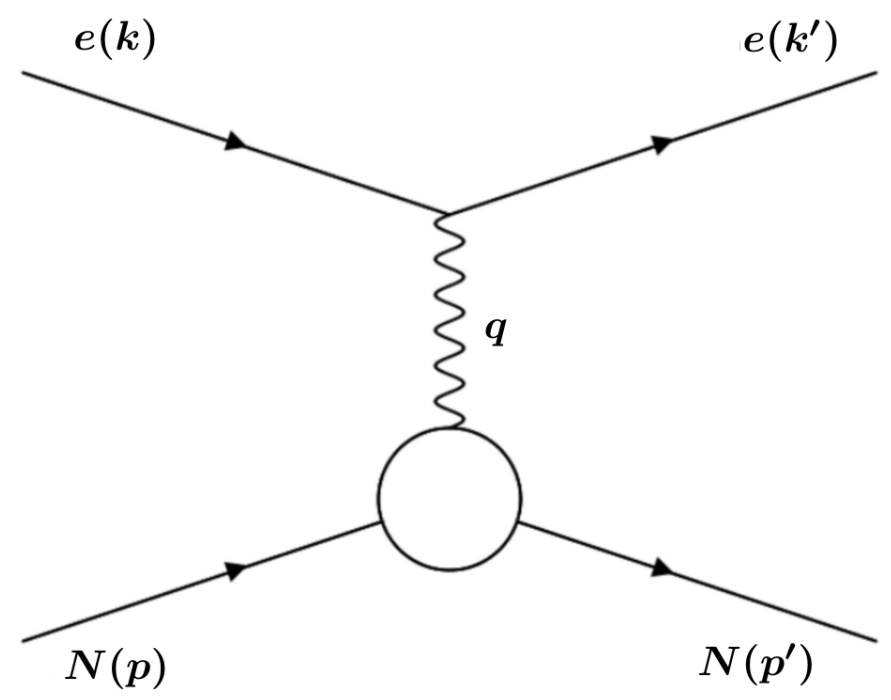

Fig. 2.1: Tree level elastic $e N$ diagram; the circle represents the structure of $N$.

is transferred from the electron to the nucleon. In the lab frame and if the nucleon is initially at rest, then the momentum of the recoiling nucleon $\mathbf{p}^{\prime}$ is in the direction of momentum transfer $\mathbf{q}$.

The electromagnetic process may be calculated within the framework of quantum electrodynamics (QED), a renormalizable quantum field theory that is suitable for all electromagnetic interactions. The theory is well tested, and perhaps the most successful theory in regards to precision measurements, for example the anomalous magnetic moment of the electron or bound-state QED. A desirable property of QED is that the perturbation series converges rapidly, which is due to the smallness of the fine structure constant $\alpha$, or $\alpha=\frac{e^{2}}{2 \pi} \approx \frac{1}{137}$. As a result, tree level calculations are typically a good approximation.* Following the Feynman rules for QED, the invariant amplitude for this

\footnotetext{
${ }^{*}$ Radiative corrections are discussed in Section 3.1 .4 and play a crucial role in measuring form factors.
} 
process may be written down as

$$
\begin{aligned}
i \mathcal{M} & =-i \frac{\ell_{\mu}^{e} \mathcal{J}_{N}^{\mu}}{q^{2}} \\
& =\left[\bar{u}\left(k^{\prime}\right)\left(-i e \gamma^{\mu}\right) u(k)\right]\left(-i \frac{g_{\mu \nu}}{q^{2}}\right)\left[\bar{u}\left(p^{\prime}\right)\left(-i e \Gamma^{\nu}\right) u(p)\right],
\end{aligned}
$$

where the four momenta of the incoming and outgoing electron (nucleon) are denoted by $k$ and $k^{\prime}\left(p\right.$ and $\left.p^{\prime}\right)$, respectively. The momentum transfer squared $q^{2}=\left(k-k^{\prime}\right)^{2}=$ $\left(p^{\prime}-p\right)^{2}<0$ is space-like, and consequently the convention is to define the positive invariant $Q^{2}=-q^{2}$. The $\gamma$ matrices are the Dirac matrices in the so-called Dirac representation in which $\gamma^{0}$ is diagonal, and $g_{\mu \nu}$ is the Minkowski tensor; see Appendix A for the chosen conventions. The objects $u$ and $\bar{u}$ are Dirac spinors and adjoints, namely $\bar{u} \equiv u^{\dagger} \gamma^{0}$, which are the positive energy solutions to the free Dirac equation. Note that the invariant amplitude $\mathcal{M}$ may be generically expressed as the product of an electronic and hadronic current, denoted by $\ell_{\mu}^{e}$ and $\mathcal{J}_{N}^{\mu}$, respectively, both of which satisfy the continuity equation, i.e. the currents are conserved during the interaction.

The photon-nucleon vertex $-i e \Gamma^{\nu}$ is a correction to the typical photon-lepton vertex factor and parametrizes the structure of the nucleon. Instead of simply using $-i e \gamma^{\mu}$ as in the case for spin- $\frac{1}{2}$ point particles, a linear combination of all possible ways to construct a four vector obeying Lorentz invariance is used (e.g. see Ref. [8]):

$$
\Gamma^{\nu}=\gamma^{\nu} \cdot A+\left(p+p^{\prime}\right)^{\nu} \cdot B+\left(p-p^{\prime}\right)^{\nu} \cdot C,
$$

where due to conservation of four momentum $A, B$ and $C$ must be functions of $q^{2}$ and the mass of the nucleon $M$. Note that terms involving $\gamma^{5}=i \gamma^{0} \gamma^{1} \gamma^{2} \gamma^{3}$ are not allowed as QED is believed to conserve parity unlike the weak theory for example. The Ward 
identity, or $q_{\nu} \Gamma^{\nu}=0$, is a statement of current conservation; it forces $C=0$ as the first two terms vanish once sandwiched with the Dirac spinors and simplified using the Dirac equation, or $\not p u(p)=M u(p)$ and $\bar{u}\left(p^{\prime}\right) \not p^{\prime}=\bar{u}\left(p^{\prime}\right) M$ where the conventional Feynman slash notation is used: $\not p=p^{\mu} \gamma_{\mu}$. The $B$ term that is symmetric in $p$ may be rearranged by studying the following object:

$$
\begin{aligned}
\sigma^{\nu \alpha} q_{\alpha} & =\sigma^{\nu \alpha}\left(p^{\prime}-p\right)_{\alpha}, \\
& =\frac{i}{2}\left[\gamma^{\nu}, \gamma^{\alpha}\right]\left(p^{\prime}-p\right)_{\alpha}, \\
& =\frac{i}{2}\left(\gamma^{\nu} \gamma^{\alpha}-2 g^{\nu \alpha}+\gamma^{\nu} \gamma^{\alpha}\right)\left(p^{\prime}-p\right)_{\alpha}, \\
& =2 i\left(\gamma^{\nu}\left(\not p^{\prime}-\not p\right)-\left(p^{\prime}-p\right)^{\nu}\right), \\
& =i\left(\left(p^{\prime}+p\right)^{\nu}-2 M \gamma^{\nu}\right),
\end{aligned}
$$

where the commutation relation $\sigma^{\nu \alpha}=\frac{i}{2}\left[\gamma^{\nu}, \gamma^{\alpha}\right]$ has been used in the second line. The anticommutation relation $\left\{\gamma^{\nu}, \gamma^{\alpha}\right\}=2 g^{\nu \alpha}$ has been used twice, lines three and four, in order to swap the ordering of $\gamma^{\nu}$ and $\gamma^{\alpha}$. Remembering that this object is meant to be sandwiched between Dirac spinors, then the Dirac equation may be used to produce a factor of $M$. Rearranging this object and explicitly inserting the Dirac spinors results in the useful Gordon identity, or

$$
\bar{u}\left(p^{\prime}\right) \gamma^{\nu} u(p)=\bar{u}\left(p^{\prime}\right)\left[\frac{\left(p+p^{\prime}\right)^{\nu}}{2 M}+\frac{i \sigma^{\nu \alpha} q_{\alpha}}{2 M}\right] u(p)
$$

Therefore, we may exchange the $\left(p+p^{\prime}\right)^{\nu}$ term within Eqn 2.3 with a $\sigma^{\nu \alpha} q_{\alpha}$ term using this identity. Swapping terms, and relabeling the coefficients results in the familiar form of the vertex correction:

$$
\Gamma^{\nu}=\gamma^{\nu} F_{1}\left(q^{2}\right)+\frac{i \sigma^{\nu \alpha} q_{\alpha}}{2 M} F_{2}\left(q^{2}\right)
$$


where $F_{1}$ and $F_{2}$ are the nucleon form factors known as the Dirac and Pauli form factors, respectively. These real functions only depend on the Lorentz invariant variable $q^{2}$, and are therefore relativistically invariant. Eqn 2.6 is the form typically seen in the literature; however, it may be conveniently rearranged using Eqn 2.4 .

$$
\Gamma^{\nu}=\gamma^{\nu}\left(F_{1}+F_{2}\right)-\frac{\left(p+p^{\prime}\right)^{\nu}}{2 M} F_{2}
$$

Substituting this result into Eqn 2.2 and contracting indices yields a useful form of the invariant amplitude,

$$
\mathcal{M}=\frac{e^{2}}{q^{2}}\left[\bar{u}\left(k^{\prime}\right) \gamma^{\mu} u(k)\right]\left[\bar{u}\left(p^{\prime}\right)\left(\left(F_{1}+F_{2}\right) \gamma_{\mu}-\frac{\left(p+p^{\prime}\right)_{\mu}}{2 M} F_{2}\right) u(p)\right]
$$

which is as far as one can go without considering spin states of the incoming and outgoing particles. There are two situations that will be considered within this thesis: (1) unpolarized elastic $e N$ scattering, and (2) polarized elastic $e N$ scattering in which both the electron and nucleon are initially polarized. In the latter process, an explicit form of the Dirac spinors is needed as the initial spin of the electron and nucleon are now specified. In any case, the final results are presented as a cross section which is proportional to the invariant amplitude squared, $\overline{|\mathcal{M}|^{2}}$.

\subsection{Unpolarized Scattering}

The initial spin states are averaged while the final spin states are summed, which reflects the fact that the experimental detectors are blind to polarization. Squaring Eqn 2.8 and organizing the result in terms of an electronic tensor and a hadronic tensor gives 
the following generic expression:

$$
\overline{|\mathcal{M}|^{2}}=\frac{e^{4}}{q^{4}} L_{e}^{\mu \nu} W_{\mu \nu}^{N}
$$

where $L_{e}^{\mu \nu} \equiv \frac{\ell^{\mu} \ell^{\nu *}}{e^{2}}$ and $W_{\mu \nu}^{N} \equiv \frac{\mathcal{J}_{\mu} \mathcal{J}_{\nu}^{*}}{e^{2}}$, and each tensor may be simplified individually. The leptonic tensor evaluates to

$$
\begin{aligned}
L_{e}^{\mu \nu} & =\frac{1}{2} \sum_{s, s^{\prime}}\left[\bar{u}^{\left(s^{\prime}\right)}\left(k^{\prime}\right) \gamma^{\mu} u^{(s)}(k)\right]\left[\bar{u}^{\left(s^{\prime}\right)}\left(k^{\prime}\right) \gamma^{\nu} u^{(s)}(k)\right]^{*}, \\
& =\frac{1}{2} \sum_{s, s^{\prime}}\left[\bar{u}^{\left(s^{\prime}\right)}\left(k^{\prime}\right) \gamma^{\mu} u^{(s)}(k)\right]\left[\bar{u}^{(s)}(k) \gamma^{\nu} u^{\left(s^{\prime}\right)}\left(k^{\prime}\right)\right] \\
& =\frac{1}{2} \operatorname{Tr}\left[\left(\not k^{\prime}+m_{e}\right) \gamma^{\mu}\left(\not k+m_{e}\right) \gamma^{\nu}\right] \\
& =\frac{1}{2}\left(\operatorname{Tr}\left[\not k^{\prime} \gamma^{\mu} \not k \gamma^{\nu}\right]+m_{e}^{2} \operatorname{Tr}\left[\gamma^{\mu} \gamma^{\nu}\right]\right) \\
& =2\left(k^{\prime \mu} k^{\nu}+k^{\prime \nu} k^{\mu}-g^{\mu \nu}\left(k \cdot k^{\prime}-m_{e}^{2}\right)\right) .
\end{aligned}
$$

The factor of $\frac{1}{2}$ in line 1 originates from averaging over initial spin states, and the completeness relation for summing over spin states $\sum_{s} u^{(s)}(k) \bar{u}^{(s)}(k)=\not k+m_{e}$ has been used twice in line 3. Standard trace identities have been utilized in the last line, see Appendix B for more details. The unpolarized leptonic tensor is symmetric and real. The hadronic tensor may be evaluated in a similar manner:

$$
\begin{aligned}
W_{\mu \nu}^{N}= & \frac{1}{2} \sum_{s, s^{\prime}}\left[\bar{u}^{\left(s^{\prime}\right)}\left(p^{\prime}\right)\left(\left(F_{1}+F_{2}\right) \gamma_{\mu}-\frac{\left(p+p^{\prime}\right)_{\mu}}{2 M} F_{2}\right) u^{(s)}(p)\right] \\
& \times\left[\bar{u}^{(s)}(p)\left(\left(F_{1}+F_{2}\right) \gamma_{\nu}-\frac{\left(p+p^{\prime}\right)_{\nu}}{2 M} F_{2}\right) u^{\left(s^{\prime}\right)}\left(p^{\prime}\right)\right], \\
= & \left(F_{1}+F_{2}\right)^{2} \operatorname{Tr}\left[\left(\not p^{\prime}+M\right) \gamma_{\mu}(\not p+M) \gamma_{\nu}\right] \\
& -2 \times\left(F_{1}+F_{2}\right) F_{2} \frac{\left(p+p^{\prime}\right)_{\nu}}{2 M} \operatorname{Tr}\left[\left(\not p^{\prime}+M\right) \gamma_{\mu}(\not p+M)\right] \\
& -F_{2}^{2} \frac{\left(p+p^{\prime}\right)_{\mu}\left(p+p^{\prime}\right)_{\nu}}{4 M^{2}} \operatorname{Tr}\left[\left(\not p{ }^{\prime}+M\right)(\not p+M)\right],
\end{aligned}
$$


where the $\left(F_{1}+F_{2}\right) F_{2}$ term has been simplified producing a factor of 2 . In this case, there are three traces that need to be evaluated:

$$
\begin{aligned}
\operatorname{Tr}\left[\left(\not p^{\prime}+M\right) \gamma_{\mu}(\not p+M) \gamma_{\nu}\right] & =4\left[p_{\mu} p_{\nu}^{\prime}+p_{\mu}^{\prime} p_{\nu}-g_{\mu \nu}\left(p \cdot p^{\prime}-M^{2}\right)\right], \\
\operatorname{Tr}\left[\left(\not p^{\prime}+M\right) \gamma_{\mu}(\not p+M)\right] & =4 M\left(p+p^{\prime}\right)_{\mu}, \\
\operatorname{Tr}\left[\left(\not p^{\prime}+M\right)(\not p+M)\right] & =4\left(p \cdot p^{\prime}+M^{2}\right),
\end{aligned}
$$

where the standard trace identities have been utilized again. Substituting the trace results into the tensor and dividing both sides by 4 gives the following:

$$
\begin{aligned}
\frac{W_{\mu \nu}^{N}}{4}= & \left(F_{1}+F_{2}\right)^{2}\left[p_{\mu} p_{\nu}^{\prime}+p_{\mu}^{\prime} p_{\nu}-g_{\mu \nu}\left(p \cdot p^{\prime}-M^{2}\right)\right] \\
& -\left(F_{1}+F_{2}\right) F_{2}\left(p+p^{\prime}\right)_{\mu}\left(p+p^{\prime}\right)_{\nu} \\
& +\frac{F_{2}^{2}}{4 M^{2}}\left(p+p^{\prime}\right)_{\mu}\left(p+p^{\prime}\right)_{\nu}\left(p \cdot p^{\prime}+M^{2}\right) .
\end{aligned}
$$

Since the form factors are real functions, the hadronic tensor for the unpolarized nucleon is also real and symmetric. The leptonic and hadronic tensors can be simplified further, but a particular frame of reference is required. A common choice that often reflects experimental conditions is to work in the rest frame of the nucleon, henceforth the lab frame.* In this frame, the incoming electron bombards a nucleon that is initially at rest. The kinematics of the lab frame will be exhaustively explored before returning to the unpolarized derivation. Furthermore, the QED result must be related to an observable for a measurement; therefore, the cross section and its relationship to the invariant amplitude squared will also be introduced.

\footnotetext{
* The nucleon is initially at rest; therefore, the rest frame of the nucleon and the lab frame coincide.
} 


\subsubsection{Lab Frame Kinematics}

In the lab frame, an electron of initial energy $E_{e}$ scatters from a nucleon that is initially at rest. The coordinate system is oriented such that the initial relativistic electron travels in the $z$-direction, or $\mathbf{k}=E_{e} \hat{\boldsymbol{z}}$. The scattering is defined to take place in the $x z$-plane, therefore the $y$-dimension is irrelevant due to conservation of momentum. Assuming that the electron scatters into the $+\hat{\boldsymbol{x}}$ direction, and using the notation of Figure 2.1, the four momenta are the following:

$$
k^{\mu}=\left(\begin{array}{c}
E_{e} \\
0 \\
0 \\
E_{e}
\end{array}\right), \quad k^{\prime \mu}=\left(\begin{array}{c}
E_{e}^{\prime} \\
E_{e}^{\prime} \sin \theta_{e} \\
0 \\
E_{e}^{\prime} \cos \theta_{e}
\end{array}\right), \quad p^{\mu}=\left(\begin{array}{c}
M \\
0 \\
0 \\
0
\end{array}\right), \quad p^{\prime \mu}=\left(\begin{array}{c}
E_{p}^{\prime} \\
-p_{p}^{\prime} \sin \theta_{p} \\
0 \\
-p_{p}^{\prime} \cos \theta_{p}
\end{array}\right),
$$

where the energy of the outgoing electron (nucleon) is $E_{e}^{\prime}\left(E_{p}^{\prime}\right)$. The polar angles $\theta_{e}$ and $\theta_{p}$ are the scattering angles of the electron and nucleon, respectively. Conservation of four momenta reveals the following relationships:

$$
\begin{aligned}
E_{e}+M & =E_{e}^{\prime}+E_{p}^{\prime}, \\
E_{e}^{\prime} \sin \theta_{e} & =p_{p}^{\prime} \sin \theta_{p}, \\
E_{e}-E_{e}^{\prime} \cos \theta_{e} & =-p_{p}^{\prime} \cos \theta_{p} .
\end{aligned}
$$

For the remainder of this thesis, unless otherwise stated, the mass of the electron is assumed to be negligible, i.e. $m_{e}=0$, as the energies encountered in this thesis are on the order of $1.5 \mathrm{GeV}$; therefore, the electron mass is $\frac{1.5 \times 10^{3} \mathrm{MeV}}{0.511 \mathrm{MeV}} \sim 3 \times 10^{3}$ times smaller and may be ignored. Exploiting momentum conservation and the energy-momentum 
relation, many useful expressions may be derived:

$$
\begin{aligned}
E_{p}^{\prime} & =E_{e}-E_{e}^{\prime}+M=\nu+M \text { where } \nu \equiv E_{e}-E_{e}^{\prime}, \\
q^{2} & =\left(k-k^{\prime}\right)^{2}=-2 E_{e} E_{e}^{\prime}\left(1-\cos \theta_{e}\right)=-4 E_{e} E_{e}^{\prime} \sin ^{2} \frac{\theta_{e}}{2} \\
& \Rightarrow Q^{2}=4 E_{e} E_{e}^{\prime} \sin ^{2} \frac{\theta_{e}}{2}, \\
q^{2} & =\left(p^{\prime}-p\right)^{2}=-2 M E_{e}+2 M E_{e}^{\prime}=-2 M \nu, \\
& \Rightarrow E_{e}^{\prime}=E_{e}+\frac{q^{2}}{2 M} \text { and } Q^{2}=2 M \nu \\
& \Rightarrow \frac{E_{e}^{\prime}}{E_{e}}=\frac{1}{1+\frac{2 E_{e}}{M} \sin ^{2} \frac{\theta_{e}}{2}},
\end{aligned}
$$

where Eqn 2.23 is the result of combining Eqn 2.22 and Eqn 2.19. The ratio $\frac{E_{e}^{\prime}}{E_{e}}$ is commonly referred to as the recoil factor. Rewriting the Lorentz scalers of the problem and recalling $p^{2}=p^{\prime 2}=M^{2}$ and $k^{2}=k^{\prime 2}=0$ results in the following relationships:

$$
\begin{aligned}
p^{\prime 2} & =M^{2}=(q+p)^{2}=q^{2}-M^{2}+2 p \cdot q, \\
k^{\prime 2} & =0=(k-q)^{2}=q^{2}-2 k \cdot q, \\
k^{2} & =0=\left(q+k^{\prime}\right)^{2}=q^{2}+2 k^{\prime} \cdot q, \\
& \Rightarrow k \cdot k^{\prime}=-\frac{q^{2}}{2} \\
& \Rightarrow p \cdot p^{\prime}=M^{2}-\frac{q^{2}}{2} \\
& \Rightarrow k \cdot p^{\prime}=k^{\prime} \cdot p=M E_{e}+\frac{q^{2}}{2} \\
& \Rightarrow k \cdot p=k^{\prime} \cdot p^{\prime}=M E_{e}
\end{aligned}
$$

which will be useful when simplifying the leptonic and hadronic tensors. 


\subsubsection{Differential Cross Section}

An expression for the lab frame differential cross section is needed, and can be computed by beginning with the most general form for two body scattering (e.g. page 106 of Ref. [8]), or

$$
d \sigma=\frac{\overline{|\mathcal{M}|^{2}}}{2 E_{e} 2 E_{p}\left|v_{p}-v_{e}\right|}\left(\prod_{f} \frac{d^{3} p_{f}}{(2 \pi)^{3}} \frac{1}{2 E_{f}}\right)(2 \pi)^{4} \delta^{(4)}\left(k+p-\sum p_{f}\right),
$$

where $\left|v_{p}-v_{e}\right|$ is the relative velocity between the nucleon and electron as viewed in the lab frame, $E_{p}$ is the initial energy of the nucleon, the subscript $f$ denotes the final states and the integral over final-state momenta, or

$$
\left(\prod_{f} \int \frac{d^{3} p_{f}}{(2 \pi)^{3}} \frac{1}{2 E_{f}}\right)(2 \pi)^{4} \delta^{(4)}\left(k+p-\sum p_{f}\right)
$$

is a relativistically invariant phase space factor. This equation takes into account that particles can be created during the scattering process, but lab frame elastic scattering simplifies the differential cross section considerably:

$$
d \sigma=\frac{\overline{|\mathcal{M}|^{2}}}{64 \pi^{2} M E_{e} E_{e}^{\prime} E_{p}^{\prime}} d^{3} k^{\prime} d^{3} p^{\prime} \delta^{(4)}\left(k-k^{\prime}+p-p^{\prime}\right),
$$

where $\left|v_{p}-v_{e}\right|=1,{ }^{*} E_{p}=M$ and recalling that only two final states are produced. The integral over outgoing nucleon momenta $\mathbf{p}^{\prime}$ may be done immediately:

$$
d \sigma=\frac{\overline{|\mathcal{M}|^{2}}}{64 \pi^{2} M E_{e} E_{e}^{\prime} E_{p}^{\prime}} d^{3} k^{\prime} \delta\left(E_{e}+M-E_{e}^{\prime}-E_{p}^{\prime}\right)
$$

where the $\delta$-function effectively sets $\mathbf{p}^{\prime} \rightarrow \mathbf{p}+\mathbf{q}$. The argument of the $\delta$-function needs to be expressed in terms of $E_{e}^{\prime}$ which requires an expression for $E_{p}^{\prime}$ utilizing the

\footnotetext{
* The electron approaches the stationary nucleon at roughly the speed of light, and $c=1$ in natural units.
} 
energy-momentum relation:

$$
\begin{aligned}
E_{p}^{\prime} & =\sqrt{M^{2}+(\mathbf{p}+\mathbf{q})^{2}}, \\
& =\sqrt{M^{2}+\mathbf{q}^{2}}, \\
& =\sqrt{M^{2}+E_{e}^{2}+E_{e}^{\prime 2}-2 E_{e} E_{e}^{\prime} \cos \theta_{e}} .
\end{aligned}
$$

Substituting this into the argument of the $\delta$-function, setting $d^{3} k^{\prime}=E_{e}^{\prime 2} d E_{e}^{\prime} d \Omega$ and evaluating the integral yields the following:

$$
\begin{aligned}
d \sigma & =\frac{\overline{|\mathcal{M}|^{2}} E_{e}^{\prime} d E_{e}^{\prime} d \Omega}{64 \pi^{2} M E_{e} E_{p}^{\prime}} \delta\left(E_{e}+M-E_{e}^{\prime}-\sqrt{M^{2}+E_{e}^{2}+E_{e}^{\prime 2}-2 E_{e} E_{e}^{\prime} \cos \theta_{e}}\right), \\
& =\frac{\overline{|\mathcal{M}|^{2}}}{64 \pi^{2} M} \frac{E_{e}^{\prime}}{E_{e}} \frac{1}{E_{p}^{\prime}}\left(1+\frac{E_{e}^{\prime}-E_{e} \cos \theta_{e}}{E_{p}^{\prime}}\right)^{-1} d \Omega, \\
& =\frac{\overline{|\mathcal{M}|^{2}}}{64 \pi^{2} M} \frac{E_{e}^{\prime}}{E_{e}}\left(\frac{1}{M+E_{e}\left(1-\cos \theta_{e}\right)}\right) d \Omega, \\
& =\frac{\overline{|\mathcal{M}|^{2}}}{64 \pi^{2} M^{2}} \frac{E_{e}^{\prime}}{E_{e}}\left(\frac{1}{1+\frac{E_{e}}{M}\left(1-\cos \theta_{e}\right)}\right) d \Omega, \\
& =\frac{\overline{|\mathcal{M}|^{2}}}{64 \pi^{2} M^{2}}\left(\frac{E_{e}^{\prime}}{E_{e}}\right)^{2} d \Omega,
\end{aligned}
$$

where the delta function property $\delta[g(x)]=\sum \frac{\delta\left(x-x_{i}\right)}{\left|g^{\prime}\left(x_{i}\right)\right|}$ has been used in order to compute the integral over $d E_{e}^{\prime}$, Eqn 2.18 is used to rewrite $E_{p}^{\prime}$ and Eqn 2.23 has been used to simplify the last line. Therefore, the differential cross section in the lab frame becomes

$$
\frac{d \sigma}{d \Omega}=\frac{\overline{|\mathcal{M}|^{2}}}{64 \pi^{2} M^{2}}\left(\frac{E_{e}^{\prime}}{E_{e}}\right)^{2}
$$

and is used to relate the invariant amplitude squared to a physical observable in the unpolarized and polarized derivations. 


\subsubsection{Rosenbluth Cross Section}

The kinematics and differential cross section have been specified, therefore the unpolarized derivation, which results in the Rosenbluth formula, may resume. Inserting the relation $p \cdot p^{\prime}=M^{2}-\frac{q^{2}}{2}(\mathrm{Eqn} 2.25$ into the hadronic tensor (Eqn 2.14 gives

$$
\begin{aligned}
\frac{W_{\mu \nu}^{N}}{2}= & \left(p+p^{\prime}\right)_{\mu}\left(p+p^{\prime}\right)_{\nu}\left(F_{1}^{2}-\frac{q^{2}}{4 M^{2}} F_{2}^{2}\right) \\
& -\left[\left(p-p^{\prime}\right)_{\mu}\left(p-p^{\prime}\right)_{\nu}+g_{\mu \nu}\left(p \cdot p^{\prime}-M^{2}\right)\right]\left(F_{1}+F_{2}\right)^{2}
\end{aligned}
$$

At this stage, the kinematic relation is only substituted into the $F_{2}^{2}$ term, but not the $\left(F_{1}+F_{2}\right)^{2}$ term; the reasoning will soon become clear. Substituting the leptonic and hadronic tensors into the invariant amplitude squared, or Eqn 2.9, results in

$$
\begin{aligned}
\overline{|\mathcal{M}|^{2}}= & \frac{e^{4}}{q^{4}} L_{e}^{\mu \nu} W_{\mu \nu}^{N}, \\
= & \frac{2 e^{4}}{q^{4}}\left[k^{\mu} k^{\prime \nu}+k^{\prime \mu} k^{\nu}-g^{\mu \nu}\left(k \cdot k^{\prime}\right)\right] \\
& \times\left\{\left(p+p^{\prime}\right)_{\mu}\left(p+p^{\prime}\right)_{\nu}\left(F_{1}^{2}-\frac{q^{2}}{4 M^{2}} F_{2}^{2}\right)\right. \\
& \left.\quad-\left[\left(p-p^{\prime}\right)_{\mu}\left(p-p^{\prime}\right)_{\nu}+g_{\mu \nu}\left(p \cdot p^{\prime}-M^{2}\right)\right]\left(F_{1}+F_{2}\right)^{2}\right\}, \\
= & \frac{2 e^{4}}{q^{4}}\left[\mathcal{A}\left(F_{1}^{2}-\frac{q^{2}}{4 M^{2}} F_{2}^{2}\right)+\mathcal{B}\left(F_{1}+F_{2}\right)^{2}\right] .
\end{aligned}
$$

$\mathcal{A}$ and $\mathcal{B}$ are kinematic factors that are easier to digest individually:

$$
\begin{aligned}
\mathcal{A} & \equiv\left[k^{\mu} k^{\prime \nu}+k^{\prime \mu} k^{\nu}-g^{\mu \nu}\left(k \cdot k^{\prime}\right)\right]\left[\left(p+p^{\prime}\right)_{\mu}\left(p+p^{\prime}\right)_{\nu}\right], \\
& =\left(k^{\mu} k^{\prime \nu}+k^{\prime \mu} k^{\nu}\right)\left(p_{\mu} p_{\nu}+p_{\mu} p_{\nu}^{\prime}+p_{\mu}^{\prime} p_{\nu}+p_{\mu}^{\prime} p_{\nu}^{\prime}\right)-\left(k \cdot k^{\prime}\right)\left(p+p^{\prime}\right)^{2}, \\
& =2\left[\left(k \cdot p+k \cdot p^{\prime}\right)\left(k^{\prime} \cdot p+p^{\prime} \cdot k^{\prime}\right)\right]-\left(k \cdot k^{\prime}\right)\left(p+p^{\prime}\right)^{2}, \\
& =\left(2 M E_{e}+\frac{q^{2}}{2}\right)\left(2 M E_{e}+\frac{q^{2}}{2}\right)+\left(\frac{q^{2}}{2}\right)\left(4 M^{2}-q^{2}\right),
\end{aligned}
$$




$$
\begin{aligned}
& =8 M^{2} E_{e}^{2}+4 M E_{e} q^{2}+2 M^{2} q^{2}, \\
& =2 \frac{4 M^{2} E_{e}^{2}\left(1+\frac{2 E_{e}}{M} \sin ^{2} \frac{\theta_{e}}{2}\right)-8 M E_{e}^{3} \sin ^{2} \frac{\theta_{e}}{2}-4 M^{2} E_{e}^{2} \sin ^{2} \frac{\theta_{e}}{2}}{1+\frac{2 E_{e}}{M} \sin ^{2} \frac{\theta_{e}}{2}}, \\
& =\frac{8 M^{2} E_{e}^{2}\left(1-\sin ^{2} \frac{\theta_{e}}{2}\right)}{1+\frac{2 E_{e}}{M} \sin ^{2} \frac{\theta_{e}}{2}}, \\
& =\frac{8 M^{2} E_{e}^{2}}{1+\frac{2 E_{e}}{M} \sin ^{2} \frac{\theta_{e}}{2}} \cos ^{2} \frac{\theta_{e}}{2},
\end{aligned}
$$

where kinematic relations Eqn 2.25- Eqn 2.27 have been used in the fourth line, and relations Eqn 2.19 and Eqn 2.23 have been used in the sixth line. Simplifying $\mathcal{B}$ in a similar manner:

$$
\begin{aligned}
\mathcal{B} & \equiv\left[k^{\mu} k^{\prime \nu}+k^{\prime \mu} k^{\nu}-g^{\mu \nu}\left(k \cdot k^{\prime}\right)\right]\left[\left(p-p^{\prime}\right)_{\mu}\left(p-p^{\prime}\right)_{\nu}+g_{\mu \nu}\left(p \cdot p^{\prime}-M^{2}\right)\right] \\
& =2\left[\left(k \cdot p-k \cdot p^{\prime}\right)\left(k^{\prime} \cdot p-p^{\prime} \cdot k^{\prime}\right)\right]-\left(k \cdot k^{\prime}\right)\left(p-p^{\prime}\right)^{2}-4\left(k \cdot k^{\prime}\right)\left(p \cdot p^{\prime}-M^{2}\right), \\
& =2\left[\left(k \cdot p-k \cdot p^{\prime}\right)\left(k^{\prime} \cdot p-p^{\prime} \cdot k^{\prime}\right)\right]+\left(k \cdot k^{\prime}\right)\left(p-p^{\prime}\right)^{2} \\
& =2\left[\left(-\frac{q^{2}}{2}\right)\left(\frac{q^{2}}{2}\right)\right]-\frac{q^{2}}{2} q^{2}, \\
& =-q^{2} \cdot q^{2} \\
& =-\frac{q^{2}}{2 M^{2}} \frac{8 M^{2} E_{e}^{2} \sin ^{2} \frac{\theta_{e}}{2}}{1+\frac{2 E_{e}}{M} \sin ^{2} \frac{\theta_{e}}{2}}
\end{aligned}
$$

where the kinematic relations $p \cdot p^{\prime}=M^{2}-\frac{q^{2}}{2}$ and $q^{2}=\left(p-p^{\prime}\right)^{2}$ have been used in order to combine the second and third terms in the second line, and a factor of $\frac{2 M^{2}}{2 M^{2}}$ has been introduced in the last line. Substituting these results (Eqn 2.38 and Eqn 2.39) into Eqn 2.37 yields $\overline{|\mathcal{M}|^{2}}$ in terms of Dirac and Pauli form factors, or

$$
\overline{|\mathcal{M}|^{2}}=\frac{16 e^{4} M^{2} E_{e}^{2}}{q^{4}\left(1+\frac{2 E_{e}}{M} \sin ^{2} \frac{\theta_{e}}{2}\right)} \cos ^{2} \frac{\theta_{e}}{2}\left[\left(F_{1}^{2}-\frac{q^{2}}{4 M^{2}} F_{2}^{2}\right)-\frac{q^{2}}{2 M^{2}}\left(F_{1}+F_{2}\right)^{2} \tan ^{2} \frac{\theta_{e}}{2}\right]
$$


Another common set of form factors are linear combinations of $F_{1}$ and $F_{2}$, or

$$
\begin{aligned}
& G_{E}\left(q^{2}\right)=F_{1}\left(q^{2}\right)-\tau F_{2}\left(q^{2}\right), \\
& G_{M}\left(q^{2}\right)=F_{1}\left(q^{2}\right)+F_{2}\left(q^{2}\right),
\end{aligned}
$$

where $\tau \equiv-\frac{q^{2}}{4 M^{2}}=\frac{Q^{2}}{4 M^{2}}$. The functions $G_{E}$ and $G_{M}$ are referred to as the Sachs electric and magnetic form factors, respectively [9, 10]. Expressing $\overline{|\mathcal{M}|^{2}}$ in terms of the Sachs form factors gives

$$
\overline{|\mathcal{M}|^{2}}=\frac{16 e^{4} M^{2} E_{e}^{2}}{q^{4}\left(1+\frac{2 E_{e}}{M} \sin ^{2} \frac{\theta_{e}}{2}\right)} \cos ^{2} \frac{\theta_{e}}{2}\left[\frac{G_{E}^{2}+\tau G_{M}^{2}}{1+\tau}+2 \tau G_{M}^{2} \tan ^{2} \frac{\theta_{e}}{2}\right]
$$

The use of the Sachs form factors is advantageous because there are no cross terms, e.g Eqn 2.40 has a term that is $\propto F_{1} F_{2}$. Additionally, the Sachs form factors have a physical interpretation as being related to the charge and magnetic current densities (hence the names), but this statement only holds within a particular reference frame and must be interpreted carefully; this is explicitly shown in Section 2.3.1. Substituting the invariant amplitude squared in terms of Sachs form factors into Eqn 2.34 results in the final form of the cross section,

$$
\begin{aligned}
\left.\frac{d \sigma}{d \Omega}\right|_{\mathrm{LAB}} & =\frac{4 \alpha^{2} E_{e}^{\prime 2} \cos ^{2} \frac{\theta_{e}}{2}}{q^{4}\left(1+\frac{2 E_{e}}{M} \sin ^{2} \frac{\theta_{e}}{2}\right)}\left[\frac{G_{E}^{2}+\tau G_{M}^{2}}{1+\tau}+2 \tau G_{M}^{2} \tan ^{2} \frac{\theta_{e}}{2}\right] \\
& =\frac{\alpha^{2}}{4 E_{e}^{2} \sin ^{4} \frac{\theta_{e}}{2}} \frac{E_{e}^{\prime}}{E_{e}} \cos ^{2} \frac{\theta_{e}}{2}\left[\frac{G_{E}^{2}+\tau G_{M}^{2}}{1+\tau}+2 \tau G_{M}^{2} \tan ^{2} \frac{\theta_{e}}{2}\right]
\end{aligned}
$$

This expression is known as the Rosenbluth formula after Marshall Rosenbluth's work in the early 1950s [11], but note that the expression wasn't written down in terms of two form factors until 1957 [12, 13]. Recall that this formula is the unpolarized elastic $e N$ cross section in the one photon exchange approximation where the nucleon structure has 
been taken into account with the most general expression given by the symmetries of QED. The cross section is calculated to leading order in $\alpha$, and represents an observable that may be measured from which the Sachs form factors may be extracted and used for a physics interpretation in regards to nucleon structure.

The structure of Eqn 2.44 may be made more transparent by studying less complicated elastic scattering processes, for example if nucleon structure and/or spin is removed from the calculation. If the calculation is redone in exactly the same manner for a structureless nucleon, or $\Gamma^{\nu} \rightarrow \gamma^{\nu}$, then the results may be outlined as follows:

$$
\begin{aligned}
i \mathcal{M} & =\frac{i e^{2}}{q^{2}}\left[\bar{u}\left(k^{\prime}\right) \gamma^{\mu} u(k)\right]\left[\bar{u}\left(p^{\prime}\right) \gamma_{\nu} u(p)\right], \\
\frac{1}{4} \sum \overline{|\mathcal{M}|^{2}} & =\frac{8 e^{4}}{q^{4}}\left[\left(k \cdot p^{\prime}\right)\left(k^{\prime} \cdot p\right)+(k \cdot p)\left(k^{\prime} \cdot p^{\prime}\right)-M^{2}\left(k \cdot k^{\prime}\right)\right], \\
\frac{d \sigma}{d \Omega} & =\frac{\alpha^{2}}{4 E_{e}^{2} \sin ^{4} \frac{\theta_{e}}{2}} \frac{E_{e}^{\prime}}{E_{e}} \cos ^{2} \frac{\theta_{e}}{2}\left(1+2 \tau \tan ^{2} \frac{\theta_{e}}{2}\right),
\end{aligned}
$$

where the mass of the electron has been neglected, initial particle spins are averaged, final particle spins are summed and the expression is evaluated in the lab frame. This also could have been realized immediately by recalling the form of $\Gamma^{\nu}$ :

$$
\Gamma^{\nu}=\gamma^{\nu}\left(F_{1}+F_{2}\right)-\frac{\left(p+p^{\prime}\right)^{\nu}}{2 M} F_{2},
$$

where $\Gamma^{\nu} \rightarrow \gamma^{\nu}$ if $F_{1}=1$ and $F_{2}=0$. In terms of Sachs form factors, the cross section for a structureless nucleon may be acquired by setting $G_{E}=G_{M}=1$ in the Rosenbluth formula. If the calculation is redone another time, removing nucleon structure and spin, then the results may be summarized by the following:

$$
L_{e}^{\mu \nu} \rightarrow L_{e}^{\mu \nu}, \quad W_{\mu \nu}^{N} \rightarrow\left(p+p^{\prime}\right)_{\mu}\left(p+p^{\prime}\right)_{\nu}
$$




$$
\left.\frac{d \sigma}{d \Omega}\right|_{\text {Mott }} \equiv \frac{\alpha^{2}}{4 E_{e}^{2} \sin ^{4} \frac{\theta_{e}}{2}} \frac{E_{e}^{\prime}}{E_{e}} \cos ^{2} \frac{\theta_{e}}{2} .
$$

This cross section is commonly referred to as the Mott formula, and to be complete it represents the elastic scattering of spin- $\frac{1}{2}$ electrons from a structureless, spinless charged particle. The simplification seen in Eqn 2.48 reflects the fact that the nucleon spin has been removed from the calculation, and consequently the $\sin ^{2} \frac{\theta_{e}}{2}$ dependence has vanished from the cross section. With this information at hand and recalling the Gordon identity Eqn 2.5, it can be inferred that spin, and hence the magnetic moment, of the nucleon is contained in the $\sigma^{\nu \alpha} q_{\alpha}$ term. By comparing the Mott formula to the cross sections with spin incorporated, it can be deduced that the $\sin ^{2} \frac{\theta_{e}}{2}$ dependence is a consequence of the electron scattering from the magnetic moment of the nucleon target. Using the same argument but in reverse, the $\cos ^{2} \frac{\theta_{e}}{2}$ large angle suppression seen in the Mott formula reflects the fact that a spinless target, i.e. a target with no magnetic moment, cannot flip the helicity of the incoming electron resulting in a situation where forward scattering is preferred. Therefore, the $\sin ^{2} \frac{\theta_{e}}{2}$ term allows for backwards scattering by flipping the helicity of the electron. In this light, $F_{1}\left(Q^{2}\right)$ and $F_{2}\left(Q^{2}\right)$ are often referred to as the helicity-conserving (chirality) and helicity-flip form factors, respectively.

If the spin of the electron is removed in addition to neglecting the nucleon structure, spin and recoil, then Rutherford scattering is obtained in the non-relativistic limit:

$$
\left.\frac{d \sigma}{d \Omega}\right|_{\text {Rutherford }} \propto \frac{\alpha^{2}}{4 E_{k}^{2} \sin ^{4} \frac{\theta_{e}}{2}},
$$

where $E_{k}$ is the kinetic energy of the electron and the famous $1 / \sin ^{4} \frac{\theta_{e}}{2}$ behavior can be attributed to the Coulomb force. This yields a divergent total cross section, and 
reflects the fact that the Coulomb potential has infinite range originating from the $r^{-2}$ dependence of the Coulomb force. The $\cos ^{2} \frac{\theta_{e}}{2}$ term and the recoil term $\frac{E_{e}^{\prime}}{E_{e}}$ have both disappeared, a consequence of assuming that the incoming electron has no spin and that the target is immovable.

\subsubsection{Rosenbluth Separation Technique}

Specialized techniques have been developed in order to experimentally separate the Sachs form factors. The Rosenbluth formula is commonly written as

$$
\left.\frac{d \sigma}{d \Omega}\right|_{\mathrm{LAB}}=\left.\frac{d \sigma}{d \Omega}\right|_{\mathrm{Mott}}\left[\frac{G_{E}^{2}+\tau G_{M}^{2}}{1+\tau}+2 \tau G_{M}^{2} \tan ^{2} \frac{\theta_{e}}{2}\right]
$$

where the terms that parametrize the structure of the nucleon represent the deviation from the Mott expectation. A reduced cross section, denoted by $\sigma_{R}$, is defined in an attempt to isolate the nucleon structure dependence which may be expressed as

$$
\begin{aligned}
\sigma_{R} & =\frac{\left.\frac{d \sigma}{d \Omega}\right|_{\mathrm{LAB}}}{\left.\frac{d \sigma}{d \Omega}\right|_{\operatorname{Mott}}}\left(\frac{\epsilon(1+\tau)}{\tau}\right)=\frac{\epsilon}{\tau} G_{E}^{2}+G_{M}^{2}, \\
\epsilon & \equiv\left(1+2(1+\tau) \tan ^{2} \frac{\theta_{e}}{2}\right)^{-1},
\end{aligned}
$$

where $\epsilon$ is commonly referred to as the polarization of the virtual photon with $\epsilon=1$ $(\epsilon \rightarrow 0)$ corresponding to forward (backward) scattering. An experiment can separate the square of the Sachs form factors by varying $E_{e}$ and $\theta_{e}$ in such a way to keep $Q^{2}$ fixed while varying $\epsilon$. In other words, a plot of $\sigma_{R}$ vs. $\epsilon$ results in a linear dependence with $\frac{G_{E}^{2}}{\tau}$ as the slope and $G_{M}^{2}$ as the y-intercept. This procedure of separating out the form factors is known as the Rosenbluth separation method, ${ }^{*}$ and was the method of choice

\footnotetext{
* Often referred to as longitudinal-transverse or $\mathrm{L} / \mathrm{T}$ technique within the literature.
} 
from the 1950s to the 1990s. The implications and results of the Rosenbluth technique with respect to the proton and neutron will be discussed in great detail in Section 3.1. In the 1990s, facilities were able to sufficiently polarize the beam and/or target allowing for alternative extraction techniques to be discussed in an upcoming section.

\subsubsection{Initial Form Factor Discussion}

As of now, nucleon form factors are simply real-valued functions that depend on $Q^{2}$, and have originated by expressing the most general form of the photo-nucleon vertex based on QED symmetry arguments; the vertex correction is often synonymous with "parametrizing the structure of the nucleon." While an entire chapter is devoted towards nucleon form factors, an initial discussion may shed light on the importance of form factors, starting with the low $Q^{2}$ expectation. As $Q^{2} \rightarrow 0$, the virtual photon probe is no longer able to resolve the substructure of the nucleon. In this case, the virtual

photon should only see a point particle of charge $e$ and magnetic moment $\frac{e(1+\kappa)}{2 M}$ where $\kappa$ is the anomalous magnetic moment, experimentally measured to be $\kappa_{p}=1.79$ and $\kappa_{n}=-1.91$ for the proton and neutron, respectively. Furthermore, by comparing the point-like requirements to the vertex correction Eqn 2.7, the Dirac, Pauli and Sachs form factors for the proton and neutron in the static limit are

$$
\begin{array}{lr}
F_{1}^{p}(0)=1, & F_{2}^{p}(0)=\kappa_{p}=\mu_{p}-1=1.79, \\
F_{1}^{n}(0)=0, & F_{2}^{n}(0)=\kappa_{n}=\mu_{n}=-1.91, \\
G_{E}^{p}(0)=1, & G_{M}^{p}(0)=\kappa_{p}+1=\mu_{p}=2.79, \\
G_{E}^{n}(0)=0, & G_{M}^{n}(0)=\kappa_{n}=\mu_{n}=-1.91,
\end{array}
$$


where the nuclear magneton unit is assumed. Note that the notation $F_{1 j}, F_{2 j}, G_{E j}$ and $G_{M j}$ where $j=p, n$ is also common within the literature.

A question yet to be addressed is "why is $G_{E}\left(G_{M}\right)$ associated with the electric (magnetic) charge distribution of the nucleon?" The nucleon current $\mathcal{J}_{\mu}$ separates beautifully into individual contributions from $G_{E}$ and $G_{M}$, where the zeroth component is proportional to $G_{E}$ while the vectoral components are proportional to $G_{M}$ hence the names electric and magnetic Sachs form factors. The derivation is explicitly shown in Section 2.3.1, which is intentionally delayed as it concerns polarized $e N$ scattering. However, the interpretation is a result of calculating the nucleon current in the so-called Breit reference frame, a mathematically convenient frame in which the relation between incoming and outgoing nucleon momenta is $\mathbf{p}^{\prime}=-\mathbf{p}$. There is a frame for each value of $Q^{2}$ resulting in an infinite number of Breit frames; additionally, if $Q^{2}$ is large enough then the nucleon moves relativistically in which the shape and distribution become distorted and non-trivial. While the interpretation is physically satisfying, the Breit frame result must not be taken too seriously.

Despite the dangers of a physics interpretation in the Breit frame, a single form factor may be used to parametrize the deviation from the Mott scattering expectation, and in the early days was commonly expressed as the following [14]:

$$
\left.\frac{d \sigma}{d \Omega}\right|_{\mathrm{LAB}}=\left.\frac{d \sigma}{d \Omega}\right|_{\mathrm{Mott}}\left|\int_{\mathrm{V}} \rho(\mathbf{r}) e^{i \mathbf{q} \cdot \mathbf{r}} d^{3} \mathbf{r}\right|^{2}=\left.\frac{d \sigma}{d \Omega}\right|_{\mathrm{Mott}}|F(q)|^{2},
$$

where $F$ is simply some generic function of $q$ not to be confused with $F_{1}$ or $F_{2}$. If $|\mathbf{q}|$ is small, then this corresponds to a situation where the terms multiplied by $\tau$ in Eqn 2.52 
may be neglected, or

$$
\left.\frac{d \sigma}{d \Omega}\right|_{\mathrm{LAB}}=\left.\frac{d \sigma}{d \Omega}\right|_{\mathrm{Mott}} G_{E}^{2},
$$

where the factor $(1+\tau)^{-1} \approx 1$. As $\tau \rightarrow 0$, or equivalently as $Q^{2} \rightarrow 0$, the electric Sachs form factor dominates the cross section, and $G_{E}$ may be written as the Fourier transform of a static charge distribution of the nucleon. Expanding the exponential as the argument is assumed to be small and assuming that the charge density only depends on the radial coordinate yields the following relationship:

$$
\begin{aligned}
G_{E} & =\int_{\mathrm{V}} \rho(\mathbf{r}) e^{i \mathbf{q} \cdot \mathbf{r}} d^{3} \mathbf{r} \\
& =2 \pi \int_{0}^{\infty} d r \rho(r) r^{2} \int_{0}^{\pi} d \theta \sin \theta\left(1+i|\mathbf{q}| r \cos \theta-\frac{1}{2} \mathbf{q}^{2} r^{2} \cos ^{2} \theta+\ldots\right), \\
& =1-\frac{1}{6} \mathbf{q}^{2}\left\langle r^{2}\right\rangle+\ldots \\
\Rightarrow\left\langle r_{E}^{2}\right\rangle & =\left.6\left(\frac{d G_{E}\left(q^{2}\right)}{d q^{2}}\right)\right|_{q^{2} \rightarrow 0}=-\left.6\left(\frac{d G_{E}\left(Q^{2}\right)}{d Q^{2}}\right)\right|_{Q^{2} \rightarrow 0}
\end{aligned}
$$

where in the second line the $\theta$ integral is performed only to remove the $\cos ^{2} \theta$ term. In the low-energy limit, the charge form factor $G_{E}$ is related to the mean square charge radius of the nucleon. In practice, form factor data is typically fit to a model, perhaps physically motivated, and extrapolated to $Q^{2}=0$ such that a derivative may be performed. An analogous definition exists for the mean square magnetic radius using $G_{M}$ and a magnetization density, except the expression is normalized by the magnetic moment to retain the low-energy expectations. Recently, the value of the RMS charge radius of the proton has been subject to intense scrutiny due to the so-called proton radius puzzle, a concept that will be introduced within Section 3.1.5. 


\subsection{Polarized Scattering}

The Rosenbluth formula served as the basis for elastic $e N$ scattering experiments for decades starting with Robert Hofstadter and collaborators [15, 16] at Stanford in the early 1950s. The unpolarized cross section experiments extracted the form factors squared using the Rosenbluth separation technique. A drawback of the Rosenbluth

method is that as $Q^{2}$ increases, $G_{E}^{2}$ is suppressed by a factor of $\tau$. In other words, the magnetic form factor is enhanced by a factor of $\tau$ which completely dominates the cross section making a $G_{E}$ extraction problematic. Historically, extractions of the electric form factor even at modest $Q^{2}$ proved to be difficult, particularly for the neutron. In fact and as will be discussed in Section 3.1.2, extracting $G_{E}^{n}$ through unpolarized observables without suffering from large systematic uncertainties is futile. In the interest of exploring differing experimental techniques, polarized observables, which require polarized beams and/or targets, began to be used to extract nucleon form factors starting in the early-1990s utilizing theoretical tools developed by Refs. [17-23]. Two new classes of $e N$ scattering experiments, often collectively referred to as double polarization experiments, were now possible where the type depends on the polarization of the incoming and outgoing particles: recoil polarimetry (or polarization transfer) and beam-target asymmetry measurements. The first class, or recoil polarimetry, refers to experiments where the beam is polarized but the target is unpolarized; in this situation, the polarized beam is able transfer polarization to the unpolarized nucleon and results in a polarized recoiling nucleon from which the polarization components may be measured through a secondary analyzing reaction. The second class, or a beam-target asymmetry measure- 
ment, requires both the beam and the target to be polarized; if the polarization of the beam or target is pseudorandomly flipped while keeping the other polarization fixed, then a measurable helicity-dependent asymmetry arises. Both techniques are capable of extracting the form factor ratio $G_{E} / G_{M}$ in a single measurement whereas a Rosenbluth separation requires a minimum of two in order to do a linear fit. In order to fully appreciate the differences, both positive and negative, between the Rosenbluth technique and the double polarization experiments, the form factor experimental landscape must be presented which is delayed until Chapter 3 .

In the context of E02-013, a polarized electron beam quasielastically scatters from a polarized neutron within a ${ }^{3} \mathrm{He}$ target, and the helicity of the initial electron is pseudorandomly flipped between \pm 1 , or parallel and antiparallel with respect to the beam direction, introducing a cross section asymmetry. The form factor ratio $G_{E}^{n} / G_{M}^{n}$ may be extracted from the measured beam-target asymmetry by a comparison to the theoretical form; the goal of this section is to present the theoretical details. The outgoing polarizations are not measured, and a simple summation over final spin states is sufficient. The calculation proceeds in a similar manner as the Rosenbluth formula, but now the leptonic and hadronic tensors will be boosted to the so-called Breit frame where $\mathbf{p}^{\prime}=-\mathbf{p} \Rightarrow \nu=0$ which simplifies the calculation. The validity of this procedure is simple: $\mathcal{M} \propto L_{\mu \nu}^{e} W_{N}^{\mu \nu}$ where $\mathcal{M}$ is a Lorentz invariant; therefore, the product of the leptonic and hadronic tensor is a Lorentz invariant which means that these tensors together may be evaluated in any inertial frame just as long as they are evaluated in the same inertial frame. The expressions needed to perform the transformation from 
the Breit frame to the lab frame, where the interpretation of the Sachs form factors is safe, will be derived prior to the derivation of the beam-target asymmetry.

\subsubsection{Breit Frame Kinematics}

The Breit frame is commonly called the brick wall frame or the infinite momentum frame. The four momentum transfer squared is space-like, i.e. $q^{2}<0$, therefore a boost along $\hat{\boldsymbol{q}}$ such that $\nu=E_{e}-E_{e}^{\prime}=0$ is possible. The result is a frame in which the nucleon initially travels towards the virtual photon with momentum $\mathbf{p}$ and then recoils

with the same momentum magnitude but in reverse, or $\mathbf{p}^{\prime}=-\mathbf{p}$. If $Q^{2}$ is large, then the incoming and outgoing nucleons are relativistic resulting in non-trivial interpretations of observables and kinematics within this frame. The direction of $\hat{z}$ is defined to point along the direction of momentum transfer, and $\hat{\boldsymbol{x}}$ is perpendicular to $\hat{\boldsymbol{z}}$ and in the $e N$ scattering plane as seen in Figure 2.2. The irrelevant $y$-axis is formed by taking the cross product $\hat{\boldsymbol{z}} \times \hat{\boldsymbol{x}}$ which forms a standard right-handed coordinate system. Using this coordinate system, and working out the consequences of $\mathbf{p}^{\prime}=-\mathbf{p}$ :

$$
\begin{aligned}
\nu & =0 \Rightarrow E_{p}=E_{p}^{\prime}, \\
q^{2} & =\left(p^{\prime}-p\right)^{2}=q_{0}^{2}-\mathbf{q}^{2}=-\left(\mathbf{p}^{\prime}-\mathbf{p}\right)^{2}=-4 \mathbf{p}^{2}, \\
Q^{2} & =-q^{2}=4 \mathbf{p}^{2} \Rightarrow \mathbf{p}=-\frac{Q}{2} \hat{\boldsymbol{z}}, \\
E_{p} & =\sqrt{M^{2}+\mathbf{p}^{2}}=M \sqrt{1+\tau},
\end{aligned}
$$

with initial and final nucleon four momenta equal to the following:

$$
p^{\mu}=\left(\begin{array}{cccc}
E_{p} & 0 & 0 & -Q / 2
\end{array}\right), \quad p^{\prime \mu}=\left(\begin{array}{llll}
E_{p} & 0 & 0 & Q / 2
\end{array}\right)
$$




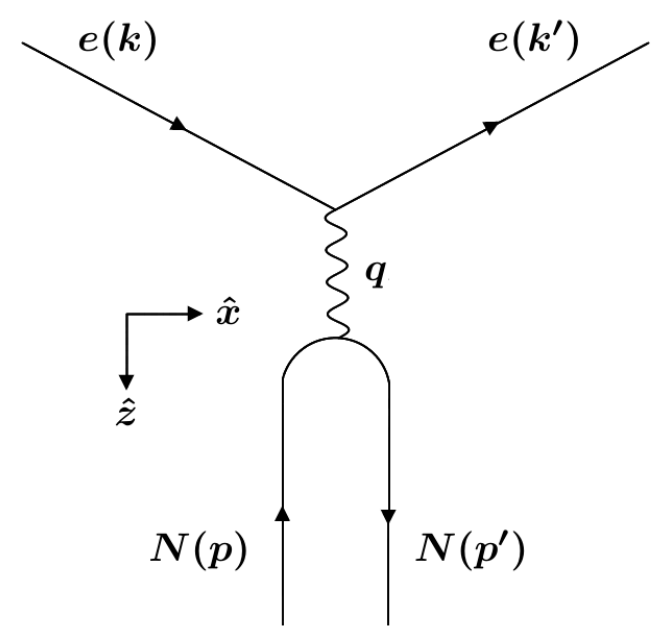

Fig. 2.2: Breit frame diagram displaying the chosen coordinate system.

The initial and final electron four momenta may be generically defined as $k^{\mu}=\left(k_{0}, k_{x}, k_{y}, k_{z}\right)$ and $k^{\prime \mu}=\left(k_{0}^{\prime}, k_{x}^{\prime}, k_{y}^{\prime}, k_{z}^{\prime}\right)$; note that $k_{y}=k_{y}^{\prime}=0$ as scattering is defined to take place in the $x z$-plane. The $z$-components must be equal and opposite, and by conservation of four momentum is $k_{z}=\frac{Q}{2}=-k_{z}^{\prime}$. The $x$-component is non-trivial:

$$
\begin{aligned}
& \cos \theta_{B}=\frac{\mathbf{k} \cdot \mathbf{k}^{\prime}}{|\mathbf{k}|\left|\mathbf{k}^{\prime}\right|}=\frac{k_{x}^{2}-\frac{Q^{2}}{4}}{k_{x}^{2}+\frac{Q^{2}}{4}}, \\
& \Rightarrow k_{x}=\frac{Q}{2} \cot \frac{\theta_{B}}{2},
\end{aligned}
$$

where $\theta_{B}$ corresponds to the electron polar scattering angle in the Breit frame not to be confused with the lab frame polar angle $\theta_{e}$. The energy component of the electron four momenta takes the following form:

$$
k_{0}=k_{0}^{\prime}=\sqrt{\mathbf{k}^{2}+m_{e}^{2}}=\sqrt{k_{x}^{2}+\frac{Q^{2}}{4}}=\frac{Q}{2} \csc \frac{\theta_{B}}{2}
$$


where the mass of the electron is neglected. Therefore, the four momenta of the incoming and outgoing electron may be written as

$$
\begin{aligned}
k^{\mu} & =\left(\begin{array}{llll}
\frac{Q}{2} \csc \frac{\theta_{B}}{2} & \frac{Q}{2} \cot \frac{\theta_{B}}{2} & 0 & Q / 2
\end{array}\right), \\
k^{\prime \mu} & =\left(\begin{array}{llll}
\frac{Q}{2} \csc \frac{\theta_{B}}{2} & \frac{Q}{2} \cot \frac{\theta_{B}}{2} & 0 & -Q / 2
\end{array}\right) .
\end{aligned}
$$

A relationship between the Breit and lab frames is required, and can be acquired by finding a expression that relates $\theta_{e}$ to $\theta_{B}$. The desired relation can be obtained by recalling that the $y$-dimension is irrelevant and the boost occurs solely in the $\hat{\boldsymbol{z}}$-direction. The perpendicular component $k_{x}$ is unaffected and therefore may be exploited to find an expression between the scattering polar angles in both frames. Recalling that the momentum transfer is only in the $\hat{\boldsymbol{z}}$-direction, then an expression for $k_{x}$ is

$$
\begin{aligned}
\mathbf{k} \cdot \mathbf{q} & =k_{x} q_{x}+k_{y} q_{y}+k_{z} q_{z}=k_{z} q_{z}, \\
q_{z} & =|\mathbf{q}| \\
\Rightarrow k_{z} & =\frac{\mathbf{k} \cdot \mathbf{q}}{|\mathbf{q}|}, \\
\mathbf{k}^{2} & =k_{x}^{2}+k_{z}^{2}, \\
\Rightarrow k_{x}^{2} & =\mathbf{k}^{2}-k_{z}^{2}=\frac{\mathbf{k}^{2} \mathbf{q}^{2}-(\mathbf{k} \cdot \mathbf{q})^{2}}{\mathbf{q}^{2}} .
\end{aligned}
$$

Evaluating $k_{x}^{2}$ in the lab frame and equating it to Breit frame expression of $\frac{Q^{2}}{4} \cot ^{2} \frac{\theta_{B}}{2}$ yields the desired relationship between the two frames. Explicitly solving for each factor within Eqn 2.71 in the lab frame results in the following:

$$
\begin{aligned}
& \mathbf{k}^{2}=E_{e}^{2} \\
& \mathbf{q}^{2}=\left(\mathbf{k}-\mathbf{k}^{\prime}\right)^{2}=E_{e}^{2}+E_{e}^{\prime 2}-2 E_{e} E_{e}^{\prime} \cos \theta_{e},
\end{aligned}
$$




$$
\begin{aligned}
q^{2} & =-Q^{2}=\nu^{2}-\mathbf{q}^{2}=-4 E_{e} E_{e}^{\prime} \sin ^{2} \frac{\theta_{e}}{2} \\
\mathbf{q}^{2} & =Q^{2}+\nu^{2}=Q^{2}+\frac{Q^{4}}{4 M^{2}}=Q^{2}(1+\tau) \\
\mathbf{k} \cdot \mathbf{q} & =E_{e}^{2}-E_{e} E_{e}^{\prime} \cos \theta_{e} \\
\mathbf{k}^{2} \mathbf{q}^{2}-(\mathbf{k} \cdot \mathbf{q})^{2} & =E_{e}^{2} Q^{2}+E_{e}^{2}\left(E_{e}-E_{e}^{\prime}\right)^{2}-\left(E_{e}^{2}-E_{e} E_{e}^{\prime} \cos \theta_{e}\right)^{2} \\
& =E_{e}^{2} E_{e}^{\prime 2} \sin ^{2} \theta_{e} \\
& =4 E_{e}^{2} E_{e}^{\prime 2} \sin ^{2} \frac{\theta_{e}}{2} \cos ^{2} \frac{\theta_{e}}{2} \\
& =\frac{Q^{4}}{4} \cot ^{2} \frac{\theta_{e}}{2}, \\
\Rightarrow k_{x}^{2} & =\frac{\mathbf{k}^{2} \mathbf{q}^{2}-(\mathbf{k} \cdot \mathbf{q})^{2}}{\mathbf{q}^{2}}=\frac{Q^{2}}{4(1+\tau)} \cot ^{2} \frac{\theta_{e}}{2}
\end{aligned}
$$

The relationship between frames may now be realized and conveniently may be expressed in multiple forms for a variety of needs:

$$
\begin{aligned}
\cot ^{2} \frac{\theta_{B}}{2} & =\frac{\cot ^{2} \frac{\theta_{e}}{2}}{1+\tau}, \\
\Rightarrow \cot \frac{\theta_{B}}{2} & =\frac{\cot \frac{\theta_{e}}{2}}{\sqrt{1+\tau}}, \\
\Rightarrow \csc \frac{\theta_{B}}{2} & =\frac{\cot \frac{\theta_{e}}{2}}{\sqrt{1+\tau}} \sqrt{1+(1+\tau) \tan ^{2} \frac{\theta_{e}}{2}} .
\end{aligned}
$$

It is useful to rewrite Eqn 2.73 as

$$
\frac{\cos ^{2} \frac{\theta_{B}}{2}}{1-\cos ^{2} \frac{\theta_{B}}{2}}=\frac{\cot ^{2} \frac{\theta_{e}}{2}}{1+\tau} \Rightarrow \frac{1}{\cos ^{2} \frac{\theta_{B}}{2}}=\tan ^{2} \frac{\theta_{e}}{2}\left(\csc ^{2} \frac{\theta_{e}}{2}+\tau\right) .
$$

The last expression may be simplified by rearranging the lab frame $E_{e}^{\prime}$ formula Eqn 2.23 .

$$
\begin{aligned}
\frac{E_{e}}{E_{e}^{\prime}}=1+\frac{2 E_{e}}{M} \sin ^{2} \frac{\theta_{e}}{2} \Rightarrow \csc ^{2} \frac{\theta_{e}}{2}=\frac{2 E_{e}}{M} \frac{E_{e}^{\prime}}{E_{e}-E_{e}^{\prime}}, \\
\csc ^{2} \frac{\theta_{e}}{2}+\tau=\frac{2 E_{e}}{M} \frac{E_{e}^{\prime}}{E_{e}-E_{e}^{\prime}}+\frac{E_{e}-E_{e}^{\prime}}{2 M}=\frac{\left(E_{e}+E_{e}^{\prime}\right)^{2}}{Q^{2}}, \\
\Rightarrow \frac{1}{\cos \frac{\theta_{B}}{2}}=\frac{E_{e}+E_{e}^{\prime}}{Q} \tan \frac{\theta_{e}}{2},
\end{aligned}
$$


where the last line is a useful form for the recoil polarimetry derivation explicitly done in Ref. 24 for example. The kinematics of the Breit frame have been introduced, and the necessary relations to transform between reference frames are available for use.

There is one last piece that needs to be addressed: the nucleon current $\mathcal{J}_{N}^{\mu}$ evaluated in the Breit frame. The vertex correction of the nucleon is

$$
\Gamma^{\mu}=\gamma^{\mu}\left(F_{1}+F_{2}\right)-\frac{\left(p+p^{\prime}\right)^{\mu}}{2 M} F_{2}=G_{M} \gamma^{\mu}+\frac{G_{E}-G_{M}}{2 M(1+\tau)}\left(p+p^{\prime}\right)^{\mu}
$$

which has been expressed in terms of the Sachs form factors. Sandwiching the vertex correction $\Gamma^{\mu}$ in between Dirac spinors gives the nucleon current from which the hadronic tensor is formed by squaring, or

$$
\begin{gathered}
\mathcal{J}_{N}^{\mu}=\bar{u}\left(p^{\prime}\right) \Gamma^{\mu} u(p)=\bar{u}\left(p^{\prime}\right) \\
\left(G_{M} \gamma^{\mu}+\frac{G_{E}-G_{M}}{2 M(1+\tau)}\left(p+p^{\prime}\right)^{\mu}\right) u(p), \\
\Rightarrow W_{N}^{\mu \nu}=\mathcal{J}_{N}^{\mu} \mathcal{J}_{N}^{\nu *}
\end{gathered}
$$

In order to transform $\mathcal{J}_{N}^{\mu}$ to the Breit frame, the explicit Dirac free particle spinors defined in Appendix A.2 need to be evaluated in the Breit frame as well. The spinors for the incoming and outgoing nucleons have the following forms, respectively,

$$
\begin{aligned}
& u(p)=\sqrt{E_{p}+M}\left(\begin{array}{c}
\chi \\
\frac{\boldsymbol{\sigma} \cdot \mathbf{p}}{E_{p}+M} \chi
\end{array}\right), \\
& \bar{u}\left(p^{\prime}\right)=u^{\dagger}\left(p^{\prime}\right) \gamma^{0}=\sqrt{E_{p}^{\prime}+M}\left(\chi^{\prime \dagger}, \quad \chi^{\prime \dagger} \frac{\boldsymbol{\sigma} \cdot \mathbf{p}^{\prime}}{E_{p}^{\prime}+M}\right) \gamma^{0},
\end{aligned}
$$

where $\boldsymbol{\sigma}$ is meant to be interpreted as a vector, i.e. $\mathbf{p} \cdot \boldsymbol{\sigma}=p_{x} \sigma_{1}+p_{y} \sigma_{2}+p_{z} \sigma_{3}$ which utilizes the standard $2 \times 2$ Pauli matrices for a spin- $\frac{1}{2}$ particle. Note that $\left(\boldsymbol{\sigma} \cdot \mathbf{p}^{\prime}\right)^{\dagger}=$ $\boldsymbol{\sigma} \cdot \mathbf{p}^{\prime}$ is a useful relation. Recalling some of the Breit frame relations yields significant 
simplifications:

$$
\begin{aligned}
E_{p} & =E_{p}^{\prime}=M \sqrt{1+\tau} \\
\mathbf{p} & =-\mathbf{p}^{\prime} \\
\left(p+p^{\prime}\right)^{\mu} & =\left(\begin{array}{llll}
2 M \sqrt{1+\tau} & 0 & 0 & 0
\end{array}\right)^{T} .
\end{aligned}
$$

By examining Eqn 2.79, there are two types of spinor products required: $\bar{u}\left(p^{\prime}\right) u(p)$ and $\bar{u}\left(p^{\prime}\right) \gamma^{\mu} u(p)$. Calculating the former product:

$$
\begin{aligned}
\bar{u}\left(p^{\prime}\right) u(p) & =\left(E_{p}+M\right)\left(\chi^{\prime \dagger},-\chi^{\prime \dagger} \frac{\boldsymbol{\sigma} \cdot \mathbf{p}}{E_{p}+M}\right)\left(\begin{array}{cc}
1 & 0 \\
0 & -1
\end{array}\right)\left(\begin{array}{c}
\chi \\
\frac{\boldsymbol{\sigma} \cdot \mathbf{p}}{E_{p}+M} \chi
\end{array}\right) \\
& =\left(E_{p}+M\right)\left(\chi^{\prime \dagger} \chi+\chi^{\prime \dagger} \chi \frac{\left(E_{p}-M\right)\left(E_{p}+M\right)}{\left(E_{p}+M\right)^{2}}\right) \\
& =2 E_{p} \chi^{\prime \dagger} \chi
\end{aligned}
$$

where the identity $(\boldsymbol{\sigma} \cdot \mathbf{a})(\boldsymbol{\sigma} \cdot \mathbf{b})=\mathbf{a} \cdot \mathbf{b}+i \boldsymbol{\sigma} \cdot(\mathbf{a} \times \mathbf{b})$ has been used in the second line, or more explicitly $(\boldsymbol{\sigma} \cdot \mathbf{p})(\boldsymbol{\sigma} \cdot \mathbf{p})=p^{2}=E_{p}^{2}-M^{2}$. The second product containing $\gamma^{\mu}$ is easier to handle by dealing with the time and space components separately. The time component $(\mu=0)$ is

$$
\begin{aligned}
\bar{u}\left(p^{\prime}\right) \gamma^{0} u(p) & =u^{\dagger}\left(p^{\prime}\right) u(p) \\
& =\left(E_{p}+M\right)\left(\chi^{\prime \dagger},-\chi^{\prime \dagger} \frac{\boldsymbol{\sigma} \cdot \mathbf{p}}{E_{p}+M}\right)\left(\begin{array}{c}
\chi \\
\frac{\boldsymbol{\sigma} \cdot \mathbf{p}}{E_{p}+M} \chi
\end{array}\right), \\
& =\left(E_{p}+M\right) \chi^{\prime \dagger} \chi\left(1-\frac{p^{2}}{\left(E_{p}+M\right)^{2}}\right), \\
& =2 M \chi^{\prime \dagger} \chi,
\end{aligned}
$$


where the same techniques from the first product have been implemented. The spatial components are $(k \neq 0)$

$$
\begin{aligned}
\bar{u}\left(p^{\prime}\right) \gamma^{k} u(p) & =\left(E_{p}+M\right)\left(\chi^{\prime \dagger}, \quad-\chi^{\prime \dagger} \frac{\boldsymbol{\sigma} \cdot \mathbf{p}}{E_{p}+M}\right)\left(\begin{array}{cc}
0 & \sigma^{k} \\
\sigma^{k} & 0
\end{array}\right)\left(\begin{array}{c}
\chi \\
\frac{\boldsymbol{\sigma} \cdot \mathbf{p}}{E_{p}+M}
\end{array}\right) \\
& =\left(E_{p}+M\right)\left(\chi^{\prime \dagger}, \quad-\chi^{\prime \dagger} \frac{\boldsymbol{\sigma} \cdot \mathbf{p}}{E_{p}+M}\right)\left(\begin{array}{c}
\sigma^{k} \frac{\boldsymbol{\sigma} \cdot \mathbf{p}}{E_{p}+M} \chi \\
\sigma^{k} \chi
\end{array}\right) \\
& =\chi^{\prime \dagger}\left(\sigma^{k} \boldsymbol{\sigma} \cdot \mathbf{p}-\boldsymbol{\sigma} \cdot \mathbf{p} \sigma^{k}\right) \chi \\
& =\chi^{\prime \dagger}\left[\sigma^{k}, \sigma^{i} p^{i}\right] \chi \\
& =\chi^{\prime \dagger}\left(2 i \epsilon^{k i j} p^{i} \sigma^{j}\right) \chi \\
& =2 i \chi^{\prime \dagger}(\mathbf{p} \times \boldsymbol{\sigma}) \chi .
\end{aligned}
$$

The calculations Eqn 2.86-Eqn 2.88 may be substituted into the nucleon current density Eqn 2.79. Considering the time and space components of the nucleon current $\mathcal{J}_{N}^{\mu}$ separately, then the final form of the nucleon current in the Breit frame is

$$
\mathcal{J}_{N}^{\mu}=\left(\begin{array}{c}
\mathcal{J}_{N}^{0} \\
\overrightarrow{\mathcal{J}}_{N}
\end{array}\right)=\left(\begin{array}{c}
2 M G_{E} \chi^{\prime \dagger} \chi \\
2 i G_{M} \chi^{\prime \dagger}(\mathbf{p} \times \boldsymbol{\sigma}) \chi
\end{array}\right)=\left(\begin{array}{c}
2 M G_{E} \chi^{\prime \dagger} \chi \\
i Q G_{M} \chi^{\prime \dagger} \sigma_{y} \chi \\
-i Q G_{M} \chi^{\prime \dagger} \sigma_{x} \chi \\
0
\end{array}\right) .
$$

It is noted that $\mathcal{J}_{N}^{0} \propto G_{E}$ and can be physically thought of as the nucleon charge density $\rho$ within the Breit Frame. The other components are proportional to the magnetic form factor, or $\overrightarrow{\mathcal{J}}_{N} \propto G_{M}$, which may be compared to the nucleon current density. For these reasons, the Sachs form factors $G_{E}$ and $G_{M}$ are associated to the electric and magnetic distributions of the nucleon. 


\subsubsection{Beam-Target Asymmetry}

In this section, the beam-target helicity asymmetry, namely the formula that serves as the basis of this thesis and E02-013, is derived in the lab frame. The procedure starts by using the Breit frame nucleon current (Eqn 2.89) and its adjoint in order to calculate the hadronic tensor $W_{N}^{\mu \nu}$. The initial nucleon is polarized with completeness relation equal to $\sum_{s} \chi^{(s)} \chi^{(s) \dagger}=\frac{1}{2}[1+\boldsymbol{\sigma} \cdot \mathbf{P}]$ where $\mathbf{P}$ is the nucleon polarization vector. The outgoing nucleon spin states are simply summed as the experiment does not measure the polarization of the nucleon, i.e. $\sum_{s^{\prime}} \chi^{\prime \dagger} \chi^{\prime}=2$ where the spin index is assumed. The leptonic tensor is calculated by allowing the initial electron to be polarized and summing over outgoing electron spin states. And finally, the invariant amplitude squared is calculated by contracting the hadronic tensor with the leptonic tensor.

The adjoint of Eqn 2.89 is

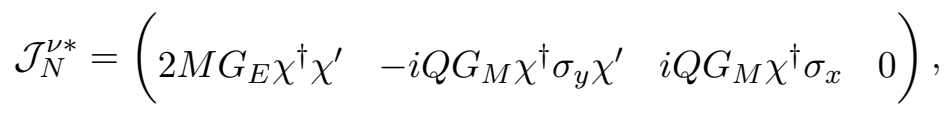

and the hadronic tensor may be calculated as $W_{N}^{\mu \nu}=\mathcal{J}_{N}^{\mu} \mathcal{J}_{N}^{\nu *}$. In order to conserve space, only one element is going to be explicitly calculated, and then the tensor result will simply be stated. For example, the element $\mu \nu=01$ may be calculated as follows:

$$
\begin{aligned}
W_{N}^{01} & =-2 i M Q G_{E} G_{M} \sum_{s, s^{\prime}}\left(\chi^{\prime \dagger} \chi \chi^{\dagger} \sigma_{y} \chi^{\prime}\right), \\
& =-i M Q G_{E} G_{M} \sum_{s^{\prime}} \chi^{\prime \dagger}(1+\boldsymbol{\sigma} \cdot \mathbf{P}) \sigma_{y} \chi^{\prime}, \\
& =-i M Q G_{E} G_{M} \sum_{s^{\prime}} \chi^{\prime \dagger}(\boldsymbol{\sigma} \cdot \mathbf{P}) \sigma_{y} \chi^{\prime},
\end{aligned}
$$




$$
\begin{aligned}
& =-i M Q G_{E} G_{M} \sum_{s^{\prime}} \chi^{\prime \dagger}\left(\begin{array}{cc}
P_{y}+i P_{x} & -P_{z} \\
-i P_{z} & P_{y}-i P_{x}
\end{array}\right) \chi^{\prime}, \\
& =-i M Q G_{E} G_{M} \operatorname{Tr}\left(\begin{array}{cc}
P_{y}+i P_{x} & -P_{z} \\
-i P_{z} & P_{y}-i P_{x}
\end{array}\right), \\
& =-2 i Q M G_{E} G_{M} P_{y} .
\end{aligned}
$$

The other elements may be calculated using a similar technique, and the result is

$$
W_{N}^{\mu \nu}=\left(\begin{array}{cccc}
4 M^{2} G_{E}^{2} & -2 i M Q G_{E} G_{M} P_{y} & 2 i M Q G_{E} G_{M} P_{x} & 0 \\
2 i M Q G_{E} G_{M} P_{y} & Q^{2} G_{M}^{2} & -i Q^{2} G_{M}^{2} P_{z} & 0 \\
-2 i M Q G_{E} G_{M} P_{x} & i Q^{2} G_{M}^{2} P_{z} & Q^{2} G_{M}^{2} & 0 \\
0 & 0 & 0 & 0
\end{array}\right)
$$

The diagonal elements are real, spin-independent and proportional to the form factors squared. The off-diagonal elements are imaginary and depend on components of the polarization vector $\mathbf{P}$; in other words, the information regarding the polarization of the nucleon during the scattering process is contained in these elements. Note that $W_{N}^{\mu \nu}$ may be separated into a sum of a symmetric and an antisymmetric tensor, or

$$
W_{N}^{\mu \nu}=W_{N, S}^{\mu \nu}+W_{N, A}^{\mu \nu},
$$

which effectively separates the unpolarized components from the polarized components, respectively.

The leptonic tensor may be calculated utilizing a similar method, i.e. explicitly using spinors with the polarized and unpolarized completeness relations found in Eqn A.2. However, there is another method which is valid for massless fermions. Recall 
that the incoming electron is polarized, and a simple spin summation is going to be performed on the outgoing electron spin states as the experiment has no sensitivity to this observable. Reusing the electronic tensor Eqn 2.10, and inserting the so-called projection operator in order to isolate a particular spin component of the electron spinors results in the following:

$$
\begin{aligned}
L_{\mu \nu}^{e} & =\bar{u}\left(k^{\prime}\right) \gamma_{\mu} u(k) \bar{u}(k) \gamma_{\nu} u\left(k^{\prime}\right), \\
\Rightarrow & \bar{u}\left(k^{\prime}\right) \gamma_{\mu}\left(\frac{1-h_{e} \gamma^{5}}{2}\right) u(k) \bar{u}(k) \gamma_{\nu} u\left(k^{\prime}\right), \\
& =\frac{1}{2} \operatorname{Tr}\left[\not k^{\prime} \gamma_{\mu}\left(1-h_{e} \gamma^{5}\right) \not k \gamma_{\nu}\right], \\
& =\frac{1}{2}\left(\operatorname{Tr}\left[\not k^{\prime} \gamma_{\mu} \not k \gamma_{\nu}\right]-h_{e} \operatorname{Tr}\left[\not k^{\prime} \gamma_{\mu} \gamma^{5} \not k \gamma_{\nu}\right]\right),
\end{aligned}
$$

where $h_{e}$ represents the helicity state of the electron, either + or - , and $\gamma^{5}=i \gamma^{0} \gamma^{1} \gamma^{2} \gamma^{3}$. The use of the projection operator is justified in the high-energy limit, or equivalently in the limit where the mass of the electron is assumed to be zero. If this is true, then the basis of polarization states may be chosen to be oriented along the direction of propagation, or $\pm \hat{\boldsymbol{k}}$, resulting in a right-handed or left-handed spinor. Therefore, the helicity of the electron points longitudinally with respect to the direction of propagation. The first term is identical to the unpolarized leptonic tensor in the massless limit, and therefore the results are simply reused. The second term contains the polarization information and evaluates to

$$
\begin{aligned}
\operatorname{Tr}\left[\not k^{\prime} \gamma_{\mu} \gamma^{5} k \gamma_{\nu}\right] & =k^{\alpha} k^{\prime \lambda} \operatorname{Tr}\left[\gamma_{\lambda} \gamma_{\mu} \gamma^{5} \gamma_{\alpha} \gamma_{\nu}\right] \\
& =-4 i \epsilon_{\mu \nu \alpha \lambda} k^{\alpha} k^{\prime \lambda},
\end{aligned}
$$

where the last line uses trace identity Eqn B.6 and $\epsilon_{\mu \nu \alpha \lambda}$ is the totally antisymmetric 
Levi-Civita tensor. Combining results gives the form of the leptonic tensor as

$$
L_{\mu \nu}^{e}=2\left(k^{\prime \mu} k^{\nu}+k^{\prime \nu} k^{\mu}-g^{\mu \nu}\left(k \cdot k^{\prime}\right)+i h_{e} \epsilon_{\mu \nu \alpha \lambda} k^{\alpha} k^{\prime \lambda}\right)=L_{\mu \nu}^{e, S}+L_{\mu \nu}^{e, A},
$$

where again the tensor may be separated into a sum of a symmetric tensor and an antisymmetric tensor, effectively compartmentalizing the unpolarized components from the polarized components. Each component may now be computed in the Breit frame, and in an effort to conserve space only two components, one from the symmetric part and one from the antisymmetric part, are going to be explicitly shown. Recall that the Breit frame electron four momenta are

$$
\begin{aligned}
k^{\mu} & =\frac{Q}{2}\left(\begin{array}{llll}
\csc \frac{\theta_{B}}{2} & \cot \frac{\theta_{B}}{2} & 0 & 1
\end{array}\right)^{T}, \\
k^{\prime \mu} & =\frac{Q}{2}\left(\begin{array}{llll}
\csc \frac{\theta_{B}}{2} & \cot \frac{\theta_{B}}{2} & 0 & -1
\end{array}\right)^{T},
\end{aligned}
$$

and explicitly calculating an element from the symmetric and antisymmetric part results in the following:

$$
\begin{aligned}
L_{\mu \nu}^{e, S} & =2\left[k^{\mu} k^{\prime \nu}+k^{\prime \mu} k^{\nu}-g^{\mu \nu}\left(k \cdot k^{\prime}\right)\right], \\
& =2\left(k^{\mu} k^{\prime \nu}+k^{\prime \mu} k^{\nu}-Q^{2} g^{\mu \nu}\right), \\
\Rightarrow L_{01}^{e, S} & =Q^{2} \csc \frac{\theta_{B}}{2} \cot \frac{\theta_{B}}{2} \\
L_{\mu \nu}^{e, A} & =2 i h_{e} \epsilon_{\mu \nu \alpha \lambda} k^{\alpha} k^{\prime \lambda} \\
\Rightarrow L_{02}^{e, A} & =2 i h_{e} \epsilon_{0213} k^{1} k^{\prime 3} \\
& =2 i h_{e}\left(k^{1} k^{\prime 3}-k^{3} k^{\prime 1}\right), \\
& =2 i h_{e}\left(-\frac{Q^{2}}{2} \cot \frac{\theta_{B}}{2}\right), \\
& =-i Q^{2} h_{e} \cot \frac{\theta_{B}}{2} .
\end{aligned}
$$


Evaluating each element in this manner gives the following tensor calculated in the Breit frame, or

$$
L_{\mu \nu}^{e}=Q^{2}\left(\begin{array}{cccc}
\cot ^{2} \frac{\theta_{B}}{2} & \csc \frac{\theta_{B}}{2} \cot \frac{\theta_{B}}{2} & i h_{e} \cot \frac{\theta_{B}}{2} & 0 \\
\csc \frac{\theta_{B}}{2} \cot \frac{\theta_{B}}{2} & \csc ^{2} \frac{\theta_{B}}{2} & -i h_{e} \csc \frac{\theta_{B}}{2} & 0 \\
-i h_{e} \cot \frac{\theta_{B}}{2} & i h_{e} \csc \frac{\theta_{B}}{2} & 1 & 0 \\
0 & 0 & 0 & 0
\end{array}\right) .
$$

The symmetric and antisymmetric parts of the leptonic tensor are explicitly realized. Note that the totally imaginary antisymmetric components vanish when summed, and this must be the case as the polarized terms do not show up in the unpolarized calculation; the polarized terms are only revealed once a particle is put into a particular spin state. The required tensors have both been calculated in the Breit frame, and the invariant amplitude squared may be calculated:

$$
\begin{aligned}
\overline{|\mathcal{M}|^{2}} & =\frac{e^{4}}{q^{4}} L_{\mu \nu}^{e} W_{N}^{\mu \nu}, \\
& =\frac{e^{4}}{q^{4}}\left(L_{\mu \nu}^{e, S}+L_{\mu \nu}^{e, A}\right)\left(W_{N, S}^{\mu \nu}+W_{N, A}^{\mu \nu}\right), \\
& =\frac{e^{4}}{q^{4}}\left(L_{\mu \nu}^{e, S} W_{N, S}^{\mu \nu}+L_{\mu \nu}^{e, A} W_{N, A}^{\mu \nu}\right),
\end{aligned}
$$

where the last line is a useful consequence of separating the two tensors into symmetric and antisymmetric parts, namely the contraction of a symmetric with an antisymmetric tensor always vanishes. The symmetric contraction gives

$$
\begin{aligned}
\overline{|\mathcal{M}|^{2}}{ }_{S} & =\frac{e^{4}}{q^{4}} L_{\mu \nu}^{e, S} W_{N, S}^{\mu \nu}, \\
& =\frac{e^{4}}{q^{4}}\left\{4 M^{2} Q^{2} G_{E}^{2} \cot ^{2} \frac{\theta_{B}}{2}+Q^{4} G_{M}^{2}\left(\csc ^{2} \frac{\theta_{B}}{2}+1\right)\right\}, \\
& =\frac{4 e^{4} M^{2} Q^{2}}{q^{4}}\left[\left(G_{E}^{2}+\tau G_{M}^{2}\right) \cot ^{2} \frac{\theta_{B}}{2}+2 \tau G_{M}^{2}\right] .
\end{aligned}
$$


Substituting in Eqn 2.73 transforms the result to the lab frame, and the familiar form for unpolarized scattering is recovered:

$$
\begin{aligned}
\overline{|\mathcal{M}|^{2}} & =\frac{4 e^{4} M^{2} Q^{2}}{q^{4}}\left[\frac{G_{E}^{2}+\tau G_{M}^{2}}{1+\tau} \cot ^{2} \frac{\theta_{e}}{2}+2 \tau G_{M}^{2}\right], \\
& =\frac{4 e^{4} M^{2} Q^{2}}{q^{4}} \cot ^{2} \frac{\theta_{e}}{2}\left(\frac{\epsilon G_{E}^{2}+\tau G_{M}^{2}}{\epsilon(1+\tau)}\right) .
\end{aligned}
$$

The antisymmetric contraction in the Breit frame gives

$$
\begin{aligned}
\overline{|\mathcal{M}|^{2}} & =\frac{e^{4}}{q^{4}} L_{\mu \nu}^{e, A} W_{N, A}^{\mu \nu} \\
& =-h_{e} \frac{e^{4}}{q^{4}}\left[2 Q^{4} G_{M}^{2} P_{z} \csc \frac{\theta_{B}}{2}+4 M Q^{3} G_{E} G_{M} P_{x} \cot \frac{\theta_{B}}{2}\right]
\end{aligned}
$$

where recall that $h_{e}$ and $\mathbf{P}$ refer to the helicity and polarization of the initial electron and nucleon, respectively. The expressions needed to transform from the Breit frame to the lab frame are Eqn 2.74 and Eqn 2.75, and written down again for convenience:

$$
\cot \frac{\theta_{B}}{2}=\frac{\cot \frac{\theta_{e}}{2}}{\sqrt{1+\tau}}, \quad \csc \frac{\theta_{B}}{2}=\frac{\cot \frac{\theta_{e}}{2}}{\sqrt{1+\tau}} \sqrt{1+(1+\tau) \tan ^{2} \frac{\theta_{e}}{2}} .
$$

In the Born approximation, the invariant amplitude squared and hence the cross section may be written as a sum of two terms: an unpolarized part (symmetric) and a polarized part (antisymmetric), often denoted by $\Sigma$ and $\Delta$, respectively, within the literature. The helicity-dependent cross section may then be written as

$$
\sigma_{h_{e}}=\Sigma+h_{e} \Delta
$$

The physical asymmetry, namely the observable of interest which will eventually be compared to the experimental data in order to extract the form factor ratio of the neutron, is defined as

$$
A_{\mathrm{phys}}=\frac{\sigma_{+}-\sigma_{-}}{\sigma_{+}+\sigma_{-}}=\frac{\Delta}{\Sigma}=\frac{1}{h_{e}} \frac{\overline{|\mathcal{M}|^{2}} A}{\overline{|\mathcal{M}|^{2}}}
$$


where $\sigma_{+}$and $\sigma_{-}$are the cross sections for the two beam helicity states. The physical asymmetry may be calculated by taking the ratio of the polarized invariant amplitude squared to the unpolarized invariant amplitude squared, both evaluated in the lab frame:

$$
\begin{aligned}
A_{\text {phys }} & =-\frac{2 Q^{3}\left[Q G_{M}^{2} P_{z} \csc \frac{\theta_{B}}{2}+2 M G_{E} G_{M} P_{x} \cot \frac{\theta_{B}}{2}\right]}{4 M^{2} Q^{2} \cot ^{2} \frac{\theta_{e}}{2}\left(\frac{\epsilon G_{E}^{2}+\tau G_{M}^{2}}{\epsilon(1+\tau)}\right)}, \\
& =-\frac{Q(1+\tau)}{2 M^{2} \cot ^{2} \frac{\theta_{e}}{2} I_{0}}\left\{Q \sqrt{1+(1+\tau) \tan ^{2} \frac{\theta_{e}}{2}} G_{M}^{2} P_{z}+2 M G_{E} G_{M} \frac{\cot \frac{\theta_{e}}{2}}{\sqrt{1+\tau}} P_{x}\right\}, \\
& =-\frac{Q \sqrt{1+\tau \tan \frac{\theta_{e}}{2}}}{2 M^{2} I_{0}}\left\{Q \sqrt{1+(1+\tau) \tan ^{2} \frac{\theta_{e}}{2}} G_{M}^{2} P_{z}+2 M G_{E} G_{M} P_{x}\right\}, \\
& =-\frac{2 \sqrt{\tau(1+\tau)} \tan \frac{\theta_{e}}{2}}{I_{0}}\left\{\sqrt{\tau\left[1+(1+\tau) \tan ^{2} \frac{\theta_{e}}{2}\right]} G_{M}^{2} P_{z}+G_{E} G_{M} P_{x}\right\},
\end{aligned}
$$

where $I_{0} \equiv G_{E}^{2}+\frac{\tau}{\epsilon} G_{M}^{2}$.

In order to equate the theoretical physical asymmetry to a measured asymmetry, experimental realities need to be taken into account to scale the calculation with ideal assumptions:

$$
A_{\exp }=h_{e} P_{e} P_{n} D A_{\mathrm{phys}},
$$

where the helicity of the electron can change the sign of the measured asymmetry. Additionally, $P_{e}$ and $P_{n}$ represent the degree of polarization of the electron and nucleon, respectively, and may range from $[0,1]$ where 1 represents a perfect polarization of $100 \%$. The polarization of the beam and target are inevitably going to be less than $100 \%$, and the measured experimental asymmetry needs to be scaled by a factor equal to the product of the experimental polarizations. Furthermore, events other than quasielastic scattering dilute or contaminate the signal of interest; these corrections are all contained within the dilution factor $D$, which also takes a value of $[0,1]$. A large portion of the 


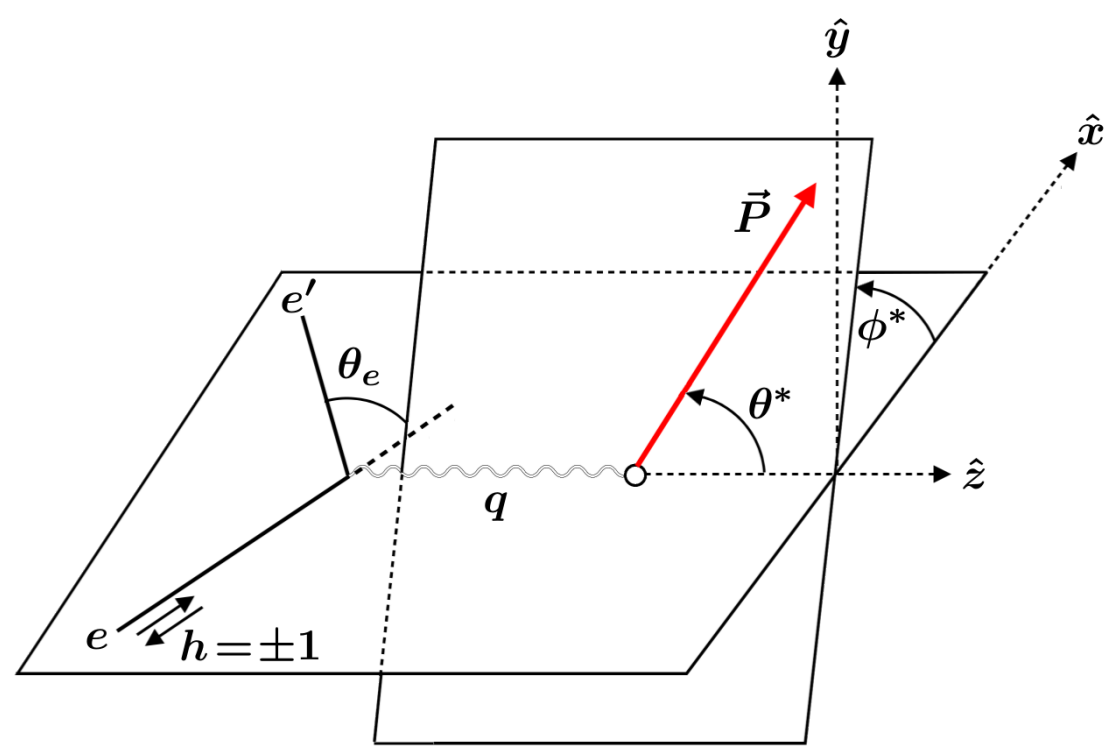

Fig. 2.3: Beam-target asymmetry diagram displaying the definitions of $\mathbf{P}, \theta^{*}$ and $\phi^{*}$. The incoming electron is longitudinally polarized with a helicity that is pseudorandomly flipped and scatters off of a polarized $N$. Use permitted by [25].

analysis is dedicated towards identifying and estimating various contributions to $D$. In order to finalize the expression for the physical asymmetry, the components of the nucleon polarization $\mathbf{P}$ in spherical coordinates are defined as

$$
P_{x}=\sin \theta^{*} \cos \phi^{*}, \quad P_{z}=\cos \theta^{*},
$$

where $\theta^{*}$ and $\phi^{*}$ are the laboratory polar and azimuthal angles of the target polarization relative to the momentum transfer $\mathbf{q}$, and are defined in Figure 2.3 .

The physical asymmetry $A_{\text {phys }}$ is particularly useful in extracting the Sachs form factor ratio under certain experimental conditions, namely if $\theta^{*}=\pi / 2$ and $\phi^{*}=0$ or $\pi$, which corresponds to the situation where the target polarization is perpendicular to 
q and within the reaction plane. In this case, the $G_{M}^{2}$ term vanishes, and the physical asymmetry, often referred to as the perpendicular or transverse asymmetry, reduces to

$$
\begin{aligned}
A_{\perp} & =-\frac{2 \sqrt{\tau(1+\tau)} \tan \frac{\theta_{e}}{2}}{G_{E}^{2}+\frac{\tau}{\epsilon} G_{M}^{2}} G_{E} G_{M}, \\
& =-\frac{2 \sqrt{\tau(1+\tau)} \tan \frac{\theta_{e}}{2} \frac{G_{E}}{G_{M}}}{\left(\frac{G_{E}}{G_{M}}\right)^{2}+\frac{\tau}{\epsilon}} \\
& =-\frac{\epsilon}{\tau} \frac{2 \sqrt{\tau(1+\tau)} \tan \frac{\theta_{e}}{2} \frac{G_{E}}{G_{M}}}{1+\frac{\epsilon}{\tau}\left(\frac{G_{E}}{G_{M}}\right)^{2}} \\
& =-2 \epsilon \sqrt{\frac{1+\tau}{\tau}} \tan \frac{\theta_{e}}{2} \frac{G_{E}}{G_{M}}\left[1-\frac{\epsilon}{\tau}\left(\frac{G_{E}}{G_{M}}\right)^{2}+\mathcal{O}\left(\frac{G_{E}}{G_{M}}\right)^{4}+\ldots\right], \\
& \approx-2 \epsilon \sqrt{\frac{1+\tau}{\tau}} \tan \frac{\theta_{e}}{2}\left(\frac{G_{E}}{G_{M}}\right),
\end{aligned}
$$

where $\left(\frac{G_{E}}{G_{M}}\right)^{2}$ is assumed to be small for the neutron. The value of $\epsilon / \tau$ is of order 2 for the kinematics relevant to this thesis, i.e. $Q^{2}=1.16 \mathrm{GeV}^{2}$ and $\theta_{e}=56.26^{\circ} \Rightarrow \tau \approx 0.3$ and $\epsilon \approx 0.6$, which further justifies the expansion choice. At leading order, the form factor ratio $G_{E} / G_{M}$ is proportional to the perpendicular component of the physical asymmetry which may be exploited by a carefully designed experiment, namely orienting the target polarization such that $P_{z}=0$. This is the theoretical basis of E02-013 in which the form factor ratio of the neutron $G_{E}^{n} / G_{M}^{n}$ was extracted by measuring the beam-target asymmetry at four kinematic points. The magnetic form factor of the neutron $G_{M}^{n}$ is known with relatively high precision up to a $Q^{2}$ well-beyond E02-013; therefore, the form factor $G_{E}^{n}$ may be separated from the ratio.

In practice, though, an experiment can never achieve a vanishing $P_{z}$ component due to the finite-sized nature of experimental detectors and target magnetic field imperfections. The remaining contribution to $A_{\text {phys }}$, often referred to as the parallel or 
longitudinal component, may be isolated if $\theta^{*}=0$ or $\pi$ which corresponds to a situation where the target is polarized parallel or antiparallel relative to the momentum transfer:

$$
\begin{aligned}
A_{\|} & =-\frac{2 \sqrt{\tau(1+\tau)} \tan \frac{\theta_{e}}{2}}{G_{E}^{2}+\frac{\tau}{\epsilon} G_{M}^{2}} \sqrt{\tau\left[1+(1+\tau) \tan ^{2} \frac{\theta_{e}}{2}\right]} G_{M}^{2}, \\
& =-\frac{2 \epsilon \sqrt{1+\tau+(1+\tau)^{2} \tan ^{2} \frac{\theta_{e}}{2}} \tan \frac{\theta_{e}}{2}}{1+\frac{\epsilon}{\tau}\left(\frac{G_{E}}{G_{M}}\right)^{2}} \\
& \approx-2 \epsilon \sqrt{1+\tau+(1+\tau)^{2} \tan ^{2} \frac{\theta_{e}}{2}} \tan \frac{\theta_{e}}{2}
\end{aligned}
$$

where $\left(\frac{G_{E}}{G_{M}}\right)^{2}$ is assumed to be small. At leading order, the parallel component of the physical asymmetry only depends on the kinematics of the experiment.

In addition to measuring $A_{\perp}$ for a form factor ratio extraction, another common strategy is to measure both $A_{\perp}$ and $A_{\|}$in order to take the ratio. A measurement of $A_{\perp} / A_{\|}$is advantageous as absolute polarization determinations are unnecessary which significantly reduces systematics. For example, the uncertainty in the beam and target polarization measurements for E02-013, which was optimized for $A_{\perp}$, dominate the systematic uncertainty in the form factor ratio determination. A drawback to $A_{\perp} / A_{\|}$ is that multiple measurements are required, e.g. the orientation of the target needs to be adjusted to optimize the components at any given kinematic setting, affecting the statistical uncertainty due to beamtime concerns. The literature often uses $A_{\perp} \rightarrow A_{t}$ and $A_{\|} \rightarrow A_{l}$ which represents the transverse and longitudinal components, respectively.

The elastic scattering process $\vec{e}(k)+\vec{n}(p) \rightarrow e\left(k^{\prime}\right)+n\left(p^{\prime}\right)$ at leading order has been studied in detail in which a $100 \%$ longitudinally polarized electron scatters from a $100 \%$ polarized neutron; therefore, the calculation polarizes the initial states of the electron and neutron but sums over final spin states. The structure of the neutron 
has been parametrized by two invariant functions, namely form factors $G_{E}^{n}$ and $G_{M}^{n}$, which encode information regarding the spatial and momentum distributions of charge and current of the nucleon. Additionally, the form factors can be used to calculate nucleon radii in the non-relativistic limit. Recall that these form factors parametrize the structure at leading order within QED, but the underlying strong dynamics, or strong interactions between the quarks and gluons, are contained within these functions. The physical observable, the beam-target asymmetry, is defined as Eqn 2.106 which may be evaluated by taking the ratio of the antisymmetric invariant amplitude squared (the polarized information) to the symmetric invariant amplitude squared (the unpolarized information) resulting in Eqn 2.107. If the target polarization is oriented such that it is transverse to the momentum transfer vector $\mathbf{q}$ but within the scattering plane, then the electric form factor of the neutron may be extracted by the perpendicular component of the asymmetry (Eqn 2.110); this is the theoretical concept behind E02-013.

The formalism assumes many ideal conditions that are simply not possible to replicate in an experimental hall. Perhaps the most difficult condition to overcome is that no free neutron targets exist as the neutron decays with a mean lifetime of approximately 880 seconds [26]. Common substitutes include deuterium or ${ }^{3} \mathrm{He}$ with the latter being the target of choice for E02-013 as the ground state is dominated by a configuration where the proton spins anti-align, resulting in a nucleus where the neutron carries $\sim 86 \%$ of the spin [27 34]. However, this is a bound system and complications arise due to nuclear effects and final state interactions. For example, the recoiling neutron may interact with the spectator protons before being completely knocked-out of the 
nucleus, or the recoiling neutron may knock-out a spectator proton altogether which is commonly referred to as charge-exchange. For $G_{E}^{n}$, nuclear corrections are handled with the generalized eikonal approximation (GEA) [35] which is an extension to the Glauber approximation [36]. Other complications arise when one attempts to interpret the asymmetry measured during an experiment. The asymmetry that is measured does include quasielastic electron-neutron events, i.e. the events of interest, but the measurement also includes other events that need to be removed or suppressed. Examples include but are not limited to the following: random accidental background, inelastics and proton misidentification. A misidentified proton event occurs if a proton originating from the

${ }^{3}$ He nucleus is observed as a neutron by the detectors which effectively dilutes the neutron count. The theoretical formalism also assumes that the electron and neutron are perfectly polarized; therefore, methods to monitor the beam and target polarization are required during the asymmetry measurements to correct for this experimental reality. Lastly, due to the finite-nature of detectors, a range of $Q^{2}\left(\theta_{e}\right.$ and $\left.E_{e}^{\prime}\right)$ data are accepted which is not accounted for in the ideal formalism; therefore, an averaging method must be introduced. A large portion of the analysis is devoted towards overcoming the above challenges, see Chapter 5 and Chapter 6 .

\subsubsection{Recoil Polarimetry}

The remaining class of double polarization experiments is known as recoil polarization or polarization transfer. In this case, the polarized electron scatters off an unpolarized target transferring polarization to the recoiling nucleon, and a diagram may be seen by Figure 2.4. The derivation is similar to the beam-target asymmetry calculation 


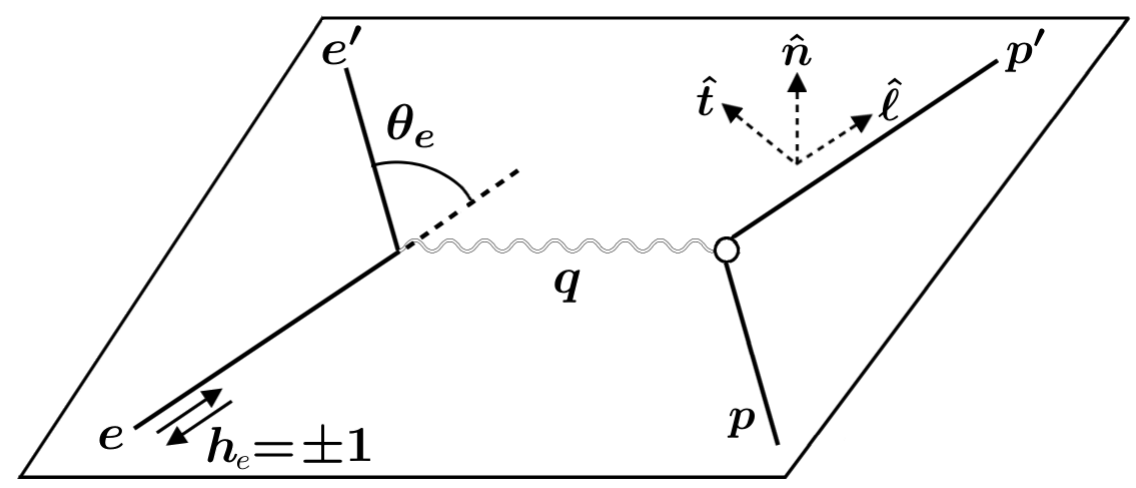

Fig. 2.4: Polarization transfer diagram displaying the coordinate system.

and only begins to deviate when one wants to handle spin within the hadronic tensor. For example, the leptonic tensor defined in Eqn 2.94 may be reused exactly, but the hadronic tensor needs to be recalculated using the unpolarized completeness relations. The calculation resumes identically and utilizes the Breit frame relations, particularly Eqn 2.77, to get the result back to the lab. The results are summarized as

$$
\begin{aligned}
I_{0} P_{l} & =h_{e} \sqrt{\tau(1+\tau)} \tan ^{2} \frac{\theta_{e}}{2} \frac{E_{e}+E_{e}^{\prime}}{M} G_{M}^{2}, \\
I_{0} P_{t} & =-2 h_{e} \sqrt{\tau(1+\tau)} \tan \frac{\theta_{e}}{2} G_{E} G_{M}, \\
I_{0} P_{n} & =0, \\
\Rightarrow \frac{G_{E}}{G_{M}} & =-\frac{P_{t}}{P_{l}} \frac{E_{e}+E_{e}^{\prime}}{2 M} \tan \frac{\theta_{e}}{2}=-\frac{P_{t}}{P_{l}} \sqrt{\frac{\tau(1+\epsilon)}{2 \epsilon}},
\end{aligned}
$$

where $I_{0} \equiv G_{E}^{2}+\frac{\tau}{\epsilon} G_{M}^{2}$ and $h_{e}$ is the electron helicity. The polarization of the recoiling nucleon is typically expressed in a $(\hat{\boldsymbol{t}}, \hat{\boldsymbol{n}}, \hat{\boldsymbol{\ell}})$ basis, representing the transverse $\left(P_{t}\right)$, normal $\left(P_{n}\right)$, and longitudinal $\left(P_{\ell}\right)$ components, where $\hat{\boldsymbol{t}}$ is perpendicular to the momentum transfer and within the electron scattering plane, $\hat{\boldsymbol{n}}$ is normal to the scattering plane, and $\hat{\ell}$ is in the direction of momentum transfer. The transverse polarization observable 
is proportional to $G_{E} / G_{M}$ at leading order; more commonly, though, the longitudinal and transverse polarization observables are simultaneously measured, but the recoiling nucleon must travel through a magnetic field that is oriented in such a way to precess $P_{l}$ into a normal component. A nucleon polarization measurement requires a secondary scattering process, and by flipping the beam helicity a measurable azimuthal asymmetry distribution arises that is related to both the polarization observables $P_{t}$ and $P_{l}$. Since $P_{t}$ and $P_{l}$ may be measured simultaneously, the ratio $P_{t} / P_{l}$ only requires a single measurement which is proportional to the form factor ratio, but also has the benefit of canceling systematics in the ratio.

\subsubsection{Closing Remarks on Double Polarization}

In summary, double polarization experiments generally fall into two categories: beamtarget asymmetry and recoil polarimetry.* The formalism to calculate the former at leading order has been presented in detail while the latter is discussed in broad strokes, but the results of the two calculations are related. In the Born approximation, the polarization components $\left(P_{t}\right.$ and $\left.P_{l}\right)$ are related to the beam-target asymmetry components $\left(A_{\perp}\right.$ and $\left.A_{\|}\right)$through a time-reversal symmetry [20, 22, 23]: $P_{t}=A_{\perp}$ and $P_{l}=-A_{\|}$. Both $P_{t}$ and $A_{\perp}$ are proportional to $G_{E} / G_{M}$ at leading order. Measurements of the type $A_{\perp} / A_{\|}$and $P_{t} / P_{l}$ are common strategies to reduce systematics as details related to an absolute polarization determination and the analyzing power of the secondary reaction cancel in the ratio. This method is often referred to as double ratio experiments as extractions come from ratios of ratios.

\footnotetext{
* Note that an unpolarized beam on a polarized target is a possibility but not included here.
} 


\section{Chapter 3}

\section{Nucleon Form Factors}

The purpose of this chapter is to explore the experimental status of nucleon form factors where topics of current excitement will be emphasized. In addition to lattice QCD, there are many phenomenological models capable of predicting nucleon form factors, and a brief overview of a select few will be presented highlighting the role that form factors play in regards to describing the structure of the nucleon. Several simple parametrizations useful for form factor calculations will be presented.

\subsection{Previous Experiments}

Elastic $e N$ experiments have been performed for over 60 years, starting with the pioneer of the field R. Hofstadter and collaborators at the High Energy Physics Laboratory in Stanford in the 1950s. The early electron scattering experiments tested the validity of cross section formulas derived in Chapter 2, namely Eqn 2.49, Eqn 2.47, and Eqn 2.44. When the data did not follow that of electron scattering off structureless protons or the Mott cross section, attention was directed towards understanding the deviations in terms of form factor measurements. The first measurement of a proton form factor was in the mid-1950s [14 16, 37, 38] where various fit shapes were used to estimate the 
proton radius; a value of $0.77 \pm 0.10 \mathrm{fm}$ was quoted in Ref. [14. The neutron magnetic form factor was first measured in the late-1950s using a liquid deuterium target [39], and again the possibility of a radius was explored but the neutron is technically and experimentally much more difficult to handle, which are details that would take decades to understand. The electron scattering experiments of the 1950s solidified the concept that the nucleon is a finite-sized object with an internal structure giving rise to properties such as a radius.* The striking results of the early electron studies on the nucleon led to a proliferation in the construction of other facilities and accelerators around the world with similar goals of studying the nucleon. Over the next several decades, nucleon form factors (now written in terms of two form factors, $F_{1}$ and $F_{2}$ or $G_{E}$ and $G_{M}$ ) were measured to $Q^{2}$ values that were technically and statistically feasible by variations of the Rosenbluth separation technique. In the early-1990s, accelerator, target and detector technology matured to the point where polarized electron scattering from polarized or unpolarized targets was possible. In 2000, Jefferson Lab's Hall A Collaboration published double polarization proton form factor data that disagreed with decades of previous Rosenbluth measurements [40, a result that rejuvenated the form factor field and sparked intense theoretical and experimental effort. More double polarization data emerged [41 52], confirming the disagreement between the two methods. The purpose of this section is to explore the experimental results of the Rosenbluth and double polarization methods, and then to discuss a possible source of the discrepancy. For clarity, form factors obtained from polarization observables and cross section measurements will be differentiated as polarized and unpolarized extractions, respectively. A proton target

\footnotetext{
* Hofstadter received the Nobel Prize in 1961 for his work on nucleon structure.
} 
was easier to develop, and correspondingly the proton form factors were historically studied first; therefore, the proton status will proceed prior to the neutron. Note that Refs. [53, 54] contain convenient and exhaustive tables of form factor measurements up to 2005.

\subsubsection{Unpolarized Proton Results}

The formalism of Section 2.2 applies to elastic unpolarized $e N$ scattering assuming that the nucleon is free, and may be used to extract proton form factors; the most common reaction is $\mathrm{H}\left(e, e^{\prime}\right)$, but $\mathrm{H}\left(e, e^{\prime} p\right)$ and $\mathrm{H}(e, p)$ have also been used.* Early proton form factor measurements discovered the approximate low- $Q^{2}$ scaling relationships, $G_{E}^{p} / G_{D} \sim 1$ and $G_{M}^{p} /\left(\mu_{p} G_{D}\right) \sim 1$ where the dipole form factor, denoted by $G_{D}$, has the following form (more details may be found in Section 3.2.1):

$$
G_{D}\left(Q^{2}\right)=\frac{1}{\left(1+\frac{Q^{2}}{\Lambda^{2}}\right)^{2}},
$$

which fit the early data well for $\Lambda^{2}=0.71 \mathrm{GeV}^{2} .^{\dagger}$ The functional form of $G_{D}$ naively corresponds to the inverse Fourier transform of a radially, exponentially decaying density. In other words, the electric and magnetic distributions of the proton are similar if the form factors $G_{E}^{p}$ and $G_{M}^{p}$ scale according to this parametrization. ${ }^{\ddagger}$ By equating the two relations, an empirical relationship between the Sachs form factors may be realized as $\mu_{p} G_{E}^{p} / G_{M}^{p} \sim 1$. As a result of this important empirical finding of the time, it is conventional to normalize form factor data by the dipole parametrization as seen in

\footnotetext{
* The so-called "Super-Rosenbluth" extraction of Qattan et al. [55] detected the outgoing proton.

${ }^{\dagger}$ Albrecht et al. [56] published the fit parameter in 1966 and is now fixed by convention.

$\ddagger$ See the recent review of Ref. [57] for a modern discussion of $G_{D}$ and form factors.
} 
Figure 3.1. note that the $G_{M}^{p}$ data is also scaled by the magnetic moment of the proton in order to retain unity as $Q^{2}$ approaches zero. The electric form factor is consistent with the dipole parametrization up to $Q^{2} \sim 1 \mathrm{GeV}^{2}$. As $Q^{2}>1 \mathrm{GeV}^{2}$, the data quality worsens, reflecting the fact that $G_{E}^{p}$ becomes increasingly more difficult to extract as $Q^{2}$ increases. This can be explicitly seen in the formula for the reduced cross section (Eqn 2.52 where $G_{E}$ is suppressed by a factor of $\frac{1}{\tau}$. As such, no definitive statements regarding $G_{E}^{p}$ scaling as $G_{D}$ may be made for $Q^{2}>1 \mathrm{GeV}^{2}$. On the other hand, $G_{M}^{p}$ dominates the cross section at large $Q^{2}$ allowing for high-precision measurements over a large $Q^{2}$ range. Note that Sill et al. 63] quotes measurements up to $Q^{2}=31.20 \mathrm{GeV}^{2}$ with a combined uncertainty ranging from $3.6 \%$ to $19 \%$ for the lowest and highest $Q^{2}$ points, respectively. The magnetic form factor data begins to strongly disagree with the dipole parametrization around $Q^{2} \gtrsim 8 \mathrm{GeV}^{2}$. And finally, Figure 3.2 observes the scaling $\mu_{p} G_{E}^{p} / G_{M}^{p} \sim 1$ up to $Q^{2} \sim 1 \mathrm{GeV}^{2}$, but as $Q^{2}>1 \mathrm{GeV}^{2}$ then the uncertainties make it impossible to make such a statement.

\subsubsection{Unpolarized Neutron Results}

On top of all the challenges of unpolarized electron scattering, extracting the neutron form factors has additional technicalities, both experimentally and theoretically. There are no free neutron targets available as the neutron decays* weakly via $\beta^{-}$decay, or $n \rightarrow p+e^{-}+\bar{\nu}_{e}$. Therefore, an appropriate substitute would be to use deuterium or helium, but now the neutron is bound to a nucleus and corrections must be applied. Additionally, since the neutron is chargeless, the value of $G_{E}^{n}$ is inherently small. The

\footnotetext{
* The PDG mean lifetime of the neutron is $880.2 \pm 1.0$ seconds [26].
} 

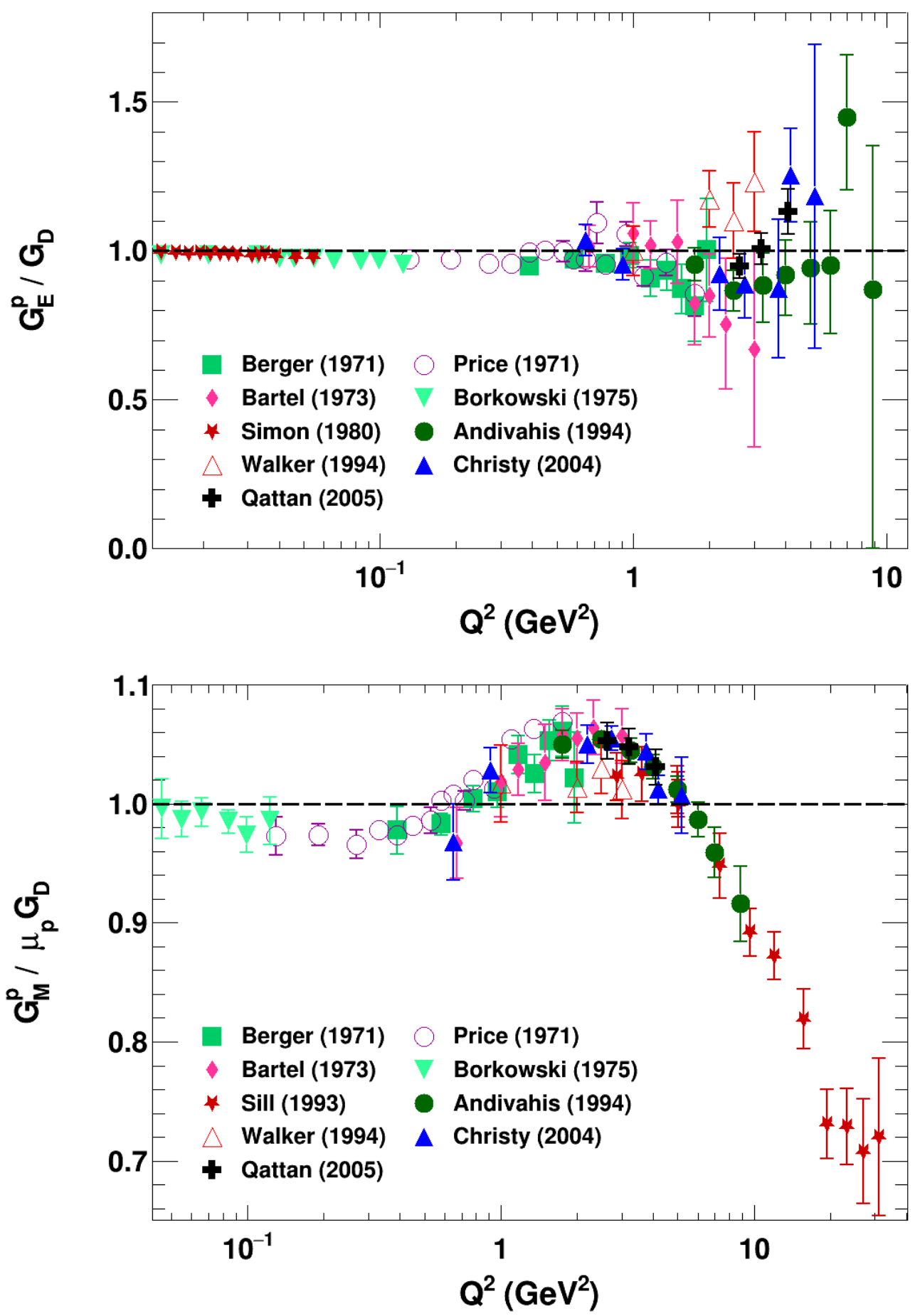

Fig. 3.1: Selected electric (top) and magnetic (bottom) form factor data for the proton obtained by a Rosenbluth separation [55, 58, 66]. 


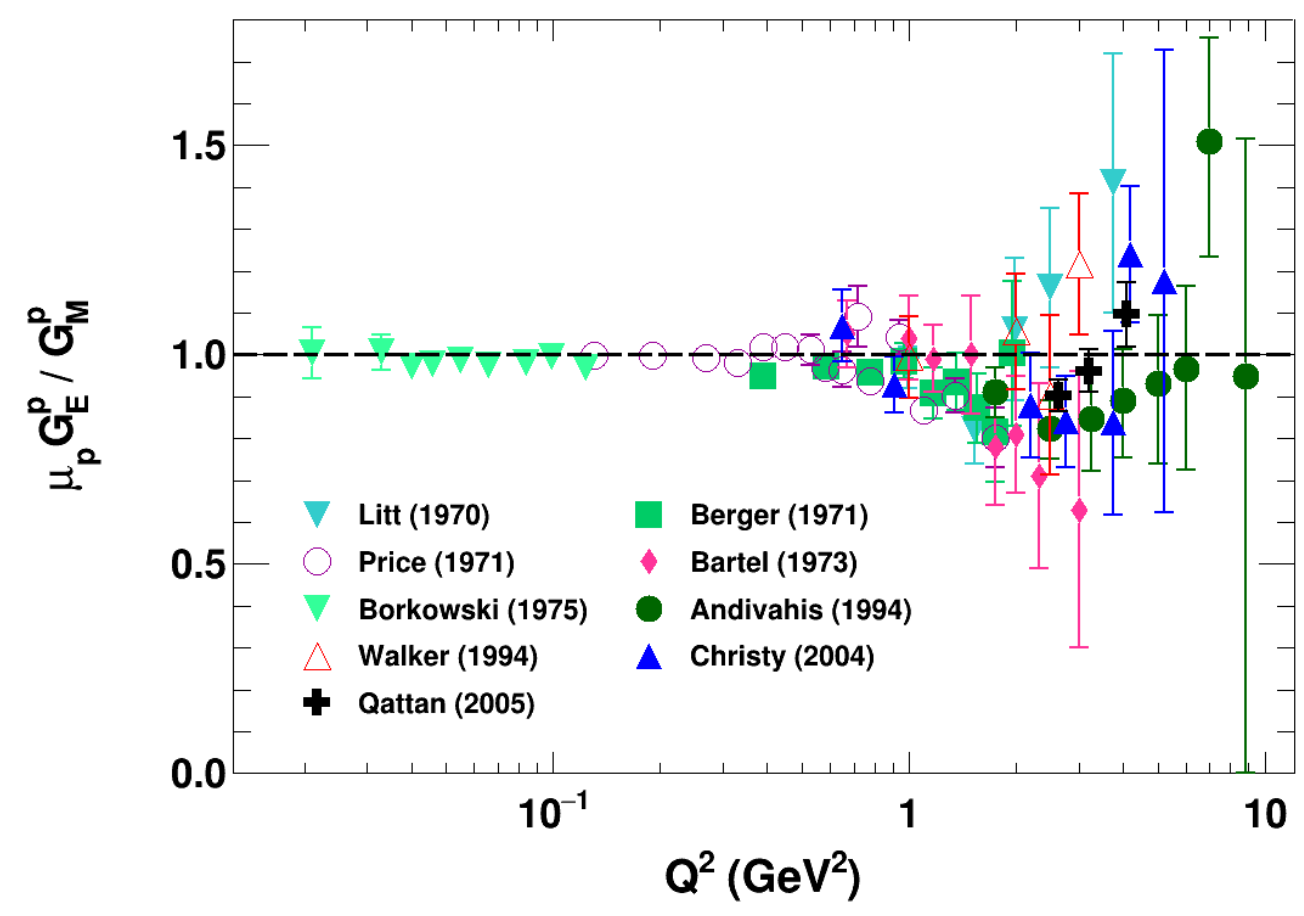

Fig. 3.2: Selected proton form factor ratio data extracted via a Rosenbluth separation where the $Q^{2}$ range is limited by $G_{E}^{p}[55,58,61,64,67$.

smallness of $G_{E}^{n}$ combined with property that $G_{M}^{n}$ scales with $\tau$ in the cross section makes a $G_{E}^{n}$ measurement more difficult for a range of $Q^{2}$. On top of these difficulties, the lack of a charge makes it challenging to detect; for example, there are exclusive measurements where the neutron detection efficiency needs to be precisely known. As a result, unpolarized experiments throughout the early-1960s to early-1990s* attempted to extract the neutron form factors utilizing two theoretical techniques, both requiring a Rosenbluth-like separation: unpolarized quasielastic inclusive and semi-exclusive electron-deuteron $(e d)$ cross section measurements and unpolarized elastic ed cross sec-

\footnotetext{
* The most recent unpolarized $G_{E}^{n}$ measurement was published in 1993 [68.
} 
tion measurements. Note that presence of a deuterium target requires alterations to the formalism of Section 2.2 which will be introduced as necessary.

Neutron extractions using the former technique, quasielastic ed scattering first suggested by Hofstadter in 1956 [14, proved to be difficult due to the Rosenbluth decomposition of the neutron from the deuteron. Early attempts used a technique that was not much different than the proton case:

$$
\left(\frac{d \sigma}{d \Omega}\right)_{e d}=\left(\frac{d \sigma}{d \Omega}\right)_{e p}+\left(\frac{d \sigma}{d \Omega}\right)_{e n}
$$

where the differential cross sections are given by Eqn 2.44. The formalism requires knowledge of the proton form factors. In the impulse approximation, or assuming that the virtual photon only interacts with one nucleon while the remaining nucleon is simply a non-interacting spectator, separating the neutron form factors may be done by a difference in an inclusive unpolarized quasielastic ed cross section measurement from an elastic unpolarized $e p$ cross section measurement where a Rosenbluth separation is performed in both situations. In practice, though, history has shown that to reliably extract $G_{E}^{n}$ information utilizing this technique, or variations of this technique such as $d\left(e, e^{\prime} n\right), d\left(e, e^{\prime} \bar{p}\right), d\left(e, e^{\prime} n\right) / d\left(e, e^{\prime} p\right), d\left(e, e^{\prime}\right) / p\left(e, e^{\prime}\right)$ or $d\left(e, e^{\prime} \bar{p}\right) / d\left(e, e^{\prime} p\right)$ where $\bar{p}$ indicates anti-coincidence, is difficult. For example, the most recent $G_{E}^{n}$ unpolarized extraction by Lung et al. 68] in 1993 used inclusive quasielastic ed scattering, and reported values of $\left(G_{E}^{n}\right)^{2}$ consistent with zero in the $1<Q^{2}<4 \mathrm{GeV}^{2}$ range with large model-dependent systematic uncertainties that were not under control. Furthermore, the early unpolarized $G_{E}^{n}$ extractions established the importance of nuclear corrections (to be discussed), a necessary correction as the neutron is bound to a nucleus which 
introduces technical difficulties. Since there exists no reliable extractions of $G_{E}^{n}$ using quasielastic ed scattering, no data will be shown. On the other hand, this formalism is commonly used to extract $G_{M}^{n}$, and post-1994 extractions commonly use the ratio method, or $d\left(e, e^{\prime} n\right) / d\left(e, e^{\prime} p\right)$.

The latter extraction method, unpolarized elastic ed cross section measurements, requires three deuteron form factors in the impulse approximation (IA). First derived by Gourdin [69], the form of Eqn 2.51 may be used but rewritten as

$$
\begin{aligned}
\left.\frac{d \sigma}{d \Omega}\right|_{\mathrm{LAB}} & =\left.\frac{d \sigma}{d \Omega}\right|_{\mathrm{Mott}}\left[A\left(Q^{2}\right)+B\left(Q^{2}\right) \tan ^{2} \frac{\theta_{e}}{2}\right], \\
A\left(Q^{2}\right) & =G_{C}^{2}\left(Q^{2}\right)+\frac{8}{9} \tau_{D}^{2} G_{Q}^{2}\left(Q^{2}\right)+\frac{2}{3} \tau_{D}\left(1+\tau_{D}\right) G_{M}^{2}, \\
B\left(Q^{2}\right) & =\frac{3}{4} \tau_{D}\left(1+\tau_{D}\right)^{2} G_{M}^{2}\left(Q^{2}\right),
\end{aligned}
$$

where $\tau_{D} \equiv Q^{2} / 4 M_{D}^{2}$ and $M_{D}$ is the mass of the deuteron. The three functions $G_{C}$, $G_{Q}$, and $G_{M}$ are the charge, quadrupole and magnetic form factors of the deuteron, respectively, and have the following normalization conditions,

$$
G_{C}(0)=1, \quad G_{Q}(0)=M_{D}^{2} Q, \quad G_{M}(0)=2 M_{D} \mu_{D}
$$

To be clear and in this context, $G_{M}$ represents the magnetic form factor of the deuteron not to be confused with the Sachs magnetic form factor. The deuteron form factors may be written in terms of the isoscaler electric and magnetic form factors of the nucleon, denoted by $G_{E}^{S}$ and $G_{M}^{S}$, as follows:

$$
G_{C}=2 G_{E}^{S} C_{E}, \quad G_{Q}=2 G_{E}^{S} C_{Q}, \quad G_{M}=\frac{M_{D}}{M_{p}}\left(2 G_{M}^{S} C_{S}+G_{E}^{S} C_{L}\right)
$$

where the coefficients $C_{E}, C_{Q}, C_{S}$, and $C_{L}$ are computed by considering the explicit forms of the deuteron $S$ and $D$-state wave functions. The Sachs nucleon form factors 
are then related to the isoscaler form factors by

$$
\begin{aligned}
G_{E}^{S} & =\frac{1}{2}\left(G_{E}^{n}+G_{E}^{p}\right), \\
G_{M}^{S} & =\frac{1}{2}\left(G_{M}^{n}+G_{M}^{p}\right) .
\end{aligned}
$$

The formalism contains many details, but the overall procedure is identical to what has already been discussed by Eqn 2.52 . The functions $A\left(Q^{2}\right)$ and $B\left(Q^{2}\right)$ within the cross section are analogously separated using the Rosenbluth method which are then related to the isoscaler functions. The $C$ coefficients require theoretical input and a particular $N N$ interaction, but can be calculated in a limited $Q^{2}$ range. Therefore, $G_{E}^{n}$ may be isolated if knowledge of the other form factors exists at that particular $Q^{2}$. The formalism is very sensitive, particularly as $Q^{2}$ increases, to which deuteron wave function is used as the structure functions depend on the $C$-integrals. In other words, the formalism of unpolarized elastic ed is highly model-dependent as a deuteron structure "unfolding" must be done in order to separate $G_{C}$ and $G_{Q}$, requiring a deuteron wave function derived from a particular $N N$ potential which introduces a major systematic uncertainty that was never fully under control. Furthermore, $G_{E}^{n}$ extraction attempts quickly noticed that the IA was insufficient in characterizing the shape of the $A\left(Q^{2}\right)$ structure function which firmly established the importance of nuclear corrections. The nuclear effects that need to be accounted for are the following:

1. it is possible to have rescattering, i.e. the struck nucleon may rescatter off of the spectator, known as a final state interaction (FSI);

2. the virtual photon may couple to a virtual meson that is exchanged between the two nucleons, commonly referred to as meson exchange currents (MEC); 
3. the nucleon may go into an excited state and then couple to the virtual photon, called an isobar configuration (IC);

4. the nucleons are not stationary within the nucleus, resulting in Fermi smearing which broadens all spectra relative to the $e p$ data.

In addition to nuclear corrections, relativistic effects such as wave function distortion need to considered. The most recent $G_{E}^{n}$ extraction using this technique was published in 1990 by Platchkov et al. [70] in which there are more than 40 extractions in the $0<$ $Q^{2}<0.7 \mathrm{GeV}^{2}$ range with significantly smaller statistical uncertainties than previous measurements. Unpolarized elastic ed extractions demonstrated that $G_{E}^{n}>0$ whereas quasielastic ed extractions could not; however, the model-dependence related to the choice of the $N N$ potential yielded prohibitively large systematic uncertainties of $\pm 40 \%$ in the data of Ref. [70], revealing the limitations of a $G_{E}^{n}$ extraction via elastic ed cross section measurements. For completeness, while it has been done it is uncommon to use this method to extract $G_{M}^{n}[71$.

A unique extraction of $G_{E}^{n}$ has been reported by Schiavilla and Sick in 2001 [72] by analyzing unpolarized elastic ed cross section data and exploiting the quadrupole deuteron form factor $G_{Q}$ rather than a combination of $G_{C}$ and $G_{Q}$ as was conventionally done in cross section analyses. In order to separate the deuteron form factors $G_{C}$, $G_{Q}$ and $G_{M}$, a third observable such as the deuteron tensor polarization observable $t_{2 i}$ is required [21]. Ref. [72] used $t_{20}$ to disentangle the three form factors from the Rosenbluth-separated $A\left(Q^{2}\right)$ and $B\left(Q^{2}\right)$ structure functions, resulting in more than 10 additional $G_{E}^{n}$ extractions up to $Q^{2}=1.6 \mathrm{GeV}^{2}$. While the extraction exploits 


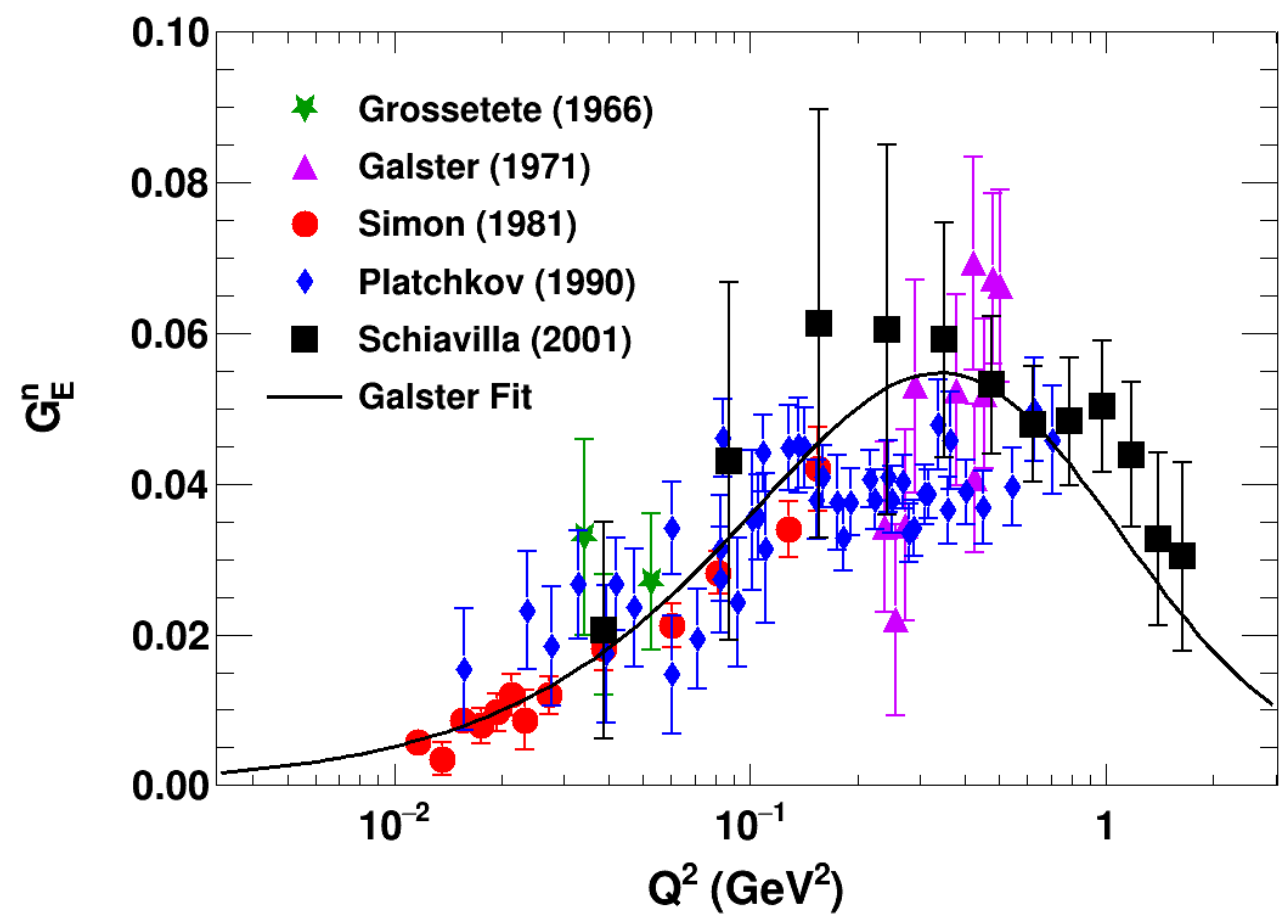

Fig. 3.3: Selected electric form factor data for the neutron extracted from unpolarized elastic ed cross section measurements [70, 72,75]. Note that Ref. [72] is a reanalysis of elastic ed cross section data in which polarization observables are exploited. The black curve is the Galster parametrization [74].

polarization observations, the analysis primarily makes use of elastic ed cross section data and is categorized as such.

A representative sample of $G_{E}^{n}$ extractions from unpolarized elastic ed scattering measurements may be seen by Figure 3.3 , and no scaling relations are observed or even expected given the very different low $Q^{2}$ behavior. The well-known Galster parametrization has the form $G_{E}^{n}\left(Q^{2}\right)=-\left[\mu_{n} \tau /(1+5.6 \tau)\right] G_{D}\left(Q^{2}\right)$ where the functional form when coupled to the Feshbach-Lomon deuteron wave function [76] produced the lowest $\chi^{2}$ when fit to the 1971 and earlier data [74. The form of the Galster parametrization is 


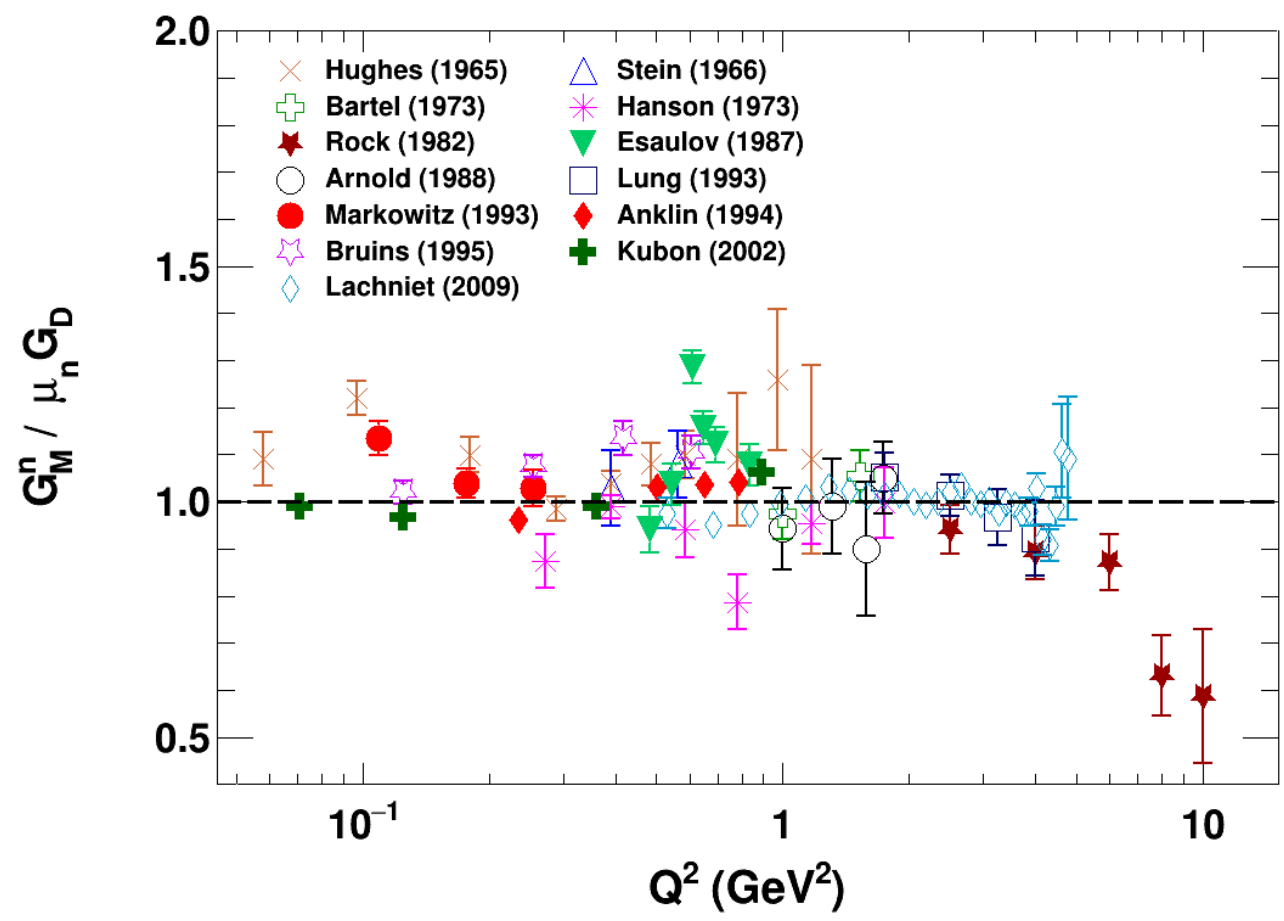

Fig. 3.4: Selected magnetic form factor data for the neutron extracted from unpolarized quasielastic ed cross section measurements [60, 68, 77, 87 ].

$\propto Q^{2}\left(1+a Q^{2}\right)^{-1}$, or $Q^{2} \times($ monopole term $)$, and the monopole term is loosely connected to the structure of the propagator; however, the parametrization is largely unphysical and simply corresponds to a functional form producing a low $\chi^{2}$ when paired with a particular wave function.

The magnetic form factor is not as elusive as $G_{E}^{n}$ and is most commonly extracted using unpolarized quasielastic ed reactions. The data shown in Figure 3.4 corresponds to experiments ran in the mid-1960s to 2009. The most recent extractions of Lachniet et al. [87] obtained high precision using the ratio technique, or $d\left(e, e^{\prime} n\right) / d\left(e, e^{\prime} p\right)$, while simultaneously calibrating the neutron detection efficiency in situ. In this case "scaling" is observed in the quantity $G_{M}^{n} \sim \mu_{n} G_{D}$, but begins to break down as $Q^{2} \gtrsim 6 \mathrm{GeV}^{2}$ if 
the data of Rock et al. is to be believed.

\subsubsection{Polarized Results}

Unpolarized electron scattering off of hydrogen and deuterium targets has proven to be an invaluable tool, and consequently the nucleon form factors are no longer a complete mystery with perhaps the exception of $G_{E}^{n}$. It is inherently interesting, though, to extract the form factors via another observable as a cross-check between experimental methods. Recall that double polarization experiments require a polarized electron beam and/or a polarized target, giving a new reaction from which the form factor ratio may be extracted in a single measurement. The benefits of double polarization experiments are multi-fold: (1) it is possible to extract the form factor ratio in one measurement as opposed to a Rosenbluth separation which requires a minimum of two measurements in order to fit the data to a straight-line; (2) measuring a ratio (or a ratio of ratios in the case of $P_{t} / P_{l}$ or $\left.A_{\perp} / A_{\|}\right)$dramatically decreases the systematic uncertainties, a necessity for $G_{E} ;(3)$ double polarization measurements extract the form factor ratio rather than the form factors squared allowing for an unambiguous determination of the sign; and (4) radiative corrections affect Rosenbluth data significantly while negligibly affecting the double polarization data, which is a current topic of interest to be discussed in the next section. Double polarization experiments began to be used for form factor extractions as early as 1991 and contrary to the history of the Rosenbluth separation technique, the neutron was studied first.

The electric form factor of the neutron never observed any empirical scaling as in the case of the remaining form factors that gained significant attention in the early days, 
i.e. $G_{E}^{p} \approx G_{M}^{p} / \mu_{p} \approx G_{M}^{n} / \mu_{n} \approx G_{D}$. Unpolarized cross section measurements while establishing that $G_{E}^{n}>0$ provided little information on the distribution as a function of $Q^{2}$ due to large systematic uncertainties in addition to the difficulties associated to a bound neutron. Out of shear necessity, the neutron required alternative experimental techniques exploiting polarized degrees of freedom. Following the lead of Refs. [53, 54, an exhaustive chronological summary of neutron form factor extractions via double polarization techniques may be seen by Table 3.1; note that Ref. [72] is not included despite using the $t_{20}$ observable. Double polarization extractions of $G_{E}^{n}$ generally fall into three categories: asymmetry measurements using polarized ${ }^{3} \mathrm{He}$ or polarized deuterium, or recoil polarimetry with an unpolarized deuterium target. The formalism to handle both situations in the ideal case, namely the rest frame of a free neutron, has been detailed in Section 2.3. however, the neutron is now bound and corrections to handle nuclear effects, imperfect polarization of the beam and/or target and other experimental realities must be taken into account. Figure 3.5 displays $G_{E}^{n}$ double polarization extractions and is organized by technique: (1) recoil polarimetry with a deuterium target (red marker), (2) a beam-target asymmetry using a polarized ${ }^{3} \mathrm{He}$ target (blue marker), and (3) a beam-target asymmetry using a polarized deuterium target (green marker). The dark blue square represents the subject of this thesis, and has been plotted for comparison at $Q^{2}=1.16 \mathrm{GeV}^{2}$. The fit by Riordan et al. is a Galster-like fit using two parameters and takes the following form: $G_{E}=[a \tau /(1+b \tau)] G_{D}$ where $a=1.39$ and $b=2.00$. The pre-1999 Refs. [88 90] are ignored as nuclear corrections are not handled, and the 1994 extraction of Eden et al. [91] is omitted due to the large error bars. Golak et al. [109] 


\begin{tabular}{|c|c|c|c|c|c|}
\hline Publication & Facility & Year & Reaction & $Q^{2}(\mathrm{GeV})^{2}$ & Extraction \\
\hline 88 & MIT-Bates & 1991 & ${ }^{3} \overrightarrow{\mathrm{He}}\left(\vec{e}, e^{\prime}\right)$ & 0.16 & $A_{\perp} \rightarrow G_{E}^{n}$ \\
\hline 89 & MIT-Bates & 1992 & ${ }^{3} \overrightarrow{\mathrm{He}}\left(\vec{e}, e^{\prime}\right)$ & 0.2 & $A_{\perp}, A_{\|} \rightarrow G_{E}^{n}$ \\
\hline 90 & MAMI & 1994 & ${ }^{3} \overrightarrow{\mathrm{He}}\left(\vec{e}, e^{\prime} n\right)$ & 0.31 & $A_{\perp}, A_{\|} \rightarrow G_{E}^{n}$ \\
\hline 91 & MIT-Bates & 1994 & ${ }^{2} \mathrm{H}\left(\vec{e}, e^{\prime} \vec{n}\right)$ & 0.255 & $P_{t}^{(h)} \rightarrow G_{E}^{n}$ \\
\hline 92 & MIT-Bates & 1994 & ${ }^{3} \mathrm{He}\left(\vec{e}, e^{\prime}\right)$ & 0.19 & $A_{\|} \rightarrow G_{M}^{n}$ \\
\hline 93 & MAMI & 1999 & ${ }^{3} \overrightarrow{\mathrm{He}}\left(\vec{e}, e^{\prime} n\right)$ & 0.40 & $A_{\perp}, A_{\|} \rightarrow G_{E}^{n}$ \\
\hline [94, 95] & MAMI & 1999 & ${ }^{2} \mathrm{H}\left(\vec{e}, e^{\prime} \vec{n}\right)$ & 0.34 & $P_{t}^{(h)}, P_{l}^{(h)} \rightarrow G_{E}^{n}$ \\
\hline 96 & NIKHEF & 1999 & ${ }^{2} \overrightarrow{\mathrm{H}}\left(\vec{e}, e^{\prime} n\right)$ & 0.21 & $A_{\text {ed }}^{V} \rightarrow G_{E}^{n}$ \\
\hline 97, 98 & MAMI & $1999 / 2003$ & ${ }^{3} \overrightarrow{\mathrm{He}}\left(\vec{e}, e^{\prime} n\right)$ & 0.67 & $A_{\perp}, A_{\|} \rightarrow G_{E}^{n}$ \\
\hline 99,100 & JLab & $2000 / 2003$ & ${ }^{3} \overrightarrow{\mathrm{He}}\left(\vec{e}, e^{\prime}\right)$ & $0.1-0.6$ & $A_{\|} \rightarrow G_{M}^{n}$ \\
\hline 101 & JLab & 2001 & ${ }^{2} \overrightarrow{\mathrm{H}}\left(\vec{e}, e^{\prime} n\right)$ & 0.495 & $A_{\mathrm{ed}}^{V} \rightarrow G_{E}^{n}$ \\
\hline 54,102 & JLab & $2003 / 2006$ & ${ }^{2} \mathrm{H}\left(\vec{e}, e^{\prime} \vec{n}\right)$ & $0.45-1.45$ & $P_{t}^{(h)}, P_{l}^{(h)} \rightarrow G_{E}^{n}$ \\
\hline 103 & JLab & 2004 & ${ }^{2} \overrightarrow{\mathrm{H}}\left(\vec{e}, e^{\prime} n\right)$ & $0.5,1.0$ & $A_{\mathrm{ed}}^{V} \rightarrow G_{E}^{n}$ \\
\hline 104 & MAMI & 2005 & ${ }^{2} \mathrm{H}\left(\vec{e}, e^{\prime} \vec{n}\right)$ & $0.30-0.79$ & $P_{t}^{(h)}, P_{l}^{(h)} \rightarrow G_{E}^{n}$ \\
\hline 105 & JLab & 2007 & ${ }^{3} \overrightarrow{\mathrm{He}}\left(\vec{e}, e^{\prime}\right)$ & $0.1-0.6$ & $A_{\perp} \rightarrow G_{M}^{n}$ \\
\hline 106 & MIT-Bates & 2008 & ${ }^{2} \overrightarrow{\mathrm{H}}\left(\vec{e}, e^{\prime} n\right)$ & $0.14-0.42$ & $A_{\mathrm{ed}}^{V} \rightarrow G_{E}^{n}$ \\
\hline [7] & JLab & 2010 & ${ }^{3} \overrightarrow{\mathrm{He}}\left(\vec{e}, e^{\prime} n\right)$ & $1.72-3.41$ & $A_{\perp}, A_{\|} \rightarrow G_{E}^{n}$ \\
\hline 107 & MAMI & 2013 & ${ }^{3} \overrightarrow{\mathrm{He}}\left(\vec{e}, e^{\prime} n\right)$ & 1.58 & $A_{\perp}, A_{\|} \rightarrow G_{E}^{n}$ \\
\hline 108 & JLab & 2017 & ${ }^{3} \overrightarrow{\mathrm{He}}\left(\vec{e}, e^{\prime}\right)$ & 0.98 & $A_{\perp}, A_{\|} \rightarrow G_{E}^{n}$ \\
\hline
\end{tabular}

Table 3.1: Summary of neutron form factor extractions via double polarization. 


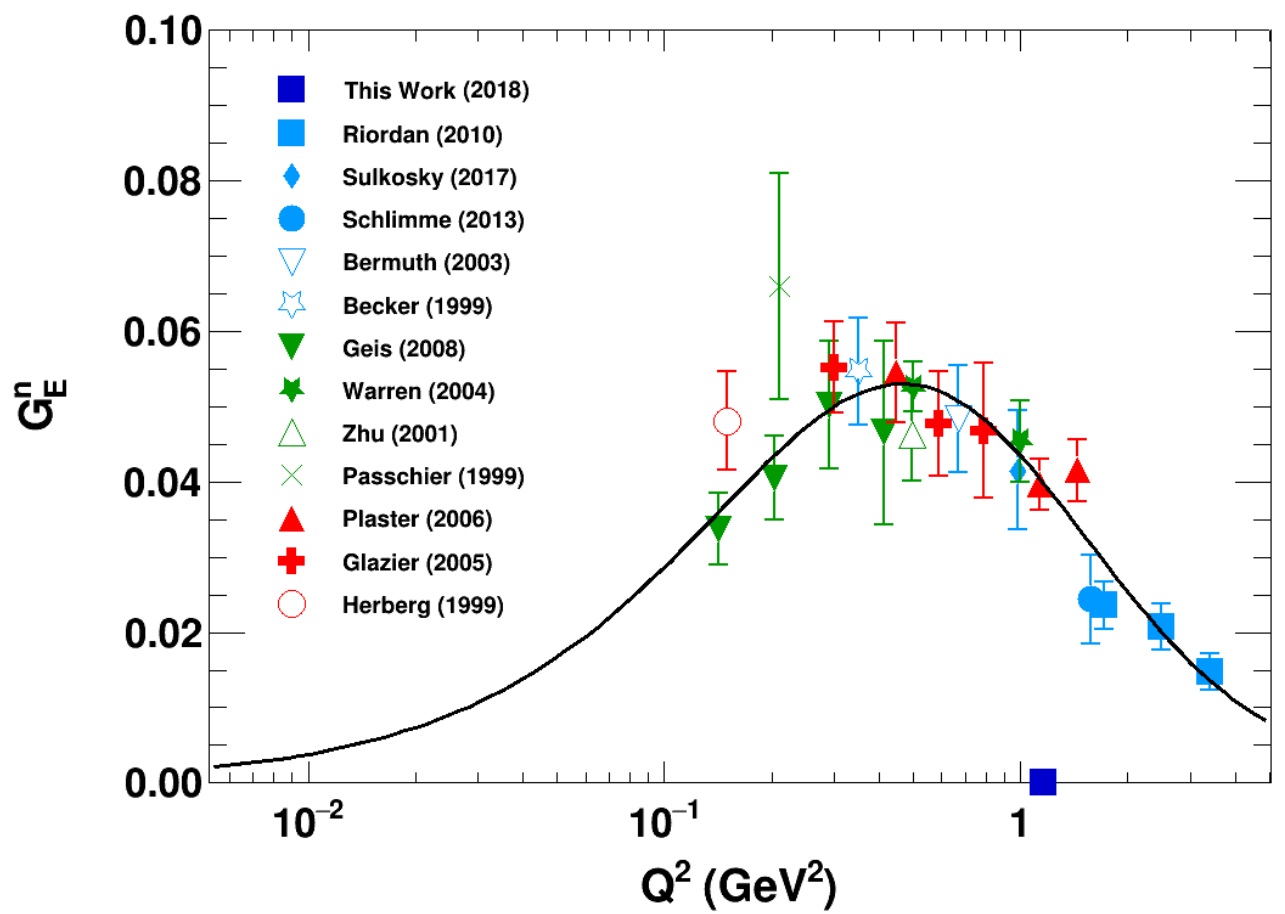

Fig. 3.5: Post-1999 $G_{E}^{n}$ extractions via polarization observables: ${ }^{3} \mathrm{He}$ asymmetry (blue markers) [7, 93, 98, 107, 108, deuterium asymmetry (green markers) [96, 101, 103, 106] and deuterium recoil polarimetry (red markers) [54, 95, 104]. The curve is a Galster-like fit reported by Ref. [7].

applied FSI corrections to Ref. [93], but ignored systematic experimental errors. A variety of deuterium targets have been used: solid deuterated ammonia ([101, 103]), gaseous deuterium ([96, 106]) and liquid deuterium ([54, 91, 95, 104]). The 2017 data of Sulkosky et al. is the only modern extraction using inclusive ${ }^{3} \overrightarrow{\mathrm{He}}\left(\vec{e}, e^{\prime}\right)$, which is a measurement that is feasible in a limited $Q^{2}$ region as neutron detection becomes increasingly more important in order to suppress pion contamination at larger $Q^{2}$. The neutron form factor ratio $\mu_{n} G_{E}^{n} / G_{M}^{n}$ may be seen by Figure 3.6 with the same color- 


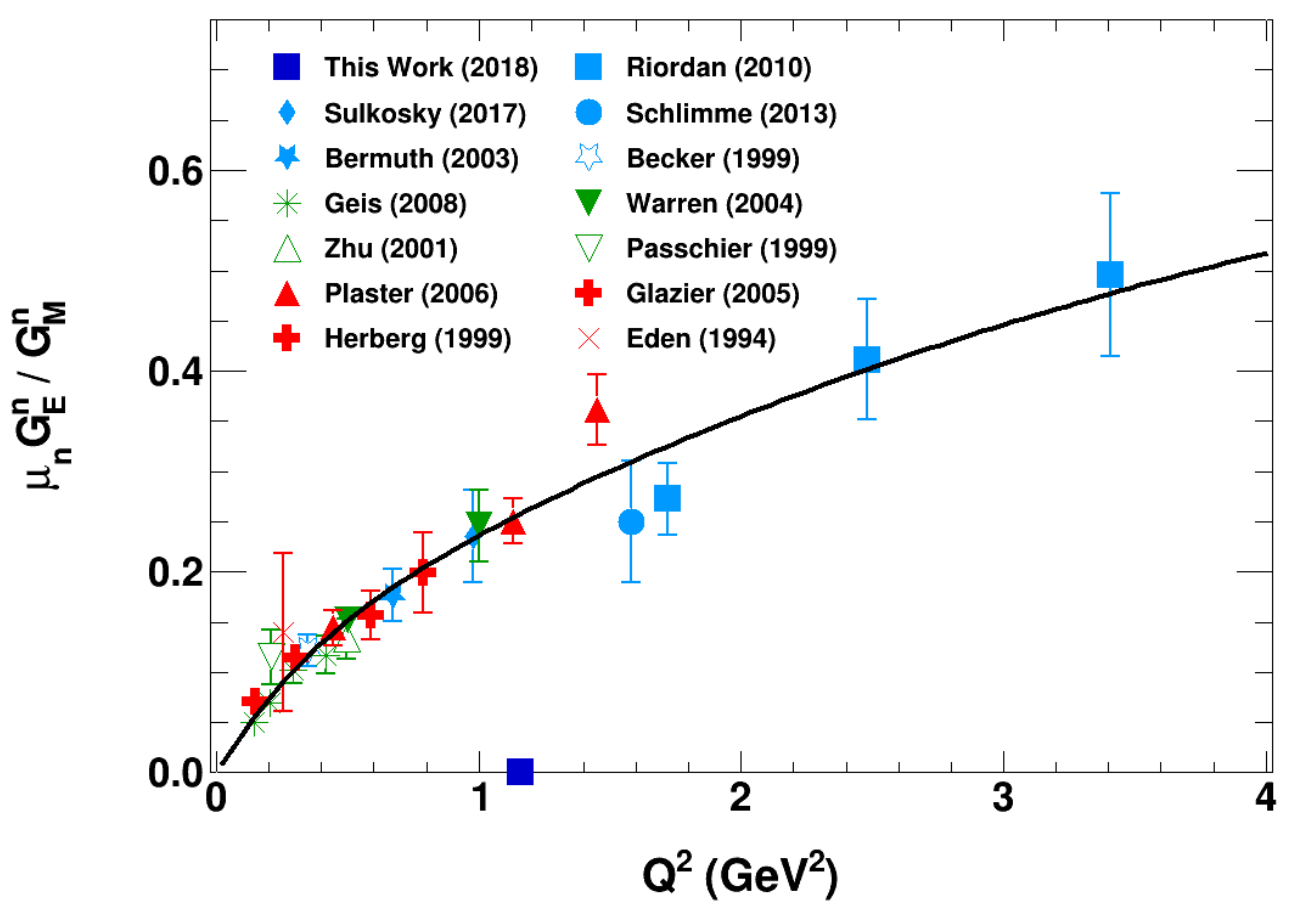

Fig. 3.6: Double polarization extractions of the neutron form factor ratio $\mu_{n} G_{E}^{n} / G_{M}^{n}$. The references are the same as Figure 3.5 with the addition of Ref. 91].

coding as the previous figure. The large $Q^{2}$ data is limited, but there is a noticeable discrepancy between the Plaster data at $Q^{2}=1.45 \mathrm{GeV}^{2}$ and the results of Riordan (and the reanalysis) and Schlimme at $Q^{2}=1.6$ and $1.7 \mathrm{GeV}^{2}$, respectively.

For the proton, it is more common to extract $G_{E}^{p} / G_{M}^{p}$ using the reaction $p\left(\vec{e}, e^{\prime} \vec{p}\right)$; however, the reaction $\vec{p}\left(\vec{e}, e^{\prime} p\right)$ has been used. Figure 3.7 displays recent polarization transfer data with post-1994 Rosenbluth cross section measurements (green) for comparison. In sharp contrast, the more precise polarization data clearly and unambiguously deviates from the scaling relation $\mu_{p} G_{E}^{p} / G_{M}^{p} \sim 1$ as observed with the Rosenbluth extracted data. In an attempt to resolve the discrepancy, the so-called "Super-Rosenbluth" 


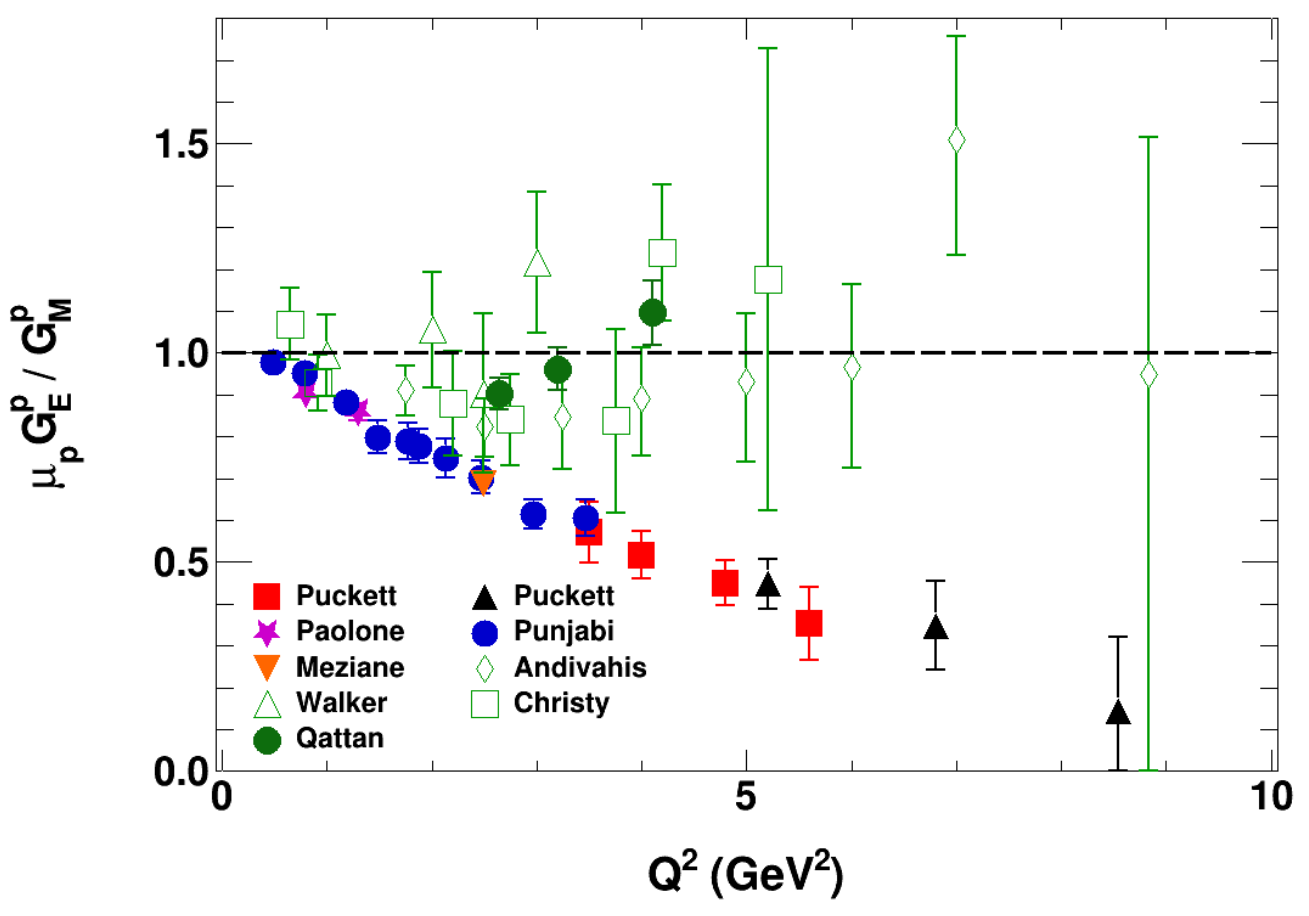

Fig. 3.7: Selected data of $\mu_{p} G_{E}^{p} / G_{M}^{p}$ displaying the differences between Rosenbluth separated data [55, 64,66] and the polarization transfer data: [40, 45] (filled blue circle), 47] (filled magenta star), [48, 52] (filled inverted orange triangle), [43, 51] (filled red square), and [52] (filled black triangle).

experiment of Qattan et al. [55] (dark green filled circle) paid special attention to reducing systematic uncertainties and determined the form factor ratio to a total uncertainty of $4 \%-8 \%$, but the results are consistent with previous Rosenbluth data with much smaller uncertainties. The authors concluded that the two techniques must have some systematic differences, and as a result theoretical investigations looked towards higherorder QED corrections that were neglected from the "standard" radiative corrections applied to Rosenbluth analyses; this issue is addressed further in Section 3.1.4. 


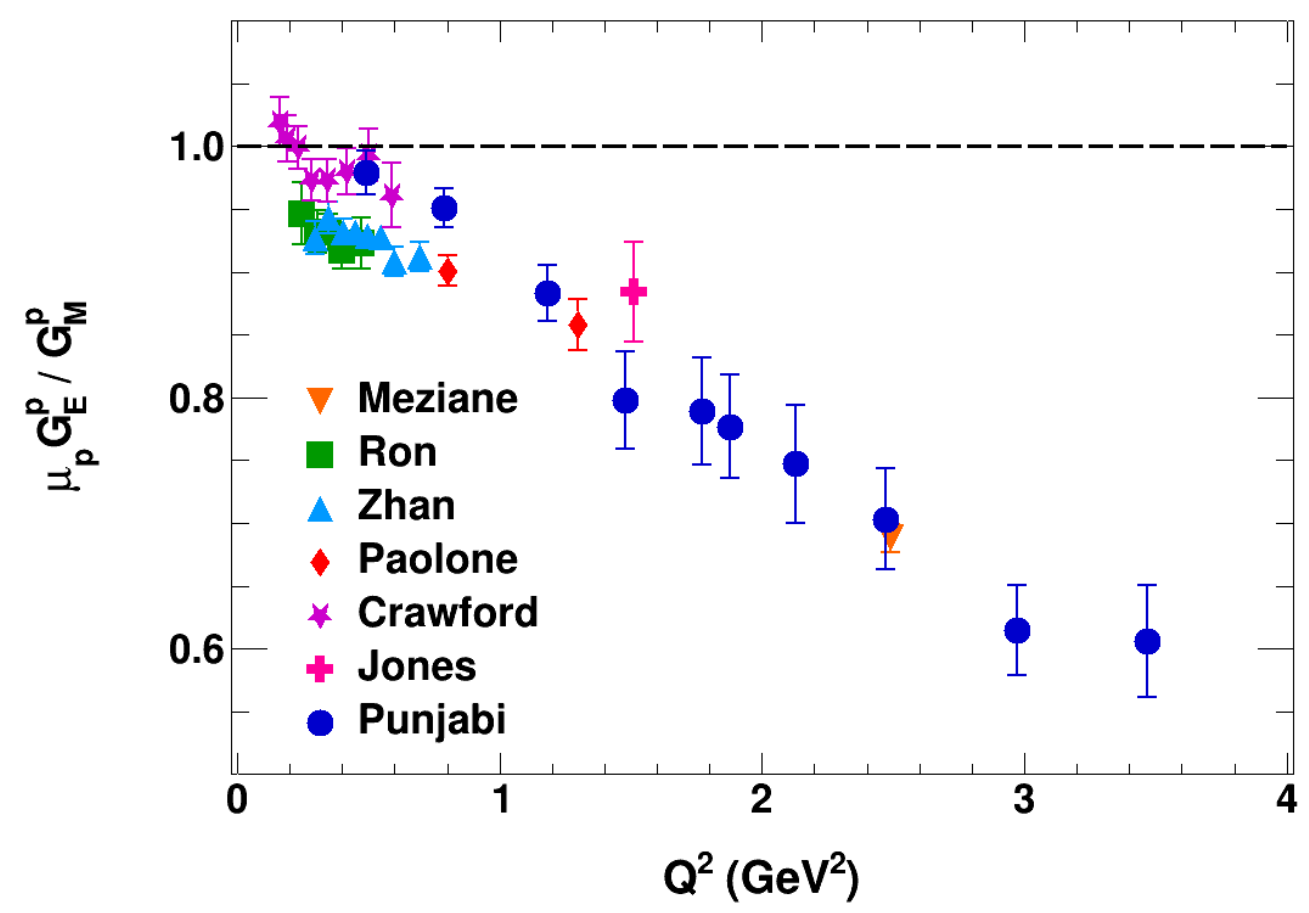

Fig. 3.8: Selected $\mu_{p} G_{E}^{p} / G_{M}^{p}$ data highlighting the discrepancy of low $Q^{2}$ data. Data extracted using polarization transfer: Punjabi 2005 [40, 45], Paolone 2010 [47, Zhan 2011 [49], Ron 2011 [50] and Meziane 2011 [48, 52]. Data extracted via a beam-target asymmetry: Jones 2006 [110] and Crawford 2007 [111].

Recent low $Q^{2}$ data has been plotted in Figure 3.8 where all data extracted the proton form factor ratio using the polarization transfer method except for the data of Crawford et al. (MIT-Bates) and Jones et al. (JLab Hall C) which measured the beamtarget asymmetry using polarized hydrogen and polarized frozen ammonia $\left({ }^{15} \mathrm{NH}_{3}\right)$, respectively. While the Crawford data tends to agree with the two low $Q^{2}$ points of Punjabi, the 2010-2011 data of Ron, Zhan and Paolone taken in Jefferson Lab's Hall A appear to be systematically lower. The reasoning for the apparent difference is currently 
unknown, but is likely due to unaccounted systematics.

At the moment, $G_{E}^{n}$ and therefore the neutron form factor ratio are only known to $Q^{2}=3.4 \mathrm{GeV}^{2}$ while the proton is known to $Q^{2}=8.5 \mathrm{GeV}^{2}$, a factor of 2.5 difference. Pushing $G_{E}^{n}$ measurements to comparable $Q^{2}$ values as its nucleon counterpart is fruitful from an experimental and theoretical point of view; note that a list of upcoming form factor experiments focusing on high $Q^{2}$ may be seen by Table 6.14. For example, flavor decomposition, which provides insight to the $u$ and $d$ quark distributions within the nucleon, is currently limited by the lack of knowledge in $G_{E}^{n}$. Recall that nucleon form factors can be accessed through pure electromagnetic process, but the functions contain fundamental information about the quark and gluon interactions; therefore, theoretical efforts handling the non-perturbative regime must be able to predict nucleon form factors which provides stringent tests on a multitude of theories currently on the market. Before exploring nucleon form factors and theoretical predictions, though, the proton form factor discrepancy, namely the differences in extractions from polarized and unpolarized observables summarized by Figure 3.7, must be addressed.

\subsubsection{Radiative Corrections and Proton Data}

If a Rosenbluth analysis took radiative corrections into account, the "standard" prescription developed by Mo and Tsai [112], published in 1969, or an update to this procedure developed by Maximom and Tjon in 2000 [113] was typically used. Recall that in Chapter 2 the Rosenbluth formula has been derived in the one photon exchange approximation which neglects higher order contributions that are suppressed by additional factors of $\alpha$. Radiative corrections, though, have been shown to affect the 


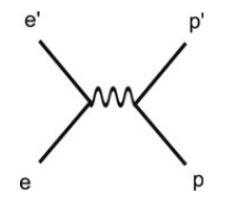

(a) Born term

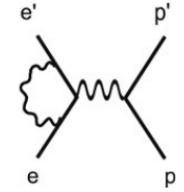

(b) Vertex.

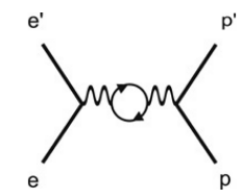

(c) Vacuum.

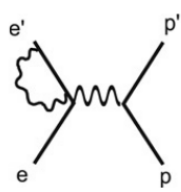

(d) Self energy.
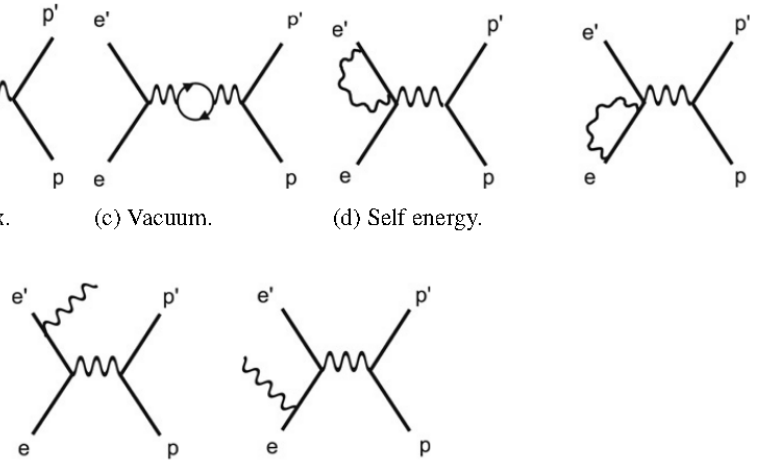

(e) Bremsstrahlung.

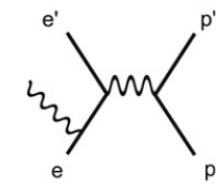

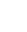

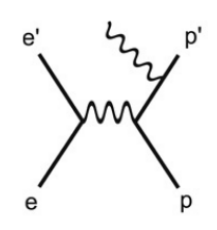

(a) Bremsstrahlung.

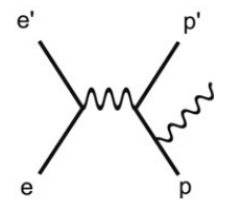

(b) Vertex

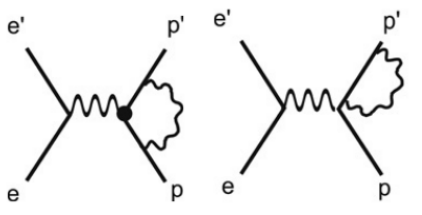

(c) Self energy.

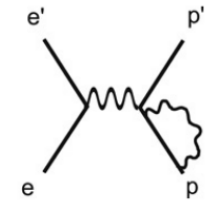

p

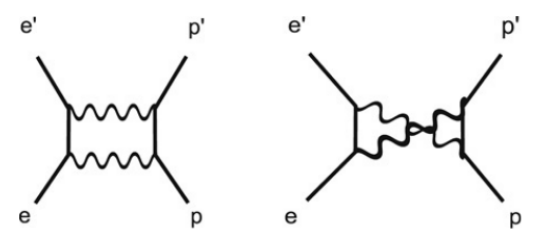

(d) Two-photon.

Fig. 3.9: One loop radiative corrections to elastic $e N$ scattering where the top (bottom) set correspond to the electron (nucleon). Use permitted by Ref. [25].

cross section measurements more so than the double polarization measurements due to the ratio (or ratios of ratios) nature of the asymmetries; corrections tend to cancel when taking the ratio. The corrections to tree-level elastic $e N$ scattering can be seen schematically in Figure 3.9, where the top (bottom) set of diagrams corresponds to the electron (proton), and can be grouped into two categories: 1) one virtual photon has been exchanged, or 2) two virtual photons have been exchanged. In regards to the 
Rosenbluth data, radiative corrections that are important to the form factor extraction effectively change the slope and/or the $y$-intercept of the reduced cross section within Eqn 2.52, Recall that if $\sigma_{R}$ is plotted versus the kinematic parameter $\epsilon$, then a straightline should appear with $\frac{G_{E}^{2}}{\tau}$ as the slope and $G_{M}^{2}$ as the $y$-intercept. It turns out that radiative corrections can change the slope by as much as $\sim 30 \%$ while affecting the double polarization data minimally [48]. Therefore, the canonical radiative correction procedure have made approximations that possibly do not apply at the momentum transfer squared where the discrepancy begins to become large. One such contribution that is a possible source of the discrepancy corresponds to the two-photon exchange (TPEX) contribution (see Refs. [114 119], and the recent review [120]), specifically the "box" and "cross" diagrams in Figure 3.9. If TPEX corrections are important, then nonlinearities may possibly be seen within a $\sigma_{R}$ vs. $\epsilon$ plot; however, Ref. [13] mentions that separating the non-linearity may be difficult experimentally.

In the case where at least two virtual photons are exchanged, the elastic $e N$ scattering amplitude takes the form $[114]^{*}$

$$
i \mathcal{M}=i \frac{e^{2}}{Q^{2}}\left[\bar{u}\left(k^{\prime}\right) \gamma_{\mu} u(k)\right] \times \bar{u}\left(p^{\prime}\right)\left[\tilde{G}_{M} \gamma^{\mu}-\tilde{F}_{2} \frac{P^{\mu}}{M}+\tilde{F}_{3} \frac{\gamma \cdot K P^{\mu}}{M^{2}}\right] u(p),
$$

where $P=\frac{p+p^{\prime}}{2}$ and $K=\frac{k+k^{\prime}}{2}$, and $\tilde{G}_{M}, \tilde{F}_{2}$ and $\tilde{F}_{3}$ are the complex Lorentz invariant structure functions that are not only functions of $Q^{2}$ (as in the case in the Born approximation), but also functions of the longitudinal polarization of the virtual photon $\epsilon$. The generalized form factors are related to the Sachs form factors:

$$
\tilde{G}_{M}=G_{M}\left(Q^{2}\right)+\delta \tilde{G}_{M}\left(Q^{2}, \epsilon\right),
$$

* No attempt will be made to derive radiative corrections as it is well beyond the scope of this thesis. 


$$
\begin{aligned}
\tilde{G}_{E}\left(Q^{2}, \epsilon\right)\left(Q^{2}, \epsilon\right) & \equiv \tilde{G}_{M}-(1+\tau) \tilde{F}_{2}=G_{E}\left(Q^{2}\right)+\delta \tilde{G}_{E}\left(Q^{2}, \epsilon\right), \\
\tilde{F}_{3}\left(Q^{2}, \epsilon\right) & =\delta \tilde{F}_{3}\left(Q^{2}, \epsilon\right) .
\end{aligned}
$$

The complex amplitudes $\delta \tilde{G}_{M}\left(Q^{2}, \epsilon\right), \delta \tilde{G}_{E}\left(Q^{2}, \epsilon\right)$ and $\delta \tilde{F}_{3}\left(Q^{2}, \epsilon\right)$ only exist at the TPEX level or more, and therefore vanish in the Born approximation. Additionally, given the similarities of the form of the generalized vertex correction to the form of $\Gamma^{\mu}$ (Eqn 2.6), the function $\tilde{F}_{3}$ must also vanish at tree-level. Using the above invariant amplitude, the reduced cross section and polarization observable ratio may be written as

$$
\begin{aligned}
\sigma_{r} & =G_{M}^{2}+\frac{\epsilon}{\tau} G_{E}^{2}+\frac{2 \epsilon}{\tau} G_{E} \Re\left(\delta \tilde{G}_{E}+\frac{\nu}{M^{2}} \tilde{F}_{3}\right)+2 G_{M} \Re\left(\delta \tilde{G}_{M}+\frac{\epsilon \nu}{M^{2}} \tilde{F}_{3}\right), \\
R & \equiv-\mu_{p} \frac{P_{t}}{P_{l}} \sqrt{\frac{\tau(1+\epsilon)}{2 \epsilon}}=\mu_{p} \frac{G_{E}}{G_{M}} \Re\left[1-\frac{\delta \tilde{G}_{M}}{G_{M}}+\frac{\delta \tilde{G}_{E}}{G_{E}}+\frac{\nu \tilde{F}_{3}}{M^{2}}\left(\frac{1}{G_{E}}-\frac{2 \epsilon}{1+\epsilon} \frac{1}{G_{M}}\right)\right],
\end{aligned}
$$

where $R \equiv \mu G_{E} / G_{M}, E_{e}\left(E_{e}^{\prime}\right)$ is the energy of the incident (scattered) electron, $\nu=M \frac{E_{e}+E_{e}^{\prime}}{2}, \frac{\nu}{M^{2}}=\sqrt{\tau(1+\tau) \frac{1+\epsilon}{1-\epsilon}}$, and the polarization components $\left(P_{t}\right.$ and $\left.P_{l}\right)$ are calculated using the invariant amplitude defined by Eqn 3.10. The polarization components in the Born approximation have been introduced in Section 2.3.3 and are differentiated here by a "Born" superscript. The normal polarization component denoted by $P_{n}$ is completely imaginary which vanishes in the Born approximation. The notation $\Re$ stands for the real part of the generalized form factors. The generalized corrections to the reduced cross section are additive, i.e. it is the Born result plus TPEX corrections; therefore, even small corrections can affect the slope and/or y-intercept and thus significantly damage the form factor extraction even at modest $Q^{2}$. On the other hand, the correction to the double polarization expression takes the form $R=\mu \frac{G_{E}}{G_{M}}(1+\mathcal{O}(\alpha))$, which is the Born value plus small relative corrections. These considerations, namely the TPEX affecting Rosenbluth extractions while negligibly affecting double polariza- 
tion extractions, could possibly resolve the differences observed in the $G_{E}^{p}$ data, and needs to be demonstrated experimentally.

Recall that at the $2 \gamma$ level, the complex amplitudes depend on $Q^{2}$ and $\epsilon$, and as a result the TPEX contribution can be investigated by searching for an $\epsilon$ dependence within $\sigma_{R}$. In regards to recoil polarimetry, TPEX signatures may be explored by searching for an $\epsilon$ dependence in the ratio $R$ or $\frac{P_{l}}{P_{l}^{\text {Born }}}$, or by an induced normal recoil polarization component as the Born expectation is zero. A high precision recoil polarimetry experiment referred to as GEp-2 $\gamma$ [48] (reanalyzed by Ref. [52]) ran in Jefferson Lab's Hall $\mathrm{C}$ in an attempt to find such an $\epsilon$ dependence in the measured ratio $R=-\mu_{p} \frac{P_{t}}{P_{l}} \sqrt{\frac{\tau(1+\epsilon)}{2 \epsilon}}$ where $P_{t}$ and $P_{l}$ are calculated using the form of the invariant amplitude above, or Eqn 3.10, In the reanalysis in which the uncertainties are significantly smaller than the original publication, Ref. [52] finds $d R / d \epsilon=-0.017 \pm 0.017$. In other words, while the ratio $\frac{P_{l}}{P_{l}^{\text {Born }}}$ does show a small $\epsilon$ dependence, this implies that the transverse component $P_{t}$ experiences a similar effect which ultimately cancels in the ratio $R$; the result is $R$ that is consistent with a constant as predicted in the Born approximation.

A more direct way to study the hard TPEX contribution is to study the cross section ratio $\frac{\sigma\left(e^{+} p\right)}{\sigma\left(e^{-} p\right)}$ which exploits the charge of the lepton, and may be written as

$$
\begin{aligned}
R & =\frac{\sigma\left(e^{+} p\right)}{\sigma\left(e^{-} p\right)} \\
& \approx \frac{1+\delta_{\text {even }}-\delta_{2 \gamma}-\delta_{\text {brem }}}{1+\delta_{\text {even }}+\delta_{2 \gamma}-\delta_{\text {brem }}} \\
& \approx 1-2 \frac{\delta_{2 \gamma}+\delta_{\text {brem }}}{1+\delta_{\text {even }}}
\end{aligned}
$$

where the subscripts even, $2 \gamma$ and brem correspond to the charge-even radiative correc- 
tion factor, the two photon radiative contribution and the bremsstrahlung correction, respectively [121]. The ratio may be corrected for $\delta_{\text {brem }}$ using the prescription by Mo and Tsai, and the bremsstrahlung-corrected ratio denoted by $R^{\prime}$ may be expressed as

$$
R^{\prime} \approx 1-\frac{2 \delta_{2 \gamma}}{1+\delta_{\text {even }}}
$$

As the leading order two photon contribution is due to an interference term between the Born amplitude and the $2 \gamma$ amplitude, $\delta_{2 \gamma}$ may be written as

$$
\delta_{2 \gamma}=\frac{2 \operatorname{Re}\left(\mathcal{M}_{1 \gamma}^{\dagger} \mathcal{M}_{2 \gamma}^{\text {hard }}\right)}{\left|\mathcal{M}_{1 \gamma}\right|^{2}},
$$

where $\mathcal{M}_{1 \gamma}$ is the Born approximation amplitude and $\mathcal{M}_{2 \gamma}^{\text {hard }}$ is the model-dependent "hard" part of the two photon amplitude, $\mathcal{M}_{2 \gamma}=\mathcal{M}_{2 \gamma}^{\text {hard }}+\mathcal{M}_{2 \gamma}^{\text {soft }}$. Note that the "standard" radiative corrections calculate the infrared divergent "soft" part which is needed in order to cancel the bremsstrahlung infrared divergences [122]. Many attempts at calculating the model-dependent hard $2 \gamma$ amplitude* under a variety of assumptions and approximations have been published recently (Refs. [117, 119, 123 125]), some of which partially resolve the Rosenbluth-double polarization discrepancy by reducing the Rosenbluth extracted ratio such that the corrected result is comparable to double polarization data given the uncertainties. In order to search for a TPEX signature using this formalism, namely measuring and correcting $R$ for bremsstrahlung (Eqn 3.15), then an $\epsilon$ dependence in $R^{\prime}$ at a constant $Q^{2}$ would reveal contributions due to the hard TPEX correction. In this case, an $R^{\prime}$ of unity represents the limit of no TPEX contribution; therefore, deviations from this limit indicate hard TPEX contributions. There have been three experiments that used this formalism and recently published: Rachek et al. in 2015

\footnotetext{
* A model-independent calculation is currently unavailable.
} 
[122], Adikaram et al. in 2015 [121], and Henderson et al. in 2017 [126]. While Rachek and Adikaram conclude that the two-photon exchange correction partially resolves the discrepancy, the recently published conclusions of Henderson (Olympus collaboration) disagree with their claims.

In summary, the Rosenbluth-double polarization discrepancy has sparked ongoing intense theoretical and experimental effort ever since the early 2000s. While the disagreement between the two methods is still not fully understood, the TPEX correction at least partially resolves the discrepancy. An issue that has evaded the theory community is that the hard TPEX contribution can only be calculated in a model-dependent manner, resulting in numerous approaches attempting to understand the effect of TPEX to form factor data. What is clear, though, is that the TPEX corrections affect the Rosenbluth data significantly while negligibly modifying the double polarization data. A general consensus among the community has emerged in which double polarization data is viewed to be more reliable than the Rosenbluth data for the reasons described throughout this section, a fact that will continue to be true until the discrepancy is fully resolved.

\subsubsection{Nucleon Charge Radii}

The nucleon radii, more specifically the RMS charge and magnetic radii, can be extracted by analyzing form factor data at low $Q^{2}$ (Eqn 2.61), but for the purposes of this thesis only the charge radius will be discussed in detail. Note if the phrase charge radius is used, RMS charge radius is understood henceforth unless stated otherwise. As there has been tremendous excitement recently in regards to the proton radius, it will 
be discussed first.

The proton charge radius has been extracted from elastic ep scattering experiments ever since the early days of Hofstadter and collaborators, and has been measured many times throughout the years [10, 61, 62, 127]. The 1980 Simon et al. result of $\left\langle r_{E p}^{2}\right\rangle^{1 / 2}=0.862 \pm 0.012 \mathrm{fm}$ determined via elastic $e p$ scattering is highly cited. Recall that the expression for the charge radius requires the slope of the proton charge form factor at $Q^{2}=0$, and as a result analyses fit the data with various functional forms, e.g polynomials or continued fractions.* Furthermore, many theoretically inspired fits have been used, examples include vector meson dominance (VMD) and the more general approach of dispersion relations. Recent extractions from ep scattering [49, 128] and various reanalyses [129 131] have continued to get more precise, resulting in a charge radius that has settled around $0.88 \mathrm{fm}$. Hydrogen spectroscopy also can extract the proton radius with better precision than ep scattering utilizing bound-state QED, and recent measurements agree with scattering extractions. The often quoted 2012 CODATA $^{\dagger}$ value is $0.8775(51) \mathrm{fm}$ [132], and corresponds to electronic measurements coming from ep scattering and hydrogen spectroscopy. However, in 2010 Pohl et al. [133] published a muonic hydrogen $(\mu \mathrm{H})$ extraction exploiting the Lamb shift ${ }^{\ddagger}$ with unprecedented precision. The result of $0.84087(39) \mathrm{fm}$ (2013 Annual Review [134]), a value that differs from electronic measurements by $4 \%$ corresponding to $7 \sigma$, has sparked intense experimental and theoretical discussion amongst the community, and correspondingly the discrepancy has been coined the "proton radius puzzle." One of the defining features

\footnotetext{
* Higher order terms can be added to Eqn 2.61 as well.

$\dagger$ Committee on Data for Science and Technology

$\ddagger$ The $2 \mathrm{~S}_{1 / 2}^{F=1}-2 \mathrm{P}_{3 / 2}^{F=2}$ transition that is dominated by the vacuum polarization diagram for $\mu \mathrm{H}$.
} 
of $\mu \mathrm{H}$ is that the muon is roughly 200 times more massive than the electron, yielding a Bohr radius that is 200 times smaller as $a_{0} \propto \frac{1}{m}$ where $m$ is the mass of the lepton. As a result, the $\mu \mathrm{H}$ S-states are shifted while the P-states are not significantly affected; therefore, the Lamb shift separation in $\mu \mathrm{H}$ is enhanced which provides better sensitivity than traditional hydrogen spectroscopy. The collaboration has extended the technique to muonic deuterium $(\mu d)$ and obtained a more accurate value but $7.5 \sigma$ smaller than the CODATA-2010 value, $r_{d}=2.12562(78)$ fm compared to $r_{d}=2.1424(21) \mathrm{fm}$, respectively [135], which now implies that the puzzle extends beyond just simple hydrogen. There are many attempts to explain the proton radius puzzle, e.g. unexpected QCD corrections, a violation of lepton universality or systematic errors associated to a particular measurement technique (see Refs. 134, 136]), but an explanation is currently unknown. For completeness, the magnetic radius of the proton has also been calculated, e.g. a dispersion analysis [137] and a continued fractions approach [138, to be approximately $0.85 \mathrm{fm}$.

While the neutron charge radius may be extracted in an identical manner by extrapolating charge form factor extractions to $Q^{2}=0$, it leads to results with large uncertainties. Alternatively, the charge radius of the neutron may be calculated by precision measurements of the scattering length between electrons and thermal neutrons denoted by $b_{\text {ne }}$, which takes the following form:

$$
b_{\mathrm{ne}}=\frac{M}{3 m_{e} a_{0}}\left\langle r_{\mathrm{En}}^{2}\right\rangle
$$

where $m_{e}(M)$ is the mass of the electron (neutron), $a_{0}$ is the Bohr radius and $\left\langle r_{\text {En }}^{2}\right\rangle \approx$ 
$-\left.6 \frac{d G_{E}^{n}}{d Q^{2}}\right|_{Q^{2} \rightarrow 0}[139,140] *^{*}$ Foldy showed that the electron-neutron interaction may be written as

$$
b_{\mathrm{ne}}=\frac{M}{3 m_{e} a_{0}}\left(\int d^{3} \mathbf{r} r^{2} \rho_{\mathrm{ch}}(\mathbf{r})+\frac{3 \mu_{n}}{2 M^{2}}\right)
$$

where $\rho_{\text {ch }}$ represents the neutron's intrinsic charge density and $\mu_{n}$ is the magnetic moment of the neutron. Therefore, by combining Eqn 3.17 and Eqn 3.18 the expression for the neutron charge radius becomes

$$
\left\langle r_{\mathrm{En}}^{2}\right\rangle=\left\langle r_{\mathrm{ch}}^{2}\right\rangle+\frac{3 \mu_{n}}{2 M^{2}}
$$

where $\left\langle r_{\mathrm{ch}}^{2}\right\rangle$ is the mean square radius of the intrinsic charge density. In order to understand the difference between $r_{\mathrm{ch}}$ and $r_{\mathrm{En}}$, this expression can be derived without any mention of the neutron-electron interaction length. Starting with $G_{E}^{n}=F_{1}^{n}-\frac{Q^{2}}{4 M^{2}} F_{2}^{n}$ (Eqn 2.41) and recalling the Fourier transform interpretation, then the form factors may be expanded in powers of $Q^{2}$, or

$$
\begin{aligned}
G_{E}^{n} & =F_{1}^{n}-\frac{Q^{2}}{4 M^{2}} F_{2}^{n}, \\
-\frac{1}{6}\left\langle r_{\mathrm{En}}^{2}\right\rangle Q^{2} & \approx-\frac{1}{6}\left\langle r_{1 \mathrm{n}}^{2}\right\rangle Q^{2}-\frac{Q^{2}}{4 M^{2}} \mu_{n}, \\
\Rightarrow\left\langle r_{\mathrm{En}}^{2}\right\rangle & =\left\langle r_{1 \mathrm{n}}^{2}\right\rangle+\frac{3 \mu_{n}}{2 M^{2}},
\end{aligned}
$$

where only terms $\propto Q^{2}$ are kept. It is explicit that $\left\langle r_{1 \mathrm{n}}^{2}\right\rangle=\left\langle r_{\mathrm{ch}}^{2}\right\rangle$, and therefore $\left\langle r_{\mathrm{ch}}^{2}\right\rangle$ corresponds to the intrinsic electric form factor of the neutron, or $F_{1}^{n}$. The so-called Foldy term, or the $\frac{3 \mu_{n}}{2 M^{2}}$ term, has a numerical value of $-0.126 \mathrm{fm}^{2}$. The value of the mean-square charge radius of the neutron has been stable for decades, and the 2014 Particle Data Group average value is $-0.1161 \pm 0.0022 \mathrm{fm}^{2}$ [6]. However, the

\footnotetext{
* Fermi and Marshall attempted the first measurement of $b_{\text {ne }}$ in 1947 [141].
} 
interpretation of this number has not been stable which is partly due to the numerical closeness of the Foldy term and the mean-square charge radius. Is the slope of the Sachs electric form factor data evaluated at zero really a measure of how the neutron charge is distributed radially, or is this value dominated by the anomalous magnetic moment Foldy term? If the contribution is mostly attributed to the Foldy term, then this would imply that the mean-charge radius is much smaller than expected and that the measured value is dominated by relativistic effects. ${ }^{*}$ In 1999, Isgur addressed this question and showed that in a relativistic approximation to the constituent quark model, the Foldy term is exactly cancelled by a relativistic correction to the Dirac form factor $F_{1}^{n}[142$, and has been independently confirmed by [143, 144]. In this light, this result suggests that $r_{E n}^{2}$ may safely be interpreted as arising from the internal charge distribution of the neutron within its rest frame, and the numerical closeness of the Foldy term and the mean-square charge radius of the neutron is simply a coincidence. Consequently, experiments measuring the electron-neutron interaction length $b_{\text {ne }}$ are in fact extracting information about the charge radius of the neutron, and not the Foldy term. For completeness, the RMS magnetic radius has been extracted to be $0.873 \pm 0.015 \mathrm{fm}$ using a continued fractions approach [138] and $0.862_{-0.008}^{+0.009}$ fm from a dispersion analysis [145].

\subsection{Parametrizations}

There are situations where it is convenient to have a simple parametrization in order to quickly calculate the four nucleon form factors. Many parametrizations exist as updates typically occur when additional form factor data become available, but only a handful

\footnotetext{
* The origin of the Foldy term comes from the well-known phenomenon of Zitterbewegung.
} 
are discussed here.

\subsubsection{Dipole Form Factor}

The ubiquitous dipole form factor continues to exist primarily for historical reasons and convenience; the form has been introduced by Eqn 3.1. The literature commonly normalizes form factor data by the dipole form factor where $\Lambda^{2}=0.71 \mathrm{GeV}^{2}$ is fixed by convention, a relic of the empirical scaling relations observed in the early days.

In the non-relativistic limit, it has been shown in Eqn 2.60 that the electric and magnetic Sachs form factors may be interpreted as a Fourier transform of the charge and magnetic density of the nucleon, respectively. In this intuitively naive picture, the dipole form factor corresponds to the following spherically symmetric charge distribution, which is normalized such that the volume integral equates to unity:*

$$
\rho(r)=\frac{\Lambda^{3}}{8 \pi} e^{-\Lambda r}
$$

The dipole form factor may be obtained by taking the Fourier transform of this charge density:

$$
\begin{aligned}
G_{D} & =2 \pi \frac{\Lambda^{3}}{8 \pi} \int_{0}^{\infty} d r r^{2} e^{-\Lambda r} \int_{-1}^{1} d(\cos \theta) e^{i q r \cos \theta} \\
& =\frac{\Lambda^{3}}{4 i q} \int_{0}^{\infty} d r r e^{-\Lambda r}\left(e^{i q r}-e^{-i q r}\right), \\
& =\frac{1}{\left(1+\frac{Q^{2}}{\Lambda^{2}}\right)^{2}} .
\end{aligned}
$$

The low $Q^{2}$ behavior of $G_{E}^{p}$ is decently described by the dipole form factor as seen by Figure 3.1 while the magnetic form factors roughly follow the dipole scaling law. On the

\footnotetext{
* This exercise is done on page 155 of Ref. 146] for example.
} 
other hand, $G_{E}^{n}$ is not described by this parametrization at all since $G_{E}^{n} \rightarrow 0$ as $Q^{2} \rightarrow 0$. By inserting the expression of $G_{D}$ into Eqn 2.61, the RMS proton charge radius may be estimated:

$$
\left\langle r^{2}\right\rangle=-\left.6\left(\frac{d G_{D}\left(Q^{2}\right)}{d Q^{2}}\right)\right|_{Q^{2} \rightarrow 0}=\frac{12}{\Lambda^{2}} \quad \Rightarrow \sqrt{\left\langle r^{2}\right\rangle} \approx 0.8 \mathrm{fm}
$$

where $1 \mathrm{fm}^{-1}=197.3 \mathrm{MeV}$. Remarkably, a simple model assuming a radially symmetric charge density yields a RMS proton charge radius that is roughly the measured size.

\subsubsection{Galster Fit}

Since the dipole form factor does not describe $G_{E}^{n}$, an alternative parametrization was proposed by Galster et al. [74] and takes the following form:

$$
G_{E}^{n}\left(Q^{2}\right)=-\frac{\mu_{n} \tau}{1+b \tau} G_{D}\left(Q^{2}\right)
$$

where $b=5.6, \tau=\frac{Q^{2}}{4 M^{2}}$ and the fit may be seen amongst unpolarized elastic ed cross section data within Figure 3.3 . Evaluating the neutron mean square charge radius using this parametrization results in the following:

$$
\left\langle r^{2}\right\rangle=-\left.6\left(\frac{d G_{E}^{n}\left(Q^{2}\right)}{d Q^{2}}\right)\right|_{Q^{2} \rightarrow 0}=\frac{3 \mu_{n}}{2 M^{2}}
$$

which is equal to the Foldy term discussed in Section 3.1 .5

\subsubsection{Kelly and Galster-like Fits}

In 2004, Kelly provided fits for $G_{E}^{p}, G_{M}^{p}$ and $G_{M}^{n}$ using a ratio of polynomials in $\tau$, and focused on the limiting behavior for both $Q^{2} \rightarrow 0$ and $Q^{2} \rightarrow \infty$ [147]. Motivated by 
pQCD dimensional scaling rules, the parametrization takes the following form:

$$
G\left(Q^{2}\right) \propto \frac{\sum_{k=0}^{n} a_{k} \tau^{k}}{1+\sum_{k=1}^{n+2} b_{k} \tau^{k}},
$$

where the degree in the denominator is $n+2$ such that $G \propto Q^{-4}$ for large $Q^{2}$. If the magnetic form factors are being calculated then a factor of $\mu$ needs to be introduced on the right-hand side so the low $Q^{2}$ behavior is retained. This approach did not work well for $G_{E}^{n}$; therefore, Kelly used a Galster-like parametrization:

$$
G_{E}^{n}\left(Q^{2}\right)=\frac{A \tau}{1+B \tau} G_{D}\left(Q^{2}\right)
$$

where $A=1.70 \pm 0.04, B=3.30 \pm 0.32$ and $G_{D}\left(Q^{2}\right)$ is the typical dipole form factor. The coefficients of the Galster-like fit have been updated to $A=1.39$ and $B=2.00$ by Riordan et al. in 2010 [7] when higher $Q^{2}$ data was available.

\subsubsection{Diehl and Kroll}

A recent paper by Ref. [57] approached a nucleon form factor parametrization in terms of flavor form factors and used a product of two fractional power laws:

$$
F_{i}^{q}\left(Q^{2}\right)=\frac{F_{i}^{q}(0)}{\left(1+\frac{a_{i, q}}{p_{i, q}} Q^{2}\right)^{p_{i, q}}\left(1+\frac{b_{i, q}}{q_{i, q}} Q^{2}\right)^{q_{i, q}}},
$$

where the flavor is denoted by $q=u, d$ and $i=1,2$. In other words, $F_{i}^{q}\left(Q^{2}\right)$ parametrizes the Dirac and Pauli form factor of the $u$ and $d$ quarks if the strangeness contribution is neglected. The remaining terms, or $a_{i}, b_{i}, q_{i}$ and $p_{i}$, are the fit parameters. Contrary to the motivations of Kelly, Diehl and Kroll did not impose the asymptotic scaling behaviors predicted by dimensional scaling. This argument is motivated by a Generalized Parton Density (GPD) fit, suggesting that dimensional scaling is not relevant for the 
Sachs form factors in the $Q^{2}$ range that is currently experimentally accessible. The flavor form factors have the following $Q^{2} \rightarrow 0$ behavior:

$$
\begin{array}{ll}
F_{1}^{u}(0)=2, & F_{2}^{u}(0)=2\left(\mu_{p}-1\right)+\mu_{n}, \\
F_{1}^{d}(0)=1, & F_{2}^{d}(0)=\left(\mu_{p}-1\right)+2 \mu_{n},
\end{array}
$$

where the $Q^{2} \rightarrow 0$ behavior of the nucleon form factors is defined in Eqn 2.54. The nucleon Dirac and Pauli form factors are related to the flavor form factors by

$$
\begin{aligned}
& F_{i}^{p}=e_{u} F_{i}^{u}+e_{d} F_{i}^{d}, \\
& F_{i}^{n}=e_{u} F_{i}^{d}+e_{d} F_{i}^{u},
\end{aligned}
$$

where $e_{u}=2 / 3$ and $e_{d}=-1 / 3$. Lastly, the Sachs form factors may be calculated by the following linear combinations (Eqn 2.41 and Eqn 2.42):

$$
\begin{aligned}
& G_{E}^{i}=F_{1}^{i}-\tau_{i} F_{2}^{i}, \\
& G_{M}^{i}=F_{1}^{i}+F_{2}^{i},
\end{aligned}
$$

where $i=p, n$ for the proton or neutron. It is noted that Diehl and Kroll paid special

attention to the data sets included in the fits. Additionally, more high $Q^{2}$ data was available making this parametrization more suitable for calculations that are out of the scope of Kelly's parametrization.

\subsection{Theoretical Predictions}

In the asymptotically free regime, QCD may be solved perturbatively; however, when the strong coupling constant becomes appreciable then non-perturbative methods are 
required. The cross over region for hard exclusive processes such as elastic $e N$ scattering is believed to occur at high- $Q^{2}$, much larger than what has already been explored. Over the years, many phenomenological models have been introduced, which is a reflection of the difficulty of the non-perturbative region. Currently, the only technique of solving the QCD equations for the nucleon from first principles is lattice QCD, and as of 2018 there are a fair amount of caveats that come along with that statement. Recall that historically there were many theories attempting to explain the strong interaction prior to the introduction of QCD, some of which had considerable success. The development of QCD combined and inherited concepts firmly established in existing theories, e.g. symmetries of the quark model and the success of a renormalizable field theory like QED. Nucleon form factors put strong constraints on the fundamental theory of strong interactions, and the correct model must explain properties of the nucleon as well as the nucleon form factors, both space-like and time-like. In this light, several successful phenomenological models will be discussed in the context of nucleon form factors.

\subsubsection{Vector Meson Dominance}

An early model attempting to describe the interaction between hadrons and photons is within the framework of vector meson dominance (VMD), originally developed by Sakurai in the early 1960s [148 [149 and has continued to be extended by others. The fundamental idea rests upon the fact that the photon has the same $J^{P C}$ quantum numbers as the following lightest vector mesons: $\rho(770), \omega(782)$, and $\phi(1020)$. In this model, the photon may couple to the hadron via the exchange of these low lying vector mesons. As these mesons frequently show up in $e^{-} e^{+} \rightarrow$ hadrons in the form of resonances, 
it is expected that in the low space-like $Q^{2}$ scattering process $e N \rightarrow e N$ the nucleon electromagnetic form factors receive dominant contributions by these poles [25]. In the framework of VMD, the isoscaler and isovector Pauli and Dirac form factors may be written as

$$
F_{1,2}^{\mathrm{is}, \mathrm{iv}}\left(Q^{2}\right)=\sum_{i \text { mesons }} \frac{m_{i}^{2} C_{\gamma V_{i}}}{Q^{2}+m_{i}^{2}} F_{V_{i} N}\left(Q^{2}\right)
$$

where $C_{\gamma V_{i}}$ is the photon-meson coupling strength to be determined experimentally or kept as free parameters in a fit to the nucleon form factors, $F_{V N}$ is the meson-nucleon vertex form factors, ${ }^{*}$ and lastly the sum includes the mesons of mass $m_{i}$ that have been incorporated into the calculation [53. While early VMD models typically used the three lowest-lying vector mesons, additions are made in VMD extensions which is reflected by the summation. A functional form for the meson-nucleon vertex form factor, $F_{V N}$, is required. For example, the monopole and dipole forms are commonly used within the early literature, or $F_{V N}=\left(1+\frac{Q^{2}}{\Lambda^{2}}\right)^{-n}$ where $n=1$ and 2 , respectively. Furthermore, the formalism above neglects the widths of the vector mesons, but extensions to the basic VMD model take this into account. The experimentally observed Sachs form factors may then be calculated by the following linear combinations:

$$
\begin{aligned}
G_{E} & =\frac{1}{2}\left(F_{1}^{\mathrm{is}} \pm F_{1}^{\mathrm{iv}}\right)-\tau \frac{1}{2}\left(F_{2}^{\mathrm{is}} \pm F_{2}^{\mathrm{iv}}\right), \\
G_{M} & =\frac{1}{2}\left(F_{1}^{\mathrm{is}} \pm F_{1}^{\mathrm{iv}}\right)+\frac{1}{2}\left(F_{2}^{\mathrm{is}} \pm F_{2}^{\mathrm{iv}}\right)
\end{aligned}
$$

where the $+(-)$ is specific to the proton (neutron).

VMD has had considerable success in explaining features of low $Q^{2}$ nucleon form factor data. Perhaps most notably, VMD fits to nucleon form factor data by Iachello,

\footnotetext{
* Sometimes called the intrinsic nucleon form factor.
} 
Jackson, and Lande (IJL) in 1973 predicted the almost linear decrease of the proton form factor ratio $\left(Q^{2}>1 \mathrm{GeV}^{2}\right)$ almost three decades before the Jefferson Lab Hall A data initiated the disagreement [150]. This early VMD model included the $\rho, \omega$, and $\phi$ mesons, and the width of the $\rho$ is incorporated by making the following alteration to the $F_{1,2}^{\text {is,iv }}$ prototype:

$$
\frac{m_{\rho}^{2}}{m_{\rho}^{2}+Q^{2}} \rightarrow \frac{m_{\rho}^{2}+8 \Gamma_{\rho} m_{\pi} / \pi}{\left(m_{\rho}^{2}+Q^{2}\right)+\left(4 m_{\pi}^{2}+Q^{2}\right) \Gamma_{\rho} \alpha\left(Q^{2}\right) / m_{\pi}}
$$

where $\Gamma_{\rho}=112 \mathrm{MeV}$ and $\alpha\left(Q^{2}\right)$ is

$$
\alpha\left(Q^{2}\right)=\frac{2}{\pi}\left[\frac{4 m_{\pi}^{2}+Q^{2}}{Q^{2}}\right]^{1 / 2} \ln \left[\frac{\sqrt{4 m_{\pi}^{2}+Q^{2}}+\sqrt{Q^{2}}}{2 m_{\pi}}\right] .
$$

The widths of the $\omega$ and $\phi$ mesons are small compared to the $\rho$ and are consequently neglected in this work. Several function forms of the meson-nucleon vertex form factors were used including the monopole and dipole. While the fits to the then-existing proton form factor data was in good agreement, the fits to the neutron data were not as good; recall that the $G_{E}^{n}$ data in 1973 was sparse and subject to large uncertainties.

In the mid-1980s, Gari and Krümpelmann were able to analyze nucleon form factors with the belief that QCD correctly describes the strong interaction [151]. In their extended VMD (EVMD) model, ${ }^{*}$ VMD techniques are invoked while enforcing large $Q^{2}$ perturbative QCD asymptotic predictions:

$$
\begin{aligned}
& F_{1} \sim\left[\frac{1}{Q^{2} \log \left(Q^{2} / \Lambda_{\mathrm{QCD}}^{2}\right)}\right]^{2}, \\
& F_{2} \sim \frac{F_{1}}{Q^{2}} .
\end{aligned}
$$

\footnotetext{
* Sometimes referred to as QCD-VM model within the literature.
} 
In other words, the model connects the low $Q^{2}$ behavior in which the nucleon form factors are dominated by meson dynamics and attempts to interpolate to high $Q^{2}$ where $F_{1}$ and $F_{2}$ are completely dominated by quark dynamics. Furthermore, in 1991 the authors reanalyzed the nucleon form factor data within the QCD-VM framework, focusing on the strange quark and its role in the electric form factor of the neutron, $G_{E}^{n}[151$. The analysis finds that the decrease in $G_{E}^{n}$ around $Q^{2} \approx 1 \mathrm{GeV}^{2}$, or in other words the relative suppression of the Pauli form factor, may be explained by the effect of the strange quarks, modeled by the coupling of the $\phi$ meson to the nucleon in a two step process. To be more specific, due to the OZI rule, the Dirac form factor of the nucleon does not see strange quark contributions at low $Q^{2}$; however, at intermediate $Q^{2}$, there is a possible two-step process that allows for a coupling between the $\phi$ meson and the nucleon, highlighting the importance of the strange meson cloud. While a small $G_{E}^{n}$ at low $Q^{2}$ may indicate the importance of strange quarks within the nucleon, it appears not to significantly affect the remaining three nucleon form factors in this model. Various fits were performed to $G_{E}^{n}$ but it is noted that the data quality at the time for the neutron was quite poor, most of the data points originate from Platchkov [70.

The model of Gari and Krümpelmann (GK) was further extended by Lomon [152] [153] after the publication of Jefferson Lab's Hall A $G_{E}^{p} / G_{M}^{p}$ data in 2000 [40] and 2002 [43]. The 2001 paper [152] extends the GK approach in which the $\rho$ meson width contribution is obtained by the result of dispersion relations; additionally, the isovector $\rho^{\prime}(1450)$ vector meson pole has been added to the analysis. The 2002 reanalysis [153] incorporated the $\omega^{\prime}(1419)$ isoscaler vector meson pole and found a better agreement to 
the then-newly released JLab proton form factor data.

The 1973 IJL model was developed prior to the development of QCD and consequently the predictions of pQCD; therefore, a "reanalysis" was released in 2004 by Iachello and Wan [154 and Bijker and Iachello [155] utilizing the extensions of GK among others. It is noted that the Madey [102], or the Plaster reanalysis [54], neutron form factor ratio data via recoil polarimetry was available for these analyses. The former paper [154] analyzes the then-current status of extended VMD, namely the approach of GK with a dipole form of the intrinsic form factor, and highlights the disagreement between the model and the neutron form factor data. In order to explore the discrepancy further, the authors focus on the calculation of time-like nucleon form factors, obtainable theoretically by analytic continuation of the intrinsic form factor and the vector meson form factors. Experimentally, the time-like nucleon form factors may be accessed in the following reactions: $e^{+} e^{-} \rightarrow p \bar{p}, e^{+} e^{-} \rightarrow n \bar{n}$, and $p \bar{p} \rightarrow e^{+} e^{-}$. The authors conclude that additional contributions to the model are required in order to explain the neutron data which is presented in the latter manuscript, [155]. In this paper, the intrinsic structure of the nucleon is assumed to be given by a three valence quark structure following $\mathrm{SU}(6)$, namely that the three-quark contribution to the anomalous moment is purely isovector. Additionally, the intrinsic form factor in this analysis is assumed to be dipole which is consistent with pQCD. The authors acquire more consistent fits to the double polarization neutron form factor data while maintaining good agreement to the other form factors.

VMD has a long history with quite a few differing attempts and reanalyses as 
newer data became available. At the end of the day, the model does require fitting to data and overall has little predictive power; this may be trivially seen by comparing Lomon's attempts from 2001-2006 to the $2010 G_{E}^{n} / G_{M}^{n}$ extractions of Riordan et al. The theory did predict, perhaps fortuitously, the fall-off in the proton form factor ratio in the early 1970s and continues to provide a useful physics interpretation of low $Q^{2}$ nucleon form factors. Some of the successful VMD fits to the form factor ratio of the proton and neutron may be seen by Figure 3.10 and Figure 3.11, respectively. Note that the VMD models are a special case of the more general dispersion relation approach which is not discussed here.

\subsubsection{Perturbative QCD}

As introduced in Chapter 1. QCD has unique behavior at large and small energy scales, namely asymptotic freedom and color confinement, respectively. These properties are built into the renormalizable, non-Abelian field theory where the color SU(3) symmetry is identified as the gauge group. The ability to solve the theory in a perturbative manner depends on the value of the strong coupling constant $\alpha_{s}$, given by Eqn 1.2 , For elastic nucleon scattering and at large energy scales $(100 \mathrm{GeV}-\mathrm{TeV})$, the value of the strong couple constant is roughly $\alpha_{s} \sim 0.1$ and perturbation theory is applicable; however, at small energy scales, or below $\sim 1 \mathrm{GeV}$, the theory becomes strongly interacting and non-perturbative methods are required. The energy scales encountered within this thesis are on the order of a couple $\mathrm{GeV}$ which is within the region connecting the strongly interacting to the asymptotically free regime. In this light, it is instructive to discuss the predictions of pQCD in the context of nucleon form factors. 


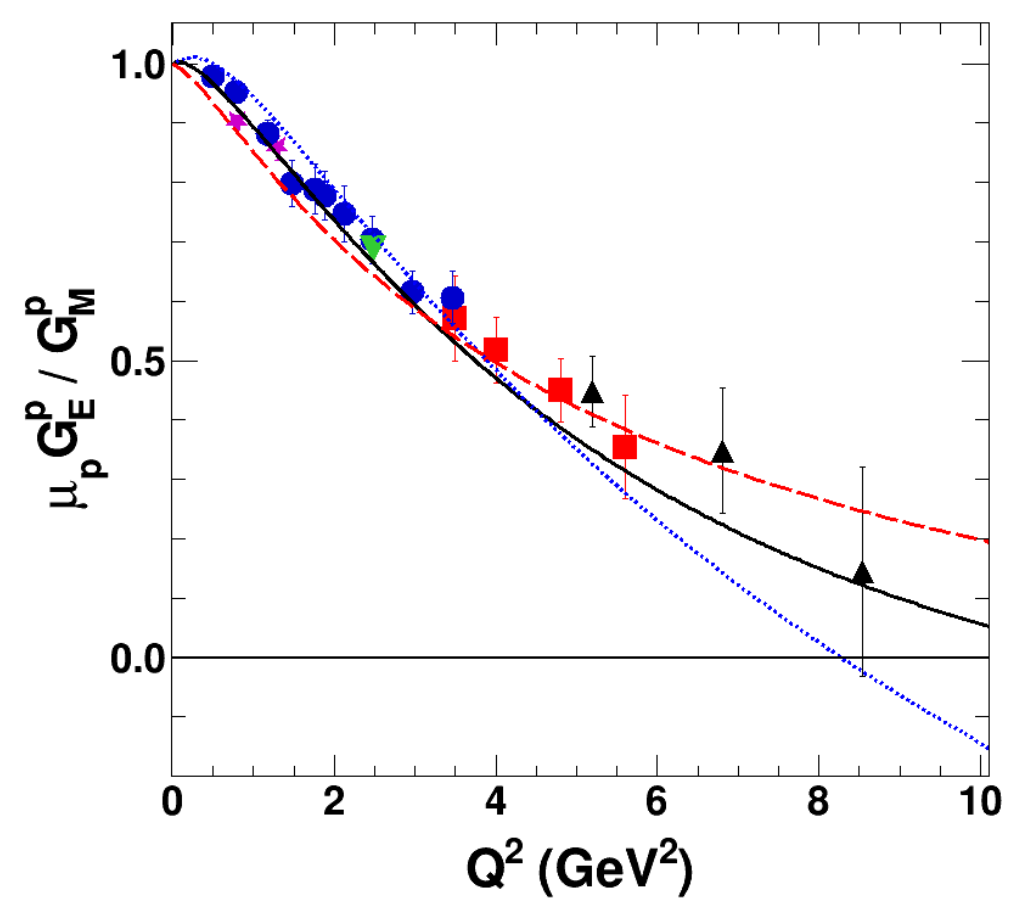

Fig. 3.10: Selected VMD fits to the proton form factor ratio data extracted via double polarization and referenced in Figure 3.7. The dotted curve (blue) is the early fit of IJL [150], the dashed curve (red) is that of Bijker and Iachello [155] and lastly the solid curve (black) is a fit of Lomon [156].

In the limit of large momentum transfer, early pQCD analyses predict scaling in the ratio of the nucleon form factors $F_{2} / F_{1}$. Assuming the nucleon is comprised of three valence quarks and in the context of elastic scattering, then in this limit the virtual photon makes a hard collision with only one quark. In order for elastic scattering to occur, the struck quark must interact with the remaining two nearly collinear quarks via the exchange of two hard gluons. The predictions for such an exclusive process yield the following form factor scaling relations for asymptotically large $Q^{2}: Q^{4} F_{1} \sim$ constant and 


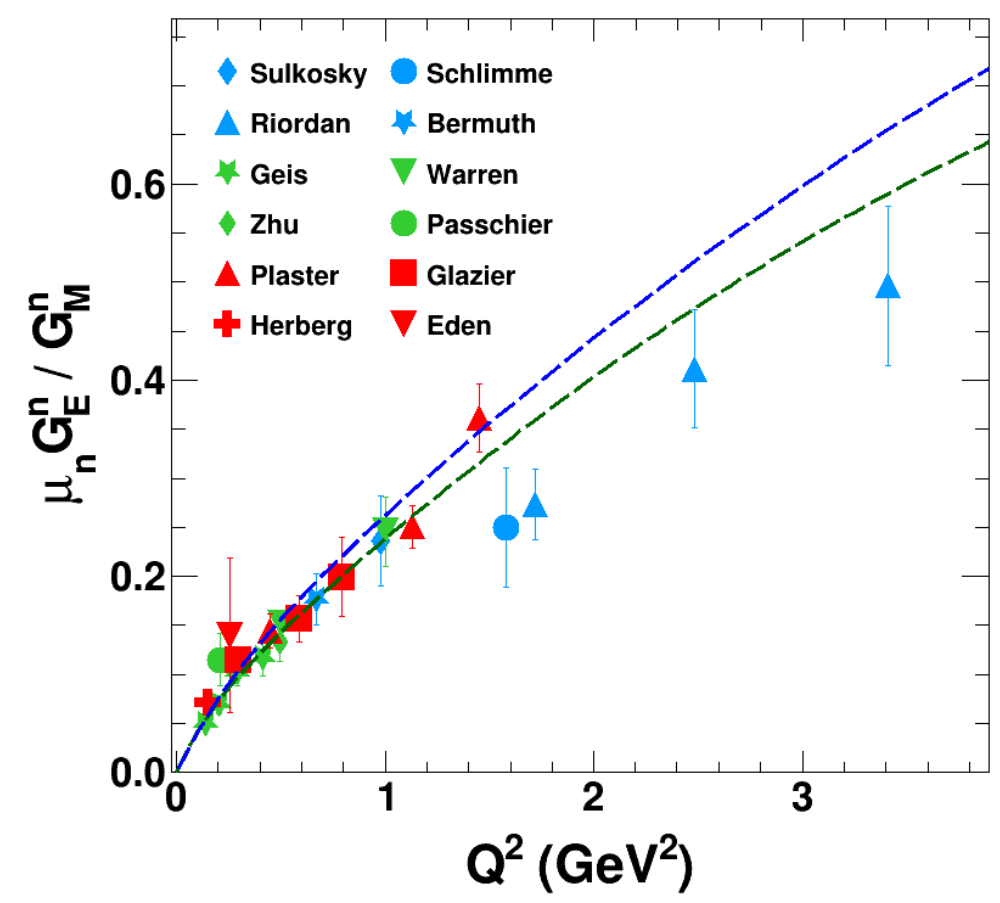

Fig. 3.11: Selected VMD fits to the form factor ratio of the neutron extracted via double polarization, see Figure 3.5 for the references. The blue dashed curve is by Bijker and Iachello [155] and the green dashed curve is the fit of Lomon [156], both of which were published prior to the data of Refs. [7, 107].

$Q^{2} \frac{F_{2}}{F_{1}} \sim$ constant as calculated by Brodsky and Farrar [157, 158] and Matveen et al. [159] in 1973. In other words, the hadron helicity-conserving form factor $F_{1}\left(Q^{2}\right)$ is predicted to have a dominant scaling behavior of $Q^{-4}$ while $F_{2}\left(Q^{2}\right)$, which is related to the hadron helicity-flip amplitude, is expected to fall like $Q^{-6}$. As many recent experiments extract the Sachs form factor ratio, it is beneficial to have a relation between $F_{2} / F_{1}$ and the form factor ratio $r \equiv G_{E} / G_{M}$, and may be obtained by a simple rearrangement of 
Eqn 2.41 and Eqn 2.42;

$$
\frac{F_{2}}{F_{1}}=\frac{1-r}{\tau+r},
$$

where $\tau=Q^{2} / 4 M^{2}$.

Further contributions to this framework were provided by Belitsky et al. in 2003 [160]. The authors argue the importance of quark orbital angular momentum in regards to the spin-flip form factor; therefore, the pQCD framework is extended to handle quark orbital angular momentum utilizing light-cone wave functions. The authors claim that in $e p$ scattering, the virtual photon cannot flip the spin of massless (or close to massless) quarks, it must be put in a state in which the angular momentum is nonzero with projection $\left|L_{z}\right|=1$. The result of such a calculation gives the coefficient of the dominant power term to the helicity-flip form factor and depends on twist-three and twist-four light-cone wave functions. The dominant scaling agrees with previous pQCD calculations, namely a $Q^{-6}$ scaling is observed in $F_{2}$, but modified by a logarithmic coefficient:

$$
\frac{F_{2}\left(Q^{2}\right)}{F_{1}\left(Q^{2}\right)} \sim \frac{\log ^{2}\left(Q^{2} / \Lambda^{2}\right)}{Q^{2}}
$$

where $\Lambda$ is a non-perturbative mass scale and typically chosen to be $200-400 \mathrm{MeV}$ in the literature.

The quantity $Q^{2} \frac{F_{2}}{F_{1}}$ is compared to recent proton form factor data extracted via double polarization or the Rosenbluth method (green data points), and may be seen by the top panel of Figure 3.12. While the Rosenbluth data begins to show constant behavior around $Q^{2}=2 \mathrm{GeV}^{2}$, the double polarization data displays no flattening in 

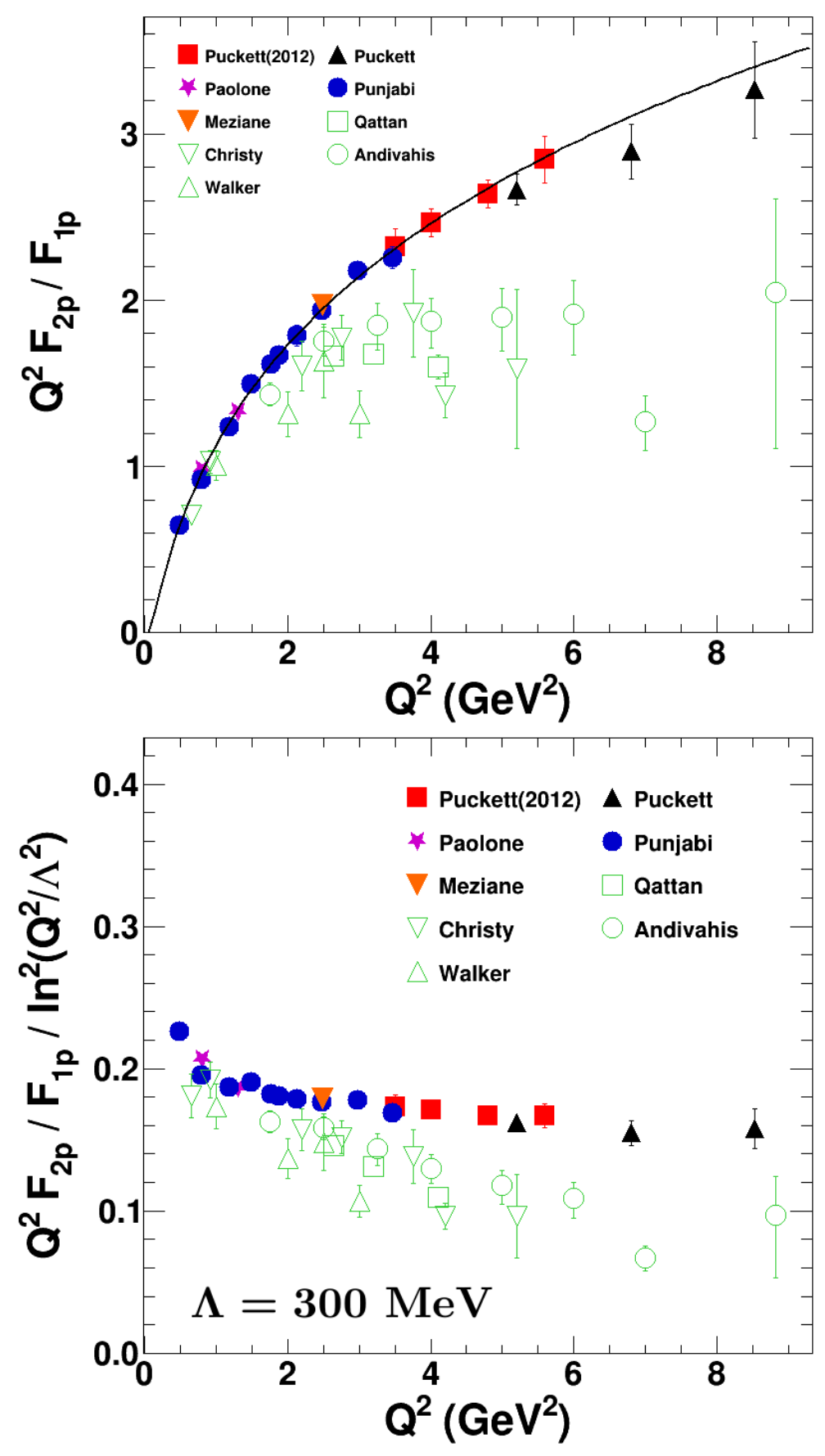

Fig. 3.12: Proton data are plotted as $Q^{2} F_{2}^{p} / F_{1}^{p}$ and $Q^{2} F_{2}^{p} / F_{1}^{p} / \ln ^{2}\left(Q^{2} / \Lambda^{2}\right)$ in the top and bottom panels, respectively, and highlighting the difference between double polarization and Rosenbluth data (green data points). For details about the fit in the top panel, see Eqn 3.45 . 
the $Q^{2}$ range in which data exists. The black curve is from Belitsky et al. [160], or

$$
Q^{2} \frac{F_{2}^{p}}{F_{1}^{p}}=\frac{A}{\kappa_{p}} \ln ^{2}\left(\frac{Q^{2}}{\Lambda^{2}}\right)
$$

and has been fit to the double polarization data resulting in $A=0.238$ and $\Lambda=$ $232 \mathrm{MeV}$. The logarithmically modified behavior appears to describe the proton data reasonably well. Furthermore, the bottom panel of Figure 3.12 plots the proton data as $Q^{2} F_{2}^{p} / F_{1}^{p} / \ln ^{2}\left(Q^{2} / \Lambda^{2}\right)$ in an attempt to show the extended pQCD prediction, or

$$
Q^{2} \frac{F_{2}^{p}}{F_{1}^{p}} \frac{1}{\ln ^{2}\left(Q^{2} / \Lambda^{2}\right)} \sim \text { constant }
$$

with a mass scale parameter of $300 \mathrm{MeV}$. The data does seem to follow the extended prediction, and it will be interesting to see if higher $Q^{2}$ data continues to support the modified scaling behavior.

The neutron, on the other hand, has a sparse data selection in order to perform a

similar analysis. The results for the quantity $Q^{2} \frac{F_{2}}{F_{1}}$ may be seen by Figure 3.13 , and the low $Q^{2}$ behavior obviously does not follow the modified logarithmic scaling prediction. The higher $Q^{2}$ data of Riordan et al. inconclusively follows the scaling behavior. Due to the lack of data, this type of behavior cannot be definitively ruled out as it is possible that higher $Q^{2}$ data will support the extended pQCD predictions. The black curve uses the same fitting parameters as Eqn 3.45, and has been normalized to match the data.

\subsubsection{Generalized Parton Distributions}

An alternative way to view the nucleon may be given within the framework of Generalized Parton Distributions (GPDs) in which concepts from parton distributions and 


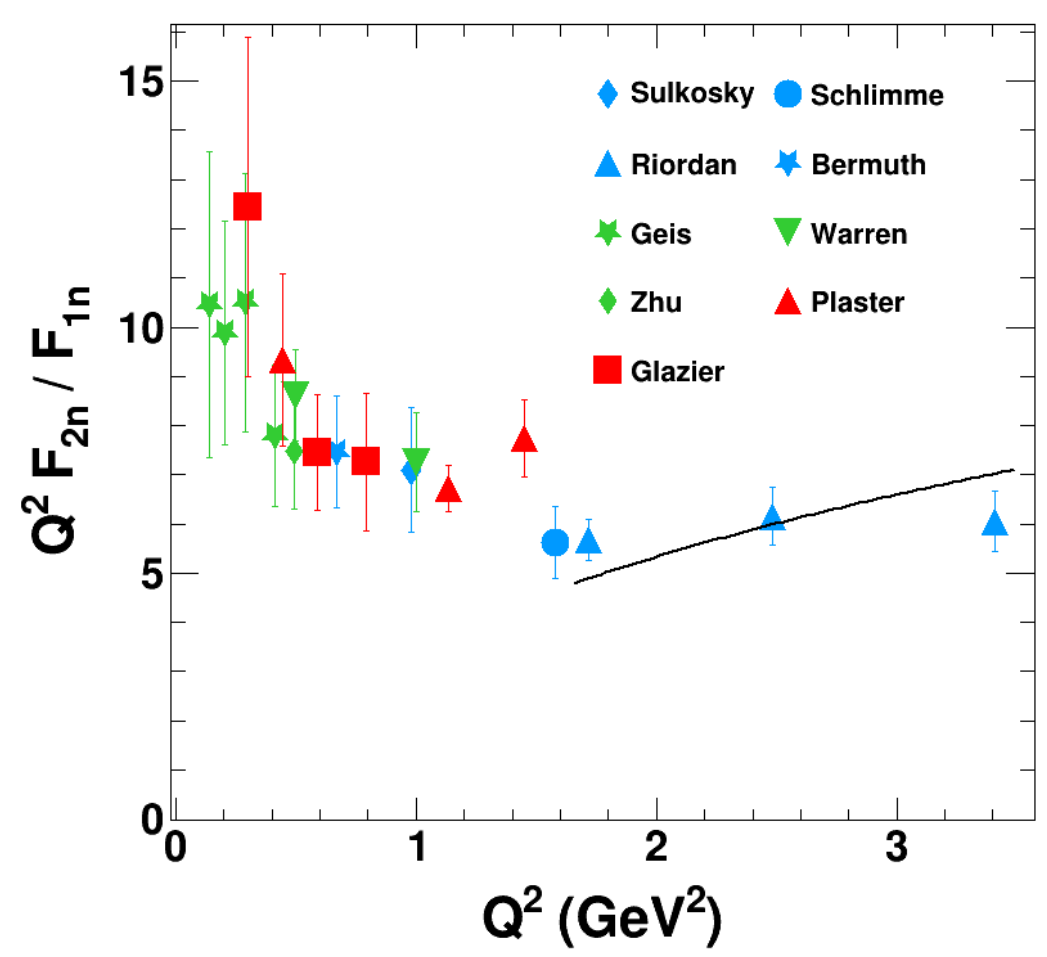

Fig. 3.13: Neutron data plotted as $Q^{2} F_{2}^{n} / F_{1}^{n}$. The black curve is from [160] with the same fitting parameters as Eqn 3.45 and normalized to match the data.

hadronic form factors are unified, and yields a method to access non-perturbative information about quarks and gluons in the context of hard exclusive processes. Deep virtual Compton scattering (DVCS), or $\gamma^{*}\left(q_{h}\right)+N(p) \rightarrow \gamma\left(q^{\prime}\right)+N\left(p^{\prime}\right)$, is a hard exclusive process in which the amplitudes may be experimentally accessed, e.g. ep $\rightarrow$ e $\gamma p$. The DVCS process may be seen by Figure 3.14 where $q$ is the momentum transfer to the nucleon, $P=\left(p+p^{\prime}\right) / 2, q^{2}=0$ and $Q_{h}^{2}=-q_{h}^{2}>0$. The top half of the diagram represents a hard collision between the virtual photon and a single quark and represents a process calculable using perturbation theory. The remaining complexities, namely the 


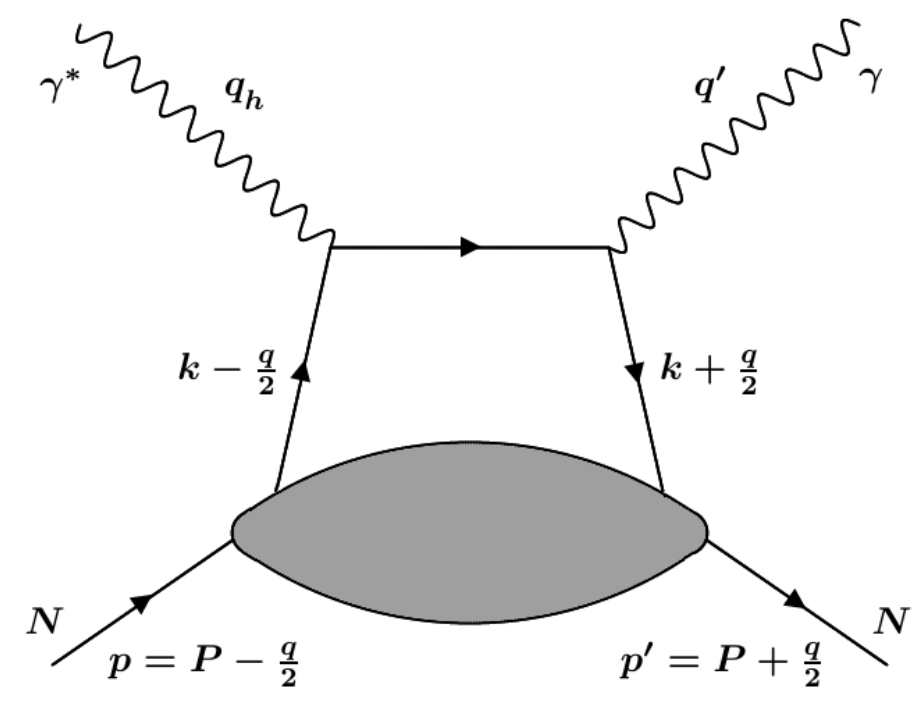

Fig. 3.14: The so-called "handbag" diagram for the nucleon DVCS process, note that the diagram in which the photons are crossed is also necessary. The process may be factorized if the four momentum of the virtual photon is large.

interactions and dynamics of the quarks (sea and valence) and gluons, that collectively make up the nucleon structure are represented by the grey "blob" and may be described by GPDs. QCD factorization theorems allows for the two sections to be factorized; in other words, the total amplitude is a convolution of the quark perturbative process and a non-perturbative amplitude parametrized by GPDs. The non-perturbative GPDs contain global, or universal, information regarding the structure of the nucleon in the context of $\gamma$ or $e^{ \pm}$induced hard exclusive processes [13]. As the GPDs contain nucleon structure information, then it is no surprise that nucleon form factors are related to the GPDs. To be more explicit, the first moments of the $H$ and $E$ GPDs are related to the 
quark Dirac and Pauli form factors via the sum rules [161]:

$$
\begin{aligned}
F_{1}^{q}\left(Q^{2}\right) & =\int_{0}^{1} d x H_{v}^{q}\left(x, Q^{2}\right), \\
F_{2}^{q}\left(Q^{2}\right) & =\int_{0}^{1} d x E_{v}^{q}\left(x, Q^{2}\right), \\
H_{v}^{q}\left(x, Q^{2}\right) & =H^{q}\left(x, 0, Q^{2}\right)+H^{q}\left(-x, 0, Q^{2}\right), \\
E_{v}^{q}\left(x, Q^{2}\right) & =E^{q}\left(x, 0, Q^{2}\right)+E^{q}\left(-x, 0, Q^{2}\right),
\end{aligned}
$$

where $q$ is the quark flavor, $x$ is the momentum fraction of the struck quark, ${ }^{*} \xi$ is the skewedness or asymmetry between the quark momenta and $H^{q}\left(x, \xi, Q^{2}\right)$ and $E^{q}\left(x, \xi, Q^{2}\right)$ are the proton GPDs for unpolarized quarks. Therefore, Eqn 3.49 and Eqn 3.50 are defined with a $\xi=0$ and contain contributions of quarks in addition to antiquarks. The $v$ subscript refers to the "valence GPDs" since in the limit of $Q^{2} \rightarrow 0$, then $H_{v}^{u}\left(x, Q^{2}=\right.$ $0)=u_{v}(x)$ and $H_{v}^{d}\left(x, Q^{2}=0\right)=d_{v}(x)$ which are the usual valence quark densities of the proton. Once the quark form factors are known, then the nucleon form factors may be constructed using a procedure described in Ref. [57] which has been summarized in Section 3.2.4. In practice, though, this typically means a parametrization for $H$ and $E$ must be chosen, see Guidal et al. [162] or Diehl and Kroll [57] for example ansatzes. It is noted that the situation may be viewed in reverse; if nucleon form factors are well-known, then GPD models may be constrained.

\subsubsection{Summary}

Only several theories (or phenomenological models) have been presented, but many more exist which are not discussed in the interest of space. The shear number of pre-

\footnotetext{
${ }^{*}+x(-x)$ for quarks (antiquarks)
} 
dictions should be a measure of the difficulty of the problem, namely connecting the non-perturbative to the perturbative regime such that strongly interacting systems like the nucleon may be fully described theoretically. Other successful models not discussed include but are not limited to relativistic constituent quark models (RCQM) [163], Dyson-Schwinger equations (DSE) [164 167], diquark correlations and the Nambu-JonaLasinio (NJL) model [168], quark-diquark model with a pion cloud [169], dispersively improved Chiral Effective Field Theory [170], light-front holographic QCD [171], and many more. Furthermore, QCD on the lattice is making exciting progress in determining large $Q^{2}$ form factors in which an extension of the Feynman-Hellmann theorem is used to access non-forward matrix elements [172. ${ }^{*}$ While there are quite a few models that predict a zero crossing in the proton form factor ratio, only the DSE calculations predict a zero crossing in $G_{E}^{n} / G_{M}^{n}$ at $Q^{2} \approx 10 \mathrm{GeV}^{2 \dagger}$ which may be seen by Figure 3.15 among various other models. An important goal of experimental nuclear physics is to measure the nucleon form factors to higher $Q^{2}$ in order to help constrain predictions. The JLab $12 \mathrm{GeV}$ upgrade will push the neutron (proton) form factor ratio extractions up to approximately $Q^{2}=10 \mathrm{GeV}^{2}\left(14 \mathrm{GeV}^{2}\right)^{\ddagger}$ which may or may not reveal new behavior, such as a zero crossing or to what extent perturbative QCD scaling is exhibited. ${ }^{\S}$ Additionally, as the neutron data will be pushed to roughly $10 \mathrm{GeV}^{2}$, flavor decomposition of nucleon form factors will be possible up to $10 \mathrm{GeV}^{2}$, which will reveal more on the nature of $u$ and $d$ quark contributions to the nucleon.

\footnotetext{
${ }^{*}$ The neutron is particularly difficult on the lattice, but see Fig. 7.16 of Ref. 173 for $G_{E}^{n} / G_{M}^{n}$.

${ }^{\dagger}$ Ref. [167] updated the prediction of Ref. [165], and predicts a zero-crossing at $Q^{2} \approx 12 \mathrm{GeV}^{2}$.

¥ See Table 6.14 for upcoming nucleon form factor experiments at Jefferson Lab.

$\S$ For elastic $e N$ scattering, pQCD is expected to be applicable when $Q^{2}$ is on the order of $\sim 100 \mathrm{GeV}^{2}$.

『 For example, see [174] which was published shortly after the high $Q^{2} G_{E}^{n} / G_{M}^{n}$ data of Riordan et al.
} 


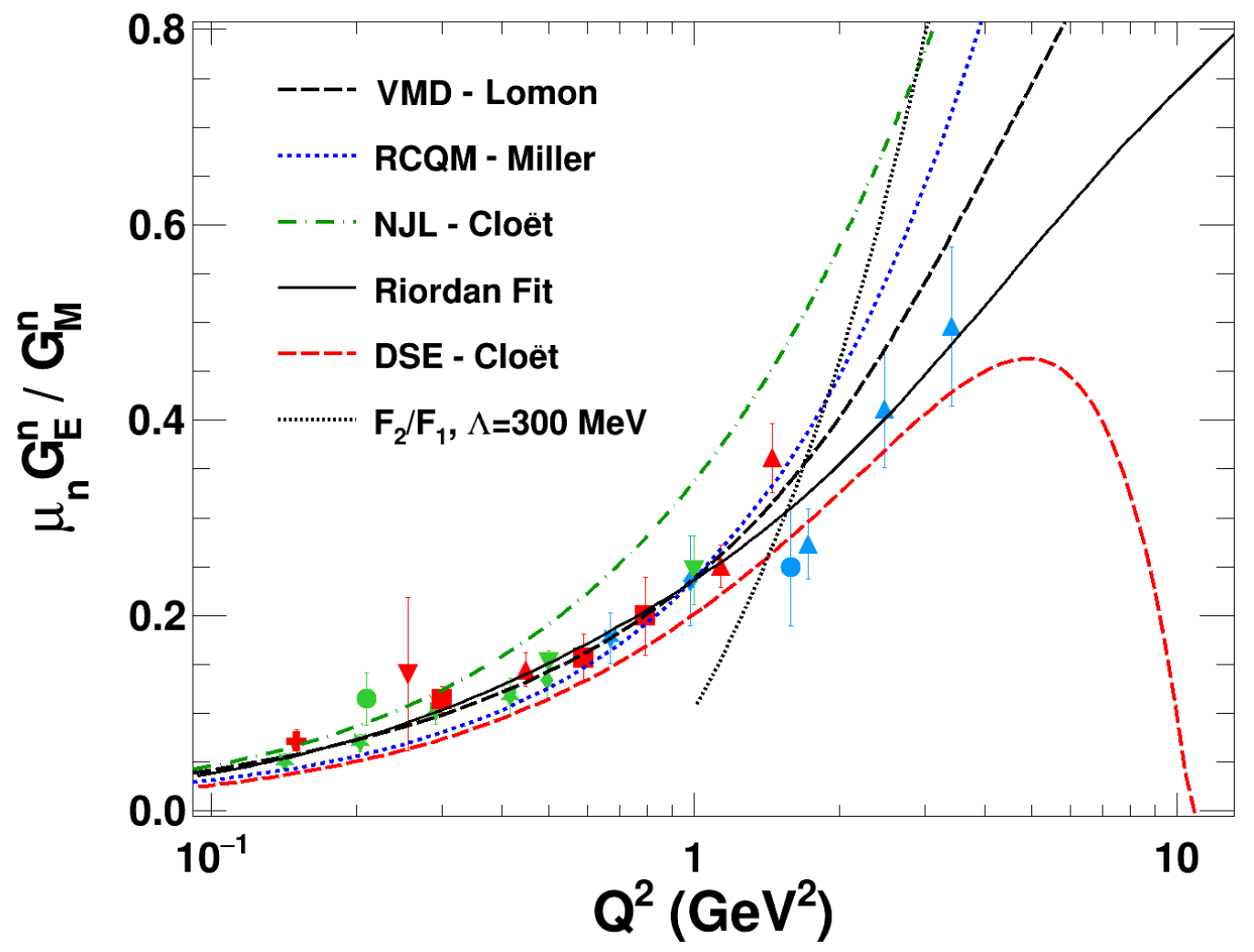

Fig. 3.15: Selected models and $G_{E}^{n} / G_{M}^{n}$. VMD and pQCD predictions $\left(F_{2} / F_{1}\right)$ have been discussed in previous sections. The remaining fits have not been discussed but are included to give a sense of other models on the market: RCQM by Gerald Miller (blue dots) [163, a DSE calculation by Ian Cloët et al. (red dashes) [165], and lastly the NJL model by Cloët et al. [168]. 


\section{Chapter 4}

\section{Experimental Description}

From late-February to mid-May of 2006, the Jefferson Laboratory (JLab) ${ }^{\dagger}$ experiment E02-013 extracted the neutron form factor ratio $G_{E}^{n} / G_{M}^{n}$ by measuring the beam-target asymmetry in quasielastic scattering of longitudinally polarized electrons from a polarized ${ }^{3} \mathrm{He}$ target via the semi-exclusive reaction ${ }^{3} \mathrm{He}\left(\vec{e}, e^{\prime} n\right) p p$ at four $Q^{2}$ points. The extraction theory is summarized by Eqn 2.106- Eqn 2.108, Eqn 2.110, and Eqn 2.111. Riordan et al. published the results of $G_{E}^{n} / G_{M}^{n}$ at $Q^{2}=1.72,2.48$ and $3.41 \mathrm{GeV}^{2}$ in 2010 [7]. The remaining kinematic, or $Q^{2}=1.16 \mathrm{GeV}^{2}$, had not been analyzed as the data was taken during the commissioning phase; therefore, the newly constructed detector subsystems were not fully understood yet. Now that the analysis is mature enough, the last kinematic point may be revisited. For completeness, a number of independent analyses were performed by the Ph.D. students of E02-013, and their respective expertise is documented: Sergey Abrahamyan, ${ }^{\ddagger}$ Aidan Kelleher [175], Ameya Kolarkar [176], Jonathan Miller [177] and Seamus Riordan [178]. A Master's student Tim Ngo documented valuable information regarding the geometry of the neutron detector [179].

\footnotetext{
$\dagger$ Thomas Jefferson National Accelerator Facility (TJNAF)

$\ddagger$ His thesis is written in Russian.
} 
The purpose of this chapter is to present the experimental apparatus of E02-013 with a focus on the unpublished $Q^{2}$ point. The chapter will begin with a broad overview of the detector layout and a description of the coordinate systems used throughout $G_{E}^{n}$, followed by an in-depth discussion of the accelerator and how an experiment measures the kinematics of the incoming electrons. The targets and custom target chamber will be presented in detail, which includes a discussion of the novelty of the experiment: hybrid spin-exchange optical pumping. The systems used to detect the quasielastically scattered electron in coincidence to the recoiling nucleon will be presented. Lastly, a description of how an experiment bridges the gap between the detector hardware, the electronics chain, the data acquisition system and the software in order to produce the raw data files necessary for the data analysis phase will be detailed.

\subsection{Introduction to E02-013}

E02-013 was a two-arm coincidence experiment located in JLab's Hall A and utilized the $6 \mathrm{GeV}$ Continuous Electron Beam Accelerator Facility (CEBAF) for its longitudinally polarized electrons.* The electrons were extracted from CEBAF where the kinematic properties, e.g. energy, position and polarization, were measured to a resolution that met or exceeded E02-013 requirements; the electrons then entered the target box and scattered from a polarized ${ }^{3} \mathrm{He}$ target. The quasielastically scattered electrons were detected by a large angular acceptance spectrometer known as BigBite. The purpose of BigBite was to measure the four momentum of the electron by tracking the trajectory using multi-wire drift chambers (MWDCs) and measuring the energy in an electromag-

\footnotetext{
* Construction of the $12 \mathrm{GeV}$ upgrade began in 2010, and unfortunately is not relevant to E02-013.
} 


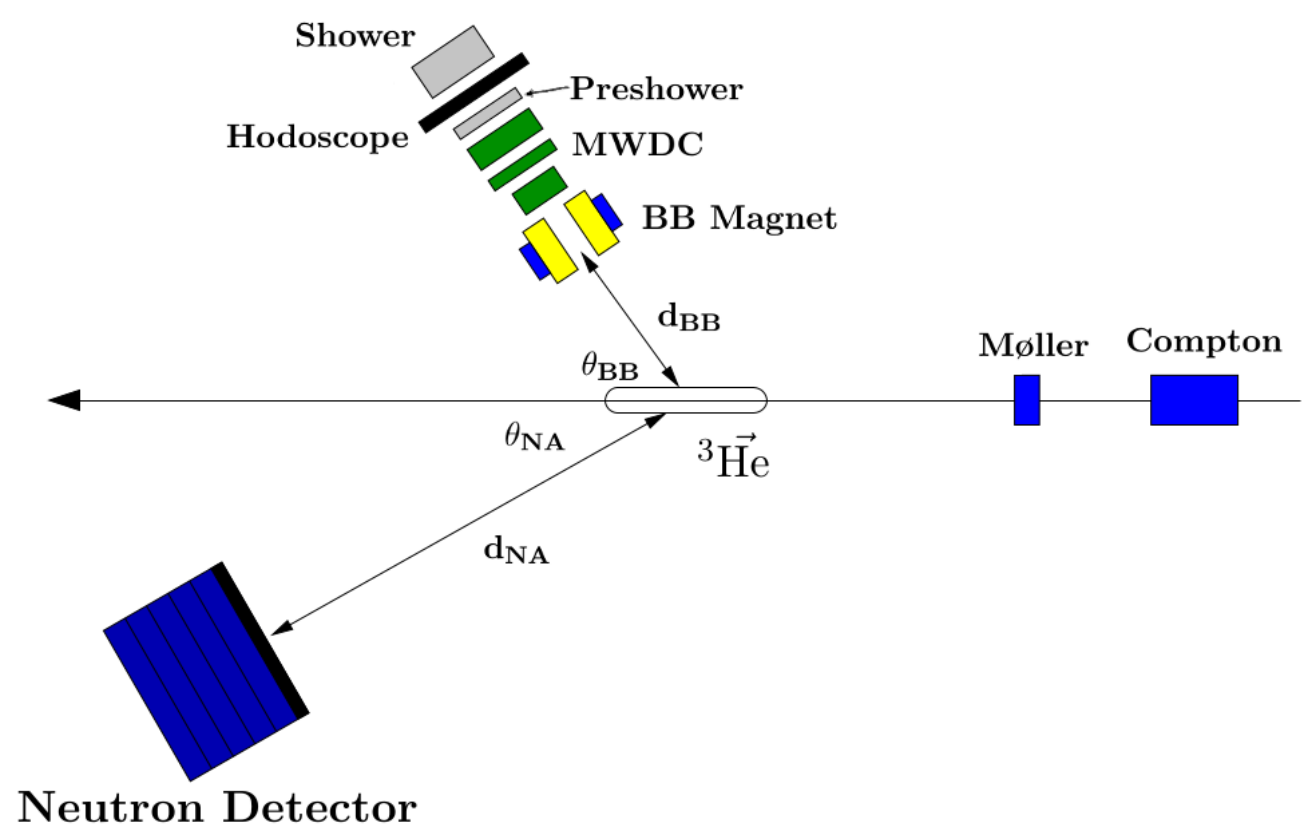

Fig. 4.1: Layout of the major detectors of experiment E02-013, not to scale. The geometric settings of the four kinematics are summarized in Table 4.1 .

netic calorimeter package which consisted of a preshower, a timing hodoscope and a shower. The recoiling nucleons were detected in coincidence within the neutron arm (NA) using planes of arrayed scintillator interlaced with iron and lead, or converter plates, which had the purpose of increasing the probability of a hadronic shower. The collection of scintillator and converter plates was called the Big Hall A Neutron Detector (BigHand) or simply the neutron detector (ND); the goal of the ND was to determine the momentum of the recoiling nucleon by measuring the time of flight (ToF) and to differentiate between protons and neutrons via a charge identification scheme. The novel technique known as hybrid spin-exchange optical pumping (hSEOP) was implemented to polarize the ${ }^{3} \mathrm{He}$ nucleus resulting in a highly polarized ${ }^{3} \mathrm{He}$ target from which 


\begin{tabular}{ccccccc}
\hline Kin & $\boldsymbol{Q}^{\mathbf{2}}\left[\mathrm{GeV}^{\mathbf{2}}\right]$ & Days & $\boldsymbol{E}_{\mathrm{b}}[\mathrm{GeV}]$ & $\boldsymbol{\theta}_{\mathrm{BB}}[\mathbf{d e g}]$ & $\boldsymbol{\theta}_{\mathbf{N A}}[\mathbf{d e g}]$ & $\mathbf{d}_{\mathbf{N A}}[\mathbf{m}]$ \\
\hline 1 & 1.16 & 8 & 1.519 & -56.3 & 35.74 & 8.2 \\
2 & 2.48 & 19 & 2.640 & -51.6 & 30.25 & 11 \\
3 & 3.41 & 33 & 3.291 & -51.6 & 25.63 & 11 \\
4 & 1.72 & 9 & 2.079 & -51.6 & 35.74 & 8.2 \\
\hline
\end{tabular}

Table 4.1: Experimental configurations of the four kinematic points. See Figure 4.1 for definitions of geometric variables; $E_{\mathrm{b}}$ represents the electron beam energy. The green $Q^{2}$ points were published in 2010 [7].

the neutron carries the vast majority of it. The degree to which the target, or more specifically the neutron within a ${ }^{3} \mathrm{He}$ nucleus within the target cell, was polarized scales the desired measured asymmetry; therefore, the fraction of polarized nuclei within the ${ }^{3} \mathrm{He}$ target ensemble needs to be optimized for a precision measurement. The theory and difficulties of overcoming such a challenging experimental hurdle will be discussed in great detail. Equally as important as the large detector equipment and hardware was the trigger and data acquisition (DAQ) systems. The standard JLab systems, e.g. $\mathrm{CODA}^{*}$ and $\mathrm{EPICS}^{\dagger}$, were implemented, and specifically CODA was used to handle the event-by-event retrieval of data. The CODA raw data output then needed to be decoded and analyzed using an extension of the Hall A analyzing software from which users may then analyze and interpret the data; these aspects of the experiment will be discussed after the detectors.

\footnotetext{
* CEBAF Online Data Acquisition

${ }^{\dagger}$ Experimental Physics and Industrial Control System
} 


\begin{tabular}{ccccccc}
\hline Kin. & 1 & $2(\mathrm{a})$ & $3(\mathrm{a})$ & $2(\mathrm{~b})$ & $3(\mathrm{~b})$ & 4 \\
\hline Date & $3 / 01-3 / 08$ & $3 / 08-3 / 23$ & $3 / 23-4 / 17$ & $4 / 17-4 / 24$ & $4 / 24-5 / 01$ & $5 / 01-5 / 09$ \\
Tgt. Cell & Barbara & Dolly & Edna & Edna & Edna & Edna \\
\hline
\end{tabular}

Table 4.2: The four kinematic run times of E02-013, and the target cells used.

The four beam-target asymmetry measurements required different experimental configurations, and are summarized in Table 4.1. The angles and distances are described in Figure 4.1, a simplified, not-to-scale diagram displaying the detector locations. An aerial view of the Kinematic 1 setup as viewed within a simulation utilizing GEANT4 [180] is displayed by Figure 4.3. Among other things, the design choices were motivated by acceptance matching between BigBite and the neutron detector; the desired elastic en cross section is small, therefore optimizing the number of accepted quasielastic events is crucial for a precision measurement. Each experimental setup is colloquially referred to as Kinematic \#, for example the subject of this thesis analyzes data obtained by the Kinematic 1 configuration, or simply Kin 1 . Table 4.2 displays the chronological ordering of the four kinematic points and the name of the polarized ${ }^{3} \mathrm{He}$ cell used for production. While Kinematics 2-4 will not be discussed in detail within this thesis, it is noted that the two high $Q^{2}$ points, namely Kin. 2 and 3, recorded data over two time intervals due to accelerator scheduling considerations; subsequently, these time periods are differentiated by Kin \#(a) and \#(b). The remainder of this chapter is dedicated to the experimental details of E02-013. 


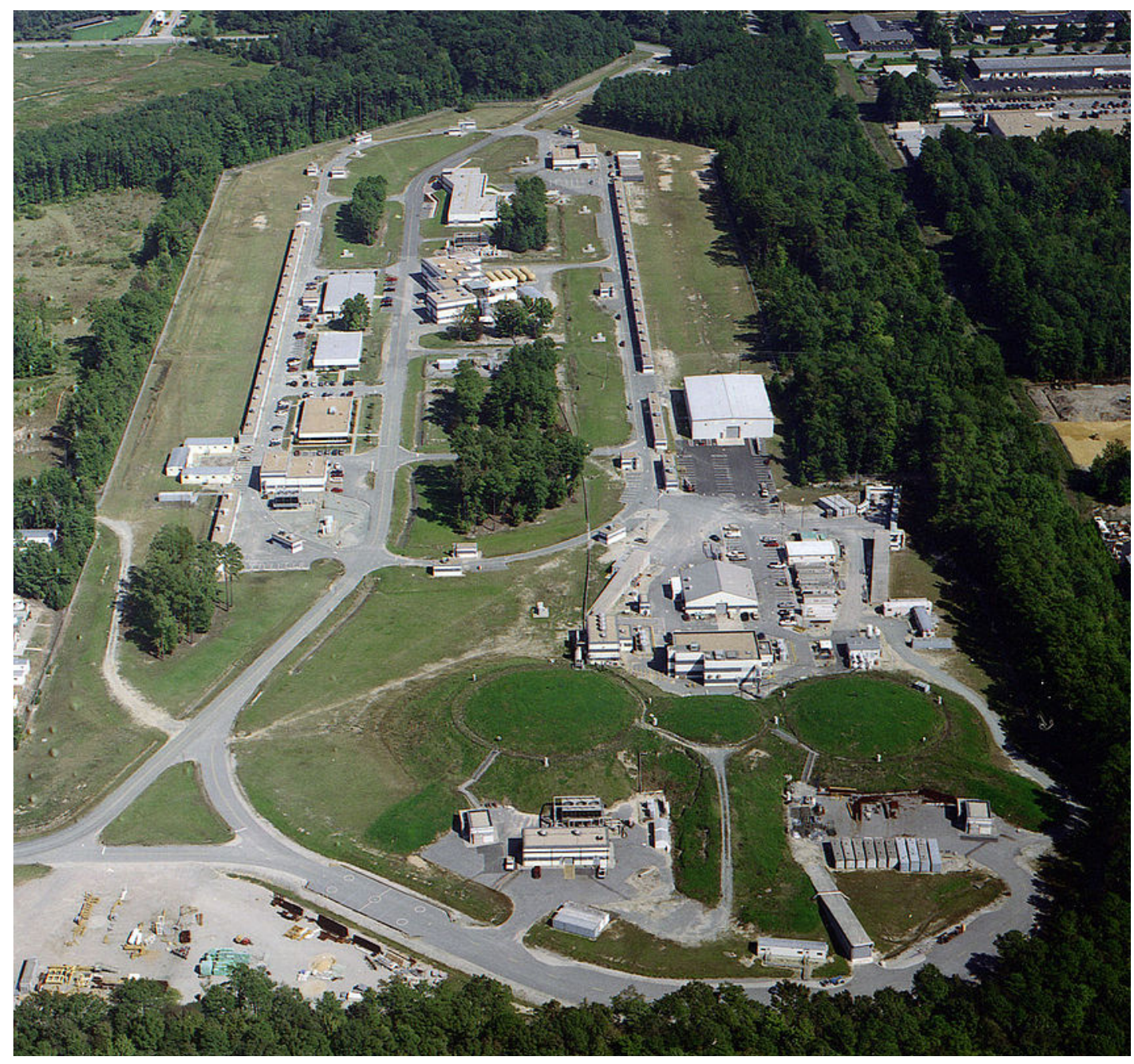

Fig. 4.2: Aerial view of Jefferson Laboratory prior to the $12 \mathrm{GeV}$ upgrade. CEBAF is located underneath the asphalt racetrack shape, and the three experimental Halls are located underneath the three dirt mounds; Hall A is associated with the left mound [181]. 


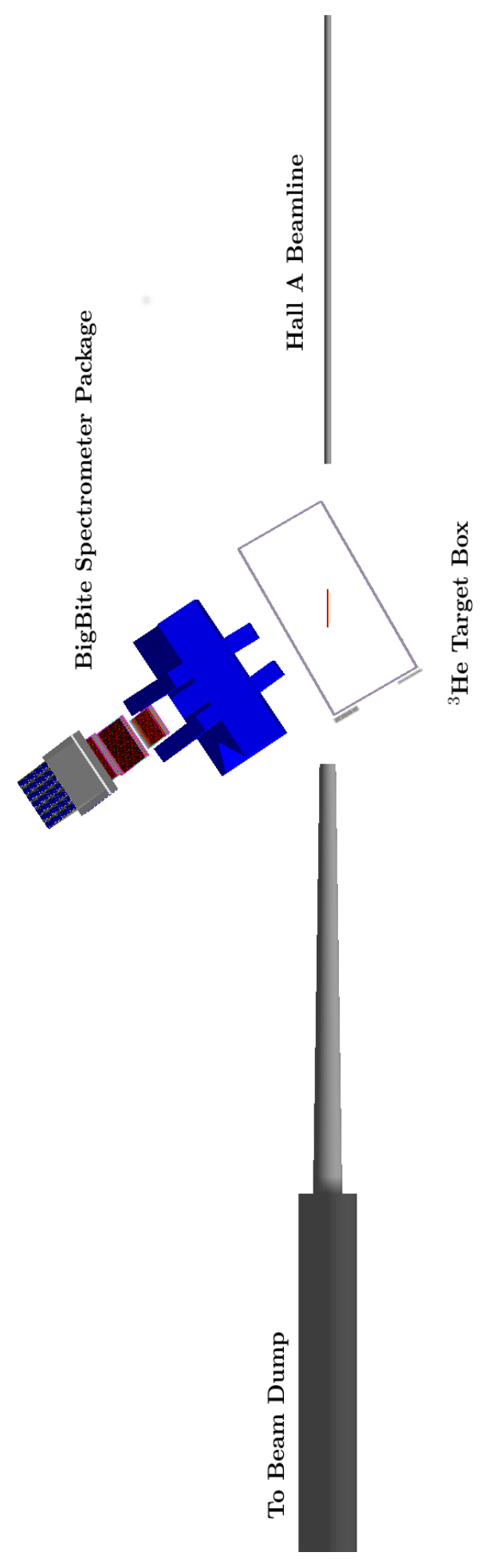

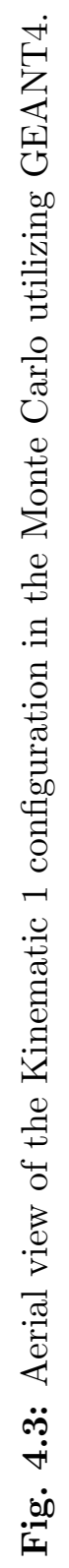




\subsection{Coordinate Systems}

There are four major coordinate systems defined and are named as follows: the lab or Hall coordinates, the target coordinates, BigBite or spectrometer coordinates and neutron detector coordinates. All systems are used in the analysis phase, see Figure 4.4 for a summary of the coordinate systems.

1. The origin of the lab system is located at the center of the target cell with $\hat{z}$ pointing along the beamline (downstream), $\hat{\boldsymbol{y}}$ pointing towards the Hall ceiling (vertical and against gravity), and $\hat{\boldsymbol{x}}=\hat{\boldsymbol{y}} \times \hat{\boldsymbol{z}}$ or "beam-left" forming a standard right-handed coordinate system.

2. The target coordinate system ideally has the origin at the target center with $\hat{\boldsymbol{z}}$ pointing along the BigBite central unit vector and parallel to the ground, $\hat{\boldsymbol{x}}$ pointing towards the floor (vertical and with gravity) and $\hat{\boldsymbol{y}}=\hat{\boldsymbol{z}} \times \hat{\boldsymbol{x}}$.

3. The BigBite coordinate system has the origin at the center of the first plane of the MWDCs. The $\hat{z}$-direction is pitched "upwards" ideally by 10 degrees relative to the Hall floor, and is normal to the detector stack. The $\hat{\boldsymbol{x}}$-direction points in the magnetic dispersive direction, or "down" along the surface of the drift chamber face, and $\hat{\boldsymbol{y}}$ forms a right-handed system.

4. The neutron detector is displaced transversely relative to the central ray running down the neutron arm, or in other words the ND central normal vector does not point towards the Hall origin, but roughly $17 \mathrm{~cm}$ upstream. The $\hat{z}$ points "into" the detector, or along the depth dimension. The $\hat{\boldsymbol{y}}$ points along the width 


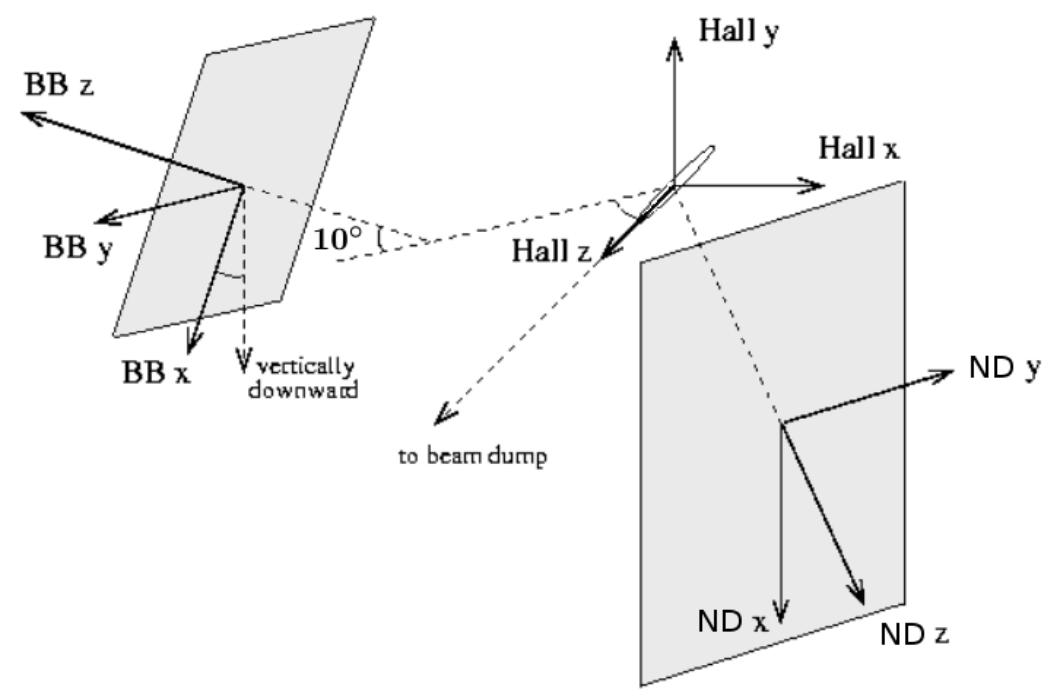

(a) Hall and detectors coordinate systems.

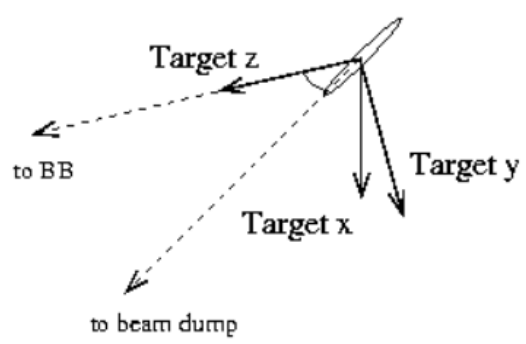

(b) Target coordinate system.

Fig. 4.4: The coordinate systems of $G_{E}^{n}$. Diagram has been altered from [182].

dimension and away from the beamline, and $\hat{\boldsymbol{x}}$ points along the height dimension in the direction of gravity. The origin is located on the target side of the detector shielding, and centered with respect to the height and weight dimensions. 


\subsection{CEBAF: The Electron Beam}

In 2006, CEBAF was a continuous-wave (cw) electron accelerator capable of delivering highly polarized electrons to three experimental areas: Halls A, B and C. The approximately $4.5 \mathrm{~km}$ "race-track" shaped accelerator was composed of two antiparallel superconducting radio frequency linear accelerators (linacs) connected by recirculation beamline arcs allowing for simultaneous beam delivery to all three Halls during passes one to a maximum of five. Each linac is capable of producing an energy gain of roughly $565 \mathrm{MeV}$ per pass resulting in a maximum electron energy of approximately $5.7 \mathrm{GeV}$. Hall A was able to receive a maximum average current of $150 \mu \mathrm{A}$ in which the RMS transverse beam size was $\sim 80$ microns and the relative energy spread was $2.5 \times 10^{-5}$. The high-quality, high-duty factor beam sustained polarizations greater than $85 \%$ for the duration of E02-013. These unique qualities made (and continue to make) Jefferson Lab an ideal facility to probe the structure of the nucleon.

The polarized electrons that make it to the experimental Halls are sourced from within a mechanically strained gallium arsenide photocathode. Electrons are liberated from the photocathode by three gain-switched circularly polarized diode lasers pulsed at a frequency of $499 \mathrm{MHz}$; each laser pulse corresponds to photoemitted electron bunches destined for one of the three Halls. The laser pulses are phase shifted by $120^{\circ}$ relative to each other which effectively produces an electron bunch "train" that has a frequency of $1497 \mathrm{MHz}$ which matches the fundamental frequency of the linacs. The helicity of the circularly polarized lasers, either left or right, is controlled by a so-called Pockels cell (and the voltage polarity applied) prior to electron photoemission which 


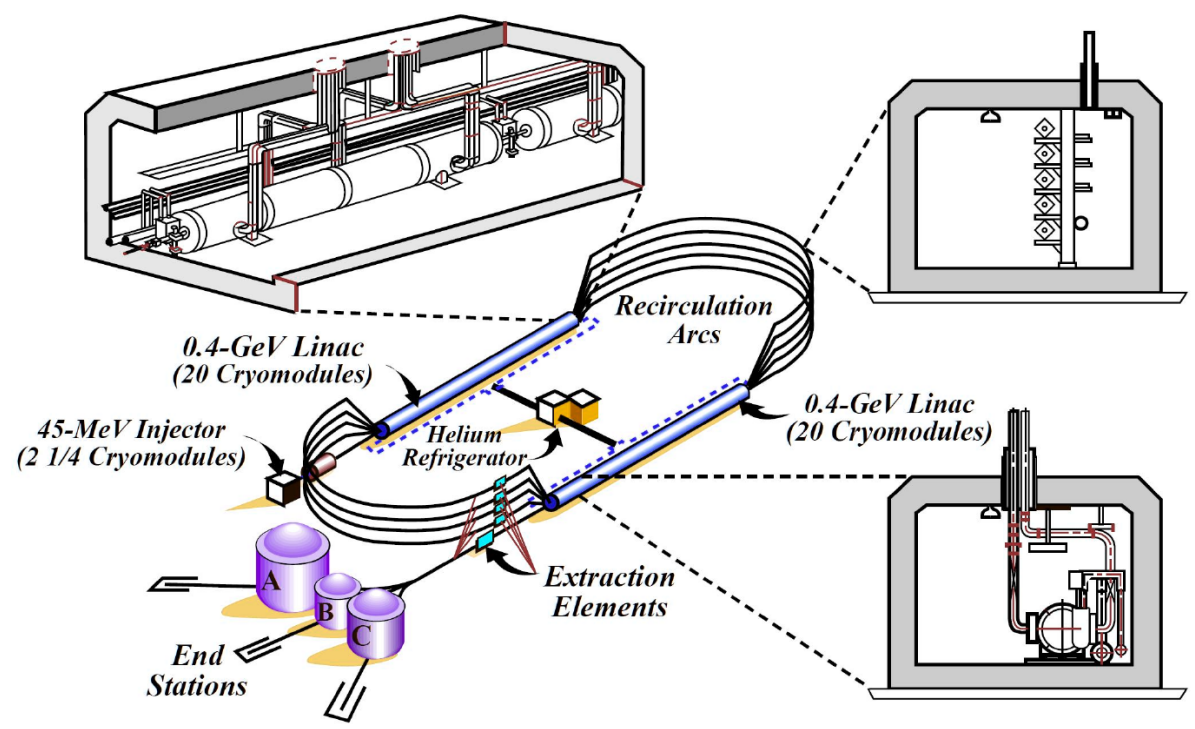

Fig. 4.5: The layout of CEBAF circa 2006.

directly determines the helicity of the longitudinally polarized electrons, either parallel or antiparallel to the direction of the beam. The polarity of the voltage applied to the Pockels cell, or effectively the helicity of the electron beam, is flipped at a frequency of $30 \mathrm{~Hz}$ according to a pseudorandom scheme, and is described in more detail within Section 4.8.5. There is a $500 \mu$ s transition time as the Pockels cell settles after reversal in which the helicity is simply undefined. Flipping the beam helicity results in the desired beam-target asymmetry; additionally, the method is an important practice to reduce systematic uncertainties associated with the asymmetry measurement. Over periods of data taking, the pseudorandom algorithm will produce roughly equal amounts of "plus" and "minus" helicity electrons, and consequently the total accumulated beam charge for each helicity state should also be roughly equal. The helicity of the beam may be reversed post Pockels cell with the insertion of a $\lambda / 2$ plate, or half-wave plate (HWP). 
The half-wave plate is one of three sources that can potentially change the sign of the beam-target asymmetry measurement, and must be accounted for during the analysis.

The GaAs photocathode and $100 \mathrm{keV}$ electron gun are housed in ultrahighvacuum conditions, a technological necessity to prevent deterioration of the photocathode quantum efficiency from atomic collisions. The photoemitted longitudinally polarized electrons are then accelerated by the $100 \mathrm{keV}$ DC gun into the injector from which an additional acceleration up to $67 \mathrm{MeV}$ is possible using superconducting radio frequency (rf) technology. The injector then "injects" the sufficiently relativistic electrons into the North Linac. By the time the electrons make it into the main racetrack, they are already moving near $c$ and are consequently in phase with the fundamental frequency of the accelerator $(1497 \mathrm{MHz})$. Each linac pass now increases the energy of the electron, and Figure 4.5 displays nine arcs, five (four) in the North (South) end, which is a requirement to account for the Lorentz force of a charged particle of varying energy. Electrons may be sent to any one of the three experimental Halls during pass one through five using $499 \mathrm{MHz}$ rf separator magnets; the correct electron bunch is effectively extracted from the three pulse train. For electrons destined for Hall A, the beam needs to traverse an additional eight dipole magnets within the arc-section* before entering the Hall which includes the beam diagnostics area, the target, the necessary detection equipment and the beam dump.

The injector and linacs are constructed out of niobium cavities where two connected cavities are referred to as a cryounit. Four cryounits make up a $8.25 \mathrm{~m}$ long cryomodule, simply consisting of eight cavities. The cyromodules are kept at $2 \mathrm{~K}$,

\footnotetext{
* The nominal bend angle of the arc section is $34.3^{\circ}$.
} 
hence the helium refrigerator in the middle of the racetrack. The injector contains 2.25 cryomodules while the linacs contain 20 each. The cavities operate at $1497 \mathrm{MHz}$ and have accelerating gradients of $7 \mathrm{MeV} / \mathrm{m}$.

The spin polarization of the electrons is monitored in the injector and measured with a $5 \mathrm{MeV}$ Mott polarimeter. In short, the counting rate asymmetry of elastically scattered electrons off of gold nuclei is measured by the polarimeter. If the asymmetry is nonzero, then the beam polarization is related to the asymmetry via the so-called Sherman function resulting in a method that can determine the polarization of the beam with an instrumental precision of $0.5 \%$. The Mott polarimetry measurements relevant to $G_{E}^{n}$ are summarized within Table 4.4. See [183] for more information.

The electrons spin precess when steered by the accelerator dipole magnets, and consequently the longitudinal polarization of the beam electrons measured at the Halls depends on the total precession angle. The total precession in the accelerator $\phi_{\text {accel }}$ after $n$ passes is

$$
\phi_{\text {accel }}=\left(\frac{E_{l}}{m_{e}}\right)\left(\frac{g-2}{2}\right)(n+\alpha)(2 n-1) \pi,
$$

where $g$ is the typical electron $g$-factor, $E_{l}$ is the single linac energy, and the constant $\alpha$ is the ratio of injector energy to $2 E_{l}$ equalling 0.1125 for CEBAF. The factor of $\pi$ accounts for the fact that each recirculation arc is $180^{\circ}$. The expression assumes that the linacs are running in balanced mode where the North and South linac energy gains are the same. The precession through the arc-section on the way to the Halls is

$$
\phi_{\operatorname{arcs}}= \pm\left(\frac{E_{l}}{m_{e}}\right)\left(\frac{g-2}{2}\right)(2 n+\alpha)\left(\frac{\pi b}{2}\right)
$$

where the $+(-)$ corresponds to Hall A (C). The constant $b$ depends on the Hall arc 
bend angles; therefore, $b=-\frac{1}{2.4}, 0$ and $\frac{1}{2.4}$ for Halls A, B and C, respectively. The total precession of Hall A electrons is simply the sum:

$$
\phi_{\text {spin }}=E_{l} \frac{g-2}{2 m_{e}}\left[2 n^{2}-n(1-2 \alpha+b)-\alpha\left(1+\frac{b}{2}\right)\right] \times 180^{\circ} .
$$

The polarization of the beam entering any one of the three experimental Halls may be manipulated by the orientation of a Wien filter located within the injector. With the introduction of a Wien filter, Eqn 4.3 gets altered by the addition of $\theta_{\text {Wien }}$, or $\phi_{\text {spin }}=\phi_{\text {accel }}+\phi_{\text {arcs }}+\theta_{\text {Wien }}$. As the Hall beam polarization varies sinusoidally with the Wien angle, an optimization may be performed by scanning over a number of Wien angles. For a small number of end station energy combinations, simultaneous delivery of longitudinally polarized electrons to all three Halls for a single Wien angle is possible. There are a large number of energy combinations to simultaneously deliver polarized electrons to Halls A and C. At the time of E02-013 production, the Wien angle was optimized for the higher priority parity violating experiment $G^{0}$ running in Hall C. Consequently, $G_{E}^{n}$ had to run with less than optimized beam polarization in Hall A. Note that it is possible to tweak another accelerator parameter in order to optimize the longitudinal polarization simultaneously to Halls A and C. Typically the linacs run in balanced mode; however, by running in unbalanced mode but keeping the total energy gain per pass fixed (e.g. 555 and $575 \mathrm{MeV}$ ), the degree of simultaneous delivery of polarized electrons to Halls A and C may be further enhanced [184]. See [185] for more information about the accelerator, and for more information regarding polarized electron sources and guns, see [186, 187]. 


\subsection{Hall A Beam Monitoring and Diagnostics}

In order to reconstruct a quasielastic scattering event, the position, direction and energy of the incoming electrons prior to scattering must be known precisely and accurately. The Hall A beamline consists of various diagnostic instrumentation required for determination of beam parameters to a resolution that meets or exceeds experimental needs. The basic Hall A instrumentation is summarized within Figure 4.6 .

- Beam Position Monitors (BPMs);

- Beam Charge Monitors (BCMs);

- instrumentation to raster the beam;

- energy diagnostics, eP and the Arc method;

- two methods of polarimetry, Compton and Møller.

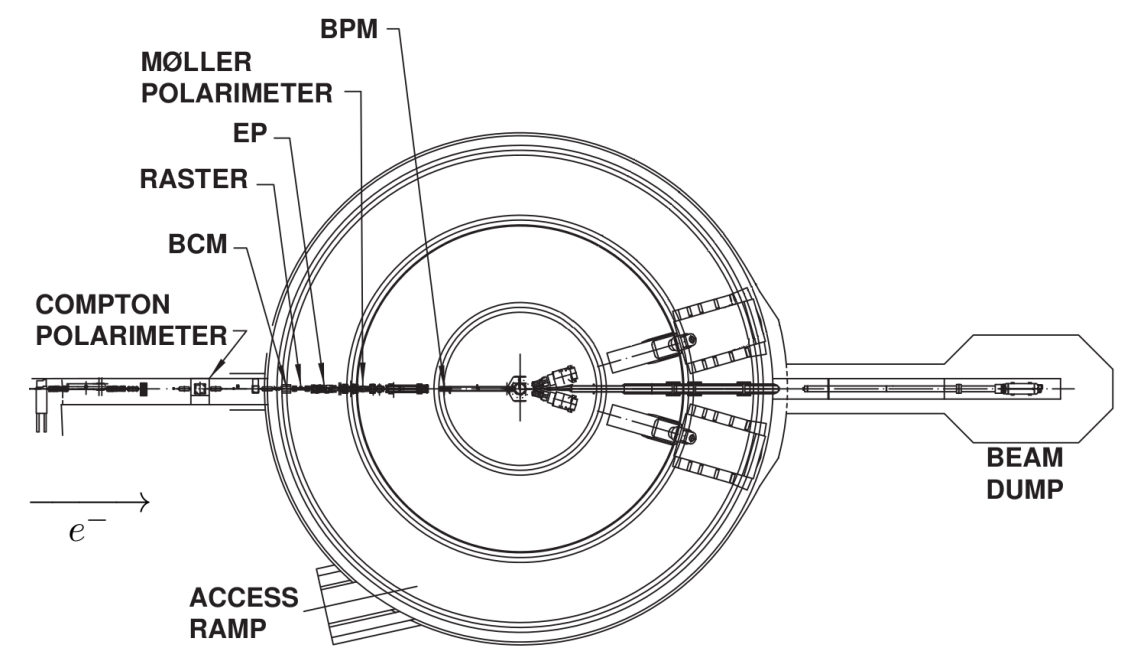

Fig. 4.6: Aerial view of Hall A displaying the instrumentation along the beamline [188]. 


\subsubsection{Beam Position}

The Beam Position Monitors (BPMs) nonintrusively monitor the position of the incoming electron beam. There are two BPMs located at $7.52 \mathrm{~m}$ and $1.29 \mathrm{~m}$ upstream of the target, each consisting of a four-wire antenna array tuned to the fundamental frequency of

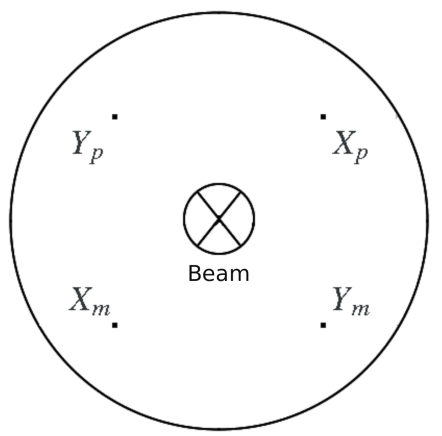

Fig. 4.7: The antenna array.

the accelerator, or $1.497 \mathrm{GeV}$. The wire array is oriented parallel to the beamline but individual antennae are placed at a distance $\pm 45^{\circ}$ away from the beam in the lab $x y$-plane, diagrammatically seen by Figure 4.7 where $X_{i}$ and $Y_{i}(\mathrm{i}=p, m)$ represent the pedestal corrected ADC signals induced by the beam. When the beam electrons are in the vicinity of the antennae, a measurable signal is induced that is inversely proportional to the electron's distance from the wires. Therefore, the standard difference over sum technique may be used in order to calculate the relative position of the beam, or more explicitly:

$$
x_{p}=k \frac{X_{p}-X_{m}}{X_{p}+X_{m}} \quad \text { and } \quad y_{p}=k \frac{Y_{p}-Y_{m}}{Y_{p}+Y_{m}}
$$

where $k$ is a calibration coefficient [188]. The relative measurement is able to determine the position of the beam to within $100 \mu \mathrm{m}$ for currents $\geq 1 \mu \mathrm{A}$. The absolute position of the beam may also be determined using the BPMs, but it requires an invasive calibration of the BPMs using wire scanners, or superharps, which are positioned near the BPMs* at $7.35 \mathrm{~m}$ and $1.12 \mathrm{~m}$ upstream. Briefly, a wire scanner consists of three tungsten wires

\footnotetext{
* Hall A actually had six harps, 2 near the BPMs and 4 along the arc-section.
} 
and a motor which can accurately move the wires through the electron beam. When a tungsten wire comes in contact with the beam electrons, an electromagnetic shower is initiated which then induces a signal in a photomultiplier tube (PMT) located a few meters downstream towards the target. Using the known positions of the tungsten wires and the output voltage of the PMT, the beam may be surveying from which an absolute position may then be obtained. This procedure is also used during the energy measurements using the Arc method. Note that the direction of the beam may then be calculated by knowing its position at two points in space via the two BPMs, and can be determined to within $30 \mu \mathrm{rad}$. The position of the beam is recorded in two ways for use in subsequent analysis. In the first, the BPM position averaged over 0.3 seconds is logged into EPICS every second. The second, the BPM antennae information is recorded in the CODA data stream on the event-by-event level.

\subsubsection{Beam Current}

Hall A has two Beam Current Monitors (BCMs) located roughly 25 m upstream from the target, and are designed to provide a non-invasive, low noise measurement of the beam current with an accuracy of $\leq 0.5 \%$ down to a current of $1 \mu \mathrm{A}$. A BCM consists of an Unser monitor [189, 190] and two rf cavities tuned to $1497 \mathrm{MHz}$, all housed in a magnetically shielded and temperature controlled box; these are requirements that help to reduce noise and signal drift of the Unser monitor's output signal. The Unser monitor is a parametric current transformer. It is capable of measuring the beam current and providing an absolute reference which may then be used to calibrate the BCM rf cavities. As the output signal drifts significantly on the order of minutes, the Unser monitor is 
not designed to monitor the beam current in real time; however, calibration runs may measure the drift which may then be removed during calibrations of the rf cavities. The two stainless steel, cylindrical rf cavities are located on either side of the Unser monitor. These waveguides are tuned to the fundamental frequency of the accelerator, and are capable of producing an output voltage that is proportional to the beam current. The rf output signals from the two cavities are then further processed being split into two parts leading to sampled and integrated data. The sampled data come from a high-precision digital AC voltmeter which effectively produces a digital output that is representative of the input signal RMS over a one second interval. The digital output is proportional to the accumulated beam charge over that one second, and is sent to the CODA data stream every 2-5 seconds. The other cavity signal, leading to integrated data, is sent to an RMS-to-DC converter which eliminates noise with a bandpass filter and then produces an analog DC voltage level; this level drives a voltage-to-frequency (VTOF) converter and the resulting outputs are then sent to $200 \mathrm{MHz}$ VME scalers. The scaler outputs are sent to the data stream and are allowed to accumulate. The integrated data more accurately represents the total beam charge of a particular run. For more information regarding BCMs, see [188, 191].

\subsubsection{Rastering the Beam}

A set of field coils positioned $23 \mathrm{~m}$ upstream of the target are used to produce small transverse magnetic deflections of the beam, resulting in a beam square of $\sim 3 \times 3 \mathrm{~mm}^{2}$ at the location of the target in the Hall $x y$-plane. The technique of spreading the beam out before it hits the target is known as rastering. For $G_{E}^{n}$, the fast raster system uses 
a $25 \mathrm{kHz}$, triangular waveform for the $x$ and $y$ directions [176]. Rastering is used to prevent significant overheating of the target cells which can reduce the lifetime, damage it, or even rupture it. Since rastering alters the position of the beam, the current used to drive the coils is sent to CODA on an event-by-event level as it must be used with the BPM position information in order to reconstruct the initial kinematics of the electrons.

\subsubsection{Absolute Energy Measurements}

There are two methods of determining the energy of the beam electrons absolutely: the Arc and eP method. The first method to be discussed is the Arc method, and the instrumentation is set up along the arc-region; this is the beamline section that connects the accelerator exit (the beam switch yard) to Hall A. The nominal bend angle of the beam within the arc is $34.3^{\circ}$, and is accomplished by eight dipole magnets.* The momentum of the beam is related to the total field integral of the dipoles and the bend angle within the arc section, or more explicitly:

$$
p_{\text {beam }}=k \frac{\int \vec{B} \cdot d \vec{l}}{\theta_{\text {bend }}},
$$

where $\vec{B} \cdot d \vec{l}$ is the net field integral, $\theta_{\text {bend }}$ is the net bend angle, and $\mathrm{k}$ is a constant. ${ }^{\dagger}$ In order to calculate $p_{\text {beam }}$ using this method, the field integral and bend angle need to be measured simultaneously. The net bend angle is measured using four wire scanners, two of which are positioned at the entrance and exit, respectively, of the arc. The eight arc magnets are housed in a vacuum, therefore in order to perform a field integral measurement a ninth identical dipole magnet is powered in series as a reference magnet;

\footnotetext{
${ }^{*}$ Each dipole magnet is paired with focusing and correcting magnets as well.

${ }^{\dagger} k=0.299792 \mathrm{GeV} \cdot \mathrm{rad} /(\mathrm{T} \cdot \mathrm{m})[188$
} 
the so-called " $9^{\text {th }}$ dipole." By averaging several bend angle measurements, an average energy may then be calculated with a systematic uncertainty of $6.8 \times 10^{-5}$ for $4.0 \mathrm{GeV}$ electrons.

As with all Jefferson Lab beam diagnostic equipment, there is a second, redundant method of measuring the energy of the beam. The eP method utilizes the elastic reaction $\mathrm{H}\left(e, e^{\prime} p\right)$, and the stand-alone device is located roughly $17 \mathrm{~m}$ upstream of the target. Therefore, the energy of the incoming electrons is calculated by measuring the outgoing angles of the electron and recoiling protons, $\theta_{e}$ and $\theta_{p}$, respectively, using the following kinematic expression obeying conservation of four momenta:

$$
E_{e}=M \frac{\cos \theta_{e}+\sin \theta_{e} / \tan \theta_{p}-1}{1-\cos \theta_{p}}
$$

where $E_{e}$ is the beam energy and $M$ is the mass of the proton. The actual device uses a thin polyethylene tape, or $\mathrm{CH}_{2}$, as the target and constantly moves during a measurement to prevent damage and prolong the lifetime. The detector has two identical detector arms which are placed symmetrically about the beam axis and in the Hall $x z$-plane. A two arm design is chosen as it cancels the uncertainties associated with the direction and position of the beam to first order. Each arm consists of a proton and electron detection system positioned in the reaction plane, utilizing silicon micro-strip detector arrays (SDD) and scintillator detectors for timing and trigger information. The proton detectors are mounted at a fixed angle of $60^{\circ}$ while the SSDs for the electrons are installed to cover a larger acceptance reflecting the variation in possible beam energies. Behind the electron scintillator is a $\mathrm{CO}_{2}$ Cherenkov which serves as a trigger. For more information regarding the Arc and eP measurements, see [188, 191]. 
$G_{E}^{n}$ used a variation of the Arc method known as the Tiefenback method. This method is calibrated using the invasive Arc method, and then the beam energy may be continuously monitored by using the relationship between the dipole field integrals and the current setpoint, yielding the same relative accuracy of $5 \times 10^{-4}$ but with the advantage of being non-invasive.

\subsubsection{Polarimetry}

Hall A has two methods of determining the longitudinal beam polarization prior to scattering off the target: Møller and Compton polarimetry. The Møller polarimeter operates on the principle of polarized Møller scattering, $\vec{e}^{-}+\vec{e}^{-} \rightarrow e^{-}+e^{-}$, in which a helicity driven asymmetry arises. A polarized QED calculation results in a cross section evaluated in the center-of-mass (CM) frame that depends on the polarizations of the beam and target:

$$
\sigma \propto 1+\sum_{i} A_{i i} \cdot P_{i}^{\mathrm{t}} \cdot P_{i}^{\mathrm{b}}
$$

The sum is over $i=X, Y, Z$, and are directions that correspond to the polarization projections assuming that the incoming electrons are oriented along the $\hat{z}$-direction and the reaction occurs within the $x z$-plane. The polarizations of the target and beam are $P_{i}^{\mathrm{t}}$ and $P_{i}^{\mathrm{b}}$, respectively, and $A_{i i}(\theta)$ is the analyzing power of the reaction and solely depends on the CM angle $\theta$. The components of the analyzing power are

$$
\begin{aligned}
& A_{x x}=-\frac{\sin ^{4} \theta}{\left(4-\sin ^{2} \theta\right)^{2}}=-\left.\frac{1}{9}\right|_{\theta=\frac{\pi}{2}}, \\
& A_{z z}=-\sin ^{2} \theta \frac{8-\sin ^{2} \theta}{\left(4-\sin ^{2} \theta\right)^{2}}=-\left.\frac{7}{9}\right|_{\theta=\frac{\pi}{2}},
\end{aligned}
$$


and $A_{y y}=-A_{x x}$. The longitudinal component of the analyzing power $A_{z z}$ is maximized if $\theta=\pi / 2$, and it follows that the transverse asymmetry components are smaller by a factor of 7 . Therefore, the beam polarization may be calculated by measuring the counting rate asymmetry, the target polarization, and target angle. In practice, the polarized electron target consists of a magnetized ferromagnetic foil. The foil may be rotated relative to the beam in the horizontal plane, resulting in a target with longitudinal and transverse polarization components. By measuring the counting rate asymmetry at target angles of $\sim \pm 20^{\circ}$ and averaging, systematic uncertainties and false asymmetries may be reduced. Averaging also tends to cancel the transverse components as rotating the target introduces a relative minus sign. The detector package to measure the outgoing electrons in coincidence consists of a magnetic spectrometer and a two-arm lead-glass calorimeter. The Møller polarimeter is invasive, and takes roughly one hour for a measurement with a statistical error around $0.2 \%$. The Møller polarimetry measurements taken throughout the duration of $G_{E}^{n}$ are summarized in Table 4.3 , the data was re-analyzed in 2009 when more precise target information was available, effectively reducing the uncertainties.

The second method of measuring the beam polarization employed a Compton polarimeter in order to measure the counting rate asymmetry associated with polarized Compton scattering, or $\vec{e}+\vec{\gamma} \rightarrow e^{\prime}+\gamma^{\prime}$. An asymmetry arises by scattering longitudinally polarized electrons from a circularly polarized laser beam when the beam helicity is flipped, resulting in a non-invasive process to which the polarization of the beam may 


\begin{tabular}{lcc}
\hline Date of Measurement & $Q^{\mathbf{2}}\left(\mathrm{GeV}^{2}\right)$ & Møller \pm stat \pm sys (\%) \\
\hline $02 / 28 / 2006$ & 1.16 & $-88.43 \pm 0.08 \pm 2.0$ \\
$03 / 04 / 2006$ & 1.16 & $+87.81 \pm 0.10 \pm 2.0$ \\
$03 / 09 / 2006$ & 2.48 & $-85.05 \pm 0.08 \pm 2.0$ \\
$03 / 25 / 2006$ & 3.41 & $-81.65 \pm 0.09 \pm 2.0$ \\
$05 / 10 / 2006$ & 1.72 & $-85.27 \pm 0.06 \pm 2.0$ \\
$05 / 12 / 2006$ & 1.72 & $+84.77 \pm 0.20 \pm 2.0$ \\
\hline Average & & $85.45 \pm 0.04 \pm 2.0$ \\
\hline
\end{tabular}

Table 4.3: The Møller polarimetry results for the duration of the experiment.

be extracted via the standard formalism:

$$
P_{e}=\frac{A_{\mathrm{exp}}}{P_{\gamma} \cdot A_{\mathrm{th}}},
$$

where $A_{\text {th }}$ is the QED polarized asymmetry calculation, $P_{e}$ and $P_{\gamma}$ are the polarizations of the incoming electrons and photons, respectively, and $A_{\exp }$ is the corrected counting rate asymmetry measured during polarimetry. A rotatable quarter-wave plate may be used in order to reverse the polarization of the photon beam; therefore, the asymmetry may be measured for right and left circularly polarized photons which reduces false asymmetries associated with the laser. The apparatus to measure this asymmetry may schematically seen within Figure 4.8. The polarimeter consists of a four-dipole magnetic chicane, a photon source, silicon strip detectors for electron detection, and an electromagnetic calorimeter for scattered photon detection. In this design, beam electrons are magnetically deflected towards the photon source in which scattering may 


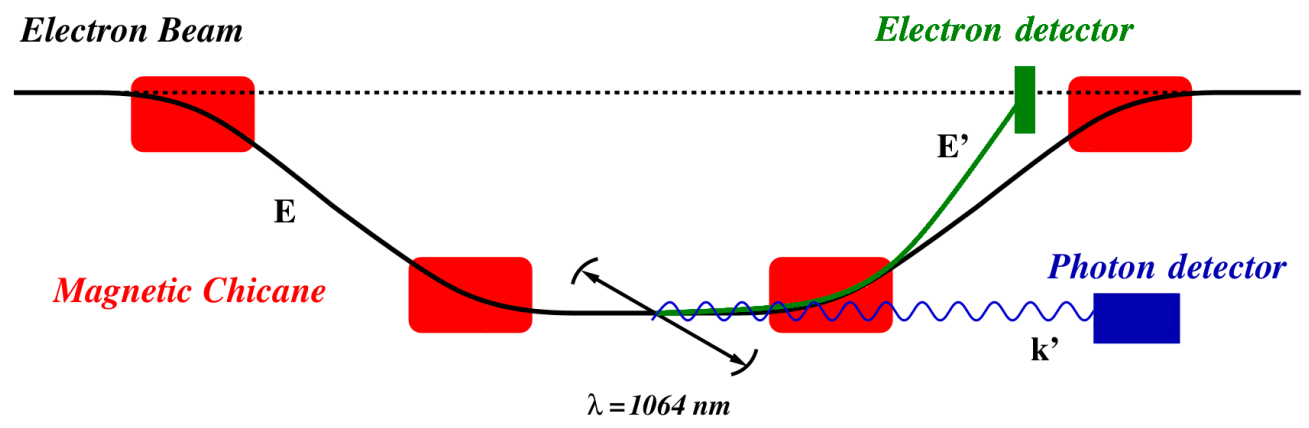

Fig. 4.8: Hall A Compton polarimetry schematic [191].

take place; the backscattered photons are detected in a $5 \times 5 \mathrm{PbWO}_{4}$ calorimeter in coincidence to the scattered electrons which are momentum analyzed within the third dipole and detected using four planes of silicon strips. The photon source employs a resonant, high-finesse Fabry-Pérot cavity in which the primary $230 \mathrm{~mW}, \lambda=1064 \mathrm{~nm}$ laser beam* is amplified by a factor of 7300 , resulting in circularly polarized light beam of power around $1680 \mathrm{~W}$ inside the cavity. The result is a non-invasive method of measuring the beam polarization to a statistical uncertainty of around $1 \%$ in less than an hour of data taking. The Compton polarimetry measurements for $G_{E}^{n}$ are summarized in Figure 4.9, and represents polarimetry measurements for Kinematic 2 and 3. While Kinematic 4 data is available, some of the data has been excluded as the statistical errors are prohibitively large. For more information regarding the Møller and Compton polarimetry at Jefferson Lab, see [188, 191-194].

While it is not Hall A beamline equipment, the Mott polarimeter located in the accelerator injector was able to provide valuable information regarding the polarization; the results are summarized Table 4.4 for completeness.

* A CW Nd:YaG laser is used. 


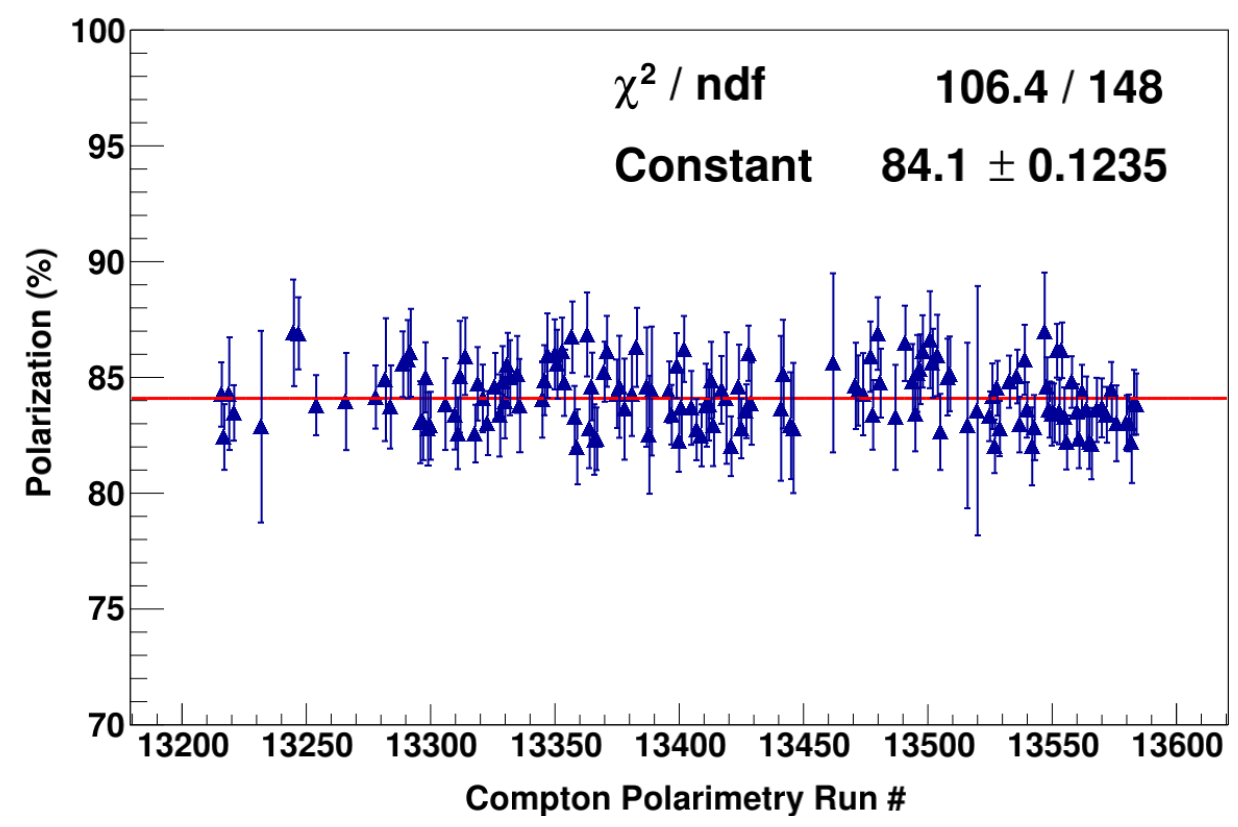

Fig. 4.9: Compton polarization measurements taken over the period 3/30 - 5/01 of 2006 corresponding to Kinematics 2-4. Some Kin. 4 data has been removed due to prohibitively large error bars. The error bars are statistical.

\begin{tabular}{lccc}
\hline Date of Meas. & $\boldsymbol{Q}^{\mathbf{2}}\left(\mathrm{GeV}^{\mathbf{2}}\right)$ & Time & Polarization (\%) \\
\hline $03 / 30 / 2006$ & 3.41 & $18: 44$ & $83.32 \pm 1.45 \pm 1.00$ \\
$03 / 30 / 2006$ & 3.41 & $18: 50$ & $81.62 \pm 1.45 \pm 0.98$ \\
$04 / 13 / 2006$ & 3.41 & $09: 43$ & $84.12 \pm 1.11 \pm 1.01$ \\
$04 / 13 / 2006$ & 3.41 & $09: 49$ & $83.25 \pm 1.11 \pm 1.00$ \\
\hline Average & & & $83.24 \pm 0.67 \pm 1.00$ \\
\hline
\end{tabular}

Table 4.4: Measurements of the beam polarization using the $5 \mathrm{MeV}$ Mott polarimeter. 


\subsection{Targets}

There were several targets used throughout the experiment:

- three polarized ${ }^{3} \mathrm{He}$ cells for production runs;

- an empty target for background studies and beam-tuning;

- carbon and beryllium-oxide foils for calibrations and corrections;

- a reference cell that may be filled with

- $\mathrm{H}_{2}$ for elastic ep events and asymmetry corrections,

- $\mathrm{N}_{2}$ for asymmetry corrections, ${ }^{*}$

- nothing and is simply an empty cell for calibrations and background studies.

A target ladder consisted of the above elements in that order, and had the ability to move vertically up-and-down to toggle the target. The ladder was mounted to the roof within a 0.25 " thick iron box, and the whole unit was simply referred to as the target box. As $G_{E}^{n}$ is a beam-target asymmetry experiment, then aspects related to the polarization of the ${ }^{3} \mathrm{He}$ target are of the utmost importance. This section will begin with a discussion of the principles behind polarizing ${ }^{3} \mathrm{He}$, and in particular the novel approach of hybrid spin-exchange optical pumping which was a technique first used for this experiment to polarize a nuclear target. The design to magnetize the target box in order to provide a uniform magnetic field over the target region will be described in detail, which includes the compass apparatus used to measure the magnetic field direction. Furthermore, the

\footnotetext{
* Kinematic 1 did not take any $\mathrm{N}_{2}$ data, therefore carbon foils must serve as a substitute.
} 
methods to measure and monitor the target polarization will be presented, and lastly the relevant details of the remaining targets will be described.

\subsubsection{Helium-3 as a Neutron Target}

The data of the neutron electric form factor is sparse when compared to the other three nucleon form factors for primarily for two reasons: 1) $G_{E}^{n}$ is small and 2) significant experimental and theoretical efforts are needed in order to overcome the difficulty of no free neutron sources. The latter complication is a consequence of a free neutron weakly decaying via $n \rightarrow p+e^{-}+\bar{\nu}_{e}$ with a mean lifetime of $880.2 \pm 1.0$ seconds [26].* Stable neutrons only exist within the nucleus, and as a result light, bound-state nuclei must be chosen as a substitute target if one wants to study the neutron; natural candidates are deuterium and helium. Neutron Rosenbluth extractions exclusively used deuterium while double polarization experiments typically use deuterium for recoil polarimetry and both polarized deuterium and polarized ${ }^{3} \mathrm{He}$ for beam-target asymmetry measurements. Polarized ${ }^{3} \mathrm{He}$ is an advantageous choice as an effective neutron target for an asymmetry experiment as the majority of the ground state is dominated by the spatially symmetric S-state in which the protons are restricted to a spin-singlet state due to Pauli exclusion; consequently, the effect of their magnetic moments tend to cancel resulting in a nucleus where the neutron carries $\sim 86 \%$ of the spin [27, 29, 31, 33, 34]. The contribution of the $\mathrm{P}$-state is negligible and ignored, but the $\mathrm{D}$ and $\mathrm{S}^{\prime}$-state contributions are roughly $10 \%$ and $1.5 \%$, respectively, and subsequently there is a small but non-negligible spin contribution of the protons. The dominant spin contributions of ${ }^{3} \mathrm{He}$ are summarized

\footnotetext{
* The discrepancy between beam and bottle measurements is a current topic of interest.
} 


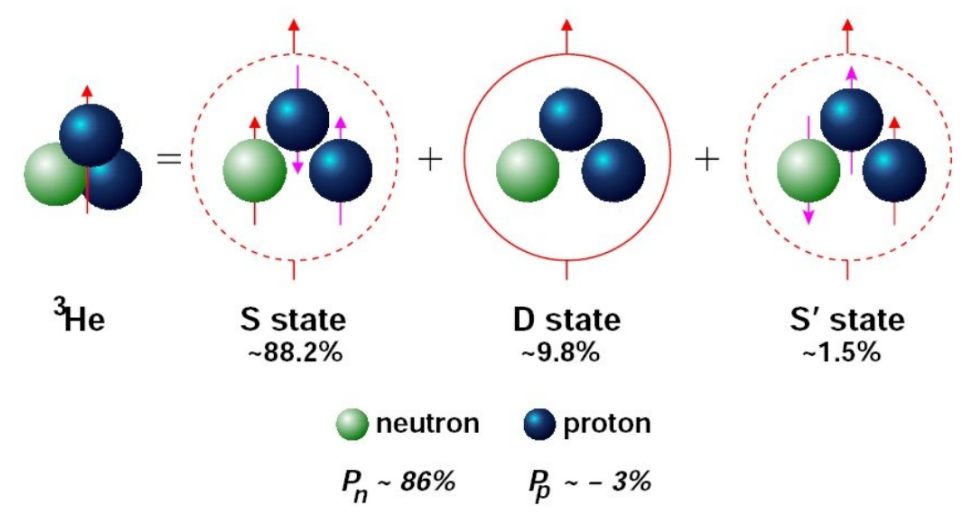

Fig. 4.10: The dominant spin contributions of the ${ }^{3} \mathrm{He}$ nucleus.

in Figure 4.10. The downside, though, is that the neutron is bound to a nucleus and complications arise due to various nuclear corrections, e.g. Fermi motion and final state interactions. In other words, the initial momentum of the nucleons within the nucleus is non-trivial, and the initially struck nucleon may interact with the remaining two spectator nucleons. Furthermore, the naive ratio of protons to neutrons for ${ }^{3} \mathrm{He}$ is 2 ; therefore, a method needs to be introduced to differentiate between the nucleons. The data analysis will need to take these considerations into account by accounting for the less than $100 \%$ polarization of the neutron, correcting the measured asymmetry for the possibility of protons contaminating the neutron signal, and calculating the contributions due to nuclear effects.

\subsubsection{Principles of Polarizing a ${ }^{3} \mathrm{He}$ Target}

A common technique to polarize noble gas atoms is to use spin-exchange optical pumping (SEOP) which is relatively well-understood and has been used in industry and R\&D for decades. Conceptually, SEOP is a two step process: 1) spin-polarize alkali metal 


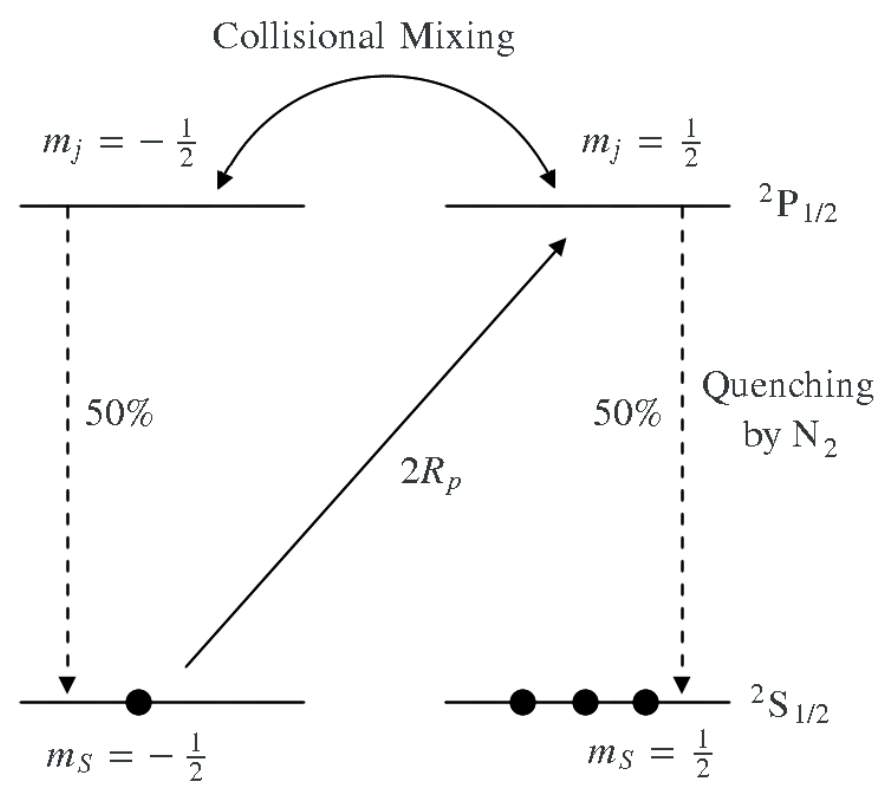

Fig. 4.11: Rb atoms interacting with circularly polarized light.

atoms by optical pumping; and 2) transfer the polarization from the alkali metal atoms to the noble gas atoms through spin-exchange. In practice, this requires a glass cell to be filled with an alkali-metal vapor and the noble gas to be polarized, or ${ }^{3} \mathrm{He}$ for $G_{E}^{n}$. Most commonly, the alkali metal used is rubidium $(\mathrm{Rb})$ as vaporization occurs at modest temperatures $\left(<130^{\circ} \mathrm{C}\right)$, has a large Zeeman splitting, and can be optically pumped with readily available lasers such as dye, titanium sapphire and gallium aluminum arsenide injection lasers.* The optical pumping of the Rb atoms is diagrammatically summarized by Figure 4.11 in which circularly polarized resonance light can excite atoms from the spin-down ${ }^{2} \mathrm{~S}_{1 / 2}$ state to the spin-up ${ }^{2} \mathrm{P}_{1 / 2}$ state with some absorption rate $R_{p}$. From here, both sublevels of the excited ${ }^{2} \mathrm{P}_{1 / 2}$ state equalize through collisional mixing. The atoms in the excited ${ }^{2} \mathrm{P}_{1 / 2}$ states can de-excite by emitting photons; one photon may

\footnotetext{
* For these reasons, the target cell must be placed in an oven with a magnetic field present.
} 
possibly depolarize multiple atoms. In order to suppress this de-excitation channel, the diatomic molecule $\mathrm{N}_{2}$ is introduced in order to quench the excited atoms. In other words, the vibrational and rotational degrees of freedom of $\mathrm{N}_{2}$ allow for $\mathrm{Rb}$ to deexcite through a collision rather than the emission of a photon. As a result, the ground state sublevels are repopulated via quenching collisions with $\mathrm{N}_{2}$ with approximately equal probability, and overtime the alkali-metal acquires a net polarization. The now polarized $\mathrm{Rb}$ atoms can spin-exchange, or partially transfer spin polarization, with the ${ }^{3} \mathrm{He}$ through a hyperfine interaction between the $\mathrm{Rb}$ valence electrons and the ${ }^{3} \mathrm{He}$ nucleus, or more explicitly

$$
H_{\mathrm{SE}}=\alpha(r)\left(\mathrm{K}^{3} \mathrm{He} \cdot \mathrm{S}^{\mathrm{Rb}}\right)
$$

where $\mathrm{S}^{\mathrm{Rb}}$ is the electron spin and $\mathrm{K}^{3} \mathrm{He}$ is the spin of the ${ }^{3} \mathrm{He}$ nucleus. The coupling function $\alpha(r)$ depends on the distance $r$ between the $\mathrm{Rb}^{3}{ }^{3} \mathrm{He}$ pair, and is dominated by the Fermi-contact interaction.

There are spin relaxation mechanisms that limit or destroy the acquired polarization of ${ }^{3} \mathrm{He}$ in SEOP. The excited $\mathrm{Rb}$ atoms can emit unpolarized photons which may be reabsorbed by other atoms, and possibly depolarizing multiple Rb atoms before the photon eventually leaves the target cell; the result is optically depumping the Rb atoms. This mechanism has been mitigated with the introduction of the $\mathrm{N}_{2}$ quenching gas.* Adding more rubidium atoms to the system can increase the spin-exchange rate, but there are limitations. Spin-polarized rubidium atoms may collide providing a mechanism to spin-relax in target cells where the $\mathrm{Rb}$ vapor pressure is high. The spin

\footnotetext{
* An electron can scatter from $\mathrm{N}_{2}$ rather than a ${ }^{3} \mathrm{He}$ nucleon, and must be corrected during the analysis phase.
} 
destructive cross section for Rb-Rb is large, $\sigma \sim 1.6 \times 10^{-17} \mathrm{~cm}^{2}$, which is a factor of 10 smaller (larger) than other alkali metals such as Cs-Cs (K-K) for comparison [195]. Another possibility is a relaxation due to ${ }^{3} \mathrm{He}-{ }^{3} \mathrm{He}$ collisions which is dominated by the dipole-dipole interaction. Furthermore, the polarization of ${ }^{3} \mathrm{He}$ may be lost by interacting with the target cell walls, and extreme care and precautions are taken during cell fabrication such that this relaxation time is on the order of hours or even days. There are a large number of spin relaxation mechanisms originating from subtle and delicate physical interactions; the goal is to minimize spin-relaxation while maximizing spin-exchange such that the result is a highly polarized target. The constant battle between the rates of spin-exchange and spin-relaxation may be explicitly summarized by the following equations which have the benefit of being physically intuitive while absorbing many of the difficulties [196]:

$$
\begin{aligned}
\frac{d P^{3} \mathrm{He}}{d t} & =k_{\mathrm{SE}}[\mathrm{Rb}]\left(P_{\mathrm{Rb}}-P^{3} \mathrm{He}\right. \\
P_{\mathrm{Rb}} & =\frac{R(z)}{R(z)+\Gamma_{\mathrm{Rb}}}, \\
\Gamma_{\mathrm{Rb}} & =\left(k_{\mathrm{SE}}+k_{\mathrm{SR}}\right)\left[{ }^{3} \mathrm{He}\right]+\Gamma_{0}, \\
\frac{d R(z)}{d z} & =-[\mathrm{Rb}] \sigma_{L} \Gamma_{\mathrm{Rb}} P_{\mathrm{Rb}} .
\end{aligned}
$$

Starting from Eqn 4.11 and working downwards:

1. the $\mathrm{Rb}$ and ${ }^{3} \mathrm{He}$ polarizations, denoted by $P_{\mathrm{Rb}}$ and $P_{3^{3} \mathrm{He}}$, are constantly being transferred depending on the spin-exchange rate $k_{\mathrm{SE}}[\mathrm{Rb}]$ which depends on the density of $\mathrm{Rb}$ vapor; the ${ }^{3} \mathrm{He}$ polarization rate is slowed down by the ${ }^{3} \mathrm{He}$ spinrelaxation rate denoted by $\Gamma_{3} \mathrm{He}$ 
2. the polarization of $\mathrm{Rb}$ atoms depends on the optical pumping rate $R(z)$ at position $z$ within the target cell, and can be reduced by spin-relaxation mechanisms associated to $\mathrm{Rb}$ denoted by $\Gamma_{\mathrm{Rb}}$;

3. the Rb spin-relaxation mechanisms depend on processes associated to spin-exchange $\left(k_{\mathrm{SE}}\right)$ and spin-relaxation $\left(k_{\mathrm{SR}}\right)$ collisions with ${ }^{3} \mathrm{He}$; other spin-relaxation processes such as $\mathrm{Rb}-\mathrm{Rb}$ or $\mathrm{Rb}$ interacting with the cell walls are absorbed into the $\Gamma_{0}$ term;

4. the $\mathrm{Rb}$ optical pumping rate depends on the $\mathrm{Rb}$ vapor density [Rb], the light absorption cross section $\sigma_{L}$ and the Rb spin-relaxation mechanisms $\Gamma_{\mathrm{Rb}}$; the minus sign indicates that the rate decreases when the light needs to propagate deeper into the target cell.

While Eqn 4.11 - Eqn 4.14 are physically satisfying, the results tend to be optimistic. For example, if $k_{\mathrm{SE}}[\mathrm{Rb}]=0.1 / \mathrm{hr}$ and $\Gamma_{3} \mathrm{He}=0.005 / \mathrm{hr}$ then it is a simple integral exercise to solve for $P^{3} \mathrm{He}$ in Eqn 4.11 assuming temperature independence; the steady-

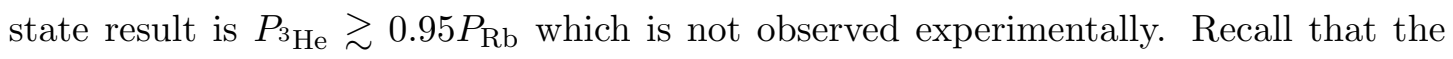
$G_{E}^{n}$ experiment observed target polarizations in the $45-50 \%$ range. Additionally, the spin-exchange rates vary wildly from mixture to mixture, and are strongly temperature dependent; therefore, the above exercise is grossly simplified.

A significant amount of effort has been dedicated towards optimizing the SEOP process; the technique has wide commercial applications, but recall that in the context of a form factor measurement the beam-target asymmetry measurement gets scaled by the target polarization. In the late 1990s, it was shown that SEOP using $\mathrm{K}-{ }^{3} \mathrm{He}$ results in spin-exchange and spin-relaxation mechanisms that are considerably more favorable 
than the traditional $\mathrm{Rb}^{3}{ }^{3} \mathrm{He}$ mixture [197]. Specifically, the spin-exchange efficiency $\eta_{\mathrm{SE}}$, defined to be the ratio of the spin-exchange rate to the rate at which polarization is lost, is limited to about $2 \%$ for $\mathrm{Rb}^{3}{ }^{3} \mathrm{He}$ mixtures; therefore, about 50 photons are required to produce a single polarized ${ }^{3} \mathrm{He}$ nucleus. On the other hand, $\mathrm{K}-{ }^{3} \mathrm{He}$ should only require four photons per polarized nucleus [198]. There is a major drawback, though, as diode lasers for potassium are not as readily available as for rubidium. Subsequently, a mixture including $\mathrm{Rb}$ and $\mathrm{K}$ began to be used in an attempt to combine the best of both worlds. A low density $\mathrm{Rb}$ vapor is optically pumped and polarized, then the polarization is transferred to the higher density $\mathrm{K}$ vapor via rapid spin-exchange collisions; note that the K-Rb spin-exchange cross section is roughly $200 \times 10^{-16} \mathrm{~cm}^{2}$ and much larger than the $\mathrm{Rb}-\mathrm{Rb}$ cross section resulting in high spin-exchange rates at typical cell densities. Finally, the ${ }^{3} \mathrm{He}$ is polarized primarily by efficient collisions with $\mathrm{K}$ atoms, but also to some extent by Rb spin-exchange collisions. In this case, Eqn 4.11 gets the following alterations:

$$
\begin{aligned}
k_{\mathrm{SE}}[\mathrm{Rb}] & \longrightarrow k_{\mathrm{Rb}}[\mathrm{Rb}]+k_{\mathrm{K}}[\mathrm{K}] \\
\Gamma_{\mathrm{Rb}} & \longrightarrow \Gamma_{\mathrm{Rb}}+\mathcal{D} \Gamma_{\mathrm{K}}+q_{\mathrm{K}, \mathrm{Rb}}[\mathrm{K}]
\end{aligned}
$$

where the SE subscript in the first line has been suppressed, $\mathcal{D}=[\mathrm{K}] /[\mathrm{Rb}], \Gamma_{\mathrm{K}}$ is the total relaxation rate for $\mathrm{K}$ atoms, and $q_{\mathrm{K}, \mathrm{Rb}}[\mathrm{K}]$ is the $\mathrm{K}-\mathrm{Rb}$ loss rate. This technique is known as hybrid SEOP and represents a significant advancement in the SEOP field. $G_{E}^{n}$ was the first experiment to utilize this novel approach to polarize a nuclear target, and obtained polarizations of $\sim 50 \%$ for the three highest $Q^{2}$ points. For example, the target cell Edna was used for 48 days with a polarization that sometimes exceeded $50 \%$. 


\begin{tabular}{cccccc}
\hline Cell & Pol (\%) & $\mathbf{V}_{\boldsymbol{t}}\left[\mathbf{c m}^{\mathbf{3}}\right]$ & $\boldsymbol{\rho}[\mathbf{a m g}]$ & Spinup $\left(\mathbf{2 3 5} \mathbf{C}^{\circ} \mathbf{C}\right)[\mathbf{h r}]$ & $\mathbf{K} / \mathbf{R b}$ \\
\hline Barbara & 53 & 393.5 & $7.56 \pm 0.07$ & 6 & 20 \\
Dolly & 42 & 378.2 & $7.39 \pm 0.07$ & 5.6 & 18.6 \\
Edna & 54 & 374.9 & $7.40 \pm 0.07$ & 4.9 & 5.1 \\
\hline
\end{tabular}

Table 4.5: Selected parameters of ${ }^{3} \mathrm{He}$ production cells, see text for description.

Kinematic 1 used Barbara, 2(a) used Dolly, remainder used Edna [199].

\subsection{3 $\quad{ }^{3}$ He Target Cells}

There were a multitude of cells designed for experimental use, but only three were used for production: Barbara, Dolly and Edna. The cells were handblown at Princeton by Mike Souza, and then filled with the appropriate mixtures at UVA [176]. Table 4.5 displays some of the operational parameters of the ${ }^{3} \mathrm{He}$ target cells used during Kinematics 1-4 where the Pol column refers to the maximum obtained polarization, $\mathrm{V}_{t}$ is the total volume of the cell, $\rho$ is the density in amagats, ${ }^{*}$ spinup refers to the time it takes to sufficiently polarize the cell and $\mathrm{K} / \mathrm{Rb}$ is the alkali density ratio at $235^{\circ} \mathrm{C}$. Recall that the benefits of hybrid SEOP occur when the $\mathrm{K}$ vapor density is greater than the $\mathrm{Rb}$ vapor density, therefore the ratios used for Barbara and Dolly are 20:1 and 5:1 for Edna. Each cell consists of a small amount of $\mathrm{N}_{2}\left(\sim 2 \%\right.$ of the ${ }^{3} \mathrm{He}$ volume $)$ as the quenching gas, and corrections will be made during the analysis. For more technical details of the polarized targets, see the UVA target inventory [199] and the recent Ref. [200].

A polarized ${ }^{3} \mathrm{He}$ target cell is constructed out of GE180 aluminosilicate glass and

\footnotetext{
${ }^{*} 1 \mathrm{amg}=44.615 \mathrm{~mol} / \mathrm{m}^{3}$
} 


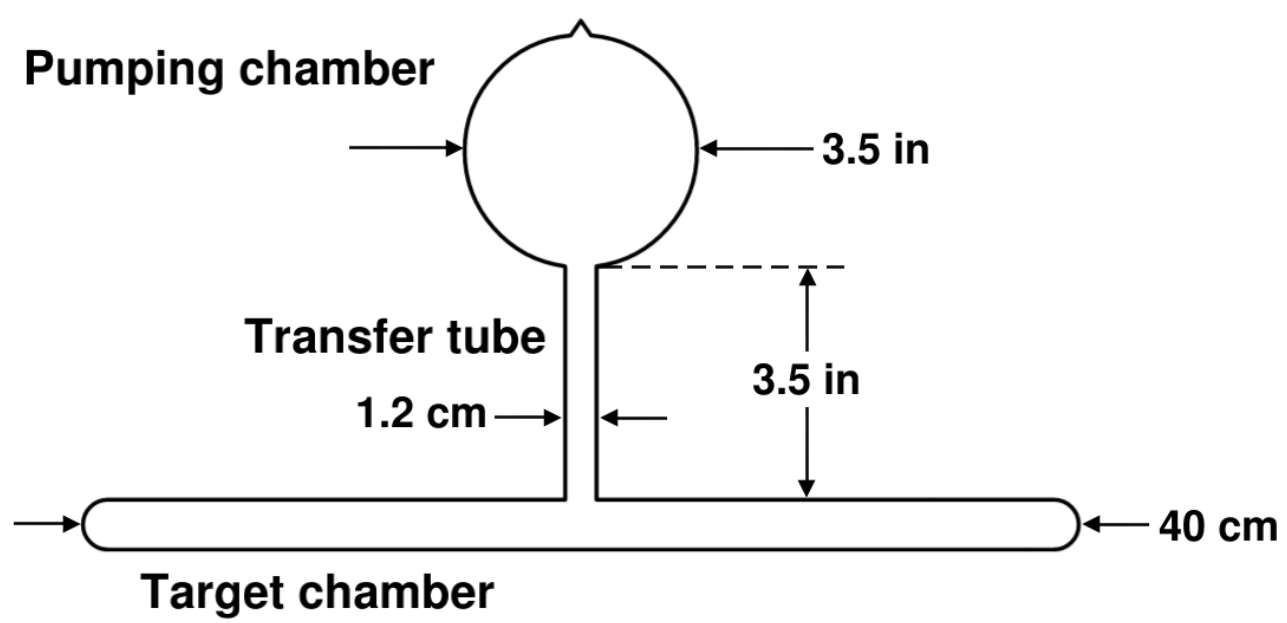

Fig. 4.12: A $40 \mathrm{~cm}$ long target cell consisting of three sections: the pumping chamber, the transfer tube and the target chamber. Hybrid SEOP occurs within the pumping chamber, and then polarized ${ }^{3} \mathrm{He}$ diffuses towards the lower temperature target chamber where scattering takes place.

consists of three sections as seen by Figure 4.12 the pumping chamber, the transfer tube, and the target chamber. The roughly 3.5 inch diameter pumping chamber is where the spin-exchange optical pumping occurs, and must be exposed to circularly polarized laser light and heated in an oven to a temperature of $\sim 235^{\circ} \mathrm{C}$ in order to achieve the necessary $\mathrm{K}$ vapor density. The purpose of the transfer tube is to separate the high temperature pumping chamber from the target chamber, and allows the polarized ${ }^{3} \mathrm{He}$ to diffuse towards the lower temperature target chamber, or the region of the cell where nuclei interact with the electron beam. All $G_{E}^{n}$ production targets had a $40 \mathrm{~cm}$ cylindrical target chamber design. The entry and exit region of the cylindrical target chamber are commonly referred to as the upstream and downstream windows, 


\begin{tabular}{lcccc}
\hline Cell & Left $(\mathbf{m m})$ & Right $(\mathbf{m m})$ & Upstream $(\mathbf{m m})$ & Downstream $(\mathbf{m m})$ \\
\hline Anna & 1.568 & 1.690 & 0.131 & 0.127 \\
Barbara & 1.568 & 1.690 & 0.151 & 0.134 \\
Dolly & 1.648 & 1.584 & 0.121 & 0.152 \\
Edna & 1.610 & 1.610 & 0.126 & 0.138 \\
Reference & 0.836 & 0.877 & 0.128 & 0.122 \\
\hline
\end{tabular}

Table 4.6: Summary of target cell wall thicknesses. Left (right) denotes side closest to $\mathrm{ND}(\mathrm{BB})$, and upstream/downstream refer to the target windows [175].

respectively. The cell walls and windows are a source of energy loss for charged particles, and due to the nature of glassblowing careful measurements of the wall thicknesses as a function of location are needed. Laser interferometry was used to measure the cell wall thickness at twelve places, ${ }^{*}$ and a summary may be seen by Table 4.6 . In order to reduce the counting rate in BigBite due to events originating from the cell end-windows, collimators were constructed out of tungsten powder combined with an epoxy with no measurable conductivity. More details on the cell thickness measurements may be found in Ref. [175].

\subsubsection{Monitoring the Polarization: NMR and EPR}

The polarization of the target is measured using two methods: adiabatic fast passage (AFP) nuclear magnetic resonance (NMR) and electron paramagnetic resonance (EPR). AFP NMR provides a relative polarization measurement and is less disruptive than the

\footnotetext{
* The two windows and five locations along each side of the cell.
} 
absolute EPR technique. In this case, a disruptive polarization measurement is one that causes significant depolarization of the target. Therefore, EPR is used to calibrate the NMR method, and then NMR is used to frequently check the polarization of the target. In the context of $G_{E}^{n}$, AFP NMR was used approximately once every four hours, while EPR was performed about once per day.

AFP NMR refers to the technique of changing the magnetic holding field which effectively reverses the spins of the ${ }^{3} \mathrm{He}$ nuclei, and applying an orthogonal $91 \mathrm{kHz} \mathrm{rf}$ magnetic field. The criteria for Adiabatic Fast Passage is satisfied if the reversal, or passage of the spins, is fast compared to spin-relaxation times, but slow compared to the Larmor precession. By operating in field sweep mode, the magnetic holding field is swept backwards and forwards from the nominal field value of $25 \mathrm{G}$ to $32 \mathrm{G}$ at a rate of $0.9 \mathrm{G} / \mathrm{s}$ while keeping a fixed rf field perpendicular to the holding field direction [176]. The $91 \mathrm{kHz}$ rf corresponds to a ${ }^{3} \mathrm{He}$ resonance at a magnetic holding field of around 28.1 G. Therefore, the sweep eventually picks up the resonance which then induces an EMF within pickup coils; the result is a readout signal $S$ which is proportional to the polarization of the target:

$$
S \propto \frac{\omega_{1}}{\sqrt{\left(\omega-\omega_{0}\right)^{2}+\omega_{1}^{2}}},
$$

where $\omega$ and $\omega_{1}$ are the precession frequencies due to the holding field and applied rf field, respectively. The target polarization is determined by the amplitude of the a Lorentzian-like fit to the readout signal providing an absolute measurement of the target polarization assuming prior calibration with the EPR method. The target depolarization due to AFP NMR was found to be $\sim 1 \%$ [177]. The major required components of this 


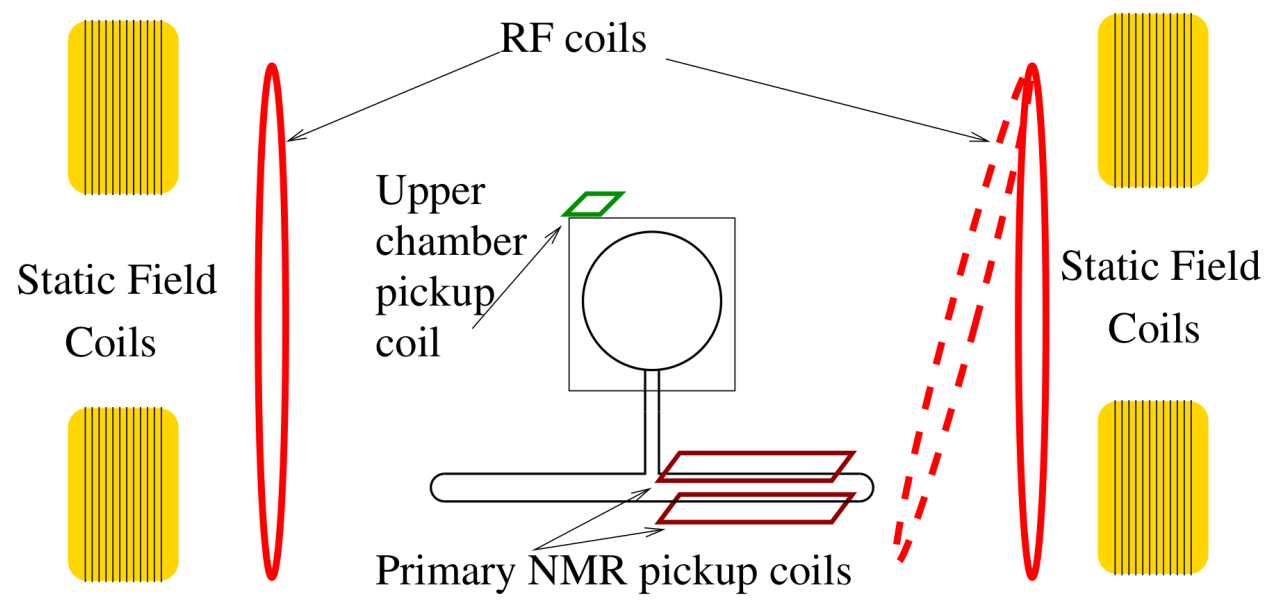

Fig. 4.13: Schematic displaying components of the AFP NMR technique [175].

technique are diagrammatically displayed in Figure 4.13 in which there are pickup coils located at the pumping and target chambers. The pickup coils and one of the RF coils could be slightly adjusted in order to maintain orthogonality with the holding field.

The second method, or EPR, takes advantage of the hSEOP alkali metals in which energy levels are split due to the external magnetic fields. The dominant splitting contribution is due to the holding magnetic field corresponding to a frequency of $\nu_{0}=$ $k_{z} B_{0}$ where $B_{0}$ is the holding magnetic field and $k_{z}$ is a constant that depends on the alkali. However, there are contributions that shift $\nu_{0}$, denoted by $\Delta \nu_{\text {other }}$, and originate from small effective magnetic fields from the spin-exchange interactions between $\mathrm{Rb}-\mathrm{K}$ and ${ }^{3} \mathrm{He}-\mathrm{K}$. Additionally, there are energy shifts due to the classical magnetic field of the polarized ${ }^{3} \mathrm{He}$ itself, and the contributions are denoted by $\Delta \nu_{\mathrm{He}}$. The shift $\Delta \nu_{\mathrm{He}}$ can be isolated by a procedure similar to AFP; however, frequency sweeping rather than field sweeping is used. In this case, the magnetic holding field is fixed but an oscillating 
rf field is applied that is in resonance with the precession of the ${ }^{3} \mathrm{He}$ nuclei due to the constant holding field. By reversing the rf field in resonance, the spins of the ${ }^{3} \mathrm{He}$ change direction, or in other words are parallel and antiparallel to the magnetic holding field direction. The energy shift corresponding to this flip is twice the frequency shift due to the ${ }^{3} \mathrm{He}$ polarization, and is proportional to the polarization, the density, and a parametrization $\kappa_{0}$ that is related to the imaginary part of the $\mathrm{K}_{-}{ }^{3} \mathrm{He}$ spin-exchange cross section. The pertinent $\Delta \nu_{\mathrm{He}}$ may be experimentally separated from the other contributions by determining the shift when the ${ }^{3} \mathrm{He}$ spins are aligned and antialigned with the holding magnetic field:

$$
\begin{aligned}
\Delta \nu_{ \pm} & =\Delta \nu_{0} \pm \Delta \nu_{\mathrm{He}}+\Delta \nu_{\mathrm{other}}, \\
\Rightarrow 2 \times \Delta \nu_{\mathrm{He}} & =\Delta \nu_{+}-\Delta \nu_{-} .
\end{aligned}
$$

The energy shift due to polarized ${ }^{3} \mathrm{He}$ is related to its magnetization $M_{\mathrm{He}}$ which is proportional to the target polarization $P_{\mathrm{He}}$ [201]:

$$
\begin{aligned}
\Delta \nu_{\mathrm{He}} & =\frac{d \nu_{\mathrm{EPR}}(F, m)}{d B} C M_{\mathrm{He}}, \\
& =\frac{d \nu_{\mathrm{EPR}}(F, m)}{d B} C n_{\mathrm{He}} \mu_{\mathrm{He}} P_{\mathrm{He}} \\
& =\frac{8 \pi}{3} \frac{d \nu_{\mathrm{EPR}}(F, m)}{d B} \kappa_{0} \mu_{\mathrm{He}} P_{\mathrm{He}}
\end{aligned}
$$

where $n_{\mathrm{He}}$ is the number density of ${ }^{3} \mathrm{He}$ nuclei, $\mu_{\mathrm{He}}$ is the magnetic moment of ${ }^{3} \mathrm{He}$ and $\nu_{\mathrm{EPR}}(F, m)$ is the EPR transition frequency associated with the quantum numbers $F$ and $m$. The constant $C$ is a dimensionless quantity, and depends on the geometry of the polarized ${ }^{3} \mathrm{He}$ cell; note that in going from the second line to the third line, the shape of the sample is assumed to be spherical. The temperature-dependent parametrization $\kappa_{0}$ 
must be obtained via measurement and does not depend on the polarization or density of the ${ }^{3} \mathrm{He}$. As a bonus, it is possible to determine the magnitude of the magnetic holding field $B_{0}$ and the direction of the target polarization relative to $B_{0}$. In the former, if it is assumed that other contributions are small then

$$
\begin{aligned}
\Delta \nu_{0} & =\frac{\Delta \nu_{+}+\Delta \nu_{-}}{2}, \\
\Rightarrow B_{0} & =-\frac{h \Delta \nu_{0}}{\mu_{B}}
\end{aligned}
$$

where $h$ is Planck's constant and $\mu_{B}$ is the Bohr magneton. If the direction of the target polarization is already known, then the EPR measurements can determine if the target polarization is aligned or antialigned relative to the holding field direction.

In regards to handling the systematic uncertainties associated to the target polarization, there are a handful of measurements that must be considered: $\kappa_{0}$, EPR, magnetic flux, temperature, density and NMR. The dominant contribution to the target polarization systematic for target cells Dolly and Edna is in the measurement of $\kappa_{0}$ and has a relative uncertainty contribution of $4 \%$. For Barbara, however, the largest contributions are due to the EPR, magnetic flux and density measurements, accounting for a relative uncertainty of 3\%. See Table 4.12 of Ref. [175] for the target error budget.

\subsubsection{Measuring the Polarization Direction}

The physical asymmetry has been introduced by Eqn 2.107 and is a function of the target polarization vector $\mathbf{P}$, specifically $P_{x}=\sin \theta^{*} \cos \phi^{*}$ and $P_{z}=\cos \theta^{*}$. Recall that $\theta^{*}$ is the laboratory polar angle of the target polarization vector with the momentum transfer oriented in the $\hat{\boldsymbol{z}}$ direction and $\hat{\boldsymbol{y}}$ is normal to the electron scattering plane, 
see Figure 2.3. In order to maximize the perpendicular physical asymmetry for a form factor ratio extraction, the ideal orientation of the target polarization is perpendicular to momentum transfer and within the reaction plane, or more explicitly $\theta^{*}=\pi / 2$ and $\phi^{*}=0$ or $\pi$. As the direction of momentum transfer is approximately $30^{\circ}$ for quasielastic scattering, then the polar angle of the target polarization needs to be $90^{\circ}+30^{\circ}=120^{\circ}$ and contained within the scattering plane. In practice, the polarization direction may be optimized for a $G_{E}^{n}$ extraction, but the parallel component of the physical asymmetry can never be absolutely eliminated due to the finite nature of the detectors, a correction that is presented in Section 5.7.2. A Monte Carlo calculation has determined that the uncertainty associated with $\theta^{*}$ must be known with a precision better than $0.1^{\circ}$ such that the systematic contribution does not impact the uncertainty of $G_{E}^{n}$ [202], which requires a precise measurement of the magnetic holding field direction.

A special magnetic compass was developed for the magnetic holding field measurement which consisted of a strong cylindrical permanent magnet pivoting on an air bearing, which acted as a magnetic arrow pointing in the direction of the magnetic holding field; see Figure 4.14 for an illustration of the compass. Two compass magnets were used to estimate the systematic error associated to the volume of the magnet: a short (long) permanent magnet with a diameter of 0.5 " (0.25") and a length of 1" (2"). A 3 inch diameter mirror was attached to the north pole of the compass magnet, and both were mounted in a $\mathrm{V}$-grooved channel of a floating (due to compressed nitrogen) aluminum disk which was allowed to rotate freely about a piston. A solid aluminum reference bar was mounted on the outside of the target box in which a reference mirror was 


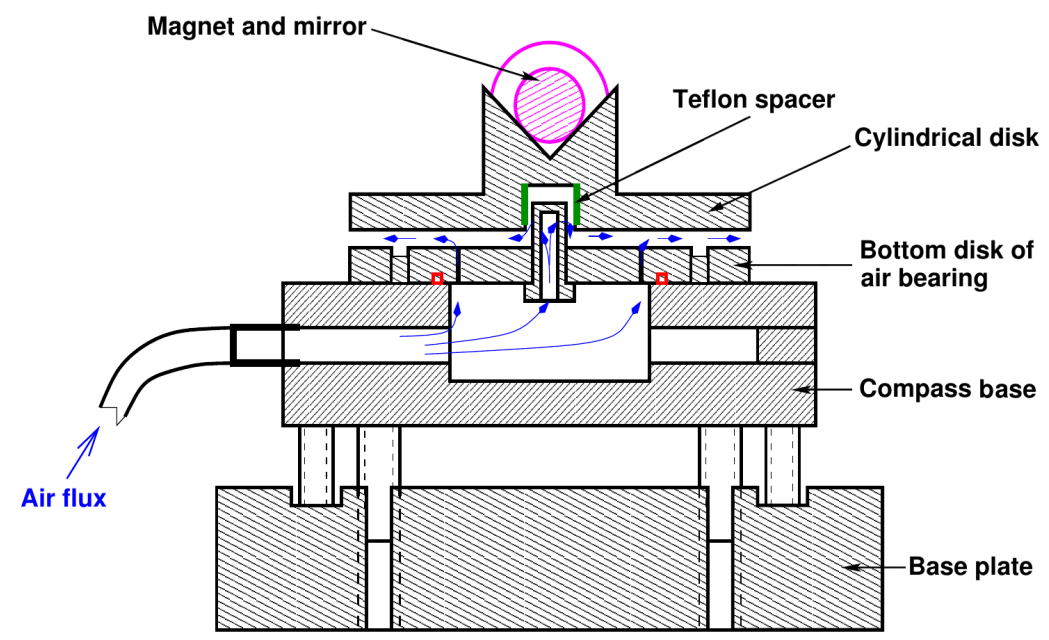

Fig. 4.14: The major components of the compass used for measurements of the magnetic holding field direction 202].

positioned at the center. A laser was mounted on a tripod that was positioned roughly $2 \mathrm{~m}$ away from the reference mirror such that the laser, reference mirror, and compass mirror were collinear. There were two relevant measurements: 1) relative measurements mapping the magnetic holding field direction within the target box; and 2) an absolute measurement to transform the relative measurements into Hall A coordinates.

The absolute measurement employed a transparent screen that was positioned in between the reference mirror and laser as seen by Figure 4.15, and two angle measurements were performed. In the first, the laser beam reflected off of the reference mirror; the light leaving the laser left a beam spot on the transparency, then reflected off of the mirror and left another beam spot depending on the angle of the mirror with respect to the transparency. In the second measurement, the reference mirror was removed and the process was repeated but in this case the compass mirror was used to reflect the laser. 


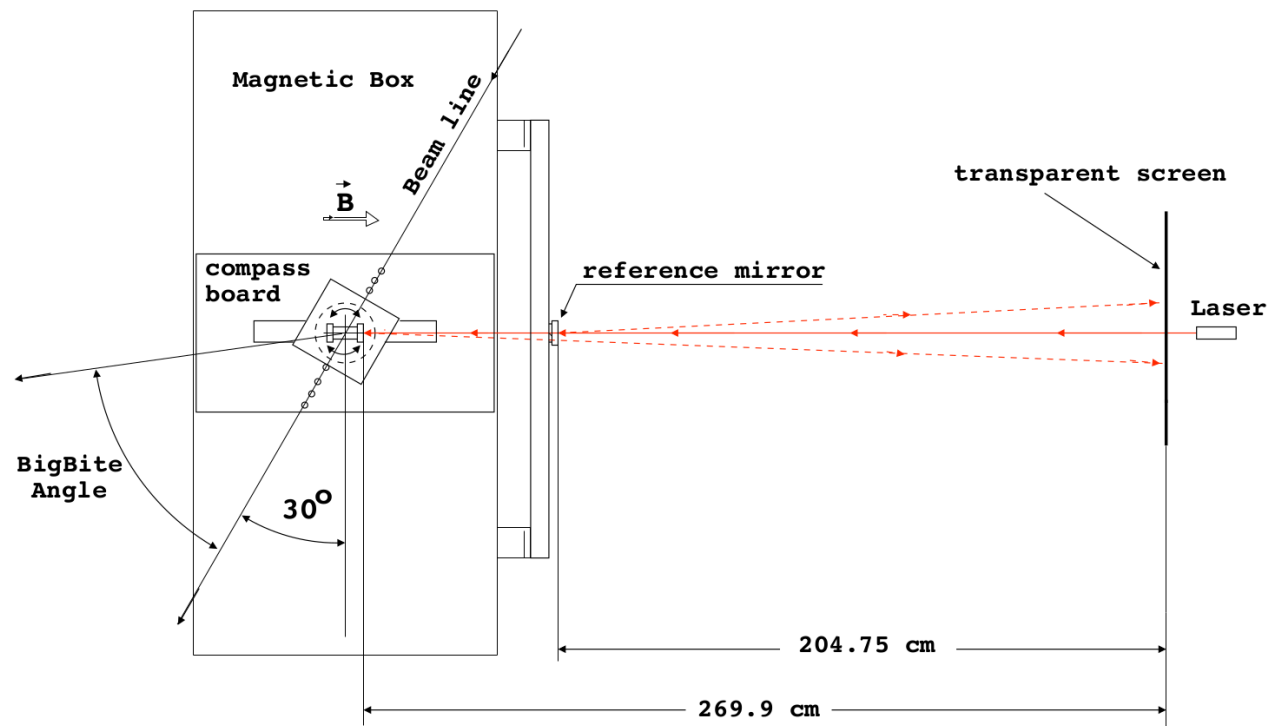

Fig. 4.15: The $G_{E}^{n}$ compass absolute measurement setup [202].

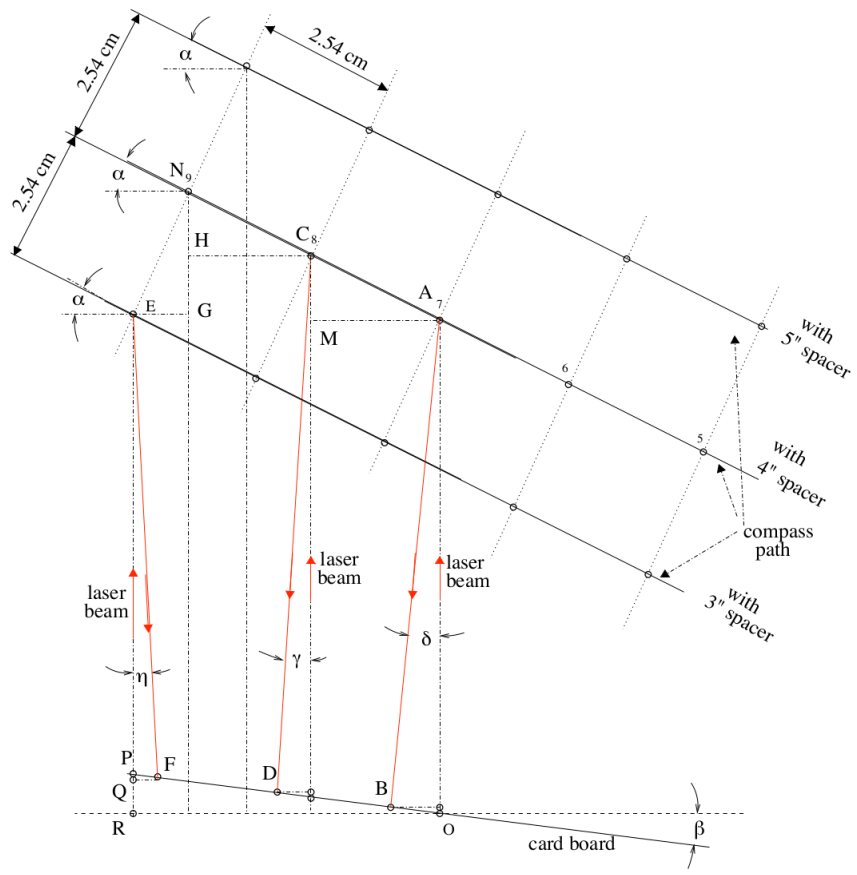

Fig. 4.16: The $G_{E}^{n}$ compass relative measurement setup [202]. 


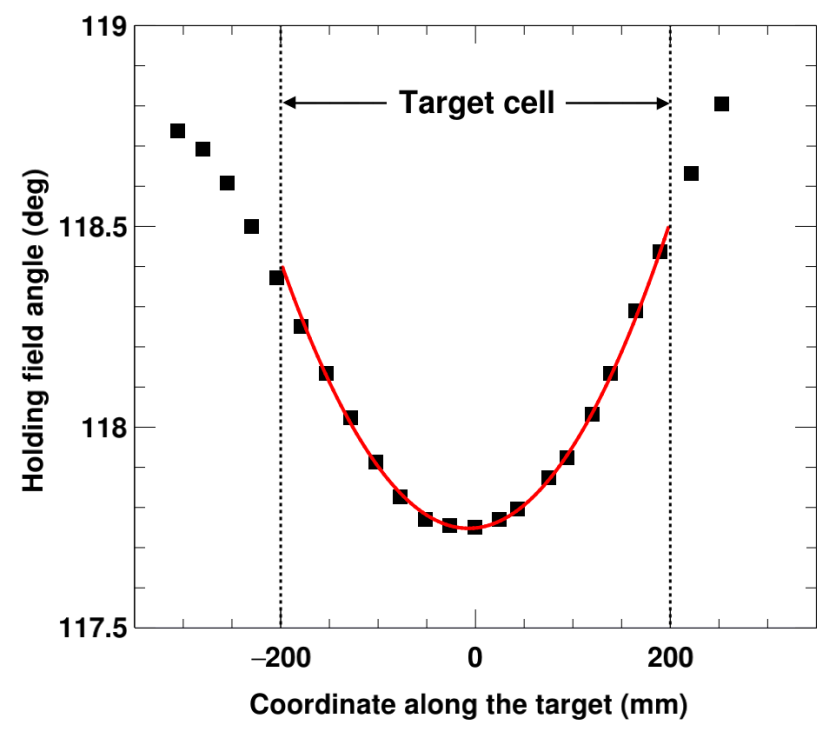

Fig. 4.17: The magnetic holding field angle, defined to be the counter clockwise angle between the beamline direction and the holding field direction, across the target cell region. See the text for information on the fit.

The two measurements provided an angle relation between the compass and reference mirror. Lastly, the position of the reference mirror within the Hall was determined by a survey performed by the Jefferson Lab Alignment Group.

The relative measurements utilized a $1 \mathrm{~mm}$ scale cardboard screen in place of the transparent screen. In this measurement, the laser beam reflected off of the compass mirror and a measurement was made using the cardboard scale. The compass was moved along the length of the target in step sizes of one inch. For consistency, the compass edge remained flush with a fixed guiding bar as it was moved along the length of the target region, see Figure 4.16 for a visualization. The measurements were repeated with different sized spacers placed in between the guiding bar and compass edge, which 
effectively mapped the magnetic holding field direction in the target box. The holding field angle, defined to be the counter clockwise angle between the beamline direction and the holding field direction, along the target cell may be seen within Figure 4.17. The parametrization $\theta=117.75^{\circ}+a\left(v_{z}+b\right)^{2}$ is used along the target cell region, or -20 to 20 $\mathrm{cm}$, where $v_{z}$ is the reconstructed vertex position along the beamline direction, $\theta$ is the holding field angle, and the fit parameters $a$ and $b$ are found to be $\left(1.78 \times 10^{-5}\right)^{\circ} / \mathrm{mm}^{2}$ and $7.0 \mathrm{~mm}$, respectively.*

\subsubsection{Description of the Target Box and Magnetic Field}

The targets were housed in a large 0.25 " iron box of dimensions $2 \times 2 \times 1 \mathrm{~m}^{3}$, which was rotated ideally by $30^{\circ}$ about the lab $y$-axis towards the neutron detector. The box had an electron exit window which was covered with $10 \mathrm{~mm}$ thick tedlar film to prevent glass particles from getting into the BigBite magnet in the event of a target cell explosion. Additionally, there were windows for target access and exiting recoiling nucleons, both of which were covered with 0.25 " thick G10 plastic sheets. The purpose of the iron box is multi-fold: (1) generate a uniform holding field for hSEOP, (2) protect the target from BigBite fringe fields, and (3) house the target oven and monitoring equipment. There were eight coils placed symmetrically at the corners of the target box in order to magnetize the iron walls and to create a uniform magnetic field of 25 Gauss across the target region; the field gradient was observed to be less than 10 mGauss/cm [202]. In addition to the magnet box itself protecting the target from BigBite fringe fields, two iron field clamps were installed in between the box and magnet, see Figure 4.18

\footnotetext{
* The measurements and analysis were originally performed by Vladimir Nelyubin.
} 


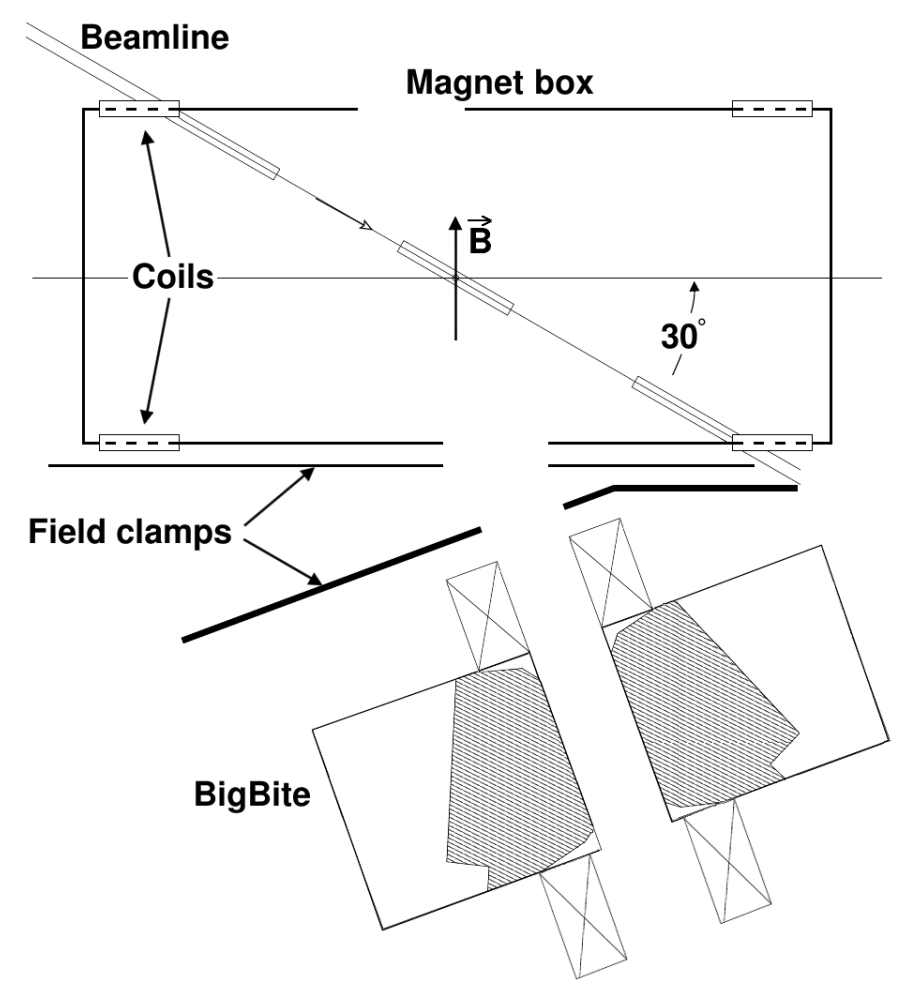

Fig. 4.18: Top down schematic of the target magnet box displaying the orientation relative to BigBite in addition to the magnetization coils and field clamps.

for a schematic of the box and the orientation relative to BigBite. Pickup coils were located at the target and pumping chambers for target polarization monitoring using AFP NMR and EPR techniques. There were RF coils inside the target box to produce a transverse magnetic field relative to the holding field for the frequency and field sweeping methods of NMR and EPR. The target temperature was monitored with eight resistive temperature devices (RTDs) located in various positions including the oven; the actual temperature in the pumping chamber, however, was generally higher than the RTD readings due to heating from the optical pumping laser light. The effect was studied by observing the change in the NMR signal with the lasers on and off which 


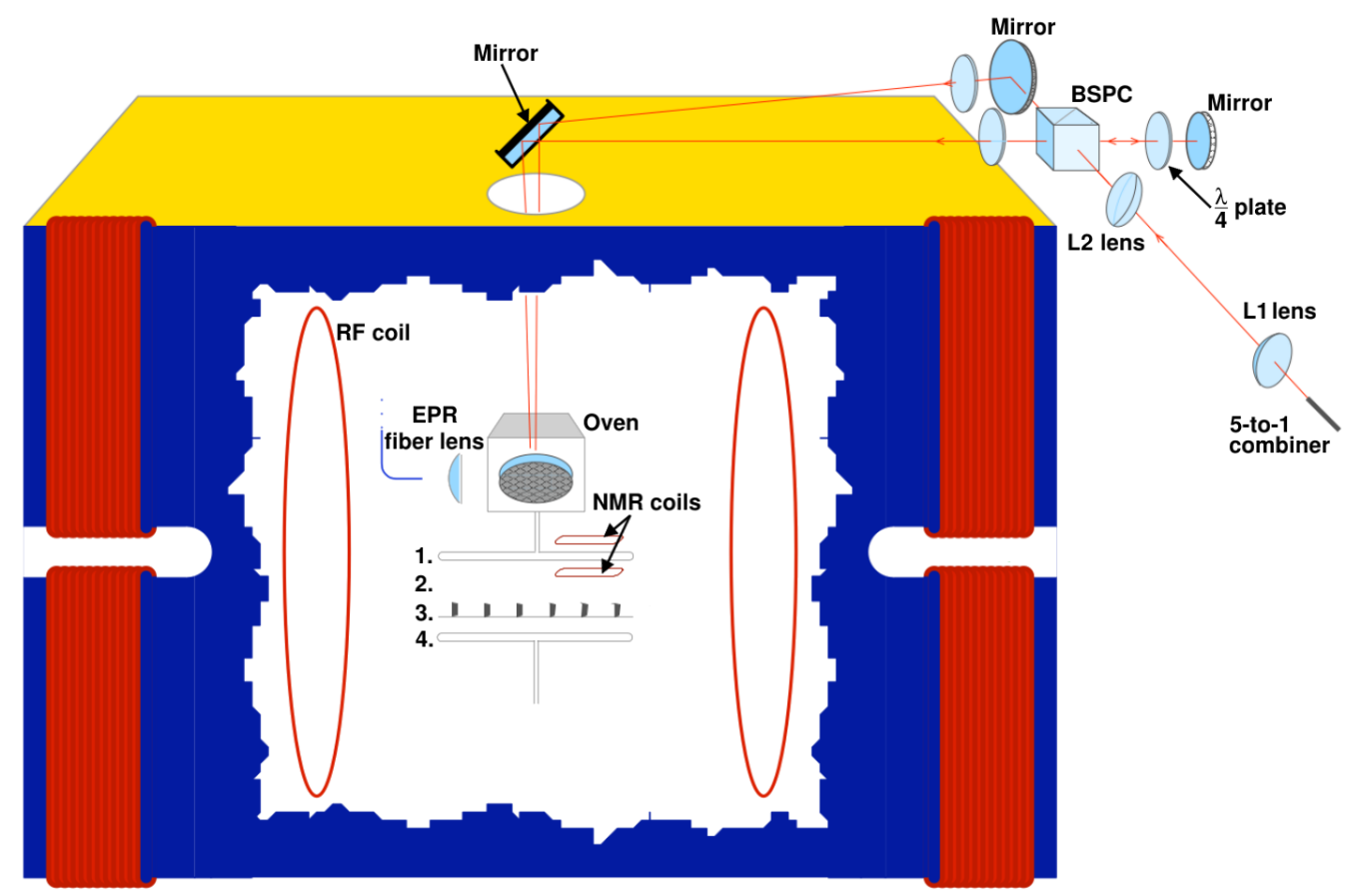

Fig. 4.19: Major components within target box including the targets, the polarizing optics, the location of the EPR and NMR measurements, and the target box magnetization coils (only 4 out of 8 are seen) [176].

gave insight to the change in density, and a temperature difference of roughly $40^{\circ}$ was determined. There was a spectrum analyzer (SA) which was used to monitor the optical pumping state of the target; furthermore, the SA could be used to determine if the laser wavelength was not optimized for optical pumping, and therefore could be adjusted accordingly. See the not-to-scale diagram in Figure 4.19 for an overview of the major equipment in addition to the polarizing optics. The five lasers were located outside of Hall $\mathrm{A}$ in the laser lab due to safety protocols, and the light was delivered to the target via $75 \mathrm{~m}$ long optical fibers. Several optical components were necessary due to 


\begin{tabular}{ccccc}
\hline Foil \# & Type & $z_{\text {lab }}[\mathbf{c m}]$ & Density $\left(\mathbf{g} / \mathbf{c m}^{\mathbf{3}}\right)$ & Thickness $\left(\mathbf{m g} / \mathbf{c m}^{2}\right)$ \\
\hline 1 & $\mathrm{C}$ & -13.3 & 2.2 & 47.8 \\
2 & $\mathrm{C}$ & -6.7 & 2.2 & 47.8 \\
3 & $\mathrm{BeO}$ & 0.0 & 3.0 & 150.1 \\
4 & $\mathrm{C}$ & 6.7 & 2.2 & 47.8 \\
5 & $\mathrm{C}$ & 13.3 & 2.2 & 47.8 \\
\hline
\end{tabular}

Table 4.7: Relevant specifications of the beryllium oxide and carbon foil target.

the five-to-one combiner and included lenses (L1 and L2), a beam-splitter-polarizingcube (BSPC) to prepare the circular polarization state necessary for optical pumping of $\mathrm{Rb}$, and quarter-wave plates. Lastly, the four targets (the ${ }^{3} \mathrm{He}$ cell and target oven, the empty setting, the beryllium oxide and carbon foils, and the reference cell) were mounted to a single milled sheet of macor, which collectively was referred to as the target ladder. The ladder was mounted to the roof of the target box, and the vertical position could then be remotely controlled in order to toggle between targets.

\subsubsection{Carbon Foil Target}

There were a set of solid foil targets utilized for event vertex reconstruction calibrations among other analyses. In total, there were six carbon foils and one beryllium-oxide foil, oriented in a C-C-C-BeO-C-C-C pattern; however, the first and last $\mathrm{C}$ foil cannot be seen in the data due to the collimators (designed to block events originating from the target end-windows to reduce the BigBite counting rate). The positions of the foil centers are summarized in Table 4.7. Towards the end of the experiment, the carbon foil 
at $-6.7 \mathrm{~cm}$ was damaged and consequently removed, which only affected the $Q^{2}=1.72$ $\mathrm{GeV}^{2}$ data. The carbon foils will be used to correct the measured asymmetry due to nitrogen contamination within the target.

\subsubsection{Reference Cell}

Many of the calibrations and analysis corrections use the data taken with the reference cell. The reference cell was ideally an exact copy of the polarized ${ }^{3} \mathrm{He}$ cell, and was connected to a gas handling system allowing the cell to be filled with different compositions such as hydrogen, nitrogen, or simply nothing. The reference cell wall measurements are summarized in Table 4.6. Special care was taken to align the reference cell as closely as possible to the ${ }^{3} \mathrm{He}$ cell in order to mitigate any rotations or offsets that could affect the data quality. Lastly, there was $1.25 \mathrm{~cm}$ of macor on the neutron side of the polarized cell, but not the reference cell; therefore, the material budgets were not identical.

\subsection{Electron Arm and the BigBite Spectrometer}

The four momentum of the quasielastically scattered electrons were measured using the BigBite spectrometer, which consisted of the following major elements: a large dipole magnet (BigBite), three multi-wire drift chambers (MWDCs), a preshower, a timing hodoscope, and a shower. BigBite was originally obtained from the Nationaal Instituut voorKernfysica en Hoge-Energiefysica-Kernfysica (NIKHEF-K) 203]. For E02-013, the large angular and momentum acceptance, non-focusing spectrometer subtended approximately $76 \mathrm{msr}$, and accepted quasielastically scattered electrons in the momentum 
range of $0.9-1.5 \mathrm{GeV} .^{*}$ BigBite could deliver a maximum field of $1.2 \mathrm{~T}$ for a current setpoint of $710 \mathrm{~A}$, and was capable of field integrals of around $1.0 \mathrm{~T} \cdot \mathrm{m}$. The magnet opening was $95 \times 25 \mathrm{~cm}^{2}$, and the magnet front-face was positioned approximately $1.1 \mathrm{~m}$ from the target center ideally along the BigBite central ray for the duration of the experiment. The spectrometer underwent one configuration change, namely a rotation from $\theta_{\mathrm{BB}}=56.3^{\circ}$ to $\theta_{\mathrm{BB}}=51.6^{\circ}$ when moving from the $Q^{2}=1.16 \mathrm{GeV}^{2}$ to the $Q^{2}=2.48 \mathrm{GeV}^{2}$ setting, respectively.

A detected quasielastically scattered electron traveled through the BigBite magnet where it was magnetically deflected upwards relative to the Hall floor. The electron then passed through the three MWDCs which were responsible for trajectory tracking, and finally the energy was deposited within the lead-glass calorimeter package. This is illustrated in Figure 4.20 where the electron is represented by the blue curve, and the green markers display registered simulation hits that may be used for event reconstruction. An electromagnetic shower is initiated within the lead-glass modules of the calorimeter, and the photons (white lines) are able to propagate to the photocathode of the PMT where detection occurs with some quantum efficiency. Figure 4.21 displays a design drawing of the detector package, which perhaps may be more easily visualized by the perspective Figure 4.22 where the support structures do not block the main detectors. The spectrometer provided a momentum resolution of $\sigma_{\delta p / p} \approx 1 \%$, an angular resolution of 0.3 and $0.7 \mathrm{mrad}$ for the horizontal and vertical directions respectively, an interaction vertex resolution of $6-7 \mathrm{~mm}$, and an electron ToF resolution of $0.25 \mathrm{~ns}$. The calorimeter observed an energy resolution of $\sigma_{\delta E / E} \approx 7.5 \%$, and was

\footnotetext{
${ }^{*}$ For the lowest (highest) $Q^{2}$ point, the elastically scattered $e^{-}$energy was $E^{\prime}=E-\frac{Q^{2}}{2 M} \approx 0.9(1.5) \mathrm{GeV}$.
} 


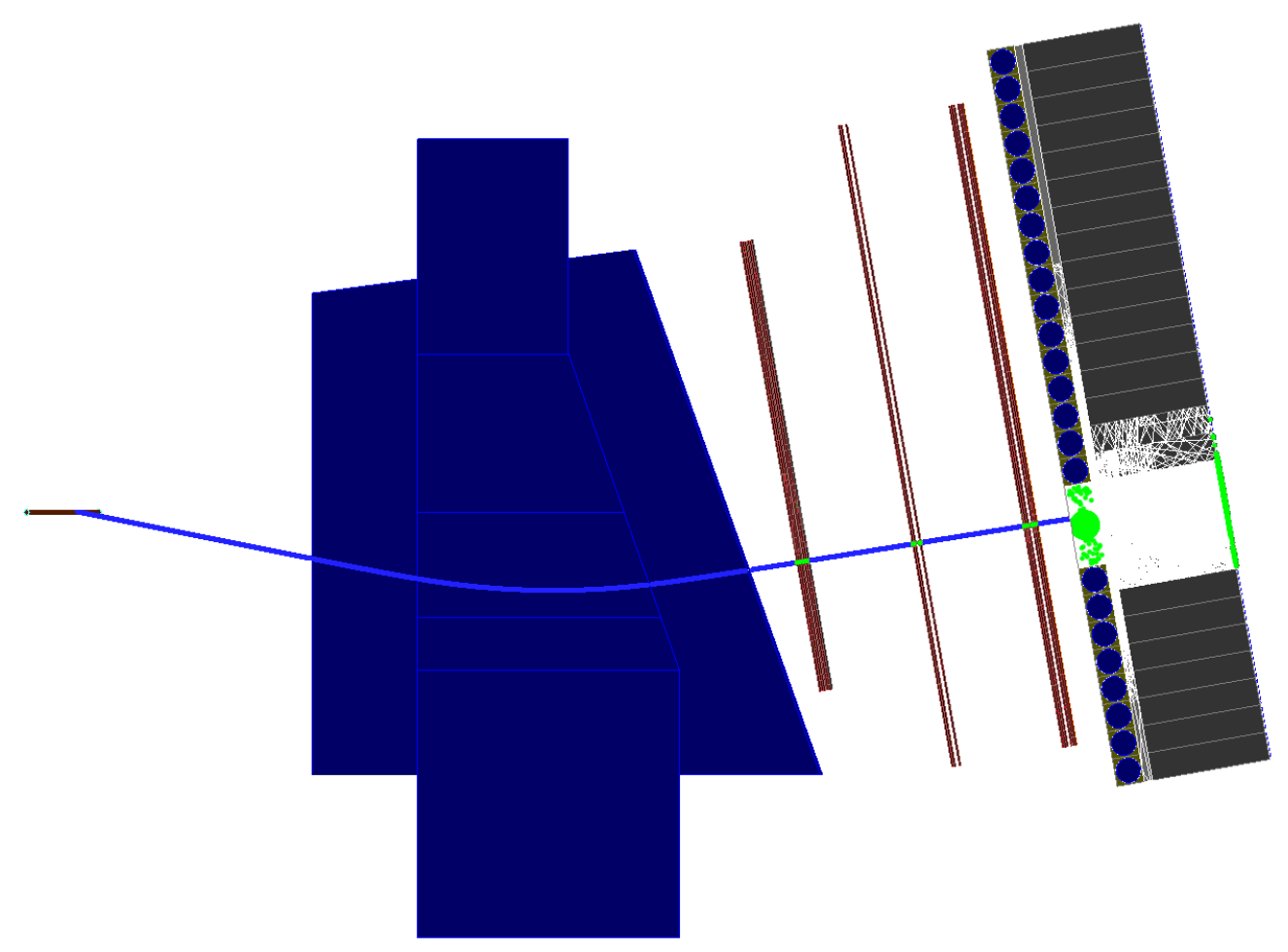

Fig. 4.20: A scattered electron traveling through the BigBite spectrometer in simulation. The electron is represented by the blue line in which it is magnetically deflected by BigBite, tracked by the three MWDCs, and finally deposits its energy within the lead-glass calorimeter package via an electromagnetic shower. The green markers represent registered hits.

used for pion-electron particle identification in addition to triggering. A major goal of the spectrometer was to reduce the background noise to acceptable levels such that the algorithms responsible for track reconstruction were fast and efficient. Many of the detectors were newly constructed for E02-013, and considerable time and effort was spent preparing the systems for production; each of the subsystems are going to be described in great detail in the next few sections. 


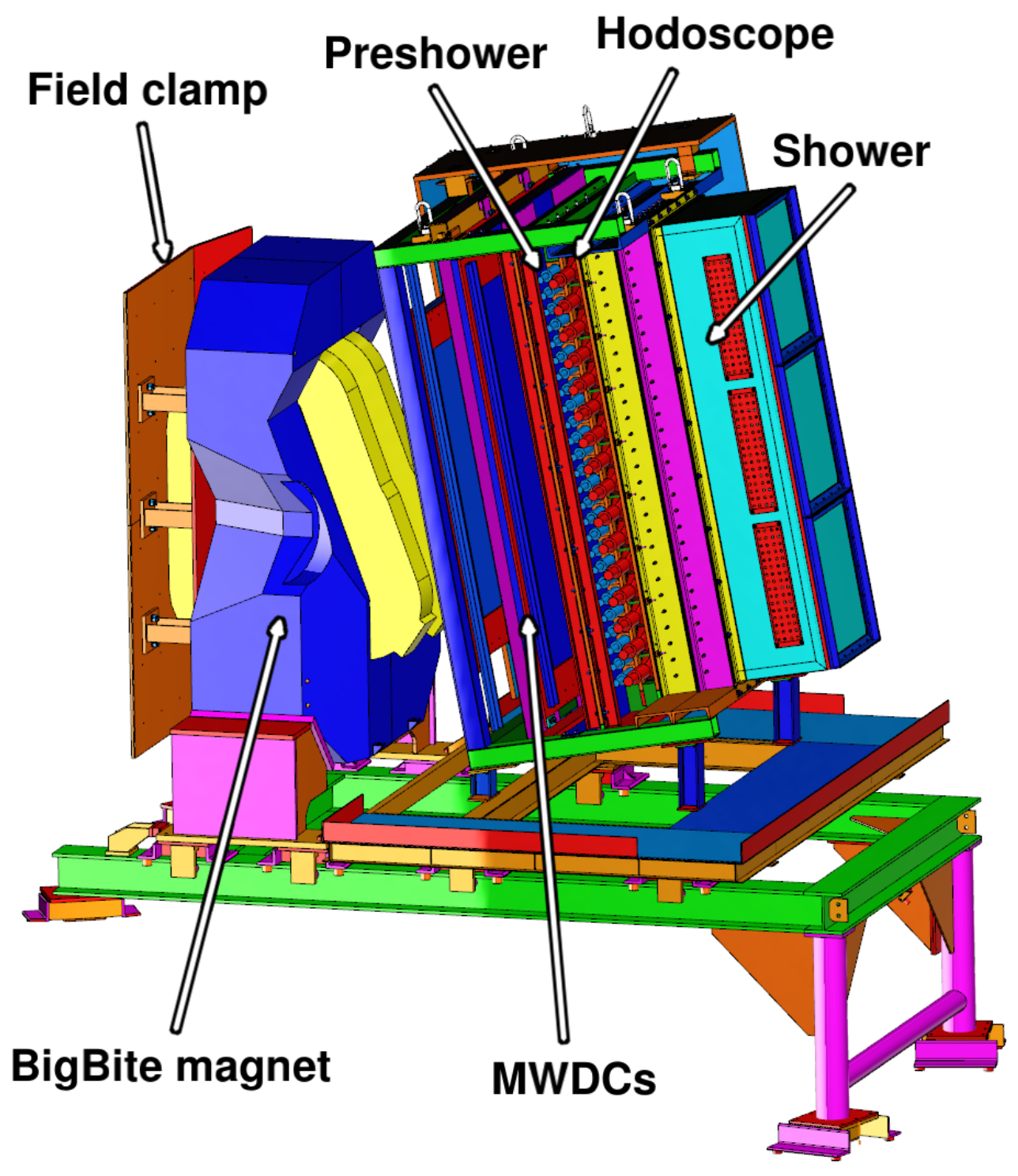

Fig. 4.21: A design drawing of the BigBite spectrometer and the support structure in which all the major components are displayed [204]. There is a field clamp on the target side of the magnet to protect the polarized ${ }^{3} \mathrm{He}$ target from BigBite fringe fields. The tracking detectors and calorimeter package are ideally pitched by $10^{\circ}$ relative to the BigBite magnet. 


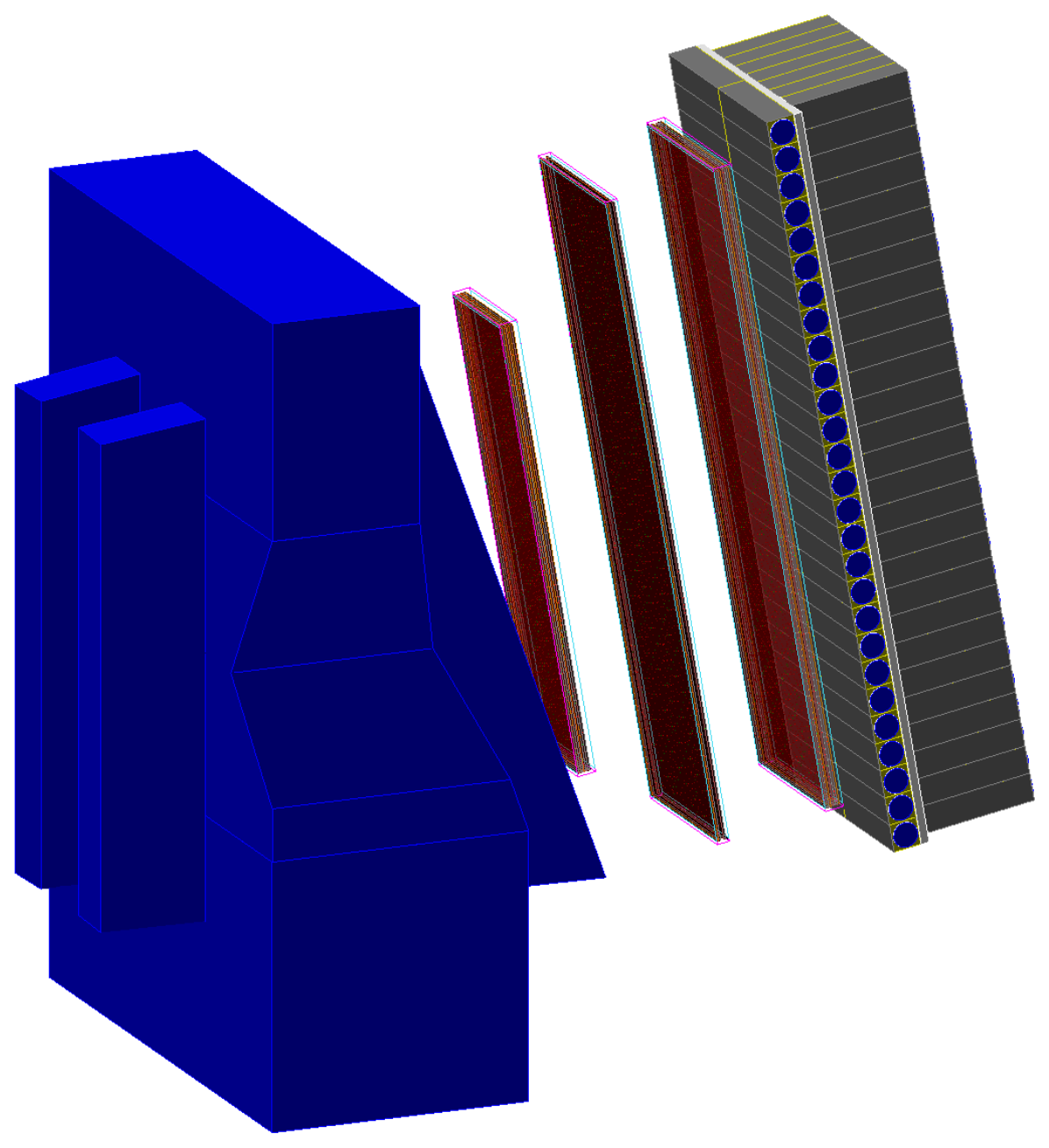

Fig. 4.22: A perspective view of the BigBite spectrometer in Monte Carlo explicitly showing the orientation of the modules within the preshower and shower. The lead-glass modules all have PMTs (blue circle) positioned at the back end of the long dimension, but the preshower modules are simply rotated with respect to the shower modules. The timing hodoscope, represented by a thin gray box, is located in between the preshower and shower. 


\subsubsection{Multi-Wire Drift Chambers}

The first set of detectors after the dipole magnet are the three multi-wire drift chambers which are capable of measuring the position of an electron with a spatial resolution of $\sim 200 \mu \mathrm{m}$ per plane. One chamber is constructed from three or six wire planes, and one plane consists of sensing wires and field wires interchangeably positioned between two cathodes. The approximate geometry may be conceptualized by Figure 4.23 in which the wires are positioned $3.0 \mathrm{~mm}$ from the cathodes; the sense (or signal) and field (or field-shaping) wires are placed in an alternating pattern, but the spacing between any two wires of the same type is $1.0 \mathrm{~cm}$. The orientation of the wires with respect to the BigBite coordinate system defines the type of plane; the pattern X has all wires parallel to $y_{\text {det }}$ while the patterns $\mathrm{V}$ and $\mathrm{U}$ have wires that are $\pm 30^{\circ}$ relative to $y_{\text {det. }}$. All wires within a particular plane are ideally positioned at the same $z_{\text {det }}$ coordinate.

For clarity, the orientations of the different patterns relative to the BigBite detector coordinates are displayed by Figure 4.24. The three drift chambers are then built out of planes of varying patterns; therefore, chambers 1, 2 and 3 have UUXXVV, UXV and

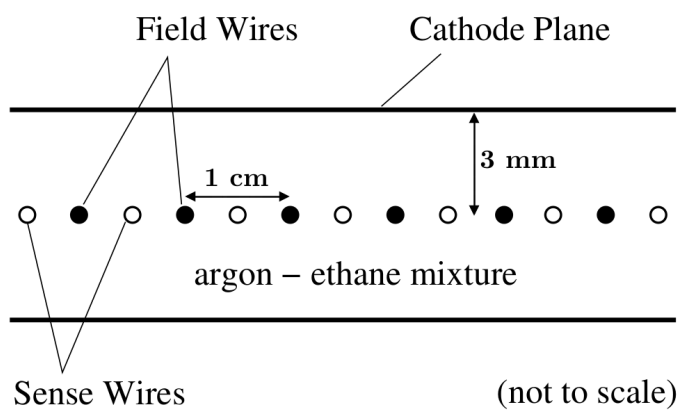

Fig. 4.23: An exaggerated diagram displaying the major features of one wire plane. 


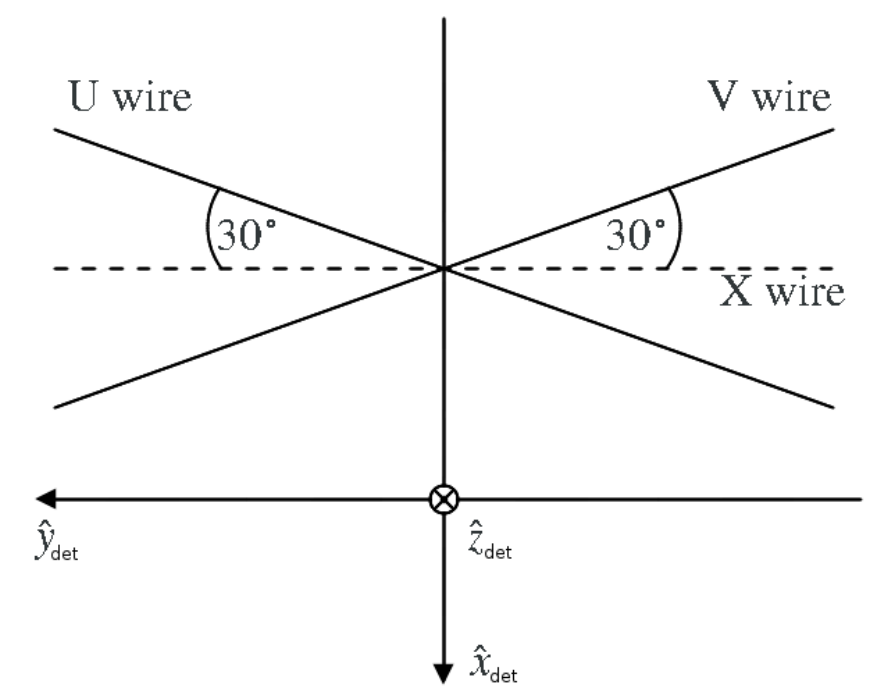

Fig. 4.24: Orientations of $\mathrm{X}, \mathrm{V}$ and $\mathrm{U}$ wires relative to $y_{\text {det }}$.

UUXXVV planes for a total of 15 wire planes. Planes of the same type are staggered by $0.5 \mathrm{~cm}$ relative to one another to resolve the left/right ambiguity. The chambers are operated with a gas mixture of $65 \%-35 \%$ argon-ethane, which flows through alcohol at $0^{\circ} \mathrm{C}$ and kept slightly above atmospheric pressure. The center of plane 1 of chamber 1 defines the origin of the BigBite detector coordinate system, and is denoted by the coordinate $z_{\mathrm{det}}=0 \mathrm{~m}$. The positions of chambers 2 and 3 are $z_{\mathrm{det}}=36$ and $71 \mathrm{~cm}$, respectively. The specifications of the MWDCs are summarized by Table 4.8 .

A relativistic electron enters a chamber and ionizes the gas mixture; the liberated electrons may initiate a localized cascade which then drift towards a sense wire due to the potential difference between the cathodes and sense wires. The field wire is designed for shaping the electric field in an attempt to minimize electron drift towards multiple wires. The sense wire signals are then directed to amplifier/discriminator cards in addition to a 


\begin{tabular}{cccccc}
\hline Chamber & Height $(\mathbf{m})$ & Width $(\mathbf{m})$ & $\boldsymbol{z}_{\text {det }}(\mathbf{m})$ & Pattern & $\begin{array}{c}\text { \# of Wires } \\
\text { per Plane }\end{array}$ \\
\hline 1 & 1.40 & 0.35 & 0.00 & UUXXVV & 142 \\
2 & 2.00 & 0.50 & 0.36 & UXV & 200 \\
3 & 2.00 & 0.50 & 0.71 & UUXXVV & 200 \\
\hline
\end{tabular}

Table 4.8: MWDC geometry specifications.

time-to-digital converter (TDC) to record a time stamp. The time relative to the trigger (to be discussed) allows a drift-time to be calculated, which may be converted into a drift-distance, i.e. a point in space that represents the distance between the electron track and the sense wire. For a candidate event, the collection of drift-times yields a collection of drift-distances which are fit to a straight line across multiple planes, and the best track is determined using $\chi^{2}$-minimization; this is track reconstruction in broad strokes. Further complications arise, though, as the drift-distance is simply a distance magnitude which is ambiguous, i.e. the hit could have occurred on either side of the sense wire; this is known as the left/right ambiguity. While planes of the same type are vertically offset by $0.5 \mathrm{~cm}$ to resolve the ambiguity associated with a drift time measurement, the technical issue persists in the context of computational resources. Reducing background to tolerable levels is imperative for successful track reconstruction as the number of track combinations for 15 possible drift planes while resolving the left/right ambiguity grows rapidly. Aggregated information obtained by the calorimeter package may be used to significantly reduce the search region for candidate tracks such that track reconstruction is successful in a large noise-to-signal environment. 


\subsubsection{Preshower and Shower}

The preshower and shower collectively act as a calorimeter and a particle identification system, and may be visualized by Figure 4.22. Additionally, the detector package is the basis of the electron arm trigger, and provides useful coordinate information that is used in conjunction with the track reconstruction algorithm to reduce the number of candidate tracks; this will be discussed in more detail in Section 5.3.5. The coordinates of the face of the preshower and shower are $z_{\text {det }}=0.85$ and $1.0 \mathrm{~m}$, respectively. Both detectors are arrays of lead-glass modules of dimension $8.5 \times 8.5 \times 35 \mathrm{~cm}^{3}$ in which there is a PMT positioned at end of the long dimension. The preshower (shower) consist of 2 columns by 27 rows ( 7 columns by 27 rows) for a grand total of $54+189=243$ modules, but the preshower modules are rotated by $\pm 90^{\circ}$ about the BigBite detector $x$-axis depending on the column.

A high energy electron radiates when penetrating a lead-glass block initiating an electromagnetic shower. Cherenkov radiation, bremsstrahlung and pair production/annihilation keep the shower developing until the energy of the primary electron falls below the critical energy, defined to be the energy at which the energy loss due to bremsstrahlung is equal to the energy loss due to ionization; when this occurs, ionization and excitation processes dominate effectively halting the longitudinal growth of the shower. The longitudinal maximum depth of the shower may be approximated by

$$
\frac{x_{\max }}{X_{0}}=\ln \frac{E_{e}}{E_{c}}-0.5,
$$

where $X_{0}$ is the radiation length of lead-glass, $E_{e}$ is the energy of the incident electron, and $E_{c}$ is the critical energy on the order of $15 \mathrm{MeV}$ for lead-glass [26]. The radiation 
length of lead-glass is small, approximately $2.7 \mathrm{~cm}$, and is defined to be the distance over which an incident electron loses all but $\frac{1}{e}$ of its initial energy. The preshower (shower) is approximately 3 (13) radiation lengths, and therefore all of the energy of the incident electron is safely deposited within the calorimeter package for $G_{E}^{n}$ kinematics. According to Eqn 4.25 and assuming $E_{e} \approx 900 \mathrm{MeV}$, an approximate maximum depth of $x_{\max } \sim 10 \mathrm{~cm}$ is obtained, and may be compared to the simulated energy deposition vs. longitudinal depth in a lead-glass module by Figure 4.25 .

The photons generated during the showering process are optically contained to a module and propagate towards the photocathode of the PMT. With some quantum efficiency, the photon may release electrons from the photocathode via the photoelectric effect; the photoemitted electrons may initiate an avalanche process where the electron multiplicative factor depends on the geometry and the dynode structure of the PMT. The result is a measurable pulse which is then amplified and sent to an analog-to-digital converter (ADC). In other words, the energy deposition of the incident electron in a BigBite calorimeter lead-glass module has been digitally converted where the unit is ADC channels, or ADC for short. A fundamental principle behind the digitization rests on the fact that the energy deposition of the incident electron is proportional to the ADC signal; the constant of proportionality is referred to as a gain coefficient and has units of $\mathrm{MeV} / \mathrm{ADC}$. As an electromagnetic shower develops in response to high-energy electrons, ${ }^{*}$ then the sum of the preshower and shower ADC signals is proportional to the energy of the incident electron, ${ }^{\dagger}$ resulting in a relatively simple method to calculate

\footnotetext{
${ }^{*}$ High-energy photons initiate an electromagnetic shower as well.

${ }^{\dagger}$ A more sophisticated method will be presented in Section 5.3.5
} 

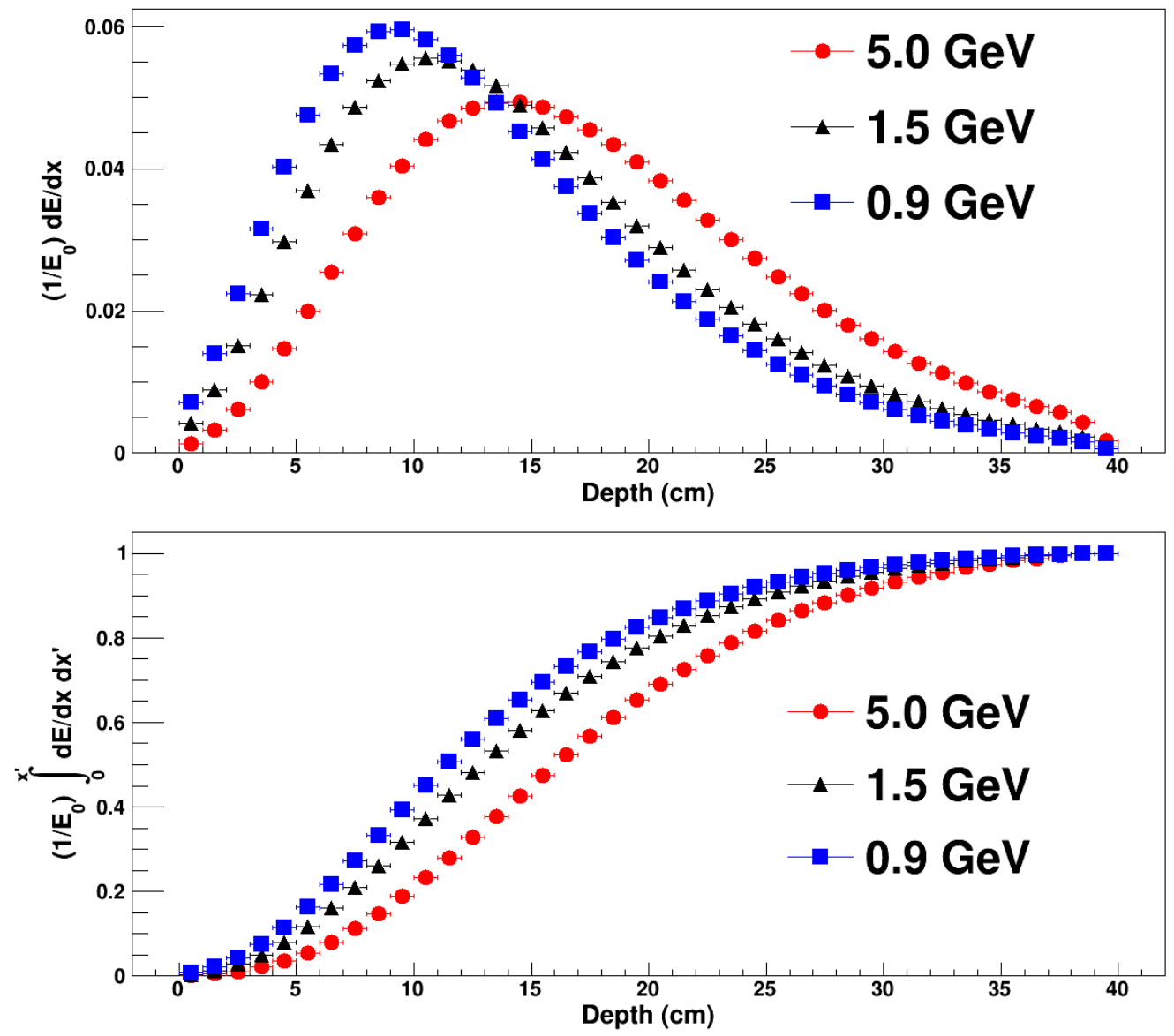

Fig. 4.25: Top: the simulated electron energy deposition $\frac{d E}{d x}$ as a function of the longitudinal depth in a BigBite $40 \mathrm{~cm}$ lead-glass module of density $\rho=3.86$ $\mathrm{g} / \mathrm{cm}^{3}$; the simulated incident energies displayed are $900 \mathrm{MeV}, 1519 \mathrm{MeV}$ and $5 \mathrm{GeV}$, and the distribution is normalized by the total energy deposition in the module, denoted by $E_{0}$. For Kin $1, E_{e} \approx 900 \mathrm{MeV}$ resulting in a $x_{\max } \approx 10 \mathrm{~cm}$ using Eqn 4.25 , which is in good agreement with the simulation. Bottom: the sum of energy deposition for a depth of 0 to $x^{\prime}$, and normalized to the total energy deposition in the lead-glass module. 
the energy of a quasielastically scattered electron. Additionally, the energy deposited within the preshower alone serves as a method of suppressing pions due the different energy signatures of pions and electrons. The analysis discards the majority of the unwanted pions with a single preshower energy threshold cut.

The calorimeter energy resolution determines how well two particles of similar energy and radiation type may be differentiated. The energy resolution of an electromagnetic calorimeter may be parametrized as

$$
\frac{\sigma_{E}}{E}=\frac{a}{\sqrt{E}} \oplus b \oplus \frac{c}{E},
$$

where $a, b$ and $c$ are constants that may be determined by the data and the symbol $\oplus$ indicates addition in quadrature [6]. Note that the equation assumes that the energy $E$ is in $\mathrm{GeV}$. The stochastic term $a$ represents phenomena that are purely governed by statistics, e.g. photoelectron and shower fluctuations. The constant systematic term, denoted by $b$, may represent detector non-uniformity or a calibration uncertainty; and the final $c$ term parametrizes the readout electronics noise. The dominant term for BigBite is the stochastic $a$ term, and the parametrization may be simplified to $\sigma_{E} \approx$ $a \sqrt{E}$. For the electromagnetic calorimeter package, an energy resolution of $\sigma_{\frac{\delta E}{E}} \approx 7.5 \%$ has been obtained.

Similar arguments may be made in regards to the position resolution which depends on the transverse granularity and the so-called Moliére radius, defined to be the radius which on average contains $\sim 90 \%$ of the shower, and scales decently well as $R_{M} \approx X_{0} E_{s} / E_{c}$ where $E_{s} \approx 21 \mathrm{MeV}$ for lead-glass [6]. The dominant contribution to transverse shower development is multiple scattering of $e^{ \pm}$which can push the en- 
ergy deposition away from the shower axis; however, electromagnetic showers tend to be narrow and on the order of a few centimeters. A position resolution of $1.5 \mathrm{~cm}$ has been obtained in both the vertical and horizontal dimensions of the calorimeter; these concepts are explored and demonstrated within Section 5.3.5.

\subsubsection{Timing Hodoscope}

The timing hodoscope, or scintillator plane, is positioned in between the preshower and shower approximately at $z_{\text {det }}=0.95 \mathrm{~m}$, and consists of 13 scintillator paddles of dimension $14 \times 60 \times 4 \mathrm{~cm}^{3}$ (vertical, horizontal, depth) with a PMT located at either end. The passage of a charged particle, e.g. a quasielastically scattered electron, in the paddle produces ionization which then excites the scintillator medium causing light to be emitted during the de-excitation; the emitted light is simply called scintillation. PMTs collect the scintillation, and produces a signal that is sent to a module responsible for duplication and $10 \times$ amplification. One copy goes to a leading-edge discriminator, and if above threshold a logic pulse is sent to a VME 775 TDC. The other signal copy is sent to a Fastbus 1881 ADC for energy deposition information. The hodoscope is capable of providing the electron ToF to a resolution of $250 \mathrm{ps,}$, which is important information in determining the start time of an event of interest. Additionally, the timing information is used as a relative time for the calculation of the time of flight of the recoiling nucleon, which is the chosen method in calculating the momentum of the nucleon. Lastly, when associated with a reconstructed track, the time may be used to determine when a candidate particle intersected the $z_{\text {det }}=0.0 \mathrm{~m}$ plane. 


\subsection{Neutron Arm and the Neutron Detector}

The neutron arm consists of one detector with two purposes related to the recoiling nucleon: nucleon (or charge) identification and momentum determination through time of flight (ToF). The position and geometry of the neutron detector (ND) have been chosen to match the acceptance of BigBite and to optimize the neutron detection efficiency at the largest $Q^{2}$ point. In short, the detector is essentially nine walls of scintillator bars of varying sizes, and is divided into two regions depending on the function:

1. two veto planes, denoted as V1 and V2, for charge identification;

2. seven neutron planes, or N1-N7, for momentum via ToF analysis.

To be more specific, the first region, or the veto layers, consists of two planes of scintillating bars arranged in 2 columns by 48 rows. Each row contains two bars, a veto long and a veto short, and are placed end-to-end with one PMT at either end. Therefore, the veto layers in total have $2 \times 96=196$ bars. Veto layer 2 is vertically offset relative to veto layer 1 by $5 \mathrm{~cm}$ towards the Hall floor. The veto short bars are physically located closer to the beamline in an attempt to equalize the random background rates between the left and right-hand sides of the veto layers. The remaining layers, the N1-N7 neutron layers, consist of scintillating bars of varying dimensions which have been contributed by three universities: Carnegie Mellon University (CMU), University of Virginia (UVA), and University of Glasgow (Glasgow). Each row within a N1-N7 plane only consists of one scintillating bar with a PMT at either end, and in total the N1 through N7 planes have $29,25,30,25,45,45$ and 45 bars, respectively, for a total of 244 bars. The dimensions 


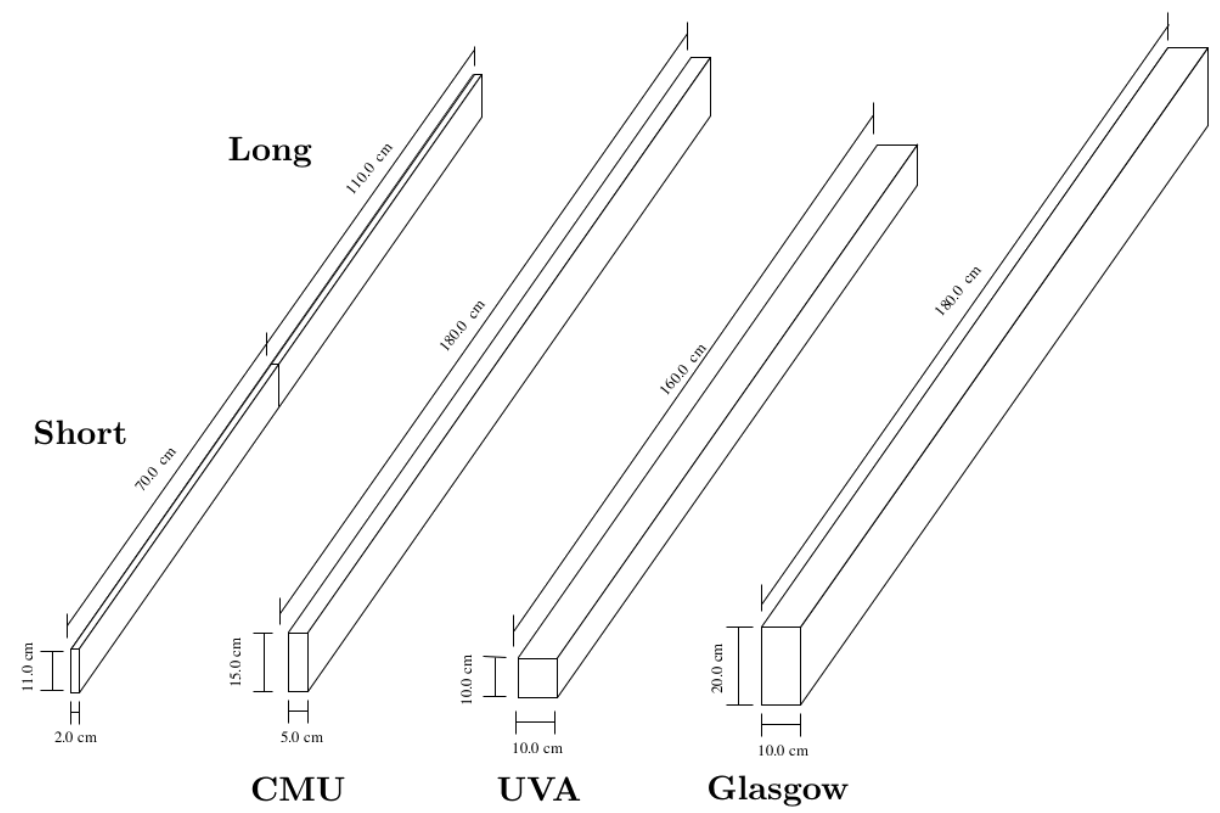

Fig. 4.26: The scintillating bars making up the neutron detector.

of each type of scintillating bar may be seen by Figure 4.26, for example, the CMU bars have a dimension of $15 \times 180 \times 5 \mathrm{~cm}^{3}$. The exact configuration and placement of every bar making up the neutron detector may be seen by Figure 4.27 and Figure 4.28 . The Glasgow bars are only located at the top of planes N1, N3, and N5-N7; the remainder of N1-N4 (N5-N7) are CMU (UVA) bars. Additionally, the diagram displays the trigger logic used during the experiment. Briefly, sums of 16 channels are formed by summing ADC signals of bars of the same color, and then sums of 32 are formed by summing adjacent sums of 16 . Therefore, all sums of 16 are included in two sums of 32 with the exception of the top and bottom sums of 16. In practice, though, scintillating bars are not simply stacked in order to form a neutron detector plane, but bars are first organized into a cassette, or a modular unit consisting of ten or less bars for structural integrity; 


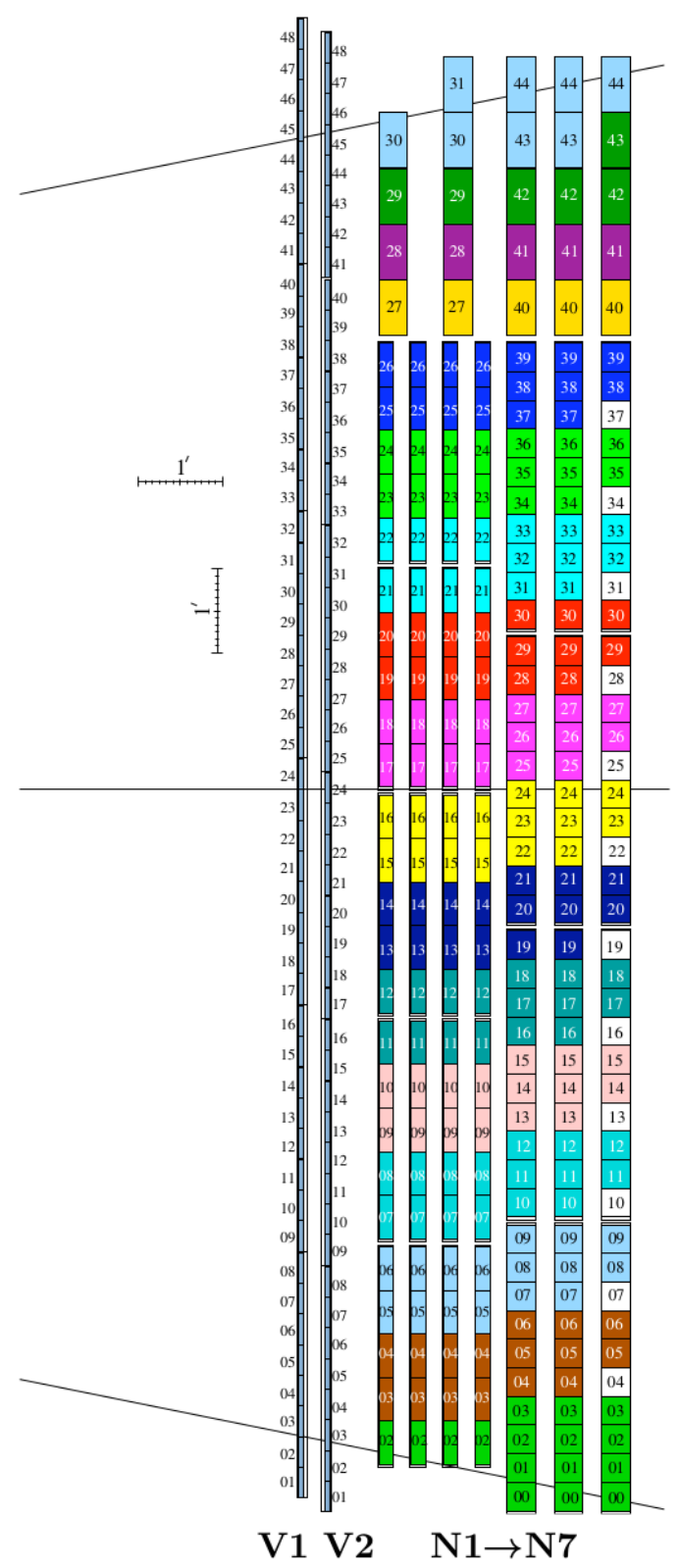

Fig. 4.27: A cross section of the ND including the 2 veto layers (charge ID) and the 7 scintillating layers $(\mathrm{ToF})$. The trigger is formed by summing on bars of the same color, then summing adjacent colors to form a sum of 32 . Note that the white bars in the N7 plane are not connected to any logic sum. There is a relative offset of around $5 \mathrm{~cm}$ between layers V1 and V2. 


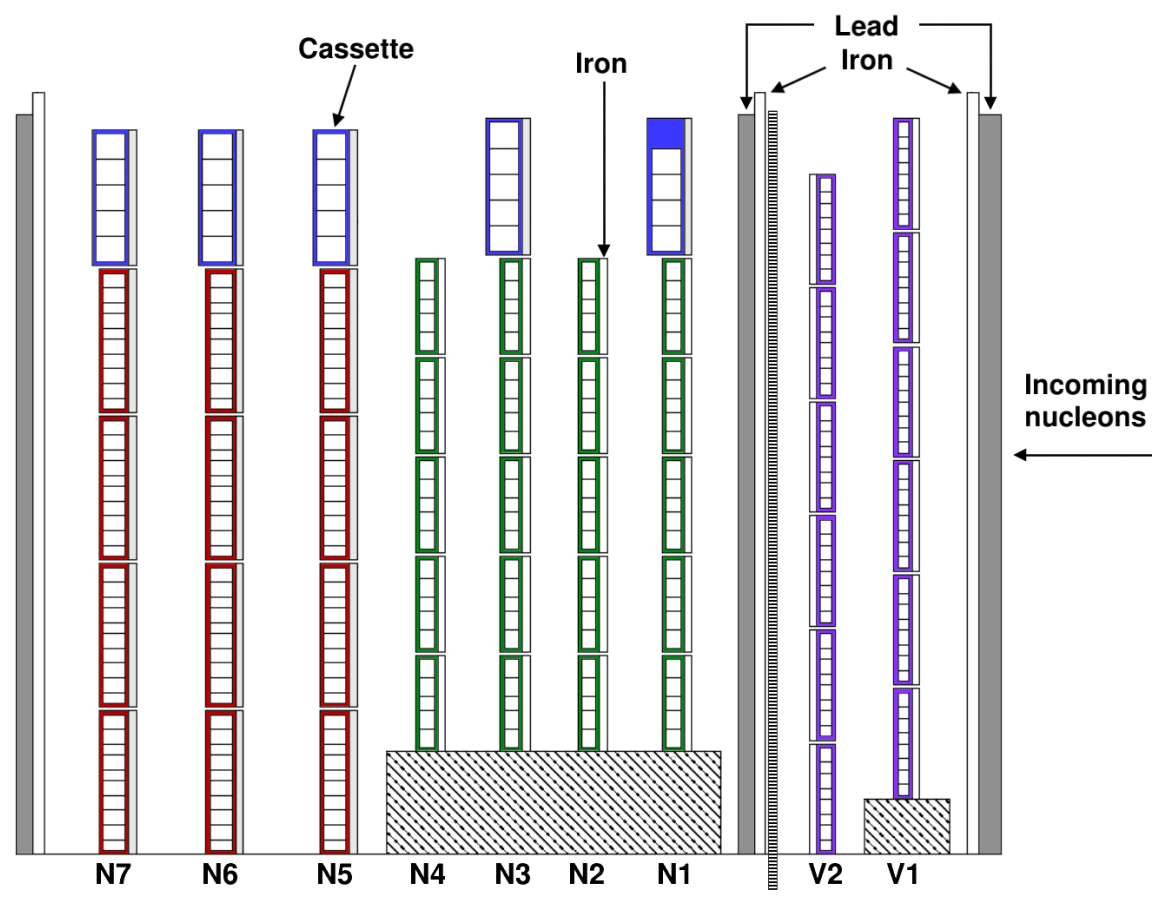

Fig. 4.28: The cassettes and shielding of BigHAND. The bars used within a cassette are color-coded: veto (purple), Glasgow (blue), CMU (green), and UVA (red). The striped box placed after the veto layers represents thin scintillating strips that ran along the vertical direction, and were used for calibrations.

cassettes are then stacked into planes. A more realistic diagram of the neutron detector may be seen by Figure 4.28 in which the cassette configuration may be seen in addition to the locations and dimensions of the shielding. Of particular importance, there is a 5 $\mathrm{cm}$ lead plate and a $1.3 \mathrm{~cm}$ iron plate shielding the detector on the target side which is labeled as "Incoming nucleons" in the diagram.

Iron and lead, henceforth referred to as converter plates, are placed in front of the scintillator bars* and is a design choice to increase the probability of a neutron undergo-

* Veto layer 2 is an exception as iron is placed behind the counters. 
ing hadronic interactions. A chargeless neutron participates in a number of interactions when traversing through the ND in which charged particles are created through strong interactions within the high $Z$ materials; the charged particles then scintillate when propagating through a bar and the scintillation is captured by a PMT. The PMT signal is sent to an amplifier and duplicated. One signal is sent to a Fastbus 1881 ADC where individual bar signals are charge integrated; the other copy is sent to a discriminator/TDC to record timing information. Since planes N1-N7 have two PMTs connected to one bar, both signals may be used to reconstruct the horizontal position along the bar corresponding to the origin of the scintillation, i.e. the position where the nucleon deposited energy within a module. The veto layers on the other hand have two bars per row, and consequently the position may not be reconstructed from the TDC signals.

The purpose of the veto layers is to differentiate between protons and neutrons originating from the polarized ${ }^{3} \mathrm{He}$ target. Conceptually, charge identification exploits the phenomena of scintillation, or in other words a luminescent material emitting light when a struck by a charged particle. If a signal is measured within the veto layers and an N1-N7 cluster has been found within the correct time and space window, then the particle is assumed to be charged, or a proton. On the other hand, if a signal is not measured within the veto layers and a cluster has been observed, the particle is assumed to be uncharged, or a neutron. The concept is illustrated by Figure 4.29 in which both the proton and neutron have a recoiling kinetic energy of $1.3 \mathrm{GeV}$ before entering the neutron detector. The green markers indicate that scintillation successfully traversed the module and has been detected by a PMT of some quantum efficiency. The proton 


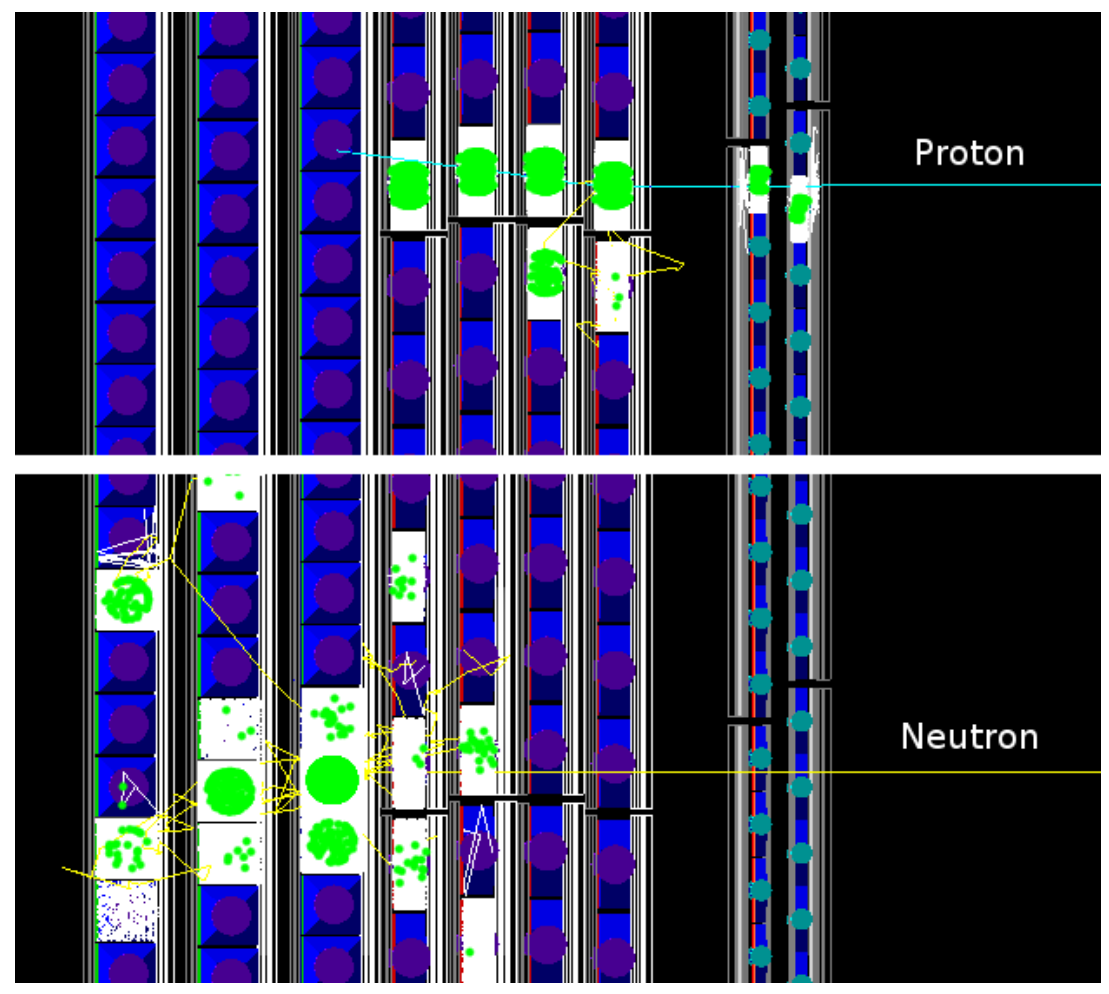

Fig. 4.29: The charge identification concept, see text for an explanation [color].

clearly deposits a large signal within both veto layers followed by a hadronic shower that is dispersed among planes N1 through N4. For the neutron, no signal is observed within the veto layers but with the help of the converter plates a hadronic shower develops and energy is deposited within modules located in planes N3 through N7. Note that this is a beautiful pedagogical example, and in practice the differentiation is not as clean.

For completeness, there also exists four scintillating bars of dimension $2.54 \times$ $304.8 \times 2.54 \mathrm{~cm}^{3}$ positioned such that the long dimension is parallel to the vertical (or height) dimension of the neutron detector. These bars are known as marker counters, and are longitudinally positioned in between V2 and N1. The purpose is to calibrate 
the horizontal dimension ( $\hat{y}$ in ND coordinates) and to obtain the speed of light within the scintillating material denoted by $c_{s}$. Recall that planes N1-N7 can reconstruct the horizontal position from TDC timing information, but the light propagation time must be corrected. Furthermore, $c_{s}$ is used when matching veto hits to a nucleon cluster during the charge identification procedure. A more complete description of the neutron detector geometry may be found in Ref. [179].

As the nucleon momentum is reconstructed through time of flight, the momentum resolution of the ND depends on the timing resolution of the TDCs used for the neutron counters. The expressions for the nucleon momentum and the dominant uncertainty are

$$
p=\gamma M \frac{\ell}{t}, \quad \delta p=\frac{M c \beta^{2}}{\ell}\left(\frac{1}{\left(1-\beta^{2}\right)^{\frac{3}{2}}}\right) \delta t
$$

where $\delta p$ is the neutron momentum resolution, $M$ is the mass of the nucleon, $\ell$ is the magnitude of the nucleon flight distance defined by the vertex position and the reconstructed nucleon coordinate, $\delta t$ is the time of flight resolution, $\beta=\frac{v}{c}=\frac{\ell}{c t}$, and $\gamma=\left(1-\beta^{2}\right)^{-\frac{1}{2}}$ is the typical Lorentz factor. As $\delta t$ is constrained by the electronics to be $300 \mathrm{ps}$, the Kinematic 1 configuration yields $\ell \sim 9 \mathrm{~m}$ and $\beta \sim 0.8$ resulting in an expected momentum resolution of $30 \mathrm{MeV}$. The calibration procedure of the neutron counters is presented in Section 5.4 in which individual bars are calibrated to an expected ToF. The result is a time of flight resolution only constrained by the electronics, or the desired $300 \mathrm{ps}$, and the expected momentum resolution of $30 \mathrm{MeV}$ is obtainable for the lowest $Q^{2}$ configuration. 


\subsection{Trigger and Data Acquisition}

There are several systems in place to acquire data from the detector electronics depending on the accepted trigger, or a signal that is associated to an event of interest, e.g. electron and/or nucleon event candidates. The types of raw data that are recorded are as follows: FastBus and VME data from the electron arm, neutron arm, beam position monitors and raster system; scaler or helicity-gated scaler* information; EPICS information; and lastly, special events that are inserted into the data stream once or infrequently during a run such as prescale factors (defined below) or detector map information. Important JLab systems and ubiquitous acronyms are summarized prior to going into the details:

\section{Trigger Supervisor (TS):}

Arguably the brain of the data acquisition system, the trigger supervisor links the experimental external triggers with the read-out controllers (ROCs) and determines if event data should be read-out in the raw data format. The ROCs handle the event-by-event retrieval, and generally means a crate of FastBus or VME modules that is connected to a detector system, for example TDC timing information associated with the neutron detector. The TS accepts up to 8 external triggers which are differentiated by simply $\mathrm{T} \#($ e.g. $\mathrm{T} 1, \mathrm{~T} 2, \ldots)$, and each trigger has a pre-set prescale factor, i.e. how often an event is read-out. A trigger with a prescale factor of 1000 means that 1 in 1000 triggers of this type are read-out and stored in a raw data file. The trigger supervisor is also responsible for the gener-

\footnotetext{
* A scaler is simply a device that counts each arriving signal such as the number of triggers.
} 
ation of the level 1 accept (L1A) signal which is critical to relate a read-out time in the electron arm to a read-out time in the neutron arm. Additionally, the TS provides a method to synchronize the data output by determining the busyness, or number of events in a front-end module buffer, of all ROCs. In the possibility of a hardware error, data will be misaligned, meaning the ROC data does not all correspond to the same event, rendering the data as useless; therefore, the idea behind synchronization is to have the TS periodically stop accepting triggers in an attempt to check and confirm that front-end module buffers are no longer processing events.

\section{CEBAF Online Data Acquisition (CODA):}

The CEBAF Online Data Acquisition, henceforth referred to as CODA [205], is the standard framework of software and hardware to acquire data in an experiment at JLab. CODA was developed at Jefferson Laboratory by the DAQ group, and can be used to implement a data acquisition system for a small experimental teststand or scaled up to handle an entire experiment such as $G_{E}^{n}$. CODA may be configured by the user in order to handle the read-out of a particular ROC into structured CODA events, or arrays of 32-bit words containing event-by-event level information. The CODA events then need to be decoded for event reconstruction, analysis and a physics interpretation. For $G_{E}^{n}$, the decoding software is called the Hall A Podd Analyzer [206] which is built utilizing CERN's ROOT [207] framework, both of which are briefly described in Section 4.9. Lastly, EPICS data (defined below) and scaler data are also sent to the CODA data stream. 


\section{Experimental Physics and Industrial Control System (EPICS):}

Experimental Physics and Industrial Control System [208], otherwise known as EPICS, provides the software infrastructure for controlling and monitoring large devices such as major telescopes and accelerators. A single control room can control hundreds of computers that have been networked together providing a robust and flexible way to satisfy the varying requests of users, e.g. differing experiments within Halls A, B and C. Therefore, EPICS may be used to record information regarding the accelerator conditions, beamline diagnostic and monitoring information, or any information that is deemed important to an experiment that typically does not change on the order of seconds. It is noted that beamline diagnostic information like BPM and rastering does change, but this information is also sent to CODA on the event-by-event level for analysis. For $G_{E}^{n}$, the type of information sent to the EPICS data stream includes beam energy, beam position, BigBite magnet settings, the orientation of the Wien filter, the status of the half-wave plane (in or out), the thresholds of the NA, the thresholds of the preshower and shower, the target oven temperature and many other experimental settings.

\subsubsection{BigBite Trigger and Read-out Electronics}

The purpose of the BigBite trigger is to minimize background events while selecting on electron candidates. Preshower and shower trigger logic ADC sums are implemented in order to roughly calculate the energy deposited by a candidate particle. If a trigger logic sum is above a pre-defined ADC threshold then a BigBite trigger signal is generated. A hardware trigger sum (TSUM) is formed by summing ADC signals of two rows of 


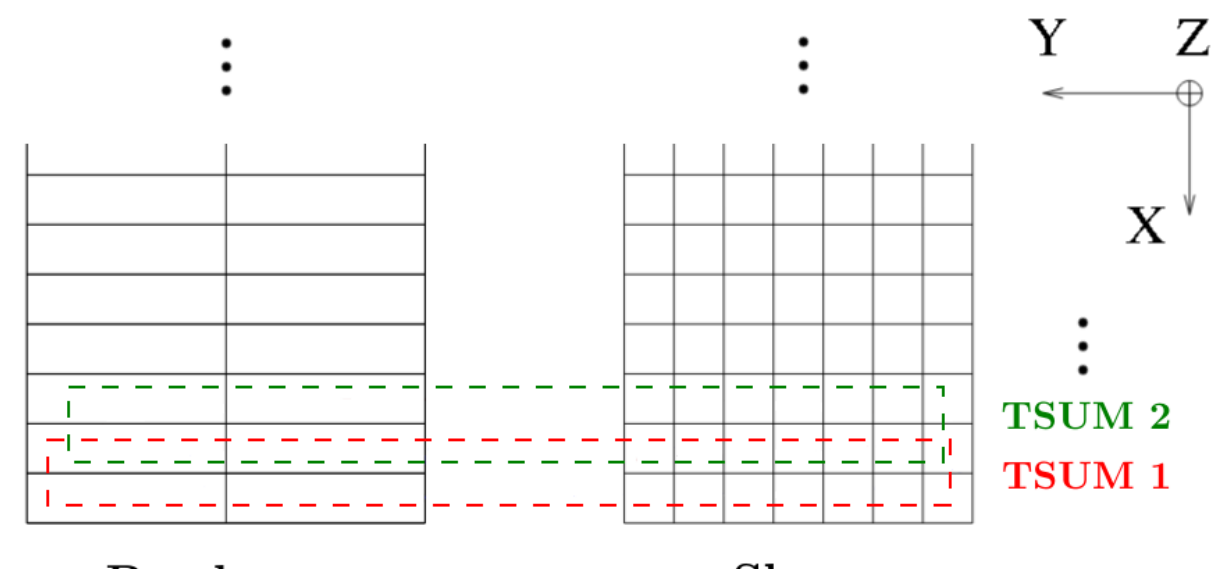

Preshower

Shower

Fig. 4.30: The BigBite trigger sum logic.

preshower $(2 \times 2=4$ blocks $)$ and shower $(2 \times 7=14$ blocks $)$; a summary of the calorimeter trigger logic may be seen by Figure 4.30 , The hardware used to implement this trigger scheme is displayed in Figure 4.31 in which there is a legend defining the important module model-numbers and its function. An explanation of TSUM 1 will be presented, and then the rest of calorimeter trigger sums may be formed iteratively. For preshower row \#1, the left and right PMT analog signals (PS1L and PS1R, respectively) are sent to a Phillips Scientific 776 unit which amplifies the signals by a factor of 10 in addition to signal duplication. An analogous operation for preshower row \#2 is performed, and together the left and right signals of rows 1 and 2 are summed by a LeCroy 428F (L428F) module. For the shower, the analog PMT signals of each row are summed with a specialized "Sum8" module, or a custom NIM module responsible for summing one row or 7 modules in addition to input signal duplication. Two "Sum8" outputs, or equivalently two rows of shower signals, are then summed with a L428F 

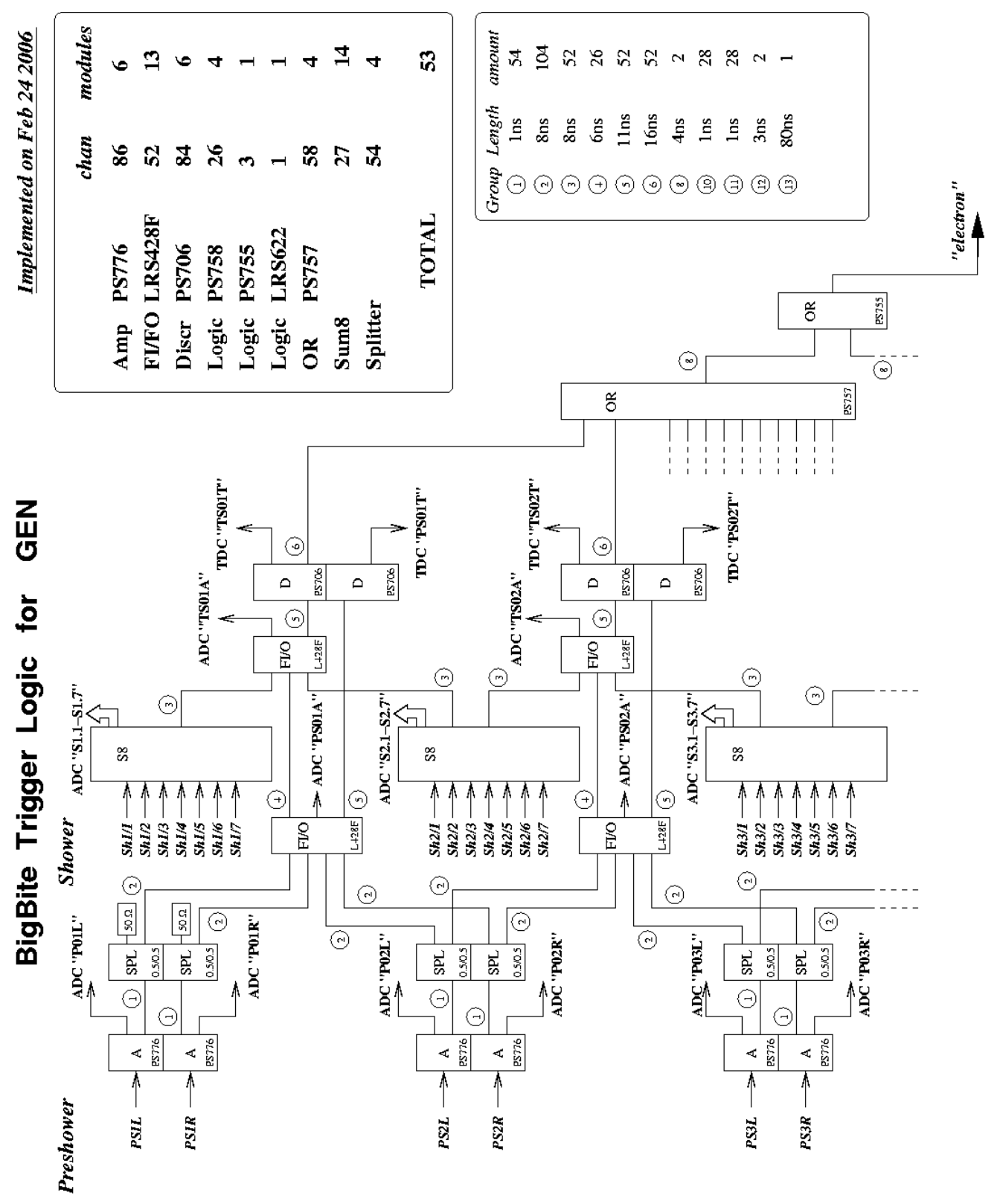

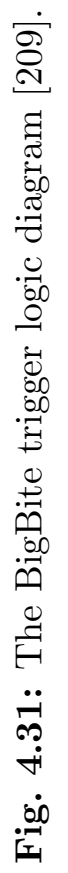

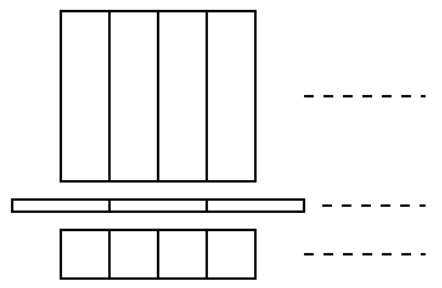


module. The preshower and shower sums, or the outputs of both L428F modules, are directed towards leading-edge discriminators, and defines a TSUM as depicted in the figure. A logic pulse is sent to a logical "OR" unit to check if the TSUM energy deposition is greater than the threshold. The implemented electron arm trigger scheme is the result of iteratively repeating the logic to cover all rows of the calorimeter. Excluding the vertical extremes of the detector, TSUM $i$ overlaps with the neighboring TSUMs $i \pm 1$. All TSUM signals are sent to logic modules in which a global "OR" is enforced to check if any of the TSUM signals are above a threshold of roughly $500 \mathrm{MeV}$; if so, then the BigBite trigger signal, denoted as T2 or Trigger 2, is initiated and awaits further processing depending on the status of the neutron arm.

The read-out electronics of the electron arm include various ADC and TDC modules stored in FastBus and VME crates. Individual amplitudes of the preshower, shower, and hodoscope in addition to the amplitude sums are recorded with LeCroy 1881 ADCs; the sums are also sent to LeCroy 1877 TDCs operating at $0.5 \mathrm{~ns} /$ channel. The hodoscope timing information is handled with Caen 775 TDCs operating at 35 ps/channel, and is used in the nucleon momentum ToF calculation hence the stricter timing requirement. Sense wire signals of the middle and back chambers are directed to Nanometric A/D cards $(\mathrm{N} 277 \mathrm{C})$ set to a threshold of $3 \mathrm{~V}$. The front chamber utilizes a new low-noise A/D card based on the Caen chip called MAD, and operates at a threshold of 5-10 times lower than the N277C. LeCroy 1877 TDCs operating at $0.5 \mathrm{~ns} /$ channel are used for the MWDC readout. The generation of a $\mathrm{T} 2$ event has been described, but a coincidence event requires a nucleon trigger candidate within the correct time-window. 


\subsubsection{Neutron Arm Trigger and Read-out Electronics}

The trigger of the neutron arm is generated in a similar manner as the electron arm. The PMT signals of each bar type are sent to a module (PS 776) that amplifies and duplicates the signal; the copies relevant to the trigger are then sent to a "Sum8" module in order to make sums of eight bars of a particular type. The trigger logic is organized into two levels: sums of sixteen (sum16) and sums of 32 (sum32). The sum16 scheme is summarized by the color-coding within Figure 4.27 or shown in explicit detail within Figure 4.32 , and consists of neutron counter signals of all planes in longitudinal patterns. Neighboring sum16s are summed by a L428F module to form a sum32 resulting in a trigger scheme with overlapping summations in complete analogy to the BigBite case. The sum32s are sent to logical "OR" modules in order to test if a signal sum is above threshold. If a sum is above the pre-defined threshold (roughly $25 \mathrm{MeV}$ ) then the neutron arm trigger T1 is generated. Note that each neutron counter has two PMTs, and the logic presented above applies to both sides of the detector, left and right, separately, which is an imperative detail.

The readout of the neutron arm is handled by VME F1 TDCs in addition to LeCroy 1881 ADCs and 1877 TDCs. The F1 TDC was developed at Jefferson Lab by members of the Fast Electronics and Data Acquisition Groups, and operated in the low resolution ( 8 channels with 120 ps/LSB each) mode. Recall that the neutron detector determines the momentum of the recoiling nucleon by the time of flight method using the neutron counters, not the veto bars. Therefore, the readout timing resolution of the neutron counters have stricter precision requirements than the readout of the veto layers. 

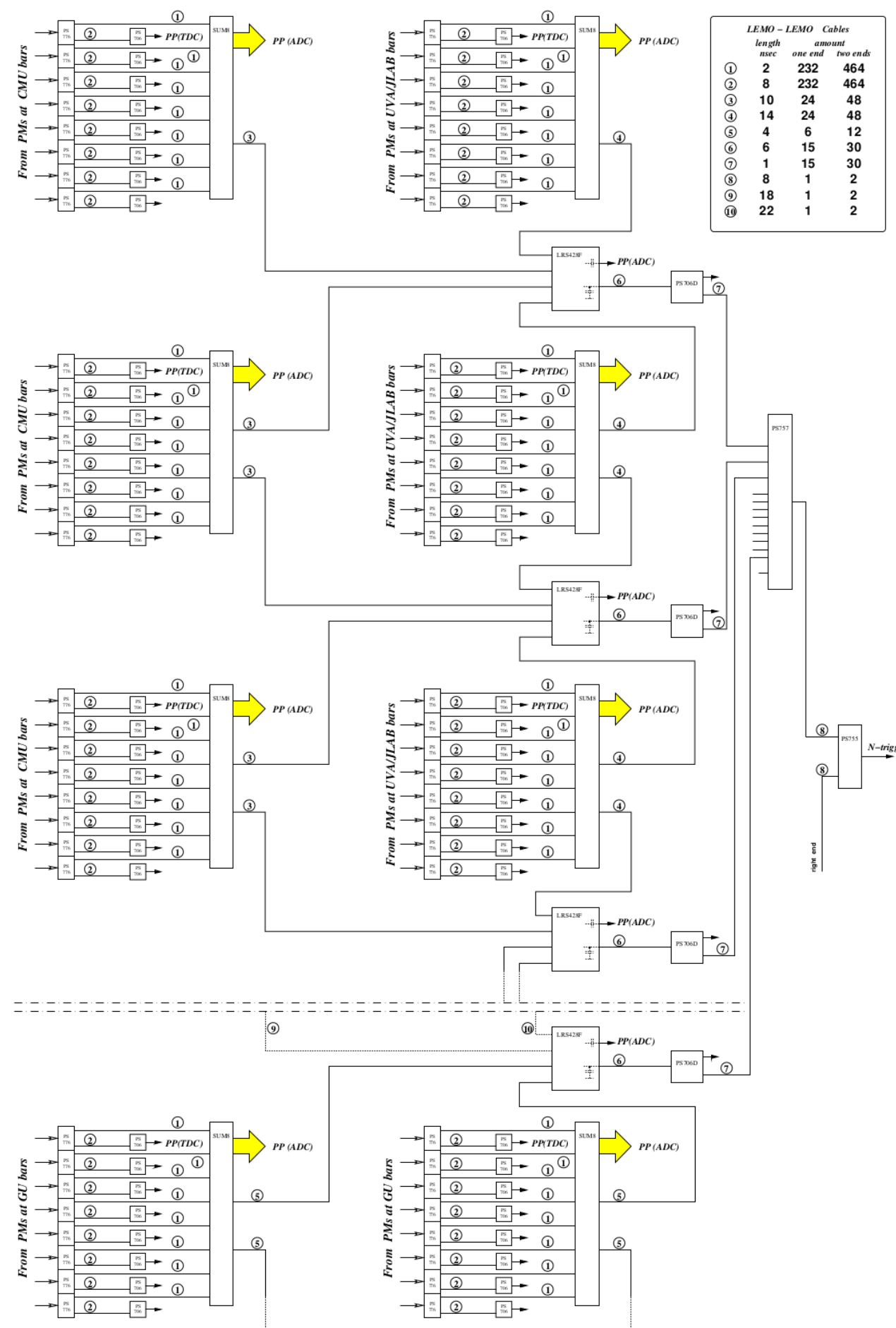
With this in mind, the individual PMT signals of all bars are sent to the LeCroy 1881 ADC modules. The timing information, however, is divided depending on the location of the PMT; veto layer signals are sent to LeCroy 1877 TDCs, but N1-N7 PMT signals are sent to the more precise F1 TDC module. The set of signal sums followed a similar electronics path; all sums are sent to the LeCroy 1881 ADCs while sums originating in the veto (N1-N7) layers are sent to LeCroy 1877 (F1) TDCs for timing information.

\subsubsection{Triggering on a Coincidence Event}

The full trigger diagram may be seen by Figure 4.33 in which the left-hand (right-hand) describes the electron (nucleon) arm of the experiment, or the electronics responsible for generating a T2 (T1) event type. In order for a coincidence event to occur, the T1 and T2 trigger signals must overlap sufficiently in time; the length of time to check for the arrival of a coincidence signal must not be too large or small. The coincidence window needs to be large enough to account for the differing time of flights of the electron and nucleon, but if it is too large then false coincidences due to random background become increasingly more common. The signal window of $\mathrm{T} 1$ and $\mathrm{T} 2$ is approximately 40 and 100 ns long, respectively. The electronics path has been designed such that the T1 signal arrives $\sim 40$ ns later than the BigBite T2 signal; each kinematic setting must have a slightly adjusted electronics path in order to account for the varying ND distance and nucleon $\beta=\frac{v}{c}$.

The T1 and T2 signals are sent to a coincidence module (PS 754) which determines the leading edge of the logical "AND" defining the start of the T3 coincidence signal which is approximately $40 \mathrm{~ns}$ long. The T3 signal is then sent to the Trigger Supervisor 


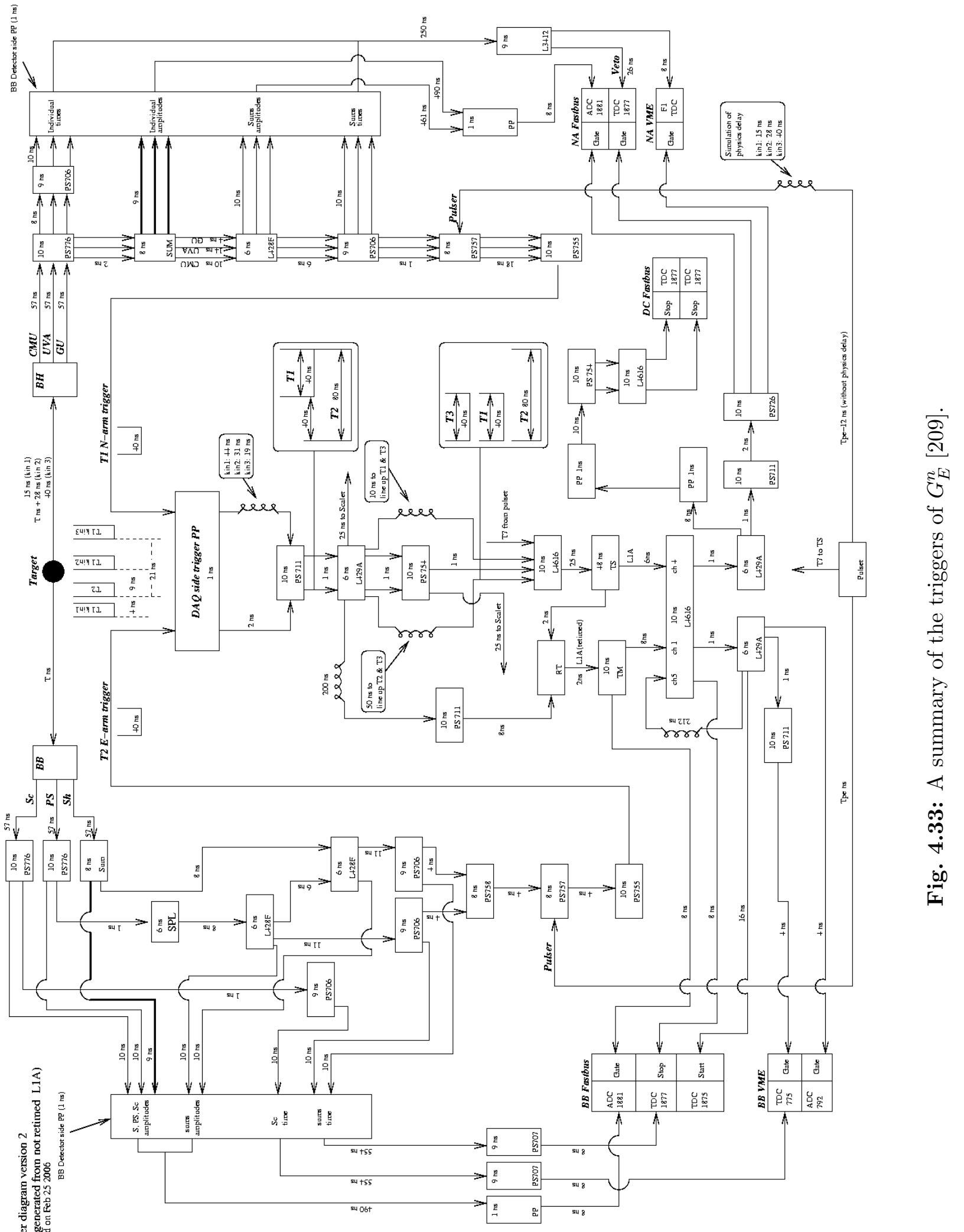


which generates and duplicates the level-1-accept (L1A) signal. One copy of L1A is recombined with the $\mathrm{T} 2$ signal in a retiming (RT) module which creates a signal with a fixed-delay relative to T2. The RT fulfills two purposes: 1) the reference signal for the BigBite arm is generated by retiming L1A and 2) the retimed L1A is sent to a LeCroy 1875A TDC (50 ps) which has a common-stop governed by the readout signal of BigBite. The latter purpose determines the difference between the L1A signal and the BigBite readout signal. The other copy of L1A originating from the Trigger Supervisor is sent to the neutron arm as a readout signal. This represents a typical event, i.e. T1 determining the start time of T3 and L1A. It is possible for T1 to arrive early, though; in this case, T2 determines the start time of T3 and L1A. These types of events are

illustrated by Figure 4.34. A single-trigger event, i.e. only T1 or T2 is generated, is also possible which may be used for calibrations. For example, in a T1 event where there is no T2 trigger available for the retiming module, the retiming module then produces a signal with a fixed-delay relative to L1A as opposed to T2.

\subsubsection{Scalers}

A copy of all trigger signals are sent to scalers providing a useful method of monitoring and calculating the various trigger rates. Triggers are also sent to helicity-gated scalers in order to monitor the raw trigger asymmetry rates; see Figure 5.51 for an example of the T2 asymmetry. Scalers are used to count BCM signals resulting in a method to calculate the accumulated beam charge which may be used for data or simulation normalizations. The trigger rates are also used in order to correct for dead time originating from within the electronics chain and data acquisition system. 


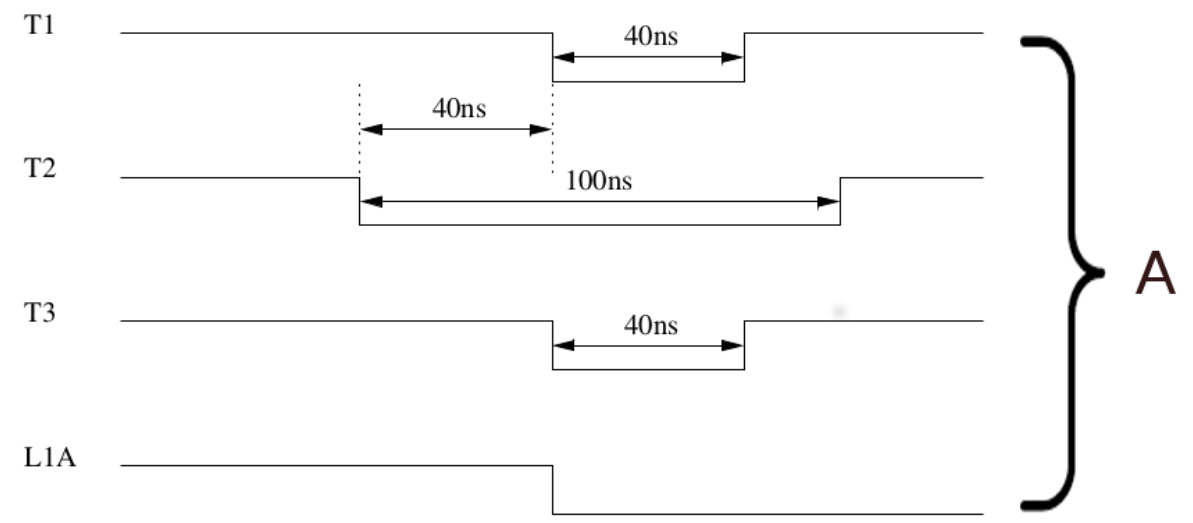

$$
\mathrm{T} 1
$$

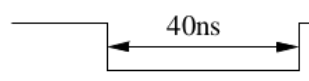

$\mathrm{T} 2$

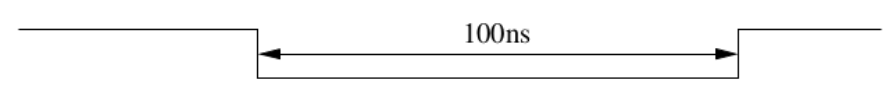

$\mathrm{T} 3$

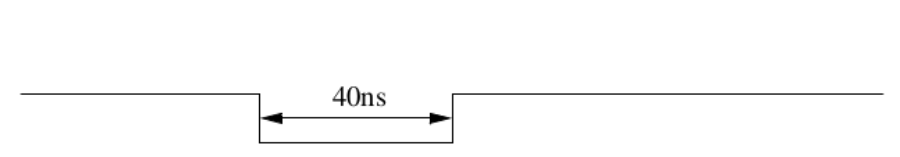

L1A
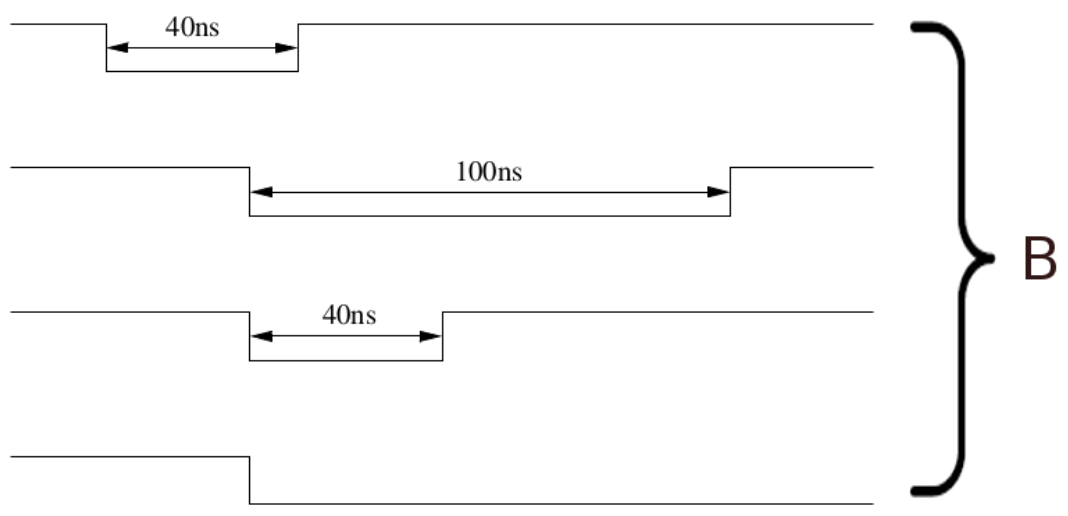

Fig. 4.34: The start of $\mathrm{L} 1 \mathrm{~A}$ is determined by the leading edge of the $\mathrm{T} 1$ and $\mathrm{T} 2$ coincidence signal. The top panel represents a typical event in which the $\mathrm{T} 1$ signal arrives after the $\mathrm{T} 2$, and therefore determines the start of the T3 and L1A signals. The bottom panels shows just the opposite, i.e. T1 arrives before T2 and consequently T3 and L1A are in-line with T2. 


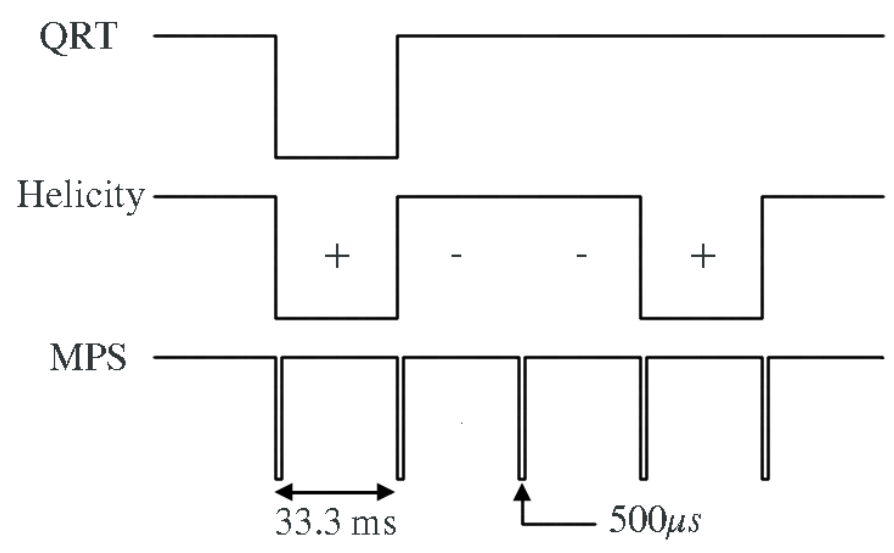

Fig. 4.35: Helicity structure and main signals used in the so-called $G^{0}$ mode [210].

\subsubsection{Beam Helicity Determination}

The source of polarized electrons has been summarized in Section 4.3 , but recall that the orientation of the Pockels cell determines the helicity state of the photoemitted electrons. JLab pseudorandomly changes the orientation of the Pockels cell at a rate of $30 \mathrm{~Hz}$ which results in beam pulses of a particular helicity state lasting for $\frac{1}{30}$ seconds. The pseudorandomly generated states come in sequences of four helicity states referred to as quartets or quads; the helicity sequence of a quad is either +--+ or -++- . A signal known as a quartet trigger (QRT) defines the beginning of a new quad. A macropulse trigger (MPS) is used as a gate to define when the helicity is defined. When the pseudorandom algorithm changes the beam helicity, there is a $500 \mu \mathrm{s}$ transition period that corresponds to the orientation of the Pockels cell being changed; the helicity during this transition period is simply undefined and denoted by a " 0 " as opposed to \pm 1 in the data. This may be visualized by Figure 4.35 where the width of the MPS signal represents the Pockels cell transition period. Additionally, $G_{E}^{n}$ used three redundant 
copies of a $105 \mathrm{kHz}$ clock signal; the purpose of this signal is to reconstruct missed QRT or MPS signals for whatever reason (e.g. DAQ deadtime, a beam trip, etc.) in order to recover the position within the helicity sequence and quad. The helicity signals are sent to the Trigger Supervisor and recognized as T8 and T9 for the $105 \mathrm{kHz}$ helicity synchronization signal and the $30 \mathrm{~Hz}$ MPS signal, respectively.

The accelerator helicity signals are delayed by a fixed 8 quads. Therefore, in the analysis when the helicity needs to be decoded, the first 1000 events of a run are used to determine the position within the helicity sequence and quad. From this event and all remaining events, the helicities are now known, but the first 1000 events of all runs are discarded due to lack of helicity knowledge. Furthermore, events corresponding to an undefined helicity state due to the Pockels cell transition period are also discarded.

\subsubsection{Summary of Triggers}

A summary of the triggers used within the $G_{E}^{n}$ experiment are presented in Table 4.9 . The electron (neutron) arm generate the signals T2 (T1) from which a coincidence T3 signal may be formed. A $8.5 \mathrm{~Hz}$ pulser is used in order to understand the total trigger logic of $G_{E}^{n}$ as seen in Figure 4.33 . The pulser is connected to both modules responsible for generating triggers on the electron and neutron arms, and is understood by the Trigger Supervisor as a T7 event. T7 events can be used in order to understand events lost due to electronics dead time. Lastly, the T8 and T9 signals are used for helicity determination as described in the previous section. 


\begin{tabular}{cl}
\hline Trigger $\#$ & Description \\
\hline T1 & Neutron Detector Trigger \\
T2 & BigBite Trigger \\
T3 & Coincidence between T1 and T2 \\
T7 & 8.5 Hz Pulser \\
T8 & $105 \mathrm{kHz}$ Helicity Synchronization Signal \\
T9 & $30 \mathrm{~Hz}$ Helicity Quad (MPS) Signal \\
\hline
\end{tabular}

Table 4.9: The trigger types of $G_{E}^{n}$.

\subsection{Analysis Software: AGen and the Hall A Analyzer}

The raw data files of CODA need to be decoded and analyzed in order to make a physics interpretation of an experiment. The Hall A standard software is known as the Hall A Analyzer which is an object-oriented, user-extensible framework that is built on top of CERN's ROOT in $\mathrm{C}++$. ROOT is a data analysis software specializing in handling large amounts of data, and provides robust and powerful facilities for analysis, visualization and the presentation of data. The Analyzer contains classes describing the standard Hall A equipment, and can perform common analysis tasks on data acquired within Hall A including the decoding of CODA raw data files into more accessible data structures that may be handled by ROOT. As many of the detectors of $G_{E}^{n}$ were newly constructed, significant effort was dedicated towards building analysis modules that coexist with the Analyzer; the result is the AGen library. 


\subsection{Monte Carlo Simulation: G4SBS}

A realistic Monte Carlo simulation has been developed in order to help answer various questions that are difficult or impossible with the experimental data alone. The MC simulation is referred to as G4SBS which utilizes the Geant4 framework [180]. G4SBS is the result of significant simulation work performed by Andrew Puckett, Seamus Riordan and collaborators for Jefferson Lab's Hall A upcoming Super BigBite (SBS) experiments, a successor set of experiments that will push nucleon form factor measurements to higher $Q^{2}$ with JLab's $12 \mathrm{GeV}$ upgrade.* The SBS MC has been configured to handle $G_{E}^{n}$; therefore, all materials and detectors relevant to E02-013 have been added to mimic reality. Images of $G_{E}^{n}$ within the G4SBS framework may be seen by the following: an aerial view (Figure 4.3), an electron traveling through the BigBite spectrometer (Figure 4.20 and a perspective view of the dipole magnet, the MWDCs and the calorimeter package (Figure 4.22). There are many event generators available for a variety of tasks which will be discussed when relevant. Additionally, the Fermi motion of the ${ }^{3} \mathrm{He}$ nucleus may be parametrized in order to partially replicate the smearing of kinematic variables; other experimental realities need to be taken into account to fully replicate observed spectra, e.g. detector resolutions, multiple scattering in air, and radiative corrections. An analysis of simulated data is capable of enforcing the realistic T1 and T2 trigger logic schemes, and the logical "AND" of the two triggers effectively produces simulated coincidence data. Lastly, the nucleon charge identification scheme may be used to differentiate between protons and nucleons in order to replicate the observed

\footnotetext{
* Details on how to use the Super BigBite MC simulation package may be found here [211].
} 
nucleon identification probabilities. The G4SBS MC package is a useful tool and much can be said about the framework; however, this is intentionally suppressed in the interest of space. Relevant details of the Monte Carlo are described in Section 5.3.6 and Section 5.4.7, and the machinery will be used as input for various corrections to the raw asymmetry described in Chapter 6 . 


\section{Chapter 5}

\section{Data Analysis}

The data analysis phase may be conceptually simplified to three major tasks: reconstruct the quasielastically scattered electron trajectory and calculate the four momentum, reconstruct the recoiling nucleon trajectory and calculate the momentum through the time of flight, and lastly determine the charge of the nucleon. With the ability to perform these three items, the kinematics of each event may be reconstructed and a neutral raw asymmetry may then be calculated. However, as the old idiom goes "the devil is in the details." The purpose of this chapter is to present the details of the data analysis flow originating from the detector electronics and hardware to the high-level kinematic information useful for a physics interpretation. The chapter begins with the raw analysis and calibration procedures for the BigBite spectrometer and the neutron arm, respectively. A large number of calibrations are required for an entire experiment, and as a result a time-dependent database of calibration coefficients and offsets must be developed. Important kinematic variables are introduced when the data and calibrations have been refined to the point where such a calculation is reliable. Once a scattering

event may be reconstructed, then the ${ }^{3} \mathrm{He}$ data needs to be examined in order to define a set of kinematic cuts to select the desired quasielastic region while suppressing un- 
wanted events. An algorithmic procedure to differentiate between recoiling protons and neutrons will be presented to select quasielastic neutron events. Lastly, the asymmetry formalism is presented, i.e. how the asymmetry measured during the experiment may be compared to the beam-target asymmetry derived in Section 2.3 .2 in order to extract the neutron form factor ratio $G_{E}^{n} / G_{M}^{n}$.

\subsection{CODA Raw Data}

The initial output of a Jefferson Lab experiment comes in the form of raw data files produced by CODA. For $G_{E}^{n}$, this includes thousands of raw data files ranging in size depending on the duration of a data-taking run, ${ }^{*}$ but the maximum size is just under 2 GB. Kinematic 1, which was a relatively short configuration compared to Kinematics 2 and 3, produced roughly $600 \mathrm{~GB}$ of data; the entire experiment produced on the order of a several TB of data. In order to remain organized and to avoid prohibitively long periods of computational time, the analysis is broken up into several stages which systematically reduces the data size of the files while transforming lower-level electronics information into higher-level variables useful for a physics extraction.

The CODA raw data structure comes in the form of an array of 32-bit words for event data; an event is determined by the Trigger Supervisor depending on the accepted trigger type. The first part of the event structure is "header" information which encodes the duration of the event, the event type or trigger type, the run number (also differentiates file name), and the event number which is a book-keeping device assigned by CODA representing time. Additionally, the raw data encodes information

\footnotetext{
* Runs have a corresponding run number which is simply a book-keeping device.
} 
regarding the read-out controllers (crates of FastBus and VME) referred to as "banks" which is how low-level information associated to the TDCs and ADCs is accessed. The Hall A Analyzer has been designed to decode CODA raw data files, and consequently integrated charge or time information associated with ADCs and TDCs, respectively, may be accessed and used in order to build and reconstruct physics events of interest.

\subsection{Available Data}

There were a number of targets used throughout the production of $G_{E}^{n}$, see Section 4.5 for a more details. The data sets used during the calibration and analysis phases of this thesis are the following: polarized ${ }^{3} \mathrm{He}, \mathrm{H}_{2}$, and the $\mathrm{C}$ and $\mathrm{BeO}$ foils. Recall that Kinematic 1 does not have any $\mathrm{N}_{2}$ data, and the carbon foil data will serve as a suitable replacement. The majority of the detector calibration procedures use the $\mathrm{H}_{2}$ data in which the elastic peak is selected; elastic ep scattering is well understood and lacks the nuclear difficulties associated to quasielastic data. There are situations where the hydrogen data cannot fill the entire acceptance of a particular detector, though, and in this case polarized helium-3 data is used to supplement the hydrogen data. In scenarios where the type of data used is irrelevant, such as calculating electronic dead time for example, hydrogen data is used unless explicitly stated otherwise. The carbon foils, or targets at discrete locations in $z_{\text {lab }}$, are useful to calibrate the vertex reconstruction procedure. All target types are utilized in the analysis phase in which the raw asymmetry needs to be corrected for various types of contamination, and in particular the calculation of the proton misidentification utilizes all three data sets. 


\subsection{BigBite: Raw Analysis and Calibration Procedures}

Recall that the primary purpose of BigBite is to reconstruct the four momentum of the quasielastically scattered electron. The analysis starts with decoded spectrometer ADC and TDC information which depends on the common-stop originating from the retiming (RT) module; this is effectively L1A retimed, see the electronics logic in Figure 4.33. The recorded TDC times of the multi-wire drift chambers must be calibrated after which the TDC information may be used in order to reconstruct the trajectories of the outgoing electrons post magnetic deflection, henceforth simply referred to as tracks. The generation of tracks and finding the most likely candidates is a significant algorithmic and computational challenge; the procedure will be summarized. Tracks need to be traced back to the target region to reconstruct the scattering vertex position and the momentum of the electron which requires an understanding of the BigBite optics. The preshower and shower ADC signals may be calibrated to represent an energy deposition in $\mathrm{MeV}$ yielding in a method to calculate the energy of the incident electron. More importantly, however, the reconstructed calorimeter information is used as input to the track reconstruction to significantly reduce the candidate track search region. The timing hodoscope, located in between the preshower and shower, may be calibrated such that the measured time accurately reflects the time of flight (ToF) of the quasielastically scattered electron; this information serves as the reference time used in the nucleon ToF calculation. The ability to perform the above tasks allows for a full reconstruction of the scattered electron. 


\subsubsection{Drift Chambers}

The drift chambers have been calibrated by Seamus Riordan [178] and Sergey Abrahamyan, and the relevant results of the calibration will be summarized. When a charged particle passes through a multi-wire drift chamber, it ionizes the argon-ethane mixture which then induces a signal on a sensing wire. A time stamp associated to this signal is recorded by a TDC; the timing signal may be further processed as it is related to the distance of closest approach between the track and the sensing wire $i$ within a wire plane. The collective goal of the three drift chambers is to then associate the TDC signals with a set of hits, and then fit straight lines to the hits resulting in candidate tracks. The best fit determined by a $\chi^{2}$-minimization procedure is taken to be the trajectory of the quasielastically scattered electron after magnetic deflection by BigBite. The bulk of the scattered electron information is obtained from the drift chambers; the remaining detectors help to reduce the noise such that track reconstruction is efficient and consequently a careful understanding and calibration is in order. The basic strategy of drift chamber calibration consists of the following: find the wire-dependent offsets denoted by $t_{0, i}$, find and calibrate the drift time to distance conversion functions, and calibrate the wire positions based on Hall A surveys.

The data acquisition of the drift chambers is designed to record the difference between the arrival of a signal associated to wire $i\left(t_{\text {signal }, i}\right)$ and the arrival time of the trigger signal $\left(t_{\text {trig }}\right)$ controlling the BigBite readout, or L1A retimed. The signal time of the $i^{t h}$ drift chamber wire may be modeled as

$$
t_{\text {signal }, i}=t_{\text {drift }}+t_{\text {propagate }, i}
$$


where $t_{\text {drift }}$ is the time for the ionization to drift from the hit position to the sensing wire and $t_{\text {propagate, } i}$ is the time for the signal to travel from the wire hit position to the TDC module. The trigger time $t_{\text {trig }}$ may be described as

$$
t_{\text {trig }}=t_{\text {travel }}+t_{\text {propagate,trig }}
$$

where $t_{\text {travel }}$ is the time that it takes the particle of interest to travel from the hit plane to the BigBite calorimeter which is responsible for forming the BigBite trigger signal T2. In complete analogy to $t_{\text {propagate }, i}$, the symbol $t_{\text {propagate,trig }}$ represents the time for the T2 trigger signal to propagate from the calorimeter to the TDC modules. Therefore, the time that is recorded is the difference between $t_{\text {signal }, i}$ and $t_{\text {trig }}$, and is

$$
\begin{aligned}
t_{\mathrm{TDC}} & \equiv t_{\text {signal }, i}-t_{\text {trig }}, \\
& =t_{\text {drift }}+\left(t_{\text {propagate }, i}-t_{\text {travel }}-t_{\text {propagate, trig }}\right) \\
& \approx t_{\text {drift }}+t_{0, i},
\end{aligned}
$$

where the last three terms have been absorbed into a single wire-dependent time offset referred to as $t_{0, i}$. If the time offsets $t_{0, i}$ are determined for each wire, then the drift time $t_{\text {drift }}$ may be extracted from the measured TDC signal.

The time offsets $t_{0, i}$ may be determined by analyzing a TDC time spectrum for every wire. Enough data is required to sufficiently populate a TDC spectra for a single wire; a typical spectra is a signal pulse on top of a flat background distribution which may be fit to a constant. The leading edge of the pulse is approximately linear, and a fit may be performed between two points corresponding to $20 \%$ and $80 \%$ of the pulse height. If the fit is extrapolated, then the intersection of the rising edge linear fit 


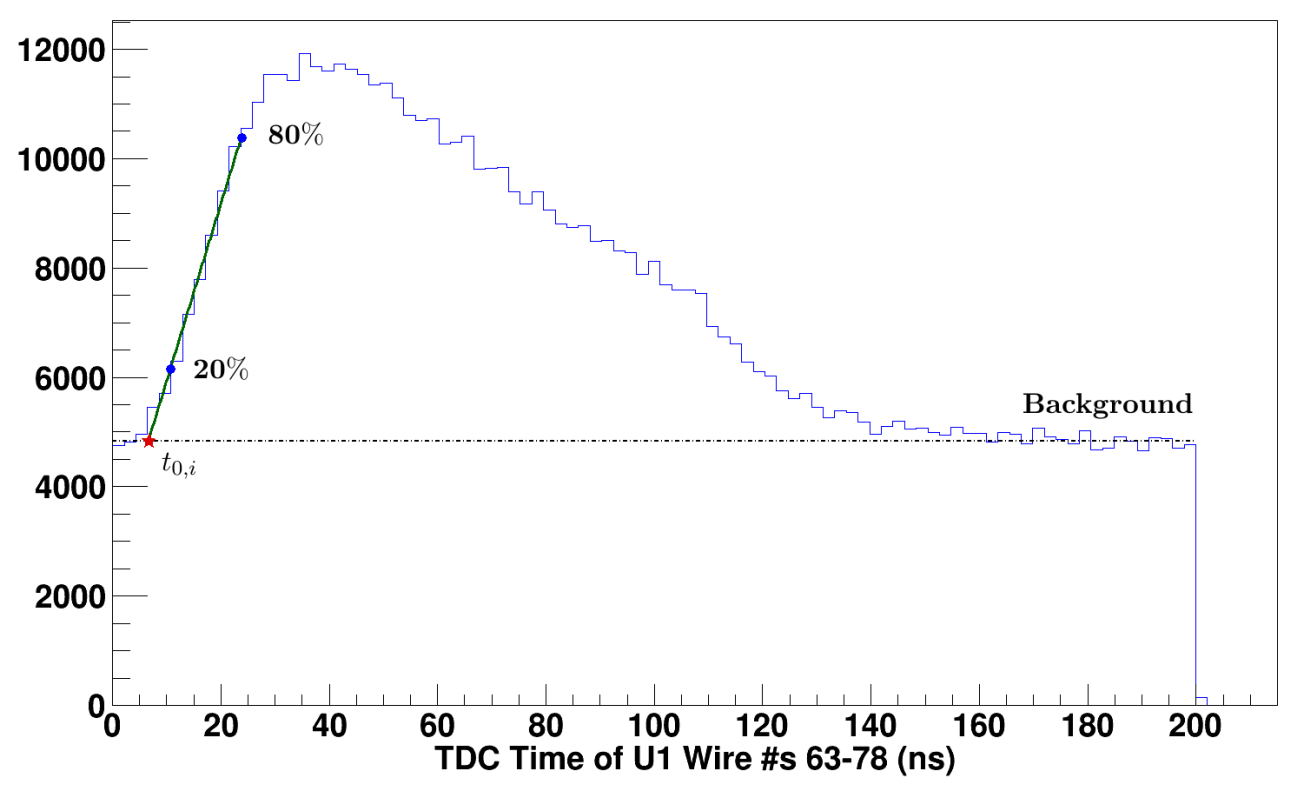

Fig. 5.1: The method to obtain drift chamber time offset for wires labeled 63-78 of the U1 plane, ${ }^{3} \mathrm{He}$ data. Calibration has already been implemented.

to the flat background constant yields a value of $t_{0, i}$ for wire $i$. It is noted that $G_{E}^{n}$ did not calibrate every wire, but rather all wires connected to the same 16 channel discriminator cards received the same calibration time offset. In Figure 5.1, the time offsets have already been applied but the procedure is demonstrated on wire numbers 63-78 of the U1 plane. The timing pulse settles to background noise well before $200 \mathrm{~ns}$. Any data set is appropriate for calibration, but ${ }^{3} \mathrm{He}$ data has been used in the figure.

A critical step towards reconstructing tracks within the BigBite drift chambers is to convert the drift time $t_{\text {drift }}$ to a drift distance in order to calculate the hit position within the wire plane. Recall that the drift time is related to the distance of closest approach between the drift chamber sensing wire and the trajectory of the electron. Assuming that the drift medium is homogeneous, the drift distance as a function of drift 
time $t_{\text {drift }}$ may be obtained by integrating a drift velocity function $v(t)$ that describes the drift time spectrum over a time window corresponding to $0 \rightarrow t_{\mathrm{drift}}$ :

$$
d\left(t_{\mathrm{drift}}\right)=\int_{0}^{t_{\mathrm{drift}}} v(t) d t
$$

If the drift time has been measured to be zero, then the drift distance should also be zero. There is an additional constraint as there is a maximum drift distance $d_{\max }$ associated to a particular wire; therefore, the desired expression may be obtained by normalizing Eqn 5.4 and multiplying the result by the maximum allowable distance:

$$
d\left(t_{\mathrm{drift}}\right)=d_{\max } \frac{\int_{0}^{t_{\mathrm{drift}}} v(t) d t}{\int_{0}^{t_{\max }} v(t) d t}
$$

In the case of the $G_{E}^{n}$ drift chambers, $d_{\max }=5 \mathrm{~mm}$ which corresponds to half the distance between two sensing wires. The maximum time $t_{\max }$ within the normalization integral is taken to be $250 \mathrm{~ns}$ which is sufficiently large compared to the typical pulse time for a set of wires as seen by Figure 5.1. The results of the calculation performed by Riordan [178 may be seen by the data points within Figure 5.2. Two conversion functions have been fitted to the data. The first is simply a linear fit which corresponds to a constant drift velocity; the drift distance is approximately linear up to drift times of $\sim 90$ ns. The second fit denoted by the black line represents a more accurate mapping function and takes the following form:

$$
d_{\mathrm{drift}}=d_{\max } \tanh \left(\frac{v_{0}\left(t_{\mathrm{drift}}-t_{0}\right)+a_{0}\left(t_{\mathrm{drift}}-t_{0}\right)^{2}}{d_{\max }}\right),
$$

where $t_{0}, v_{0}$ and $a_{0}$ are fit parameters. This fit function exhibits approximately linear behavior for $t_{\mathrm{drift}}<90 \mathrm{~ns}$ and asymptotically approaches a drift distance of $5 \mathrm{~mm}$ as $t_{\mathrm{drift}}$ approaches $t_{\max }$. Note that the definition of the drift distance is inherently ambiguous 


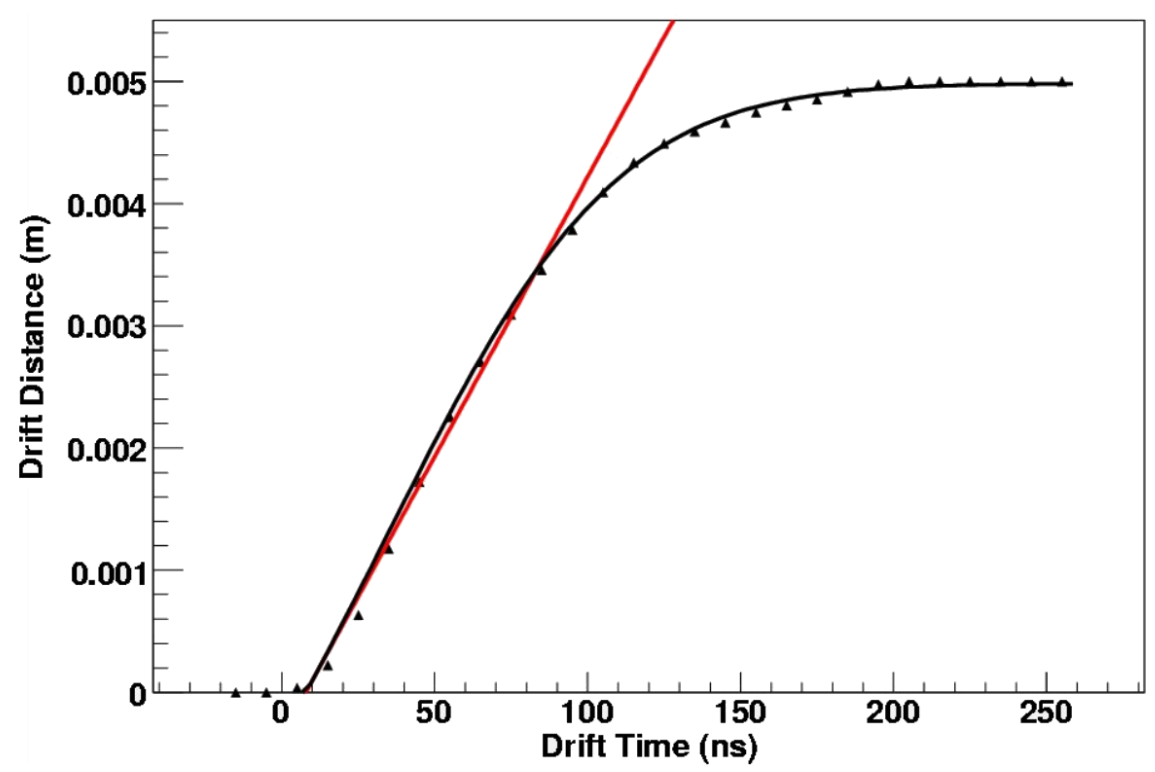

Fig. 5.2: The drift time to drift distance conversion functions shown by the red and black lines. The calculation has been performed by S. Riordan [178].

as the formalism calculates a distance magnitude and contains no information about which side of the wire the ionization originated. Resolving this technical difficulty is known as the so-called left/right differentiation, and must be accounted for within the tracking algorithm.

\subsubsection{Description of BigBite Track Reconstruction}

The BigBite tracking algorithm is the heart of the physics reconstruction of the outgoing electrons. The goal of the tracking code is to take a set of drift chamber TDC signals and to find the best combination that represents the trajectory of the electron post magnetic deflection. A major technical difficulty is to eliminate noise such that the noise to signal ratio is kept below a threshold of 10 , which is an observed value that significantly hinders 
efficiency and speed if exceeded. This has been achieved by implementing two major cuts on drift chamber hits. The first of which is a timing window cut associated with the coincidence trigger and the arrival of the drift chamber TDC signals; the cut is on the order of a couple hundred nanoseconds corresponding to the duration of a drift chamber signal pulse as seen in Figure 5.1. A second cut exploits geometric knowledge of the reconstructed calorimeter cluster. (Note that the calibration of the showers are described in detail within Section 5.3.5, and it has been organized this way as the final calorimeter energy calibration procedure requires information obtained from the track reconstruction.) A naive clustering algorithm finds the lead-glass shower module with the largest signal, and then adds signals from the geometric closest blocks within rectangular cuts to form a $3 \times 3$ cluster object. The coordinates of the cluster, which are formed by ADC-weighted coordinates of the constituent modules, then fixes an area on the calorimeter face from which a candidate track must end. As the endpoint is now fixed, and if the electrons are assumed to have originated from the beamline and the magnetic field is well understood, then the region of allowable wires associated with the calorimeter cluster is reduced significantly; the result is a reduced set of drift chamber hits. All the hits within a particular wire plane satisfying the time and space constraints are combined into a group. Groups are then sent to a coarse tracking algorithm where straight lines are fit to hit combinations. A subset is saved and sorted according to the $\chi^{2}$ fit results, and poor $\chi^{2}$ fits are discarded. If coarse tracks are within one wire spacing of each other, then the tracks are absorbed into a single track. The minimum number of active planes required to start reconstruction is 4 while the maximum is 
simply constrained by the total number of planes, or 15 .

The coarse tracks are processed further within the fine processing stage of the algorithm. Coarse tracks are correlated to the correct hit within the scintillating hodoscope. Drift times may then be corrected for the time it takes the particle to travel from a particular plane to the hodoscope; the corrected drift times are converted to drift distances. The left/right differentiation is performed for all valid plane hits of the same wire type, i.e. type $\mathrm{X}, \mathrm{U}$ or $\mathrm{V}$, and a $\chi^{2}$-minimization procedure is implemented, this time using drift distances rather than wire combinations, in order to find the best fit.

The best track after fine processing represents the trajectory of the charged particle that passed through the drift chambers. The track may be completely described by two coordinates and two directions defined in the BigBite coordinate system: $x, y$, $x^{\prime}=\frac{d x}{d z}$ and $y^{\prime}=\frac{d y}{d z}$. The origin of the BigBite coordinate system is defined to be the center of the first wire plane of the first drift chamber, or the $z_{\text {det }}=0 \mathrm{~m}$ plane. The convention is to project all tracks to the $z_{\text {det }}=0 \mathrm{~m}$ plane; therefore, the track initial coordinates, denoted by $x$ and $y$, are the offsets while $x^{\prime}$ and $y^{\prime}$ are the directional components. The concept illustrated within Figure 5.3 , and a practical calculation related to the calorimeter may be found within Section 5.3.5. The track post magnetic deflection has been reconstructed, and must now be traced through the magnetic field in order to reconstruct the scattering interaction vertex and momentum of the quasielastically scattered electron. 


\subsubsection{BigBite Optics Model: Vertex and Momentum Reconstruction}

The construction of tracks have been discussed within the previous section, and the contents of this section assumes that tracks have already been found. A track may be completely described by two coordinates and two directions within the $z_{\text {det }}=0 \mathrm{~m}$ plane, see Figure 5.3 for a visual description where the reconstructed track is labeled as back-track. In order to reconstruct the scattering vertex, the back-track may be traced backwards through the BigBite dipole field and towards the target. The front-track is defined to be the trajectory of the particle immediately after scattering; therefore,

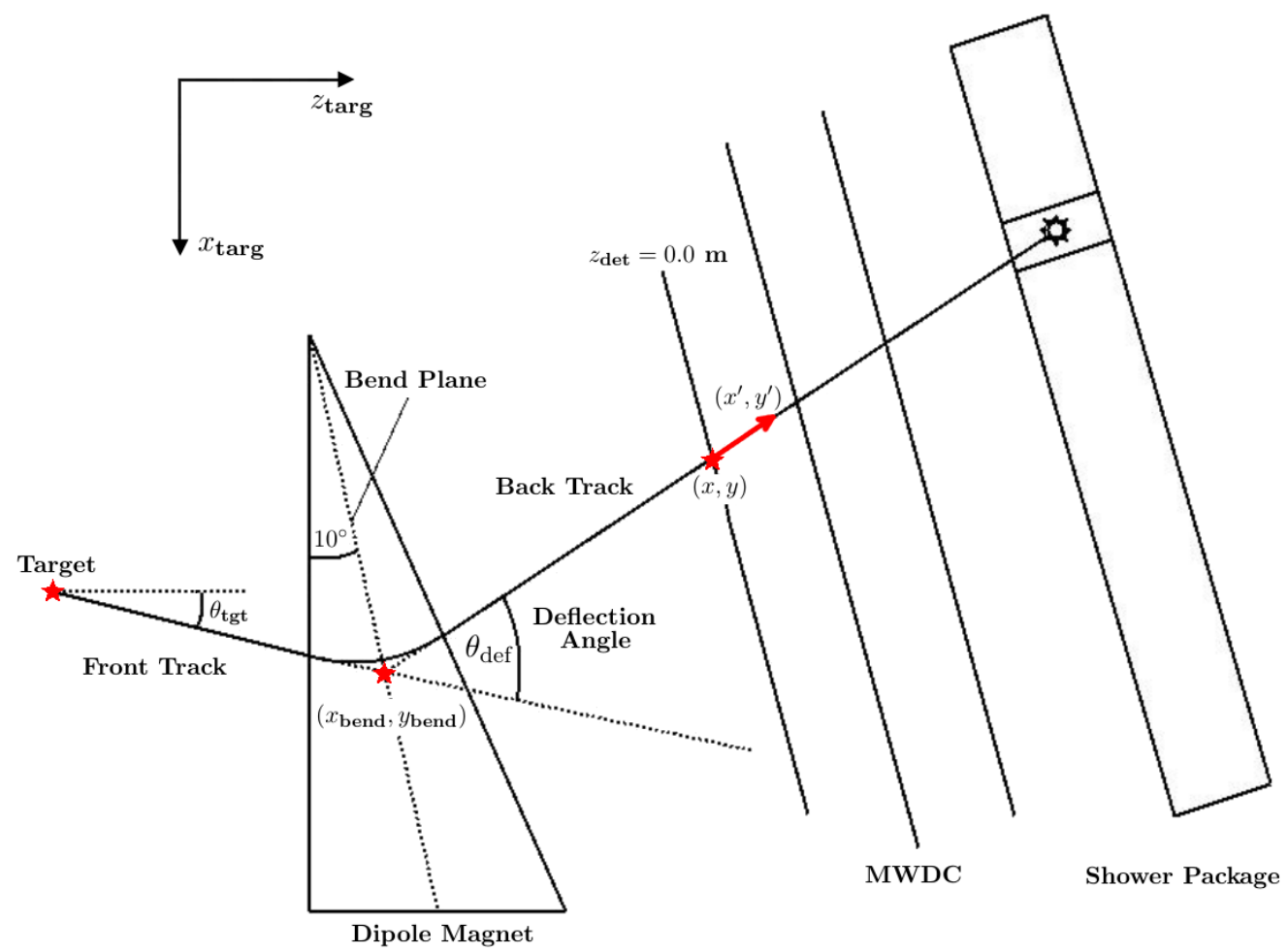

Fig. 5.3: Description of the BigBite magnetic bend plane model, viewed from the side. 
sufficient understanding of particle trajectory through the magnetic field is necessary. For $G_{E}^{n}$, a model is introduced where the front-track and back-track are constrained to the same plane and it is assumed that no magnetic deflection occurs in the $\hat{y}_{\text {det }}$ direction. The magnetic field is then modeled such that all the interaction takes place at the magnetic mid-plane; this defines the effective bend plane seen in the figure. The back-track may be projected to the bend plane which uniquely defines a point labeled as $\left(x_{\text {bend }}, y_{\text {bend }}\right)$. A plane $P$ that contains the back-track, the point $\left(x_{\text {bend }}, y_{\text {bend }}\right)$ and the front-track results in an infinite collection of possible front-tracks corresponding to the intersection of $P$ with the cylindrical target cell; the intersection defines an area bounded by the target cell denoted as $P_{\text {int }}$, which is a subspace of $P$. As the position and direction of the beam electrons are known by BPM information, then the intersection of a beam electron trajectory with $P_{\text {int }}$ defines a unique point on that surface which is taken to be the leading order vertex term denoted by $v_{0}$. Since the formalism assumes no horizontal magnetic deflection, empirical corrections are applied to $v_{0}$ and depend on the four tracking variables and $\left(x_{\text {bend }}, y_{\text {bend }}\right)$ :

$$
v_{z}=c_{0} v_{0}+c_{x} x+c_{y} y+c_{x^{\prime}} x^{\prime}+c_{y^{\prime}} y^{\prime}+a\left(x_{\text {bend }}, y_{\text {bend }}\right) .
$$

The $z$ position of the vertex is defined in the lab frame, and has five coefficients (the c's) that must be determined via calibrations to remove any residual track variable dependence. The term $a$ is needed to handle the vertical extremes of the BigBite magnet in which non-linear behavior is observed in $x_{\text {bend }}$. The calibration of $a$ requires a more careful analysis, but the result is a discrete grid of corrections in $x_{\text {bend }}$ and $y_{\text {bend }}$. The calibration of the $c$-coefficients all require a similar method; analyze data of 
targets at a known position and attempt to remove any unwanted correlations. The carbon-BeO foil data is used for vertex calibrations in which there are 5 foils in a C-CBeO-C-C pattern spaced $6.7 \mathrm{~cm}$ apart in $z_{\text {lab }}$, and the $\mathrm{BeO}$ foil is ideally centered at $z_{\text {lab }}=0.0 \mathrm{~cm}$. A plot of the vertex $z$ position and the tracking variables may or may not yield a linear correlation; if so, then the corresponding coefficient that minimizes the unwanted correlation is obtained. In order to account for the non-linear dependence on the magnetic mid-plane variables at the vertical extremes, the reconstructed vertex for $\mathrm{BeO}$ foil data is fitted in bins of equal acceptance of rectangular dimensions along the magnetic bend plane. The result is a grid of corrections from which a bilinear interpolation scheme is then implemented to extract the correction for a given ( $\left.x_{\text {bend }}, y_{\text {bend }}\right)$. The procedure is summarized within Figure 5.4 and Figure 5.5 the former (latter) representing the reconstructed vertex plotted against all tracking variables before (after) calibrations have been implemented. It is noted that within Figure 5.4, there is a small linear correlation between $v_{z}$ and all tracking variables, and the nonlinearities in the vertical extremes, namely $x_{\text {bend }}$, are seen by the red circles. After calibration, all correlations have been minimized as can be seen by Figure 5.5. The result of the calibration process yields a vertex resolution improvement from $13.5 \mathrm{~mm}$ to $6.5 \mathrm{~mm}$, and may be seen by Figure 5.6. The five foils, C-C-BeO-C-C, are clearly visible, and the resolution of the $\mathrm{BeO}$ foil has been determined with a Gaussian fit on top of a constant background. As the vertex position is now reliable, a cut of $-0.17 \mathrm{~m}<v_{z}<0.17 \mathrm{~m}$ will be implemented to future analyses in order to remove unwanted events originating from the target cell windows. 

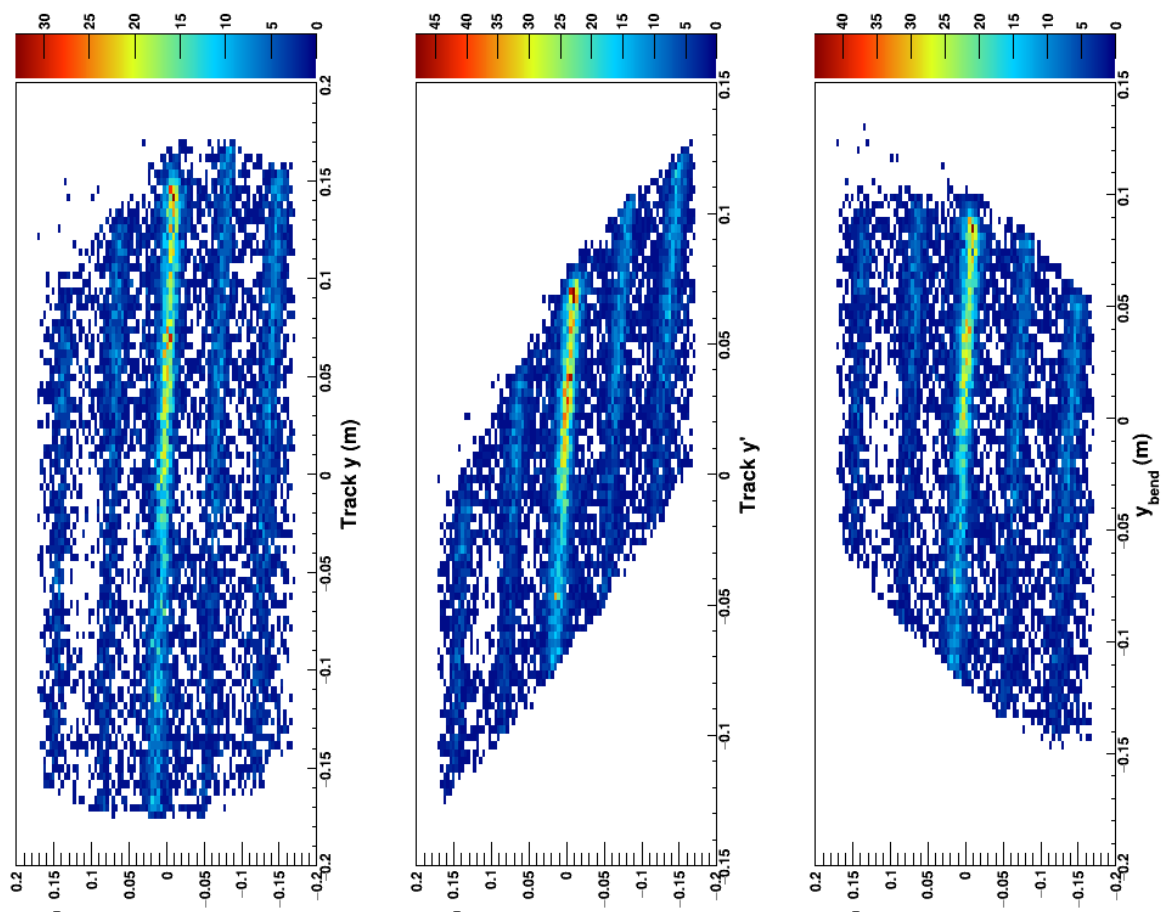

(u) ${ }^{z} \wedge$ pอเonגsuover

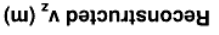

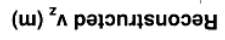

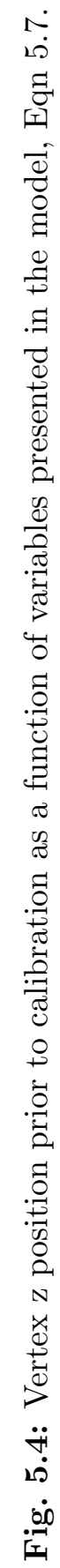
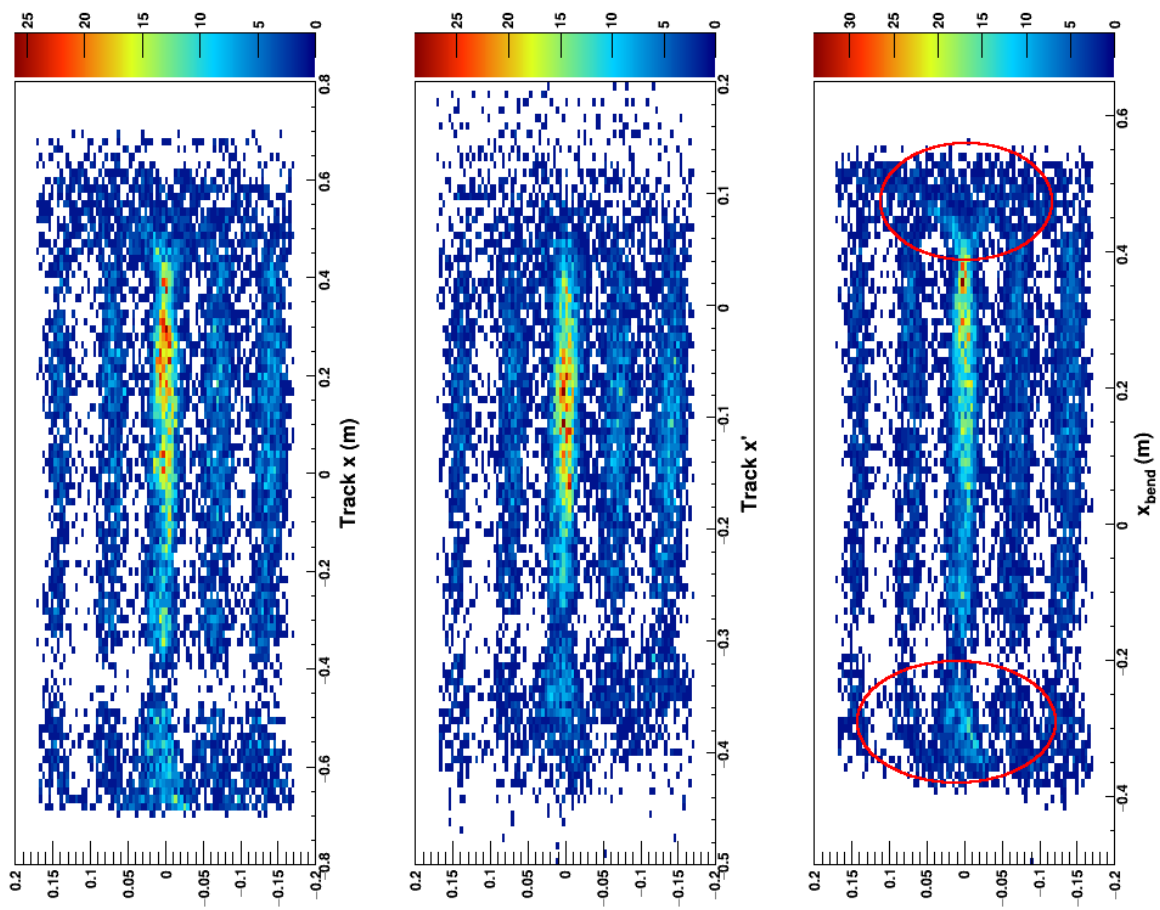

(u) ${ }^{z} \wedge$ pəəondisuover

(w) ${ }^{z} \wedge$ рәээกגsuoวay 

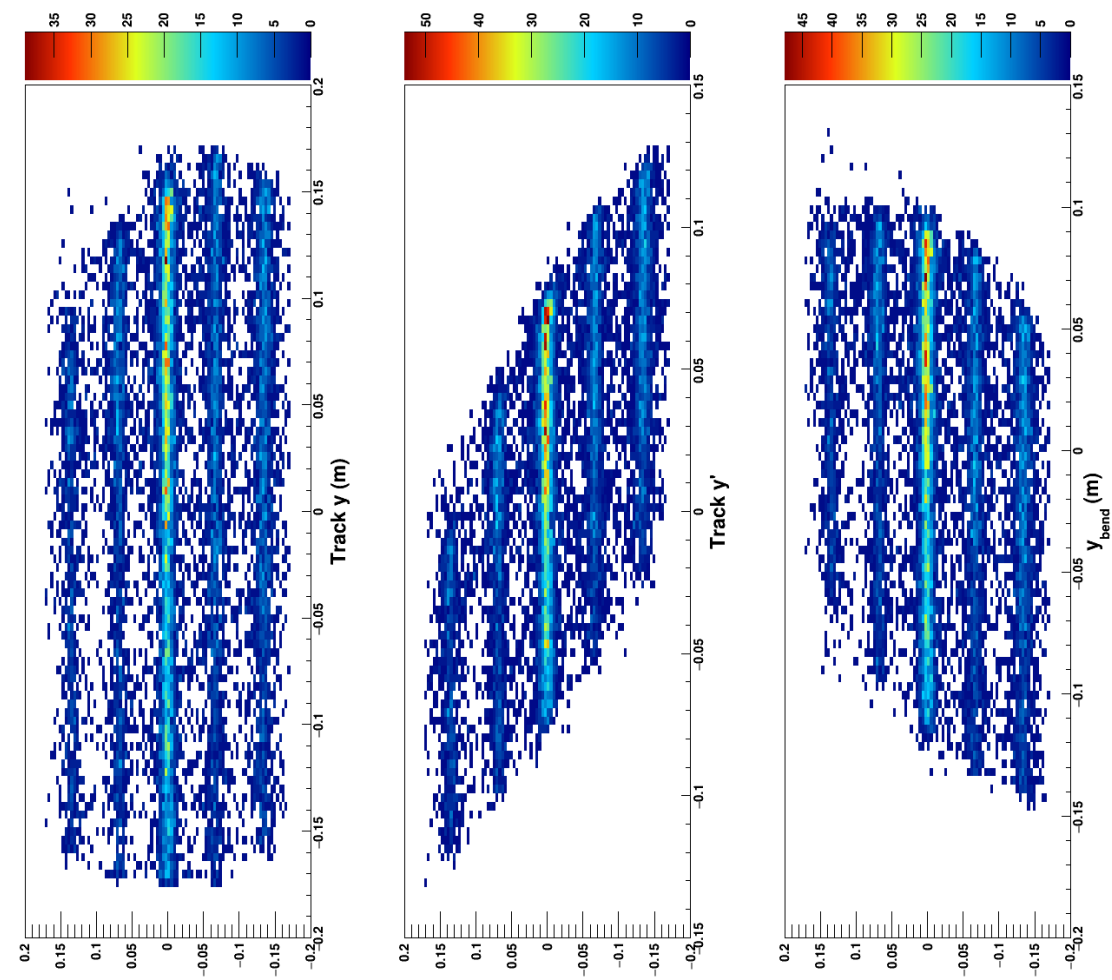

(ui) ${ }^{z} \wedge$ poเonג1suovor

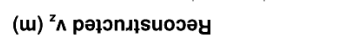

(u) ${ }^{z} \wedge$ рәээnגısuoэəy
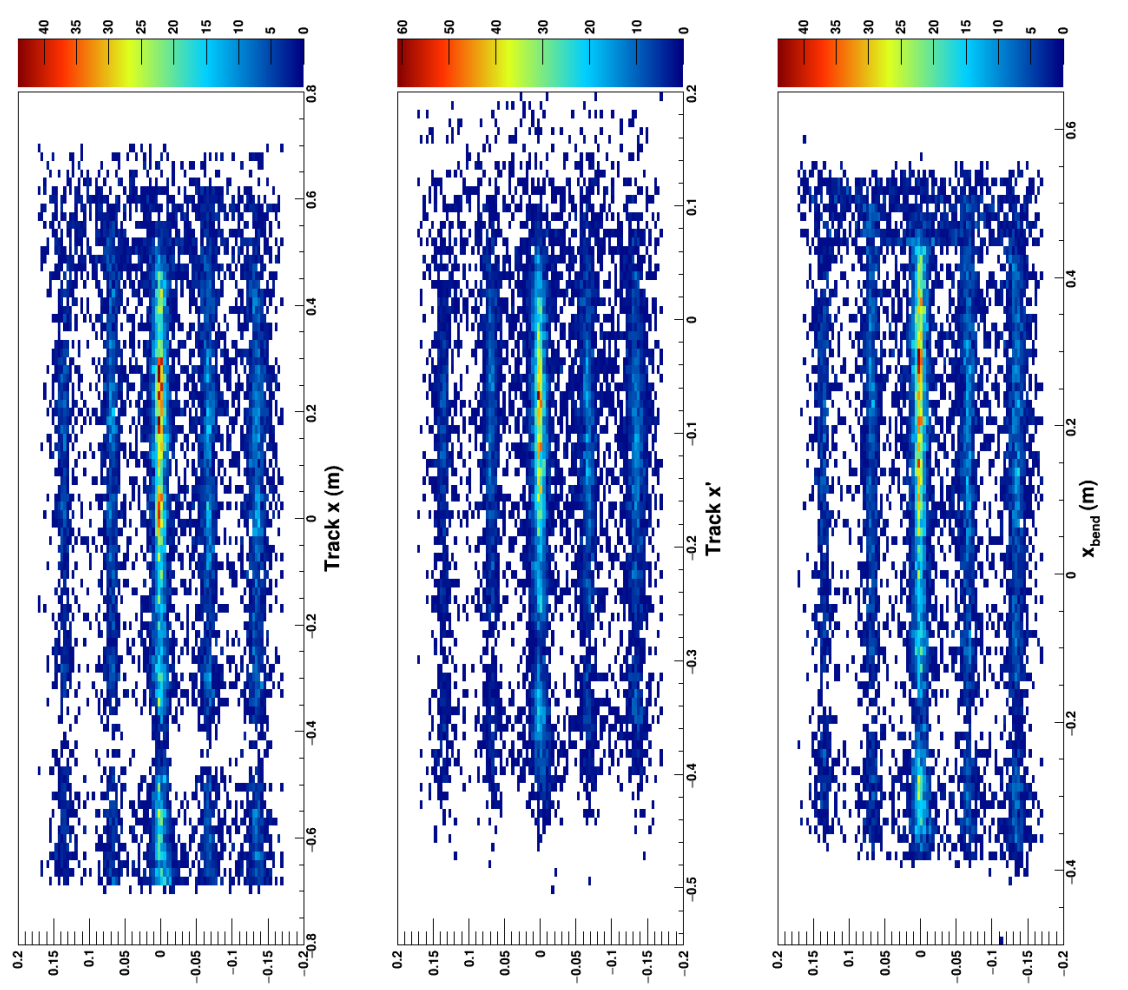

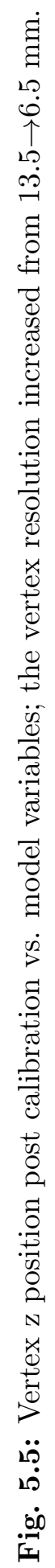

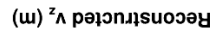

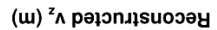

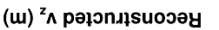




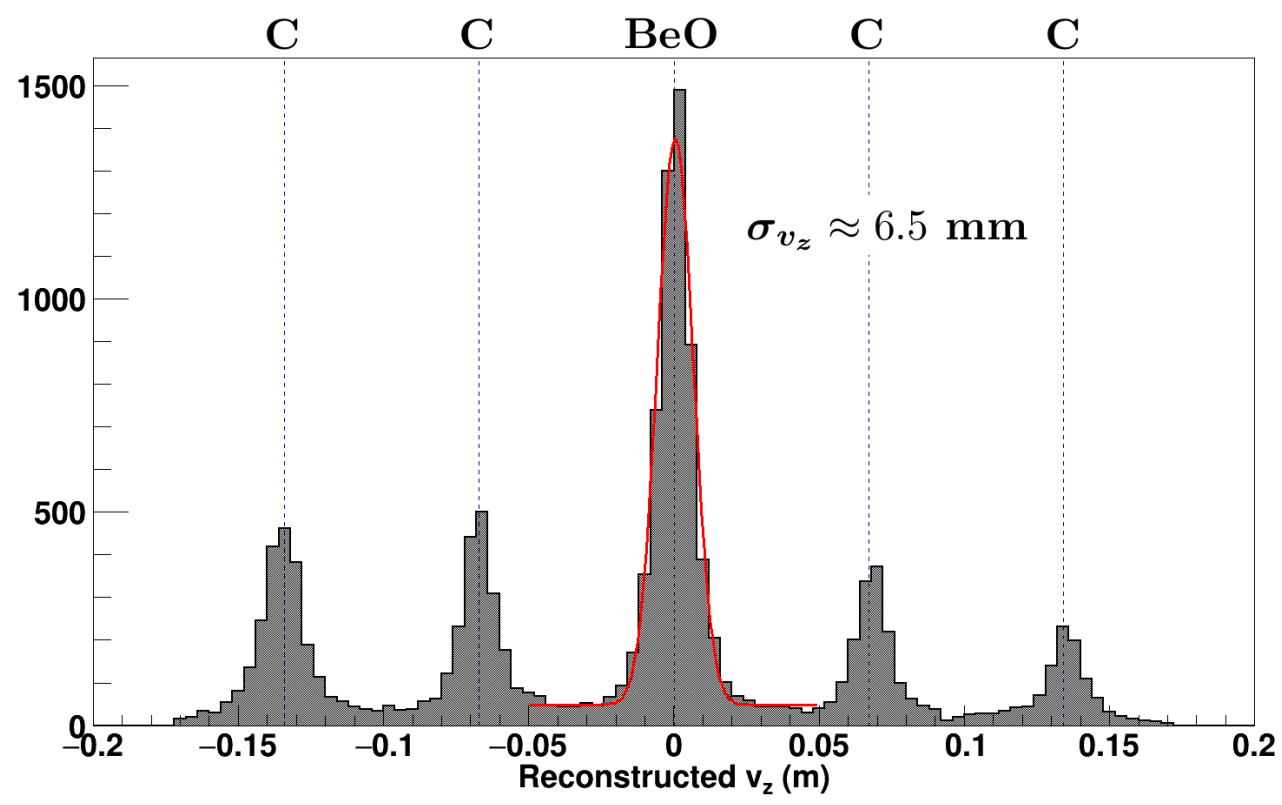

Fig. 5.6: Reconstructed vertex $z$ position within the $\mathrm{C}-\mathrm{BeO}$ foil data. A vertex cut of $-0.17 \mathrm{~m}<v_{z}<0.17 \mathrm{~m}$ is now introduced in order to exclude events originating from the target cell windows.

The momentum reconstruction and calibration for $G_{E}^{n}$ follows an analogous procedure in the sense that the leading order term is physically motivated, and then empirical first order corrections are applied. In Figure 5.3, or the effective magnetic bend model, all of magnetic interaction occurs at a point within the bend plane some distance away from ( $x_{\text {bend }}, y_{\text {bend }}$ ) defined by $R_{\text {curve }}$. A particle of momentum $p$ post scattering follows the front-track trajectory. As the front-track enters the magnet, the particle will then to leading order follow a circular trajectory determined by $R_{\text {curve }}$ which depends on the magnetic field integral and path length, recall Eqn 4.4 or $p \propto \frac{1}{\theta} \int \vec{B} \cdot d \vec{l}$. The circle defined by $R_{\text {curve }}$, or the radius of curvature, then must connect to the back-track in 
order to construct a fluid particle trajectory. Recall that the front-track, the radius of curvature, and the back-track are coplanar in this model, a consequence of treating BigBite as a perfect dipole magnet. The front-track trajectory depends on the vertex reconstruction, and the momentum model will have empirical corrections in order to handle the vertical extremes of the BigBite magnet that are outside of the scope of this model. Therefore, the form of the reconstructed momentum is

$$
p_{\text {recon }}=c_{0}\left(x_{\text {bend }}, y_{\text {bend }}\right) R_{\text {curve }}+c_{v_{z}}\left(x_{\text {bend }}, y_{\text {bend }}\right) v_{z}+c_{a}\left(x_{\text {bend }}, y_{\text {bend }}\right)+c_{y^{\prime}} y^{\prime} .
$$

It is noted that $R_{\text {curve }}$ depends on the four tracking variables. The only trivial calibration coefficient is $c_{y^{\prime}}$ which is determined in an identical manner as the $c$ coefficients within the vertex reconstruction procedure. The track $y^{\prime}$ coefficient has been added for Kinematic 1 in order to remove the unwanted correlation between $p$ and $y^{\prime}$. The nontrivial discrete grid coefficients $\left(c_{0}, c_{v_{z}}\right.$ and $\left.c_{a}\right)$ are also determined in an identical way as $a\left(x_{\text {bend }}, y_{\text {bend }}\right)$ described within the vertex reconstruction procedure, and a bilinear interpolation is then used to handle continuous coordinate inputs.

The calibration of the momentum requires $\mathrm{H}_{2}$ data in which the elastic peak is selected. It is imperative that the vertex calibration occurs prior to momentum calibration as the front-track is fixed within the target cell by $v_{z}$. By selecting the elastic peak, the reconstructed momentum may be compared to the expected (or true) elastically scattered energy which has been derived in Section 2.2.1 and rewritten for convenience:

$$
E_{e}^{\prime} \equiv p_{\text {true }}=\frac{E_{e}}{1+\frac{2 E_{e}}{M} \sin ^{2} \frac{\theta_{e}}{2}}
$$


where $E_{e}$ is the beam energy and $M$ is the mass of the proton. The notation $E_{e}^{\prime} \equiv p_{\text {true }}$ is simply a reminder that for a relativistic electron the momentum and energy are interchangeable as the mass is neglected. The polar angle $\theta_{e}$ of the elastically scattered electron depends on the incoming and outgoing electron momenta vectors which may now be calculated as the vertex calibration is assumed to be complete. The difficulty here, though, is a set of cuts required to select $\mathrm{H}_{2}$ elastic events without the availability of higher level physics variables. The accepted cuts are the following:

1. a vertex cut of $-0.17 \mathrm{~m}<v_{z}<0.17 \mathrm{~m}$ to exclude the target windows;

2. a preshower energy deposition sum $>150 \mathrm{MeV}$ in order to reduce pion contamination, and is described in Section 5.3.5:

3. a coincidence hit, or a track is found in both arms;

4. a coplanarity cut, or the $p^{\prime}$ track roughly exists in the electron scattering plane;

5. a coincidence timing cut, or the neutron arm track time and the hodoscope time difference must lie within the L1A trigger signal resulting in an effective cut to reduce background.

Cuts 2-5 are determined by examining $\mathrm{H}_{2}$ data, and must be made wide enough such that elastic events are not suppressed. The momentum resolution may be determined by comparing the reconstructed momentum $p_{\text {recon }}(\mathrm{Eqn} 5.8$ ) to the true momentum of the elastically scattered electron:

$$
\frac{\delta p}{p}=\frac{p_{\text {recon }}-p_{\text {true }}}{p_{\text {true }}}
$$




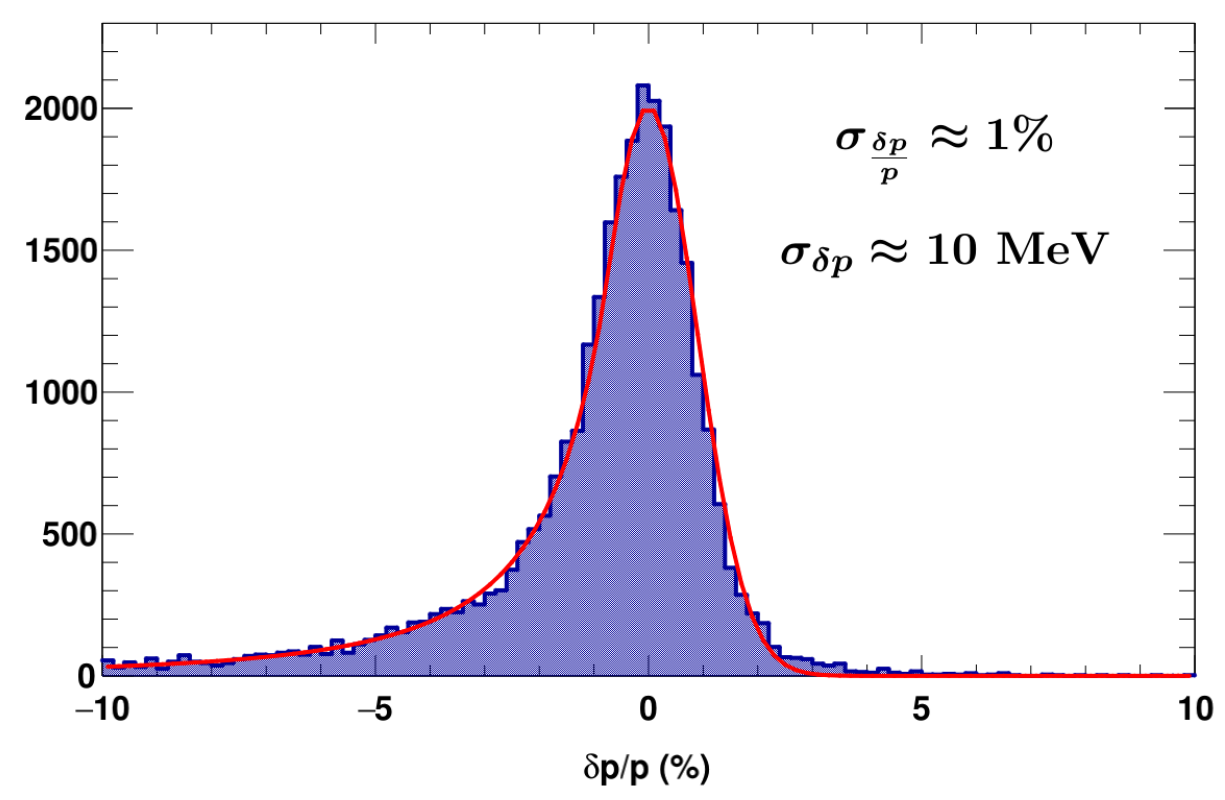

Fig. 5.7: Calibrated reconstructed momentum using $\mathrm{H}_{2}$ data and a resolution of $1 \%$ is observed. The fit is the so-called crystal ball function.

The result may be seen by Figure 5.7 in which a momentum resolution of approximately $10 \mathrm{MeV}$, or equivalently $1 \%$, has been obtained. The momentum resolution plotted against all relevant tracking variables after calibration is presented in Figure 5.8. The momentum resolution improved by a small amount for Kinematic 1, roughly $13 \mathrm{MeV}$ to $10 \mathrm{MeV}$, which is attributed to either the calibration of the $c_{y^{\prime}}$ coefficient or the energy calibration of the calorimeter (described in the next section).

The momentum resolution distribution is asymmetric and consequently a Gaussian fit alone is insufficient. A Gaussian core and a power-law low-end tail, or the so-called crystal ball function, may be used if a fit is required, which takes up to five parameters* and an example may be seen in Figure 5.7. The width of the peak is

\footnotetext{
${ }^{*}$ The parameters used for the fit: const $=3.87 \times 10^{2}, \mu=0.0, \sigma=0.89, \alpha=1.06$, and $N=1.15$.
} 
due to several aspects of the experiment: the detectors have a finite resolution, the charged particles are transported through the dipole magnet, and multiple scattering in air located between the target and drift chambers. As there is roughly $\sim 2.5 \mathrm{~m}$ of air corresponding to a mass thickness of $\sim 300 \mathrm{mg} / \mathrm{cm}^{2}$ between the target and drift chambers, multiple scattering contributes significantly to the momentum resolution. Ideally, one does not expect any events in the positive region of $\frac{\delta p}{p}$ well beyond the approximate Gaussian-width; however, events are seen which may be attributed to background events or spurious events contributing towards the width of the peak. Events outside the scope of the model, namely at the vertical extremes of the magnet, also contribute to this region and may be seen by the dispersive coordinates within Figure 5.8 , The long tail seen at negative values is due to radiative effects and energy loss of the outgoing electron prior to reaching the drift chambers. Possible sources of energy loss are the target cell, the target box window, synchrotron radiation within the dipole, or within the air. Synchrotron radiation may be estimated by knowledge of the angular deflection due to BigBite $\left(\theta_{\text {def }}\right.$ in Figure 5.3) and the value of $R_{\text {curve }}$. The angular deflection $\theta_{\text {def, }}$ or the angle between the front-track and the back-track $\left(\vec{x}_{\text {front }}\right.$ and $\vec{x}_{\text {back }}$, respectively) due to the dipole field, may be calculated as $\cos \theta_{\text {def }}=\hat{x}_{\text {front }} \cdot \hat{x}_{\text {back }}$. An average value of $\theta_{\text {def }}\left(\approx 20^{\circ}\right)$ and $R_{\text {curve }}(\approx 2.5 \mathrm{~m})$ results in an energy loss on the order of $10 \mathrm{~s}$ of $\mathrm{keV}$ and is negligible. For $G_{E}^{n}$, the energy loss due to ionization has been estimated to be small. Consequently, the tail is dominated by first-order ep radiative corrections to the Rosenbluth formula and henceforth is referred to as the radiative tail; the simulation using the ESEPP generator (see Section 5.3.6) 212] replicates the distribution. 

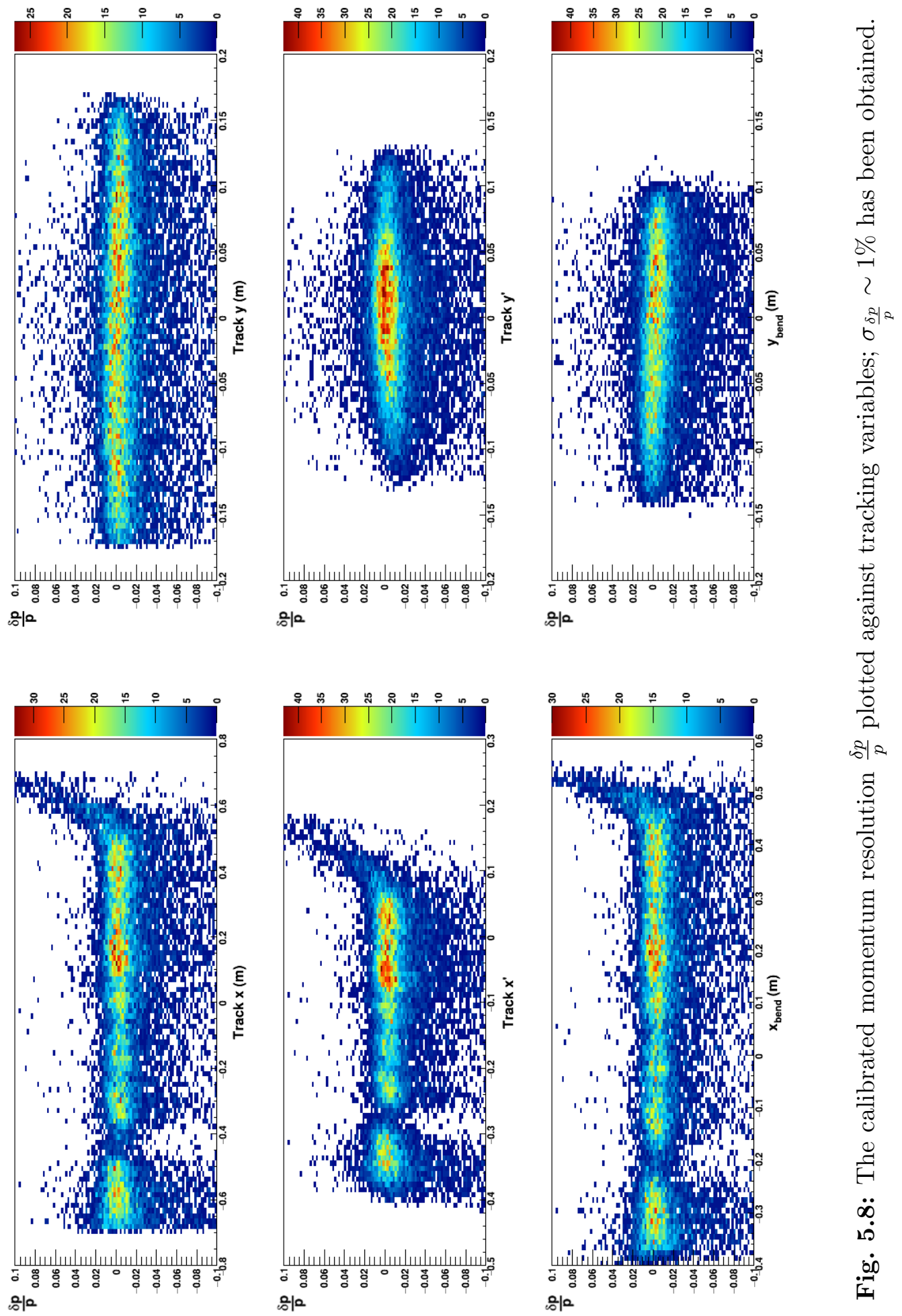

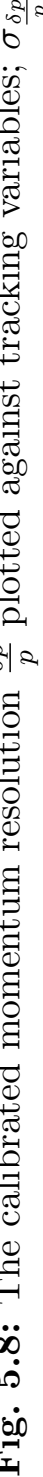




\subsubsection{Invariant Mass}

The four momentum and vertex position may now be reliably determined from the BigBite optics module. It is advantageous to enhance the understanding of the elastic region within the $\mathrm{H}_{2}$ data as it will serve as a useful guide for the ${ }^{3} \mathrm{He}$ analysis. The invariant mass $W$ of the final hadronic state is an effective kinematic quantity, and may now be defined using the same notation of Section 2.2.1:

$$
\begin{aligned}
W^{2} & =p^{2} \\
& =(p+q)^{2}, \\
& =p^{2}+q^{2}+2 p \cdot q, \\
\Rightarrow W & =\sqrt{M_{p}^{2}-Q^{2}+2 M_{p}\left(E_{e}-E_{e}^{\prime}\right)},
\end{aligned}
$$

where $p\left(p^{\prime}\right)$ and $q$ are four vectors representing the initial (outgoing) proton momentum and momentum transfer, respectively. For clarity, $M_{p}$ is the mass of the proton,* $Q^{2}=4 E_{e} E_{e}^{\prime} \sin ^{2} \frac{\theta_{e}}{2}, E_{e}$ is the beam energy and $E_{e}^{\prime}$ is the energy obtained from BigBite optics. The polar angle $\theta_{e}$ may also be obtained: $\theta_{e}=\cos ^{-1}\left(p_{z} /|\vec{p}|\right)$ where $p_{z}(|\vec{p}|)$ is the $z$ component (magnitude) of the reconstructed track momentum. For elastic $e p$ kinematics, $Q^{2}=2 M_{p} \nu=2 M_{p}\left(E_{e}-E_{e}^{\prime}\right)$ and consequently the $\mathrm{H}_{2}$ invariant mass spectrum must have a peak at $W=M_{p}$, and broadened by resolution and radiative effects; this is demonstrated by Figure 5.9. More importantly, the $W$ spectrum provides a powerful, higher-level tool for selecting elastic events. For subsequent $\mathrm{H}_{2}$ analyses, cuts as tight as $0.92<W<0.96 \mathrm{GeV}$ may be applied. It is noted that the $\Delta(1232)$ resonance is relatively small, but visible even in systems as simple as one proton. The relative am-

\footnotetext{
* The analysis cannot differentiate between the masses of the proton and neutron.
} 


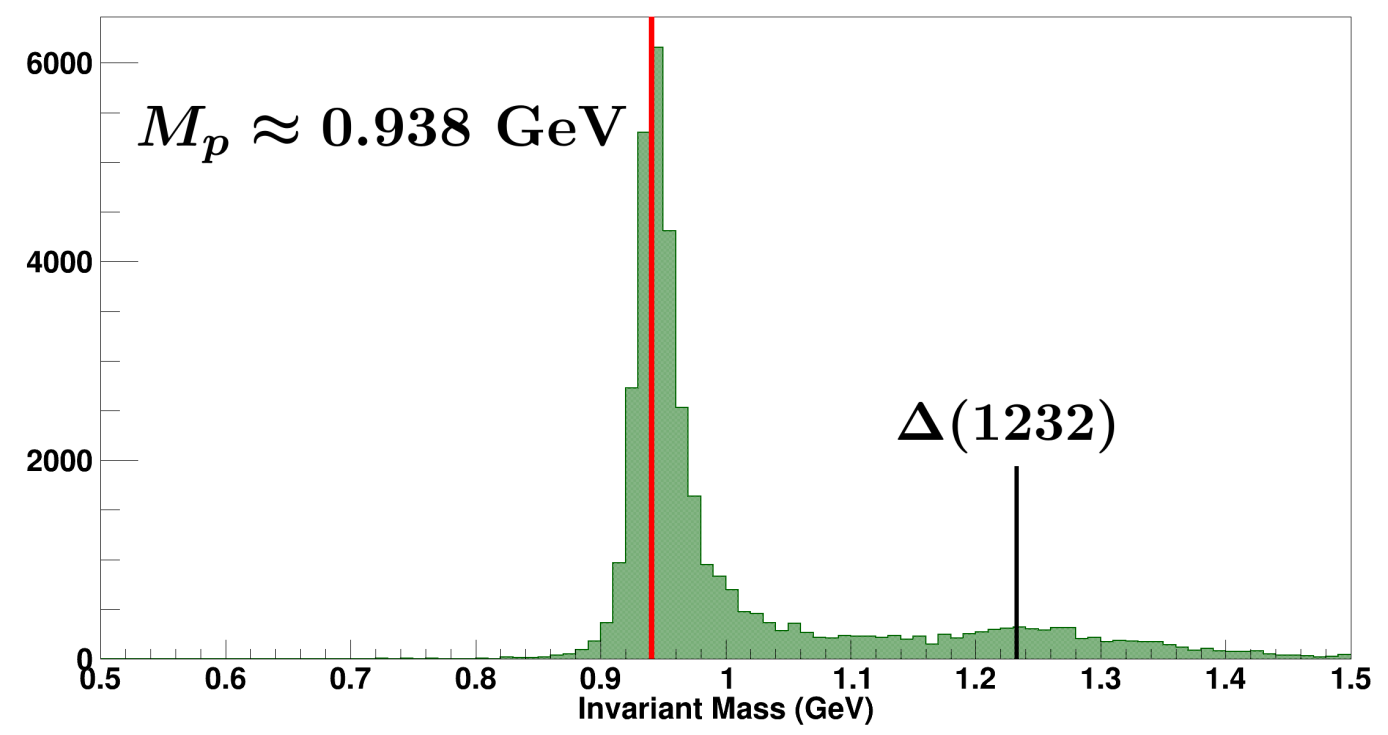

Fig. 5.9: The sharp peak at $M_{p}$ within the $\mathrm{H}_{2}$ invariant mass spectrum.

plitude of resonances becomes larger and more complicated in the polarized ${ }^{3} \mathrm{He}$ data, and the inelastic region eventually dominates as $Q^{2}$ increases for the $G_{E}^{n}$ configurations. Furthermore, the calculation of $W$ assumes that the nucleons are initially at rest, but this is not the case for ${ }^{3} \mathrm{He}$ due to the Fermi motion within the nucleus; therefore, significant broadening in the invariant mass spectrum is expected in the polarized ${ }^{3} \mathrm{He}$ data relative to Figure 5.9. Lastly, the nucleons are bound to the ${ }^{3} \mathrm{He}$ nucleus with a binding energy of roughly $2.5 \mathrm{MeV}$ per nucleon, a technicality that is not incorporated into the $W$ formula resulting in a small but noticeable shift of the quasielastic $W$ peak towards higher $W$. The ${ }^{3} \mathrm{He}$ analysis will then desire a semi-loose cut on the invariant mass; the cut needs to restrict the resonance and inelastic region, but cannot be too tight such that many quasielastic events are discarded. See Figure 5.57 and Figure 6.7 for the ${ }^{3} \mathrm{He}$ and simulated ${ }^{3} \mathrm{He}$, respectively, analog of Figure 5.9 . 


\subsubsection{Preshower and Shower}

Recall that the detectors consist of lead-glass modules, and the passage of a relativistic electron may result in an electromagnetic shower. The generated photons are then detected by the PMTs via the photoelectric effect; the resulting PMT signals are charge integrated by the ADCs over some time window defined by the trigger electronics, specifically L1A retimed. The decoding of this information represents an integrated charge for each lead-glass block in units of ADC channels. Generally, the ADC signals need to be corrected by the so-called pedestal value, or the ADC signal in the absence of any PMT signal; this is simply electronic noise and must be measured for each detector channel. The fundamental assumption of the BigBite calorimeter is that the energy deposition of the incident electron in a lead-glass module is proportional to the output signal, or

$$
E_{i}=C_{i} \cdot\left(A_{i}-P_{i}\right)
$$

where $E_{i}$ is the energy deposited within the $i^{\text {th }}$ block, $A_{i}$ is the raw ADC signal, $P_{i}$ is the pedestal value, and $C_{i}$ are proportionality constants, otherwise known as gain coefficients which have units of $\mathrm{MeV} / \mathrm{ADC}$. Henceforth, it is assumed that all ADC signals have already been corrected for the channel-dependent pedestal value. If the electron deposits all of its energy within the calorimeter, ${ }^{*}$ then the energy may be calculated by summing over all blocks with an ADC signal, or $\sum_{i=1}^{n} E_{i}$ where $n$ is the number of lead-glass blocks that fired for a given event. A more sophisticated approach is to consider a cluster rather than naively summing the energy deposition within all calorimeter blocks.

\footnotetext{
* An argument is presented in Section 4.6 .2
} 


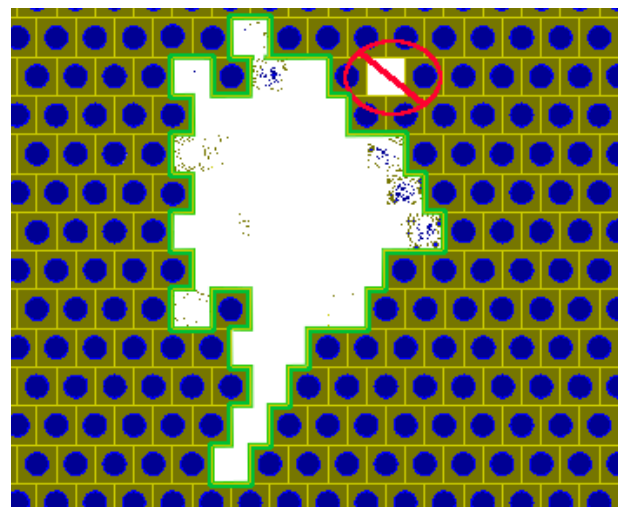

Fig. 5.10: An example of an electromagnetic shower where an algorithm combines modules outlined in green to form a cluster. Blue circles represent PMTs.

A typical clustering algorithm starts with the block with the maximum ADC signal, and continues to dynamically grow by adding nearest neighboring modules with an ADC signal until no more are found. The resulting blocks define a cluster, and an example may be seen by Figure 5.10 in which the cluster is outlined in green. The ubiquitous method of clustering is a useful strategy of isolating an electromagnetic shower while suppressing unrelated background events. In fact, the Gaussian-mean energy difference between the naive sum of all BigBite modules and the reconstructed cluster is around $200 \mathrm{MeV}$ for the low- $Q^{2}$ data (note that $E_{e}^{\prime} \approx 900 \mathrm{MeV}$ ). The $x$ and $y$ coordinates of a cluster may be found by an energy deposition weighted sum, or

$$
x_{\text {cluster }}=\frac{\sum_{i=1}^{M} E_{i} x_{i}}{\sum_{i=1}^{M} E_{i}}, \quad y_{\text {cluster }}=\frac{\sum_{i=1}^{M} E_{i} y_{i}}{\sum_{i=1}^{M} E_{i}},
$$

where the sum includes all modules within a cluster of size $M$ blocks and $E_{i}$ is the energy deposition within the $i^{\text {th }}$ cluster block. The coordinates $x_{i}$ and $y_{i}$ denote the coordinates of the block center, and $\sum_{i=1}^{M} E_{i}$ represents the energy of the cluster. Therefore, this 
method may be used to find an approximate position of a cluster on the face of the calorimeter, and the coordinates may be used with the BigBite tracking code in order to reduce the search region for candidate tracks.

The initial calibration process, namely during the commissioning phase of BigBite, included studying the response of the calorimeter to cosmic rays, or minimum ionizing particles that deposit a well-defined energy in a lead-glass block. The PMT high voltage may be chosen in such a way to align the gains of all PMTs in an iterative procedure. In order to improve upon this calibration, the gain coefficients $C_{i}$ for all preshower and shower blocks may be aligned in software by studying the calorimeter response to incident particles with kinematics similar to what is expected during quasielastic scattering. This may be done by selecting the elastic peak of the $\mathrm{H}_{2}$ data as the energy of the elastically scattered electrons is well-known (Eqn 2.23), and in this case is referred to as the "true" energy:

$$
E_{\text {true }}=\frac{E_{b}}{1+\frac{2 E_{b}}{M} \sin ^{2} \frac{\theta_{e}}{2}}=\frac{M E_{b}}{M+E_{b}\left(1-\cos \theta_{e}\right)},
$$

where $E_{\text {true }}$ is the elastically scattered electron energy, $\theta_{e}$ is the scattered polar angle of the electron, $E_{b}$ is the beam energy, $M$ is the mass of the proton and the mass of the electron has been neglected. Recall that track reconstruction has already been calibrated to $E_{\text {true }}$; therefore, the energy deposited within the lead-glass blocks may be compared to the momentum* of the reconstructed track. The best set of gain coefficients $C_{i}$ may be obtained by a $\chi^{2}$ minimization procedure where the BigBite cluster energy

\footnotetext{
* Momentum and energy are interchangeable for the relativistic outgoing electron.
} 
is compared to the energy reconstructed from the BigBite optics:

$$
\begin{aligned}
\chi^{2} & =\sum_{i=1}^{N}\left(E_{e}^{i}-\sum_{k=0}^{M} C_{k} A_{k}^{i}\right)^{2}, \\
& =\sum_{i=1}^{N}\left(\left(E_{e}^{i}\right)^{2}+\left(\sum_{k=0}^{M} C_{k} A_{k}^{i}\right)^{2}-2 E_{e}^{i} \sum_{k=0}^{M} C_{k} A_{k}^{i}\right),
\end{aligned}
$$

where $E_{e}^{i}$ is the reconstructed energy obtained from BigBite optics for the $i^{\text {th }}$ event, $N$ is the number of events, $M$ is the number of blocks constituting a calorimeter cluster, $A_{k}^{i}$ is the ADC signal corresponding to block number $k$, and $C_{k}$ represents the preshower and shower gain coefficients. Minimizing $\chi^{2}$ with respect to $C_{k}$, or $\frac{\partial \chi^{2}}{\partial C_{j}}=0$, results in a system of $243(=2 \times 27+7 \times 27)$ linear equations representing the total number of preshower and shower blocks. This may be compactly expressed in matrix form:

$$
M_{j k}=\sum_{i=1}^{N} A_{j}^{i} A_{k}^{i}, \quad C=\left(\begin{array}{c}
C_{0} \\
\vdots \\
C_{M}
\end{array}\right) \quad B=\left(\begin{array}{c}
\sum_{i=0}^{N} E_{e}^{i} A_{0}^{i} \\
\vdots \\
\sum_{i=0}^{N} E_{e}^{i} A_{M}^{i}
\end{array}\right),
$$

which are related by $M C=B$. The solution is to invert the $243 \times 243$ matrix $M$ and then the desired gain coefficients for the 243 blocks may be obtained, or $C=M^{-1} B$. The number of elastic ep events $N$ needs to be large enough to populate the entire acceptance in order to avoid problems with inverting matrix $M$. Additionally, it may be beneficial to avoid events where the block with maximum energy deposition occurs along the perimeter of the shower since in this case a large fraction of the energy may escape the calorimeter altogether; this is referred to as leakage and can be quite large. Blocks that are unable to get sufficient data, typically edge blocks located at the vertical and horizontal extremes of the detector package, are given a gain value equal to the average of the blocks with acceptable statistics. For Kinematic 1, the momentum 


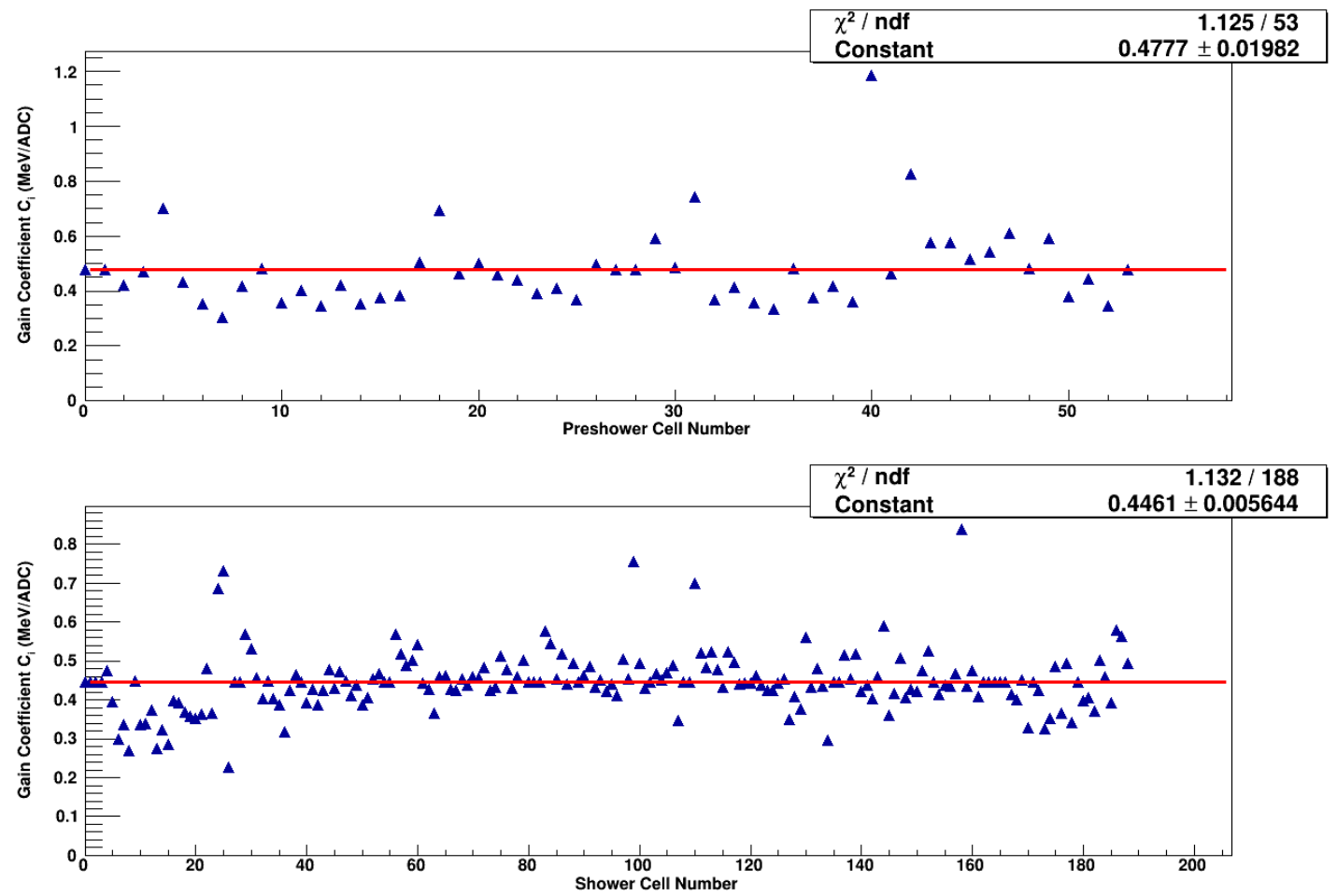

Fig. 5.11: The calibration coefficients $C_{i}$ for the preshower and shower.
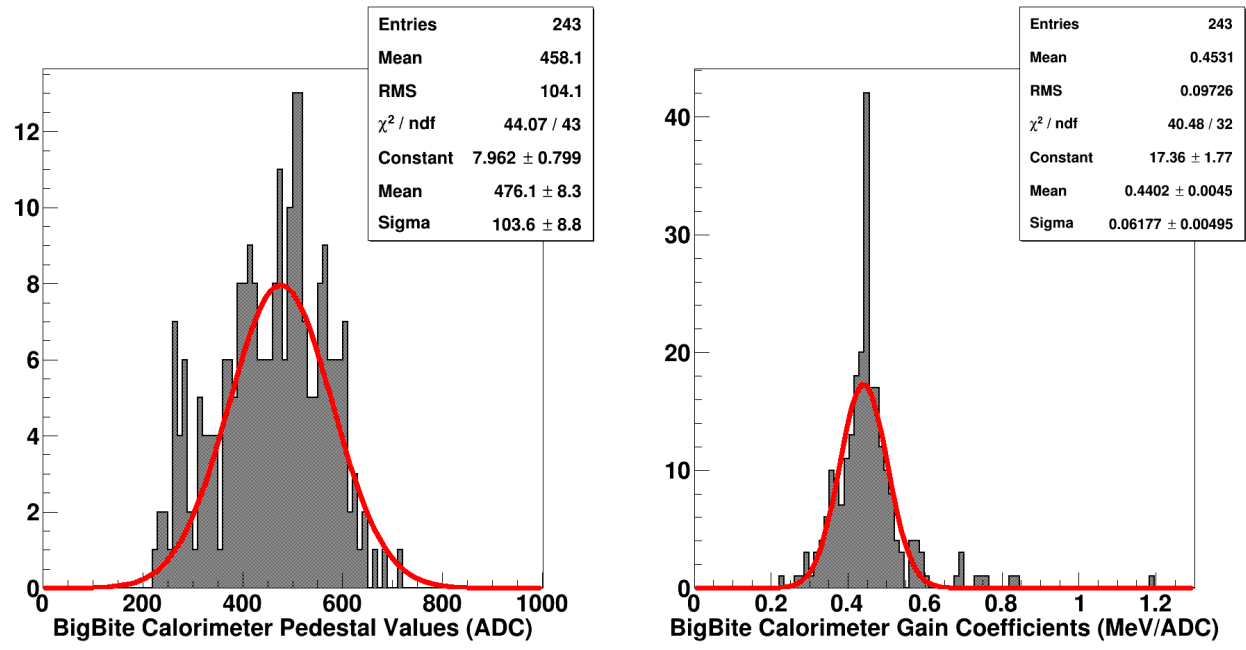

Fig. 5.12: The pedestal $P_{i}$ and gain $C_{i}$ distributions for all 243 channels. 
range of elastic ep events is approximately $0.7-1.0 \mathrm{GeV}$. In theory, the procedure can be enhanced by including data from the other $Q^{2}$ points in order to expand the momentum range which effectively fills the entire BigBite acceptance; however, this assumes that the geometric settings and run conditions are roughly the same between kinematic configurations, and this was not the case for the lowest $\mathrm{Q}^{2}$ configuration of $G_{E}^{n}$. Figure 5.11 displays the 243 calibration coefficients found for the preshower and shower using the $\chi^{2}$ minimization method and Figure 5.12 shows the distribution of the pedestal values and gain coefficients for all lead-glass blocks. The effects of aligning the gain coefficients in software may be seen by Figure 5.13 and Figure 5.15 where the improved preshower spectra for the ${ }^{3} \mathrm{He}$ and $\mathrm{H}_{2}$ data, respectively, is displayed. While the calibration uses $\mathrm{H}_{2}$ data, the before and after improvements are more impressive within the ${ }^{3} \mathrm{He}$ data.

Briefly mentioned in previous sections, BigBite is capable of primitive particle identification. By examining the preshower energy spectrum, the analysis is able to differentiate between pions and electrons as can be seen by Figure 5.13 . The lower peak may be identified as pions, and is a consequence of nucleons strongly interacting and producing both $\pi^{+}$and $\pi^{-}$. This may be checked by selecting on positively charged particles, i.e. particles that deflect "downwards" in the BigBite magnet which effectively enhances the number of $\pi^{+}$particles observed as the electron events are heavily suppressed. An alternative way to view the preshower energy spectrum may be seen by Figure 5.14, the preshower energy spectrum is plotted against the ratio $E_{\text {cluster }}$ to $E_{\text {optics }}$ where $E_{\text {cluster }}$ is the energy sum of a calorimeter cluster and $E_{\text {optics }}$ is the op- 

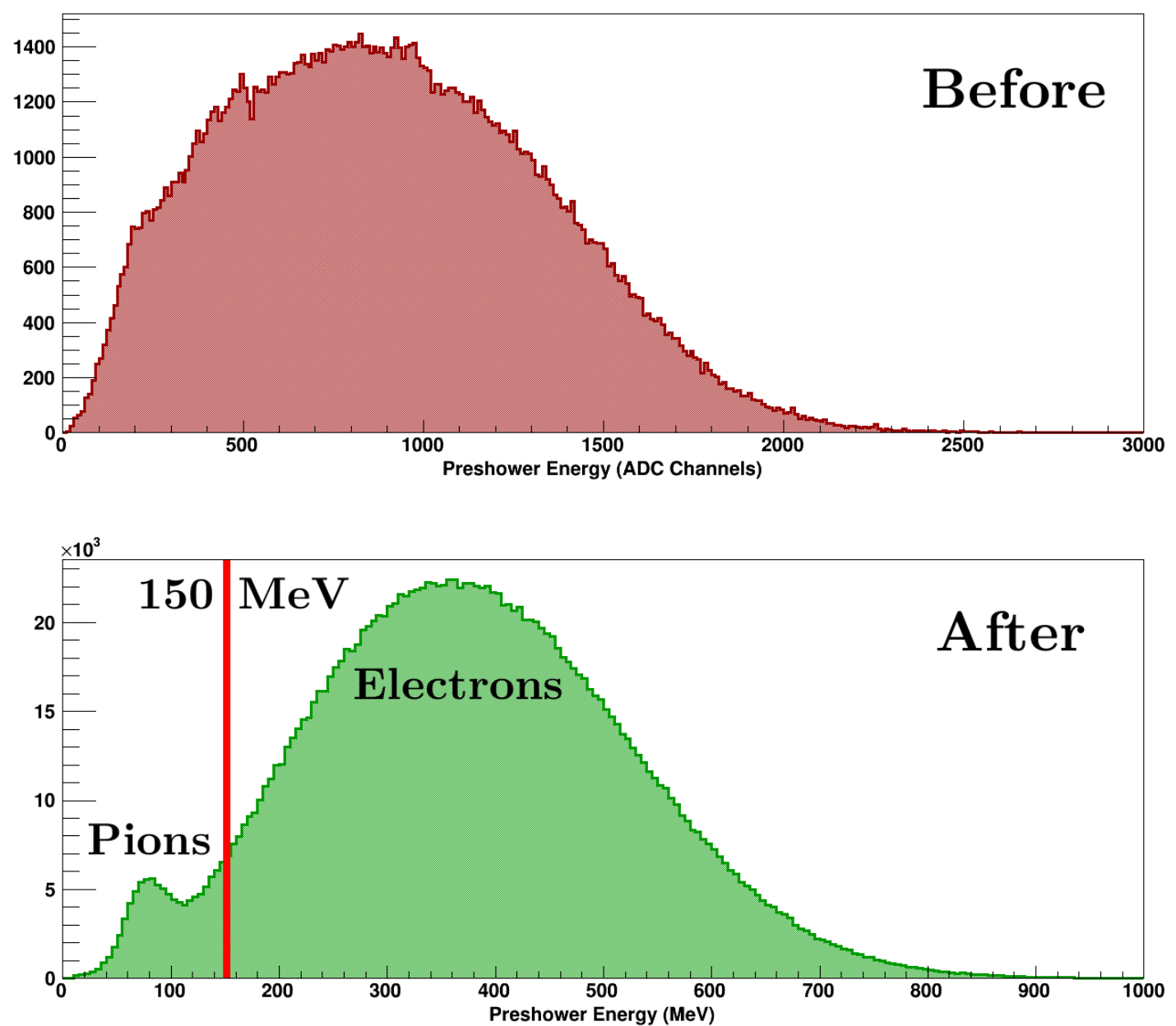

Fig. 5.13: The ${ }^{3}$ He preshower energy spectrum before and after calibrations. An energy cut of $E_{\mathrm{ps}}>150 \mathrm{MeV}$ is introduced to suppress preshower pions.

tics reconstructed energy. The two particle regions are clearly visible, and the region associated to elastically scattered electrons is centered at $E_{\text {cluster }} / E_{\text {optics }}=1$ due to the calibration process. As the lower preshower peak is understood to be pions, the observed preshower spectrum is then dominated by the sum of a pion distribution and an electron distribution. By simply introducing an energy threshold cut of $150 \mathrm{MeV}$, 


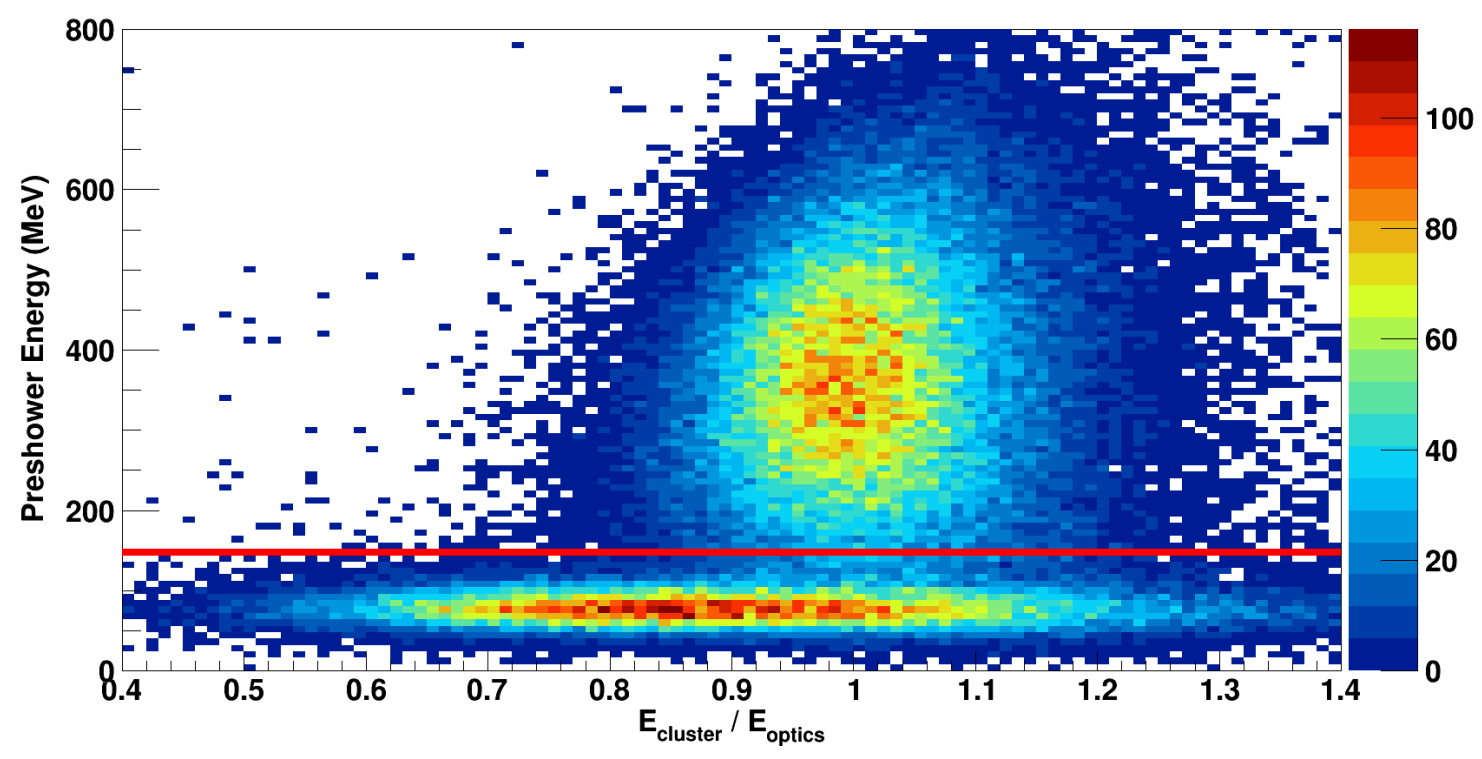

Fig. 5.14: The preshower cluster energy sum plotted against the ratio of energy obtained from clustering to the optics module. $\mathrm{H}_{2}$ data is used.

the vast majority of pions may be excluded from the analysis. However, there is a long pion tail that extends into the electron distribution which is not removed by a preshower energy cut. The preshower pion contamination has been estimated with a Monte Carlo calculation, and the correction is presented in Section 5.7.6.

The energy resolution of the BigBite calorimeter may be determined by comparing cluster energies after calibration to the energy obtained from the BigBite optics, or

$$
\frac{\delta E}{E}=\frac{E_{\text {cluster }}-E_{\text {optics }}}{E_{\text {optics }}}
$$

The $\delta E / E$ distribution may be fit to Gaussian, and the standard deviation then is a measure of the BigBite energy resolution. An energy resolution improvement from $12.5 \%$ to $7.5 \%$ has been obtained with the calibration procedure, and may be seen by Figure 5.15. The top row of Figure 5.16 shows $\delta E / E$ as a function of the reconstructed 

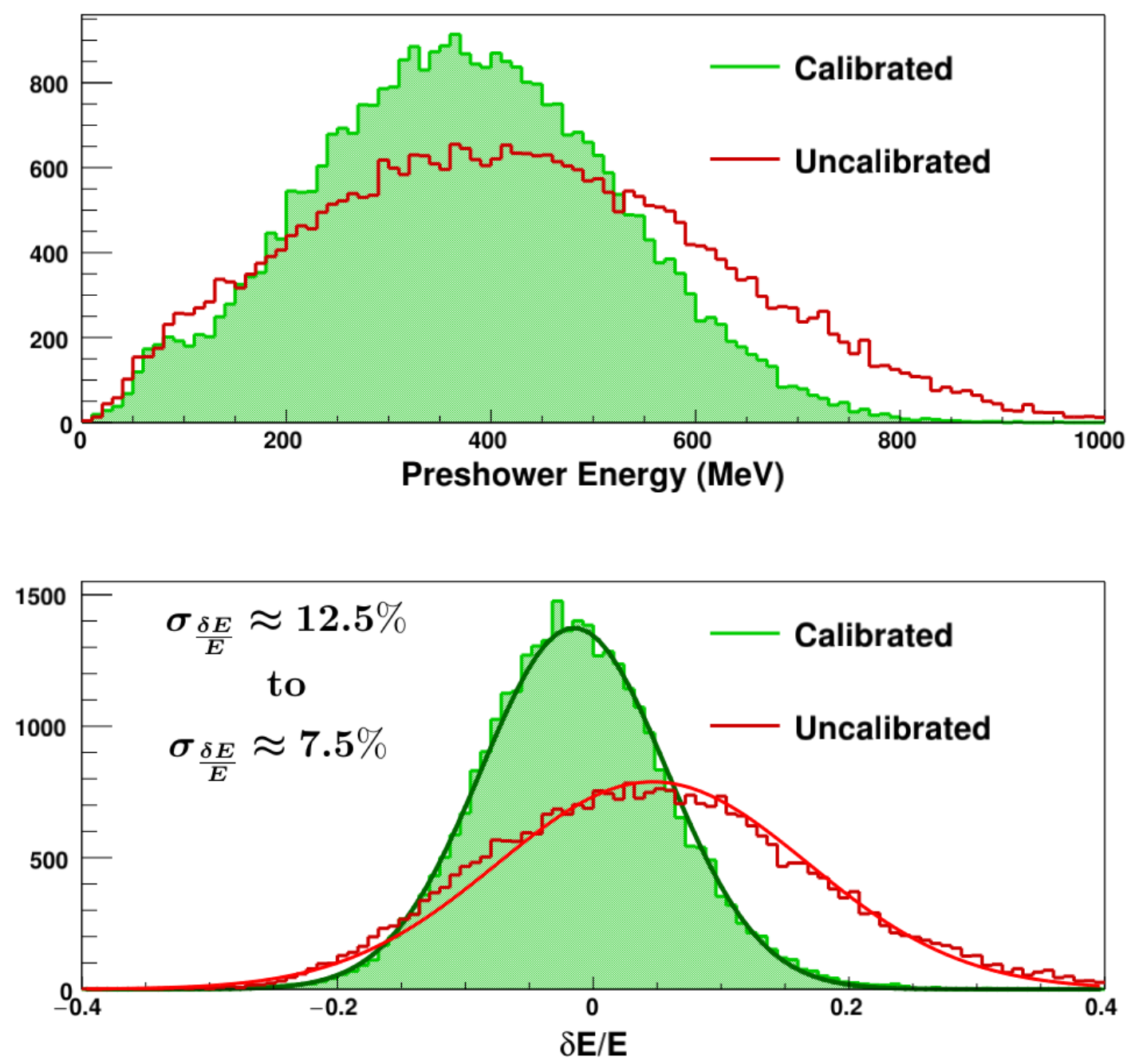

Fig. 5.15: An energy resolution improvement to $7.5 \%$ has been obtained $\left(\mathrm{H}_{2}\right.$ data).

cluster $x$ and $y$ positions, and the second and third rows display the binned Gaussian fit parameters, namely the mean and standard deviation for each bin of reconstructed cluster $x$ or $y$. The residual variations are seen to be small compared to the resolutions, therefore the quantity $\delta E / E$ is observed to be roughly centered at $\delta E / E=0$ over the $x$ and $y$ calorimeter coordinates. The elastic $\mathrm{H}_{2}$ data does not sufficiently populate the entire calorimeter acceptance, specifically the vertical and horizontal extremes are 

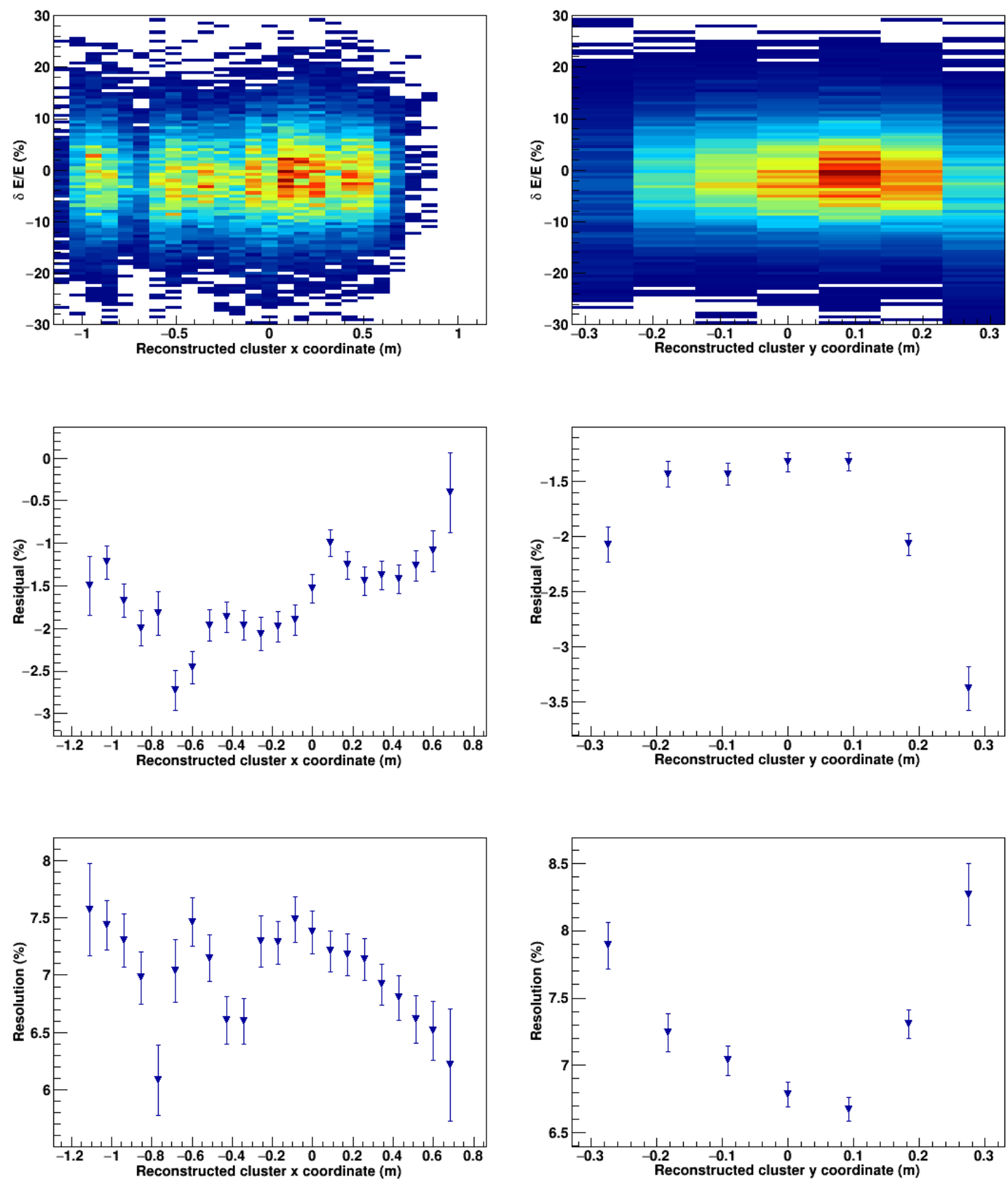

Fig. 5.16: $\frac{\delta E}{E}$ as a function of calorimeter $x$ and $y$ coordinates; no significant deviations are found as the residual variations are small compared to the resolutions. $\mathrm{H}_{2}$ data is used with a $0.92<W<0.96 \mathrm{GeV}$ cut. The efficiency, however, does vary over the $x$ dimension, and is discussed in Section 5.3.6. 
affected. As a result, fluctuations in the residuals and resolutions are observed along the perimeter of the calorimeter where statistics are low. While the $\delta E / E$ spectrum is aligned over the calorimeter coordinates, an issue still persists: the calorimeter efficiency varies over the vertical dimension significantly. For example, the $\delta E / E$ vs. cluster $x$ distribution has wildly uneven numbers of events in bins of cluster $x$ when the distribution should be relatively uniform; this issue will be addressed in the next section.

In analogy to the calculation of the BigBite calorimeter energy resolution, the $x$ and $y$ coordinate resolutions may be determined by analyzing the difference between the optics reconstructed track projected to the face of the calorimeter and the energy weighted coordinates of the largest calorimeter cluster, or $\delta \boldsymbol{x}=\boldsymbol{x}_{\text {track,proj }}-\boldsymbol{x}_{\text {cluster }}$. The bold notation contains the two relevant calorimeter coordinates in BigBite detector coordinates; therefore, $\delta \boldsymbol{x}=(\delta x, \delta y)$ denotes the vertical and horizontal coordinate differences between the projected track and the reconstructed cluster, respectively. Using the notation described in Section 5.3 .2 , the reconstructed track defined by the $z_{\text {det }}=0$ meter plane may be completely described by two coordinates and two directions: $\boldsymbol{x}_{\mathrm{o}}=(x, y)$ and $\boldsymbol{x}^{\prime}=\left(x^{\prime}, y^{\prime}\right)$. Therefore, the track may be projected to the face of the shower: $\boldsymbol{x}_{\text {track,proj }}=\boldsymbol{x}_{\mathrm{o}}+S \boldsymbol{x}^{\prime}$, where $S$ is a distance representing the length necessary for an intersection. The face of the shower may be described as $\left(\boldsymbol{x}_{\text {track,proj }}-\boldsymbol{x}_{\mathrm{sh}}\right) \cdot \hat{\boldsymbol{n}}_{\mathrm{sh}}=0$, where $\boldsymbol{x}_{\mathrm{sh}}$ is a point on the surface of the shower face and $\hat{\boldsymbol{n}}_{\mathrm{sh}}$ is a unit vector that points normal to the surface of the shower. The scaler $S$ may now be determined as the following:

$$
S=\frac{\left(\boldsymbol{x}_{\mathrm{sh}}-\boldsymbol{x}_{\mathrm{o}}\right) \cdot \hat{\boldsymbol{n}}_{\mathrm{sh}}}{\boldsymbol{x}^{\prime} \cdot \hat{\boldsymbol{n}}_{\mathrm{sh}}},
$$



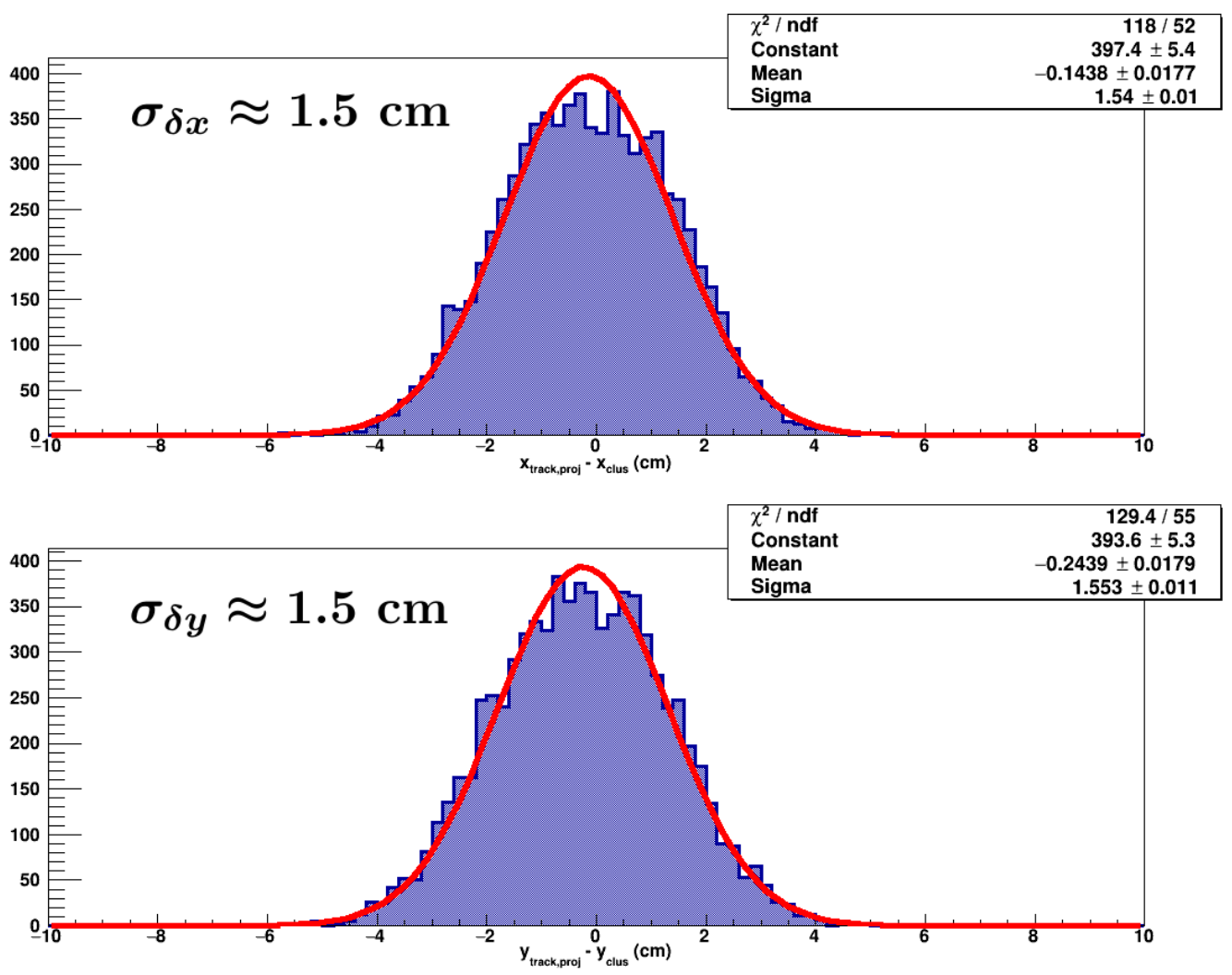

Fig. 5.17: The difference between the optics reconstructed track projected to the face of the shower and the energy weighted coordinates of the largest reconstructed shower cluster. A coordinate resolution of around $1.5 \mathrm{~cm}$ is observed in both the horizontal and vertical dimensions.

where $\boldsymbol{x}_{\mathrm{sh}}=(0,0,1)$ meters and $\hat{\boldsymbol{n}}_{\mathrm{sh}}=(0,0,1)$ in BigBite detector coordinates. This formalism assumes that the momentum and vertex of the track have been reliably reconstructed and the track must end up in the calorimeter volume, i.e. there are no situations where $S=0$ or undefined. The results of this procedure may be seen by Figure 5.17 where a vertical and horizontal coordinate resolution of approximately $1.5 \mathrm{~cm}$ is obtained. 


\subsubsection{Simulating the BigBite Calorimeter}

As the majority of the experimental apparatus was newly constructed, early data were taken prior to absolute understanding of all detector systems affecting the low $Q^{2}$ point the most. One such problem may be seen by the top left panel of Figure 5.16. The $\delta E / E$ distribution is centered at zero over the calorimeter $x$ and $y$ coordinates due to the $\chi^{2}$ calibration procedure described in the previous section; however, the efficiency varies significantly over the vertical dimension when it should be uniform which is an issue with the BigBite Kinematic 1 data that needs to be explored. The origin of the non-uniform efficiency observed in the data is likely due to two reasons: 1) the initial cosmic PMT gain alignment was poorly done, and 2) the BigBite trigger threshold was high enough to cut into the signal of some trigger logic groups. In order to investigate the non-uniform efficiency, the $\mathrm{H}_{2}$ data is analyzed to determine the value of the trigger threshold in units of ADC channels used during data taking. The G4SBS simulation, introduced in Section 4.10 , is then used where the output is analyzed using the realistic trigger logic and trigger threshold in an attempt to reproduce the observed data. The purpose of the simulation exercise is to demonstrate a deeper understanding of the detector that would not otherwise be possible with the 2006 data alone.

In order to determine the value of the trigger threshold in units of $\mathrm{ADC}$, the hydrogen data is analyzed enforcing a loose set of cuts: a vertex cut and events where BigBite triggered. If the calorimeter has been calibrated, then the gain coefficients are needed in order to convert the lead-glass energy deposition in units of $\mathrm{MeV}$ to an $\mathrm{ADC}$, or $A_{i}=E_{i} / C_{i}$ where $A_{i}$ is the $\mathrm{ADC}$ value for block $i$ for a particular event, $E_{i}$ is 


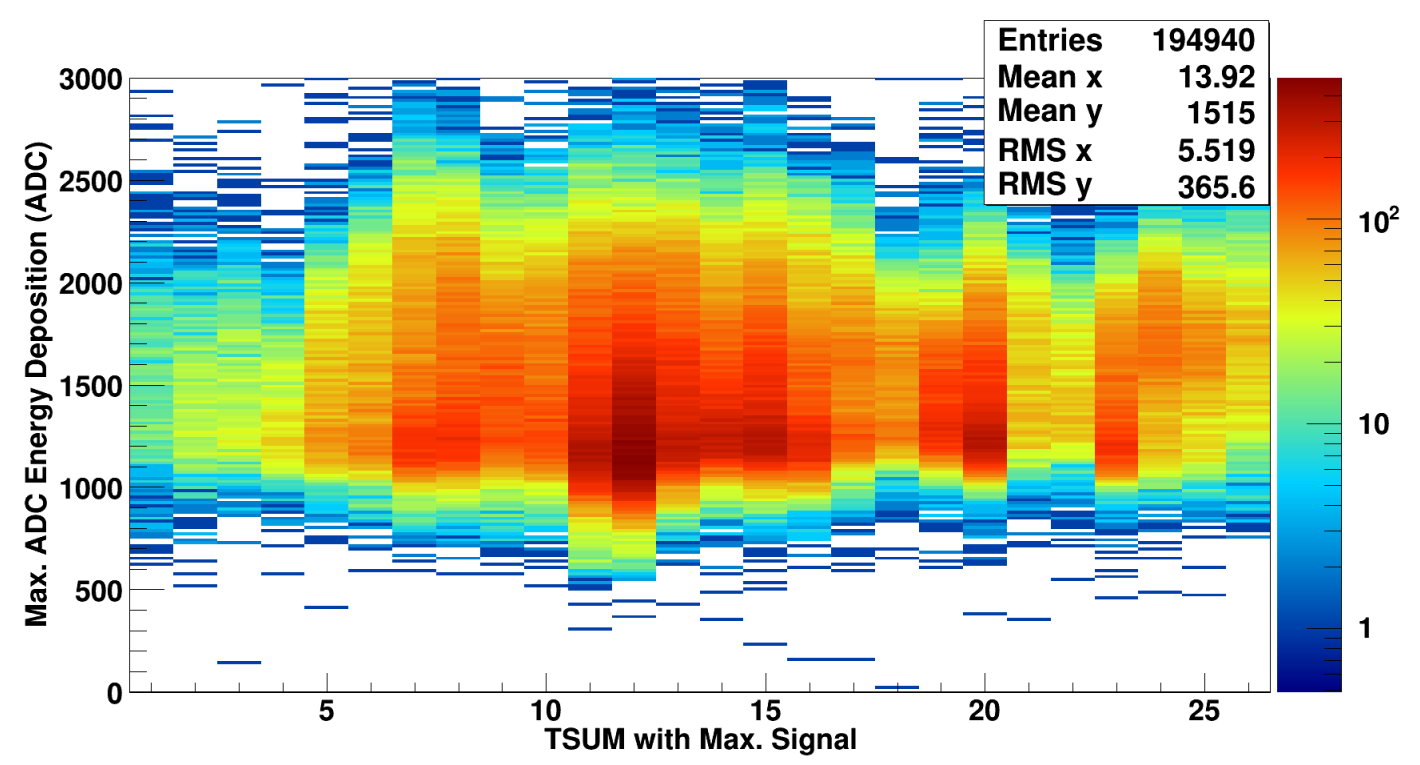

Fig. 5.18: The maximum ADC energy deposition sum for a given TSUM where the color is in a log scale. See text for definition of TSUM.

the energy deposition in units of $\mathrm{MeV}$ and $C_{i}$ are the corresponding gain coefficients as seen by Figure 5.11. The analysis requires the BigBite trigger logic as defined by Figure 4.30; for example trigger logic sum 1 (TSUM 1) consists of the bottom two rows of the preshower and shower for a total of $2 \times 2+2 \times 7=18$ calorimeter modules. There are a total of 26 TSUMs. For a given event, the ADC energy deposition sum of all TSUMs is computed. The analysis looks at the maximum ADC energy deposition sum and the corresponding TSUM; the maximum ADC sum is guaranteed to trigger BigBite as a T2 cut is enforced. The results may be seen by Figure 5.18 where the maximum ADC sum is plotted against TSUM, or the trigger logic index. The rising edge of a given TSUM is of particular interest, and in order to get a better understanding the projections, or one dimensional histograms, for a selection of TSUMs may be seen by Figure 5.19 . 

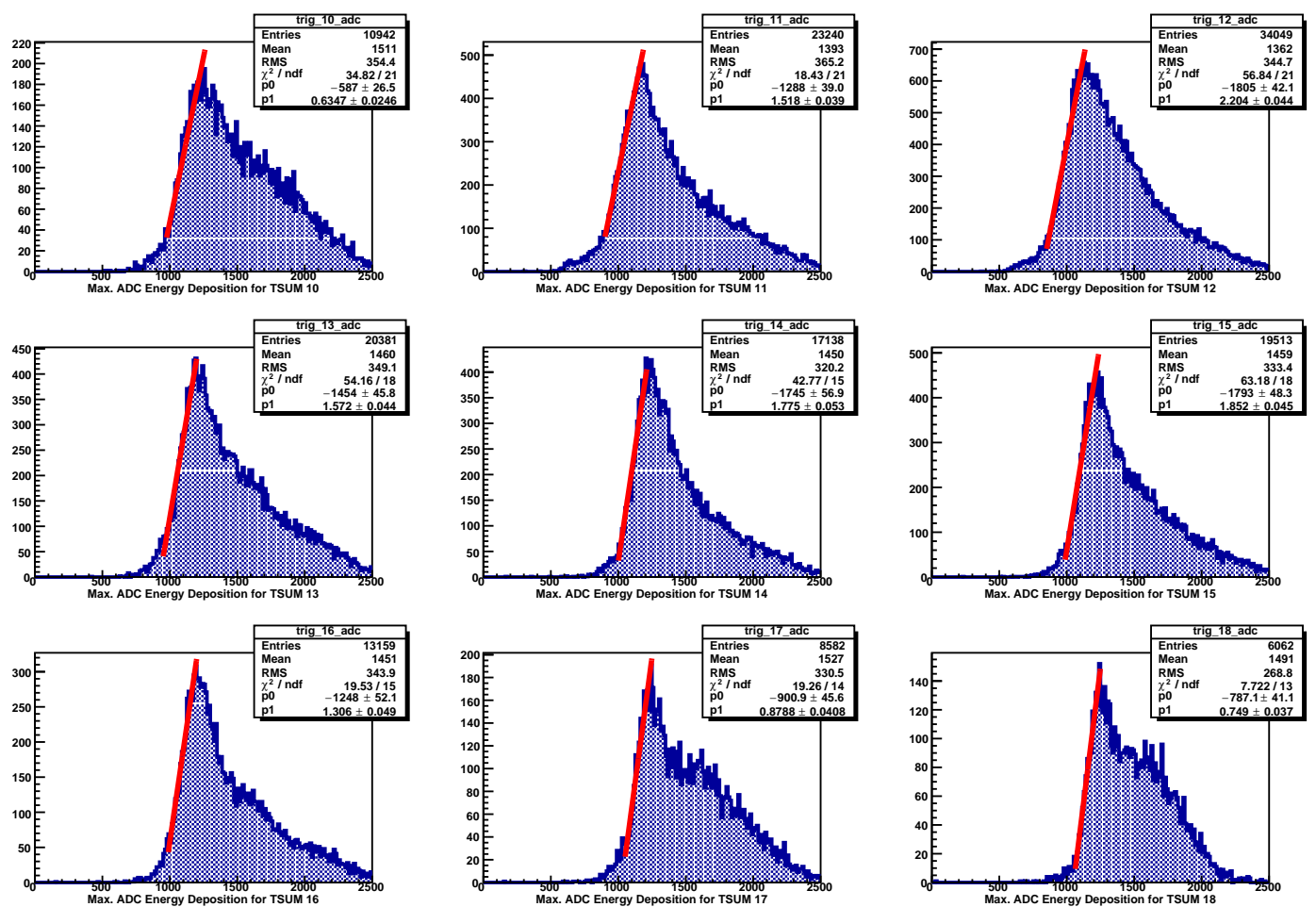

Fig. 5.19: The maximum ADC energy deposition for TSUM 10 through TSUM 18.
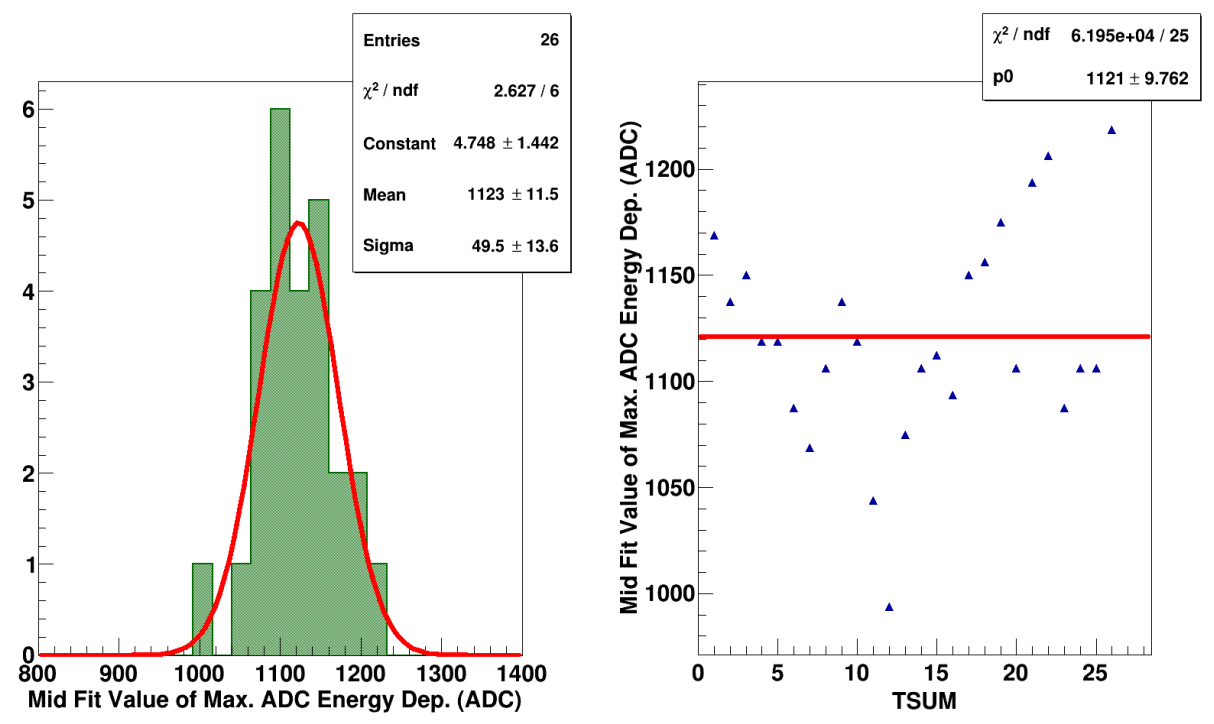

Fig. 5.20: An average ADC trigger threshold of $\sim 1120$ ADC is observed. 
In other words, the rise time for each TSUM yields information regarding the value of the ADC trigger threshold used during Kinematic 1 data taking. The procedure of extracting a threshold value for all TSUMs may be seen by Figure 5.19 where the rise time is fit to a straight line from $20 \%$ of the pulse height to the pulse height. The central value of the linear fit is extracted and may be seen by Figure 5.20 which displays the distribution of central values for all 26 TSUMs. In conclusion, an average trigger threshold of approximately 1120 ADC channels is observed which corresponds to an energy of $(1120 \mathrm{ADC}) \times(0.44 \mathrm{MeV} / \mathrm{ADC}) \approx 500 \mathrm{MeV}$ or approximately $55 \%$ of the expected elastic peak for the $Q^{2}=1.16 \mathrm{GeV}^{2}$ configuration.

In order to investigate the calorimeter inefficiencies further, a simulation utilizing a realistic elastic ep generator is required to replicate the elastic hydrogen data. The G4SBS Monte Carlo is used in conjunction with the ESEPP event generator. ESEPP, or Elastic Scattering of Electrons and Positrons on Protons, generates the outgoing kinematics of unpolarized ep scattering taking into account the lowest-order QED radiative corrections to the Rosenbluth cross section.* The ESEPP generated events are interfaced to the $G_{E}^{n}$ module of G4SBS which tracks the particles through realistic materials and detector systems designed to mimic the $G_{E}^{n}$ apparatus. The response of the calorimeter in simulation needs to be tuned to replicate the observed detector resolutions; therefore, a simplified version of Eqn 4.26 is used to Gaussian smear the simulated energy deposition in lead-glass. The momentum range for Kinematic 1 elastic hydrogen data is small, roughly $0.7-1.0 \mathrm{GeV}$, and fitting $\sigma_{E}^{2}$ to a second order polynomial in track energy yields poor results. One way to overcome this issue is to

\footnotetext{
* A user-friendly event generator that outputs the kinematics in the form of text or ROOT files, see [212].
} 


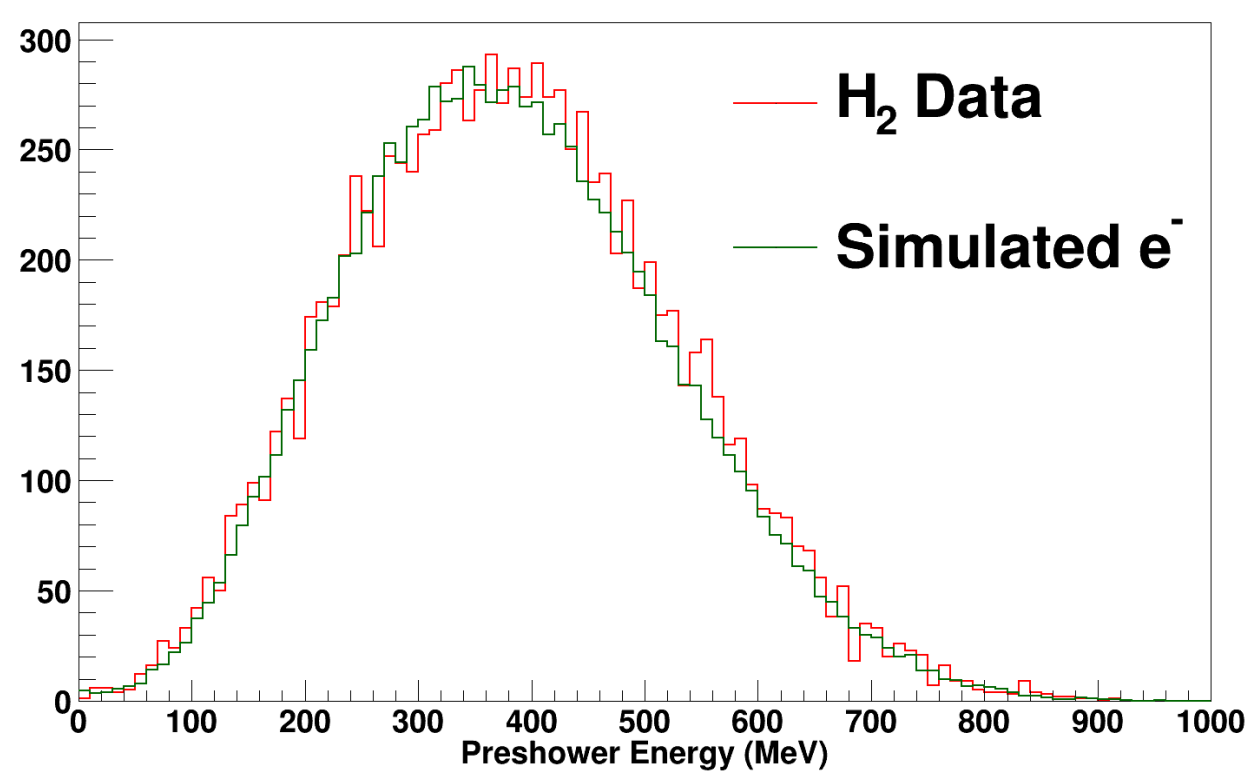

Fig. 5.21: A comparison between the $\mathrm{H}_{2}$ data and the ESEPP + G4SBS preshower spectra; the simulated energy deposition is smeared by $\sigma_{E}=0.05 \sqrt{E} \mathrm{GeV}$.

simplify the parametrization, keeping only the stochastic $a$ term resulting in $\sigma_{E} \approx a \sqrt{E}$ where $a=0.05 \mathrm{GeV}^{1 / 2}$ has been determined from the data. Using this simple smearing parametrization results in a simulated preshower spectrum that matches the $\mathrm{H}_{2}$ data well, and may be seen by Figure 5.21. Furthermore, the simulated energy resolution, which may be obtained from the simulated version of Eqn 5.17 , is roughly $7.7 \%$ in good agreement with the calibration; the results may be seen by Figure 5.22 .

In order to simulate the BigBite trigger, the simulated lead-glass energy depositions need to be converted to ADC channels using the gain coefficients determined within Section 5.3.5. Using the realistic BigBite trigger logic, the ADC energy deposition sum for all TSUMs is computed. If an ADC energy deposition sum is greater than an ADC threshold of 1120 ADC channels, then the simulated TSUM has fired 


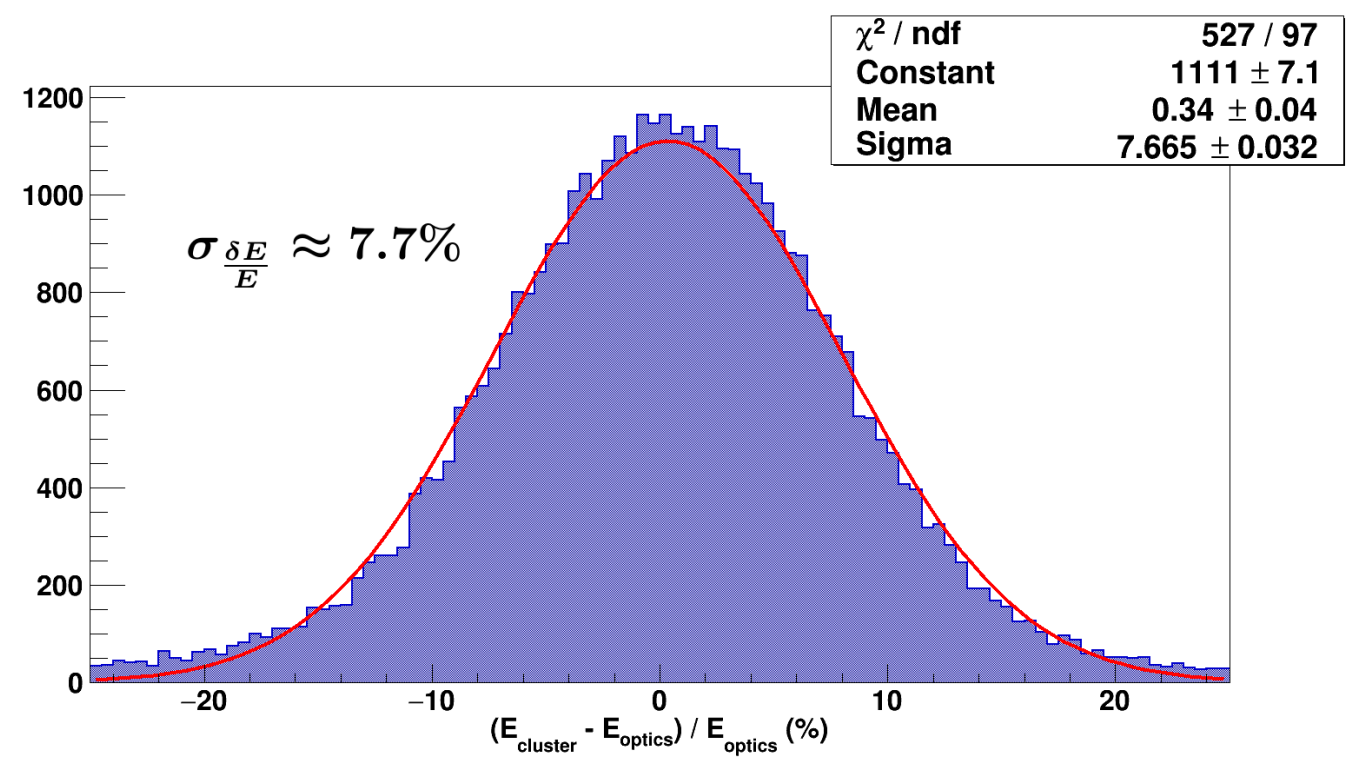

Fig. 5.22: A simulated BigBite resolution of around $7.7 \%$ is obtained.

and contributes a signal. Additionally, in order to isolate the simulated elastic peak, the following cuts are enforced: a vertex cut, $E_{\mathrm{ps}}>150 \mathrm{MeV}$ and $0.92<W<0.96$ GeV. A comparison to data may be made if the same cut selection is used, i.e. the elastic peak of the $\mathrm{H}_{2}$ data has been selected and requiring the BigBite trigger flag. The elastic ADC signal as a function of TSUM for data and simulation may be seen by Figure 5.23 . In order to make sense of the ADC energy deposition sum, a value of 2000 ADC channels corresponds to $(2000 \mathrm{ADC}) \times(0.44 \mathrm{MeV} / \mathrm{ADC}) \approx 900 \mathrm{MeV}$ which is in good agreement with the expected energy of an elastically scattered electron. A comparison of the maximum ADC sum for TSUMs 10 through 19 between data and simulation may be found in Figure 5.24. In conclusion, by using the calorimeter gain coefficients and realistic trigger logic, the simulated elastic ep events are in relatively good agreement with the observed elastic ADC signals. 

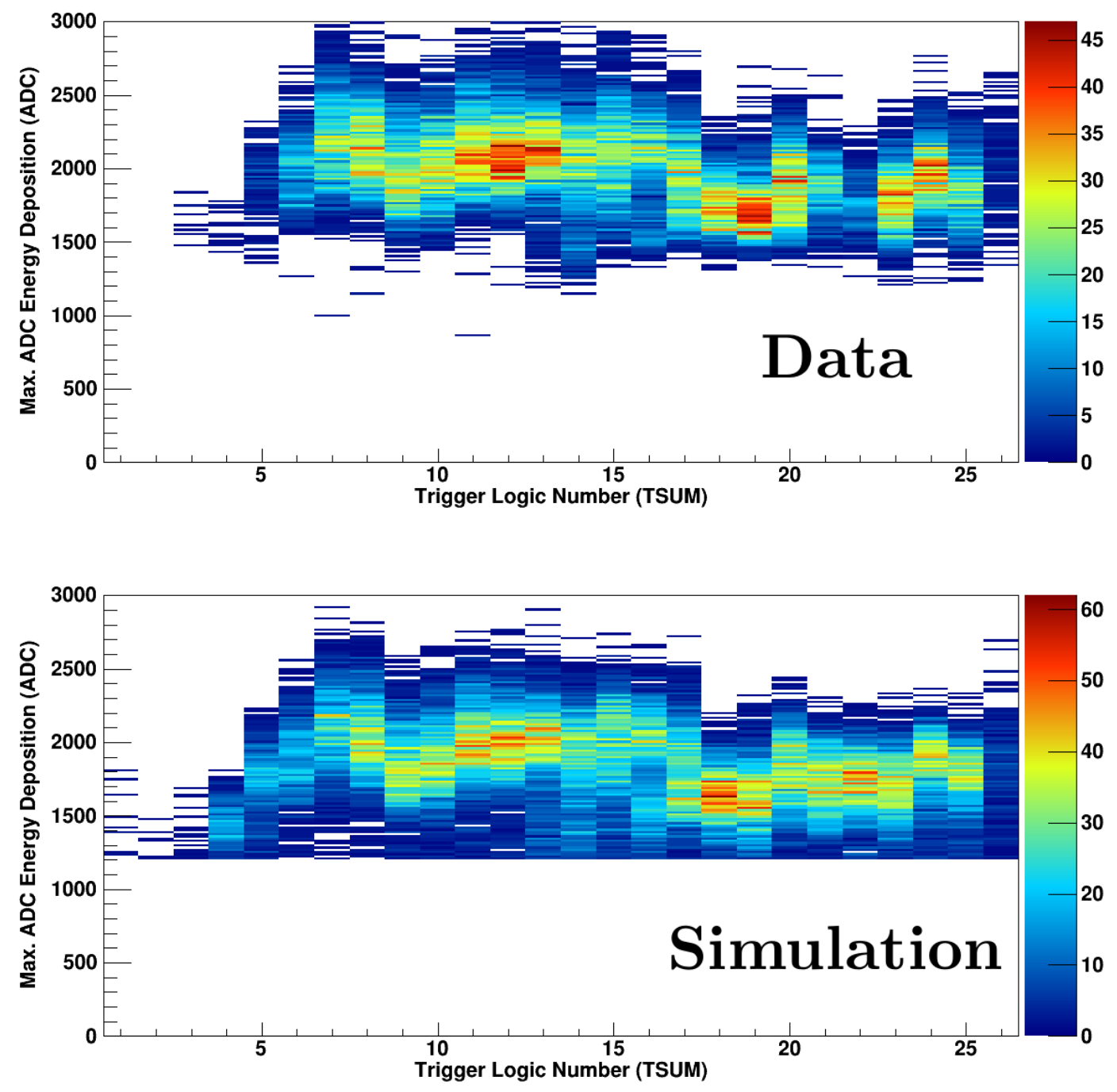

Fig. 5.23: Comparing the elastic ADC signal between the ESEPP + G4SBS simulation and the $\mathrm{H}_{2}$ data. The cut selection between data and simulation are the same with one exception: the simulated data imposes an ADC trigger threshold cut of 1120 ADC channels. 

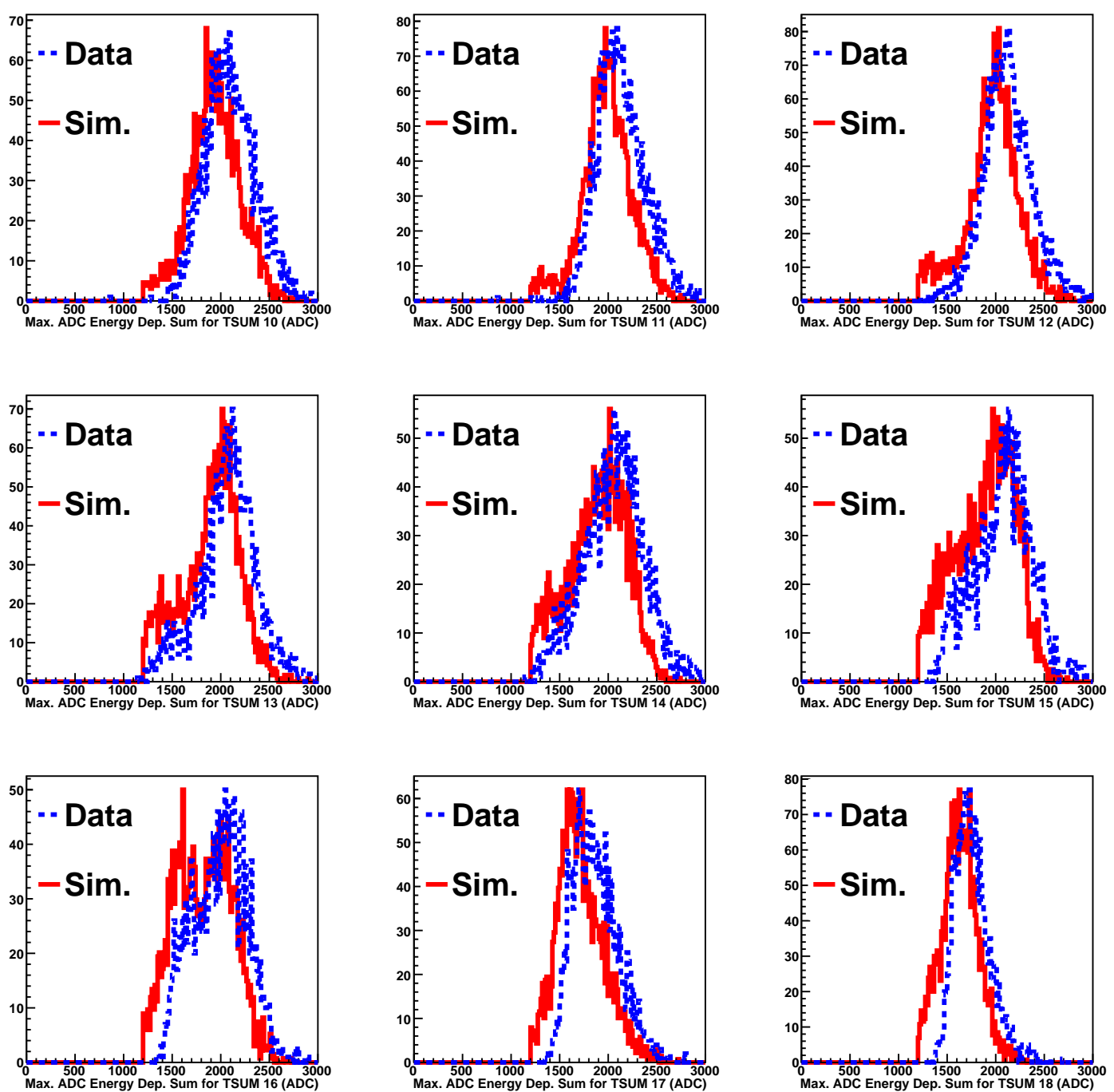

Fig. 5.24: A selection of projections of Figure 5.23 comparing the maximum ADC energy deposition sum for TSUMs 10 through 18 for data and simulation. The cut selection is identical except an ADC trigger threshold of 1120 ADC channels has been enforced on the simulated data. 


\subsubsection{Hodoscope}

Recall that the BigBite hodoscope is a scintillator wall composed of 13 scintillating paddles with a photomultiplier tube attached at either end. A particle of interest produces scintillation within a paddle corresponding to a hit location. The scintillation propagates at the speed of light in scintillator towards either PMT, and possibly initiating an electronic cascade; the resulting PMT signal is sent to a discriminator prior to the TDC electronics. In order to reconstruct the time of emitted scintillation, the TDC timing information needs to be corrected by these experimental realities. Additionally, there is an effect due to the pulse processing that is not so obvious. The discriminators emit a logic pulse once the input signal amplitude is above some threshold. As a result, there can be a time delay within this circuit that is amplitude dependent; larger amplitude pulses will reach threshold in a shorter period of time. This effect is known as "time walk" and will be denoted by the "tw" symbol when relevant.

The TDCs associated with the hodoscope scintillator record the time difference between the scintillator signal and the BigBite common-stop signal (L1A retimed), or $t_{\text {signal }}-t_{\text {trig. }}$. The time between the passage of the charged particle within a paddle and the arrival of the corresponding TDC signal associated to a PMT is

$$
t_{\text {signal }}=t_{\text {propagate }}+t_{\mathrm{tw}}
$$

where $t_{\text {propagate }}$ is the time for scintillation to propagate from the paddle hit position to the PMT and $t_{\mathrm{tw}}$ is the time walk correction. In complete analogy to the drift chambers, the trigger time may be described as

$$
t_{\text {trig }}=t_{\text {stop }}+t_{\text {travel }}+t_{\text {propagate, } \text { trig }}
$$


where $t_{\text {travel }}$ is the time it takes for the particle to travel from the scintillating paddle

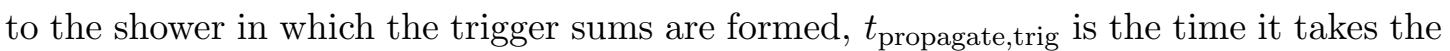
trigger signal to propagate to the TDCs, and $t_{\text {stop }}$ is the common stop time corresponding to L1A retimed. Therefore, the hodoscope TDC signal is then

$$
\begin{aligned}
t_{\mathrm{TDC}} & \equiv t_{\text {signal }}-t_{\mathrm{trig}} \\
& =t_{\text {propagate }}+t_{\mathrm{tw}}-t_{\mathrm{stop}}-t_{\text {travel }}+t_{\text {propagate,trig }} \\
& =t_{\text {propagate }}+t_{\mathrm{tw}}-t_{\mathrm{stop}}+t_{0} \\
& =\frac{d}{2 c_{s}} \pm \frac{y}{c_{s}}+t_{\mathrm{tw}}-t_{\mathrm{stop}}+t_{0}
\end{aligned}
$$

where the \pm sign depends on the right or left paddle PMT signals, respectively. In the third line, the times $t_{\text {travel }}$ and $t_{\text {propagate,trig have been absorbed into a single paddle }}$ dependent time offset $t_{0}$. The propagation time $t_{\text {propagate }}$ depends on the $y$ position relative to half of the paddle length and the speed of light within the scintillator material $c_{s}{ }^{*}$ See Figure 5.25 for a visual description, and henceforth the labels "L" and "R" will denote left and right timing signals, respectively.

A primary goal of the analysis is to eventually calculate the time of flight of the nucleon with the neutron arm times; however, time of flight is going to be calculated relative to the BigBite hodoscope. Therefore, it is advantageous to introduce $t_{\text {hit }}$ which represents the time of the scintillator interaction relative to the start of a scattering event. The average time of the hodoscope left and right times, denoted by $t_{\mathrm{avg}}$, presents a method to remove the horizontal $y$-dependence, and consequently the time of the hit

\footnotetext{
* The value of $c_{s}$ for the hodoscope is $0.146 \mathrm{~m} / \mathrm{ns}$.
} 


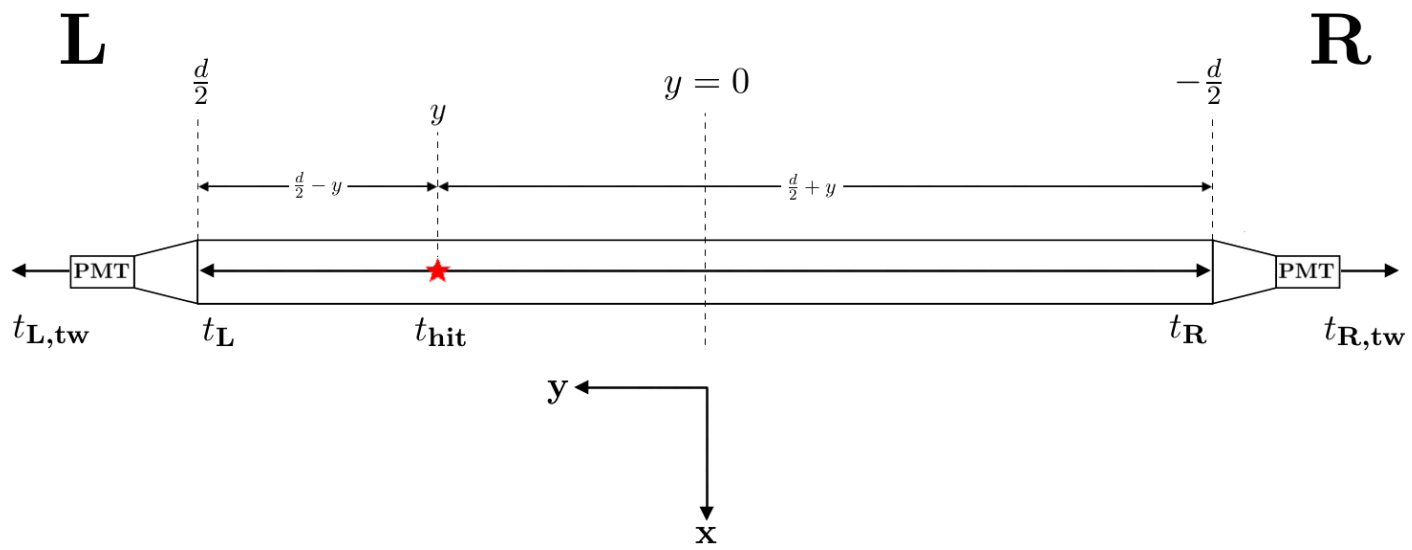

Fig. 5.25: Description of the hodoscope timing analysis in which light propagation depends on the $y$ hit position and $c_{s}$. Left and right is defined as viewing the hodoscope from the target.

may be calculated as follows:

$$
\begin{aligned}
t_{\mathrm{avg}} & =t_{\mathrm{hit}}+\frac{t_{\mathrm{L}, \mathrm{TDC}}+t_{\mathrm{R}, \mathrm{TDC}}}{2} \\
& =t_{\mathrm{hit}}+\frac{t_{\mathrm{L}, \mathrm{tw}}+t_{\mathrm{R}, \mathrm{tw}}}{2}-t_{\mathrm{stop}}+\frac{d}{2 c_{s}}+\frac{t_{\mathrm{L}, 0}+t_{\mathrm{R}, 0}}{2}, \\
& =t_{\mathrm{hit}}+\frac{t_{\mathrm{L}, \mathrm{tw}}+t_{\mathrm{R}, \mathrm{tw}}}{2}-t_{\mathrm{stop}}+t_{0, \mathrm{bar}}, \\
\Rightarrow t_{\mathrm{hit}} & =t_{\mathrm{avg}}-\frac{t_{\mathrm{L}, \mathrm{tw}}+t_{\mathrm{R}, \mathrm{tw}}}{2}+t_{\mathrm{stop}}-t_{0, \mathrm{bar}},
\end{aligned}
$$

where Eqn 5.21 has been used for the left and right side. All of the bar dependent time offsets have been absorbed into a single bar dependent term, $t_{0, \text { bar }}$. The horizontal position within the bar, or the $y$-coordinate, may be determined in a similar manner by calculating the difference between the right and left TDC times:

$$
y=\frac{c_{s}}{2}\left(\left(t_{\mathrm{R}, \mathrm{TDC}}-t_{\mathrm{L}, \mathrm{TDC}}\right)-\left(t_{\mathrm{R}, \mathrm{tw}}-t_{\mathrm{L}, \mathrm{tw}}\right)-\left(t_{\mathrm{R}, 0}-t_{\mathrm{L}, 0}\right)\right) .
$$


The hodoscope calibration process then consists of calculating the bar dependent offsets, correcting the ADC signals for attenuation, finding the ADC-dependent time walk offsets and measuring the speed of light within the scintillator. As a foreshadowing, the neutron detector also consists of scintillator bars with PMTs attached at either end, therefore the raw calculations, calibration procedures and initial analysis will be similar.

In principle, the bar dependent offsets may be different for each of the thirteen paddles; therefore, in an attempt to align the left and right PMTs in time, a simple iterative method is employed. Firstly, the times of the left and right side of the hodoscope are aligned separately. By selecting events in which adjacent paddles fired, the time difference between neighboring paddles may be aligned relative to some reference time. The middle paddle, or the paddle that is centered in the vertical direction, serves at the reference paddle. Once the left and right side of the detector are separately aligned in an iterative method, the left PMTs may be aligned to the right PMTs by analyzing the $\left(t_{\mathrm{L}, \mathrm{TDC}}+t_{\mathrm{R}, \mathrm{TDC}}\right) / 2$ distribution for all paddles, and the time differences may be zeroed if desired.

As the scintillation must propagate through the bar medium, it is subject to attenuation which ultimately affects the measured ADC signals and must be corrected. The ADC signals for the left and right side of the detector may be modeled as

$$
A_{\mathrm{L}}=G_{\mathrm{L}} A_{0} e^{\frac{-\frac{d}{2}+y}{\Gamma}} \quad \text { and } \quad A_{\mathrm{R}}=G_{\mathrm{R}} A_{0} e^{\frac{-\frac{d}{2}-y}{\Gamma}}
$$

where $\Gamma$ is the attenuation coefficient in units of length, $d$ is the length of paddle, $y$ is the reconstructed horizontal position of the hit, $G_{\mathrm{L}}$ and $G_{\mathrm{R}}$ are the gain coefficients for the left and right PMTs, respectively, and $A_{0}$ is related to the energy deposition within 
the scintillating medium. Taking the ratio of $A_{\mathrm{L}}$ to $A_{\mathrm{R}}$ and rearranging results in

$$
\ln \frac{A_{\mathrm{L}}}{A_{\mathrm{R}}}=\frac{2}{\Gamma} y+\ln \frac{G_{\mathrm{L}}}{G_{\mathrm{R}}}
$$

The attenuation coefficients and gain ratios, $G=\frac{G_{\mathrm{L}}}{G_{\mathrm{R}}}$, for all paddles may be extracted by examining a $\ln \left(A_{\mathrm{L}} / A_{\mathrm{R}}\right)$ versus $y$ distribution for each paddle. Events are selected such that both the left and right PMT within a single paddle fired, otherwise this procedure is not possible. The distributions are fit to a straight line where the slope is equal to $\frac{2}{\Gamma}$ and the y-intercept gives $\ln \frac{G_{\mathrm{L}}}{G_{\mathrm{R}}}$, assuming that $y=0$ represents the true midpoint of the paddle. While in principle the attenuation factor is different for every paddle, a single value of $0.7 \mathrm{~m}$ is used for all hodoscope paddles.

The time walk corrections occur due to the varying height of the ADC signals. A simple model in which $t_{\mathrm{tw}} \propto A^{p}$ for $-1.0<p<-0.5$ has been introduced in order to handle these effects:

$$
t_{\mathrm{L}, \mathrm{tw}}=C A_{\mathrm{L}}^{p}=\frac{C}{\sqrt{A_{0}}} e^{-\frac{y}{2 \Gamma}}
$$

where the gain and paddle length dependence have been absorbed into the constant $C$ and $p=-0.5$ has been chosen. The time walk for the right PMTs may be obtained by simply flipping the sign within the exponential argument. For the $G_{E}^{n}$ analysis, $C$ and $p$ were crudely determined in the calibration phase as the correction is quite small.

\subsubsection{Hodoscope Time Delay of Neighboring Paddles}

For the purposes of this discussion, the paddle that gets geometrically associated to a BigBite track is referred to as the primary or incident paddle. Assuming that one track has been reconstructed, a situation where only one hodoscope paddle fires is the most 
probable. However, a hodoscope cluster is possible, and in this case multiple paddles that are vertically connected to the primary paddle contribute TDC signals. For clarity, all cluster paddles that are not the primary paddle are referred to as neighbors. As the PMTs may be operating at different high voltages, a small HV dependent time delay in neighboring paddles is a possible source of time broadening. Recall that the hodoscope operates at $30 \mathrm{ps} /$ channel, therefore precise synchronization of all channels is advantageous to avoid signal smearing. The purpose of this brief analysis is to investigate if there is a time delay between the primary paddle and neighboring signals.

Hydrogen data is used in which the elastic peak is selected, i.e. the following cuts are implemented: vertex, the preshower energy must be greater than $150 \mathrm{MeV}$ and a restriction of the invariant mass to $0.9<W<0.98 \mathrm{GeV}$. In order to maximize events where neighboring signals contribute, a geometry cut is enforced:

$$
\left|x_{\text {primary }}-x_{\text {center }}\right|>0.35 h,
$$

where $x_{\text {primary }}$ is the vertical hodoscope coordinate of the hit within the primary paddle, $x_{\text {center }}$ is the vertical coordinate of the primary paddle center and $h$ is the vertical dimension, or height, of a paddle. In other words, the inner $70 \%$ of the primary paddle is excluded such that signals are more likely to be shared amongst neighbors. While it is possible to have more than one neighbor, the analysis only looks at events in which there is exactly one neighbor as it is the dominant correction. To be more explicit, an event where there is exactly one neighbor occurs roughly $\sim 30 \%$ of the time, but clusters of two or more neighbors only occur $\sim 1 \%$ of the time within the chosen cut selection and are ignored. A delay in a neighboring signal may be seen by calculating the time 

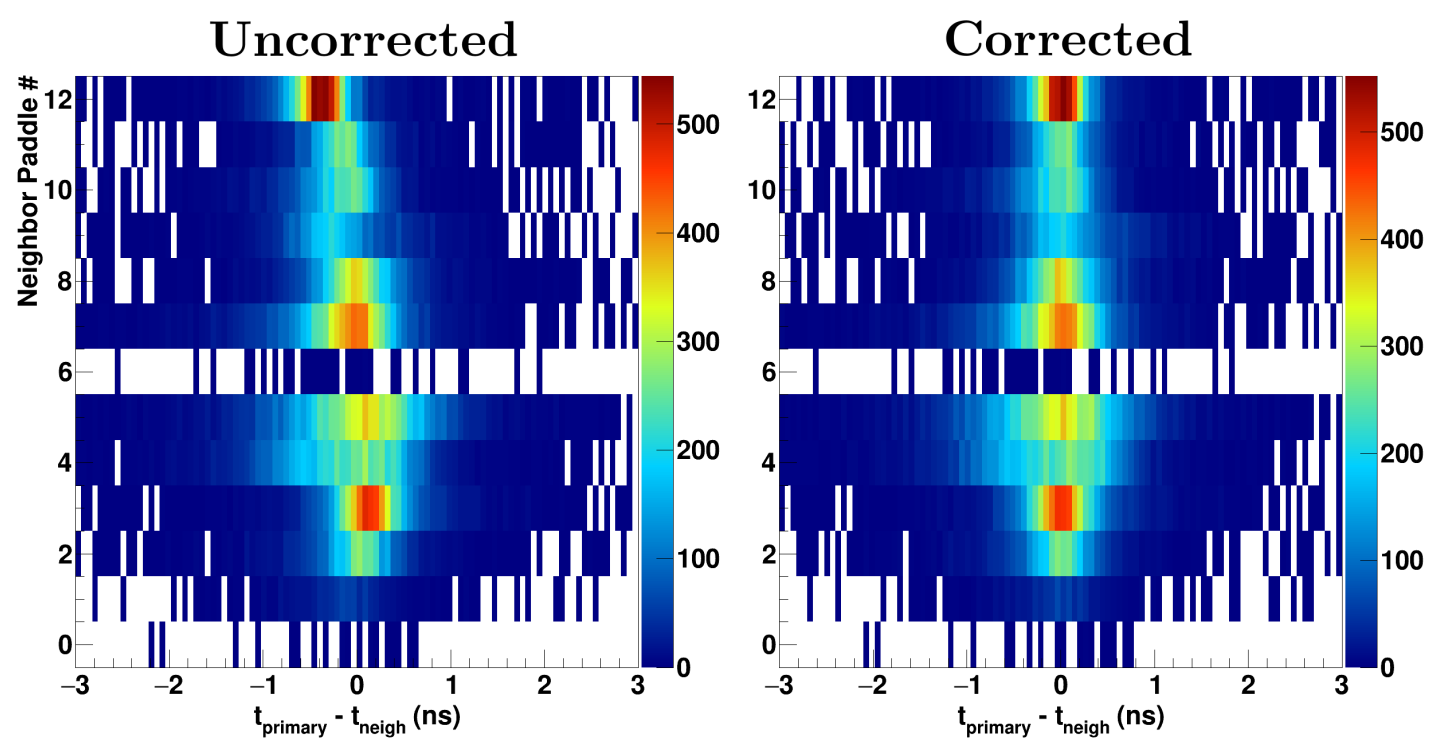

Fig. 5.26: Hodoscope neighbor paddle as a function of the time difference between the primary and neighbor signals. Left (right) panel is the data prior (post) correction. Paddle 6 data is sparse as this module is typically associated with a primary paddle.

difference between the primary hit and the neighboring hit, or $t_{\text {primary }}-t_{\text {neigh }}$. There are thirteen distributions corresponding to the paddle numbers of the neighbors, and may be seen by Figure 5.26. Paddle \#6, or the hodoscope center paddle, has a small amount of data as it is largely associated with the primary hit (meaning it is not a neighboring hit) or events in which no neighbors are found. As paddle \#6 occurs roughly in the geometric center of the hodoscope, elastic tracks are likely to enter the volume with a small vertical component and as a result the signal is less likely to be shared amongst neighbors. Lower momentum events have larger magnetic deflections and eventually end up in the upper half of the hodoscope. In these cases, the neighbors typically are located 


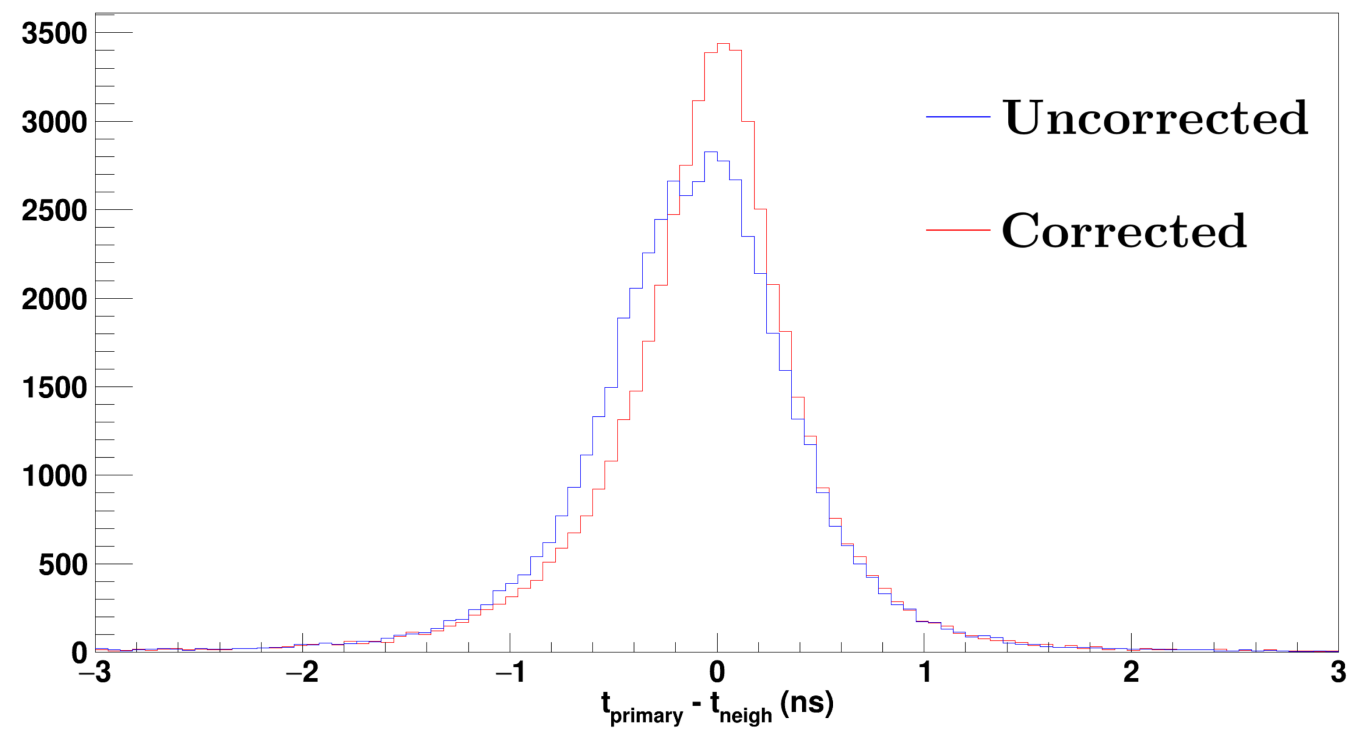

Fig. 5.27: The $t_{\text {primary }}-t_{\text {neigh }}$ distribution before and after corrections have been applied. A small improvement to delayed neighboring times is observed resulting in a tightened and more symmetric distribution that is zeroed.

vertically "above" the primary paddle. For the high momentum events in which the electron is minimally deflected by BigBite, the opposite is more likely. The result after corrections are applied may be seen by Figure 5.27. The uncorrected (blue) distribution is shifted towards the negative region which is indicative of $t_{\text {neigh }}$ being slightly delayed. The corrected distribution (red) is the result of applying the corrections to neighbors in situations where the primary paddle has at least one neighbor. 


\subsection{Neutron Detector: Raw Analysis and Calibration Procedures}

The goal of the neutron detector (ND) is to measure the time of flight of the recoiling nucleon relative to the BigBite hodoscope timing signal. Recall that the ND consists of two veto layers (V1 and V2) for charge identification and seven neutron layers (N1-N7) to capture the recoiling nucleon and measure its properties. The geometry is complicated, and has been presented in Section 4.7. There are converter plates in front of the neutron layers which are designed to increase the probability of the nucleon interacting strongly; the result is a hadronic shower and clustering must be performed to calculate meaningful quantities. While the geometry and distribution of sensitive blocks is quite different than the BigBite calorimeter, the idea of signal clustering is similar. Note that clustering is only relevant for the neutron layers. The details of the clustering algorithm will be discussed, but for now a nucleon cluster, or cluster for short, is defined to be the result of combining hits in the neutron layers. The cluster properties, e.g. the hit coordinate or time of flight, may then be reconstructed in a similar manner as handled by BigBite. Once the cluster has been found, it needs to be assigned a charge, either charged (proton) or uncharged (neutron). Recall that the polarized ${ }^{3} \mathrm{He}$ data will be used for an asymmetry calculation; therefore, differentiating between protons and neutrons is essential. In short, charge identification is the association of a signal (or lack of a signal) within the veto layers to the reconstructed nucleon cluster in space and time. With the ability to calculate the properties of the nucleon cluster and to assign a charge, the kinematics may be fully reconstructed and available for use in a raw asymmetry calculation. 
The readout of the neutron arm is controlled by the level-one accept time (L1A), and is not to be confused with BigBite's retimed L1A signal. As the ND consists of scintillating bars with two PMTs connected, the raw analysis of the TDC and ADC signals follows an identical procedure as the BigBite hodoscope (Section 5.3.7); therefore, the discussion will be in the form of a summary. There is a time offset that must be calculated from the data for each bar $\left(t_{0}\right)$, and each PMT needs to be corrected for time-walk effects $\left(t_{\mathrm{tw}}\right)$. The speed of light $c_{s}$ needs to be calculated using the marker counters (M1-M4) and a value of $c_{s}=0.1469 \mathrm{~m} / \mathrm{hs}$ has been used. The pedestal corrected ADC signals need to be corrected for attenuation by the model described by Eqn 5.25. As the neutron layers consist of two PMTs and one scintillator bar, the difference between the left and right TDC timing signals yields the horizontal, or the $y$ coordinate, of the hit. The $x$ and $z$ coordinates come from block positions relative to the Hall, and have been determined by the Hall A surveys. The hit time in a scintillator bar may be taken directly from the hodoscope discussion (Eqn 5.23):

$$
t_{\mathrm{hit}}=t_{\mathrm{avg}}-\frac{t_{\mathrm{L}, \mathrm{tw}}+t_{\mathrm{R}, \mathrm{tw}}}{2}+t_{\mathrm{stop}}-t_{0, \mathrm{bar}}
$$

Recall that $t_{\text {avg }}$ removes the $y$-dependence embedded within the TDC signal, $t_{\mathrm{tw}}$ is the time-walk correction, $t_{\text {stop }}$ is the L1A read-out time originating from the Trigger Supervisor and $t_{0, \text { bar }}$ are the bar dependent time offsets. The horizontal $y$ position within a neutron bar may be calculated by taking the difference of the corrected left and right TDC times, e.g. see Eqn 5.24. The veto layer modules, on the other hand, consist of two veto bars (short and long) and two PMTs; therefore, the horizontal position within the bar may not be determined. Recall that the primary purpose of 
the veto layers is to assign a charge to the reconstructed nucleon cluster, and precise coordinate determination is unnecessary.

\subsubsection{Raw Time of Flight Determination}

Measuring the time of flight ( $\mathrm{ToF}$ ) of the nucleon requires precise timing measurements in both arms of the experiment. The nucleon ToF $t_{\text {ToF }}$ is simply the difference between the measured cluster time $t_{\text {cluster }}$ and the start time of the event $t_{\text {event }}$. The BigBite hodoscope precisely measures the time of flight of the electron relative to the start of the event, or symbolically $t_{\text {hodo }}=t_{\text {electron }}+t_{\text {event }}$. The trigger times of the electron and neutron arm are related up to a constant. While the symbol $t_{\text {stop }}$ has been used generically to represent the readout signal, the trigger stop times may now be written as $t_{\mathrm{RT}}$ and $t_{\mathrm{L} 1 \mathrm{~A}}$ for the electron and neutron arm, respectively. Note that a "TDC" subscript represents specific time signals that are measured by a TDC as opposed to times relevant to physics studies. The time relationships are summarized as

$$
\begin{array}{r}
t_{\text {ToF }}=t_{\text {cluster }}-t_{\text {event }}, \\
t_{\text {hodo }}=t_{\text {electron }}+t_{\text {event }}, \\
t_{\mathrm{TDC}, \mathrm{L} 1 \mathrm{~A}}=t_{\mathrm{L} 1 \mathrm{~A}}-t_{\mathrm{RT}}+a .
\end{array}
$$

Recall that the TDC times of both the hodoscope and the ND modules may be expressed in a similar manner; therefore, the TDC time that is relevant is the corrected average of the left and right signals where time-walk and bar dependent offsets have been implemented. In other words, the measured TDC hodoscope or ND time may be 


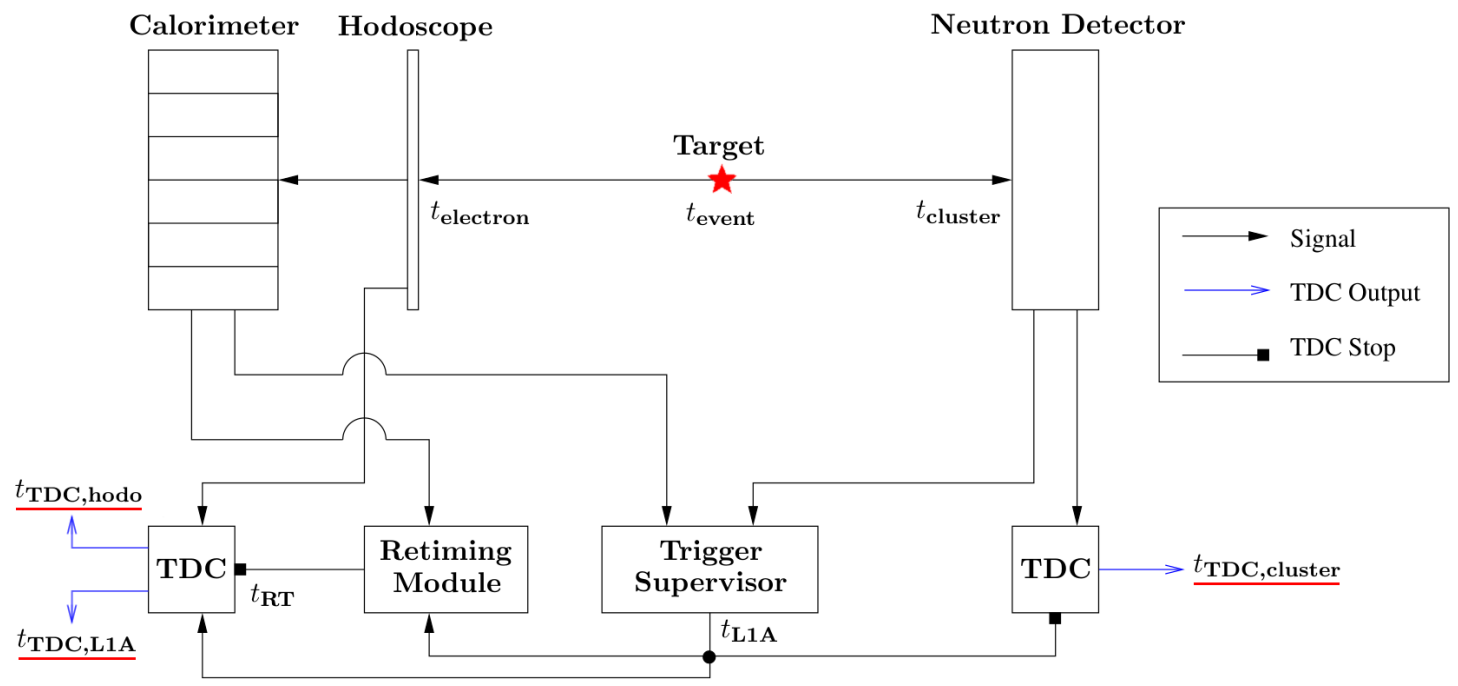

Fig. 5.28: Conceptual diagram of nucleon ToF determination. There are three required TDC times corresponding to the hodoscope, L1A and the reconstructed nucleon cluster. The original diagram by Ref. [178] has been altered.

generically expressed as

$$
t_{\mathrm{TDC}, \mathrm{corr}}=t_{\mathrm{TDC}, \text { avg }}-\frac{t_{\mathrm{L}, \mathrm{tw}}+t_{\mathrm{R}, \mathrm{tw}}}{2}-t_{0, \mathrm{bar}} .
$$

Using this notation, then the time of a hit (a nucleon cluster or a hodoscope hit) relative to the trigger is then $t_{\text {hit }}=t_{\text {TDC,corr }}+t_{\text {stop }}$ which is nothing more than a simple rewrite of Eqn 5.29. To make the notation clear, the hit time applies to both the hodoscope and the NA cluster with the appropriate stop time:

$$
\begin{gathered}
t_{\text {hodo }}=t_{\mathrm{TDC}, \text { hodo corr }}+t_{\mathrm{RT}}, \\
t_{\text {cluster }}=t_{\mathrm{TDC}, \text { cluster corr }}+t_{\mathrm{L} 1 \mathrm{~A}},
\end{gathered}
$$


where the "hit" subscript has been removed due to clutter. The ToF of the nucleon may then be expressed in terms of measured TDC times by taking the following sum:

$$
\begin{gathered}
t_{\mathrm{ToF}}+t_{\mathrm{hodo}}=t_{\text {cluster }}+t_{\text {electron }}, \\
t_{\mathrm{ToF}}=t_{\mathrm{TDC}, \text { cluster corr }}-t_{\mathrm{TDC}, \text { hodo corr }}+t_{\text {electron }}+\left(t_{\mathrm{L} 1 \mathrm{~A}}-t_{\mathrm{RT}}\right), \\
\Rightarrow t_{\mathrm{ToF}}=t_{\mathrm{TDC}, \text { cluster corr }}-t_{\mathrm{TDC}, \text { hodo corr }}+t_{\text {electron }}+t_{\mathrm{TDC}, \mathrm{L} 1 \mathrm{~A}}+a .
\end{gathered}
$$

As the cluster is made up of many bars contributing signals, the TDC time here refers to the bar with the minimum time associated with the cluster. Up to a constant $a$, the ToF depends on the time of flight of the electron from the target to the hodoscope and three TDC times: the corrected nucleon cluster time, the corrected hodoscope time, and the relative time of the two trigger signals. The constant is useful for initial calibrations of the ND; however, at a more mature stage in the analysis it may simply be absorbed into the bar dependent time offsets, $t_{0}$. In a similar manner as the BigBite optics or calorimeters, the bars of the ND then need to be calibrated to an expected time of flight using ideally the elastic peak of $\mathrm{H}_{2}$ data.

\subsubsection{RF Correction to ToF}

As seen by Eqn 5.34, the time of flight of the nucleon relative to BigBite may be determined up to a constant $a$. The nucleon ToF requires knowledge of the time of flight of the electron which is measured by the hodoscope; this may be improved by introducing the so-called RF correction. Recall that the accelerator generates electron bunches with a frequency of $499 \mathrm{MHz}$, therefore electron pulses arrive at Hall A approximately every 2 ns. The RF correction, denoted by $t_{\mathrm{RF}}$, then has the purpose of synchronizing 
the detected electron by the hodoscope to the correct electron pulse generated by the accelerator. In order to determine the RF correction, an F1 TDC is used to time a $499 \mathrm{MHz}$ signal that is generated by the accelerator and corresponds to a particular electron bunch. The F1 TDC has a resolution of 118.3 ps and is capable of determining the time of the electron at a fixed location relative to the target modulo $2 \mathrm{~ns}$. In the language of the previous section, the formula used in order to calculate the correction to the nucleon $\mathrm{ToF}$ is 178

$$
t_{\mathrm{RF}}=\text { fmod }\left[t_{\text {electron,data }}-t_{\mathrm{TDC}, \text { hodo corr }}+t_{\mathrm{TDC}, \mathrm{RF}}+t_{\mathrm{L} 1 \mathrm{~A}}-\frac{v_{z}}{c}+a+b, 2 \mathrm{~ns}\right],
$$

where $t_{\text {electron,data }}$ has been determined by the data and depends on the four tracking variables $\left(x, y, x^{\prime}\right.$, and $\left.y^{\prime}\right)$. The constant $b$ and $t_{\mathrm{TDC}, \mathrm{RF}}$ depend on the F1 TDC time relative to a fixed reference time and the electronic properties of the TDC, e.g. the time resolution and a wrap of $W=65526$ channels. Figure 5.29 displays the time correction used to correct the nucleon ToF in which a resolution of $270 \mathrm{ps}$ is observed. Corrections on the order of hundreds of picoseconds are possible which effectively allows for a more precise determination of the nucleon ToF, and henceforth will be applied in all calculations. For example in the next section, the time of flight resolution of the neutron detector is shown to be roughly $300 \mathrm{ps}$ (380 ps) with (without) the RF correction applied. Note that in an attempt to tighten up the $t_{\mathrm{RF}}$ distribution, a correlation between the hodoscope horizontal hit coordinate and the RF correction has been investigated; no dependencies are found within the Kinematic 1 data. 


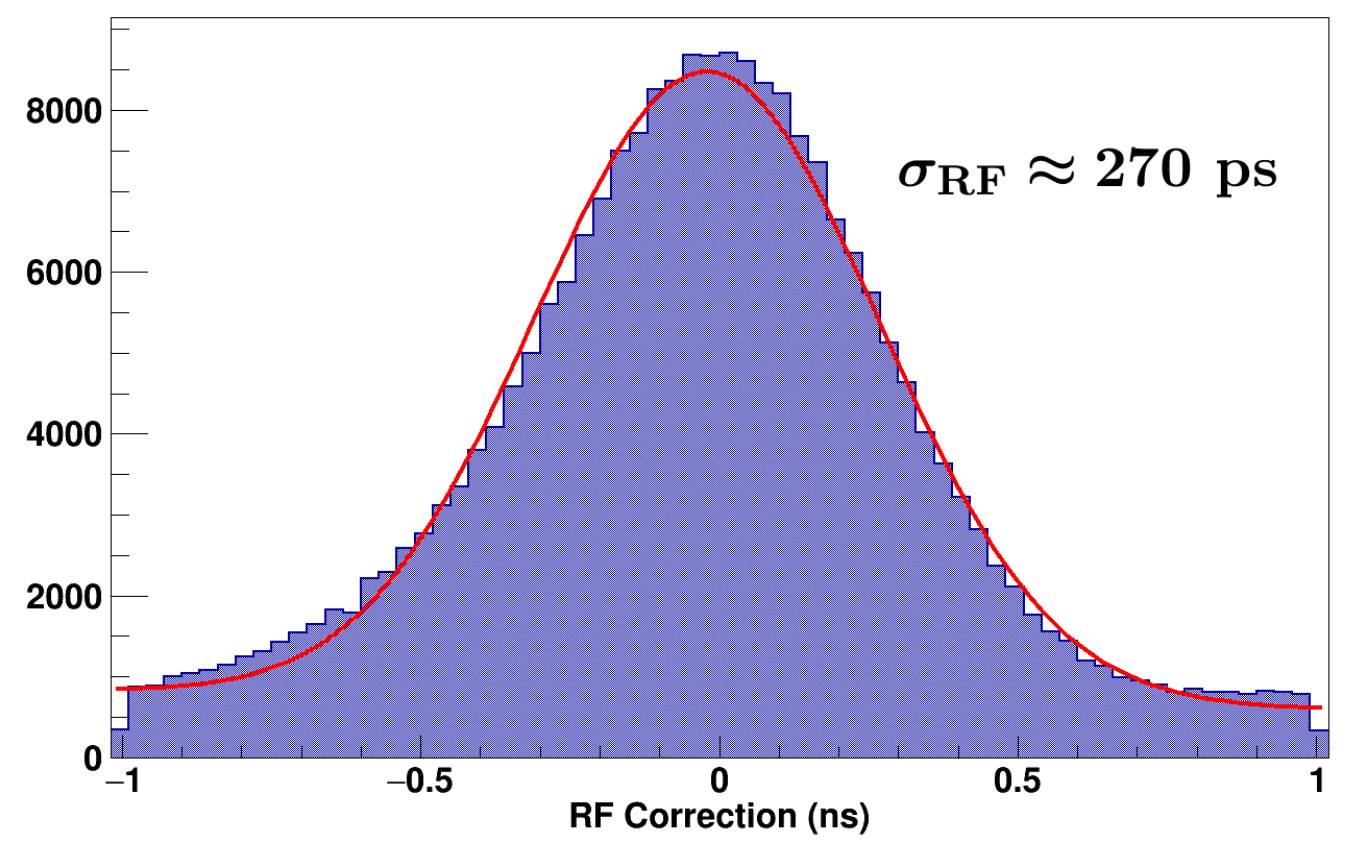

Fig. 5.29: The RF correction applied to the nucleon time of flight. The correction has the purpose of synchronizing the reconstructed track to the appropriate electron bunch originating from CEBAF. The fit is simply a Gaussian on top of a constant. 


\subsubsection{Calibration to an Expected Time of Flight}

The ND has been through many iterations of initial calibration procedures and are described in Appendix A of Ref. [177]. For the purposes of this thesis, the detector is assumed to have undergone HV tuning and left/right PMT alignment; therefore, the upcoming discussion represents the final calibration procedure that has been applied.

As the experiment relies on the ToF measurement of the recoiling nucleon, a careful calibration is required in order to achieve the optimized resolution constrained by the resolution of the TDCs. In analogy to the BigBite calibration procedures, it is desirable to compare the time of flight obtained from the neutron detector to an expected time of flight of a known sample, e.g. recoiling protons within the elastic peak of the $\mathrm{H}_{2}$ data. The ND consists of $96(=2 \times 48)$ veto bars* and $244(=29+25+30+25+3 \times 45)$ neutron detector bars for a total of 340 bars. In order to align the time measurements of the entire detector, the time obtained from each bar needs to be individually compared to an expected ToF; the result is 340 time offsets corresponding to each bar of the ND. The data used is elastic $\mathrm{H}_{2}$ data with the following cuts: a vertex cut of $-0.17<v_{z}<0.17 \mathrm{~m}$, a coincidence cut, the preshower cluster energy must be greater than $150 \mathrm{MeV}$, and an invariant mass cut of $0.9<W<0.98 \mathrm{GeV}$. The expected time of flight of a recoiling nucleon assuming elastic kinematics is

$$
t_{\mathrm{ToF}, \mathrm{ex}}=\frac{\ell}{c} \sqrt{1+\left(\frac{M}{|\vec{q}|}\right)^{2}},
$$

where $\ell$ is the distance from the vertex position to the hit coordinate within a particular bar and $|\vec{q}|$ is the magnitude of the three momentum transfer. In order to align the

\footnotetext{
* For the calibration discussion, a bar is defined to be one full row within a particular plane of the ND.
} 
times obtained from each bar such that the ND is time-synchronized, the RF corrected ToF is compared to the expected ToF:

$$
\delta t=t_{\mathrm{ToF}}-t_{\mathrm{ToF}, \mathrm{ex}}
$$

If the number of events in any given bar is sufficient, then the $\delta t$ distribution may be zeroed by a Gaussian fit; the mean represents the time offset required for calibration to an expected ToF. The hydrogen data alone is insufficient to populate all bars, and in this case a large amount of quasielastic ${ }^{3} \mathrm{He}$ data is used with the same set of cuts. However, the ${ }^{3} \mathrm{He} \delta t$ distribution has a small time offset, denoted by $\delta t_{\text {off }}$, relative to the $\mathrm{H}_{2}$ data. The origin of $\delta t_{\text {off }}$ may be conceptually seen by Figure 5.53 , the ${ }^{3} \mathrm{He}$ nucleons are bound, requiring additional energy to be liberated from the nucleus which is not accounted for in Eqn 5.36. The $\delta t_{\text {off }}$ offset may be estimated by simply comparing the ${ }^{3} \mathrm{He} \delta t$ distribution to bars where good fits are achievable using only elastic $\mathrm{H}_{2}$ data. The bars that cannot be determined from $\mathrm{H}_{2}$ alone are calibrated using the quasielastic ${ }^{3} \mathrm{He}$ data which is then modified by the average value of $\delta t_{\text {off }}$. The residual times for planes N1-N6 before and after calibration may be seen by Figure 5.31 and Figure 5.32 , note that the $x$-axis displays the bar number, a simple book-keeping device, where low indices are physically located closer to the Hall floor. The data quality of the N5-N7 planes is quite poor, indicating that the longitudinal profile of the hadronic shower typically does not exceed the N4 plane. Therefore, a careful calibration of blocks within these planes is not as critical as planes N1-N4. The cumulative result of calibrating all bars yields a ToF resolution improvement from approximately 410 ps to 300 ps, and may be seen by Figure 5.30 . The small bump around $2 \mathrm{~ns}$ is a result of the RF correction selecting 


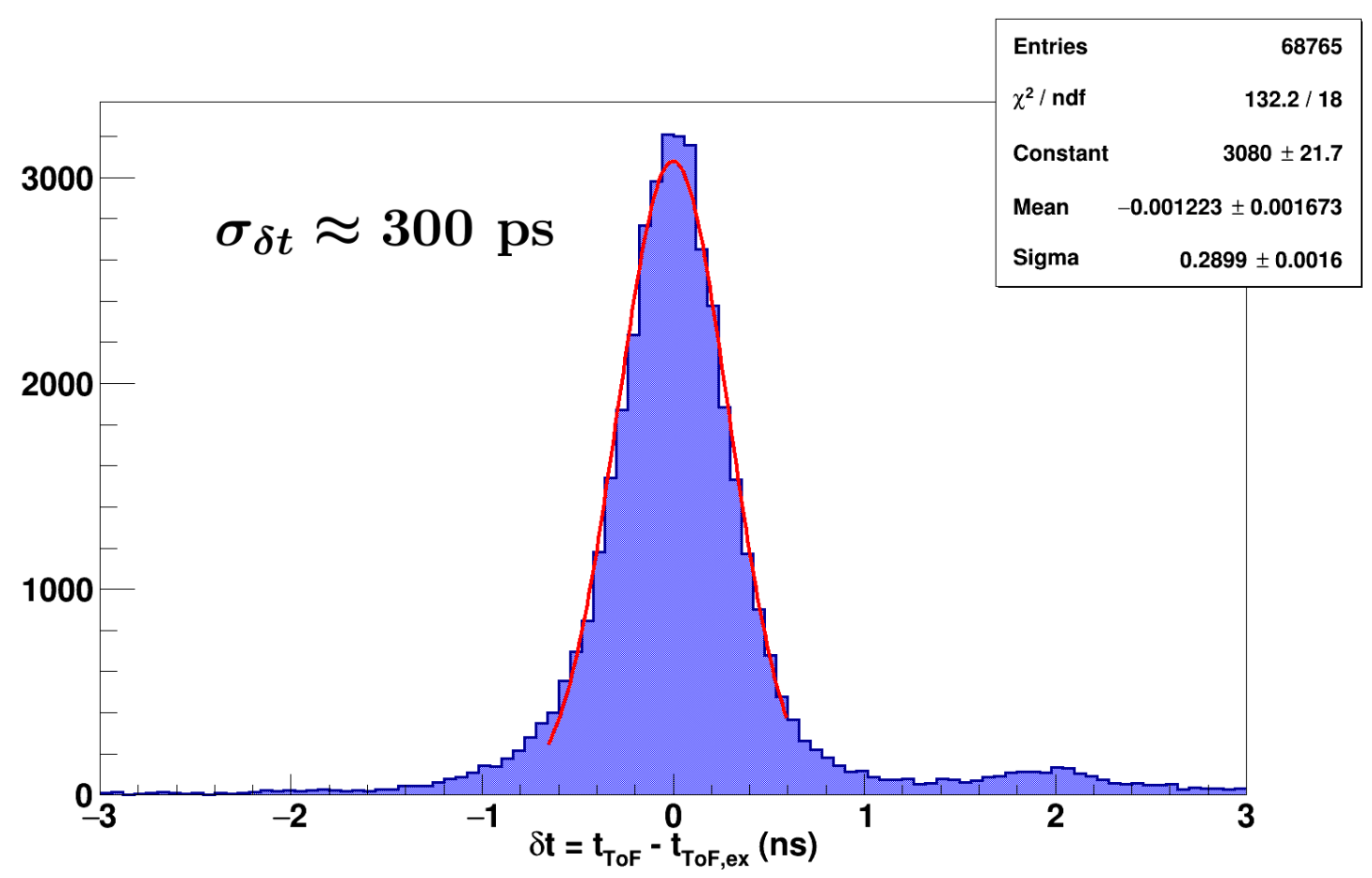

Fig. 5.30: A ToF resolution of 300 ps is obtained after the calibration procedure, and represents an improvement of approximately $35 \%$.

the incorrect electron bundle. Note that although the veto layers are also calibrated in this manner, the V1 and V2 layers do not measure the ToF; this is handled by N1N7. However, calibrating the veto layers is important as charge identification relies on time association (and space) between veto hits and the reconstructed nucleon cluster. If the veto layers are uncalibrated relative to the neutron layers, then the assignment of charge will be incorrect which dramatically affects the raw asymmetry calculation. Lastly, there are small ToF offsets that occur over experimental run time and may be investigated by analyzing $\delta t$ as a function of the ${ }^{3} \mathrm{He}$ run number; the run dependent offsets may be found and added to the calibration database if desired. 


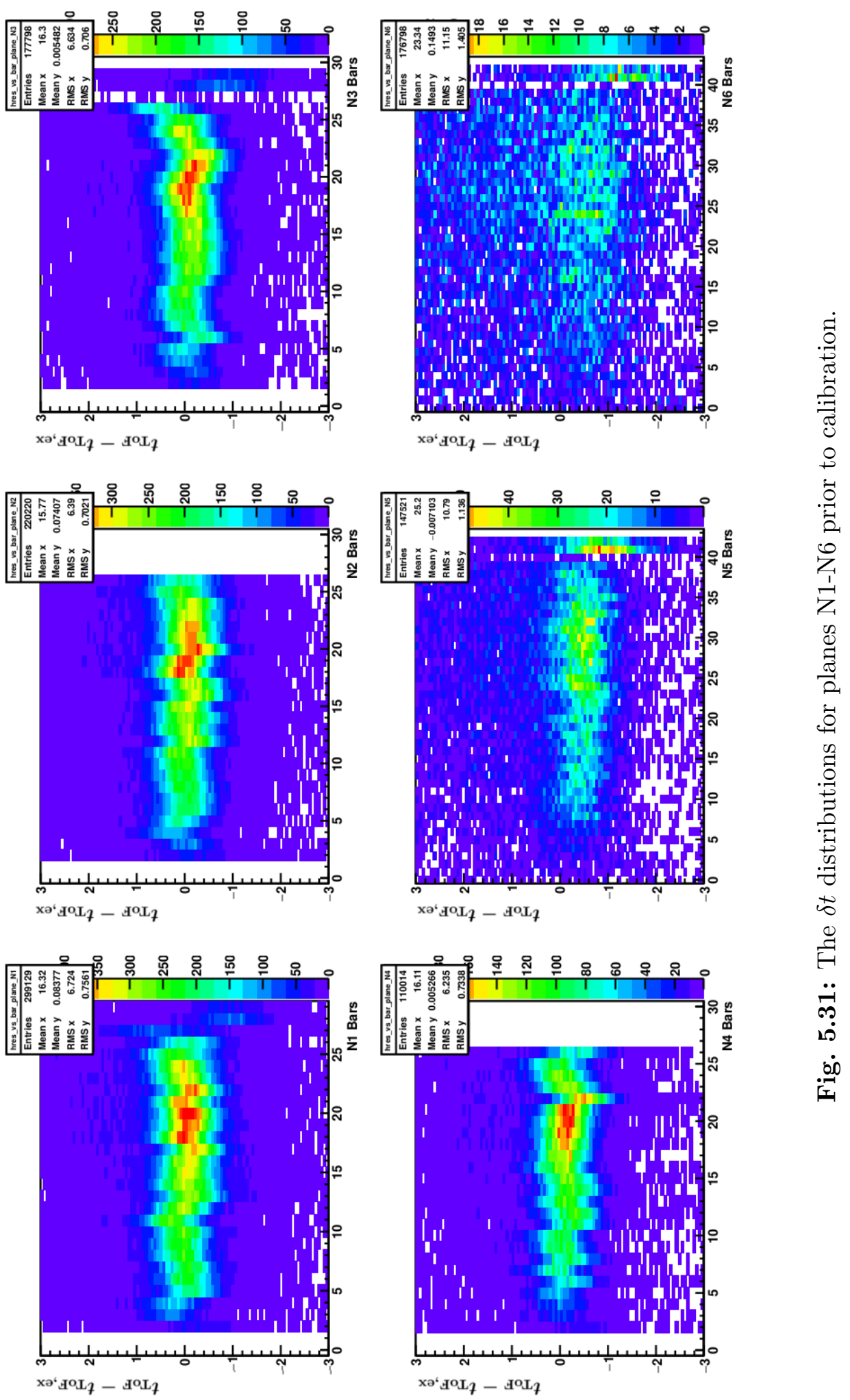



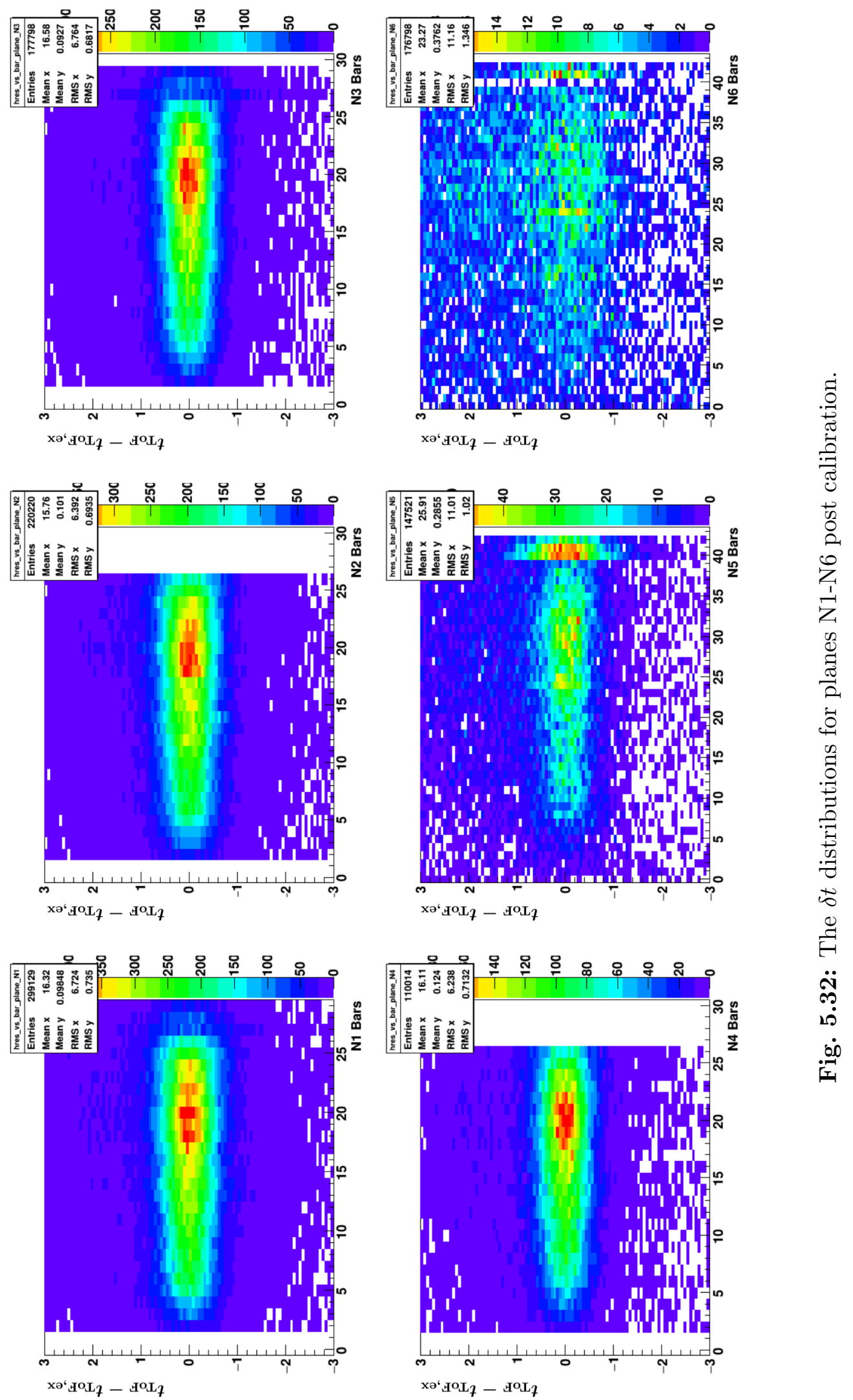


\subsubsection{Attenuation}

For the $G_{E}^{n}$ analysis, a careful determination of all attenuation factors is unnecessary; however, the analysis has been performed in order to simulate the neutron detector. Therefore, the attenuation analysis will be presented but the results are only used with respect to the simulation which is discussed in Section 5.4.7. Recall that the left and right $\mathrm{ADC}$ signals are subject to attenuation and the model to handle the effects has been presented in the context of the BigBite hodoscope, specifically by Eqn 5.25 and Eqn 5.26. For convenience, the relevant equation is

$$
\ln \frac{A_{\mathrm{L}}}{A_{\mathrm{R}}}=\frac{2}{\Gamma} y+\ln \frac{G_{\mathrm{L}}}{G_{\mathrm{R}}}
$$

where $A_{\mathrm{L}}$ and $A_{\mathrm{R}}$ are the pedestal corrected ADC signals for the left and right PMTs, respectively, $\Gamma$ is the attenuation coefficient, $y$ is the horizontal hit coordinate and $G=G_{\mathrm{L}} / G_{\mathrm{R}}$ is the ratio of the gain factors. The attenuation factors and gain ratios may be extracted from a $\ln \left(A_{\mathrm{L}} / A_{\mathrm{R}}\right)$ versus $y$ distribution for every bar of the neutron layers (N1-N7). The analysis uses elastic hydrogen data with a semi-loose cut selection: vertex and coincidence cuts. The formalism requires that both the left and right PMT contribute ADC signals. A typical distribution may be seen by Figure 5.33 which is fit to a straight line; the slope (y-intercept) is equal to $2 / \Gamma\left(\ln G_{\mathrm{L}} / G_{\mathrm{R}}\right)$. Recall that the N1-N7 layers are made up of three different types of scintillating bars; the attenuation factors for all bars organized by bar type may be seen by Figure 5.34. The layers in the back, specifically N5-N7, are mostly made up of UVA bars which explains the larger spread. Bars with poor fits are given a value equal to the average of all good bars of that particular type, which explains the spikes in the Glasgow and UVA distributions. 


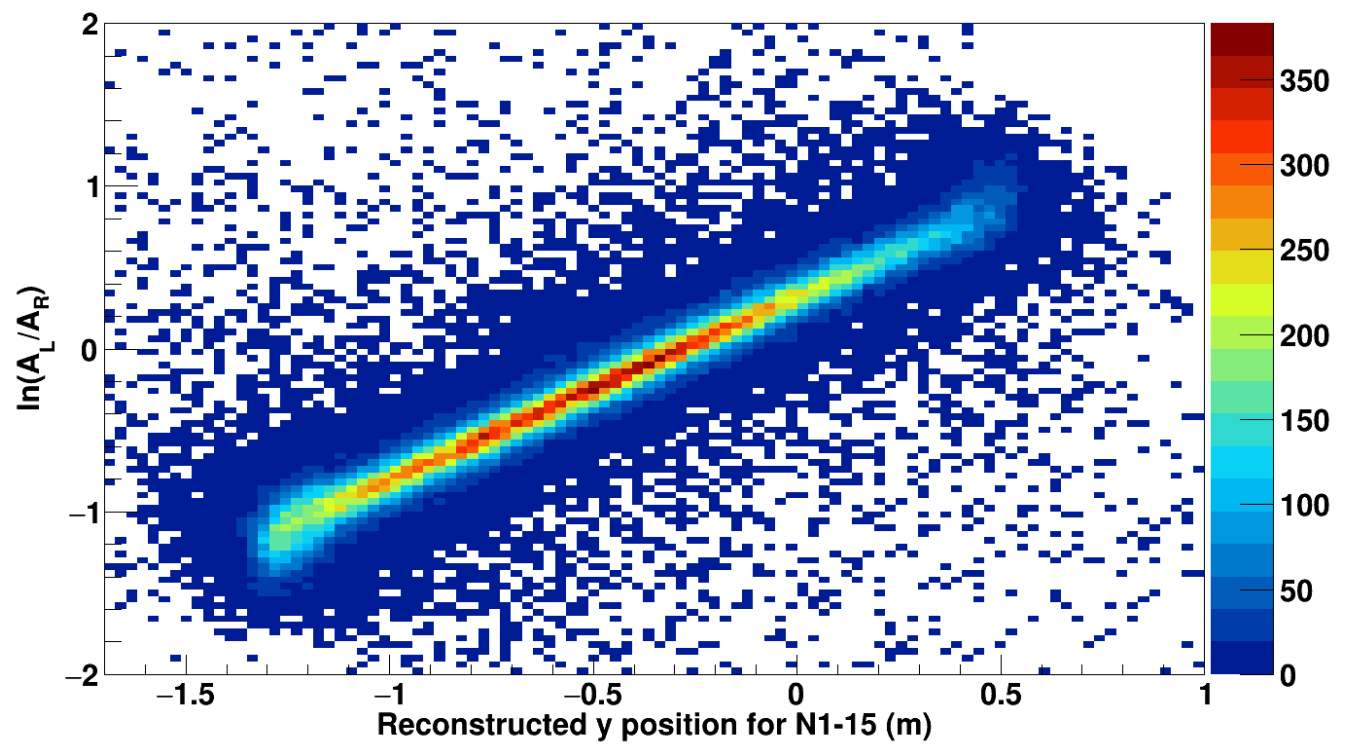

Fig. 5.33: Extracting the attenuation coefficient for bar N1-15.

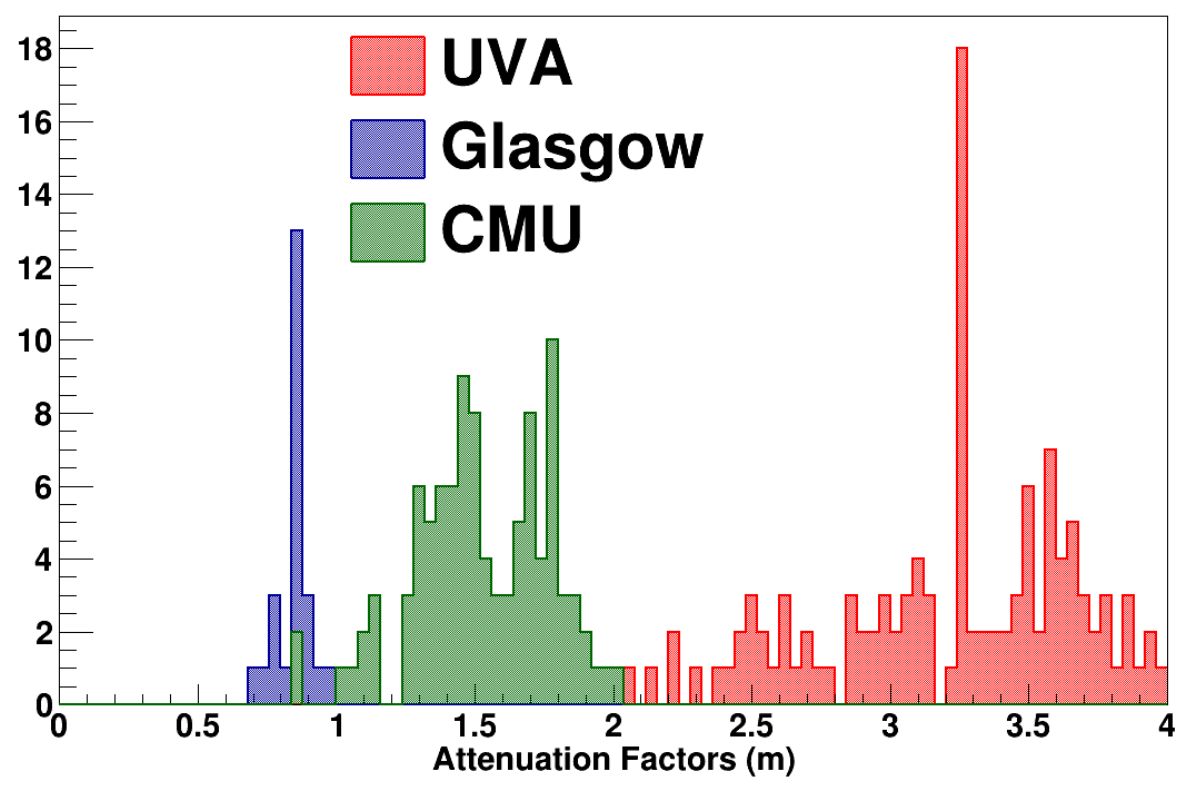

Fig. 5.34: All attenuation factors organized by bar type. 


\subsubsection{Nucleon Cluster}

A typical cluster may be visualized by Figure 4.29 in which multiple bars in multiple planes contribute signals; a sensible procedure to combine the hits into an nucleon object is required for momentum reconstruction. The raw analysis requires left and right TDC signals to reconstruct the horizontal coordinate of a hit within a bar. Within a particular plane, reconstructed hits are combined into an object referred to as a multihit. The construction of a multihit begins with the neutron counter with the earliest reconstructed TDC hit, and continues to add hits on the plane-level that satisfy a space and time criteria: the vertical coordinate must be within 1.5 bar spacings and the time must occur within a 10 ns time window, both relative to the starting hit. The multihit procedure is iterated throughout the detector. On the detector-level, multihits are then combined using a similar space and time criteria; the starting point is the multihit with the earliest time. The result of such a procedure defines a nucleon cluster object. The assigned cluster time used in subsequent momentum calculations is taken to be the RF-corrected time of the earliest multihit denoted by $t_{\text {ToF }}$. The cluster may be "converted" to a track using an energy deposition weighted sum of coordinates, described in detail within the context of the BigBite calorimeter by Eqn 5.13, during the combination of multihits; the result is an approximate coordinate of the nucleon cluster. The path length $|\vec{\ell}|$ of the recoiling nucleon represents the distance between the scattering vertex and the reconstructed cluster position in lab coordinates, and the velocity and momentum magnitudes of the nucleon may then be calculated as

$$
\beta=\frac{v}{c}=\frac{|\vec{\ell}|}{c t_{\mathrm{ToF}}} \quad \text { and } \quad p_{\mathrm{na}}=\frac{M \beta}{\sqrt{1-\beta^{2}}}
$$



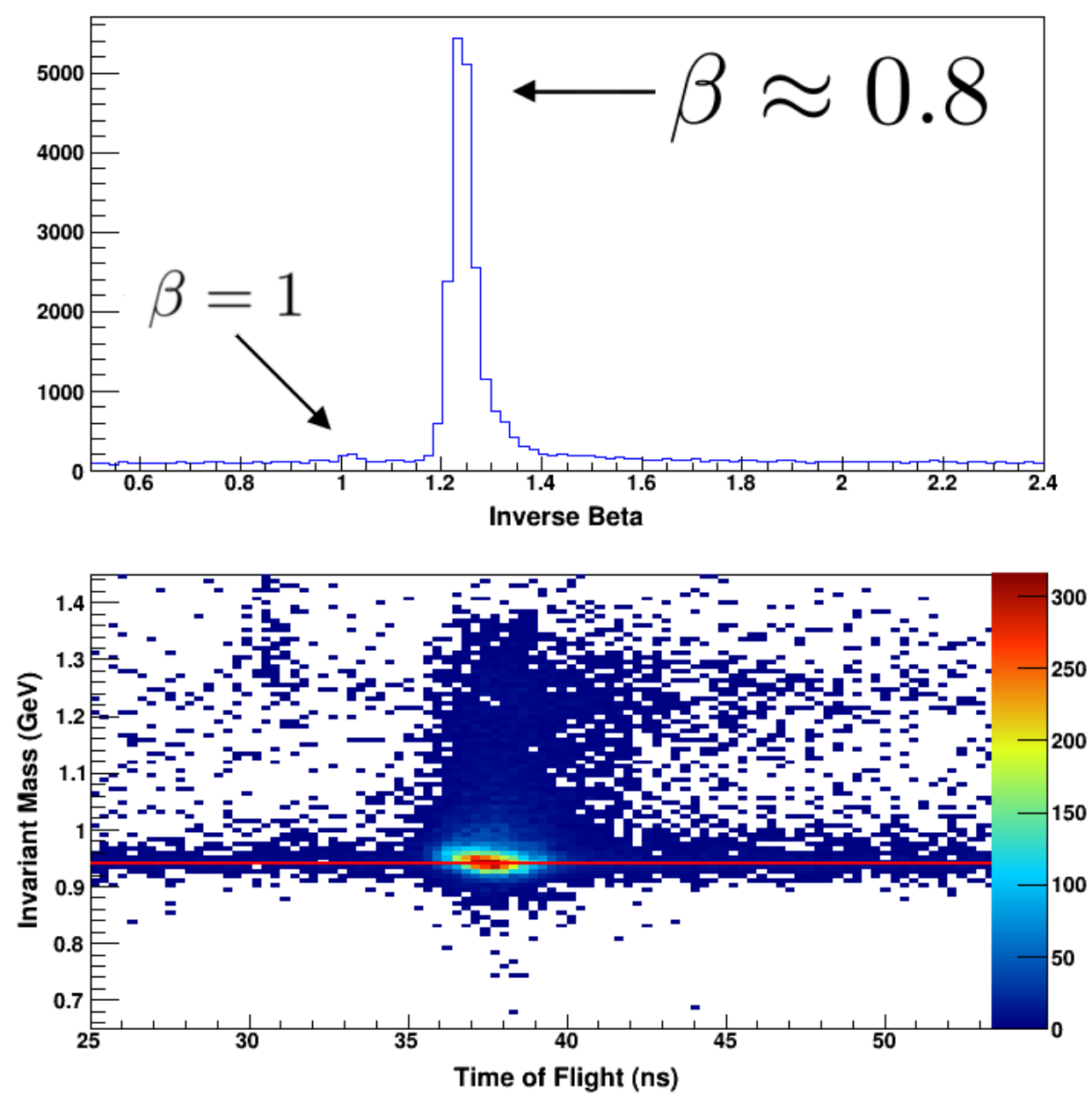

Fig. 5.35: The $\beta^{-1}$ and ToF distributions using elastic $\mathrm{H}_{2}$ data.

yielding a three momentum of $\vec{p}_{\text {na }}=p_{\text {na }} \hat{\ell}$. Assuming that the elastic peak of $\mathrm{H}_{2}$ data has been selected, the Gaussian mean of the proton time of flight spectrum is roughly 38 ns which corresponds to a $\beta \approx 0.8$ and $p_{\text {na }} \approx 1.3 \mathrm{GeV}$. The distribution of the inverse $\beta\left(=\beta^{-1}\right)$ may be seen by Figure 5.35 . The $\beta=1$ peak corresponds to photons and represents the minimum allowable time for particles associated to a particular event to be considered. Events that occur prior to $\beta^{-1}=1$ cannot have originated from the scattering vertex due to causality, and therefore correspond to the 
accidental background. The $\beta^{-1}$ may then be used to artificially shift the data in time to estimate the contamination due to random background events given a set a kinematic cuts; this is performed in Section 5.7.3. The time of flight spectrum may be seen by the bottom panel of Figure 5.35 and has been plotted against the invariant mass; the ToF signature clearly shows up at $W=M_{p}$.

At the start of an event, the nucleon is assumed to be initially at rest; therefore, after an electron transfers a momentum $\vec{q}$ to the nucleon, the recoiling nucleon is constrained by elastic kinematics to follow the direction of $\hat{q}$. As the subject of this thesis deals with quasielastic scattering from a ${ }^{3} \mathrm{He}$ nucleus, it is advantageous to define a missing momentum denoted by $\vec{p}_{\text {miss }}$, and represents the vector deviation between BigBite's prediction of $\vec{q}$ to the NA's time of flight measurement of $\vec{p}_{\text {na }}$. The missing momentum components are

$$
\begin{gathered}
\vec{p}_{\text {miss }}=\vec{q}-\vec{p}_{\text {na }}, \\
\Rightarrow p_{\text {miss }, \|}=\left(\vec{q}-\vec{p}_{\text {na }}\right) \cdot \hat{q}, \\
\Rightarrow p_{\text {miss }, \perp}=\left|\vec{q}-\vec{p}_{\text {na }}-p_{\text {miss }, \|} \hat{q}\right|,
\end{gathered}
$$

where $\vec{q}$ is the momentum transfer as calculated by BigBite. A diagram displaying the orientation of the vectors may be visualized by Figure 5.36 which is exaggerated in order to see the features. In the context of the elastic peak of the hydrogen data, the missing parallel momentum is a measure of how well the reconstructed proton cluster track aligns with the optics prediction of BigBite; therefore, the distribution should be a Gaussianlike distribution centered at zero with a standard deviation that is related to the timing resolution of the detector. For an electronics time resolution of 300 ps, Eqn 4.27 predicts 


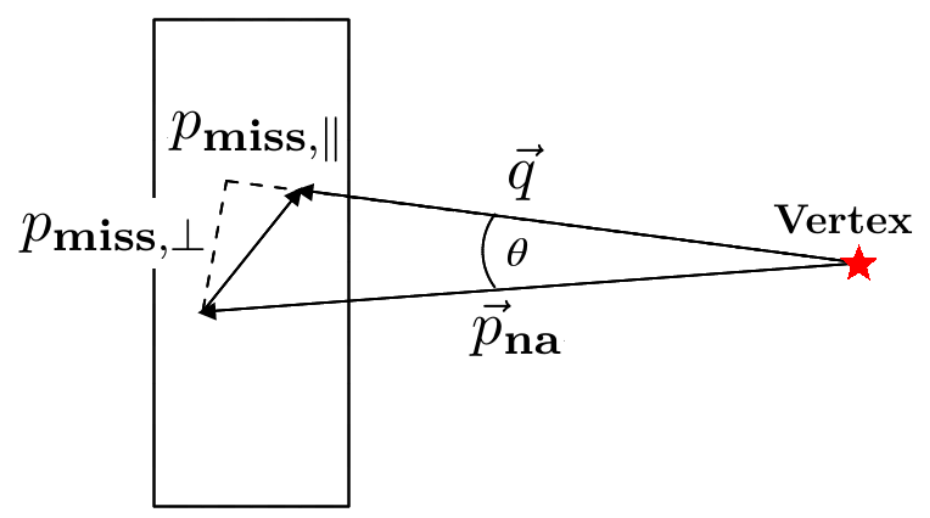

Neutron Detector

Fig. 5.36: Exaggerated diagram displaying the missing momentum components. The missing momentum is the vector difference of BigBite's prediction of $\vec{q}$ to the neutron arm's reconstructed $\vec{p}_{\text {na }}$.

a momentum resolution of $30 \mathrm{MeV}$. In good agreement, the hydrogen $p_{\text {miss, }, \|}$ spectrum yields a Gaussian standard deviation of roughly $30 \mathrm{MeV}$. The missing perpendicular momentum is a measure of the transverse distance between the cluster position and the predicted $\vec{q}$ position. Additionally, $p_{\text {miss }, \perp}$ contains information regarding the transverse momentum resolution of the ND. In this case, the distribution is a convolution of the neutron detector $x$ and $y$ dimensions where the resolutions depend on the vertical block dimension and the left/right TDC timing difference, respectively. If the distributions are assumed to be symmetric, a two-dimensional Gaussian expressed in polar coordinates then gives a single standard deviation parameter of approximately $40 \mathrm{MeV}$. The missing parallel and perpendicular momentum spectra for hydrogen elastic data may be seen by Figure 5.37, and will play an important role in the selection of quasielastic events within the ${ }^{3} \mathrm{He}$ data, e.g. to suppress inelastics and nuclear effects. 

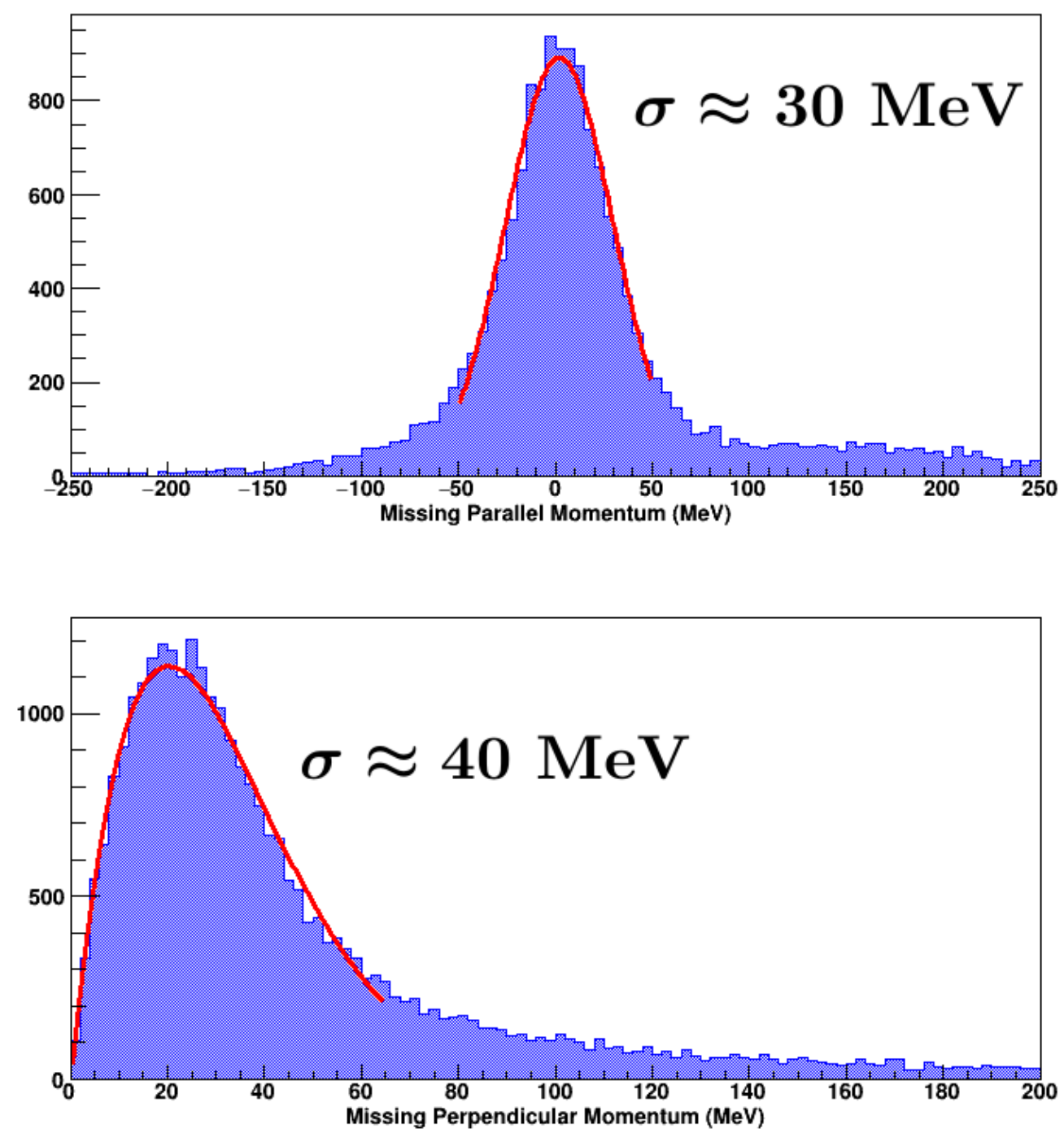

Fig. 5.37: The $p_{\text {miss }, \|}$ and $p_{\text {miss }, \perp}$ distributions for $\mathrm{H}_{2}$ elastic data. Information regarding the neutron detector may be extracted from the missing momenta distributions, see text for details. Perhaps more importantly, the missing momenta variables will play a unique role in the selection of quasielastic events within the ${ }^{3} \mathrm{He}$ data. 


\subsubsection{Matching Veto Hits to a Cluster and Charge Identification}

In order to determine the charge of the nucleon cluster, the veto hits must be matched in time and space with the reconstructed cluster. The idea is to find, or perhaps to not find, veto hits within a reasonable space and time window of a cluster of position $x_{\text {cluster }}$ and TDC time, and then decide if the cluster is a proton, neutron or undecided. The concept relies on the fact that charged particles leave signals in scintillator; therefore, if a nucleon cluster has been found and there are (are no) veto hits within a defined space and time window, then the cluster is assumed to be a proton (neutron). The concept may be visualized by Figure 4.29 .

The spatial window may be determined by analyzing the vertical $x$ direction as the $y$ coordinate of the veto counters is unable to be reconstructed. By comparing the vertical position of the reconstructed cluster $x_{\text {cluster }}$ to the vertical position of a candidate veto hit $x_{\mathrm{veto}}$, an appropriate spatial window may be found:

$$
\left|x_{\text {cluster }}-x_{\text {veto }}-x_{0}\right|<\Delta x
$$

where $x_{0}$ is a vertical offset and roughly corresponds to the center of the distribution and $\Delta x$ is the desired spatial window to look for candidate hits within the veto layers.

The values of $x_{0}$ and $\Delta x$ must be determined by analyzing the $x_{\text {cluster }}-x_{\text {veto }}$ spectra for both veto layers, and may be seen by Figure 5.38 where $\mathrm{H}_{2}$ elastic data has been used. While ${ }^{3}$ He data may also be used, the hydrogen data is preferred as the accidental rate is lower due to the reduced beam current, $5 \mu \mathrm{A}$ compared to $3 \mu \mathrm{A}$, respectively. The distributions are offset relative to each other, i.e. V1 (V2) is peaked less (greater) than zero, reflecting the fact that the veto layers are vertically offset with respect to 

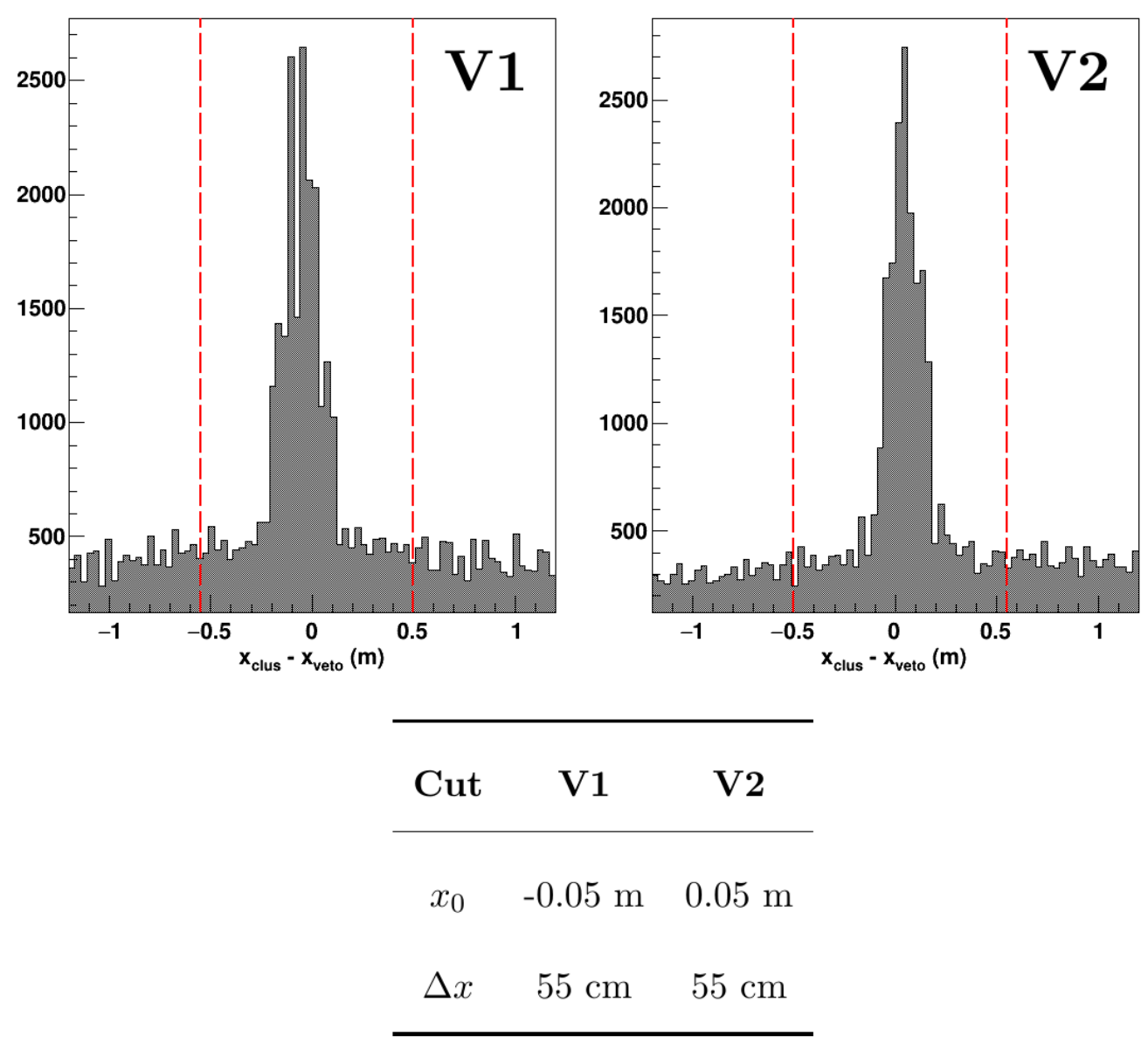

Fig. 5.38: Defining the veto-cluster space window for nucleon charge identification. The dotted red lines display the space window $\Delta x$, and the center of the distributions is roughly $x_{0}$. The table displays the chosen cut values.

one another. The values of $x_{0}$ have been chosen to be $-5 \mathrm{~cm}$ and $5 \mathrm{~cm}$ for $\mathrm{V} 1$ and $\mathrm{V} 2$, respectively. The space window $\Delta x$ is defined to be larger than required, and a value of $55 \mathrm{~cm}$ has been chosen for both layers.

A cluster-veto timing association may be found in a similar manner as the spatial association, but additional difficulties arise due to the differences between N1-N7 and 
V1-V2 modules. Recall that veto layer modules consist of two scintillating bars and two PMTs, and as a result the horizontal coordinate is not reconstructed; therefore, when analyzing the veto time spectrum the propagation time of light in the scintillating medium needs to be taken into account. The time window to associate a cluster to candidate veto hits may be found by studying the $t_{\text {cluster }}-t_{\text {veto }}$ spectra. The distribution may be tightened up significantly if light propagation time has been corrected, and this may be done by examining the cluster-veto time difference as a function of the reconstructed cluster $y$ position as seen by the top row of Figure 5.39, The distinctive "V" shape is a result of a veto module consisting of two scintillating bars: short and long. The short bar is physically located closer to the beamline or in the positive $y_{\text {cluster }}$ region. The break in the distribution is where the short bar meets the long bar, and the slope of the distributions is related to the speed of light in scintillator, $c_{s}$. The V1 and V2 distributions may be modeled by an absolute value function to handle light propagation in both bars with the vertex corresponding to the short-long boundary. The resulting time window $\Delta t$ for cluster-veto association may be defined as

$$
\Delta t=t_{\mathrm{veto}}-t_{\text {cluster }}+\frac{\left|y_{\text {cluster }}-y_{0}\right|}{c_{s}}+t_{0}
$$

where $t_{\text {cluster }}$ and $t_{\text {veto }}$ are the TDC times for the reconstructed cluster and veto hit, respectively. The absolute value function depends on the horizontal position of the reconstructed cluster $y_{\text {cluster }}$, the speed of light in scintillator $c_{s}$, an offset $y_{0}$ corresponding to the location where the short bar meets the long bar, and a time offset $t_{0}$. As in the previous case, the values must be determined by the data with the exception of $y_{0}$ as this is not a free parameter and depends on the geometry. The bottom row of Figure 5.39 

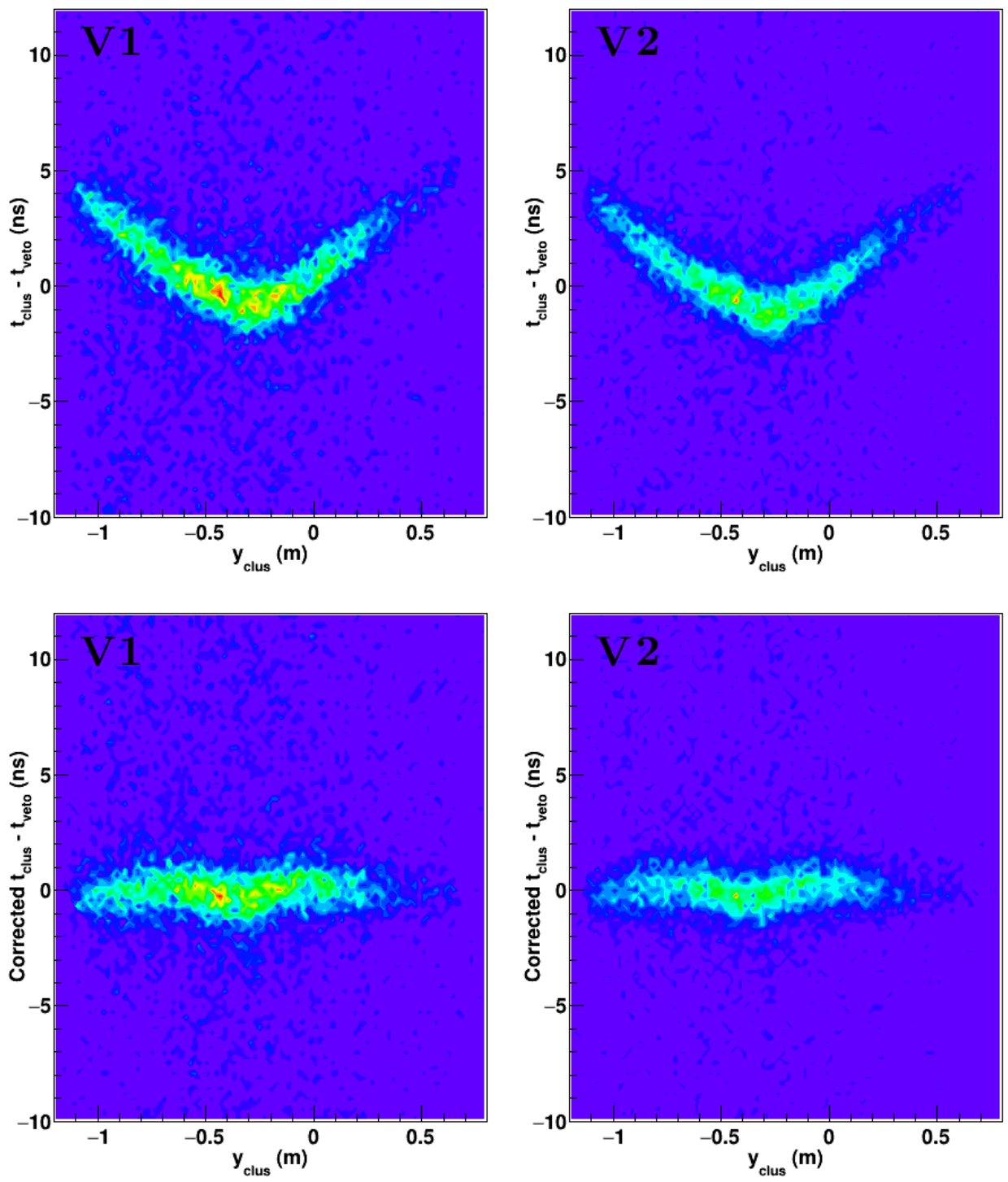

Fig. 5.39: Defining the veto-cluster time window for charge ID, see text for details.

displays the cluster-veto time difference after correcting for light propagation in the scintillating medium. Figure 5.40 displays $t_{\text {cluster }}-t_{\text {veto }}$ before and after correcting for light propagation; a resolution of 850 ps has been obtained for both veto layers, V1 and V2. Table 5.1 displays the corrections and the resulting time window $\Delta t$ to associate 

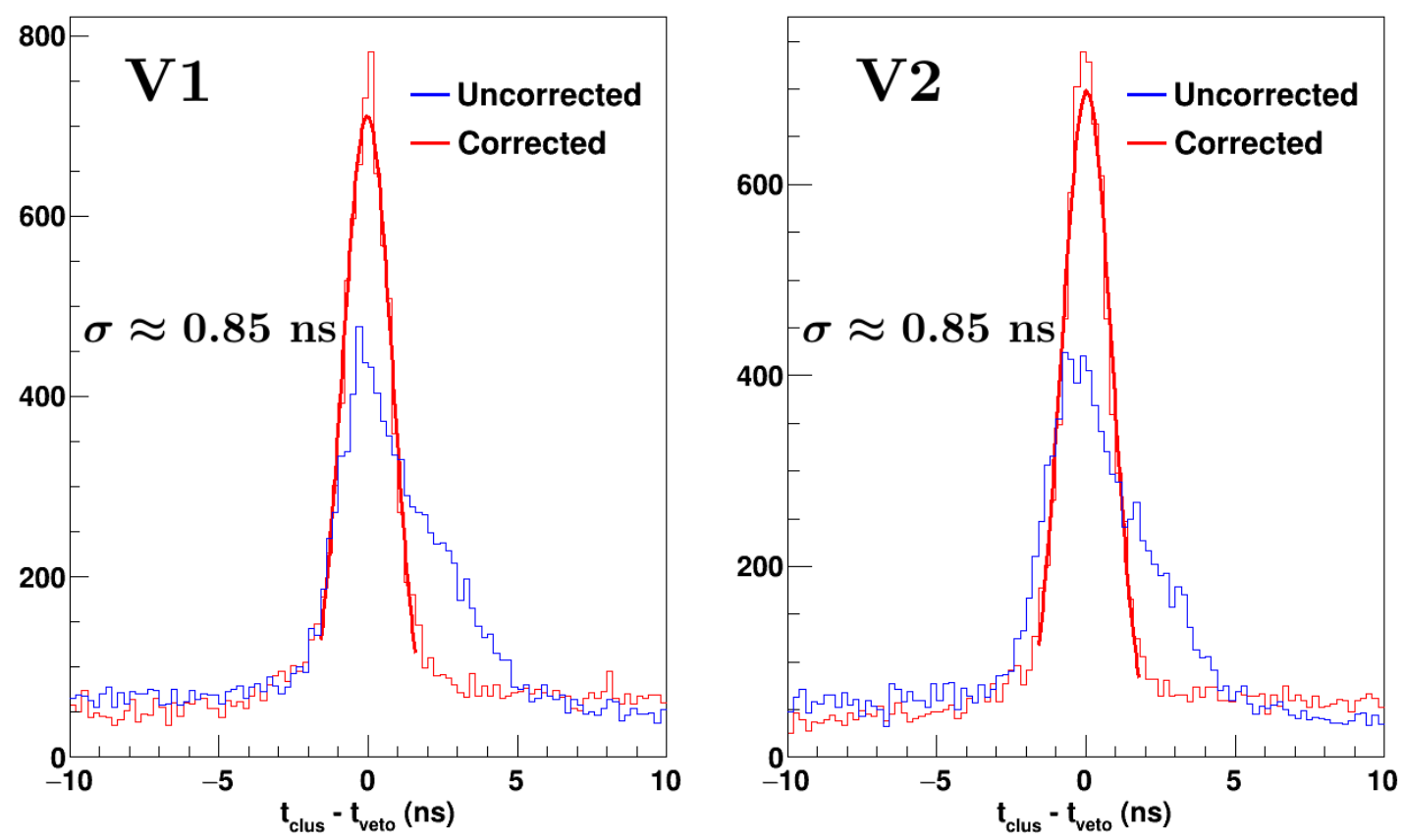

Fig. 5.40: Veto-cluster time difference before and after correcting for light propagation within the scintillating medium.

veto hits to a reconstructed cluster. A time window of 10 ns has been chosen after examination of the data; this is sufficiently large to look at events of interest but tight enough to suppress accidentals.*

The cluster-veto time association is further complicated by the electronic deadtime for each detector channel of the veto layers, and must be included in the charge identification procedure. Both left and right PMTs of all 48 rows of both veto planes $(=2 \times 2 \times 48=192$ channels $)$ have a channel specific electronic deadtime, or the period of time when no more events may be accepted as the electronics and hardware are currently busy or stimulated from previous events. In short, sources of the electronic

\footnotetext{
${ }^{*}$ Recall that a typical ToF for a recoiling nucleon is roughly $38 \mathrm{~ns}$.
} 


\begin{tabular}{ccc}
\hline Time Cut & V1 & V2 \\
\hline$t_{0}$ & $-1.2 \mathrm{~ns}$ & $-1.2 \mathrm{~ns}$ \\
$y_{0}$ & $-0.35 \mathrm{~m}$ & $-0.35 \mathrm{~m}$ \\
$c_{s}$ & $0.16 \mathrm{~m} / \mathrm{ns}$ & $0.16 \mathrm{~m} / \mathrm{ns}$ \\
$\Delta t$ & $10 \mathrm{~ns}$ & $10 \mathrm{~ns}$ \\
\hline
\end{tabular}

Table 5.1: Time cuts to associate veto hits to a nucleon cluster.

deadtime may be attributed to the rise/fall time of PMTs and the discriminators; additionally, the TDCs do not process events faster than some intrinsic rate. An example of the deadtime complications arise when thinking about background events. A hypothetical charged background particle leaves a signal within some modules of the veto layers, but does not leave a cluster. Then, while the veto modules are in the deadtime period, a true neutral particle comes in and leaves a cluster that is correlated in space and time. This is considered a blind event as the neutral event occurred within the period of the veto deadtime, and in this case the event is discarded.

The deadtime may be calculated for all veto channels by analyzing the time between successive hits within the same channel per event. Each channel has a minimum time between successive hits, and the deadtime corresponds to the region where no events are detected. A typical distribution for a sample channel of V1 may be seen by the top panel of Figure 5.42 the leading edge of the distribution is then a measure of the electronic deadtime for this particular channel. The distribution of deadtimes for all 192 channels may be seen by the bottom panel, and fall into roughly three groups corresponding to an average deadtime of 50,65 and 100 ns. An alternative way to view 


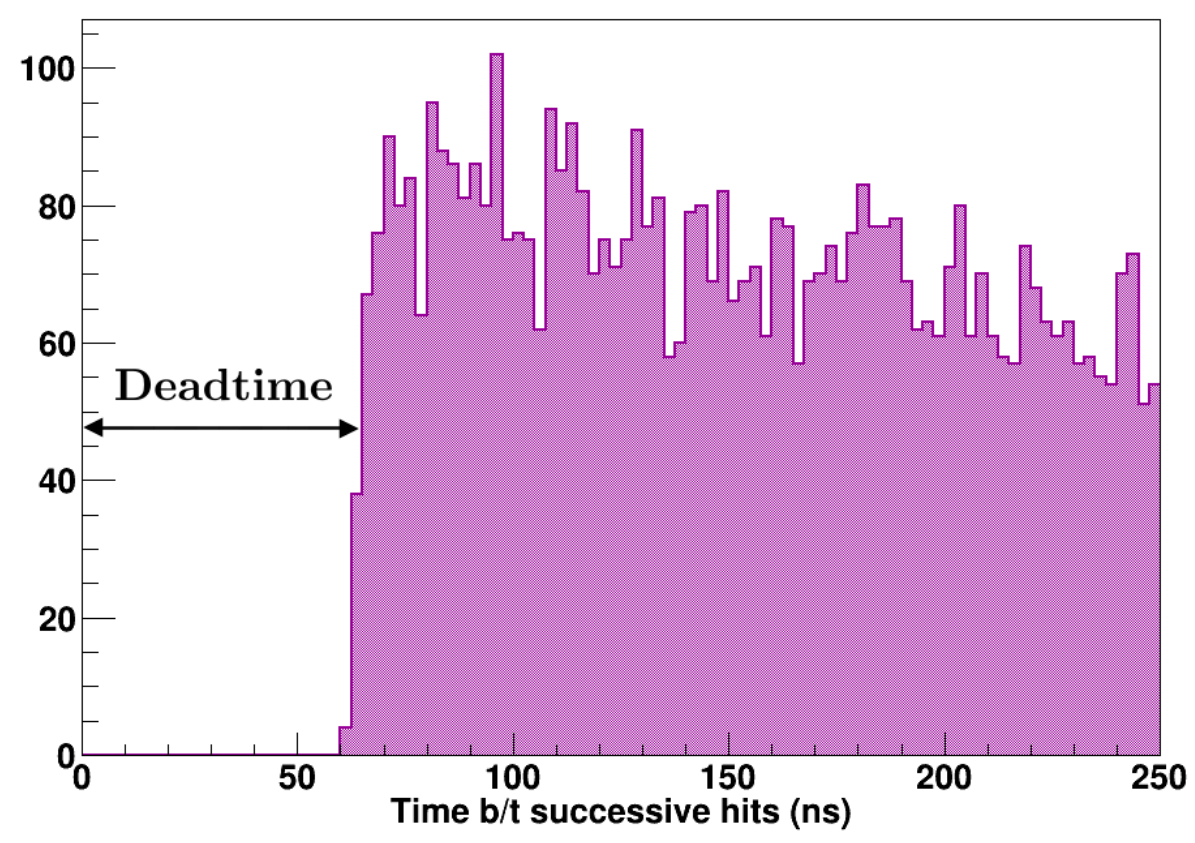

Fig. 5.41: The leading edge of the distribution corresponds to the deadtime.

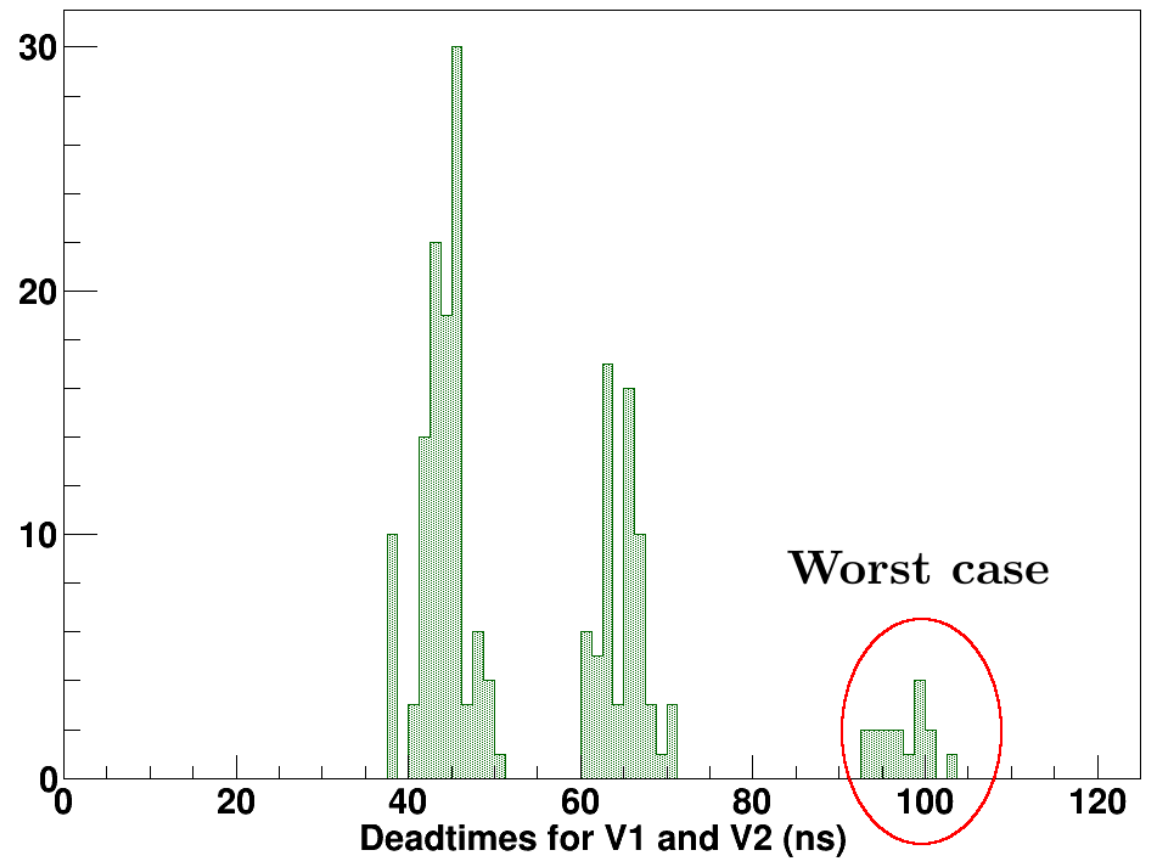

Fig. 5.42: The distribution of $\mathrm{V} 1$ and $\mathrm{V} 2$ deadtimes for left and right channels. 

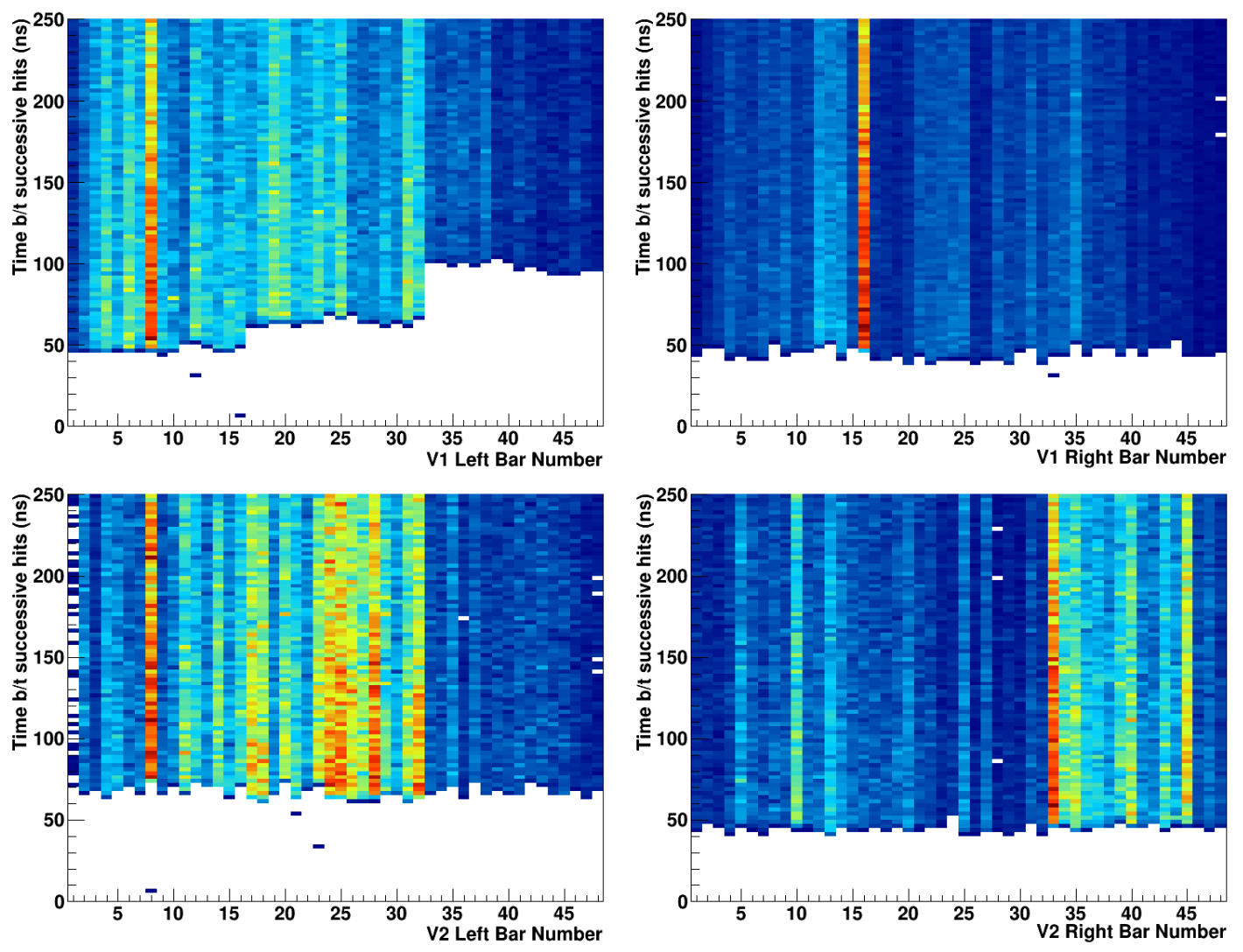

Fig. 5.43: V1 and V2 deadtime distributions for all channels. Within a particular $\mathrm{x}-$ axis bin, the deadtime is characterized by the vacant space. An example of a one dimensional projection may be seen by Figure 5.42 .

the deadtime for all channels of the two veto layers may be seen by Figure 5.43 . The locations and bar numbers making up the three deadtime groups are now obvious; for example, the channels with the largest deadtime, namely $100 \mathrm{~ns}$, all occur on the left side of V1, specifically channels 34-48. While the deadtimes for individual channels may be incorporated into the analysis, for ease of calculation a deadtime corresponding to the worst possible situation is applied to all channels; therefore, a deadtime of $100 \mathrm{~ns}$ 
is applied. The majority of channels have a deadtime much less than $100 \mathrm{~ns}$; therefore, the choice of $100 \mathrm{~ns}$ is a trade-off between simplifying future calculations and reducing statistics.

The calibration and raw analysis phase which primarily uses the hydrogen data set is now complete. At this point, the kinematics of both arms of the experiment may now be reliably determined. Furthermore, a scheme to assign a charge to the recoiling nucleon has been developed, and as a result the data may now be separated into two categories: charged and uncharged. Before moving on to the analysis of the polarized

${ }^{3} \mathrm{He}$ data, though, it is instructive to simulate the neutron detector in an attempt to understand more deeply the trigger scheme, the scintillator response to nucleons and the charge identification procedure; this is discussed in the next section.

\subsubsection{Simulating the Neutron Detector and a Coincidence Event}

Broadly speaking, simulating the neutron detector follows a similar path as Section 5.3.6 which describes the electron arm simulation analysis. For example, the neutron arm trigger, denoted by $\mathrm{T} 1$, needs to be simulated which requires a thorough trigger study of hydrogen data. More similarities include the need for clustering, i.e. combining hits of many neutron planes in order to reconstruct a nucleon cluster object from which kinematic quantities of interest may be calculated. Furthermore, a scheme to convert a simulated energy deposition in $\mathrm{MeV}$ to an $\mathrm{ADC}$ is required to replicate the $\mathrm{T} 1$ trigger in simulation. The details, however, are quite different than the electron arm, and as a result additional complications arise. The energy deposition in BigBite's leadglass block is assumed to be proportional to the output signal of the PMT as seen by 


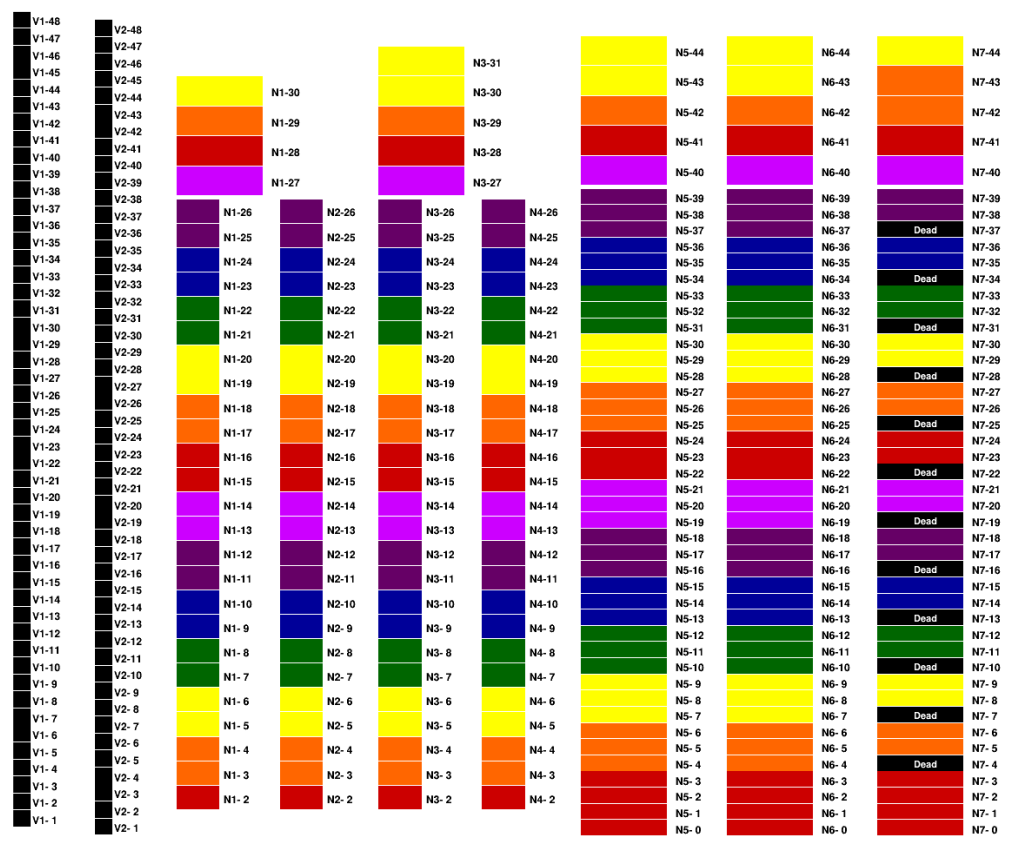

Fig. 5.44: A cross section of the neutron detector displaying the sums of 16; note that black bars are not used to generate a T1 event.

Eqn 5.12, the same cannot be said about the neutron detector in $G_{E}^{n}$. The purpose of this section is to investigate the neutron arm trigger, and to state a procedure that allows the neutron detector to be simulated. To visually see the neutron detector in simulation, see Figure 4.3 and Figure 4.29 .

The neutron arm trigger has been discussed in Section 4.8.2, but the trigger scheme will be clearly rephrased to avoid any ambiguities in the analysis. An overview of the details may be seen by Figure 5.44, the veto layers and the dead N7 modules are not incorporated into the trigger logic. Assuming that all ADC signals are pedestal corrected, the trigger is built in two levels: ADC sums of 16 blocks (sum16s) and ADC sums of 32 blocks (sum32s). The first level, or the construction of sum16s, is formed 
by summing ADC signals defined by the horizontal color-coding as seen in Figure 5.44 . resulting in a total of 17 sum16s. The second and final level, otherwise referred to as a sum32, is generated by summing adjacent sum16s. Every sum16 is included in two sum32s with the exception of the vertical extremes of the detector, totaling in 16 sums of 32 . Note that the above details apply to the left and right side of the detector individually; in other words, a T1 event may be generated by a left or a right ADC trigger sum. To make the scheme more concrete, a book-keeping device is introduced to keep track of the sum32s, and runs from $1 \rightarrow 16$ where $\# 1$ is the bottom-most sum32, comprised of the bottom red + orange blocks seen in the figure; the indexing applies to both the left and right side of the detector for a total of 32 sum32s to be considered.

In order to study the neutron arm trigger of Kinematic 1, the hydrogen coincidence data is analyzed with the usual vertex cut applied. The trigger ADC sums of 32 blocks are analyzed by simply summing the pedestal-corrected, attenuation-corrected ADC signals for all blocks making up a left or right sum32. The maximum ADC sum32 of the entire detector, namely all sum32s for the left and right side, is taken as the accepted trigger signal. The neutron arm trigger logic diagram may be seen by Figure 4.32 , and a logical "OR" between the left and right side of the detector is present; therefore, the maximum ADC sum32 for the coincidence data is guaranteed to have generated the T1 signal. The results may be seen by Figure 5.45, and the top (bottom) panel gets filled if the left (right) ADC sum32 is determined to be the maximum. The T1 ADC sum32 threshold may be determined utilizing an identical procedure as for BigBite. More specifically, the leading edge of the ADC sum32 signal may be fit to a 

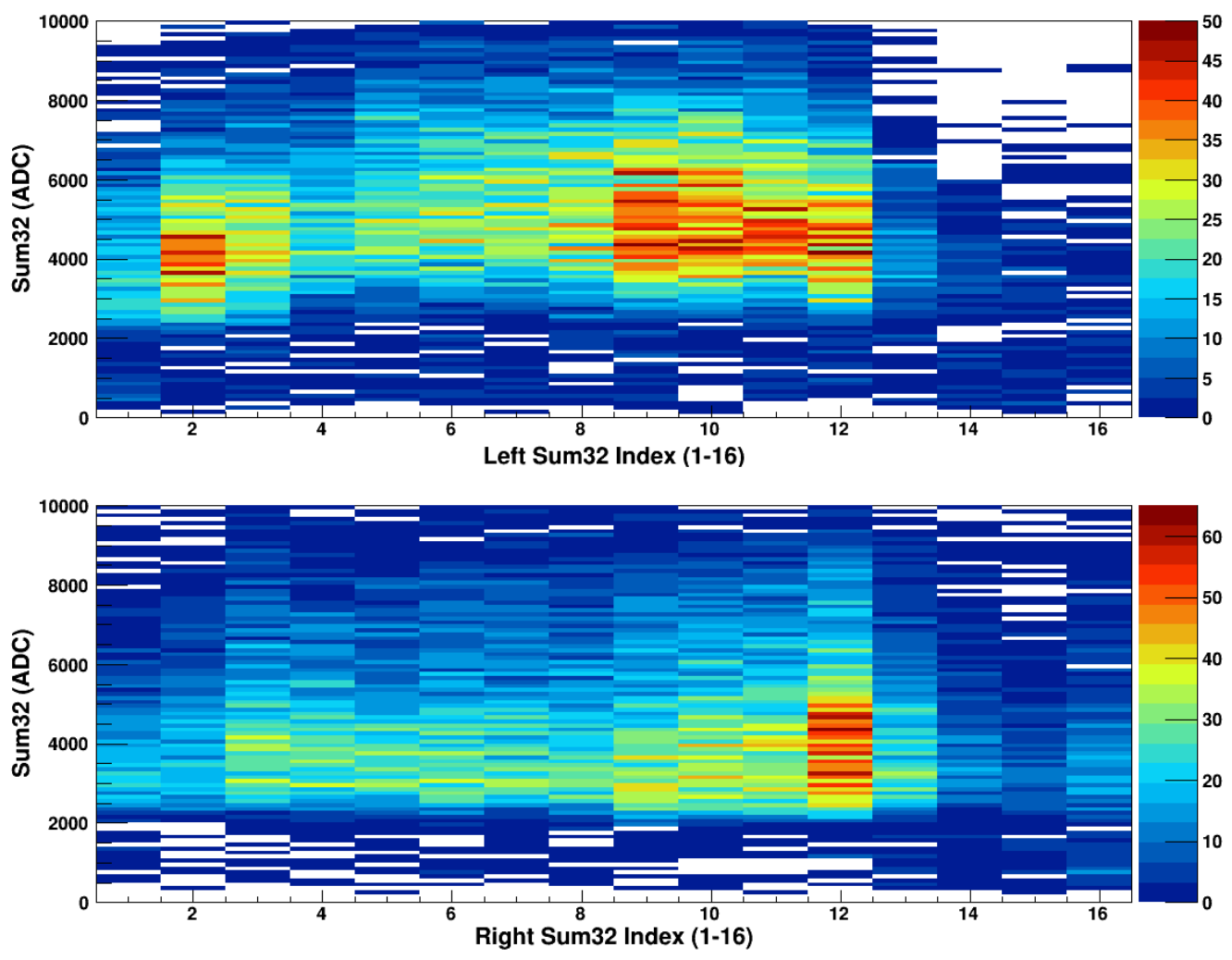

Fig. 5.45: The left and right maximum ADC sums of 32 blocks used to generate the T1 signal. The top (bottom) panel gets filled if the left (right) ADC sum32 is determined to be the maximum.

straight line from $20 \% \rightarrow 80 \%$ of the pulse height, and the threshold may be extracted as the intersection point between the fit and the $x$-axis, for example. The results of this procedure may be seen by Figure 5.46 where only reliable extractions are presented; poor fits are given a threshold of 2000 ADC channels.

Simulating the neutron arm trigger requires an appropriate method to convert a simulated energy deposition in units of $\mathrm{MeV}$ to an $\mathrm{ADC}$, or the units of the hardware. For BigBite, the conversion has been achieved by a $\chi^{2}$-minimization procedure in which 


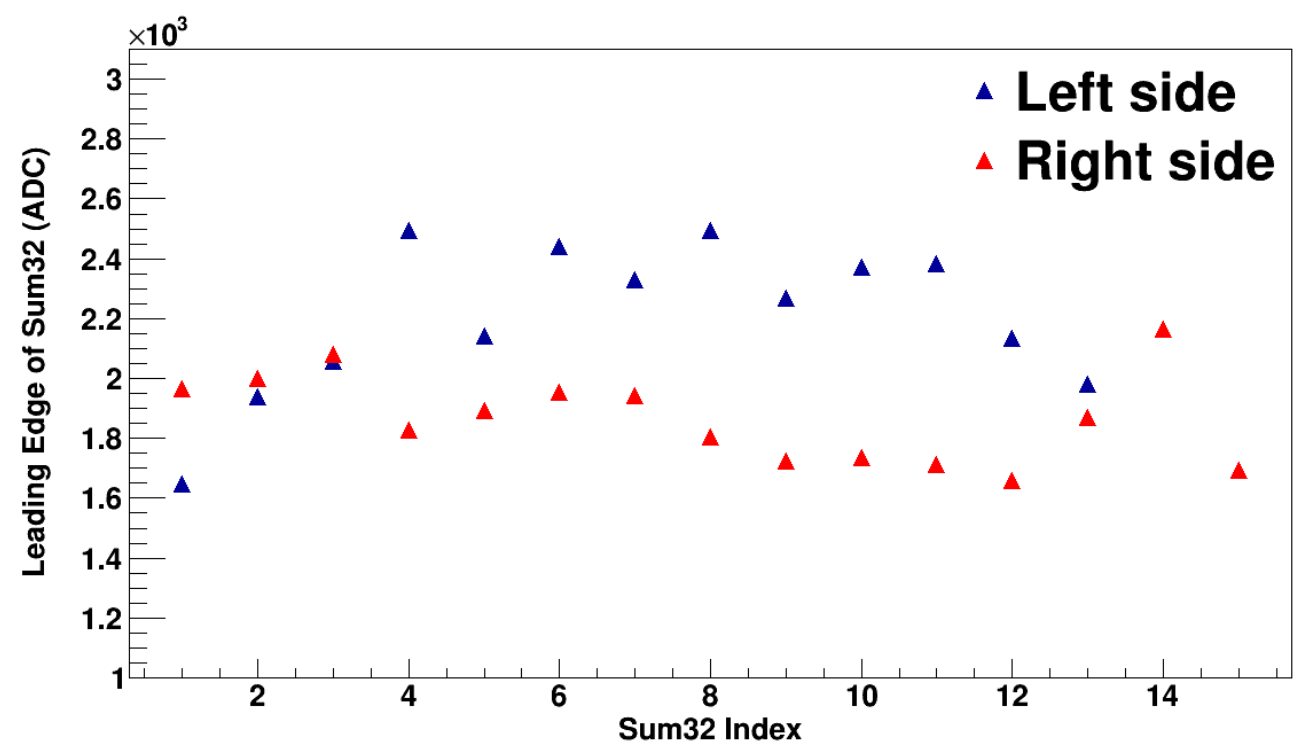

Fig. 5.46: The results of the ADC sum 32 threshold analysis for the left and right side.

the track energy is assumed to be linearly proportional to the sum of energy deposition in lead-glass, and the constants of proportionality, otherwise known as gain coefficients, may be solved using linear regression. It is tempting, then, to follow the lead of the BigBite analysis in order to calculate the gain coefficients for the left and right PMTs of the neutron detector, resulting in a method to convert a block energy deposition in $\mathrm{MeV}$ into an attenuation-corrected left and right ADC signal.* For pedagogical reasons, the attempt will be briefly discussed, but the assumption that the incident proton energy is linearly proportional to the energy deposition in scintillator is not valid for the neutron detector. The attempt may be summarized as follows:

$$
\begin{gathered}
A_{L}=G_{L} A_{0} e^{\frac{-\frac{L}{2}+y}{\Gamma}} \quad \text { and } A_{R}=G_{R} A_{0} e^{\frac{-\frac{L}{2}-y}{\Gamma}} \\
\Rightarrow \sqrt{A_{L} A_{R}}=\sqrt{G_{L} G_{R} e^{-\frac{L}{\Gamma}}} A_{0}
\end{gathered}
$$

\footnotetext{
* Note that the attenuation factors and gain ratios for all blocks have been calculated in Section 5.4.4
} 
where $A_{0}$ is the scintillator energy deposition, $L$ is the length of the bar, $\Gamma$ is the attenuation length, $y$ is the reconstructed horizontal position along the bar, $A$ is the ADC amplitude for the left/right-hand side of the detector and $G$ are the gain coefficients. To retain linearity with respect to the minimization parameters, the gain product may be rearranged as $G_{L} G_{R}=G_{L}^{2} \frac{G_{R}}{G_{L}}$, resulting in the following:

$$
\sqrt{A_{L} A_{R}}=G_{L} A_{0} \sqrt{\frac{G_{R}}{G_{L}} e^{-\frac{L}{\Gamma}}} \Rightarrow A_{0} \equiv E_{\mathrm{dep}}=\frac{1}{G_{L}} \frac{\sqrt{A_{L} A_{R}}}{\sqrt{\frac{G_{R}}{G_{L}} e^{-\frac{L}{\Gamma}}}} .
$$

The variable $A_{0}$ has been relabeled as $E_{\text {dep }}$ as an explicit reminder that the energy deposition in scintillator is the quantity that will be summed. The factor $\sqrt{\frac{G_{R}}{G_{L}} e^{-\frac{L}{\Gamma}}}$ is simply a number since $G \equiv G_{L} / G_{R}$ and $\Gamma$ have already been determined. Assuming that the total kinetic energy of the incident proton may be compared to the scintillator energy deposition sum, then the $\chi^{2}$ to minimize takes the following form:

$$
\chi^{2}=\sum_{i=1}^{N_{\text {events }}}\left(E_{\mathrm{prot}}^{i}-E_{\mathrm{dep}}^{i}\right)^{2}=\sum_{i=1}^{N_{\text {events }}}\left(E_{\mathrm{prot}}^{i}-\sum_{j=1}^{N_{\mathrm{na}}} \frac{1}{G_{L}} \sqrt{\frac{A_{L} A_{R}}{\frac{G_{R}}{G_{L}} e^{-\frac{L}{\Gamma}}}}\right)^{2},
$$

where $E_{\mathrm{prot}}^{i}$ is the total kinetic energy of the incident proton for event $i$ and $E_{\mathrm{dep}}^{i}$ is the total scintillator energy deposition. Due to clutter, the $j$ index has been intentionally suppressed from the second summation; however, the index is a book-keeping device designed to keep track of the bars making up a nucleon cluster of size $N_{\text {na }}$ for event $i$. A cluster bar must have left and right ADC signals to be considered. The expression may be minimized with respect to $1 / G_{L}$ in an identical manner as BigBite, and the result is 244 left gain factors; the right gain, or $G_{R}$, may then be unraveled from $G=G_{L} / G_{R}$. Using the results of the $\chi^{2}$-minimization, the scintillator energy deposition in simulation may be calculated as Eqn 5.43 where $A_{0}$ is in $\mathrm{MeV}$ and the gains are in $\mathrm{ADC} / \mathrm{MeV}$. 
As it turns out, though, the simulated ADC spectra are underestimated relative to the $\mathrm{H}_{2}$ coincidence data by a factor of 10 with this method. The large discrepancy originates with the fundamental assumption that the incident proton energy may be expressed solely as a sum of scintillator energy deposition. Scintillator gives a response proportional to the energy deposited by a charged particle through electronic excitation processes of the scintillator medium; however, not all energy in the complicated hadronic showering process is lost in this fashion. Furthermore, the detector has iron and lead plates in front of all neutron bars (see Figure 4.28), and a large percentage of the incident kinetic energy of the proton is in fact lost in the high- $Z$ materials. By only considering the energy deposition within scintillator, the $\chi^{2}$-minimization formalism is calculating gain coefficients assuming that the incident proton deposits all of its energy within the scintillating bar; therefore, the result of such an assumption yields ADC signals that are significantly smaller than expected.

An alternative method has been employed in order to circumvent the fundamental misunderstandings associated to the $\chi^{2}$ procedure, and may be summarized as signal peak-matching. By analyzing the $\sqrt{A_{L} A_{R}}$ spectra of the hydrogen coincidence data, the proton energy deposition signature may be extracted for all bars of the neutron detector, and then the signals in simulation may be scaled accordingly to replicate the data. Note that the $\sqrt{A_{L} A_{R}}$ distribution is more desirable than $A_{L}$ or $A_{R}$ alone as the signature is amplified, resulting in a distribution in which a Gaussian or Landau fit may be reliably performed. A fit to the $A_{L}$ or $A_{R}$ spectra is difficult due to background or low energy signals drowning the signal of interest. The result of the simple, and 

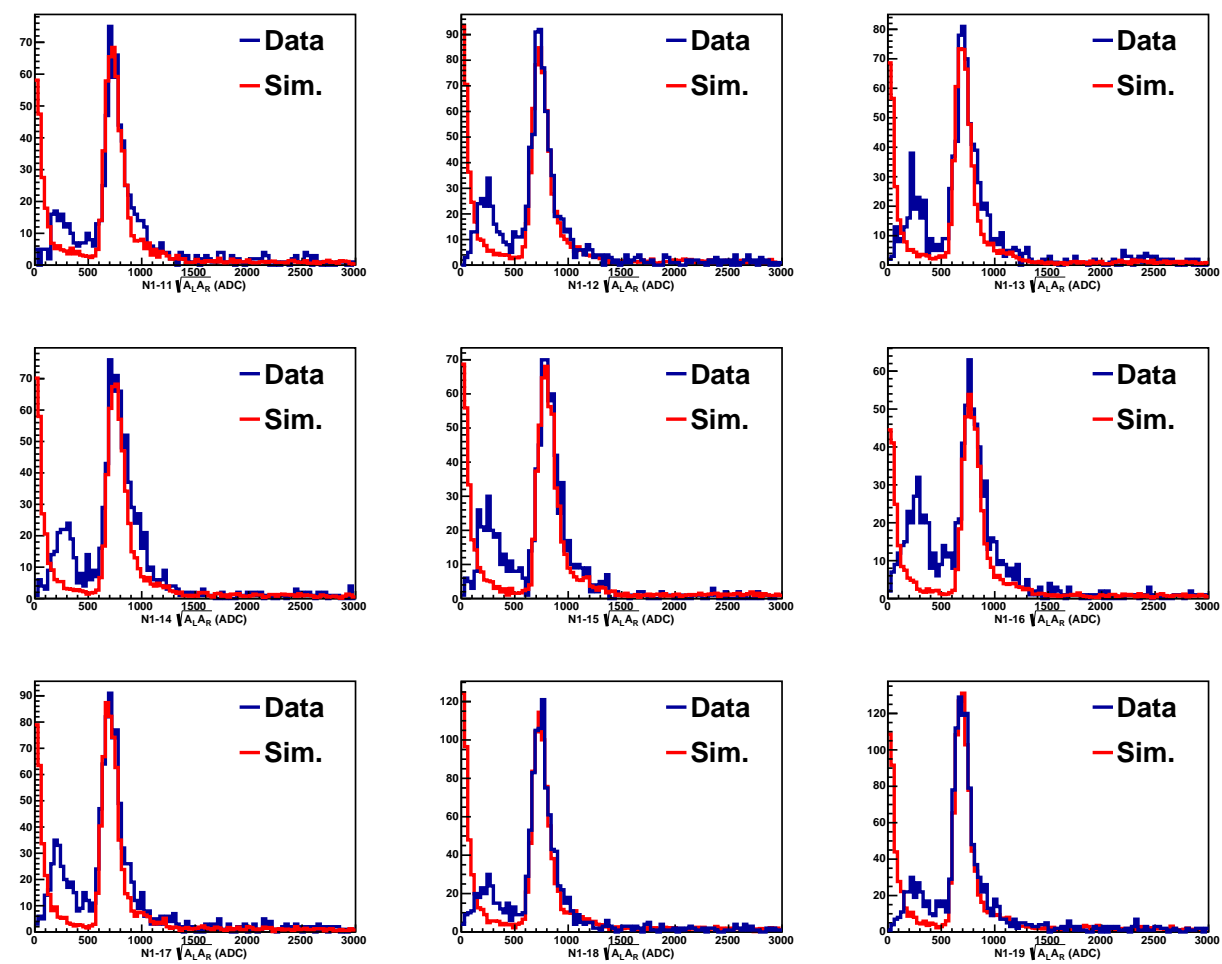

Fig. 5.47: Comparing the data to the simulation post peak-matching; the results for bars N1-11 through N1-19 are displayed.

perhaps crude, peak-matching analysis may be seen by Figure 5.47 in which only a selection of bars from the N1 plane are displayed. Note that the ESEPP generator* has been used to generate elastic ep events in which lowest-order QED radiative corrections to the Rosenbluth cross section are taken into account; the outgoing electrons and protons are propagated through the $G_{E}^{n}$ module of the G4SBS Monte Carlo package, see Section 4.10 for more information. The peak-matching coefficients may be thought of as effective gain factors and are used in place of $G_{L}$ and $G_{R}$ in Eqn 5.43 note that

\footnotetext{
${ }^{*}$ Initially discussed in Section 5.3.6 for reference see 212
} 

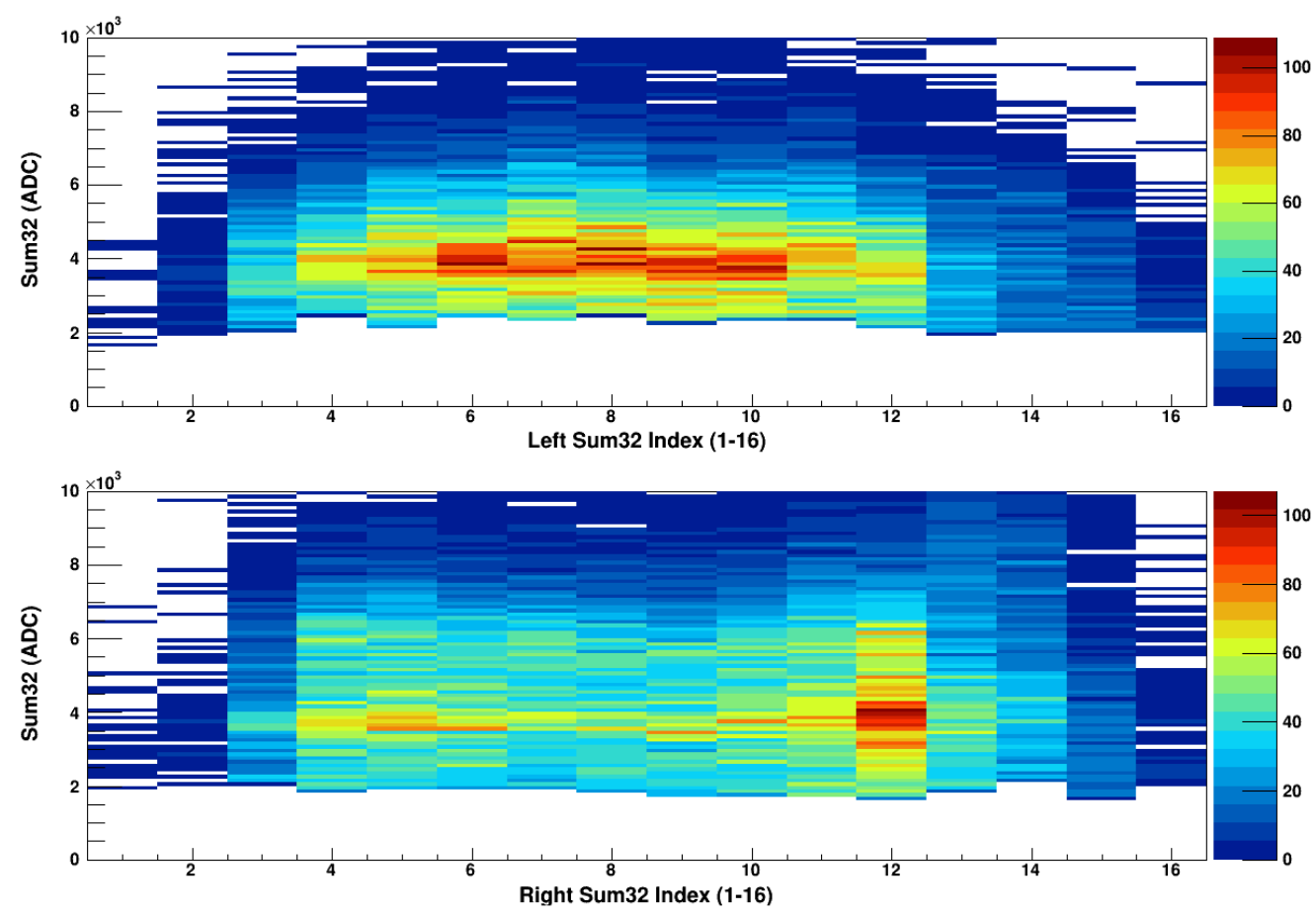

Fig. 5.48: Simulating the neutron detector left and right maximum sum 32 signals where the thresholds are motivated by Figure 5.46 .

the simulated ADC signals are still corrected for attenuation by the factor of $e^{\frac{-\frac{L}{2} \pm y}{\Gamma}}$. With the ability to simulate the response of the scintillator to Kinematic 1 protons, the ADC sum32s may be reproduced in order to simulate a T1 event. The simulated version of Figure 5.45, namely the simulated left and right maximum ADC sums of 32, may be seen by Figure 5.48. The thresholds are sum32-dependent, and if a particular sum32 for the left or right side is above threshold then a simulated T1 is generated. At this stage in the analysis, a coincidence T3 event may be simulated by a logical "AND" of a T1 and a T2 signal.*

\footnotetext{
* The generation of a T2 event in simulation is discussed in Section 5.3.6
} 
With the ability to simulate a T1 event, the hits of the neutron detector may be combined in order to simulate a nucleon cluster. Clustering is only performed on the N1-N7 planes, and the algorithm is divided into two phases. The first phase, namely the construction of a multihit, consists of combining scintillator bars with hits within a single neutron plane. The bar with the maximum energy deposition is chosen as the starting point, and adjacent bars are added to the multihit object until no more connected bars are found. The coordinate of a multihit is calculated using an energy deposition weighted sum, and the multihit time is taken to be the earliest hit time used to make up the object. The multihit construction is performed on all active neutron planes, and as a result the total number of possible multihits is 7 . Note that a multihit can range from 1 to several bars, and the only geometric constraint is that the active bar is connected to the bar of maximum energy deposition. The second phase of the algorithm consists of combining available multihits in order to construct a nucleon cluster object. The procedure takes the multihit with the minimum time as the starting point, and the remaining multihits are combined on a space and time criteria:

1. adjacent multihits are added if the vertical coordinate difference is $<10 \mathrm{~cm}$;

2. the time of the multihit must be within $10 \mathrm{~ns}$ of the minimum multihit time.

The multihits that pass the space and time criteria are combined and the result defines a nucleon cluster. The position of the cluster is calculated using an energy deposition weighted sum of multihit coordinates, and the time of the cluster is taken to be the minimum multihit time. The kinematics of the cluster, or more generally the neutron arm, are now fully reconstructable. For example, the path length may be calculated 

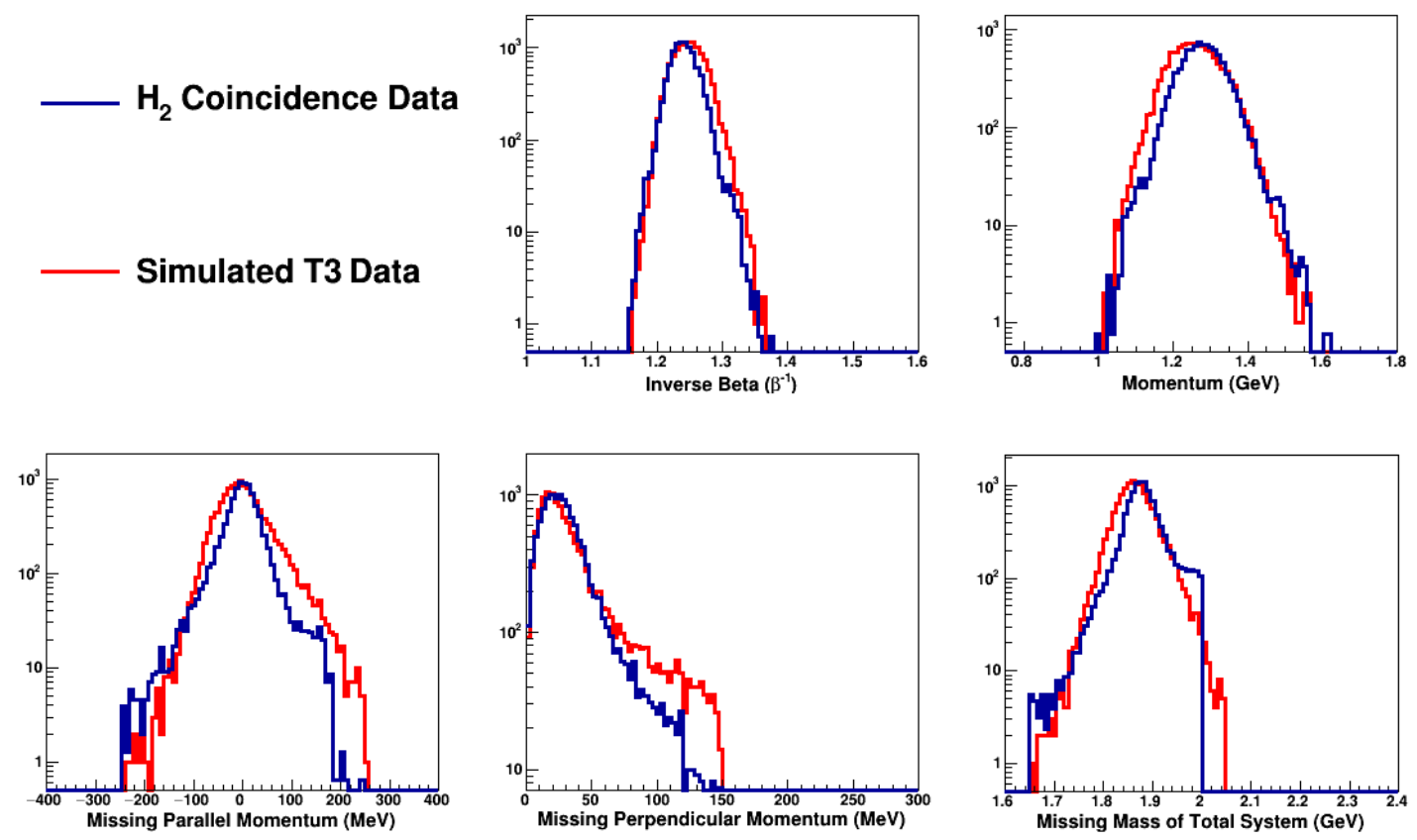

Fig. 5.49: The full simulation machinery compared to $\mathrm{H}_{2}$ coincidence data. Note that all plots are in a log scale, and see the text for the cut selection.

by taking the magnitude of the vertex-corrected cluster coordinate in Hall coordinates; $\beta$ and $p_{\text {na }}$ may be calculated using Eqn 5.38 where $t_{\text {ToF }}$ is taken to be the minimum cluster time. A comparison of the full simulation machinery, namely the generation of a T3 event using the realistic $G_{E}^{n}$ trigger logic and the ability to calculate all relevant quantities for all detectors, to the hydrogen coincidence data may be seen by Figure 5.49 . the following neutron arm specific kinematic variables are plotted: inverse $\beta$, the nucleon momentum, the missing momenta components and the missing mass of the system. The data and simulation have the full quasielastic cut selection applied, see Table 5.3 for the exhaustive list. Note that the missing mass variable is described more deeply in an upcoming section, Section 5.6. Furthermore, the purpose of the figure is to not 
discuss the quasielastic event selection, but to show the agreement between the simulated coincidence ESEPP + G4SBS data to the $\mathrm{H}_{2}$ coincidence data, both of which have all applicable cuts enforced such that an apples-to-apples comparison is possible. Only one simulation problem has yet to be addressed: nucleon charge identification.

Charge identification relies on time and space correlations between the reconstructed nucleon cluster and veto layer hits. Assuming that a T1 event has been generated, then the existence of a nucleon cluster in simulation is guaranteed. Therefore, all that remains is to check if there exists a veto hit within the space and time criteria as defined in Section 5.4.6. Additionally, if a veto hit is correlated to the nucleon cluster, the energy deposition within the veto bar must be greater than 10 ADC channels; this choice has been motivated by the data. If there is a space and time correlated hit in either veto plane (V1 or V2), then the nucleon cluster is assigned as charged; otherwise, the cluster is uncharged. Understanding the algorithm and detector performance in regards to charge identification is advantageous, and in particular will be of interest for the discussion of the corrections to the raw asymmetry, specifically the charge misidentification and inelastic corrections. The problem may be stated as follows: given protons (neutrons) that originated from the target, what fraction gets misidentified as neutrons (protons) by the neutron detector? In other words, what is the probability that a true proton (neutron) gets correctly assigned as charged (uncharged)? In order to answer this question, particles of a known type are generated in two separate simulations within the G4SBS framework:

1. protons - ESEPP generator [212], described throughout this section; and, 
2. neutrons - elastic generator with no radiative corrections utilizing a fictitious free neutron target.

Both simulations have the angle generation limits wide enough such that the detector acceptances are filled for this kinematic setting, and the beam energy has been set to $E_{\mathrm{b}}=1.519 \mathrm{GeV}$. A simulated T3 event is enforced in both simulations. The analysis then simply needs to keep track of the true particle and the result of the charge identification procedure in order to characterize the misidentification probabilities. The following probabilistic notation aids in the presentation of the results: $\eta_{\text {true }}^{\text {obs }}$ where the subscript "true" denotes the true particle or the particle that left the target, and the superscript "obs" which refers to how that particle is observed or identified by the neutron detector. Moreover, "true" and "obs" can be protons or neutrons, or $p$ and $n$ for short, resulting in four probabilistic combinations; for example, $\eta_{p}^{p}$ represents the probability that true protons are in fact assigned the correct charge by the detector and algorithm. The results of the charge identification study may be seen by Table 5.2. The proton (neutron) column represent the results of the proton and neutron simulations, respectively, in which the proton (neutron) are the true particles. The "true count" is simply the sum of the observations, and the $\eta$ parameters are ratios of the observations to the true counts. According to the proton simulation, protons are misidentified as neutrons roughly $2 \%$ of the time which is in strong agreement with the data.* The results are sensitive to the event type that is enforced, e.g. the presence of a $\mathrm{T} 1$ or T3 trigger. To be more specific, the proton misidentification probability rises to approximately $7 \%$ if

\footnotetext{
* The probabilities can also be estimated using the data, but the discussion is delayed until Section 5.7.5
} 


\begin{tabular}{cccc}
\hline Parameter & Proton $(\boldsymbol{p})$ & Neutron $(\boldsymbol{n})$ & Comments \\
\hline Observed as charged & $1.08 \times 10^{5}$ & $2.63 \times 10^{4}$ & Identified (ID) as charged \\
Observed as neutral & $2.15 \times 10^{3}$ & $4.35 \times 10^{4}$ & ID'ed as uncharged \\
True count & $1.10 \times 10^{5}$ & $6.99 \times 10^{4}$ & Sum of observations \\
$\eta_{x}^{n}$ where $x=p, n$ & $0.020 \pm 0.001$ & $0.623 \pm 0.002$ & ID'ed as $n$ over true $p$ or $n$ \\
$\eta_{x}^{p}$ where $x=p, n$ & $0.980 \pm 0.001$ & $0.377 \pm 0.002$ & ID'ed as $p$ over true $p$ or $n$ \\
\hline
\end{tabular}

Table 5.2: The results of the simulated charge identification study. The proton (neutron) column represent the results of the proton (neutron) simulations. Note that a T3 trigger event is enforced on both simulations.

the requirement of a T2 trigger is removed. Therefore, according to the simulation, the presence of a T1 and a T2 trigger is important in attempting to replicate the observed charge identification probabilities.

The remainder of the chapter will be dedicated towards analyzing the polarized ${ }^{3} \mathrm{He}$ data set, and it is comforting to know that a full simulation is available if necessary. As Kinematic 1 is more or less commissioning data, a careful and thorough analysis of the ${ }^{3} \mathrm{He}$ data set will be presented, particularly if data is excluded then a clear reason will be provided. The quasielastic cut selection will be motivated by analyzing the accepted ${ }^{3} \mathrm{He}$ data set, and lastly the formalism for calculating and correcting the raw asymmetry such that it most appropriately resembles the physical asymmetry defined by Eqn 2.107 will be presented. The final quantity of interest, namely the electric form factor of the neutron, may then be extracted from the fully corrected raw asymmetry. 


\subsection{Polarized ${ }^{3}$ He Run Selection}

As the low- $Q^{2}$ point occurred during the commissioning phase of $G_{E}^{n}$, a careful study of the ${ }^{3} \mathrm{He}$ data runs to determine an appropriate data set is in order. From a scientific point of view, all data runs should be included; however, as many detector systems were being brought online for the first time, problems and difficulties arose resulting in some data of little value for an asymmetry measurement. As a result, polarized ${ }^{3} \mathrm{He}$ runs for Kinematic 1 are discarded if and only if there is a justified and unbiased reason. The purpose of this section is to present some of the common problems faced by the early $G_{E}^{n}$ experiment, and to clearly describe the reasoning behind data run exclusion.

As with any commissioning period, experimental parameters and conditions may periodically change as the detector and detector response become more understood. Furthermore, the data as of 2018 is roughly 12 years old, and if a question arises in regards to the experimental run parameters then an analysis or a Hall A logbook entry is perhaps the only solution. For example, the high voltages of the drift chamber front and back cathodes have been changed multiple times in an attempt to increase the tracking efficiency while prolonging the lifespan of the wire planes. These changes can be seen by examining the BigBite trigger T2 rate. The BigBite total calorimeter trigger threshold has been changed several times, and one way to discover this change is to examine the preshower energy spectrum as a function of run number; the electron mean signal fluctuates depending on the value of the threshold. The NA trigger threshold has also been changed many times throughout Kinematic 1 in an attempt to optimize the neutron detection efficiency. Experimental changes are expected and are by no means 


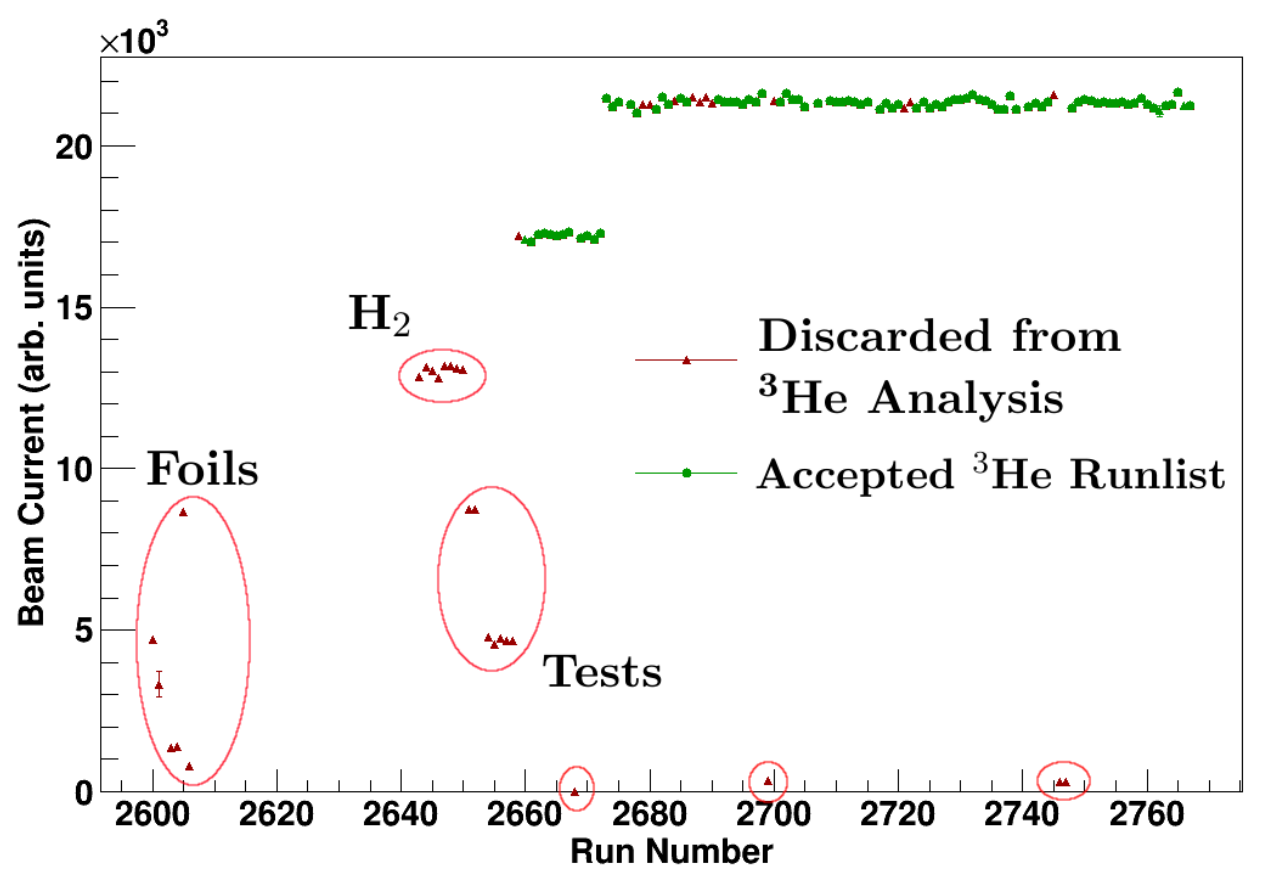

Fig. 5.50: Beam current data over the duration of the entire data set.

justification for data exclusion; however, there are examples of problems resulting in coincidence data that is not useful for a form factor extraction. As a first pass through the data files, an examination of the beam current monitor data can quickly determine if data was taken with poor beam quality, and may be seen by Figure 5.50 where the green markers represent the current ${ }^{3} \mathrm{He}$ data set. The jump in the ${ }^{3} \mathrm{He}$ data set, occurring around run number 2673 , is due to the collaboration requesting a larger beam current, namely an increase from $4 \mu \mathrm{A}$ to $5 \mu \mathrm{A}$ and is typical behavior for an experiment. While this procedure can remove obvious poor data quality runs, there are a handful of other runs that have been removed, denoted by the red markers, and must be justified.

An indicator of the performance over the duration of a data set may be explored 


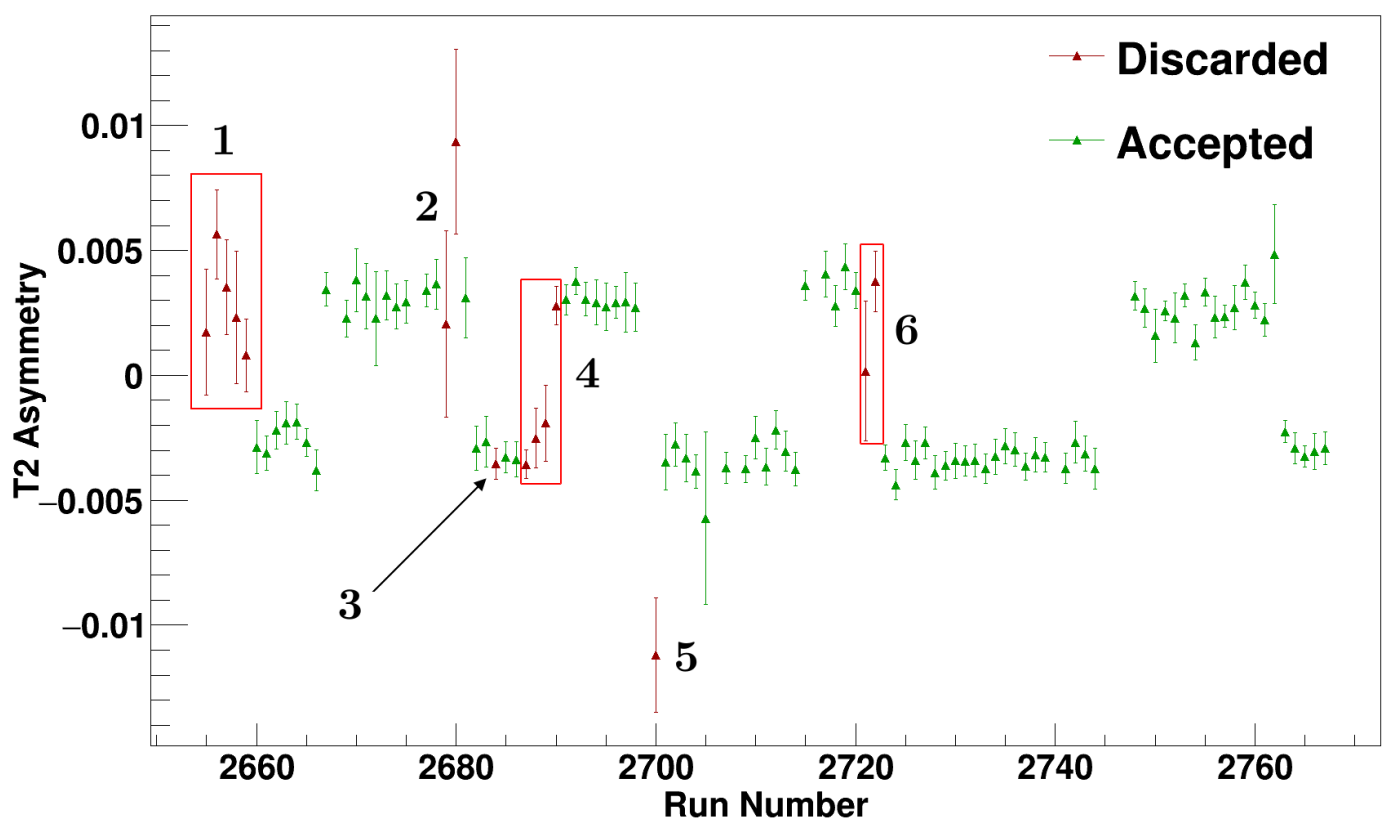

Fig. 5.51: BigBite helicity gated $\mathrm{T} 2$ asymmetry over the polarized ${ }^{3} \mathrm{He}$ data set. The sign of the asymmetry depends on the signs of the target polarization, beam precession, and the HWP.

by examining the BigBite helicity gated trigger asymmetry, defined as

$$
A_{\mathrm{T} 2}=\frac{\mathrm{T} 2^{+}-\mathrm{T} 2^{-}}{\mathrm{T} 2^{+}+\mathrm{T} 2^{-}}
$$

where $\mathrm{T} 2^{ \pm}$refers to the plus and minus helicity gated scaler counts, and may be seen by Figure 5.51. Note that the BigBite trigger logic, namely the generation of a T2 event, is introduced in Section 4.8.1. Among other things, the BigBite trigger is sensitive to an asymmetry associated to pion production; therefore, the asymmetry oscillates about zero where the sign depends on three factors: the sign of the target polarization, the beam precession sign and the sign of the half-wave plate (HWP). While the BigBite trigger alone is not an appropriate reason to exclude data, the overall sign of the asymmetry 
over the course of the ${ }^{3} \mathrm{He}$ data set may be monitored, specifically the status of the HWP as the other factors are constant for Kinematic 1. Additionally, Figure 5.51 serves as an appropriate visual tool aiding in an attempt to justify the red points which are currently discarded from the physics calculation due to various other reasons. Red data points are grouped into numbers, and have been excluded for the following reasons where the enumeration number corresponds to the group number in the plot:

1. suspicious trigger rates and initial production testing,

2. multi-wire drift chamber HV tripped,

3. BigBite and NA HV tripped,

4. a ND crate lost connection resulting in a number of channels that were off,

5. the small data file is corrupt, and

6. the ND HV tripped.

Common problems included HV trips where large sections of critical detectors were not functioning properly for a double-arm coincidence experiment, and occasionally the issue went unnoticed for consecutive runs. While the T2 asymmetry may determine an overall sign error, it is not adequate to see if the HWP changed during a run. If the HWP changed during data taking, then the run is simply discarded; this may be discovered by examining the EPICS HWP data as a function of time, more specifically the CODA event number, within a particular run. There are other useful diagnostic variables that exploit quasielastic expectations, but the presentation is delayed until after the description of the quasielastic selection. 


\subsection{Quasielastic Event Selection}

A major goal of the analysis is to mine through the polarized ${ }^{3} \mathrm{He}$ data and to select quasielastic events in order to calculate a raw asymmetry associated to the neutral sample. The following discussion assumes that BigBite tracks and nucleon clusters have been reconstructed within the coincidence window. Additionally, the beam must have a defined helicity state. Recall that the first 1000 events of every run are dedicated towards locating the correct position within the beam helicity sequence, and consequently are discarded as the helicity is unknown. Events that occur during a Pockels cell transition period result in a beam of an undefined helicity and are removed from the analysis. Events with a beam current below a certain threshold are removed, where the threshold

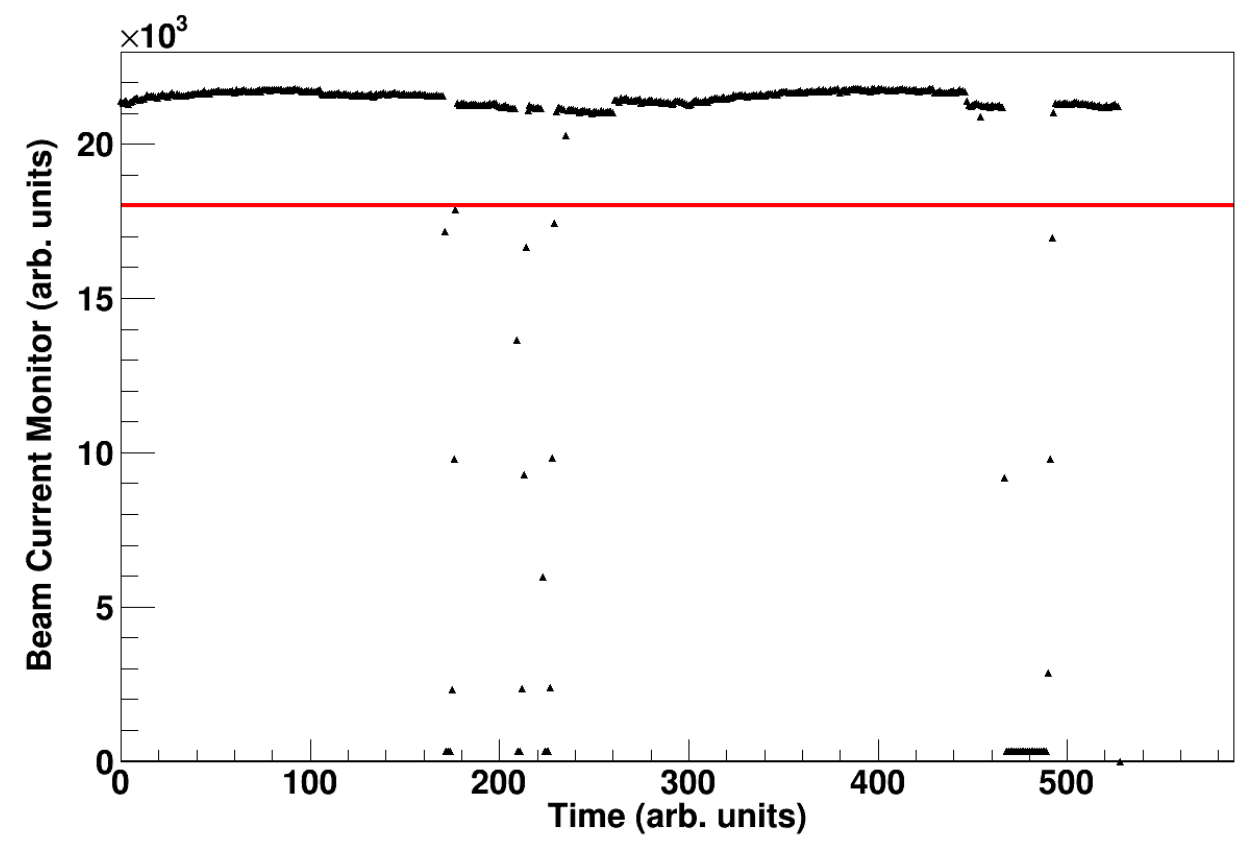

Fig. 5.52: Beam current as a function of time for an arbitrary ${ }^{3} \mathrm{He}$ run that requested a beam current of $5 \mu \mathrm{A}$. Events below the threshold are discarded. 
depends on the beam current requested by the experiment. This may be visualized by Figure 5.52, this behavior is normal and is a result of the accelerator beam tripping for a variety of technical reasons that are not relevant here.

Random background and asymmetry contamination need to be minimized while simultaneously maximizing the number of quasielastic events. A set of kinematic cuts may be introduced in order to preferentially select out the quasielastic region. The results of the elastic hydrogen data serve as a guide, and for example the invariant mass and the missing momentum have already been discussed in Section 5.3.4 and Section 5.4.5. Gross features of the ${ }^{3} \mathrm{He}$ quasielastic selection will look similar to the elastic $\mathrm{H}_{2}$ data, but significant Fermi broadening and contamination will be present. For convenience, the invariant mass and missing momentum components are

$$
\begin{aligned}
W^{2} & =\left(p_{\text {i,nuc }}+q\right)^{2}, \\
\Rightarrow W & =\sqrt{M^{2}-Q^{2}+2 M\left(E_{e}-E_{e}^{\prime}\right)}, \\
p_{\text {miss }, \|} & =\left(\vec{q}-\vec{p}_{\text {na }}\right) \cdot \hat{q}, \\
p_{\text {miss }, \perp} & =\left|\vec{q}-\vec{p}_{\text {na }}-p_{\text {miss }, \|} \hat{q}\right|,
\end{aligned}
$$

where $q$ is the four momentum transfer as determined by BigBite and $\vec{p}_{\text {na }}$ is the nucleon momentum determined by the NA through a ToF measurement. In the expression for the invariant mass, the initial nucleon of four momentum $p_{\mathrm{i} \text {,nuc }}$ is assumed to be at rest, i.e. $p_{\mathrm{i}, \mathrm{nuc}}=(M, \overrightarrow{0})$. Lastly, the missing mass of the hadronic system is introduced:

$$
m_{\text {miss }}^{2}=\left(P_{i,{ }^{3} \mathrm{He}}+q-p_{\mathrm{na}}\right)^{2},
$$

where $P_{i,{ }^{3} \mathrm{He}}$ is the initial four momentum of the ${ }^{3} \mathrm{He}$ nucleus which is assumed to be free 
and at rest. The missing mass represents the mass that is not measured; therefore, a quasielastic event should have an unmeasured mass of two times the mass of the nucleon for a ${ }^{3} \mathrm{He}$ target. Choosing an appropriate set of cuts on the invariant mass, the missing momentum and the missing mass is a powerful technique to select the quasielastic peak while suppressing the background and unwanted events originating within the resonance or inelastic region.

Recall that in the context of $\mathrm{H}_{2}$ data, the missing momentum components may be used to determine the resolution of the neutron detector; the time resolution may be computed from $p_{\text {miss, } \|}$ and the vertical/horizontal resolutions may be determined from $p_{\text {miss }, \perp}$. For the ${ }^{3}$ He data, though, it is more useful to think of the missing momentum components in regards to Fermi motion; the analysis assumes that the nucleons within the nucleus are initially at rest, but this is not entirely correct. There are longitudinal and transverse momentum components of the initial nucleon, and as a result there is a mismatch between the BigBite optics reconstructed $\vec{q}$ and the momentum of the nucleon obtained by the neutron arm via ToF. The missing momentum components characterize the mismatch, and may be conceptually realized by Figure 5.53. In the diagram, the vector $\vec{q}$ points to the center of a fictitious circle which only represents the area that may be swept by the missing momentum vector $\vec{p}_{\text {miss }}$. Due to the binding energy of ${ }^{3} \mathrm{He}, \vec{q}$ should actually point to the center plus an additional offset, but this is not diagrammatically shown to avoid clutter. The component $p_{\text {miss, } \|}$ can be aligned or antialigned with $\vec{q}$, and consequently the distribution may extend into the $p_{\text {miss, } \|}<0 \mathrm{MeV}$ region. The parallel component of the missing momentum takes a 


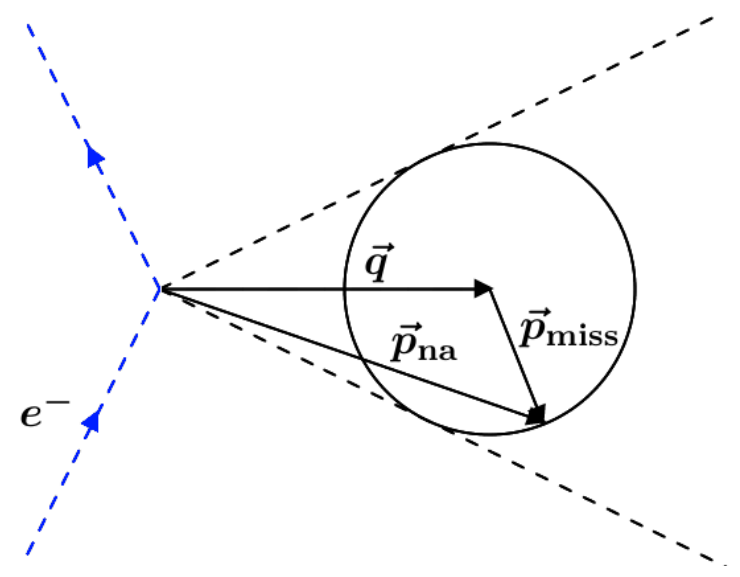

Fig. 5.53: A conceptual and exaggerated diagram displaying the relevance of missing momentum to the Fermi motion within a ${ }^{3} \mathrm{He}$ nucleus.

Gaussian-like form with a mean that is positively offset due to the binding energy of ${ }^{3} \mathrm{He}$, and is broadened by the Fermi motion. On the other hand, the perpendicular component, or the measure of the initial transverse momentum of the nucleon, is always positive. The distribution must start at zero corresponding to the situation where $\vec{q}$ and $p_{\text {miss, } \|}$ are aligned or antialigned. The transverse maximum mismatch, namely when $p_{\text {miss }, \|}=0$, then must be enhanced for the ${ }^{3} \mathrm{He}$ data relative to the hydrogen data. The missing momentum components for the ${ }^{3} \mathrm{He}$ data may be seen by Figure 5.54, and the chosen cuts are $-250<p_{\text {miss }, \|}<250 \mathrm{MeV}$ and $p_{\text {miss }, \perp}<150 \mathrm{MeV}$.

A cut on the missing mass for the reaction ${ }^{3} \overrightarrow{\mathrm{He}}\left(\vec{e}, e^{\prime} n\right) X$ is introduced to suppress inelastic events originating from pion electroproduction. While this cut is partially redundant to $p_{\text {miss }, \|}$, a cut of $2 M+m_{\pi} \approx 2 \mathrm{GeV}$ is enforced corresponding to the minimum mass necessary for pion production. The missing mass distribution of the ${ }^{3}$ He data may be seen by Figure 5.55 where a large quasielastic peak is seen to occur 

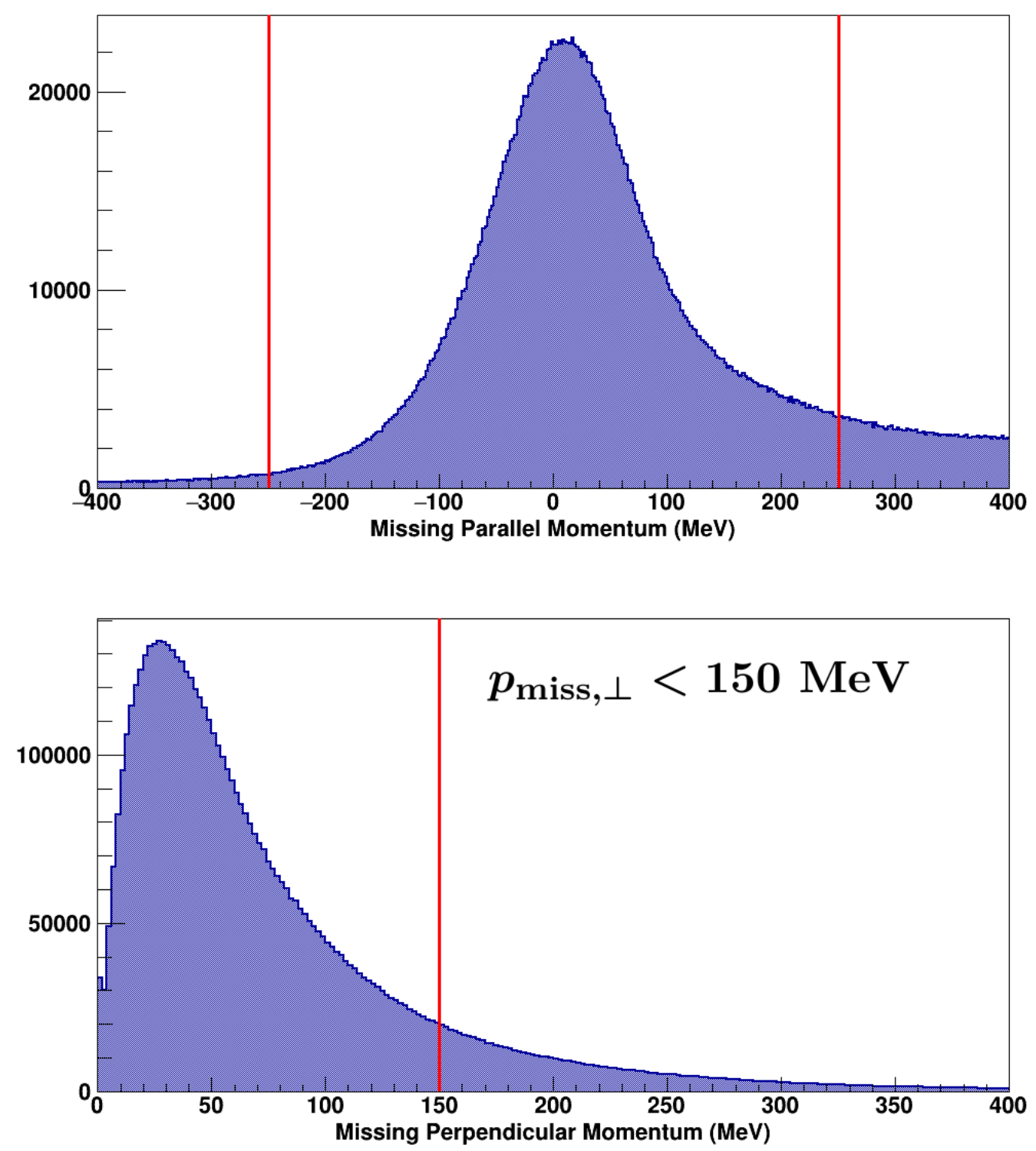

Fig. 5.54: The missing momenta components for the polarized ${ }^{3} \mathrm{He}$ data where significant Fermi broadening may be seen compared to the hydrogen data. The parallel component is not exactly centered at zero as the nucleon requires additional energy to be liberated from the ${ }^{3} \mathrm{He}$ nucleus. The chosen missing momenta cuts are $-250<p_{\text {miss }, \|}<250 \mathrm{MeV}$ and $p_{\text {miss }, \perp}<150 \mathrm{MeV}$. 


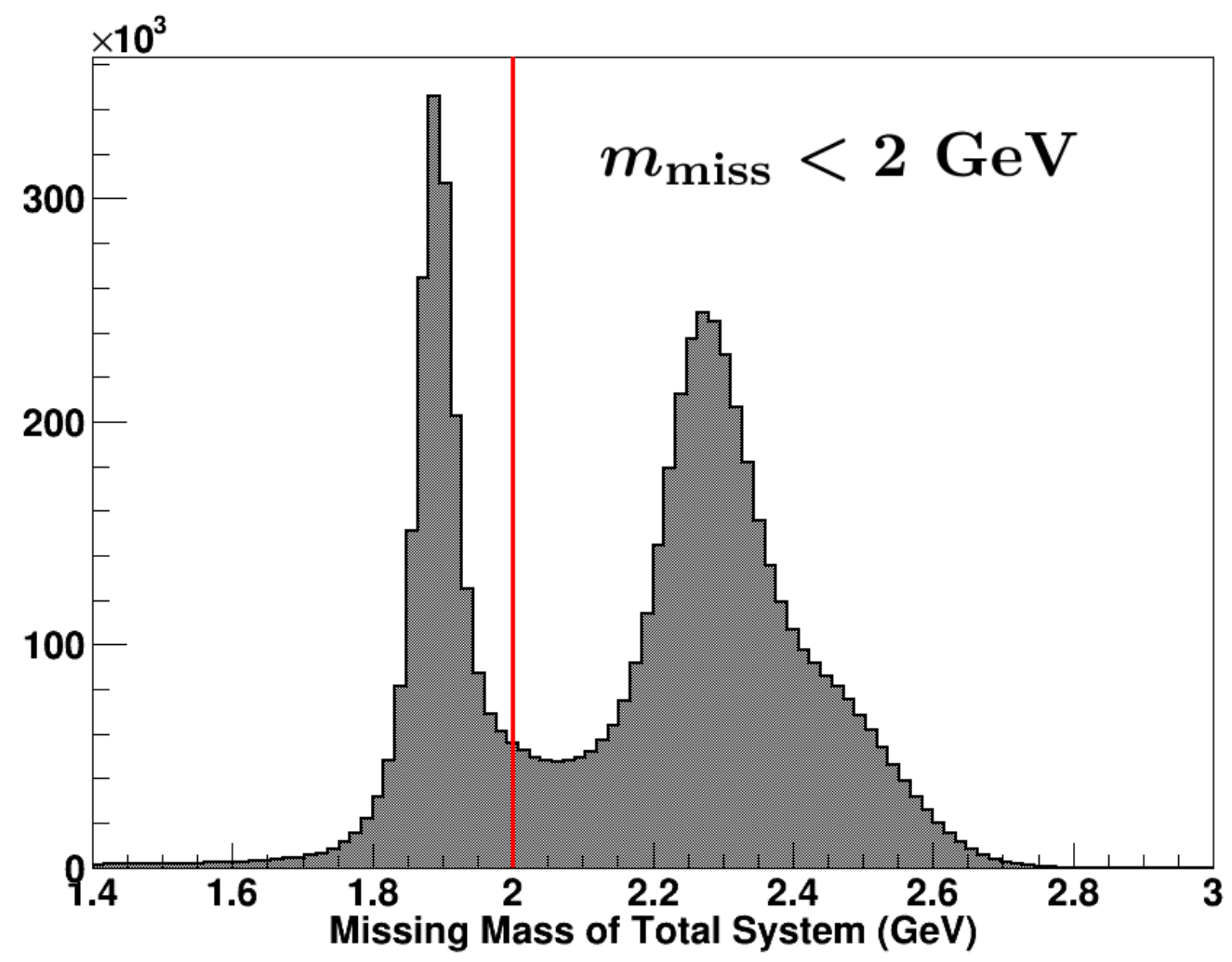

Fig. 5.55: The missing mass spectrum for the ${ }^{3} \mathrm{He}$ data set. A cut of $m_{\text {miss }}<2$ $\mathrm{GeV}$ is introduced in order to suppress inelastic events associated with pion electroproduction.

at two nucleon masses. The second peak around $m_{\text {miss }}=2.3 \mathrm{GeV}$ largely corresponds to low energy pile-up within the neutron arm likely due to the trigger gate width or a NA threshold bias. There is redundancy between the missing mass and the $p_{\text {miss, } \|}$ cuts with respect to the low energy events; these events effectively have large $p_{\text {miss, }, \|}$ values which are removed with a missing momentum cut. The shoulder of this peak, however, is a result of inelastic events that must be suppressed.

The invariant mass $W$, or the final QE cut, has a Fermi smeared peak correspond- 
ing to the mass of the nucleon $M$, and a shoulder due to the $\Delta(1232)$ resonance and inelastic events that extends into the higher $W$ regime. The $M$ peak, however, is offset relative to the hydrogen data due to the binding energy of the ${ }^{3}$ He nucleus. The binding energy per nucleon within the ${ }^{3} \mathrm{He}$ nucleus is on the order of $2.5 \mathrm{MeV}$, and consequently quasielastic scattering requires more energy than the elastic case. In order to suppress the resonance and inelastic region, an invariant mass cut of $0.8<W<1.15 \mathrm{GeV}$ is introduced into the analysis, and may be seen by Figure 5.57. In passing, it is also noted that an energy deposition cut on the BigBite preshower is also enforced, and has been introduced within section Section 5.3.5. A summary of all chosen cuts may be seen by Figure 5.56 in which the preshower energy cut has been applied. The bottom left panel displays $W$ vs. $m_{\text {miss }}$, and quasielastic events clearly show up at a $W=M$ and $m_{\text {miss }}=2 M$. Additionally, the low energy events at roughly $2.3 \mathrm{GeV}$ may been seen to show up at an invariant mass of $W=M$ further demonstrating the need for multiple kinematic cuts for QE selection; the band extends to higher $W$ which is the inelastic shoulder of the $2.3 \mathrm{GeV}$ peak. The correlation between $W$ and $p_{\text {miss, } \|}$ seen in the top left panel may be understood by the vector diagram in Figure 5.53 if $p_{\text {miss, }, \|}$ is parallel (antiparallel) to $\vec{q}$ then less (more) energy or $W$ is required for quasielastic scattering. Note that the hydrogen analog of this plot observes no such correlation.

As the invariant mass spectrum is a good indicator of the quality of the quasielastic event selection, the distribution may be seen before and after applying quasielastic cuts within Figure 5.57. In this case, the purple distribution is the $W$ spectrum with vertex and preshower energy cuts applied, and the black distribution is the result of 

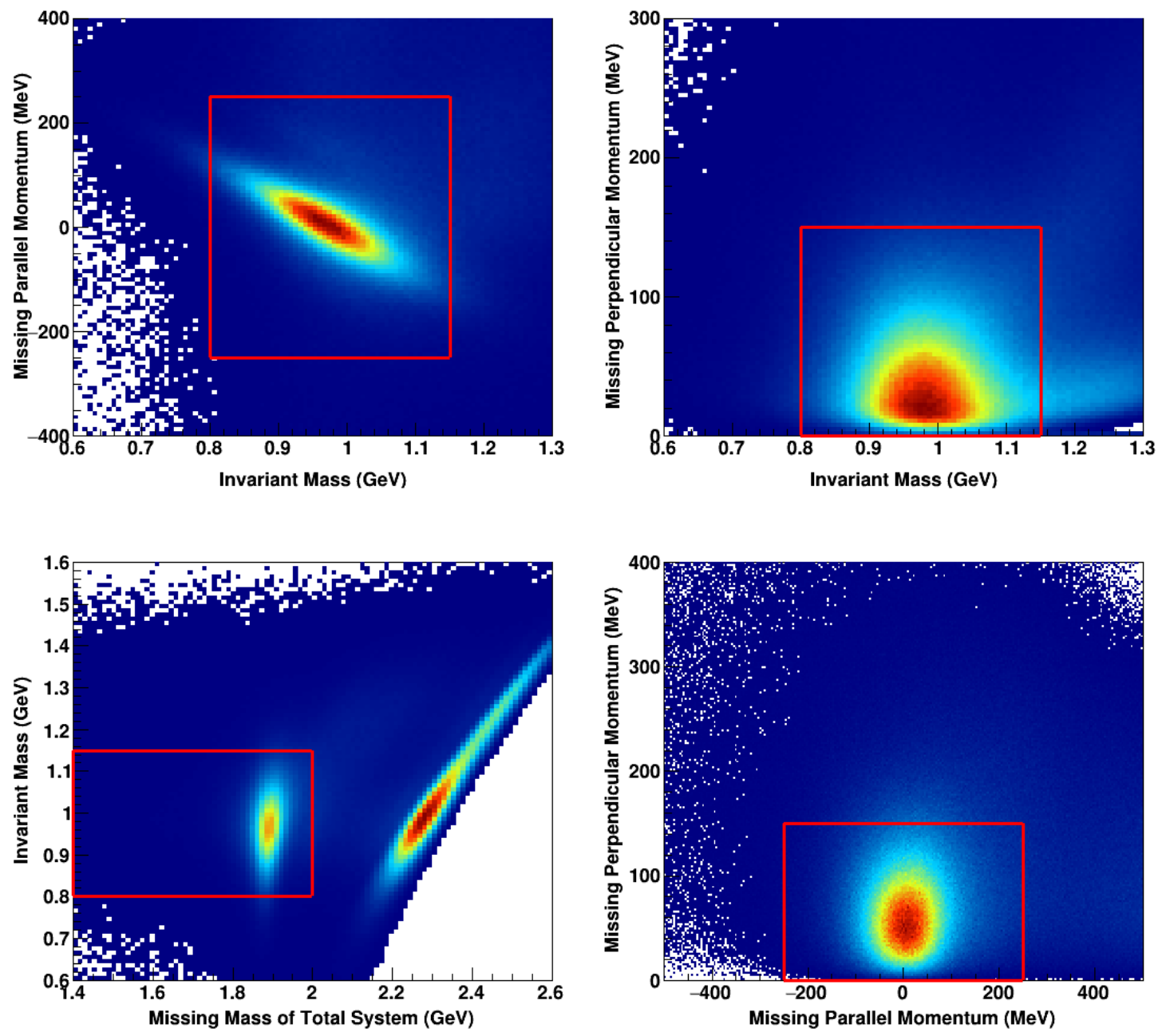

Fig. 5.56: The invariant mass $\mathrm{W}$, the missing momentum, and the missing mass are the main kinematic variables used to select the quasielastic peak. The red boxes represent the chosen cuts, and the data within these cut regions is the quasielastic selection used in the calculation of the raw asymmetry. 

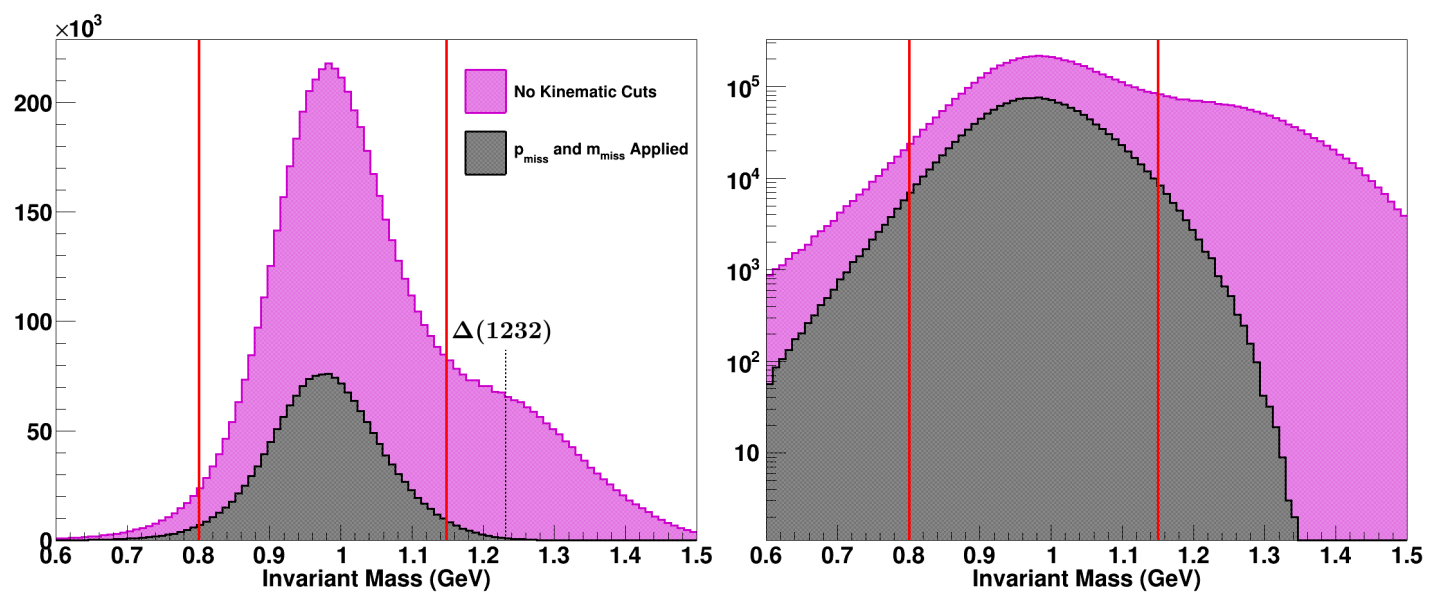

Fig. 5.57: The $W$ spectra before and after major quasielastic cuts, and highlighting the fact that the inelastic region is highly suppressed post cuts. The right panel is displaying the left panel in a log scale to see the high $W$ behavior.

applying the missing momentum and missing mass cuts in addition to the cuts of the purple distribution. The quasielastic cut selection heavily suppresses the inelastic region; therefore, the inelastic correction to the raw asymmetry is expected to be small at this $Q^{2}$. The effort to rigorously quantify this expectation is presented within Section 6.1.7 according to the simulation the events that populate the large $W$ shoulder of the black quasielastic distribution may be almost fully explained by elastic radiative corrections, a coincidence trigger, and the charge identification algorithm. Lastly, selected kinematic distributions at each stage of the quasielastic cut selection may be seen by Figure 5.58. The stages are described by the legend in the top right panel, and is to be understood as all cuts at and above the current color are applied. For example, the green distribution is a result of applying a track fiducial cut, a preshower cut, and the missing momentum cuts. The final quasielastic selection may be visualized by the 

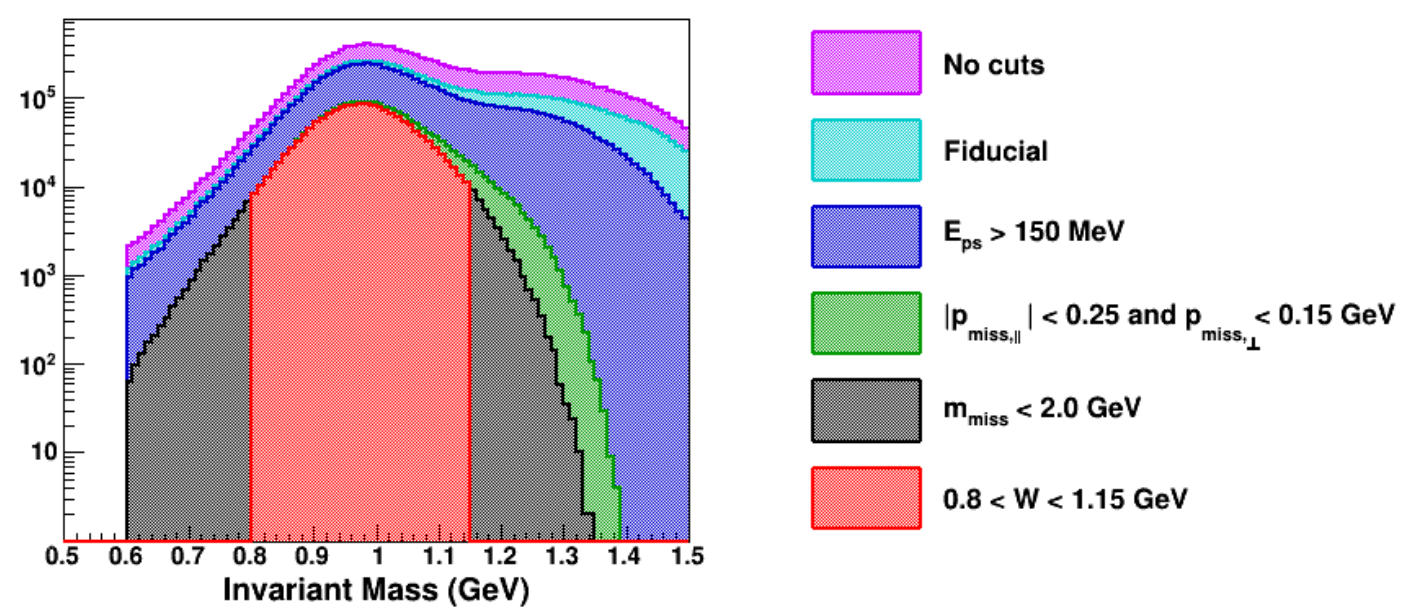

$0.8<\mathrm{W}<1.15 \mathrm{GeV}$
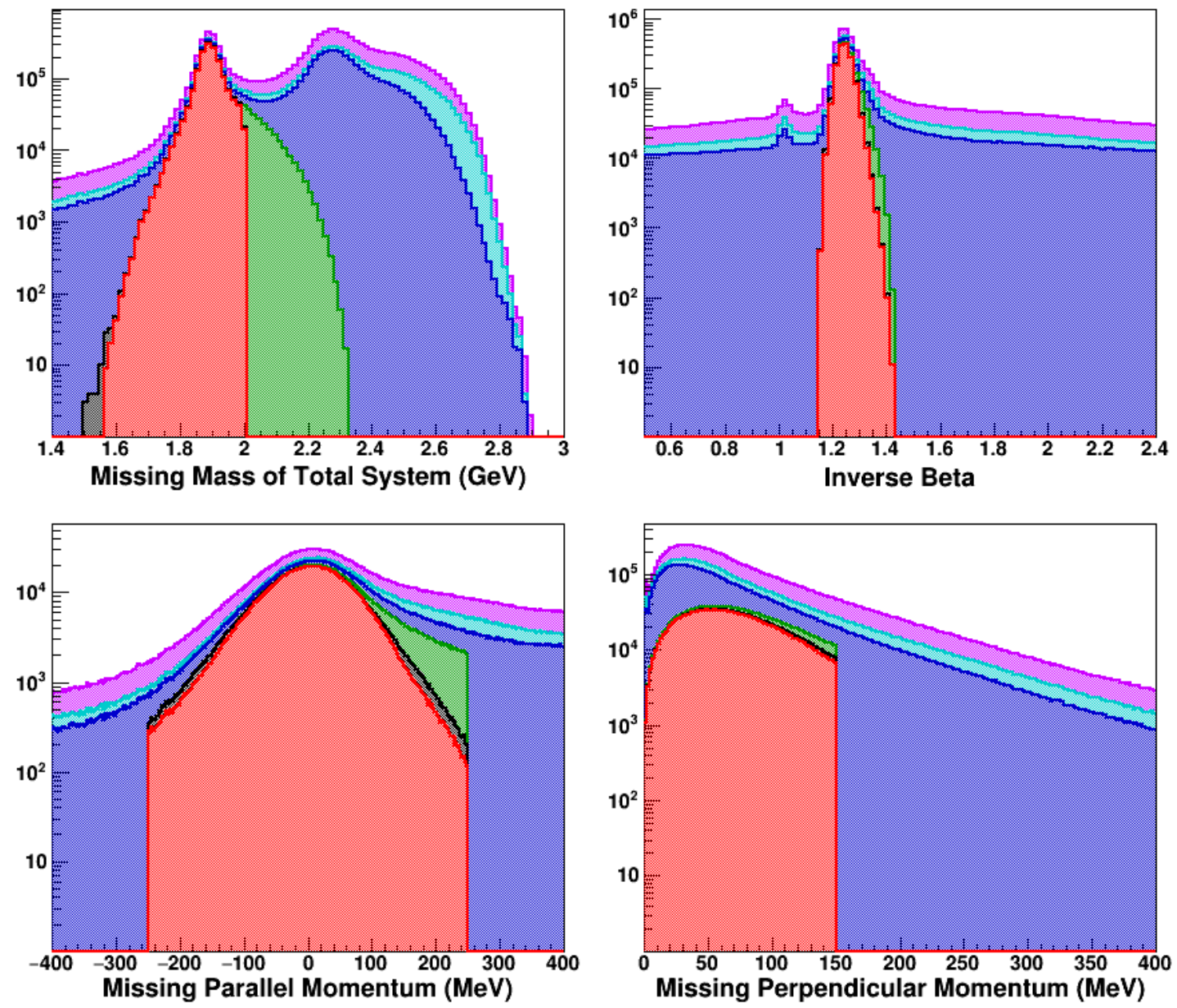

Fig. 5.58: Selected kinematic distributions at each stage of the quasielastic cut selection. The final quasielastic region after all cuts have been applied is represented by the shaded red region. 


\section{Quasielastic Cut Description}

\begin{tabular}{cc}
\hline$-0.17<v_{z}<0.17 \mathrm{~m}$ & Vertex $z$ position to remove target windows \\
Fiducial & Reconstructed track must end up in detector \\
$E_{\mathrm{ps}}>150 \mathrm{MeV}$ & Preshower cluster sum to suppress BigBite pions \\
$-250<p_{\text {miss }, \|}<250 \mathrm{MeV}$ & Parallel missing momentum to suppress inelastics \\
$p_{\text {miss }, \perp}<150 \mathrm{MeV}$ & Perpendicular missing momentum, FSI suppression \\
$m_{\text {miss }}<2 \mathrm{GeV}$ & Missing mass to suppress pion electroproduction \\
$0.8<W<1.15 \mathrm{GeV}$ & Invariant mass to suppress the inelastic region \\
\hline
\end{tabular}

Table 5.3: Summary of the chosen cuts used to select the ${ }^{3}$ He quasielastic peak.

red distribution after all the cuts that have been presented in this section have been applied. A summary of all quasielastic cuts used in the subsequent analysis may be found within Table 5.3, and henceforth will be referred to as the quasielastic selection.

As the quasielastic selection has been defined by the cuts in Table 5.3, there are additional quantities that may be examined in order to improve or solidify the ${ }^{3} \mathrm{He}$ run selection outlined in Section 5.5. Figure 5.59 displays the ratio of quasielastic events $N$ to the total accumulated beam charge $Q$ for the currently accepted ${ }^{3} \mathrm{He}$ data set. Experimental changes are clearly seen by the two jumps in the data, labeled 1 and 2, as well as the two outlier data points. The first step, denoted by 1 , in the ratio $N / Q$ is likely due to the trigger threshold of the ND being reduced by roughly $30 \%$; therefore, the ratio increases as more quasielastic events are accepted. The second step may be attributed to a $20 \%$ reduction in the trigger threshold of the BigBite calorimeter. One 


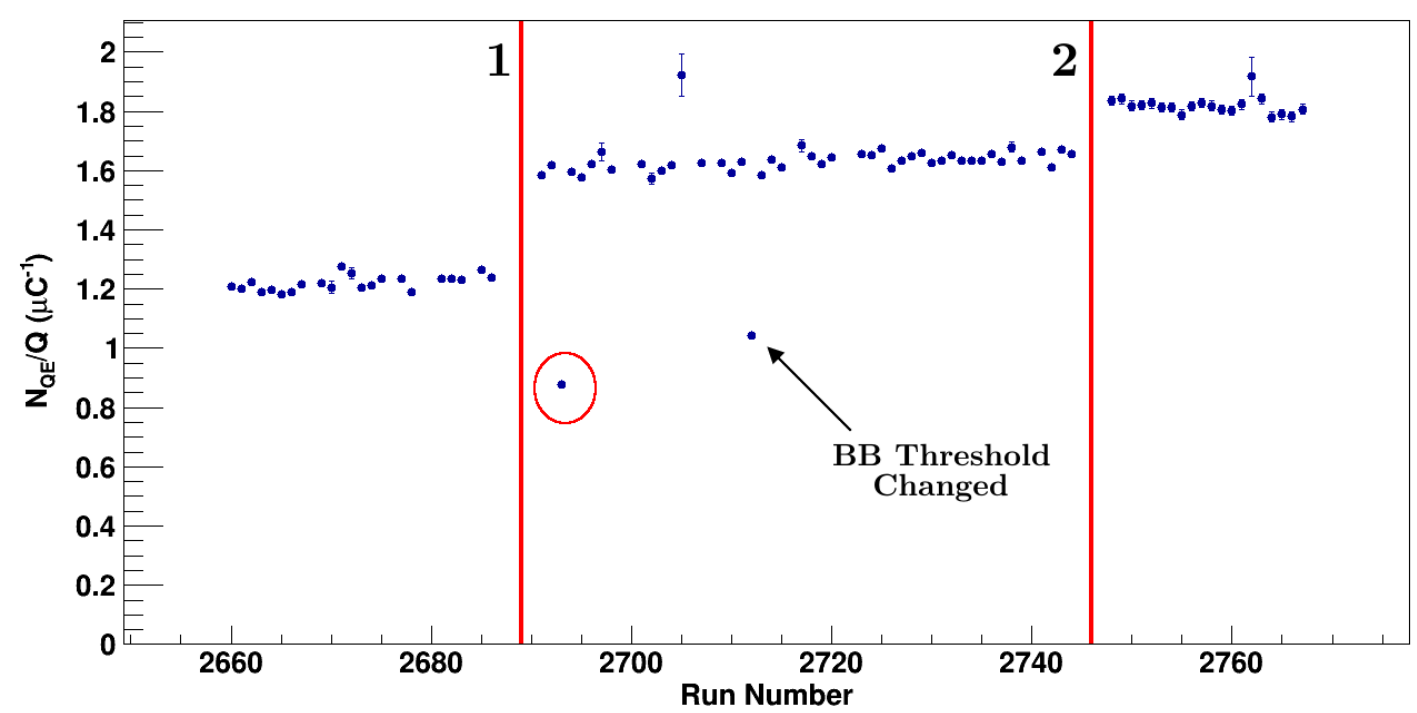

Fig. 5.59: The ratio of quasielastic counts $N$ to the accumulated beam charge $Q$ for the currently accepted ${ }^{3} \mathrm{He}$ data set.

of the outlier points is likely due to a single change in the BigBite threshold and is acceptable to keep within the ${ }^{3} \mathrm{He}$ data set. The remaining outlier which has been circled in red has suspicious BCM and Unser data and may possibly be discarded.

Recall that ${ }^{3} \mathrm{He}$ has two protons and one neutron, and as a result recorded quasielastic events consist of both charged and uncharged events depending on the nucleon cluster-veto charge identification procedure. While an asymmetry related to the neutron will inevitably be calculated, both types of quasielastic events are kept as the information is absolutely critical. The ratio of uncharged to charged quasielastic events is a useful parameter as it should be stable over the duration of the data set as seen by Figure 5.60, and note that the questionable outlier circled in red in Figure 5.59 has been included. The expected ratio may be estimated from the differential 


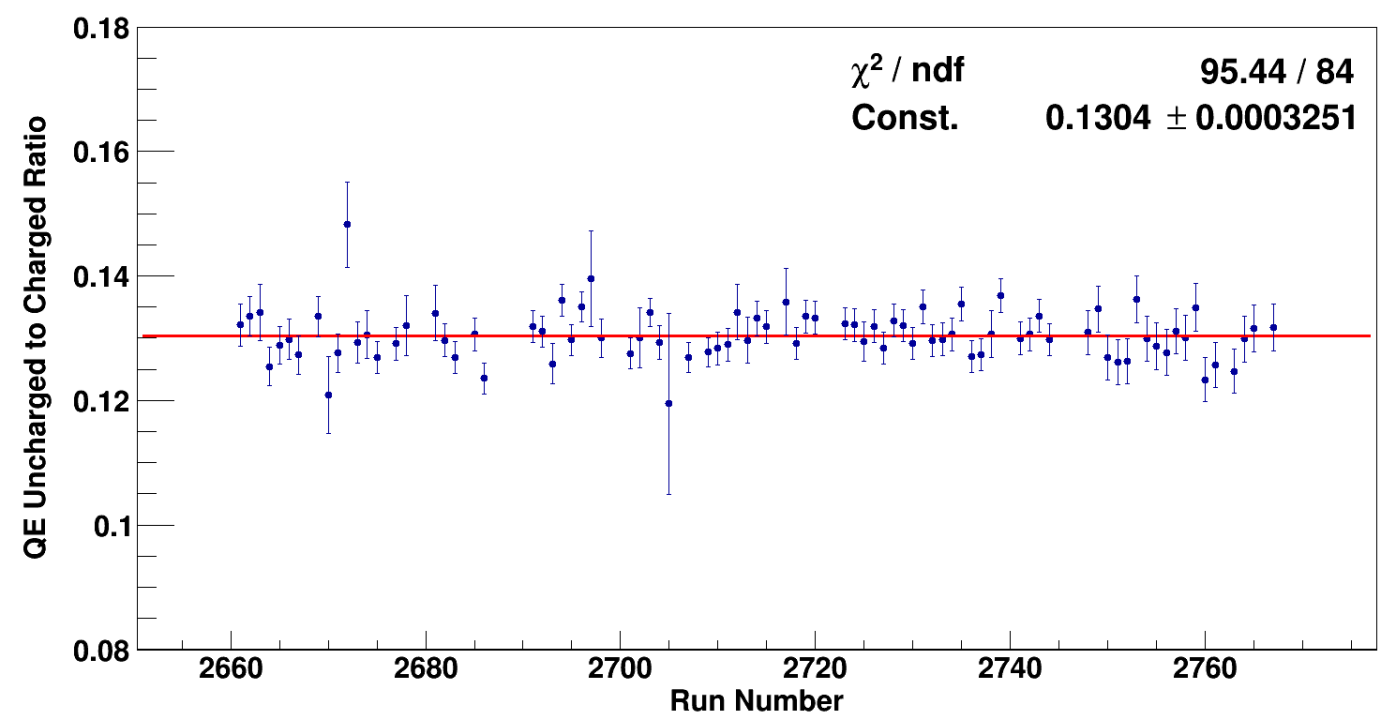

Fig. 5.60: The quasielastic uncharged to charged ratio for the accepted ${ }^{3} \mathrm{He}$ data set.

cross section of free protons and neutrons evaluated at the central values (Eqn 2.44), resulting in $R=\frac{1}{2} \frac{d \sigma_{n}}{d \sigma_{p}} \sim 0.2$. Note that this is simply an estimation as the nucleons are in reality bound within a nucleus, and the quasielastic cuts, specifically the $p_{\text {miss }}$ cuts, non-trivially reduces the ratio of neutrons to protons within the ${ }^{3} \mathrm{He}$ nucleus from the naive expectation of $\frac{1}{2}$. The observed stability in the uncharged to charged ratio over the currently accepted ${ }^{3} \mathrm{He}$ data set provides additional encouragement in the data quality which will be used in subsequent calculations. The major tasks of the initial analysis are now complete, or in other words event reconstruction is reliable, an algorithm to identify the recoiling nucleon has been presented, and lastly the quasielastic cut selection has been defined. The remainder of this thesis is devoted towards calculating and correcting the experimental raw asymmetry such that the neutron form factor ratio $G_{E}^{n} / G_{M}^{n}$ may be extracted. 


\subsection{Experimental Asymmetry Formalism}

The goal of the analysis is to extract the Sachs form factor ratio of the neutron, or $G_{E}^{n} / G_{M}^{n}$. The theoretical basis of the experiment, namely the physical asymmetry $A_{\text {phys }}$, is given by Eqn 2.107. The physical asymmetry may be decomposed into two components, $A_{\text {phys }}=A_{\perp}+A_{\|}$, and at leading order the perpendicular component is proportional to the form factor ratio under specific experimental conditions.* The calculation of the physical asymmetry assumes many ideal conditions and the most important are summarized as follows:

1. only the neutron is considered $\Rightarrow$ no ${ }^{3} \mathrm{He}$ nucleus or contaminations are present,

2. the polarizations of the incoming particles in the reaction $\vec{n}\left(\vec{e}, e^{\prime} n\right)$ are $100 \%$,

3. no HWP or beam precession to flip the sign of the asymmetry.

The asymmetry measured in experiment contains contaminations ${ }^{\dagger}$ false asymmetries and a neutron bound within a nucleus; therefore, the analysis must systematically correct the measured asymmetry such that the result most closely resembles that of a free neutron asymmetry, which allows for a comparison to theory and an extraction of the form factor ratio.

For the sake of clarity, the data that remains after the quasielastic cuts defined in Section 5.6 are referred to as the quasielastic sample. The quasielastic sample may then be divided into two categories, charged (proton) and neutral (neutron), depending on the cluster-veto charge identification. While the subsequent discussion also applies

\footnotetext{
* Recall that the target polarization must be perpendicular to $\vec{q}$ but within the reaction plane.

$\dagger$ The word contamination and dilution will be used interchangeably throughout the analysis.
} 
to the quasielastic charged sample, all variables and focus will be directed towards the quasielastic neutral sample unless explicitly stated otherwise. There are three important asymmetries associated to the quasielastic neutral sample: the measured asymmetry $\left(A_{\text {meas }}\right)$, the raw asymmetry $\left(A_{\text {raw }}\right)$ and the desired neutron asymmetry $\left(A_{\mathrm{n}}\right)$. The purpose of this section is to differentiate the three asymmetries and to bridge the gap between the theoretical physical asymmetry and the asymmetry measured by an experiment.

The asymmetry associated to the quasielastic neutral sample is known as the measured asymmetry and is denoted by $A_{\text {meas }}$. In complete analogy to the T2 asymmetry seen by Figure 5.51, the measured asymmetry changes sign (positive or negative) as a function of run number due to three factors: the target sign, the half-wave plate (HWP) state and the beam precession sign. Correcting the sign of the measured asymmetry results in the raw asymmetry:

$$
A_{\text {raw }}=\left(S_{\text {targ }} S_{\text {HWP }} S_{\text {beam }}\right) \cdot A_{\text {meas }},
$$

where $S_{\mathrm{targ}}$ is the sign of the target and depends on the polarization direction, $S_{\mathrm{HWP}}$ is the sign of the HWP and depends on whether it is inserted or removed and $S_{\text {beam }}$ is the sign of the beam precession and depends on the orientation of the Wien filter. Each factor is either \pm 1 , therefore the product $\left(S_{\text {targ }} S_{\text {HWP }} S_{\text {beam }}\right)$ determines the overall sign of the measured asymmetry. The only factor that changes and consequently alters the sign of the measured asymmetry is the state of the half-wave plate which may be explicitly seen by Figure 5.61 .

The raw asymmetry, i.e. the sign-corrected measured asymmetry of the quasielas- 


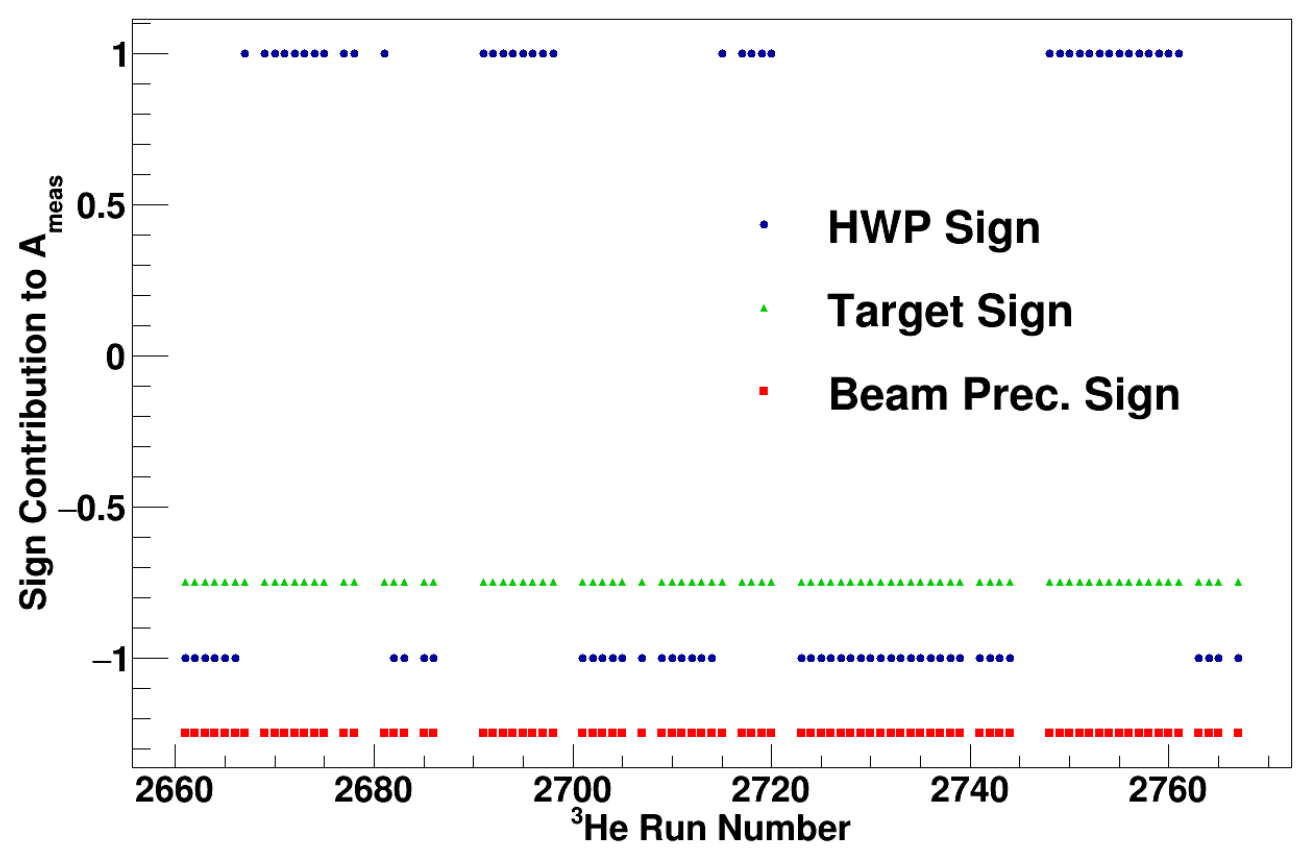

Fig. 5.61: The factors as a function of accepted ${ }^{3} \mathrm{He}$ run number that determine the sign of $A_{\text {meas }}$. Only the sign matters, not the value; therefore, the overall sign of $A_{\text {meas }}$ is determined by the insertion or removal of the HWP.

tic neutral sample, may then be formed by counting particles of a particular helicity state:

$$
A_{\text {raw }}=\frac{N^{+}-N^{-}}{N^{+}+N^{-}}
$$

where $N^{ \pm}$refers to the quasielastic neutral count of helicity + or - . As the events occur randomly in time at a constant rate, the expected count is assumed to form a Poisson distribution; therefore, the uncertainty of a count $N$ is $\delta N=\sqrt{N}$. As a result, the uncertainty of the raw asymmetry is

$$
\delta A_{\mathrm{raw}}=\frac{2 \sqrt{N^{+} N^{-}}}{\left(N^{+}+N^{-}\right)^{\frac{3}{2}}} .
$$


The raw asymmetry is formed from the quasielastic neutral sample; however, this does not mean that $N^{ \pm}$is composed of only neutron events. The raw asymmetry contains various contaminations that must be removed in order to extract a quantity associated to the neutron events only. The dominant sources of contamination are the following:

1. accidental background,

2. scattering from $\mathrm{N}_{2}$ rather than a nucleon within a ${ }^{3} \mathrm{He}$ nucleus,

3. BigBite preshower pions leaking into the electronic signal,

4. cluster-veto charge misidentification,

5. pion electroproduction inelastic events, and

6. nuclear effects including final state interactions and charge-exchange.

The ordering of corrections to apply to $A_{\text {raw }}$ must be done carefully and properly, e.g background corrections (nuclear corrections) are applied first (last). If a quasielastic neutral count, $\mathrm{N}^{+}$or $\mathrm{N}^{-}$, consists of the desired neutron events and the identified contaminations, then the count may be rewritten as the sum:

$$
N=N_{\mathrm{n}}+N_{\mathrm{bk}}+N_{\mathrm{N}_{2}}+N_{\mathrm{p}}+N_{\mathrm{other}}
$$

where $N_{\mathrm{n}}$ is the desired quasielastically scattered neutron count, $N_{\mathrm{bk}}$ is the count due to background events, $N_{\mathrm{N}_{2}}$ is the count of events that scattered off of nitrogen within the target cell rather than a ${ }^{3} \mathrm{He}$ nucleon, $N_{\mathrm{p}}$ is the number of protons that have been misidentified as neutral events and lastly $N_{\text {other }}$ is the count of all other contaminations such as BigBite pions, inelastics or FSI events. All contributions may be further 
differentiated by the helicity state, e.g. the counts due to random background within the quasielastic neutral sample for \pm helicity are denoted as $N_{\mathrm{bk}}^{ \pm}$; therefore, an asymmetry may be formed for each contamination and is referred to as a false asymmetry. The objective is to estimate the contaminations in order to correct the raw asymmetry. Once the raw asymmetry has been corrected, the result is the desired neutron asymmetry: $A_{\mathrm{n}}=\left(N_{\mathrm{n}}^{+}-N_{\mathrm{n}}^{-}\right) /\left(N_{\mathrm{n}}^{+}+N_{\mathrm{n}}^{-}\right)$where the neutron asymmetry only depends on quasielastically scattered neutral counts. The neutron asymmetry is related to the physical asymmetry $A_{\text {phys }}$ by Eqn 2.108 , and there are additional scaling corrections due to the polarizations of the experiment: the polarization of the electron beam $\left(P_{\text {beam }}\right)$, the polarization of the ${ }^{3} \mathrm{He}$ nucleus due to hSEOP $\left(P^{3} \mathrm{He}\right)$, and the polarization of the neutron within the ${ }^{3} \mathrm{He}$ nucleus $\left(P_{\mathrm{n}}\right)$. Recall that $A_{\text {phys }}$ assumes full polarizations of the electron and neutron; therefore, the fully corrected experimental asymmetry needs to be scaled by a factor equal to the product of the experimental polarizations:

$$
\frac{A_{\mathrm{n}}}{P_{\text {beam }} P_{3}{ }_{\mathrm{He}} P_{\mathrm{n}}}=A_{\text {phys }},
$$

which now provides a form factor extraction method. The general formalism and strategy of connecting the measured asymmetry to the theoretical physical asymmetry has been presented. The goal of the asymmetry corrections presented in the next few sections is to calculate the left-hand side of Eqn 5.50, i.e. to extract the asymmetry associated to neutron events only from the ${ }^{3} \mathrm{He}$ data set. The right-hand side, or $A_{\text {phys }}$, can be calculated for a single lab frame $\theta$ and $\phi$; however, a scheme must be developed to handle a range of angles as the detectors are of finite size, and this correction is presented in Section 5.7 .2 . 


\subsubsection{Corrections to the Raw Asymmetry}

The quasielastic neutral sample and raw asymmetry have been defined in the previous section by Eqn 5.49 and Eqn 5.47. The various contributions to the quasielastic neutral count, denoted by $N^{ \pm}$for helicity \pm , may be made more apparent by rewriting the raw asymmetry in terms of all appreciable experimental sources:

$$
\begin{aligned}
A_{\text {raw }} & =\frac{N^{+}-N^{-}}{N^{+}+N^{-}}, \\
& =\frac{N_{\mathrm{n}}^{+}+N_{\mathrm{bk}}^{+}+N_{\mathrm{N}_{2}}^{+}+N_{\mathrm{p}}^{+}+N_{\mathrm{other}}^{+}-N_{\mathrm{n}}^{-}-N_{\mathrm{bk}}^{-}-N_{\mathrm{N}_{2}}^{-}-N_{\mathrm{p}}^{-}-N_{\mathrm{other}}^{-}}{N_{\mathrm{n}}^{+}+N_{\mathrm{bk}}^{+}+N_{\mathrm{N}_{2}}^{+}+N_{\mathrm{p}}^{+}+N_{\mathrm{other}}^{+}+N_{\mathrm{n}}^{-}+N_{\mathrm{bk}}^{-}+N_{\mathrm{N}_{2}}^{-}+N_{\mathrm{p}}^{-}+N_{\text {other }}^{-}} .
\end{aligned}
$$

As the raw asymmetry depends on the differences and sums of all sources, then the lengthy expression may be conveniently shortened by introducing two variables:

$$
\begin{aligned}
& \Delta_{x} \equiv N_{x}^{+}-N_{x}^{-} \quad \Rightarrow \quad \Delta=\sum_{x} \Delta_{x}, \\
& \Sigma_{x} \equiv N_{x}^{+}+N_{x}^{-} \quad \Rightarrow \quad \Sigma=\sum_{x} \Sigma_{x},
\end{aligned}
$$

where the subscript $x$ refers to the source contributing to the quasielastic neutral sample. Expressing the raw asymmetry in terms of $\Delta_{x}$ and $\Sigma_{x}$ yields

$$
A_{\text {raw }}=\frac{\Delta_{\mathrm{n}}+\Delta_{\mathrm{bk}}+\Delta_{\mathrm{N}_{2}}+\Delta_{\mathrm{p}}+\Delta_{\text {other }}}{\Sigma_{\mathrm{n}}+\Sigma_{\mathrm{bk}}+\Sigma_{\mathrm{N}_{2}}+\Sigma_{\mathrm{p}}+\Sigma_{\text {other }}}=\frac{\Delta}{\Sigma}
$$

In order to relate the raw asymmetry to the physical asymmetry (Eqn 5.50), corrections to the raw asymmetry then come in three forms:

1. dilution factors which are multiplicative corrections to $A_{\text {raw }}$,

2. false asymmetries from source $x$ which are additive corrections to $A_{\text {raw }}$,

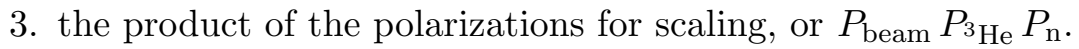


A dilution factor is simply a probability that represents the ratio of neutral quasielastic events from source $x$ to the total number of neutral quasielastic events. The chronological ordering of the dilution factor analysis for $G_{E}^{n}$ is background corrections, target $\mathrm{N}_{2}$ corrections, proton misidentification corrections and then all other dilution factors. The dilution factors are defined as follows:

$$
\begin{aligned}
D_{\mathrm{bk}} & =1-\frac{\Sigma_{\mathrm{bk}}}{\Sigma}, \\
D_{\mathrm{N}_{2}} & =1-\frac{\Sigma_{\mathrm{N}_{2}}}{\Sigma-\Sigma_{\mathrm{bk}}}, \\
D_{\mathrm{p}} & =1-\frac{\Sigma_{\mathrm{p}}}{\Sigma-\Sigma_{\mathrm{bk}}-\Sigma_{\mathrm{N}_{2}}}, \\
D_{\text {other }} & =1-\frac{\Sigma_{\mathrm{other}}}{\Sigma-\Sigma_{\mathrm{bk}}-\Sigma_{\mathrm{N}_{2}}-\Sigma_{\mathrm{p}}} .
\end{aligned}
$$

For example, the dilution factor due to random accidental background events is calculated first; therefore, the total number of events within the quasielastic neutral sample is $\Sigma$ and includes all contributions. The second calculation is the correction due to scattering from nitrogen within the target cell where the total number of neutral quasielastic counts must be background corrected, or $\Sigma-\Sigma_{\mathrm{bk}}$. The process of refining the definition of the neutral quasielastic sample continues until it equals $\Sigma_{\mathrm{n}}$, or the total number of quasielastic neutrons. The dilution factors may be rewritten using the summation found in Eqn 5.52, and the product equates to the following:

$$
\begin{aligned}
D_{\mathrm{bk}} D_{\mathrm{N}_{2}} D_{\mathrm{p}} D_{\text {other }}= & \left(\frac{\Sigma_{\mathrm{n}}+\Sigma_{\mathrm{N}_{2}}+\Sigma_{\mathrm{p}}+\Sigma_{\text {other }}}{\Sigma}\right)\left(\frac{\Sigma_{\mathrm{n}}+\Sigma_{\mathrm{p}}+\Sigma_{\text {other }}}{\Sigma_{\mathrm{n}}+\Sigma_{\mathrm{N}_{2}}+\Sigma_{\mathrm{p}}+\Sigma_{\text {other }}}\right) \\
& \times\left(\frac{\Sigma_{\mathrm{n}}+\Sigma_{\text {other }}}{\Sigma_{\mathrm{n}}+\Sigma_{\mathrm{p}}+\Sigma_{\text {other }}}\right)\left(\frac{\Sigma_{\mathrm{n}}}{\Sigma_{\mathrm{n}}+\Sigma_{\text {other }}}\right) \\
= & \frac{\Sigma_{\mathrm{n}}}{\Sigma}, \\
\Rightarrow \Sigma= & \frac{\Sigma_{\mathrm{n}}}{D_{\mathrm{bk}} D_{\mathrm{N}_{2}} D_{\mathrm{p}} D_{\text {other }}} .
\end{aligned}
$$


Substituting Eqn 5.58 into the raw asymmetry, or Eqn 5.53, results in a relationship between the raw asymmetry and the form factor ratio of the neutron:

$$
\begin{aligned}
A_{\text {raw }} & =\frac{\Delta_{\mathrm{n}}+\Delta_{\mathrm{bk}}+\Delta_{\mathrm{N}_{2}}+\Delta_{\mathrm{p}}+\Delta_{\text {other }}}{\Sigma}, \\
& =\frac{\Delta_{\mathrm{n}}}{\Sigma}+\frac{\Delta_{\mathrm{bk}}+\Delta_{\mathrm{N}_{2}}+\Delta_{\mathrm{p}}+\Delta_{\text {other }}}{\Sigma}, \\
& =D_{\mathrm{bk}} D_{\mathrm{N}_{2}} D_{\mathrm{p}} D_{\text {other }}\left(\frac{\Delta_{\mathrm{n}}}{\Sigma_{\mathrm{n}}}\right)+\frac{\Delta_{\mathrm{bk}}+\Delta_{\mathrm{N}_{2}}+\Delta_{\mathrm{p}}+\Delta_{\text {other }}}{\Sigma}, \\
& =D_{\mathrm{bk}} D_{\mathrm{N}_{2}} D_{\mathrm{p}} D_{\text {other }}\left(P_{\text {beam }} P_{{ }_{3 \mathrm{He}}} P_{\mathrm{n}} A_{\mathrm{phys}}\right)+\frac{\Delta_{\mathrm{bk}}+\Delta_{\mathrm{N}_{2}}+\Delta_{\mathrm{p}}+\Delta_{\text {other }}}{\Sigma} .
\end{aligned}
$$

In the last line, the neutron asymmetry, otherwise defined as $A_{\mathrm{n}}=\frac{\Delta_{\mathrm{n}}}{\Sigma_{\mathrm{n}}}$, is related to the physical asymmetry by Eqn 5.50, The asymmetry associated to the nitrogen within the target cell vanishes, or $A_{\mathrm{N}_{2}} \equiv \frac{\Delta_{\mathrm{N}_{2}}}{\Sigma}=0$, as it is unpolarized. In conclusion, the relationship between asymmetry theory and an E02-013 measurement is

$$
A_{\text {phys }}=\frac{A_{\text {raw }}-\left(\frac{\Delta_{\mathrm{bk}}}{\Sigma}+\frac{\Delta_{\mathrm{p}}}{\Sigma}+\frac{\Delta_{\text {other }}}{\Sigma}\right)}{P_{\text {beam }} P_{3} \mathrm{He} P_{\mathrm{n}} D_{\mathrm{bk}} D_{\mathrm{N}_{2}} D_{\mathrm{p}} D_{\text {other }}}=\frac{A_{\text {raw }}-\left(A_{\mathrm{bk}}+A_{\mathrm{p}}+A_{\text {other }}\right)}{P_{\text {beam }} P_{3} \mathrm{He} P_{\mathrm{n}} D_{\mathrm{bk}} D_{\mathrm{N}_{2}} D_{\mathrm{p}} D_{\text {other }}},
$$

where recall that the raw asymmetry represents the sign-corrected measured asymmetry of the quasielastic neutral sample.* The asymmetries within the brackets and the factors in the denominator are corrections to the raw asymmetry. The $A_{\text {other }}$ and $D_{\text {other }}$ terms represent any remaining experimental reality that can be corrected, e.g FSI, BigBite pions or inelastics; the corrections from inelastics and FSI are sourced from simulation, though, and eventually get worked into Eqn 5.59 in a slightly different manner as described in Section 5.7.7 and Section 5.7.8.

There are quite a few new definitions, and a "big picture" summary is beneficial. Once the kinematics of the experiment may be fully reconstructed and the assignment

\footnotetext{
* An identical analysis/formalism exists for the quasielastic charged sample, denoted by a "ch" superscript.
} 
of a nucleon charge is possible, then the neutral raw asymmetry may be calculated as $A_{\text {raw }}=\frac{N^{+}-N^{-}}{N^{+}+N^{-}}$where the \pm refers to the beam helicity and $N^{ \pm}$represents the neutral count post quasielastic cuts. The raw asymmetry contains contributions from all experimental sources, and must be corrected such that the result represents quasielastic neutron events. Corrections come in two forms known as dilution factors and asymmetry corrections (false asymmetries), and are multiplicative and additive, respectively, corrections to the raw asymmetry. The terms in the brackets within Eqn 5.59 have a $\frac{\Delta_{x}}{\Sigma}$ structure which is nothing more than a source $x$ asymmetry correction and may be relabeled as $A_{x}$ if desired, e.g $A_{\mathrm{bk}} \equiv \frac{\Delta_{\mathrm{bk}}}{\Sigma}$. Important notation and definitions are summarized within Table 5.4 note that $\Sigma_{x}=N_{x}^{+}+N_{x}^{-}=N_{x}$ are equivalent.

The raw asymmetry for the QE charged and uncharged samples over the entire ${ }^{3}$ He data set may be seen by Figure 5.62 . The charged raw asymmetry is expected to be much smaller than the neutral $A_{\text {raw }}$ which is observed. Recall that the ${ }^{3} \mathrm{He}$ nuclear ground state is dominated by the S-state in which the proton spins are anti-aligned; however, there are small D and $\mathrm{S}^{\prime}$-state contributions (see Figure 4.10 and the expectation for the charged sample is a small yet non-zero asymmetry. The QE neutral $A_{\text {raw }}$ is compared to kinematic variables in Figure 5.63 in which the kinematic is represented by the shaded red distribution and has been scaled to fit. The raw asymmetry does not vary significantly and is the correct sign. Note that a small correlation between $A_{\text {raw }}$ and $W$ is expected as $\theta_{e}$ is present in both $W$ and Eqn 2.107. The details of correcting $A_{\text {phys }}$ and $A_{\text {raw }}$ will now be discussed exhaustively for the remainder of the chapter starting with finite detector size corrections to $A_{\text {phys }}$. 


\begin{tabular}{|c|c|}
\hline Symbol & Description \\
\hline$\Sigma$ & Total QE neutral count, all contributions \\
\hline$\Sigma_{x}^{ \pm}$ & Neutral count for source $x$ for helicity \pm \\
\hline$\Sigma_{\mathrm{n}}$ & The count of neutrons within the QE neutral sample \\
\hline$\Sigma_{\mathrm{bk}}$ & The count due to random background events \\
\hline$\Sigma_{\mathrm{N}_{2}}$ & The count due to scattering from $\mathrm{N}_{2}$ within target cell \\
\hline$\Sigma_{\mathrm{p}}$ & The count of protons misidentified as neutrons \\
\hline$\Sigma_{\text {other }}$ & The count of all other contributions \\
\hline$P_{\text {beam }}$ & The polarization of the electron beam \\
\hline$P^{3} \mathrm{He}$ & The polarization of the ${ }^{3} \mathrm{He}$ nucleus due to hSEOP \\
\hline$P_{\mathrm{n}}$ & The polarization of the neutron within the ${ }^{3} \mathrm{He}$ nucleus \\
\hline$A_{\text {phys }}$ & Physical asymmetry $\propto \frac{G_{E}^{n}}{G_{M}^{n}}$ with ideal assumptions, Eqn 2.107 \\
\hline$A_{\text {meas }}$ & The asymmetry measured by the experiment \\
\hline$A_{\text {raw }}$ & The sign-corrected measured asymmetry \\
\hline$D_{\mathrm{bk}}\left(A_{\mathrm{bk}}\right)$ & Dilution (asymmetry) associated to accidental background \\
\hline$D_{\mathrm{N}_{2}}$ & Dilution factor associated to $\mathrm{N}_{2}$ scattering in target, $A_{\mathrm{N}_{2}}=0$ \\
\hline$D_{\mathrm{p}}\left(A_{\mathrm{p}}\right)$ & Dilution (asymmetry) associated to the proton contamination \\
\hline$D_{\text {in }}\left(A_{\text {in }}\right)$ & Dilution (asymmetry) associated to inelastic events \\
\hline$D_{\pi}\left(A_{\pi}\right)$ & Dilution (asymmetry) associated to BigBite preshower pions \\
\hline$D_{\mathrm{FSI}}\left(A_{\mathrm{FSI}}\right)$ & Dilution (asymmetry) associated to nuclear corrections \\
\hline
\end{tabular}

Table 5.4: Summary of notation used throughout the analysis. 

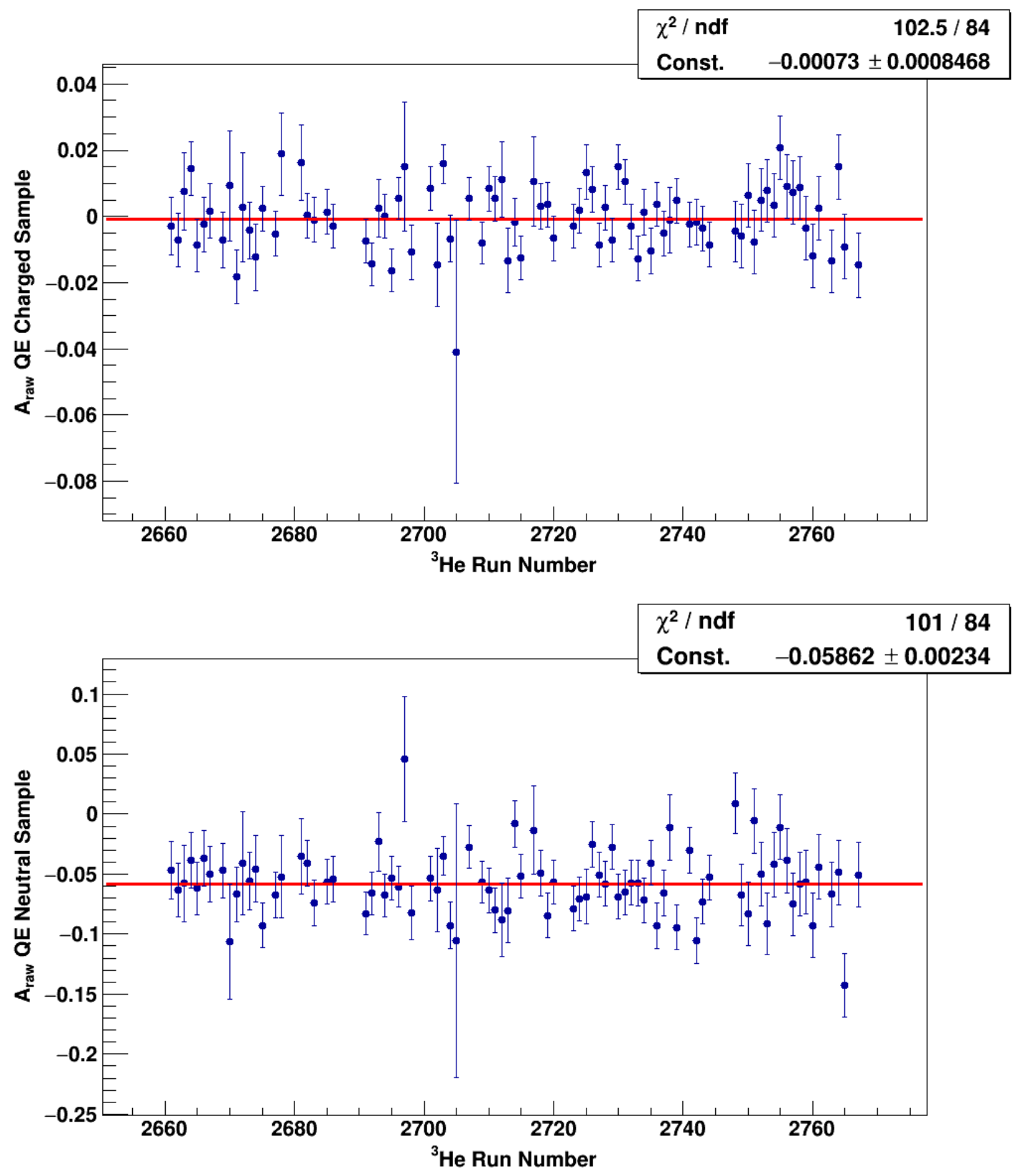

Fig. 5.62: The raw asymmetry for the QE charged (top panel) and uncharged (bottom panel) samples over the ${ }^{3}$ He data set. The raw asymmetry for the charged sample is expected to be much smaller than the uncharged sample. 

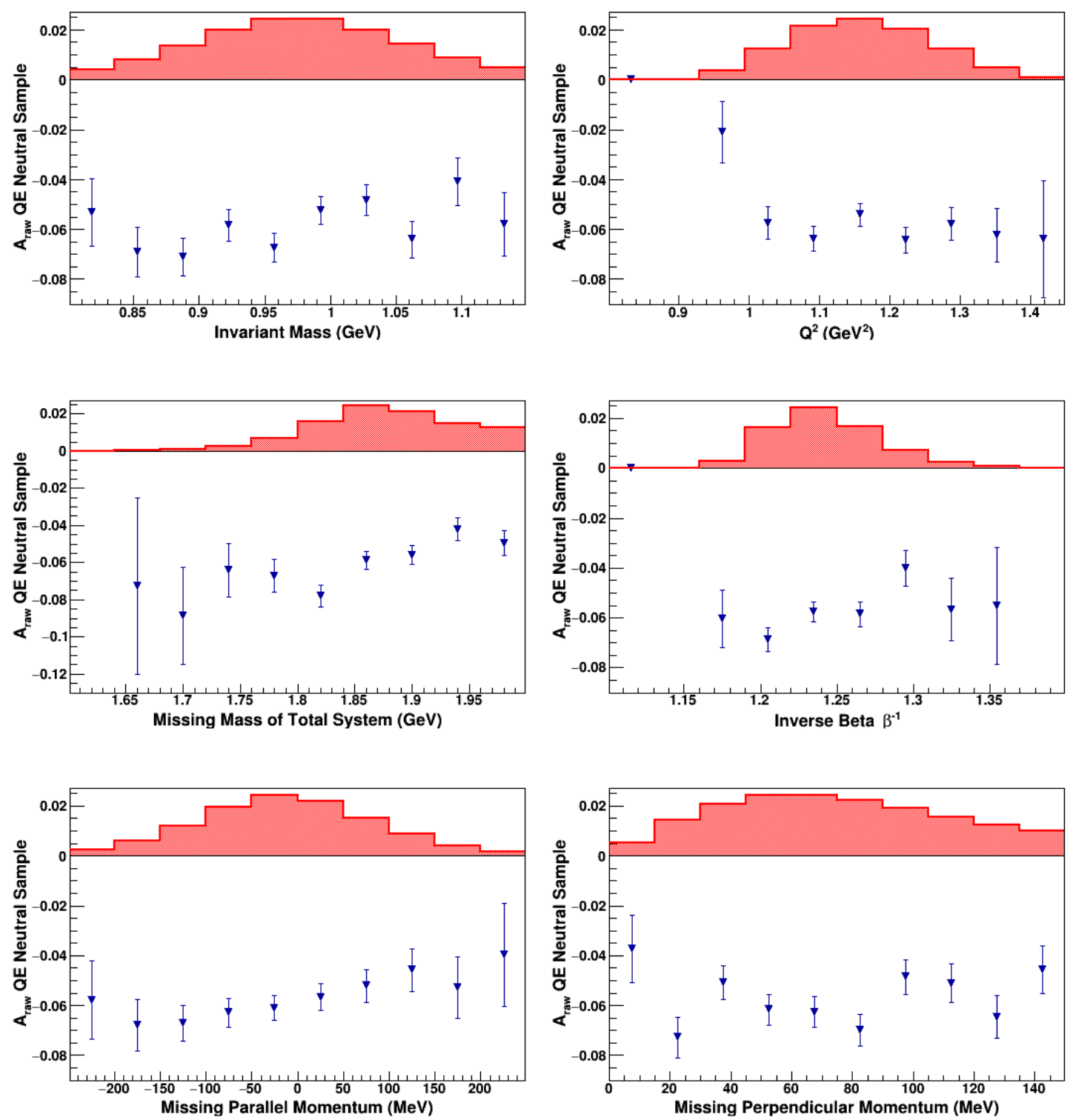

Fig. 5.63: A comparison of $A_{\text {raw }}$ to important kinematic variables which are represented by the shaded red regions and have been scaled to fit; therefore, the $y$-axis is arbitrary for kinematic relations. 


\subsubsection{Finite Acceptance Corrections to the Physical Asymmetry}

The theoretical physical asymmetry has been derived in Section 2.3 .2 .

$$
\begin{aligned}
A_{\mathrm{phys}} & =\frac{\sigma^{+}-\sigma^{-}}{\sigma^{+}+\sigma^{-}}=\frac{\Delta(\theta, \phi)}{\Sigma(\theta, \phi)}, \\
& =-\frac{2 \sqrt{\tau(1+\tau)} \tan \frac{\theta_{e}}{2}}{\frac{\tau}{\epsilon}+\Lambda^{2}}\left\{\Lambda P_{x}+\sqrt{\tau\left[1+(1+\tau) \tan ^{2} \frac{\theta_{e}}{2}\right]} P_{z}\right\},
\end{aligned}
$$

where $P_{x}=\sin \theta^{*} \cos \phi^{*}, P_{z}=\cos \theta^{*}$ and $\Lambda \equiv G_{E}^{n} / G_{M}^{n}$. The perpendicular and parallel components have been expanded to leading order under special experimental conditions. However, for the purposes of the analysis it is advantageous to rewrite the physical asymmetry more simply and then to expand the result keeping the form factor ratio $\Lambda$ to $5^{\text {th }}$-order to ensure a reliable calculation. $A_{\text {phys }}$ may be compactly written as

$$
A_{\mathrm{phys}}=\frac{\mathcal{B} \Lambda+\mathcal{C}}{\mathcal{D}+\Lambda^{2}}
$$

where $\mathcal{B}, \mathcal{C}$ and $\mathcal{D}$ contain the kinematics and are defined to be

$$
\begin{aligned}
\mathcal{B} & \equiv-2 \sqrt{\tau(1+\tau)} \tan \frac{\theta_{e}}{2} P_{x}, \\
\mathcal{C} & \equiv-2 \tau \sqrt{1+\tau+(1+\tau)^{2} \tan ^{2} \frac{\theta_{e}}{2}} \tan \frac{\theta_{e}}{2} P_{z}, \\
\mathcal{D} & \equiv \frac{\tau}{\epsilon} .
\end{aligned}
$$

The goal is to solve for $\Lambda$ in Eqn 5.62 which is a simple quadratic equation; however, a problem arises when choosing a value for the scattering kinematics: which values correctly represent the finite acceptance of the $G_{E}^{n}$ detectors? A range of values are accepted, not just the central kinematics and a scheme needs to be developed to handle this experimental reality. In order to overcome this issue, the denominator of Eqn 5.62 
may be expanded in powers of $\Lambda$ :

$$
\begin{aligned}
A_{\text {phys }} & =\frac{1}{\mathcal{D}} \cdot(\mathcal{B} \Lambda+\mathcal{C}) \cdot\left(1+\frac{\Lambda^{2}}{\mathcal{D}}\right)^{-1} \\
& \approx \frac{1}{\mathcal{D}} \cdot(\mathcal{B} \Lambda+\mathcal{C}) \cdot\left(1-\frac{\Lambda^{2}}{\mathcal{D}}+\frac{\Lambda^{4}}{\mathcal{D}^{2}}+\ldots\right) \\
& =\frac{\mathcal{C}}{\mathcal{D}}+\frac{\mathcal{B}}{\mathcal{D}} \Lambda-\frac{\mathcal{C}}{\mathcal{D}^{2}} \Lambda^{2}-\frac{\mathcal{B}}{\mathcal{D}^{2}} \Lambda^{3}+\frac{\mathcal{C}}{\mathcal{D}^{3}} \Lambda^{4}+\frac{\mathcal{B}}{\mathcal{D}^{3}} \Lambda^{5}
\end{aligned}
$$

The expression may be simplified by introducing a variable representing the six expansion coefficients, $T_{i}(\theta, \phi)$ :

$$
\begin{aligned}
& T_{0}=\frac{\mathcal{C}}{\mathcal{D}}, \quad T_{1}=\frac{\mathcal{B}}{\mathcal{D}}, \quad T_{2}=-\frac{\mathcal{C}}{\mathcal{D}^{2}}, \\
& T_{3}=-\frac{\mathcal{B}}{\mathcal{D}^{2}}, \quad T_{4}=\frac{\mathcal{C}}{\mathcal{D}^{3}}, \quad T_{5}=\frac{\mathcal{B}}{\mathcal{D}^{3}} .
\end{aligned}
$$

The physical asymmetry may then be compactly written as

$$
A_{\mathrm{phys}}=\sum_{i=0}^{5} T_{i} \cdot \Lambda^{i}
$$

which is a $5^{\text {th }}$-order polynomial with kinematic coefficients that may be calculated for a single polar angle $\theta$ and azimuthal angle $\phi$ direction in Hall coordinates. In order to handle the acceptance of the detectors, a formalism developed by Gregg Franklin (CMU) 213] is presented which utilizes the ${ }^{3} \mathrm{He}$ data. Ignoring corrective factors, e.g. polarizations and dilutions, the asymmetry measured by the experiment takes the form

$$
A_{\exp }=\frac{N^{+}-N^{-}}{N^{+}+N^{-}}=\frac{\int \Delta(\theta, \phi) \epsilon(\theta, \phi) d \Omega_{e}}{\int \Sigma(\theta, \phi) \epsilon(\theta, \phi) d \Omega_{e}},
$$

where $\epsilon(\theta, \phi)$ is the acceptance of the experiment as a function of the kinematics of the scattered electron. Only the relative acceptance is required for an asymmetry measurement; therefore, the acceptance factor $\epsilon(\theta, \phi)$ may be obtained by comparing the actual 
number of events in an angular bin obtained from the measurement to the elastic cross section, or

$$
\epsilon(\theta, \phi)=\frac{d N^{+}(\theta, \phi)+d N^{-}(\theta, \phi)}{2 \Sigma(\theta, \phi)}
$$

In other words, Eqn 5.72 is a probability that may vary over the detector acceptance, and represents the ratio of \pm counts in an acceptance bin $d N^{ \pm}(\theta, \phi)$ to the expected count via $\Sigma(\theta, \phi)$, or the unpolarized differential cross section. Substituting the detector efficiency into Eqn 5.71 results in

$$
\begin{aligned}
A_{\exp } & =\frac{\int \frac{\Delta(\theta, \phi)}{2 \Sigma(\theta, \phi)}\left[d N^{+}(\theta, \phi)+d N^{-}(\theta, \phi)\right] d \Omega_{e}}{\int \frac{1}{2}\left[d N^{+}(\theta, \phi)+d N^{-}(\theta, \phi)\right] d \Omega_{e}}, \\
& =\frac{1}{N^{+}+N^{-}} \sum_{e n \text { events }} \frac{\Delta(\theta, \phi)}{\Sigma(\theta, \phi)}, \\
& =\frac{1}{N^{+}+N^{-}} \sum_{\text {en events }} T_{0}+T_{1} \Lambda+T_{2} \Lambda^{2}+T_{3} \Lambda^{3}+T_{4} \Lambda^{4}+T_{5} \Lambda^{5},
\end{aligned}
$$

where $\Delta / \Sigma$ is given by Eqn 5.70 . The corrected measured asymmetry may be compared to the physical asymmetry in terms of the mean values of the experimentally measured quantities, i.e. the $T_{i}$ coefficients averaged over the quasielastic neutral sample. Updating Eqn 5.50 and Eqn 5.70 to reflect this experimental reality yields

$$
\begin{aligned}
& A_{\mathrm{phys}}=\sum_{i=0}^{5} \bar{T}_{i} \cdot \Lambda^{i} \\
& \Rightarrow A_{\mathrm{n}}=P_{\text {beam }} P_{{ }^{3} \mathrm{He}} P_{\mathrm{n}} \sum_{i=0}^{5} \bar{T}_{i} \cdot \Lambda^{i},
\end{aligned}
$$

where the $\bar{T}_{i}$ coefficients are kinematic factors obtained by averaging the $T_{i}$ factors over the quasielastic neutral sample. The goal of the data analysis is to calculate $A_{\text {raw }}$ and correct it such that the result most closely resembles $A_{\mathrm{n}}$, and then to compare the result to the finite acceptance corrected theory, or the right-hand side of Eqn 5.77 . 
The polarizations and $\bar{T}_{i}$ factors are simply numbers at this point, and the result is a $5^{\text {th }}$-order polynomial in $\Lambda$ which may be numerically solved for an extraction of the neutron form factor ratio.

A range of scattered electron kinematics results in a $Q^{2}$ distribution extending from approximately $0.9-1.45 \mathrm{GeV}^{2}$. Therefore, a formula representing the acceptanceaveraged value of $Q^{2}$ needs to be presented in order to report a value of the neutron form factor ratio. Expanding the form factor ratio to first order in $Q^{2}$ gives

$$
\Lambda\left(Q^{2}\right) \approx \Lambda\left(Q_{n}^{2}\right)+\left.\left(Q^{2}-Q_{n}^{2}\right) \frac{d \Lambda}{d Q^{2}}\right|_{Q^{2}=Q_{n}^{2}}
$$

where $\Lambda\left(Q_{n}^{2}\right)$ is the value of $\Lambda$ at a nominal point in the acceptance denoted by $Q_{n}^{2}$. The unknown slope evaluated at $Q_{n}^{2}$ is defined to be $\left.\alpha \equiv \frac{d \Lambda}{d Q^{2}}\right|_{Q^{2}=Q_{n}^{2}}$ for convenience. Substituting Eqn 5.78 into Eqn 5.70, but only keeping terms up to first order in $\Lambda$ gives

$$
A_{\text {phys }}\left(Q^{2}\right)=A_{\text {phys }}\left(Q_{n}^{2}\right)+T_{1}\left(Q^{2}-Q_{n}^{2}\right) \alpha \text {. }
$$

Averaging over the quasielastic neutral sample results in

$$
\bar{A}_{\text {phys }}\left(Q^{2}\right)=A_{\text {phys }}\left(Q_{n}^{2}\right)+\left(\overline{T_{1} Q^{2}}-\overline{T_{1}} Q_{n}^{2}\right) \alpha
$$

To first order, the experimentally measured asymmetry averaged over the $Q^{2}$ acceptance is the asymmetry evaluated at the acceptance-averaged value of $Q_{n}^{2}$ if the following is chosen:

$$
0=\alpha \overline{Q^{2} T_{1}}-\alpha Q_{n}^{2} \overline{T_{1}} \quad \Rightarrow \quad Q_{n}^{2}=\frac{\overline{T_{1} Q^{2}}}{\overline{T_{1}}}
$$

Henceforth, the reported value of $Q^{2}$ in any subsequent table is assumed to be the acceptance-averaged value unless explicitly stated otherwise. 


\subsubsection{Accidental Background}

Events that are observed to be detected randomly in time at some constant rate are referred to as the random background. The origin of these particles requires a Monte Carlo study, but can be largely associated with the electron beam pipe and target materials among other things. In order to estimate the contribution of the accidental background to the quasielastic region of interest, the data is artificially shifted in time prior to applying quasielastic cuts. Shifting the data in time may be accomplished by adjusting the $\beta$, or equivalently $\beta^{-1}$, of the nucleon as seen by Figure 5.64 . Recall that the inverse beta spectrum has two bumps corresponding to photons $\left(\beta^{-1}=1\right)$ and the quasielastic selection $\left(\beta^{-1} \approx 1.3\right)$, and this may be seen by the shaded red region. Additionally, a typical quasielastic nucleon has the following values for $\beta$, time of flight and path length, respectively:

$$
\beta_{\mathrm{QE}} \approx 0.8, \quad t_{\mathrm{ToF}} \approx 38 \mathrm{~ns}, \quad|\vec{\ell}| \approx 9 \mathrm{~m},
$$

where the path length is the distance from the scattering vertex to the reconstructed nucleon cluster position. Background events may be estimated by adjusting $\beta^{-1}$ such that the photon peak appears far away from the QE cut region:

$$
\beta_{\mathrm{bk}}=\frac{1}{\frac{1}{\beta_{\mathrm{QE}}}+\alpha} \approx 0.5 \Rightarrow \beta_{\mathrm{bk}}^{-1} \approx 2,
$$

where the parameter choice is $\alpha=0.8$; the result is the blue distribution in Eqn 5.64 . The shifted photon peak is estimated to arrive roughly $17 \mathrm{~ns}$ after the quasielastic region which is sufficiently far away. By shifting the reconstructed nucleon $\beta=v / c$ such that the photon peak arrives later than expected, the shifted events post quasielastic cuts are 


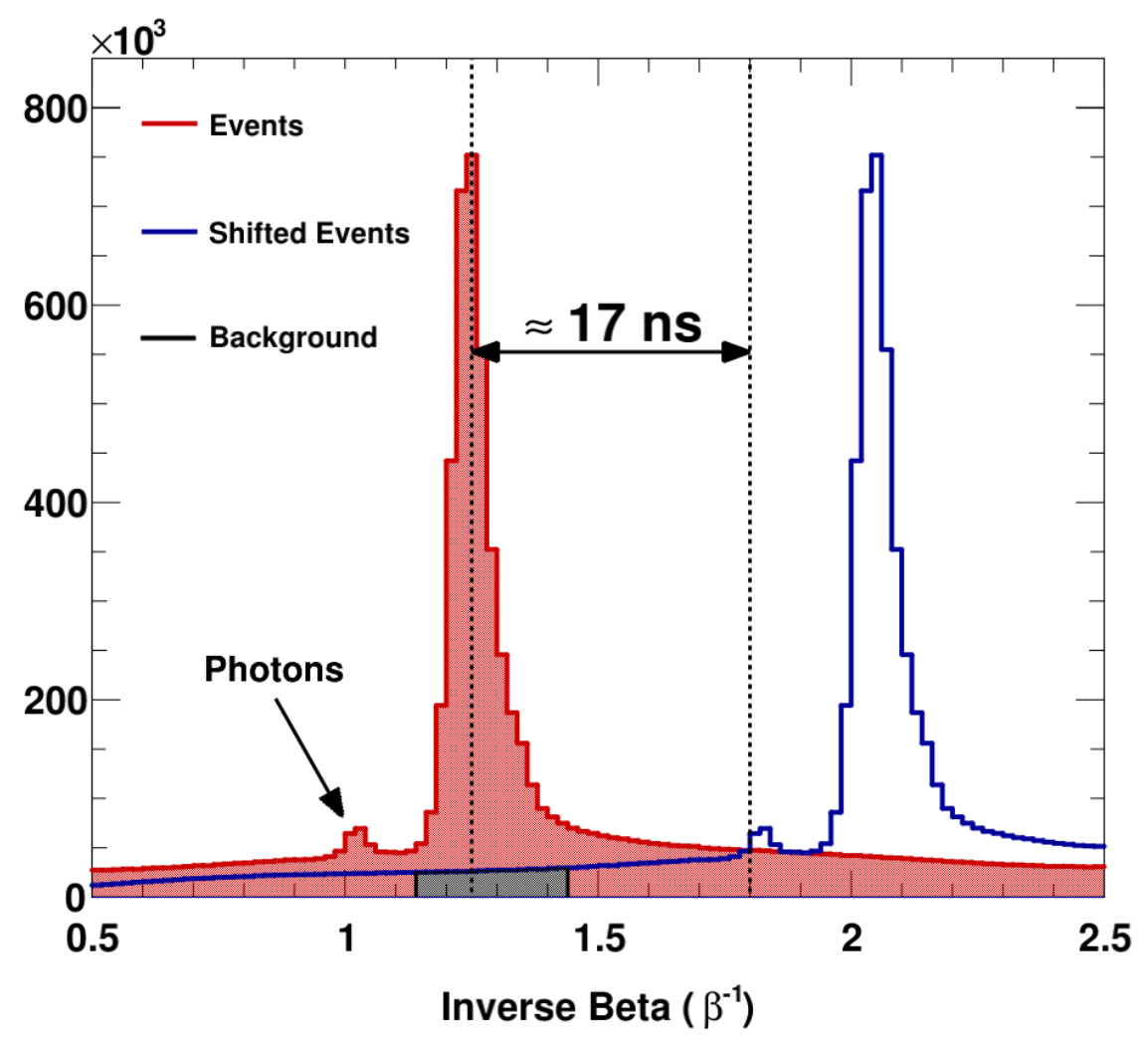

Fig. 5.64: Artificially shifting the ${ }^{3} \mathrm{He}$ data prior to applying quasielastic cuts in order to estimate the number of random background events (black).

completely unassociated to the scattering event of interest by causality, and represents the amount of background to subtract; the background contribution may be seen by the black distribution.

The overlap between the background and quasielastic selection for important variables is presented in Figure 5.65. The quasielastic cuts summarized in Table 5.3 have all been applied. Both distributions, quasielastic and background, represent the sum of uncharged and charged counts; therefore the ratio of background events to 

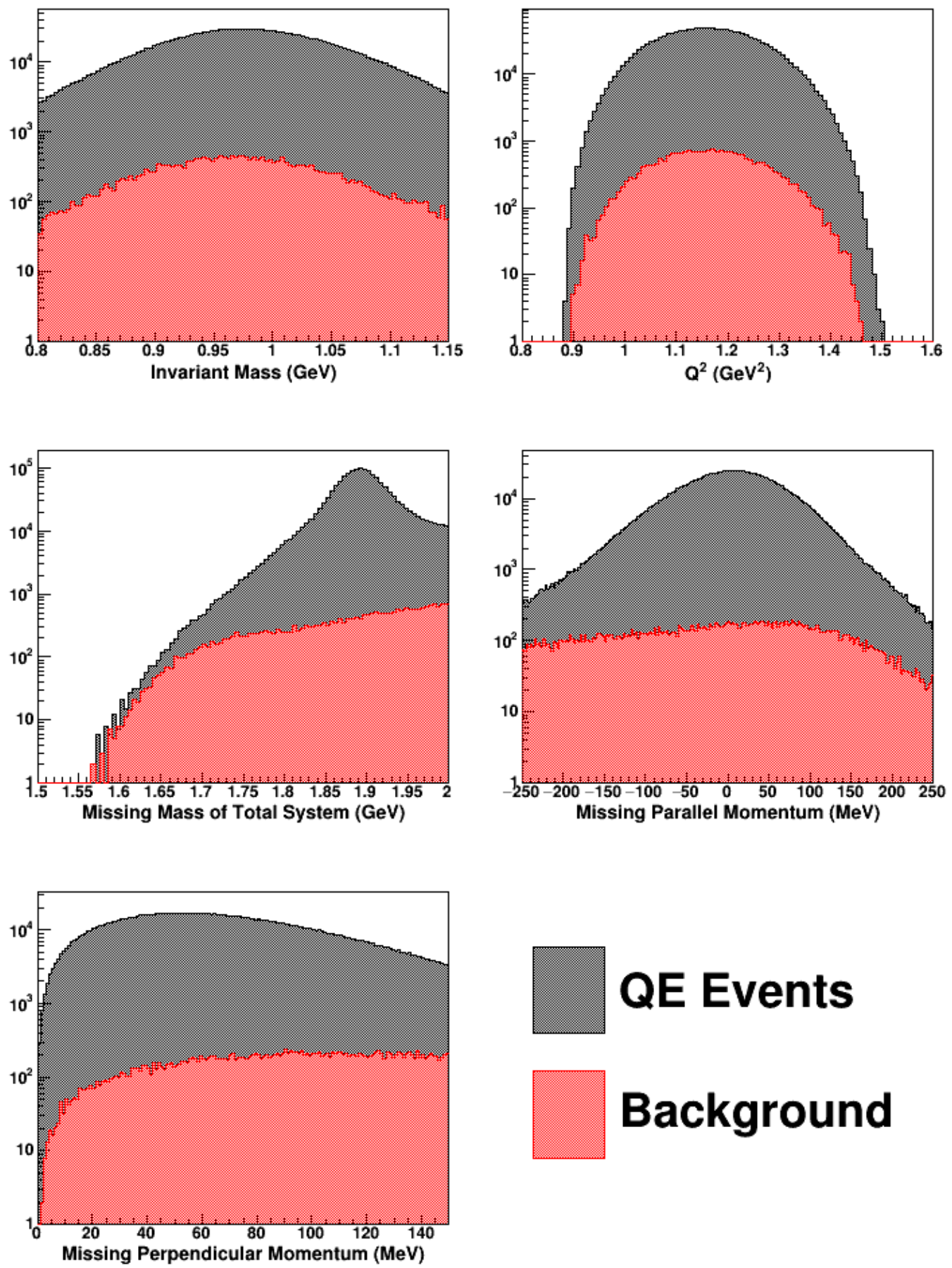

\section{QE Events}

\section{Background}

Fig. 5.65: Overlap of the quasielastic ${ }^{3} \mathrm{He}$ selection and the random background for various kinematic variables. All quasielastic cuts are defined in Table 5.3. 
quasielastic events, or

$$
\frac{\Sigma_{\mathrm{bk}}+\Sigma_{\mathrm{bk}, \mathrm{ch}}}{\Sigma+\Sigma_{\mathrm{ch}}}
$$

is calculated to be roughly $1.5 \%$. Note that the absence of a "ch" subscript refers to the neutral sample. However, a ratio of $1.5 \%$ is misleading and underestimates the true dilution as charged events are included; therefore, a proper background calculation must repeat the procedure but for both samples separately. The dilution factor and background asymmetry for the neutral sample may be calculated using the framework presented in Section 5.7.1, and may be expressed in a variety of ways:

$$
\begin{aligned}
& D_{\mathrm{bk}}=1-\frac{\Sigma_{\mathrm{bk}}}{\Sigma}=1-\frac{N_{\mathrm{bk}}^{+}+N_{\mathrm{bk}}^{-}}{N^{+}+N^{-}} \\
& A_{\mathrm{bk}} \equiv \frac{\Delta_{\mathrm{bk}}}{\Sigma}=\frac{N_{\mathrm{bk}}^{+}-N_{\mathrm{bk}}^{-}}{N^{+}+N^{-}}=A_{\mathrm{raw}, \mathrm{bk}}\left(\frac{\Sigma_{\mathrm{bk}}}{\Sigma}\right)=\left(1-D_{\mathrm{bk}}\right) A_{\mathrm{raw}, \mathrm{bk}},
\end{aligned}
$$

where $A_{\text {raw,bk }}$ is the raw asymmetry associated to the neutral background. An identical set of equations exists for the charged sample. The quasielastic background uncharged to charged ratio may be defined as $R_{\mathrm{bk}}=\frac{\Sigma_{\mathrm{bk}}}{\Sigma_{\mathrm{bk}, \mathrm{ch}}}$ which may been seen by Figure 5.66 .

There is a subtlety in the interpretation of $\Sigma_{\mathrm{bk}}$ related to the assignment of charge to a reconstructed nucleon. How much of this count is truly understood in the context of charge identification after the artificial shift in time? In other words, the charge that is assigned to the accidental background determines the amount of background to subtract from the quasielastic neutral sample.* The complication that arises, then, is charge identification of accidental background in the presence of a true quasielastic event. For example, if the nucleon has been identified as charged but the

\footnotetext{
* Charge identification is discussed and simulated in Section 5.4.6 and Table 5.2 respectively.
} 


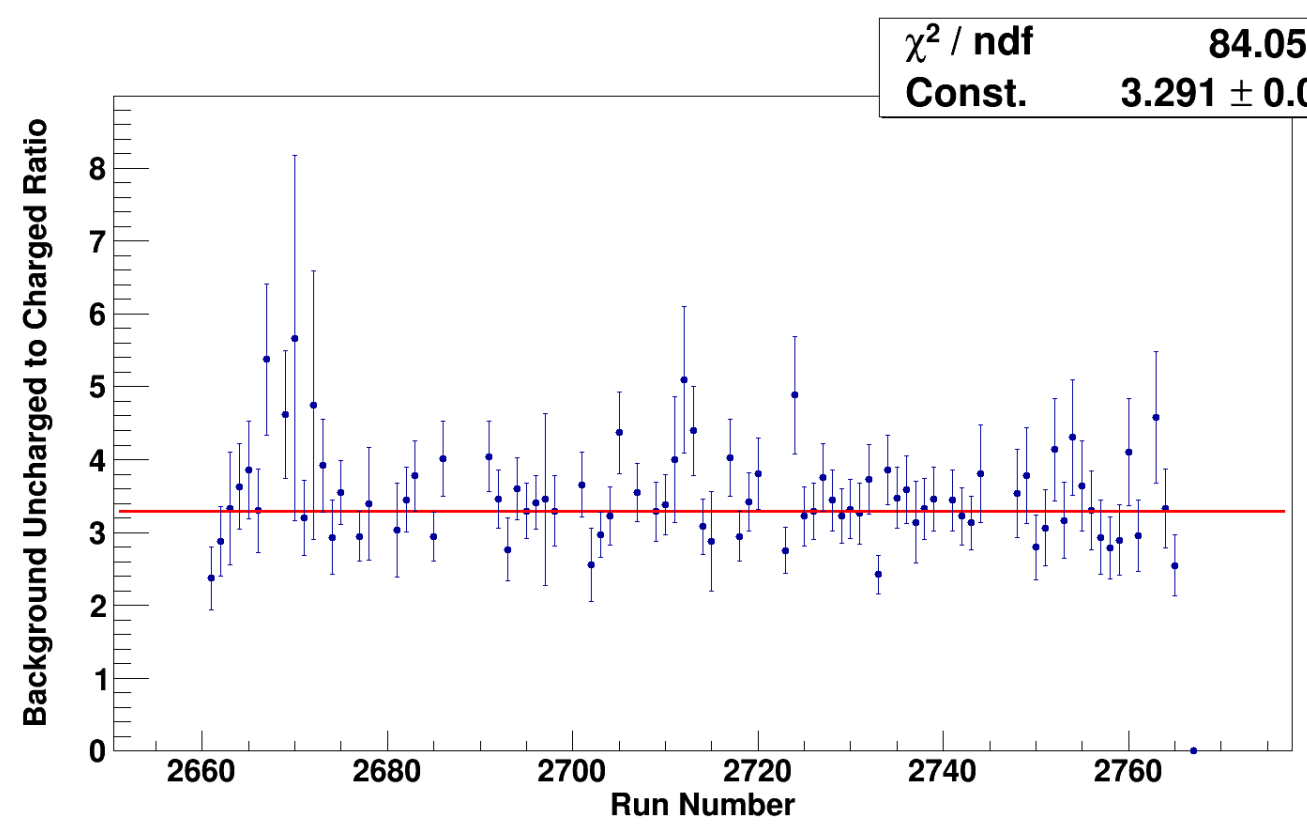

Fig. 5.66: The QE background uncharged to charged ratio for the ${ }^{3}$ He data set.

background is truly all neutral, then all the background underneath the nucleon appears to be charged as determined by the signal within the veto layers. However, the $\beta^{-1}$ shift forces the background to look neutral simply because there is no veto signal to make it look charged. In this case, all of the background has been faithfully identified as neutral. Alternatively, the opposite can happen in which all of the background is misunderstood and incorrectly identified. This is demonstrated by Figure 5.67; the event uncharged-to-charged ratio decreases in the quasielastic range to levels already presented in Figure 5.60. However, the true level of the background is represented by the background events, or the green markers, which is quite different. In regards to the big picture, the background corrections to $A_{\text {raw }}$ are small; furthermore, the uncertainties in the upper limit of the background knowledge only contributes a relatively small amount 


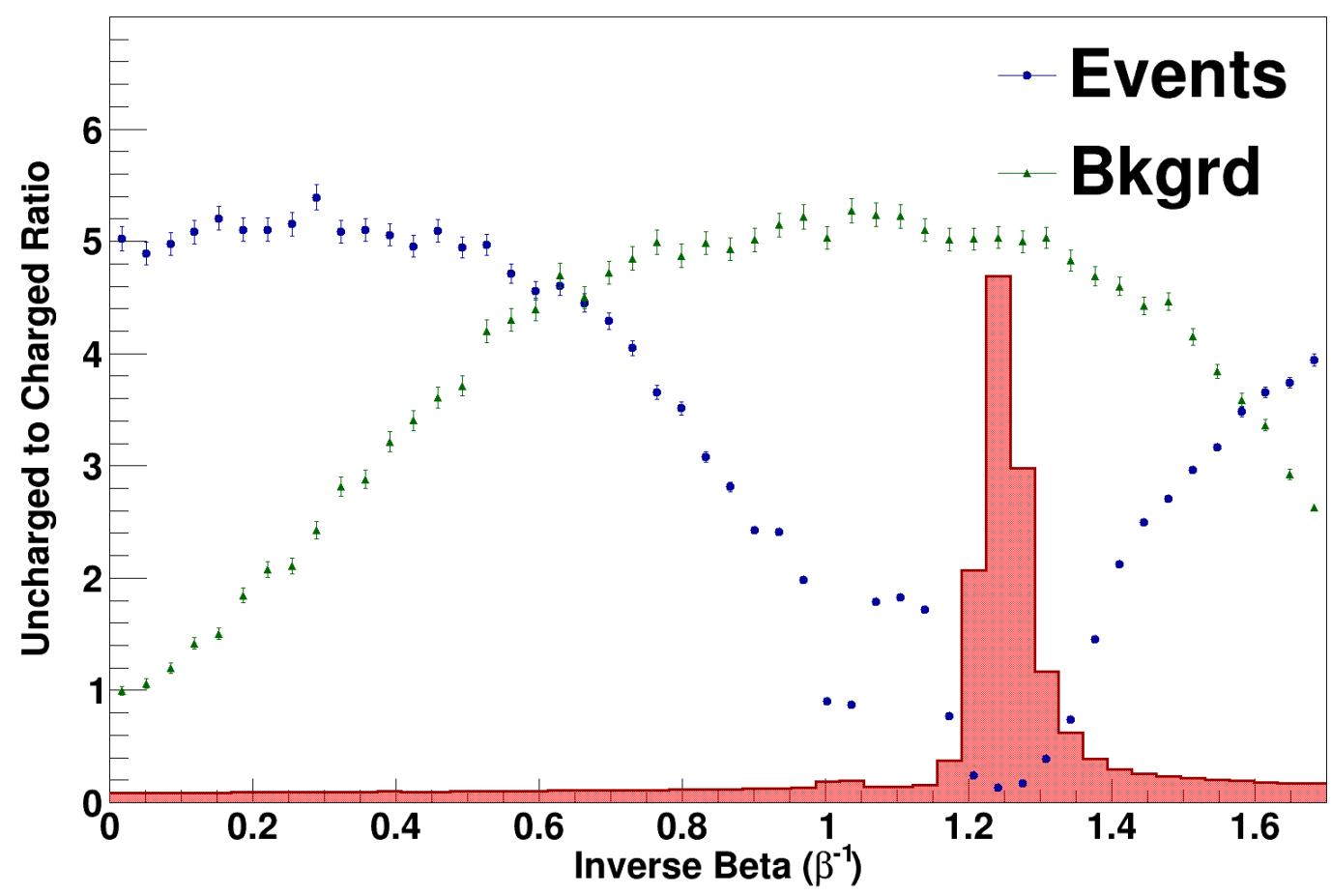

Fig. 5.67: The uncharged-to-charged ratio for events and background. The $\beta^{-1}$ spectrum has been overlaid for reference and scaled to fit. Note that the background ratio $R_{\mathrm{bk}}$ does not equal the ratio observed in Figure 5.66 as the as the restrictive quasielastic cuts are not applied in this case.

of uncertainty to the form factor ratio. Therefore, the important aspect of this analysis is to quantify an upper limit of this uncertainty. In this light, the analysis makes no assumption on the exact levels of background to subtract from the quasielastic sample. As a result, an average of the two extremes (all or nothing) is taken and the amount to subtract from the quasielastic neutral sample is $\frac{N_{\mathrm{bk}}}{2}$. The remainder then must contribute towards the background of the charged sample, and the amount to subtract is $N_{\mathrm{bk}, \mathrm{ch}}+\frac{N_{\mathrm{bk}}}{2}$. For future reference during the analysis phase, the amount of background 
to subtract for the neutral and charged samples may be summarized by the following equations:

$$
\begin{aligned}
& \text { Amount to subtract for neutral }=\frac{N_{\mathrm{bk}}}{2}, \\
& \text { Amount to subtract for charged }=N_{\mathrm{bk}, \mathrm{ch}}+\frac{N_{\mathrm{bk}}}{2},
\end{aligned}
$$

where it is understood that $N_{\mathrm{bk}}$ may be replaced by $\Sigma_{\mathrm{bk}}$.

\subsubsection{Nitrogen in the ${ }^{3} \mathrm{He}$ Cell}

The ${ }^{3} \mathrm{He}$ target cells contain a small amount of $\mathrm{N}_{2}$ for reasons described in Section 4.5.2. As a result, electron scattering off of nitrogen rather than polarized ${ }^{3} \mathrm{He}$ may occur consequently diluting the quasielastic sample. Typically, the nitrogen contamination is estimated by performing the quasielastic analysis on both nitrogen and polarized

${ }^{3} \mathrm{He}$ data sets. As the data sets consist of different targets and vary in the number of usable incoming beam electrons, two scaling factors need to be applied to the quasielastic neutral counts such that an apples-to-apples comparison can be performed. The dilution factor for nitrogen may be calculated as

$$
D_{\mathrm{N}_{2}}=1-\left(\frac{Q\left({ }^{3} \mathrm{He}\right)}{Q\left(\mathrm{~N}_{2}\right)}\right)\left(\frac{\rho_{\mathrm{N}_{2}}\left({ }^{3} \mathrm{He}\right)}{\rho_{\mathrm{N}_{2}}\left(\mathrm{~N}_{2}\right)}\right) \frac{\Sigma\left(\mathrm{N}_{2}\right)-\Sigma_{\mathrm{bk}}\left(\mathrm{N}_{2}\right)}{\Sigma\left({ }^{3} \mathrm{He}\right)-\Sigma_{\mathrm{bk}}\left({ }^{3} \mathrm{He}\right)},
$$

where $Q(t)$ is the total beam charge $Q$ for data taken with target $t, \rho_{\mathrm{N}_{2}}(t)$ is the density of $\mathrm{N}_{2}$ in target $t$, and $\Sigma(t)-\Sigma_{\mathrm{bk}}(t)$ represents the number of background corrected counts for target $t$ after quasielastic cuts have been applied. The first normalization factor, $Q\left({ }^{3} \mathrm{He}\right) / Q\left(\mathrm{~N}_{2}\right)$, allows data with different electron beam parameters, e.g. beam current, to be compared. The second factor, $\rho_{\mathrm{N}_{2}}\left({ }^{3} \mathrm{He}\right) / \rho_{\mathrm{N}_{2}}\left(\mathrm{~N}_{2}\right)$, accounts for the different number of nucleons between the two target types. If the target lengths are unequal, 
then a ratio of mass thicknesses should be used rather than a ratio of densities, or

$$
\frac{\rho_{\mathrm{N}_{2}}\left({ }^{3} \mathrm{He}\right)}{\rho_{\mathrm{N}_{2}}\left(\mathrm{~N}_{2}\right)} \longrightarrow \frac{\rho_{\mathrm{N}_{2}}\left({ }^{3} \mathrm{He}\right) \cdot l\left({ }^{3} \mathrm{He}\right)}{\rho_{\mathrm{N}_{2}}\left(\mathrm{~N}_{2}\right) \cdot l\left(\mathrm{~N}_{2}\right)},
$$

where $l(t)$ is the total length of target $t$.

While the higher $Q^{2}$ points have nitrogen data, Kinematic 1 does not; therefore, a target element with a similar number of nucleons to nitrogen may serve as an appropriate substitute. The only other target type is the BeO-C foils, see Section 4.5.7 for details about the target. The carbon nucleus has a similar number of nucleons as a nitrogen nucleus, and may act as a nitrogen substitute if the center beryllium oxide foil is removed during the analysis. An additional unobvious detail is the carbon foil target is not evacuated and the air contribution to the target mass thickness must be included. Recall that the nitrogen and carbon targets are not polarized, therefore no asymmetry corrections to $A_{\text {raw }}$ are necessary, i.e. $A_{\mathrm{N}_{2}}=0$.

\subsubsection{Misidentified Protons}

Protons that have been misidentified as neutrons and consequently dilute the neutral sample is an important correction that needs to be estimated. The procedure requires a calculation of the quasielastic uncharged to charged ratio for three targets in order to constrain three ratios known as mixing coefficients ratios, or numbers that represent probabilities of ideal expectations and the actual outcome of the veto-cluster charge identification. Naively, the uncharged to charged ratio is simply the quasielastic uncharged count over the charged count; ${ }^{*}$ however, the possibility that protons (neutrons)

\footnotetext{
* See Figure 5.60 for $R_{3} \mathrm{He}$ as an example.
} 
can contaminate the uncharged (charged) signal must be taken into account. Therefore, the ratio is reformulated:

$$
R=\frac{N^{n}}{N^{p}}=\frac{N_{p}^{n}+N_{n}^{n}}{N_{n}^{p}+N_{p}^{p}},
$$

where the subscript refers to the particle that left the target and the superscript denotes the result of charge identification, i.e. $N_{\text {true }}^{\text {observed }}$. For example, the number of neutrons $N^{n}$ has been reformulated to account for the number of true neutrons and the number of protons that have been misidentified as neutrons, and a similar explanation holds for the number of protons. Additionally, the counts may be written by introducing mixing coefficients, denoted by $\eta$, and the nucleon cross sections, $\sigma_{n}$ and $\sigma_{p}$ :

$$
\begin{array}{ll}
N_{n}^{n} \propto f \sigma_{n} \eta_{n}^{n}, & N_{p}^{n} \propto \sigma_{p} \eta_{p}^{n}, \\
N_{n}^{p} \propto f \sigma_{n} \eta_{n}^{p}, & N_{p}^{p} \propto \sigma_{p} \eta_{p}^{p} .
\end{array}
$$

To be clear, the mixing coefficients have the following probabilistic interpretations:

1. $\eta_{n}^{n}=$ true neutrons correctly identified as neutrons,

2. $\eta_{n}^{p}=$ true neutrons incorrectly identified as protons,

3. $\eta_{p}^{p}=$ true protons correctly identified as protons,

4. $\eta_{p}^{n}=$ true protons incorrectly identified as neutrons,

and have been simulated in Section 5.4.7. The parameter $f$ is target dependent and represents the effective ratio of neutrons to protons. The parameter is necessary as the ratio of neutrons to protons depends on the target and the chosen quasielastic cuts, specifically the values of $p_{\text {miss, } \|}$ and $p_{\text {miss }, \perp \text {. A naive expectation for }{ }^{3} \mathrm{He} \text { is } f^{3} \mathrm{He}}=\frac{1}{2}$, 
but this is not the case due to the cuts on missing momentum. Substituting Eqn 5.90 into the ratio $R$ which now explicitly depends on target $t$ results in the following:

$$
R_{t}=\frac{f_{t} \frac{\sigma_{n}}{\sigma_{p}}\left(\frac{\eta_{n}^{n}}{\eta_{p}^{p}}\right)+\left(\frac{\eta_{p}^{n}}{\eta_{p}^{p}}\right)}{f_{t} \frac{\sigma_{n}}{\sigma_{p}}\left(\frac{\eta_{n}^{p}}{\eta_{p}^{p}}\right)+1} .
$$

If three targets are used, then there are three target dependent $R$ ratios available to constrain the three ratios of mixing coefficients. The three targets are $\mathrm{H}_{2}$, the $C$ foils, and ${ }^{3} \mathrm{He}$ corresponding to the following target dependent ratios:

$$
\begin{aligned}
R_{\mathrm{H}_{2}} & =\frac{\eta_{p}^{n}}{\eta_{p}^{p}}, \\
R_{\mathrm{C}} & =\frac{f_{\mathrm{C}} \frac{\sigma_{n}}{\sigma_{p}}\left(\frac{\eta_{n}^{n}}{\eta_{p}^{p}}\right)+\frac{\eta_{p}^{n}}{\eta_{p}^{p}}}{f_{\mathrm{C}} \frac{\sigma_{n}}{\sigma_{p}}\left(\frac{\eta_{n}^{p}}{\eta_{p}^{p}}\right)+1}, \\
R_{{ }^{p} \mathrm{He}} & =\frac{f^{{ }^{3}} \mathrm{He}_{\frac{n}{n}} \frac{\sigma_{n}}{\sigma_{p}}\left(\frac{\eta_{n}^{n}}{\eta_{p}^{p}}\right)+\frac{\eta_{p}^{n}}{\eta_{p}^{p}}}{f_{{ }^{3} \mathrm{He}} \frac{\sigma_{n}}{\sigma_{p}}\left(\frac{\eta_{n}^{p}}{\eta_{p}^{p}}\right)+1},
\end{aligned}
$$

where it has been assumed that $f_{\mathrm{H}_{2}}=0$. The effective ratio of neutrons to protons for carbon and helium-3 are nontrivial due to the missing momentum cuts, and as a result require theoretical input. Solving for the mixing ratios yields

$$
\begin{aligned}
& \frac{\eta_{p}^{n}}{\eta_{p}^{p}}=R_{\mathrm{H}_{2}} \\
& \frac{\eta_{n}^{p}}{\eta_{p}^{p}}=\frac{\sigma_{p}}{\sigma_{n}} \frac{f_{\mathrm{C}}\left(R_{3} \mathrm{He}_{2}-R_{\mathrm{H}_{2}}\right)-f_{{ }^{3} \mathrm{He}}\left(R_{\mathrm{C}}-R_{\mathrm{H}_{2}}\right)}{f_{\mathrm{C}} f_{{ }^{3} \mathrm{He}}\left(R_{\mathrm{C}}-R_{3} \mathrm{He}_{\mathrm{e}}\right)},
\end{aligned}
$$

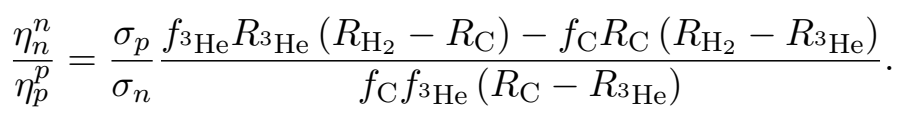

The single nucleon cross sections may be calculated using a parametrization for the nucleon form factors, see Section 3.2 . The ratios for the three targets are calculated by analyzing the data. Lastly, two theoretical values for the effective ratio of neutrons to protons is required, and then the dilution factor due to protons misidentified as neutrons (or vice versa) may be calculated. 


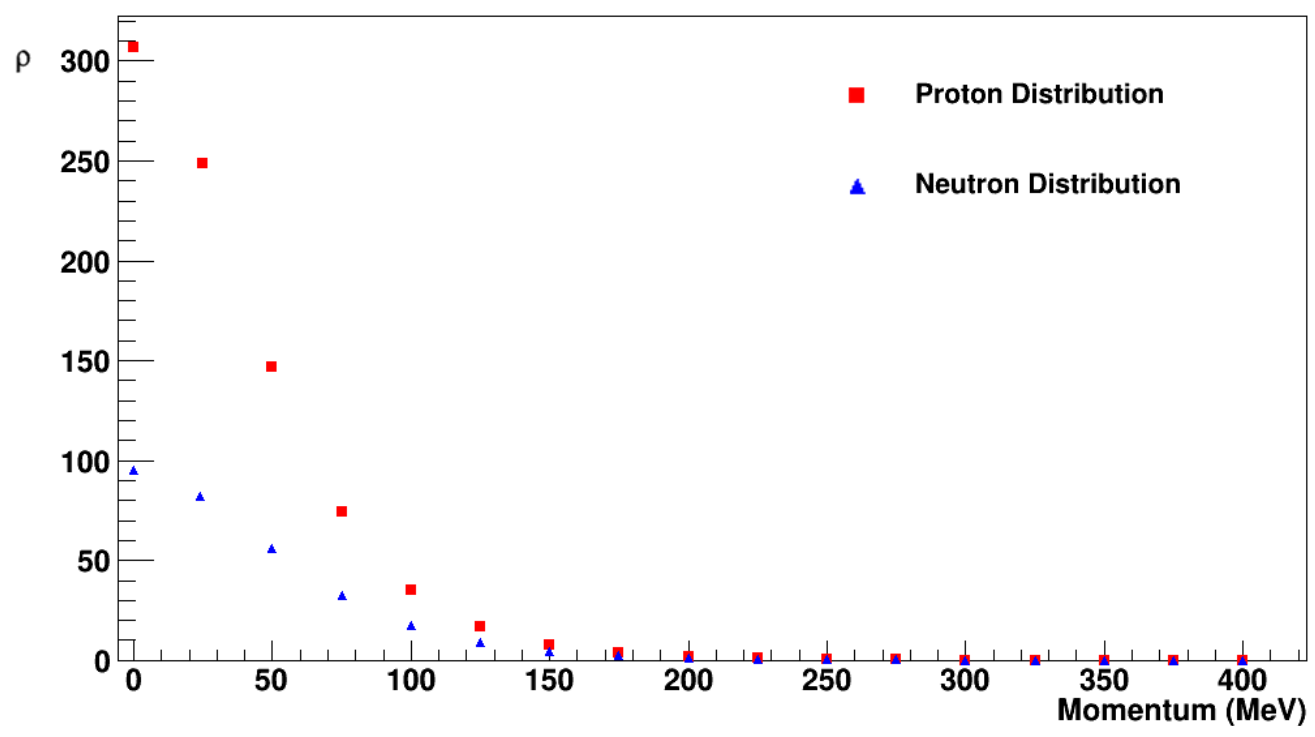

Fig. 5.68: The ${ }^{3} \mathrm{He}$ proton and neutron density distributions as a function of the momentum of the nucleon. The units of the nucleon density $\rho$ are arbitrary.

The value of the effective ratio of neutrons to protons has been calculated utilizing a ${ }^{3} \mathrm{He}$ momentum distribution provided by Rocco Schiavilla. The momentum distributions of the proton and neutron for polarized ${ }^{3} \mathrm{He}$ have been calculated using the AV18 NN potential. The nucleon density as a function of nucleon momentum using this potential may be seen by Figure 5.68. While the naive expected ratio of neutrons to protons is $\frac{1}{2}$, placing cuts on the missing perpendicular and parallel momentum no longer makes this assumption true. Figure 5.69 displays the ratio of protons to neu-

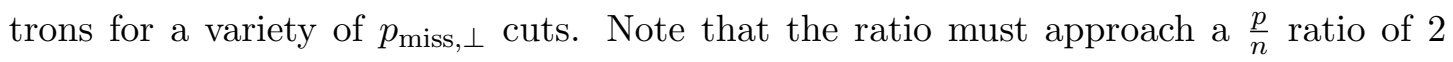
as $p_{\text {miss }, \perp} \rightarrow \infty$ and $p_{\text {miss }, \|} \rightarrow \infty$. For Kinematic 1 , the missing momentum cuts are $-250<p_{\text {miss }, \|}<250 \mathrm{MeV}$ and $p_{\text {miss }, \perp}<150 \mathrm{MeV}$, and results in a ratio of $\frac{p}{n} \approx 2.15$, or $f^{3} \mathrm{He}=\frac{1}{2.15}$ which is a $7 \%$ reduction compared to the naive expectation. An analogous 


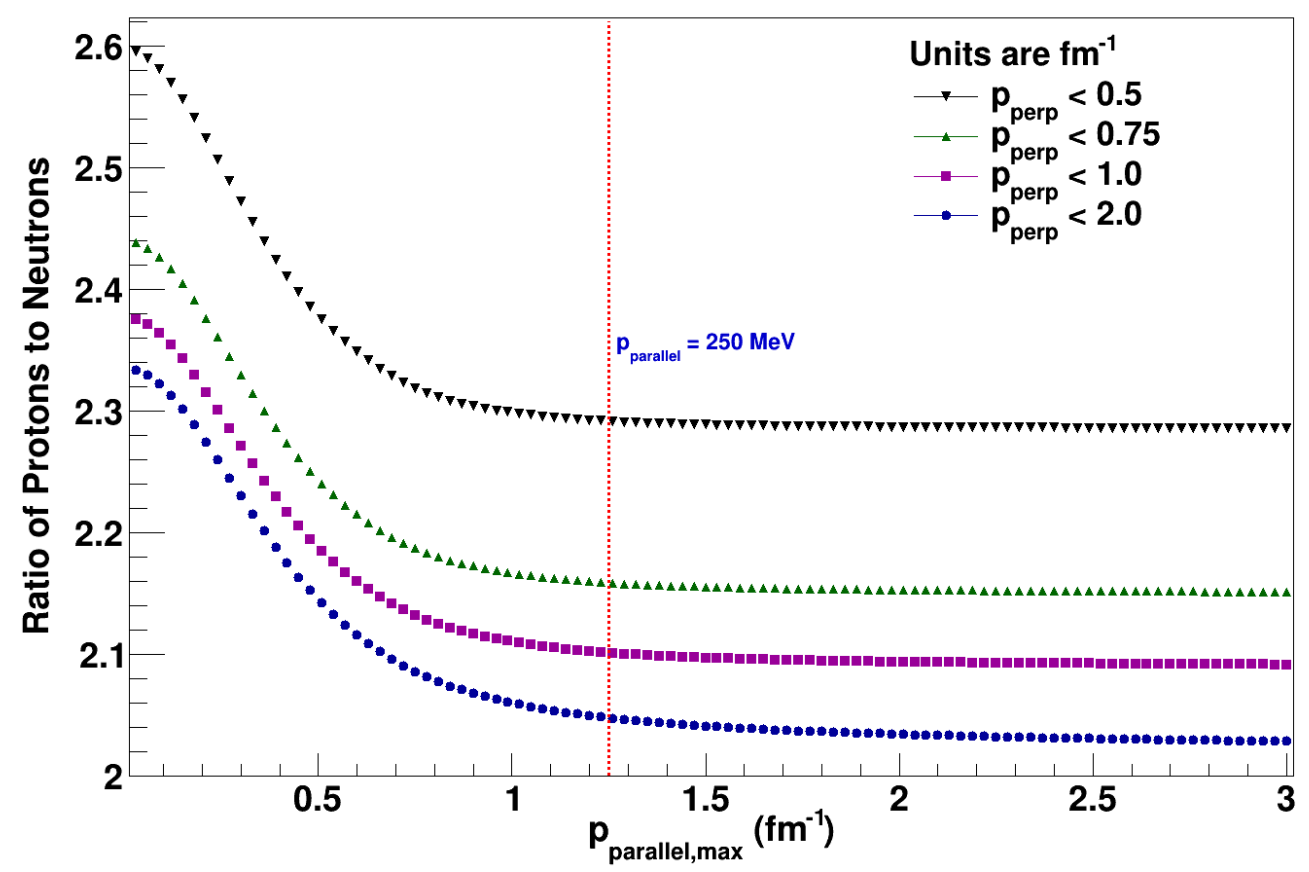

Fig. 5.69: The missing momentum dependence of the ratio of protons to neutrons for ${ }^{3}$ He. Recall that $1 \mathrm{fm}^{-1} \approx 197 \mathrm{MeV}$.

calculation was carried out for nitrogen, and the result is $f_{\mathrm{N}_{2}}=\frac{1}{0.9}$; therefore, as carbon is typically used as a proxy for nitrogen, a value of $f_{\mathrm{C}}=\frac{1}{0.9}$ is used.*

With the full information at hand, the mixing ratios may be used in order to calculate the proton dilution factors. The proton dilution factors are defined as the fraction of truly neutral (charged) to observed neutral (charged), or more explicitly as

$$
\begin{aligned}
& D_{\mathrm{p}}=\frac{N_{n}^{n}}{N_{n}^{n}+N_{p}^{n}}=\frac{f_{{ }^{3} \mathrm{He}} \frac{\sigma_{n}}{\sigma_{p}}\left(\frac{\eta_{n}^{n}}{\eta_{p}^{p}}\right)}{f_{3} \mathrm{He} \frac{\sigma_{n}}{\sigma_{p}}\left(\frac{\eta_{n}^{n}}{\eta_{p}^{p}}\right)+\left(\frac{\eta_{p}^{n}}{\eta_{p}^{p}}\right)}, \\
& D_{\mathrm{n}, \mathrm{ch}}=\frac{N_{p}^{p}}{N_{p}^{p}+N_{n}^{p}}=\frac{1}{f_{3} \mathrm{He}_{\mathrm{e}} \frac{\sigma_{n}}{\sigma_{p}}\left(\frac{\eta_{p}^{p}}{\eta_{p}^{p}}\right)+1} .
\end{aligned}
$$

It is noted that when calculating the ratios $R_{t}$ for target $t$, the charged and uncharged

\footnotetext{
* J. M. Udías provided the value of $f_{\mathrm{N}_{2}}$.
} 
quasielastic counts are corrected for the background. Furthermore, the ${ }^{3}$ He quasielastic counts are corrected for the nitrogen within the target.

The proton asymmetry, or $A_{\mathrm{p}} \equiv \frac{\Delta_{\mathrm{p}}}{\Sigma}$, is the asymmetry contamination within the quasielastic neutral sample due to protons being misidentified as neutrons. An expression may be obtained by starting with an analogous calculation as the neutron asymmetry seen in Eqn 5.50 :

$$
A_{\mathrm{p}}=\frac{\Delta_{\mathrm{p}}}{\Sigma_{\mathrm{p}}}=P_{\text {beam }} P_{3}{ }_{\mathrm{He}} P_{\mathrm{p}} A_{\mathrm{phys}}^{\mathrm{p}},
$$

where "p" is meant to explicitly describe protons rather than neutrons. The formalism for handling the physical asymmetry in regards to the finite acceptance of the detectors is presented in Section 5.7.2, specifically Eqn 5.77. In short, the physical asymmetry may be expanded to $5^{\text {th }}$-order in $\Lambda=G_{E}^{p} / G_{M}^{p}$. To account for experimental realities such as detector size, the expansion coefficients are meant to be averaged over quasielastic events, or

$$
A_{\mathrm{phys}}^{\mathrm{p}}=\sum_{i=0}^{5} \bar{T}_{i} \cdot \Lambda^{i} .
$$

The six averaged coefficients, denoted by $\bar{T}_{i}$, are defined by Eqn 5.69 and are taken to be known numbers. The proton form factor ratio, $\Lambda=G_{E}^{p} / G_{M}^{p}$, may be calculated at the acceptance averaged value of $Q^{2}$, given by Eqn 5.81, using a parametrization from Section 3.2 for example. A relationship between $\Delta_{\mathrm{p}}$ and $\Sigma$ may be realized by taking a product of Eqn 5.54 - Eqn 5.56 .

$$
\begin{gathered}
\left(1-D_{\mathrm{p}}\right) \cdot D_{\mathrm{bk}} \cdot D_{\mathrm{N}_{2}}=\left(\frac{\Sigma_{\mathrm{p}}}{\Sigma-\Sigma_{\mathrm{bk}}-\Sigma_{\mathrm{N}_{2}}}\right)\left(\frac{\Sigma-\Sigma_{\mathrm{bk}}}{\Sigma}\right)\left(\frac{\Sigma-\Sigma_{\mathrm{bk}}-\Sigma_{\mathrm{N}_{2}}}{\Sigma-\Sigma_{\mathrm{bk}}}\right) \\
\Rightarrow \Sigma_{\mathrm{p}}=\Sigma\left(1-D_{\mathrm{p}}\right) \cdot D_{\mathrm{bk}} \cdot D_{\mathrm{N}_{2}} .
\end{gathered}
$$


Substituting the expression for $\Sigma_{\mathrm{p}}$ into Eqn 5.100, and rearranging in favor of the desired proton asymmetry correction to $A_{\text {raw }}$ results in

$$
A_{\mathrm{p}} \equiv \frac{\Delta_{\mathrm{p}}}{\Sigma}=\left(1-D_{\mathrm{p}}\right) \cdot D_{\mathrm{bk}} \cdot D_{\mathrm{N}_{2}} \cdot\left(P_{\mathrm{beam}} P_{{ }^{3} \mathrm{He}} P_{\mathrm{p}}\right) \sum_{i=0}^{5} \bar{T}_{i} \cdot \Lambda^{i}
$$

In this form, the proton asymmetry contribution must take all experimental realities into account, such as the effects due to background and nitrogen dilution, the relevant polarizations of the experiment where $P_{\mathrm{p}}$ is the proton analog of $P_{\mathrm{n}}$, and the finite acceptance model.

\subsubsection{BigBite Pions}

The contamination due to BigBite $\pi^{-}$may be estimated by analyzing the preshower spectrum with no preshower energy cut, i.e. the quasielastic cut of $E_{\mathrm{ps}}>150 \mathrm{MeV}$ is removed; the result is Figure 5.13 . The observed preshower spectrum is a result of the sum of a pion and an electron preshower distribution. The pion distribution, which peaks at a preshower energy value of less than $150 \mathrm{MeV}$, has a long tail that extends well beyond the preshower cut and into the electronic distribution. Therefore, the dilution factor and asymmetry due to the preshower pion contamination within the quasielastic sample needs to be calculated.

The dilution factor, denoted by $D_{\pi}$, has been estimated using the G4SBS MC simulation, see Section 4.10 for a brief introduction. Pions are generated in an attempt to populate the acceptance of the BigBite calorimeter:

1. outgoing energy $E^{\prime}$ is randomly generated between $0.65<E^{\prime}<1.05 \mathrm{GeV}$, 
2. scattering polar angle $\theta$ is randomly generated between $\cos \theta_{\min }<\cos \theta<\cos \theta_{\max }$ where $\left(\theta_{\min }, \theta_{\max }\right)=\left(38^{\circ}, 73^{\circ}\right)$,

which are numbers motivated by the quasielastic ${ }^{3} \mathrm{He}$ data. Electrons are generated using an elastic generator with a ${ }^{3} \mathrm{He}$ target in order to match the kinematic acceptance of the data. The Fermi motion of the ${ }^{3} \mathrm{He}$ target is simulated using a parametrization in an attempt to replicate the kinematic broadening relative to the simpler $\mathrm{H}_{2}$ target. The nucleon form factors $G_{E}^{p}, G_{M}^{p}$ and $G_{M}^{n}$ are calculated using the Kelly parametrization while $G_{E}^{n}$ is calculated with a parametrization of Riordan.

The goal of the simulations is to compare the simulated preshower spectra of pions and electrons to the observed preshower spectrum. In other words, the simulated pion (electron) preshower distribution gets matched to the pion (electron) peak found in the data such that the sum of the simulated distributions matches the observed spectrum. Once this has been achieved, then the simulated pion tail may be integrated in order to estimate the $\pi^{-}$contamination making it past the quasielastic preshower energy cut of $E_{\mathrm{ps}}>150 \mathrm{MeV}$ cut. The calorimeter electronic response in simulation, however, has to describe the preshower data well in order to do this procedure; note that simulating the BigBite calorimeter has been described in detail within Section 5.3.6. The lead-glass energy deposition is Gaussian smeared by $0.05 \sqrt{E}$, and then converted to ADC channels using the gain coefficients determined from the linear regression procedure described in Section 5.3.5. Note that the Gaussian mean of all 243 gain coefficients is $0.44 \mathrm{MeV} / \mathrm{ADC}$. The realistic BigBite trigger logic is simulated in which a trigger threshold of 1120 ADC channels, or $\sim 500 \mathrm{MeV}$ which is approximately $50 \%$ of the elastic peak, is enforced. 
Furthermore, the quasielastic cuts on the vertex position and the invariant mass are applied, but it is important to ignore the $E_{\mathrm{ps}}>150 \mathrm{MeV}$ cut as the entire spectrum is required for the BigBite pion correction to $A_{\text {raw }}$. The relative energy difference of the pion to electron preshower peaks in simulation should be equal to the data; however, in this case the observed pion peak is found to occur at larger preshower energies than the simulated pion spectrum. This has been determined to be an effect of the BigBite trigger threshold which effectively biases the observed pion peak to larger values than naively expected. The trigger threshold of around 1120 ADC channels biases the peak enough such that the simulation matches the observed pion contribution.

Matching the simulation to the data is then a matter of simply scaling the amplitudes of the simulated spectra such that the sum of the pion and electron preshower distributions matches the observed spectra. In order to calculate the two normalization coefficients, several definitions need to be introduced. The output of an analysis typically comes in the form of a histogram, or a binned array of data points; therefore, in order to make this procedure more explicit, four histograms are defined representing the observed preshower spectrum, the simulated $\pi^{-}$preshower spectrum, the simulated $e^{-}$ preshower spectrum and the sum of the simulated pion and electron preshower spectra, respectively: $H_{\text {data }}, H_{\pi}, H_{e}$, and $H_{\text {sim }}$. For example, bin $i$ of $H_{\text {data }}$ consists of a count $N_{i, \text { data }}$ with a Poisson error of $\sqrt{N_{i, \text { data }}}$. For the simulated data, bin $i$ of $H_{\text {sim }}$ may be populated by

$$
N_{i, \operatorname{sim}}=C_{\pi} N_{i, \pi}+C_{e} N_{i, e}
$$

where $N_{i, \pi}$ and $N_{i, e}$ are the bin $i$ counts for the simulated pion and electron preshower 
spectra, $H_{\pi}$ and $H_{e}$, respectively. The values of the simulated pion and electron normalization coefficients, denoted by $C_{\pi}$ and $C_{e}$ respectively, may be determined by a $\chi^{2}$ minimization procedure. In this case, the $\chi^{2}$ to minimize is

$$
\chi^{2}=\sum_{i=1}^{M} \frac{\left(N_{i, \text { data }}-N_{i, \text { sim }}\right)^{2}}{N_{i, \text { data }}}
$$

where $M$ is the total number of bins. Once $C_{\pi}$ and $C_{e}$ have been determined, or in other words once $H_{\text {sim }}$ matches the data, then the dilution factor may be reliably calculated by integrating the simulated distributions scaled by the normalization coefficients:

$$
D_{\pi}=1-\frac{C_{\pi} \int_{E_{a}}^{E_{b}} H_{\pi} d E}{C_{e} \int_{E_{a}}^{E_{b}} H_{e} d E+C_{\pi} \int_{E_{a}}^{E_{b}} H_{\pi} d E}=1-\frac{C_{\pi} N_{\pi}}{C_{e} N_{e}+C_{\pi} N_{\pi}}
$$

where the integration limits are $E_{a}=150 \mathrm{MeV}$ and $E_{b}=1000 \mathrm{MeV}$. The lower limit is defined by the quasielastic cut selection while the upper limit has been defined by simply observing the data. The simulated counts prior to any scaling are denoted by $N_{\pi}$ and $N_{e}$. Note that $D_{\pi}$ has been absorbed into $D_{\text {other }}$ in Eqn 5.59 .

The asymmetry correction to $A_{\text {raw }}$, denoted by $A_{\pi} \equiv \frac{\Delta_{\pi}}{\Sigma}$, may be estimated utilizing similar techniques as $D_{\pi}$. The observed uncharged ${ }^{3}$ He preshower spectra for the \pm helicity counts after all quasielastic cuts excluding the $E_{\mathrm{ps}}>150 \mathrm{MeV}$ cut need to be obtained. Therefore, there are now two observed preshower spectra: $H_{\text {data }}^{+}$and $H_{\text {data }}^{-}$. For example, $H_{\text {data }}^{+}$is the observed preshower distribution for the quasielastic uncharged sample for the + helicity counts. The simulated pion and electron spectra may then be scaled to match the \pm observed spectra, or $H_{\text {data }}^{+}$and $H_{\text {data }}^{-}$, using the exact same procedure defined by Eqn 5.104 and Eqn 5.105. The result is four normalization coefficients, two for both \pm helicity counts, or $C_{\pi}^{ \pm}$and $C_{e}^{ \pm}$. The asymmetry due to 
BigBite preshower pions may be estimated by integrating the simulated pion spectra prior to scaling, and utilizing the normalization coefficients to match the \pm observed data such that an asymmetry may be formed:

$$
A_{\pi}=\frac{\Delta_{\pi}}{\Sigma}=\frac{C_{\pi}^{+} \int_{E_{a}}^{E_{b}} H_{\pi} d E-C_{\pi}^{-} \int_{E_{a}}^{E_{b}} H_{\pi} d E}{\Sigma}=\frac{C_{\pi}^{+} N_{\pi}-C_{\pi}^{-} N_{\pi}}{\Sigma},
$$

where $H_{\pi}$ is the simulated pion spectrum that is scaled to match the data by $C_{\pi}^{+}$or $C_{\pi}^{-}$. Recall that the normalizations $C_{\pi}^{ \pm}$are determined by the binned linear regression method through Eqn 5.105. Note that $N_{\pi}$ does not require a helicity superscript; the simulated count, i.e. the integral of the simulated pion preshower spectrum prior to scaling, gets matched to the data by the normalization coefficients $C_{\pi}^{ \pm}$. The value of $\Sigma$ is the quasielastic neutral coincidence count. The asymmetry correction is quite small and has been absorbed into the $\frac{\Delta_{\text {other }}}{\Sigma}$ term found in Eqn 5.59 . Note that an identical analysis may be done for the quasielastic charged sample.

\subsubsection{Inelastic Events}

Briefly described at the end of Section 5.6, the inelastic contributions to the raw asymmetry for the $Q^{2}=1.16 \mathrm{GeV}^{2}$ configuration are expected to be small. In particular, Figure 5.57 naively shows that the quasielastic cut selection suppresses the inelastic region to negligible levels; however, the contributions must be estimated and demonstrated to be small. In spirit, the procedure to estimate the inelastic contributions follows a similar path as the calculation of the BigBite preshower pion contamination. To be more specific, two simulated distributions are required, in this case an elastic and an inelastic invariant mass spectrum weighted by the appropriate cross sections, and 
then scaled to the quasielastic $W$ spectra by the total accumulated beam charge. Lastly, the normalization coefficients are modified by a $\chi^{2}$ minimization routine; therefore, two normalization coefficients are required, namely $C_{\text {elas }}$ and $C_{\text {inelas }}$ for the simulated elastic and inelastic spectra, respectively, which are then used to scale the simulated spectra to match the quasielastic ${ }^{3} \mathrm{He}$ data. The inelastic contributions may be calculated by integrating both the simulated elastic and inelastic scaled spectra within the quasielastic cut selection, or $0.8<W<1.15 \mathrm{GeV}$. Recall that the inelastic contributions to the raw asymmetry come in two forms, an additive asymmetry correction and a multiplicative dilution factor, which are estimated using an identical procedure as Section 5.7.6, in other words, two (four) ${ }^{3} \mathrm{He} W$ spectra are required in order to estimate the inelastic dilution factor (inelastic asymmetry correction) for the neutral and charged samples.

In order to generate the necessary simulated spectra, several event generators are employed, all of which were written by Seamus Riordan:

1. an elastic $e N$ generator with radiative methods explicitly described in Chapter 4 within the MCEEP documentation [214];

2. an inelastic generator utilizing MAID's unitary isobar model [215] which provides parametrizations of the pion electroproduction cross sections from electron scattering off of nucleons;

3. an inelastic generator in which the Christy-Bosted parametrization [216] is used to estimate the inelastic cross sections in the resonance region for $0 \leq Q^{2}<10$ $\mathrm{GeV}^{2}$ and $1.1<W<3.2 \mathrm{GeV}$.

The event generators are coupled to the $G_{E}^{n}$ module of the G4SBS Monte Carlo sim- 
ulation package. Elastic and inelastic events are generated and propagated through realistic materials representing the experimental setup of the $Q^{2}=1.16 \mathrm{GeV}^{2}$ configuration. The simulated data is analyzed using the realistic trigger schemes described in detail within Section 5.3 .6 and Section 5.4 .7 in an attempt to replicate the observed quasielastic coincidence $W$ spectra for the uncharged and charged samples. Incorporating the results of the inelastic simulation into Eqn 5.59 requires two corrections to the expression of the physical asymmetry:

$$
A_{\text {phys }}=\frac{A_{\text {raw }}-\left(\frac{\Delta_{\mathrm{bk}}}{\Sigma}+\frac{\Delta_{\mathrm{p}}}{\Sigma}+\frac{\Delta_{\pi}}{\Sigma}\right)}{P_{\text {beam }} P_{{ }_{\mathrm{He}}} P_{\mathrm{n}} D_{\mathrm{bk}} D_{\mathrm{N}_{2}} D_{\mathrm{p}} D_{\pi} D_{\text {in }}}-\frac{A_{\text {in }}}{D_{\text {in }}},
$$

where $D_{\text {in }}$ and $A_{\text {in }}$ are the multiplicative and additive corrections, respectively, for the inelastic contamination. The inelastic simulation lacks certain experimental realities and assumes a perfect $100 \%$ longitudinally polarized beam; therefore, $A_{\text {in }}$ only needs to be scaled by $D_{\text {in }}$. Note that Eqn 5.108 will undergo one more correction in the next section in order to account for nuclear corrections resulting in the final form of the physical asymmetry from which the form factor ratio may be extracted.

\subsubsection{Nuclear Corrections and Final State Interactions}

Since the neutron is one of three nucleons bound to the ${ }^{3} \mathrm{He}$ nucleus, nontrivial nuclear corrections are required. Briefly mentioned in Section 3.1.2, the semi-exclusive electronuclear reaction ${ }^{3} \mathrm{He}\left(\vec{e}, e^{\prime} N\right) X$ where the final state consists of one nucleon may be modeled by the following processes:

1. the plane-wave impulse approximation (PWIA), where one nucleon is knocked-out of the nucleus and no further interaction occurs; 
2. final state interactions (FSI), the knocked-out nucleon interacts with the residual hadronic system, e.g. single and double re-scattering;

3. meson exchange currents (MEC), the virtual photon may couple to a meson that is exchanged between two nucleons;

4. isobar current (IC), the virtual photon may produce an intermediate $\Delta$ which may interact with the residual nucleons.

The above statements may be seen pictorially by Figure 5.70 where A, B, C and D corresponds to IA, FSI, MEC and IC, respectively. For sufficiently large momentum transfer, i.e. $|\mathbf{q}|>1 \mathrm{GeV}$, the MEC and IC corrections are suppressed due to the presence of an additional factor of $Q^{-4}$ relative to the PWIA diagrams [217]. Therefore, the leading order diagrams are reduced to the PWIA diagram and the diagrams associated to FSI. The effects due to FSI are approximately computed in the so-called generalized eikonal approximation (GEA) [35], an extension to the Glauber approximation [36] which treats the nucleons as stationary scatterers; GEA allows for nonzero initial momentum of the recoiling nucleons. The core of the GEA framework resides in an assumption that the knocked-out nucleon re-scatters with a bound nucleon only once which effectively reduces an infinite number of diagrams to a finite number that may be calculated using the Feynman rules of GEA. In order words, the only FSI amplitudes that need to be calculated for $\vec{e}+{ }^{3} \overrightarrow{\mathrm{He}}$ scattering are single and double re-scattering.

A Monte Carlo code written by Misak M. Sargsian calculates the PWIA and FSI amplitudes within the GEA framework. Additionally, the initial spin of the nucleons is important as an asymmetry needs to eventually be calculated; therefore, spin dependent 


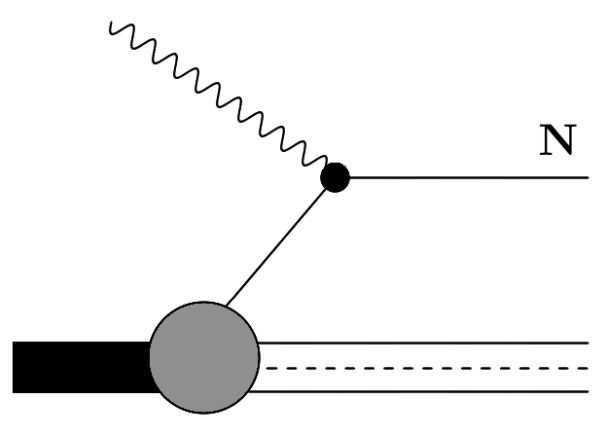

Figure A

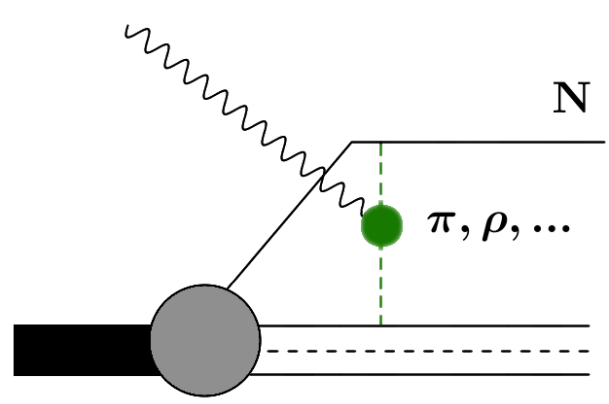

Figure C

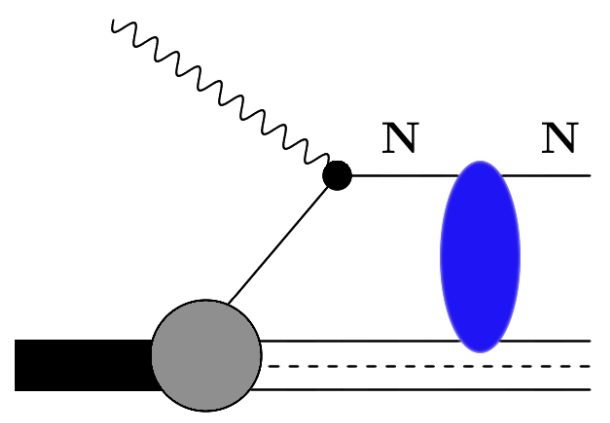

Figure B

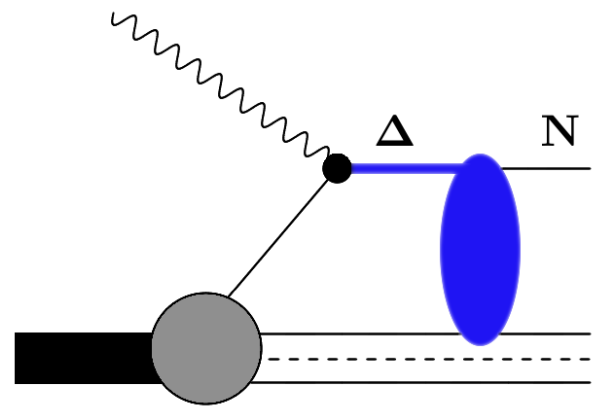

Figure D

Fig. 5.70: The lowest order dominant contributions in semi-exclusive electro-nuclear reactions: the impulse approximation (A), final state interactions (B), meson exchange currents (C) and isobar currents (D).

$N N$ scattering data and the ${ }^{3} \mathrm{He}$ wave function resulting from the AV18 $N N$ potential is used. The simulation includes the quasielastic cuts on $W, p_{\text {miss }, \|}$, and $p_{\text {miss }, \perp}$. Furthermore, the orientation of the target polarization and the finite acceptance of the experiment are taken into account. By assuming values of $\Lambda \equiv G_{E}^{n} / G_{M}^{n}$, a mapping between the asymmetry including nuclear corrections and the free neutron asymmetry may be obtained. The nuclear correction, denoted by the FSI subscript, gets worked 
into Eqn 5.59 in a similar manner as the inelastic correction. The dilution factor due to FSI has been absorbed by the $D_{\text {other }}$ term, but now may be made explicit. The asymmetry correction to $A_{\text {raw }}$ due to nuclear effects comes in the form of a simulation and does not need to be corrected for all experimental realities such as background, nitrogen or proton contamination. In this light, an additional term needs to be introduced, resulting in the final form of the experimental physical asymmetry:

$$
A_{\text {phys }}=\frac{A_{\text {raw }}-\left(\frac{\Delta_{\mathrm{bk}}}{\Sigma}+\frac{\Delta_{\mathrm{p}}}{\Sigma}+\frac{\Delta_{\pi}}{\Sigma}\right)}{P_{\text {beam }} P_{3_{\mathrm{He}}} D_{\mathrm{bk}} D_{\mathrm{N}_{2}} D_{\mathrm{p}} D_{\pi} D_{\mathrm{in}} D_{\mathrm{FSI}}}-\frac{A_{\mathrm{in}}}{D_{\mathrm{in}}}-\frac{A_{\mathrm{FSI}}}{D_{\mathrm{FSI}}} .
$$

Note that the polarization of the neutron $P_{\mathrm{n}}$ has been removed from the formula; the polarization effects of the ${ }^{3} \mathrm{He}$ nucleons are intimately embedded in the $D_{\mathrm{FSI}}$ and $A_{\mathrm{FSI}}$ terms. Consequently, $P_{\mathrm{n}}$ may not be naively separated as originally suggested. As in the inelastic simulation, the FSI corrections do not need to be scaled by $P_{\text {beam }}$.

In summary, the formalism to correct the raw asymmetry such that the result most closely resembles the asymmetry of a free neutron has been presented. The experimental physical asymmetry has been presented three times by Eqn 5.59, Eqn 5.108, and Eqn 5.109 as $A_{\text {raw }}$ continued to be refined; the latter equation represents the final form that will be used for the $G_{E}^{n} / G_{M}^{n}$ calculation. The arbitrary terms $D_{\text {other }}$ and $A_{\text {other }}$ have been updated to handle BigBite pions, inelastics and FSI corrections. Note that the polarization of the neutron within the ${ }^{3} \mathrm{He}$ nucleus, previously denoted by $P_{\mathrm{n}}$, has been removed and replaced by $D_{\mathrm{FSI}}$ as the nuclear corrections and FSI are intimately connected and cannot be naively separated. In the final chapter, all calculations necessary to evaluate the experimental and theoretical $A_{\text {phys }}$ will be presented, concluding in the extraction of the form factor ratio. 


\section{Chapter 6}

\section{Results}

The purpose of the final chapter is to present all calculations required to extract the form factor ratio of the neutron at $Q^{2}=1.16 \mathrm{GeV}^{2}$. The starting point, or the raw asymmetry acquired from the data, needs to be corrected for all contaminations as introduced in Section 5.7.1. The fully corrected raw asymmetry may then be compared to the theoretical physical asymmetry of a free neutron, see Section 5.7 .2 for the fifth order expansion and the definitions of the finite acceptance expansion coefficients. The form factor ratio may be calculated by numerically solving the resulting polynomial equation. Additionally, the error propagation is explicitly shown culminating in a final statistical and systematic uncertainty in $G_{E}^{n} / G_{M}^{n}$.

\subsection{Calculations for $G_{E}^{n} / G_{M}^{n}$}

All notation is introduced in Table 5.4 where $\Sigma_{x}=N_{x}^{+}+N_{x}^{-}=N_{x}$ are equivalent, and the quasielastic cut selection is summarized by Table 5.3. The corrections to the raw asymmetry are assumed to be constant. Furthermore, the beam polarization of the experiment is also assumed to be constant over the data set. The raw asymmetry is calculated and corrected on per run basis for the entire accepted polarized ${ }^{3} \mathrm{He}$ data set 
producing a set of physical asymmetries which then must be combined in a statistically weighted average. The purpose of this section is to explicitly show the calculations and error propagation of all corrections to the raw asymmetry.

\subsubsection{Uncertainties}

As seen in the previous chapter, the analysis truly boils down to counting events of a particular helicity after quasielastic cuts have been applied. Fluctuations in counting particles inherently arise when dealing with a random process like a scattering experiment; therefore, it is natural to expect statistical fluctuations in a finite number of counts within finite time intervals. If the counting is repeated many times, the observed values are expected to follow a Poisson distribution with a mean count $N$ and variance $\sigma^{2}=N$. The statistical uncertainty in such a count is then $\delta N=\sqrt{N}$.

Assume that there is some function $f$ of a set of $n$ variables $x_{1}, x_{2}, \ldots, x_{n}$, then the variance in $f$ may be calculated as

$$
\sigma_{f}^{2}=\mathbf{g}^{\mathbf{T}} \mathbf{V g}
$$

where $\mathbf{V}$ is the variance-covariance matrix and the $i^{\text {th }}$ element of column vector $\mathbf{g}$ of length $n$ is $\partial f / \partial x_{i}$. The notation $\mathbf{g}^{\mathbf{T}}$ represents the vector transpose of $\mathbf{g}$. If the variables are uncorrelated, then the off-diagonal terms of $\mathbf{V}$ vanish resulting in

$$
\sigma_{f}^{2}=\sum_{i=1}^{n}\left(\frac{\partial f}{\partial x_{i}}\right)^{2} \sigma_{x_{i}}^{2}
$$

In regards to notation, the variance may be denoted by $\sigma_{f}^{2}$ or $(\delta f)^{2}$ depending on the level of clutter in the naming of $f$. 


\subsubsection{Polarizations}

While the polarizations vary over time, it is assumed that all polarizations except the target polarization remain constant over the entire ${ }^{3} \mathrm{He}$ data set; the target polarization may fluctuate at the granularity of a ${ }^{3} \mathrm{He}$ run. For the dates that overlap with the $Q^{2}=1.16 \mathrm{GeV}^{2}$ point, namely the first week of March of 2006, there are three Møller measurements of the beam polarization corresponding to the first three entries of Table 4.3 . While the uncertainty in the beam polarization is dominated by systematic contributions, the three measurements are combined in a statistically weighted average and the systematic contribution is simply averaged; the quoted error combines the resulting statistical and systematic errors in quadrature. The polarization of the ${ }^{3} \mathrm{He}$ target cell (Barbara) may be seen as a function of ${ }^{3} \mathrm{He}$ run number by Figure 6.1; the error bars are systematic. In order to present a single number, a count weighted average is performed, i.e. $P^{{ }^{3} \mathrm{He}}=\frac{1}{N} \sum_{i} N_{i} P_{3} \mathrm{He}, i$ where $N$ is the total quasielastic neutral count, $N_{i}$ is the count for run $i$, and $P_{3} \mathrm{He}, i$ is the measured polarization for run $i$. Furthermore, the uncertainty in the target polarization is combined in a count weighted average. The two remaining polarizations, the polarizations of the neutron and protons within the ${ }^{3}$ He nucleus denoted by $P_{\mathrm{n}}$ and $P_{\mathrm{p}}$ respectively, require theoretical input; the spin distributions resulting from the AV18 $N N$ potential need to be integrated enforcing the quasielastic cut selection as the polarizations are sensitive to the missing momenta cuts. Note that the only correction to $A_{\text {raw }}$ that specifically requires a numerical value of $P_{\mathrm{n}}$ or $P_{\mathrm{p}}$ is the proton (neutron) asymmetry correction to the uncharged (charged) samples. The values used throughout the analysis are summarized within Table 6.1. 


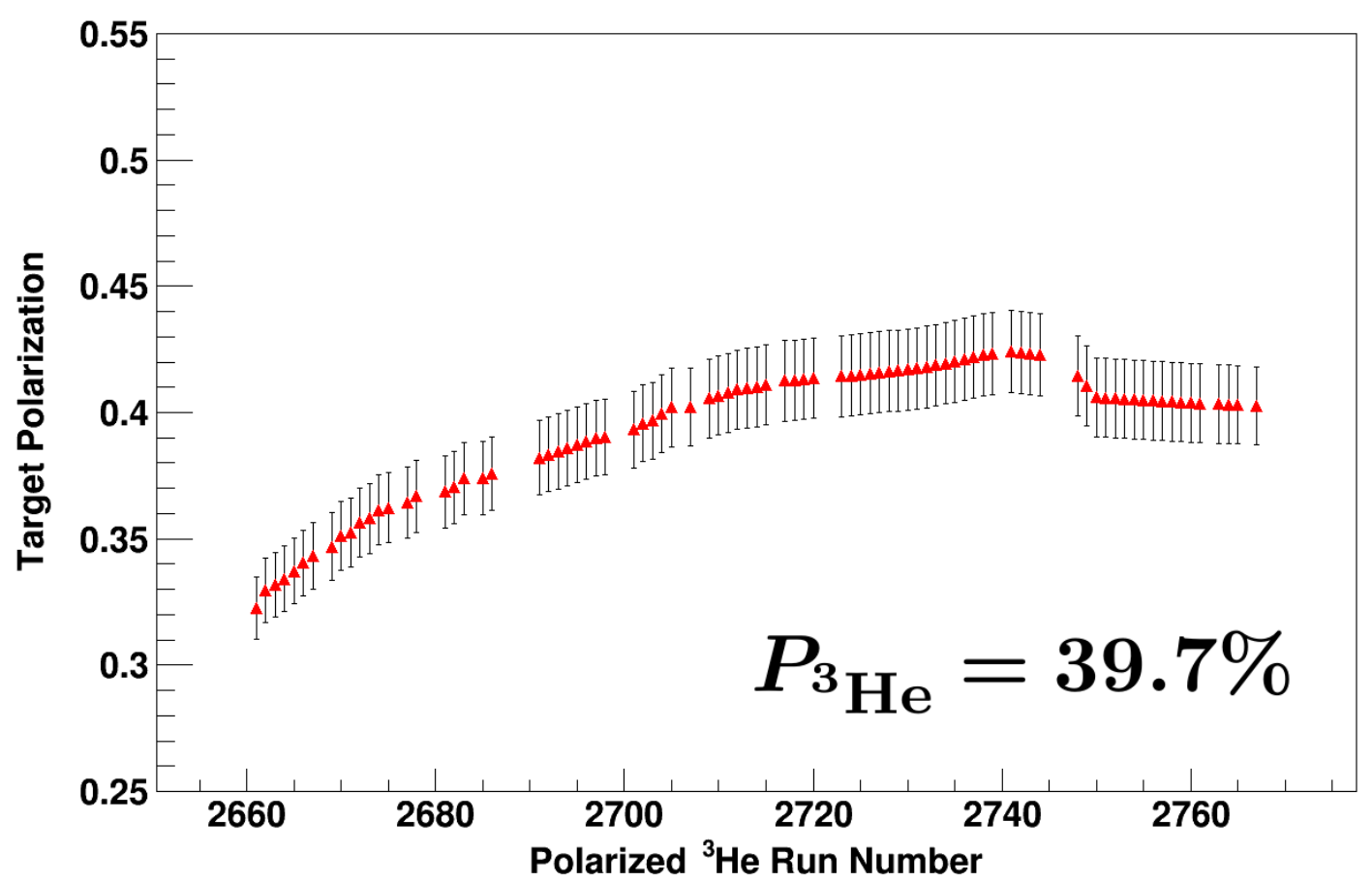

Fig. 6.1: The polarization of target cell Barbara where the error bars are systematic contributions. An uncharged count weighted average over the entire ${ }^{3} \mathrm{He}$ data set yields a target polarization of $39.7 \%$, but the analysis allows the polarization to fluctuate per run.

\begin{tabular}{ccl}
\hline Polarization & Value & Description \\
\hline$P_{\text {beam }}$ & $0.872 \pm 0.020$ & Møller beam polarization \\
$P_{3} \mathrm{He}$ & $0.397 \pm 0.015$ & ${ }^{3}$ He polarization from hSEOP \\
$P_{\mathrm{n}}$ & $0.860 \pm 0.020$ & Polarization of neutron in nucleus \\
$P_{\mathrm{p}}$ & $-0.050 \pm 0.025$ & Polarization of proton in nucleus \\
\hline
\end{tabular}

Table 6.1: A summary of the polarization values used in various calculations. 


\subsubsection{Background}

The dilution factor due to accidental background for the quasielastic neutral sample is calculated as $D_{\mathrm{bk}}=1-\frac{\Sigma_{\mathrm{bk}}}{\Sigma}\left(\mathrm{Eqn} 5.83\right.$ ). The subtleties of $\Sigma_{\mathrm{bk}}$ are presented in Section 5.7.3, or to be more specific the lack of knowledge of how a charge is assigned to the accidental background makes a true determination of $\Sigma_{\mathrm{bk}}$ difficult to calculate. For the neutral background, an average of the two extreme identification cases is used, either all or nothing: $\Sigma_{\mathrm{bk}} \rightarrow \frac{N_{\mathrm{bk}}}{2}$. There is a statistical uncertainty associated to this count:

$\sigma_{\mathrm{stat}}=\sqrt{\frac{N_{\mathrm{bk}}}{2}}$. Additionally, if the background is modeled as a uniform probability distribution denoted by $P(y)$ then a systematic uncertainty of $\sigma_{\mathrm{sys}}=\frac{N_{\mathrm{bk}}}{\sqrt{12}}$ needs to added in quadrature. To be explicit, the normalized uniform distribution is defined as

$$
P(y)=\left\{\begin{array}{cc}
\frac{1}{N_{\mathrm{bk}}} & -\frac{N_{\mathrm{bk}}}{2} \leq y \leq \frac{N_{\mathrm{bk}}}{2} \\
0 & \text { otherwise }
\end{array}\right.
$$

which results in a systematic uncertainty of

$$
\sigma^{2}=\int_{-\frac{N_{\mathrm{bk}}}{2}}^{\frac{N_{\mathrm{bk}}}{2}} d y \frac{y^{2}}{N_{\mathrm{bk}}}=\frac{N_{\mathrm{bk}}^{2}}{12} \Rightarrow \sigma_{\mathrm{sys}}=\frac{N_{\mathrm{bk}}}{\sqrt{12}} .
$$

Therefore, the variance associated to $\Sigma_{\mathrm{bk}}$ is $\sigma_{\Sigma_{\mathrm{bk}}}^{2}=\frac{N_{\mathrm{bk}}}{2}+\frac{N_{\mathrm{bk}}^{2}}{12}$. The variance in $\Sigma$ is $\sigma_{\Sigma}^{2}=N$. Even though the variables are uncorrelated, the matrix formulation is used in the calculation of the variance in the background dilution factor:

$$
\left(\delta D_{\mathrm{bk}}\right)^{2}=\left(\begin{array}{cc}
-\frac{1}{\Sigma} & -\frac{\Sigma_{\mathrm{bk}}}{\Sigma^{2}}
\end{array}\right)\left(\begin{array}{cc}
\sigma_{\Sigma_{\mathrm{bk}}}^{2} & 0 \\
0 & \sigma_{\Sigma}^{2}
\end{array}\right)\left(\begin{array}{c}
-\frac{1}{\Sigma} \\
-\frac{\Sigma_{\mathrm{bk}}}{\Sigma^{2}}
\end{array}\right)=\frac{N_{\mathrm{bk}}}{2 \Sigma^{2}}+\frac{N_{\mathrm{bk}}^{2}}{4 \Sigma^{2}}\left(\frac{1}{3}+\frac{1}{\Sigma}\right) .
$$

The background asymmetry correction to the quasielastic neutral sample is (see Eqn 5.84)

$$
A_{\mathrm{bk}}=\frac{\Delta_{\mathrm{bk}}}{\Sigma}=A_{\mathrm{raw}, \mathrm{bk}}\left(1-D_{\mathrm{bk}}\right)=\frac{N_{\mathrm{bk}}^{+}-N_{\mathrm{bk}}^{-}}{\Sigma} .
$$




\begin{tabular}{|c|c|c|c|c|c|}
\hline Parameter & \multicolumn{2}{|c|}{ Neutral } & \multicolumn{2}{|c|}{ Charged } & Description \\
\hline & + & - & + & - & Helicity \\
\hline$N$ & 85,510 & 96,113 & 696,180 & 697,216 & QE count \\
\hline$N_{\mathrm{bk}}$ & 9,173 & 9,233 & 2,681 & 2,713 & Background count \\
\hline$D_{\mathrm{bk}}$ & \multicolumn{2}{|c|}{$0.949 \pm 0.029$} & \multicolumn{2}{|c|}{$0.990 \pm 0.004$} & Background dilution \\
\hline$\sigma_{\text {stat }}$ & \multicolumn{2}{|c|}{0.0004} & \multicolumn{2}{|c|}{ negligible } & Statistical \\
\hline$\sigma_{\mathrm{sys}}$ & \multicolumn{2}{|c|}{0.0292} & \multicolumn{2}{|c|}{0.0038} & Systematic \\
\hline$A_{\text {raw }, \mathrm{bk}}$ & \multicolumn{2}{|c|}{$-0.003 \pm 0.007$} & \multicolumn{2}{|c|}{$-0.006 \pm 0.014$} & Background raw asymmetry \\
\hline$A_{\mathrm{bk}}$ & \multicolumn{2}{|c|}{$-0.0003 \pm 0.0005$} & \multicolumn{2}{|c|}{ negligible } & Background asymmetry $\frac{\Delta_{\mathrm{bk}}}{\Sigma}$ \\
\hline
\end{tabular}

Table 6.2: The results of the background correction for the neutral/charged quasielastic samples. Values that are on the order of $10^{-5}$ are denoted negligible.

The variance in $A_{\mathrm{bk}}$ may be calculated in a similar fashion as $\left(\delta D_{\mathrm{bk}}\right)^{2}$; however, the difference $N_{\mathrm{bk}}^{+}-N_{\mathrm{bk}}^{-}$introduces a systematic correlation that must be taken into account:

$$
\begin{aligned}
\left(\delta A_{\mathrm{bk}}\right)^{2} & =\left(\begin{array}{lll}
\frac{1}{\Sigma} & -\frac{1}{\Sigma} & -\frac{\Delta_{\mathrm{bk}}}{\Sigma^{2}}
\end{array}\right)\left(\begin{array}{ccc}
\frac{N_{\mathrm{bk}}^{+}}{2}+\frac{\left(N_{\mathrm{bk}}^{+}\right)^{2}}{12} & \frac{N_{\mathrm{bk}}^{+} N_{\mathrm{bk}}^{-}}{12} & 0 \\
\frac{N_{\mathrm{bk}}^{+} N_{\mathrm{bk}}^{-}}{12} & \frac{N_{\mathrm{bk}}^{-}}{2}+\frac{\left(N_{\mathrm{bk}}^{-}\right)^{2}}{12} & 0 \\
0 & 0 & N
\end{array}\right)\left(\begin{array}{c}
\frac{1}{\Sigma} \\
-\frac{1}{\Sigma} \\
-\frac{\Delta_{\mathrm{bk}}}{\Sigma^{2}}
\end{array}\right) \\
& =\frac{N_{\mathrm{bk}}^{+}+N_{\mathrm{bk}}^{-}}{2 \Sigma^{2}}+\frac{\left(N_{\mathrm{bk}}^{+}-N_{\mathrm{bk}}^{-}\right)^{2}}{4 \Sigma^{2}}\left(\frac{1}{3}+\frac{1}{\Sigma}\right) .
\end{aligned}
$$

A similar set of equations exists for the charged sample except the amount of background to subtract is now $N_{\mathrm{bk}, \mathrm{ch}}+\frac{N_{\mathrm{bk}}}{2}$ where the absence of the "ch" subscript refers to the neutral sample. The results of the background analysis are summarized Table 6.2 . 


\subsubsection{Nitrogen in the ${ }^{3} \mathrm{He}$ Cell}

In order to estimate the dilution factor due to scattering off of nitrogen in the target cell, the ratio of the background-corrected quasielastic neutral count for carbon and polarized ${ }^{3} \mathrm{He}$ data is taken and normalized according to the accumulated beam charges and target mass thicknesses for the two data types. The appropriate formula taking into account the differing target lengths is (see Eqn 5.87 and Eqn 5.88)

$$
D_{\mathrm{N}_{2}}=1-\frac{\Sigma(\mathrm{C})-\Sigma_{\mathrm{bk}}(\mathrm{C})}{\Sigma-\Sigma_{\mathrm{bk}}} \frac{Q\left({ }^{3} \mathrm{He}\right)}{Q(\mathrm{C})} \frac{m_{\mathrm{N}_{2}}\left({ }^{3} \mathrm{He}\right)}{m_{\mathrm{C}}(\mathrm{C})},
$$

where $\Sigma(\mathrm{C})-\Sigma_{\mathrm{bk}}(\mathrm{C})$ and $\Sigma-\Sigma_{\mathrm{bk}}$ are the background corrected neutral counts post quasielastic cuts for the carbon and ${ }^{3} \mathrm{He}$ data, respectively, $Q(t)$ is the accumulated beam charge for data of target type $t$ and $m_{x}(t)$ is the mass thickness of $x$ in target $t$. Recall that the carbon data has been argued to be a suitable replacement for the nitrogen data if the center $\mathrm{BeO}$ foil is removed from the analysis. Additionally, as the carbon target is not evacuated, the mass thickness for the carbon target requires a correction to account for air. In an attempt to minimize the effects due to air, vertex cuts are introduced corresponding to the carbon foil center $\pm 2.3 \mathrm{~cm}^{*}$ where the four carbon foils are located at $z$ positions of \pm 0.133 and \pm 0.067 meters. The resulting length of air that contributes to the carbon mass thickness is then $4 \times(2 \times 2.3 \mathrm{~cm})=18.4 \mathrm{~cm}$. A summary of the carbon target and cut selections may be seen by Figure 6.2 where the shaded regions are excluded from the analysis. The same set of vertex cuts are also applied to the ${ }^{3} \mathrm{He}$ data in an attempt to minimize any acceptance mismatching; this effectively reduces the length of the target from $40 \mathrm{~cm}$ to $18.4 \mathrm{~cm}$. To be absolutely

\footnotetext{
${ }^{*}$ Recall that $\sigma_{v_{z}} \approx 0.65 \mathrm{~cm} \Rightarrow$ a cut of $2.3 \mathrm{~cm}$ is approximately $3.5 \sigma_{v_{z}}$, see Figure 5.6
} 


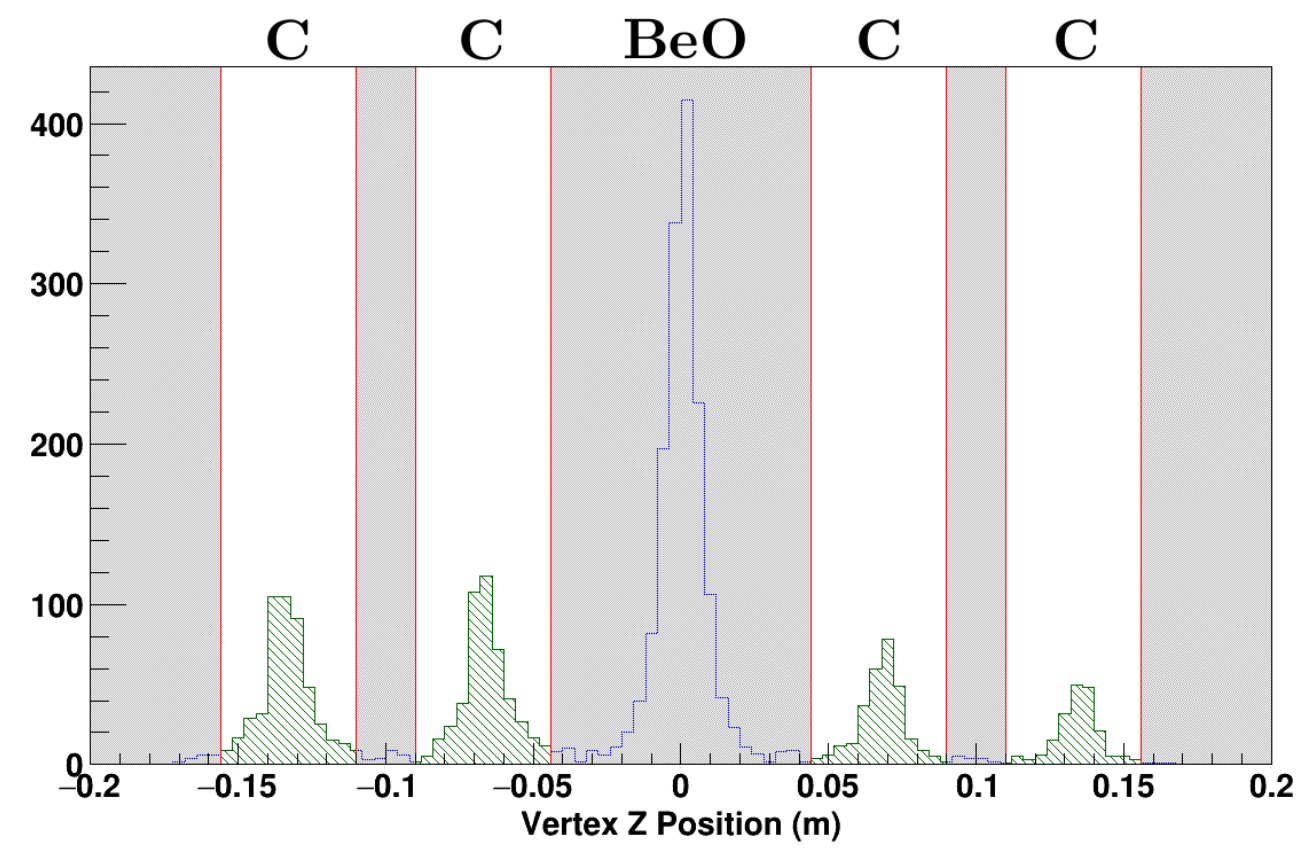

Fig. 6.2: The four carbon foils used in the $D_{\mathrm{N}_{2}}$ analysis. The dark shaded regions are excluded from the analysis which include regions of air and the $\mathrm{BeO}$ foil.

explicit, the mass thicknesses with the chosen cut selection are

$$
\begin{aligned}
m_{\mathrm{N}_{2}}\left({ }^{3} \mathrm{He}\right) & =\rho_{\mathrm{N}_{2}}\left({ }^{3} \mathrm{He}\right) l_{3} \mathrm{He}, \\
& =\left(0.203 \frac{\mathrm{mg}}{\mathrm{cm}^{3}}\right) \cdot(4 \times 2 \times 2.3 \mathrm{~cm}), \\
& =3.74 \mathrm{mg} / \mathrm{cm}^{2}, \\
m_{\mathrm{C}}(\mathrm{C}) & =\rho_{\mathrm{C}}(\mathrm{C}) l_{\mathrm{C}}+\rho_{\text {air }} l_{\text {air }}, \\
& =\left(4 \times 47.79 \frac{\mathrm{mg}}{\mathrm{cm}^{2}}\right)+\left(4 \times 2 \times 2.3 \mathrm{~cm} \times \rho_{\text {air }}\right), \\
& =(191.2+22.23) \mathrm{mg} / \mathrm{cm}^{2}, \\
& =213.4 \mathrm{mg} / \mathrm{cm}^{2},
\end{aligned}
$$


where a value of $\rho_{\text {air }}=1.21 \mathrm{mg} / \mathrm{cm}^{3}$ has been used as determined by JLab's weather archive [218].

The uncertainty in $D_{\mathrm{N}_{2}}$ may be calculated using the standard techniques of previous calculations and utilizing a short-hand notation for convenience. The derivative vector transposed is

$$
\begin{aligned}
\mathbf{g}^{\mathbf{T}} & =\left(\begin{array}{lllllll}
\frac{\partial D_{\mathrm{N}_{2}}}{\partial \Sigma(\mathrm{C})} & \frac{\partial D_{\mathrm{N}_{2}}}{\partial \Sigma_{\mathrm{bk}}(\mathrm{C})} & \frac{\partial D_{\mathrm{N}_{2}}}{\partial \Sigma} & \frac{\partial D_{\mathrm{N}_{2}}}{\partial \Sigma_{\mathrm{bk}}} & \frac{\partial D_{\mathrm{N}_{2}}}{\partial m_{\mathrm{N}_{2}}\left({ }^{3} \mathrm{He}\right)} & \frac{\partial D_{\mathrm{N}_{2}}}{\partial m_{\mathrm{C}}(\mathrm{C})}
\end{array}\right), \\
& =\frac{Q}{\mathcal{D}} \frac{m_{\mathrm{N}_{2}}\left({ }^{3} \mathrm{He}\right)}{m_{\mathrm{C}}(\mathrm{C})}\left(\begin{array}{llllll}
-1 & 1 & \frac{\mathcal{N}}{\mathcal{D}} & -\frac{\mathcal{N}}{\mathcal{D}} & -\frac{\mathcal{N}}{m_{\mathrm{N}_{2}}\left({ }^{3} \mathrm{He}\right)} & \frac{\mathcal{N}}{m_{\mathrm{C}}(\mathrm{C})}
\end{array}\right),
\end{aligned}
$$

where $\mathcal{N} \equiv \Sigma(\mathrm{C})-\Sigma_{\mathrm{bk}}(\mathrm{C}), \mathcal{D} \equiv \Sigma-\Sigma_{\mathrm{bk}}$ and $Q \equiv Q\left({ }^{3} \mathrm{He}\right) / Q(\mathrm{C})$. The variance matrix $\mathbf{V}$ is diagonal; therefore, in order to avoid unnecessarily large matrices only the diagonal components are presented:

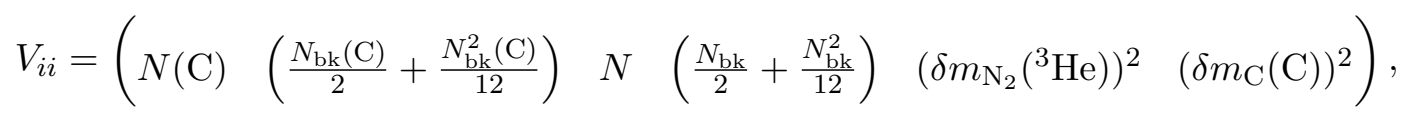

where the amount of background to subtract for both data sets is handled in an identical manner as explicitly shown in the background dilution calculation. The variance in the nitrogen dilution factor for the neutral sample is

$$
\begin{aligned}
\left(\delta D_{\mathrm{N}_{2}}\right)^{2} & =\left(\frac{Q \cdot m_{\mathrm{N}_{2}}\left({ }^{3} \mathrm{He}\right)}{\mathcal{D} \cdot m_{\mathrm{C}}(\mathrm{C})}\right)^{2}\left\{N(\mathrm{C})+\frac{N_{\mathrm{bk}}(\mathrm{C})}{2}+\frac{N_{\mathrm{bk}}^{2}(\mathrm{C})}{12}\right. \\
& \left.+\left(\frac{\mathcal{N}}{\mathcal{D}}\right)^{2}\left(N+\frac{N_{\mathrm{bk}}}{2}+\frac{N_{\mathrm{bk}}^{2}}{12}\right)+\mathcal{N}^{2}\left[\left(\frac{\delta m_{\mathrm{N}_{2}}\left({ }^{3} \mathrm{He}\right)}{m_{\mathrm{N}_{2}}\left({ }^{3} \mathrm{He}\right)}\right)^{2}+\left(\frac{\delta m_{\mathrm{C}}(\mathrm{C})}{m_{\mathrm{C}}(\mathrm{C})}\right)^{2}\right]\right\}
\end{aligned}
$$

where the short-hand notation has been used again. Note that $\delta m=l \delta \rho \Rightarrow \frac{\delta m}{m}=\frac{\delta \rho}{\rho}$ where $m$ is mass thickness. The cut size around carbon foil centers has been varied from $2.0 \rightarrow 2.75 \mathrm{~cm}$, and no significant variations in $D_{\mathrm{N}_{2}}$ are observed.

There is a systematic uncertainty associated with the acceptance mismatch between the two target types due to the vertex cuts seen in Figure 6.2. The effects of 
the acceptance mismatch in regards to the value of $D_{\mathrm{N}_{2}}$ are estimated by changing the vertex cuts applied to the data. There are three cut selections used:

1. apply the same set of vertex cuts to both data sets as defined in Figure 6.2. effectively shortening the ${ }^{3} \mathrm{He}$ target from $40 \mathrm{~cm}$ to $18.4 \mathrm{~cm}$ (the default cut);

2. no vertex cuts are applied to the ${ }^{3} \mathrm{He}$ data corresponding to a $40 \mathrm{~cm}$ long target while the cuts in Figure 6.2 are applied to the carbon data (the extreme cut);

3. cutting around the four foils individually in both data sets (the sanity cuts).

The acceptance mismatch is minimized by the default cut 1); therefore, naively the systematic uncertainty may be estimated by taking the difference of $D_{\mathrm{N}_{2}}$ after cuts 1) and 2) are applied. However, in order to make this estimate more convincing, cut selection 3) is applied resulting in four more values of $D_{\mathrm{N}_{2}}$. The result of this analysis may be seen by Figure 6.3. The blue markers correspond to cut selection 3) and the position on the $\mathrm{x}$-axis corresponds to the vertex $z$ position of a foil, i.e. either \pm 13.3 $\mathrm{cm}$ or $\pm 6.7 \mathrm{~cm}$. The red markers correspond to cut selections 1) and 2), but the positions on the $\mathrm{x}$-axis are arbitrary as the vertex cut has been expanded to included all four foils. Little variation in $D_{\mathrm{N}_{2}}$ is observed by the varying the cuts on $v_{z}$ indicating that the effects due to acceptance mismatch are small. An estimate to the systematic uncertainty is obtained by taking half the difference of $D_{\mathrm{N}_{2}}$ after applying cuts 1 ) and $2)$, corresponding to $(0.954-0.947) / 2=0.0035$, respectively.

The entire procedure presented in this section may also be done for the quasielastic charged sample where the amount of background to subtract is $N_{\mathrm{bk}, \mathrm{ch}}+\frac{N_{\mathrm{bk}}}{2}$. Recall that the absence of the "ch" subscript always refers to the neutral sample. Since the 


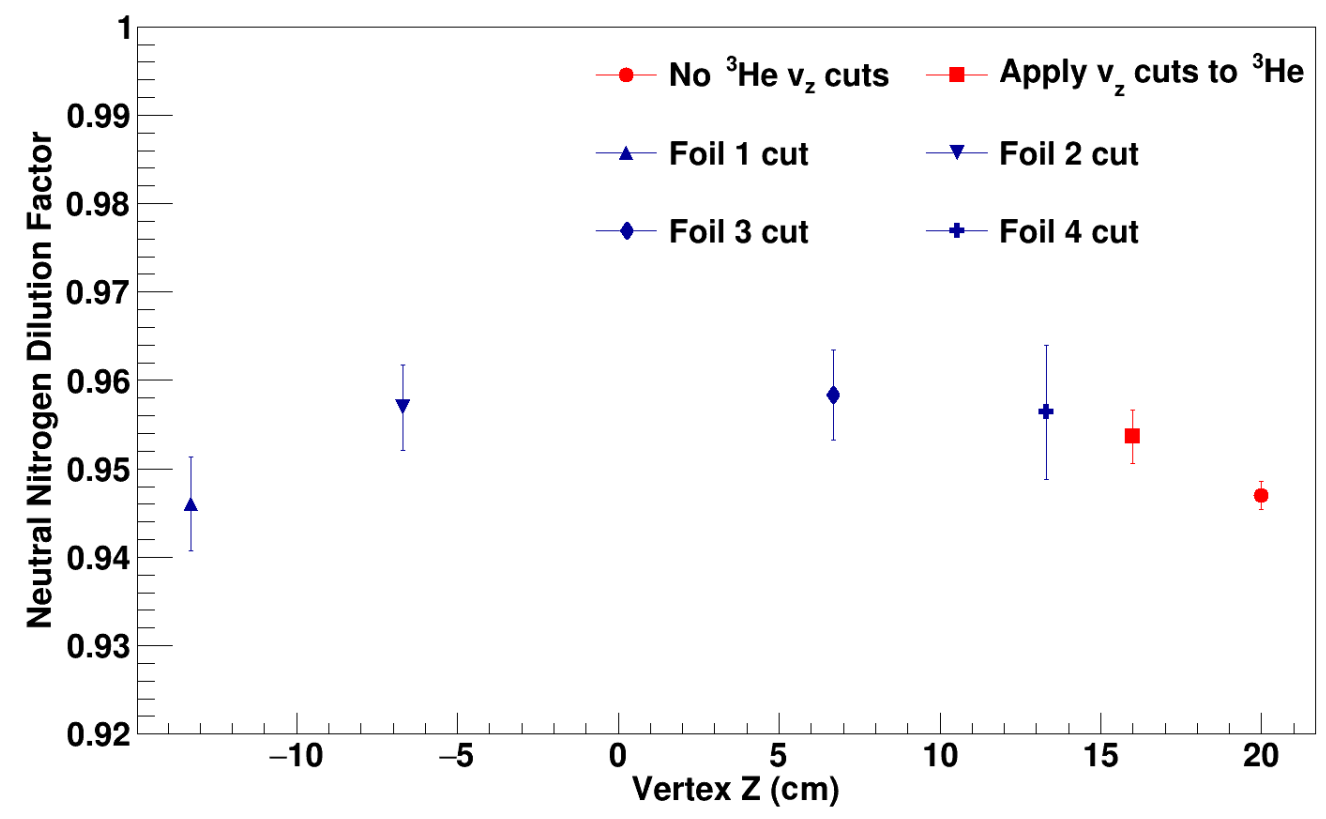

Fig. 6.3: Three sets of $v_{z}$ cuts defined in the text are applied in an attempt to quantify the effects of the acceptance mismatch in the calculation of $D_{\mathrm{N}_{2}}$. Note that the $\mathrm{x}$-values of the red data points are arbitrary as the carbon vertex cut includes all four foils.

nitrogen within the polarized target cell is unpolarized, there are no nitrogen asymmetry corrections to the raw asymmetry. Parameters that are common to both the neutral and charged calculation are presented in Table 6.3, and the results of the nitrogen dilution analysis are summarized in Table 6.4. To be clear, cut selection 1) has been used, or in other words vertex cuts of carbon foil center $\pm 2.3 \mathrm{~cm}$ have been applied to both the ${ }^{3} \mathrm{He}$ and carbon data sets. The quoted error in $D_{\mathrm{N}_{2}}$ may be obtained by combining the systematic contribution and Eqn 6.12 in quadrature. 


\begin{tabular}{cccl}
\hline Parameter & Unit & Value & Description \\
\hline$\rho_{\mathrm{N}_{2}}\left({ }^{3} \mathrm{He}\right)$ & amg & 0.162 & $\mathrm{~N}_{2}$ density within the ${ }^{3} \mathrm{He}$ cell \\
$\delta\left(\rho_{\mathrm{N}_{2}}\left({ }^{3} \mathrm{He}\right)\right)_{\mathrm{rel} .}$ & $\mathrm{N} / \mathrm{A}$ & 0.007 & Relative error in $\rho_{\mathrm{N}_{2}}\left({ }^{3} \mathrm{He}\right)$ \\
$\delta\left(\rho_{\mathrm{C}}(\mathrm{C})\right)_{\mathrm{rel} .}$ & $\mathrm{N} / \mathrm{A}$ & 0.007 & Relative error in $\rho_{\mathrm{C}}(\mathrm{C})$ \\
$m_{\mathrm{N}_{2}}\left({ }^{3} \mathrm{He}\right)$ & $\mathrm{mg} / \mathrm{cm}^{2}$ & 3.74 & Mass thickness of $\mathrm{N}_{2}$ in the ${ }^{3} \mathrm{He}$ cell \\
$m_{\text {air }}(\mathrm{C})$ & $\mathrm{mg} / \mathrm{cm}^{2}$ & 22.23 & Mass thickness of air in C target \\
$m_{\mathrm{C}}(\mathrm{C})$ & $\mathrm{mg} / \mathrm{cm}^{2}$ & 213.4 & Total mass thickness of $\mathrm{C}$ target \\
$Q\left({ }^{3} \mathrm{He}\right)$ & $\mathrm{mC}$ & 15.0 & Accum. beam charge for ${ }^{3} \mathrm{He}$ runs \\
$Q(\mathrm{C})$ & $\mathrm{mC}$ & 1.46 & Accum. beam charge for C runs \\
\hline
\end{tabular}

Table 6.3: Common parameters used in the neutral/charged calculation of $D_{\mathrm{N}_{2}}$. Note that $1 \mathrm{amg}=44.615 \mathrm{~mol} / \mathrm{m}^{3}$, and vertex cuts are foil center $\pm 2.3 \mathrm{~cm}$.

\begin{tabular}{cccl}
\hline Parameter & Neutral & Charged & Description \\
\hline$N$ & 1,467 & 11,590 & ${ }^{3} \mathrm{He}$ QE count \\
$N_{\mathrm{bk}}$ & 161 & 43 & ${ }^{3} \mathrm{He}$ QE background count \\
$N(\mathrm{C})$ & 334 & 1,122 & Carbon QE count \\
$N_{\mathrm{bk}}(\mathrm{C})$ & 3 & 1 & Carbon QE background count \\
\hline$D_{\mathrm{N}_{2}}$ & $0.954 \pm 0.005$ & $0.981 \pm 0.002$ & Nitrogen dilution factor \\
$\sigma_{\mathrm{sys}}$ & 0.0035 & 0.0016 & Sys. estimate of acceptance mismatch \\
$\delta D_{\mathrm{N}_{2}}$ & 0.0030 & 0.0006 & Uncertainty in $D_{\mathrm{N}_{2}}$ using Eqn 6.12 \\
\hline
\end{tabular}

Table 6.4: The results of the nitrogen correction for the neutral/charged QE samples. 


\subsubsection{Charge Misidentification}

Estimating the corrections due to charge misidentification requires calculating the background and $\mathrm{N}_{2}$ corrected uncharged-to-charged ratio $R_{t}$ for three target types in order to constrain the three mixing ratios defined by Eqn 5.95-Eqn 5.97 at the nominal veto rate. Once the three mixing ratios are known, then the proton dilution factor and asymmetry correction to $A_{\text {raw }}$ may be calculated.

The background and nitrogen corrected uncharged-to-charged ratio is defined as

$$
R=\left(\frac{D_{\mathrm{N}_{2}}}{D_{\mathrm{N}_{2}, \mathrm{ch}}}\right) \frac{N-\frac{N_{\mathrm{bk}}}{2}}{N_{\mathrm{ch}}-N_{\mathrm{bk}, \mathrm{ch}}-\frac{N_{\mathrm{bk}}}{2}},
$$

where $N\left(N_{\mathrm{ch}}\right)$ is the neutral (charged) count after quasielastic cuts, and recall that the correct amount of background to subtract for the uncharged and charged samples is $\frac{N_{\mathrm{bk}}}{2}$ and $N_{\mathrm{bk}, \mathrm{ch}}+\frac{N_{\mathrm{bk}}}{2}$, respectively. Note that the absence of the "ch" subscript always refers to the neutral sample. The derivative vector transposed is

$$
\begin{aligned}
& \mathbf{g}^{\mathbf{T}}=\left(\begin{array}{llllll}
\frac{\partial R}{\partial N} & \frac{\partial R}{\partial N_{\mathrm{bk}}} & \frac{\partial R}{\partial N_{\mathrm{ch}}} & \frac{\partial R}{\partial N_{\mathrm{bk}, \mathrm{ch}}} & \frac{\partial R}{\partial D_{\mathrm{N}_{2}}} & \frac{\partial R}{\partial D_{\mathrm{N}_{2}, \mathrm{ch}}}
\end{array}\right),
\end{aligned}
$$

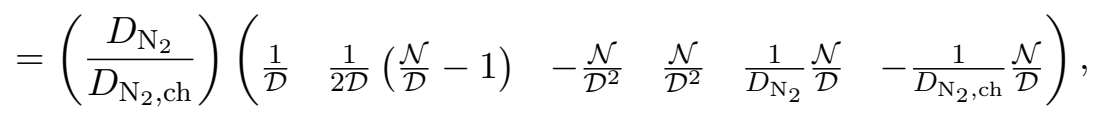

where $\mathcal{N} \equiv N-\frac{N_{\mathrm{bk}}}{2}$ and $\mathcal{D} \equiv N_{\mathrm{ch}}-N_{\mathrm{bk}, \mathrm{ch}}-\frac{N_{\mathrm{bk}}}{2}$ are defined as short-hand notation for the numerator and denominator of Eqn 6.13. All counts are uncorrelated; therefore, the diagonal components of $\mathbf{V}$ and variance in $R$ are the following:

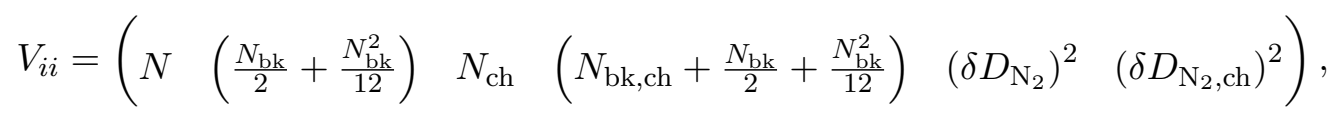

$$
\begin{aligned}
& (\delta R)^{2}=\left(\frac{D_{\mathrm{N}_{2}}}{D_{\mathrm{N}_{2}, \mathrm{ch}}}\right)^{2}\left\{\frac{N}{\mathcal{D}^{2}}+\left(\frac{N_{\mathrm{bk}}}{2}+\frac{N_{\mathrm{bk}}^{2}}{12}\right)\left[\frac{1}{2 \mathcal{D}}\left(\frac{\mathcal{N}}{\mathcal{D}}-1\right)\right]^{2}\right. \\
& \left.+\left(\frac{\mathcal{N}}{\mathcal{D}^{2}}\right)^{2}\left(N_{\mathrm{ch}}+N_{\mathrm{bk}, \mathrm{ch}}+\frac{N_{\mathrm{bk}}}{2}+\frac{N_{\mathrm{bk}}^{2}}{12}\right)+\left(\frac{\mathcal{N}}{\mathcal{D}}\right)^{2}\left(\left[\frac{\delta D_{\mathrm{N}_{2}}}{D_{\mathrm{N}_{2}}}\right]^{2}+\left[\frac{\delta D_{\mathrm{N}_{2}, \mathrm{ch}}}{D_{\mathrm{N}_{2}, \mathrm{ch}}}\right]^{2}\right)\right\},
\end{aligned}
$$




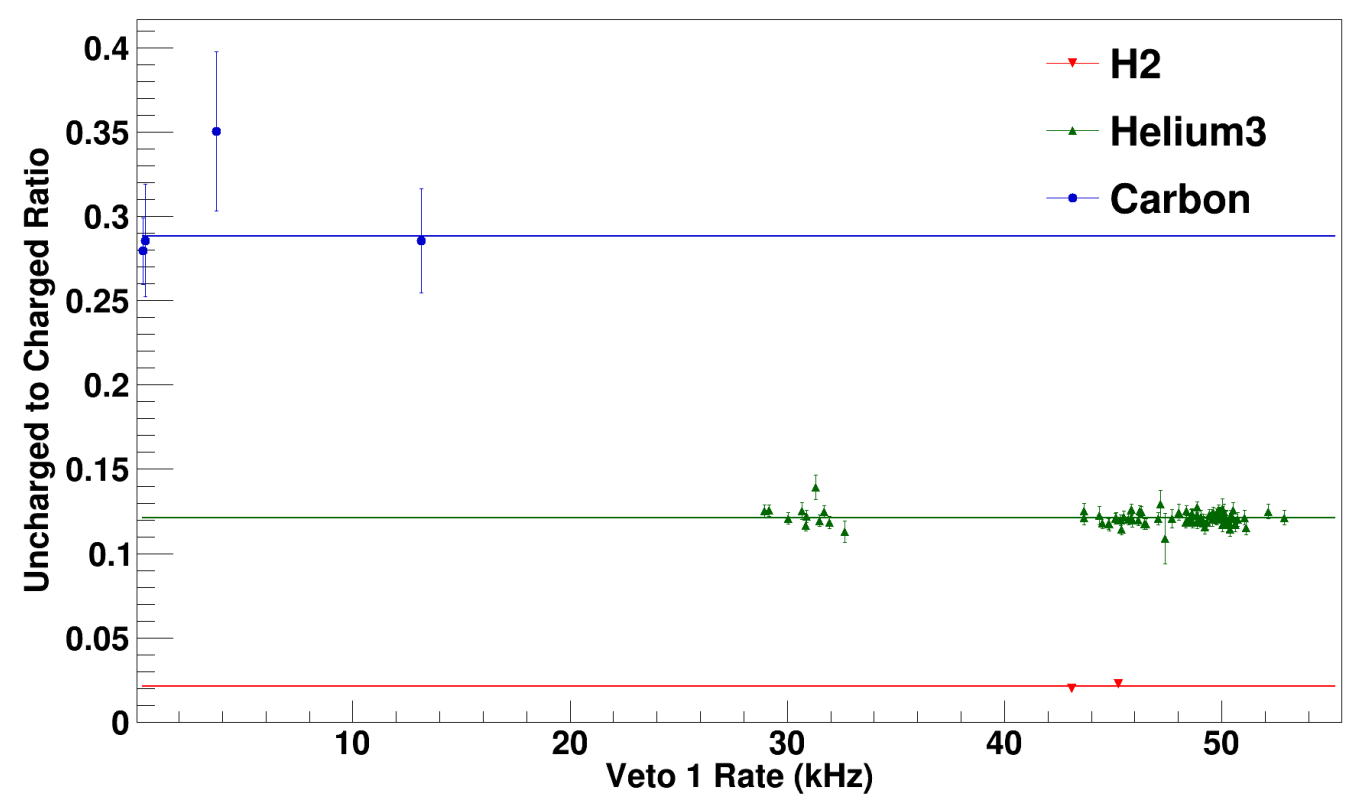

Fig. 6.4: The uncharged-to-charged ratio $R$ and fit result for the three targets.

where the short-hand notation has been used again. Eqn 6.13 and the variance Eqn 6.15 handle the most complicated situation, namely the polarized ${ }^{3} \mathrm{He}$ target where it is appropriate to correct for background and nitrogen. Since the hydrogen and carbon targets do not require an $\mathrm{N}_{2}$ correction, the correct formula to use for the unchargedto-charged ratio may be obtained by setting $D_{\mathrm{N}_{2}}=D_{\mathrm{N}_{2}, \text { ch }}=1$ and $\delta D_{\mathrm{N}_{2}}=\delta D_{\mathrm{N}_{2}, \text { ch }}=$ 0. The three uncharged-to-charged ratios and the corresponding fits may be seen by Figure 6.4. Each ratio and the uncertainty is evaluated at a veto layer 1 rate that reflects the rates during ${ }^{3} \mathrm{He}$ production; therefore, a weighted average is used on the polarized ${ }^{3} \mathrm{He}$ data yielding a nominal rate for layers V1 and V2 to be approximately $47 \mathrm{kHz}$ and $33 \mathrm{kHz}$, respectively.

Continuing to follow the procedure of Section 5.7.5, the next step is to use the 
uncharged-to-charged target ratios evaluated at the nominal V1 rate in order to constrain the three ratios of mixing coefficients, originally defined in Eqn 5.95 - Eqn 5.97 but rewritten for convenience:

$$
\begin{aligned}
\frac{\eta_{p}^{n}}{\eta_{p}^{p}} & =R_{\mathrm{H}_{2}}, \\
\frac{\eta_{n}^{p}}{\eta_{p}^{p}} & =\frac{\sigma_{p}}{\sigma_{n}} \frac{f_{\mathrm{C}}\left(R_{3_{\mathrm{He}}}-R_{\mathrm{H}_{2}}\right)-f_{3_{\mathrm{He}}}\left(R_{\mathrm{C}}-R_{\mathrm{H}_{2}}\right)}{f_{\mathrm{C}} f_{3^{\mathrm{He}}}\left(R_{\mathrm{C}}-R_{3} \mathrm{He}_{\mathrm{e}}\right)}, \\
\frac{\eta_{n}^{n}}{\eta_{p}^{p}} & =\frac{\sigma_{p}}{\sigma_{n}} \frac{f_{3_{\mathrm{He}}} R_{3_{\mathrm{He}}}\left(R_{\mathrm{H}_{2}}-R_{\mathrm{C}}\right)-f_{\mathrm{C}} R_{\mathrm{C}}\left(R_{\mathrm{H}_{2}}-R_{3} \mathrm{He}\right.}{f_{\mathrm{C}} f_{3_{\mathrm{He}}}\left(R_{\mathrm{C}}-R_{3} \mathrm{He}_{\mathrm{e}}\right)} .
\end{aligned}
$$

Recall that the subscript denotes the particle that left the target while the superscript refers to how it is identified, i.e. $\eta_{\text {true }}^{\text {observed }}$. All variables are uncorrelated and it is assumed that the uncertainties in $f^{{ }^{3} \mathrm{He}}$ and $f_{\mathrm{C}}$ are negligible. In an effort to conserve space, the variances are simply stated rather than explicitly shown:

$$
\begin{aligned}
& \left(\delta \frac{\eta_{p}^{n}}{\eta_{p}^{p}}\right)^{2}=\left(\delta R_{\mathrm{H}_{2}}\right)^{2}
\end{aligned}
$$

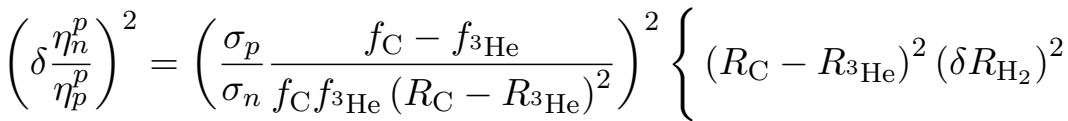

$$
\begin{aligned}
& +\left(R_{\mathrm{H}_{2}}-R_{3} \mathrm{He}\right)^{2}\left(\delta R_{\mathrm{C}}\right)^{2}+\left(R_{\mathrm{H}_{2}}-R_{\mathrm{C}}\right)^{2}\left(\delta R_{3} \mathrm{He}^{2}\right\},
\end{aligned}
$$

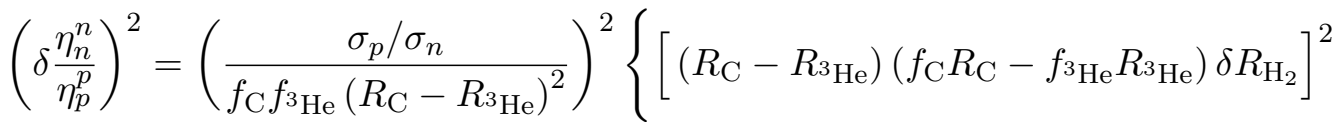

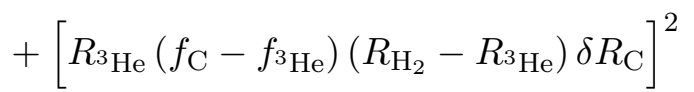

$$
\begin{aligned}
& \left.+\left[R_{\mathrm{C}}\left(f_{\mathrm{C}}-f_{{ }^{3} \mathrm{He}}\right)\left(R_{\mathrm{H}_{2}}-R_{\mathrm{C}}\right) \delta R_{3} \mathrm{He}\right]^{2}\right\} .
\end{aligned}
$$

The dilution factors associated to charge misidentification may be constructed by taking ratios of truly neutral (charged) to observed neutral (charged), and have been defined 
in Eqn 5.98 and Eqn 5.99 but rewritten for convenience:

$$
\begin{aligned}
D_{\mathrm{p}} & =\frac{f_{3} \mathrm{He}^{\frac{\sigma_{n}}{\sigma_{p}}}\left(\frac{\eta_{n}^{n}}{\eta_{p}^{p}}\right)}{f{ }_{3 \mathrm{He}} \frac{\sigma_{n}}{\sigma_{p}}\left(\frac{\eta_{n}^{n}}{\eta_{p}^{p}}\right)+\left(\frac{\eta_{p}^{n}}{\eta_{p}^{p}}\right)}, \\
D_{\mathrm{n}, \mathrm{ch}} & =\frac{1}{f^{{ }_{3} \mathrm{He}} \frac{\sigma_{n}}{\sigma_{p}}\left(\frac{\eta_{n}^{p}}{\eta_{p}^{p}}\right)+1} .
\end{aligned}
$$

To be clear, $D_{\mathrm{n}, \mathrm{ch}}$ represents the neutron contamination in the charged sample while $D_{\mathrm{p}}$ denotes the proton contamination in the neutral sample. The mixing coefficients in the expression for $D_{\mathrm{p}}$ are correlated; therefore, it is simpler to substitute in the target ratio dependent expressions for $\frac{\eta_{n}^{n}}{\eta_{p}^{p}}$ and $\frac{\eta_{p}^{n}}{\eta_{p}^{p}}$ where now the variance matrix is diagonal with respect to the variances of the three uncharged-to-charged ratios. On the other hand, the variance in $D_{\mathrm{n}, \mathrm{ch}}$ may be calculated with minimal effort as it only depends on one mixing ratio. The variances in the the two dilution factors are

$$
\begin{aligned}
& \left(\delta D_{\mathrm{p}}\right)^{2}=\left(\frac{f_{\mathrm{C}}}{\left(f_{\mathrm{C}}-f_{3_{\mathrm{He}}}\right)\left(R_{\mathrm{H}_{2}}-R_{\mathrm{C}}\right)^{2} R_{3_{\mathrm{He}}}^{2}}\right)^{2}\left\{\left[R_{\mathrm{C}} R_{3 \mathrm{He}}\left(R_{\mathrm{C}}-R_{3 \mathrm{He}}\right) \delta R_{\mathrm{H}_{2}}\right]^{2}\right. \\
& \left.+\left[R_{\mathrm{H}_{2}} R_{3} \mathrm{He}\left(R_{\mathrm{H}_{2}}-R_{3} \mathrm{He}_{\mathrm{e}}\right) \delta R_{\mathrm{C}}\right]^{2}+\left[R_{\mathrm{H}_{2}} R_{\mathrm{C}}\left(R_{\mathrm{H}_{2}}-R_{\mathrm{C}}\right) \delta R_{3} \mathrm{He}\right]^{2}\right\}, \\
& \left(\delta D_{\mathrm{n}, \mathrm{ch}}\right)^{2}=\left(\frac{\sigma_{n}}{\sigma_{p}} \frac{f^{{ }^{3} \mathrm{He}}\left(\delta \frac{\eta_{n}^{p}}{\eta_{p}^{p}}\right)}{\left[f^{f_{3} \mathrm{He}} \frac{\sigma_{n}}{\sigma_{p}}\left(\frac{\eta_{n}^{p}}{\eta_{p}^{p}}\right)+1\right]^{2}}\right)^{2} .
\end{aligned}
$$

A table displaying all the relevant results may be seen by Table 6.5. Since $R_{\mathrm{C}}-R_{\mathrm{H}_{2}}>$ $R_{3} \mathrm{He}-R_{\mathrm{H}_{2}}$, the mixing coefficient ratio $\frac{\eta_{n}^{p}}{\eta_{p}^{p}}$ is negative and denoted as undetermined; therefore, $D_{\mathrm{n} \text {,ch }}$ is also undetermined from the data. While $D_{\mathrm{n}, \mathrm{ch}}$ is not needed in the calculation of $A_{\text {phys }}$, the mixing ratios and dilution factors may be calculated using the simulation, see Section 5.4.7 for a discussion. A comparison between the data and simulation may be seen in Table 6.1.5. 


\begin{tabular}{|c|c|c|}
\hline Parameter & Value & Description \\
\hline V1 Rate $(\mathrm{kHz})$ & 46.6 & Average V1 rate, weighted by counts \\
\hline V2 Rate (kHz) & 32.8 & Average V2 rate, weighted by counts \\
\hline$R_{\mathrm{C}}$ & $0.288 \pm 0.014$ & Uncharged-to-charged ratio for $\mathrm{C}$ \\
\hline$R_{\mathrm{H}_{2}}$ & $0.021 \pm 0.002$ & Uncharged-to-charged ratio for $\mathrm{H}_{2}$ \\
\hline$R_{3} \mathrm{He}$ & $0.121 \pm 0.001$ & Uncharged-to-charged ratio for ${ }^{3} \mathrm{He}$ \\
\hline$f_{\mathrm{C}}$ & $1 / 0.9$ & Effective ratio of neutrons to protons \\
\hline$f^{f_{3} \mathrm{He}}$ & $1 / 2.15$ & Effective ratio of neutrons to protons \\
\hline$\sigma_{n} / \sigma_{p}$ & 0.38 & Nucleon x-section ratio, $Q^{2}=1.16 \mathrm{GeV}^{2}$ \\
\hline$\frac{\eta_{p}^{n}}{\eta_{p}^{p}}$ & $0.021 \pm 0.002$ & Protons observed as neutrons \\
\hline$\frac{\eta_{n}^{p}}{\eta_{p}^{p}}$ & Undetermined & Neutrons observed as protons \\
\hline$\frac{\eta_{n}^{n}}{\eta_{p}^{p}}$ & $0.559 \pm 0.027$ & Neutrons observed as neutrons \\
\hline$D_{\mathrm{n}, \mathrm{ch}}$ & Undetermined & Neutron dilution factor \\
\hline$D_{\mathrm{p}}$ & $0.812 \pm 0.017$ & Proton dilution factor, needed for $A_{\text {phys }}$ \\
\hline
\end{tabular}

Table 6.5: The dilution factors due to charge misidentification. The form factors used in the calculation of $\sigma_{n} / \sigma_{p}$ are calculated using the parametrization by Diehl and Kroll at $Q^{2}=1.16 \mathrm{GeV}^{2}$. The values of $f_{\mathrm{C}}$ and $f_{{ }^{3} \mathrm{He}}$ have been theoretically provided and are assumed to have negligible errors relative to other contributions. 


\begin{tabular}{cccl}
\hline Parameter & Data & Simulation & Description \\
\hline$\frac{\eta_{p}^{n}}{\eta_{p}^{p}}$ & $0.021 \pm 0.002$ & $0.020 \pm 0.001$ & Protons observed as neutrons \\
$\frac{\eta_{n}^{p}}{\eta_{p}^{p}}$ & Undetermined & $0.384 \pm 0.001$ & Neutrons observed as protons \\
$\frac{\eta_{n}^{n}}{\eta_{p}^{p}}$ & $0.559 \pm 0.027$ & $0.636 \pm 0.001$ & Neutrons observed as neutrons \\
\hline$D_{\mathrm{n}, \mathrm{ch}}$ & Undetermined & $0.941 \pm 0.001$ & Neutron dilution factor \\
$D_{\mathrm{p}}$ & $0.812 \pm 0.017$ & $0.839 \pm 0.001$ & Proton dilution factor \\
\hline
\end{tabular}

Table 6.6: Charge ID results for the data and the simulation.

The asymmetry correction to the raw asymmetry has been defined in Eqn 5.103 . and takes the following form:

$$
A_{\mathrm{p}}=\frac{\Delta_{\mathrm{p}}}{\Sigma}=\left(1-D_{\mathrm{p}}\right) \cdot D_{\mathrm{bk}} \cdot D_{\mathrm{N}_{2}} \cdot\left(P_{\mathrm{beam}} P_{3}{ }_{\mathrm{He}} P_{\mathrm{p}}\right) \sum_{i=0}^{5} \bar{T}_{i} \cdot \Lambda^{i}
$$

In order to calculate the proton misidentification correction to the raw asymmetry, three dilution factors, three polarizations, and the six $T_{i}$ coefficients are required. Furthermore, a proton form factor parametrization is needed in order to calculate $\Lambda=G_{E}^{p} / G_{M}^{p}$ at the acceptance averaged value of $Q^{2}$. The variance in the proton asymmetry is

$$
\begin{aligned}
\left(\delta \frac{\Delta_{\mathrm{p}}}{\Sigma}\right)^{2} & =\left(\frac{\Delta_{\mathrm{p}}}{\Sigma}\right)^{2}\left\{\left(\frac{\delta D_{\mathrm{p}}}{D_{\mathrm{p}}}\right)^{2}+\left(\frac{\delta D_{\mathrm{bk}}}{D_{\mathrm{bk}}}\right)^{2}+\left(\frac{\delta D_{\mathrm{N}_{2}}}{D_{\mathrm{N}_{2}}}\right)^{2}+\left(\frac{\delta P_{\text {beam }}}{P_{\text {beam }}}\right)^{2}+\left(\frac{\delta P_{3_{3} \mathrm{He}}}{P_{3_{\mathrm{He}}}}\right)^{2}\right. \\
& \left.+\left(\frac{\delta P_{\mathrm{p}}}{P_{\mathrm{p}}}\right)^{2}+\Lambda^{2}\left(\frac{\sum_{i=1}^{5} i \cdot \bar{T}_{i} \cdot \Lambda^{i-1}}{\sum_{i=0}^{5} \bar{T}_{i} \cdot \Lambda^{i}}\right)^{2}\left(\left[\frac{\delta G_{E}^{p}}{G_{E}^{p}}\right]^{2}+\left[\frac{\delta G_{M}^{p}}{G_{M}^{p}}\right]^{2}\right)\right\} .
\end{aligned}
$$

The results of this calculation and the values of all parameters may be found in Table 6.7 . 


\begin{tabular}{|c|c|c|}
\hline Parameter & Value & Description \\
\hline$Q^{2}\left(\mathrm{GeV}^{2}\right)$ & 1.16 & Acceptance averaged value of $Q^{2}$ \\
\hline$G_{E}^{p}$ & $0.131 \pm 0.029$ & Parametrization from Section 3.2 .4 \\
\hline$G_{M}^{p}$ & $0.424 \pm 0.035$ & Parametrization from Section 3.2.4 \\
\hline$\Lambda$ & $0.310 \pm 0.074$ & Proton form factor ratio \\
\hline $\bar{T}_{0}$ & -0.086 & $A_{\text {phys }}$ expansion coefficient, $\Lambda^{0}$ \\
\hline $\bar{T}_{1}$ & 1.198 & $A_{\text {phys }}$ expansion coefficient, $\Lambda^{1}$ \\
\hline $\bar{T}_{2}$ & 0.150 & $A_{\text {phys }}$ expansion coefficient, $\Lambda^{2}$ \\
\hline $\bar{T}_{3}$ & -2.097 & $A_{\text {phys }}$ expansion coefficient, $\Lambda^{3}$ \\
\hline $\bar{T}_{4}$ & -0.268 & $A_{\text {phys }}$ expansion coefficient, $\Lambda^{4}$ \\
\hline $\bar{T}_{5}$ & 3.743 & $A_{\text {phys }}$ expansion coefficient, $\Lambda^{5}$ \\
\hline$\sum_{i=0}^{5} \bar{T}_{i} \cdot \Lambda^{i}$ & $0.246 \pm 0.061$ & Finite acceptance model, Eqn 5.76 \\
\hline$P_{\text {beam }}$ & $0.872 \pm 0.020$ & Beam polarization \\
\hline$P^{3} \mathrm{He}$ & $0.397 \pm 0.015$ & Target polarization \\
\hline$P_{\mathrm{p}}$ & $-0.050 \pm 0.025$ & Proton polarization in ${ }^{3} \mathrm{He}$ nucleus \\
\hline$D_{\mathrm{bk}}$ & $0.949 \pm 0.029$ & Background dilution \\
\hline$D_{\mathrm{N}_{2}}$ & $0.954 \pm 0.005$ & Nitrogen dilution \\
\hline$D_{\mathrm{p}}$ & $0.812 \pm 0.017$ & Proton dilution \\
\hline$\frac{\Delta_{p}}{\Sigma}$ & $-0.0008 \pm 0.0004$ & Proton asymmetry correction \\
\hline
\end{tabular}

Table 6.7: The results of the proton asymmetry correction to $A_{\text {raw }}$ for the neutral sample. All values and uncertainties are included for convenience. 


\subsubsection{BigBite Preshower Pions}

The formalism to estimate the contribution of BigBite pions contaminating the electronic signal within the preshower has been introduced in Section 5.7.6. Recall that the procedure relies on the output of two G4SBS simulations: a pion and an electron simulation. The simulated preshower spectrum, namely the sum of the simulated electron and pion preshower distributions, is then compared to the ${ }^{3} \mathrm{He}$ quasielastic coincidence data. In order to calculate $D_{\pi}$ and $A_{\pi}$ for both quasielastic samples, there are six ${ }^{3} \mathrm{He}$ quasielastic preshower distributions that are relevant:

$D_{\pi}$ : the QE neutral and charged spectra which includes both helicities (2),

$A_{\pi}$ : the \pm helicities for both the QE neutral and charged samples (4).

Two normalization coefficients are required per data spectrum to scale the individual simulated pion and electron distributions such that the sum matches the data; therefore, in total there are 12 normalization coefficients, two for each of the six data distributions. The choice of coefficients is determined by minimizing the $\chi^{2}$ defined in Eqn 5.105 with respect to the two normalization constants for each data distribution, then the dilution factor and asymmetry correction for the quasielastic uncharged and charged samples may be calculated.

The dilution factor due to BigBite preshower pions may be calculated as

$$
D_{\pi}=1-\frac{C_{\pi} N_{\pi}}{C_{e} N_{e}+C_{\pi} N_{\pi}}
$$

where $C_{\pi}$ and $C_{e}$ are the normalization coefficients needed to scale the individual simulated distributions, and have been determined by a linear regression procedure. The 
simulated counts prior to scaling, denoted by $N_{\pi}$ and $N_{e}$, may be determined by integrating the individual simulated spectra in the preshower energy range $150<E<1000 \mathrm{MeV}$ where the lower bound has been determined by the quasielastic cut selection. The results of scaling the two individual simulations by $C_{\pi}$ and $C_{e}$ such that the sum matches the neutral and charged preshower data may be seen by Figure 6.5. Additionally, all six distributions may be seen in log scale in Figure 6.6.

The derivative vector transposed is needed to calculate the variance in $D_{\pi}$ :

$$
\begin{aligned}
\mathbf{g}^{\mathbf{T}} & =\left(\begin{array}{llll}
\frac{\partial D_{\pi}}{\partial N_{\pi}} & \frac{\partial D_{\pi}}{\partial C_{\pi}} & \frac{\partial D_{\pi}}{\partial N_{e}} & \frac{\partial D_{\pi}}{\partial C_{e}}
\end{array}\right), \\
& =\left(\begin{array}{llll}
\left(\frac{C_{\pi}^{2} N_{\pi}}{\mathcal{D}^{2}}-\frac{C_{\pi}}{\mathcal{D}}\right) & \left(\frac{C_{\pi} N_{\pi}^{2}}{\mathcal{D}^{2}}-\frac{N_{\pi}}{\mathcal{D}}\right) & \frac{C_{\pi} C_{e} N_{\pi}}{\mathcal{D}^{2}} & \frac{C_{\pi} N_{\pi} N_{e}}{\mathcal{D}^{2}}
\end{array}\right),
\end{aligned}
$$

where $\mathcal{D} \equiv C_{e} N_{e}+C_{\pi} N_{\pi}$ is a defined short-hand representing the denominator of Eqn 6.22. The normalization coefficients are correlated, and the variance matrix is

$$
\mathbf{V}=\left(\begin{array}{cccc}
N_{\pi} & 0 & 0 & 0 \\
0 & \sigma_{C_{\pi}}^{2} & 0 & \operatorname{cov}\left(C_{\pi}, C_{e}\right) \\
0 & 0 & N_{e} & 0 \\
0 & \operatorname{cov}\left(C_{\pi}, C_{e}\right) & 0 & \sigma_{C_{e}}^{2}
\end{array}\right) .
$$

Analytic formulas exist for $\sigma_{C_{\pi}}^{2}, \sigma_{C_{e}}^{2}$ and $\operatorname{cov}\left(C_{\pi}, C_{e}\right)$ as the linear regression method has been used with linear weighting parameters. The variance in the dilution factor due to BigBite pions is

$$
\begin{aligned}
\left(\delta D_{\pi}\right)^{2} & =N_{\pi}\left(\frac{C_{\pi}^{2} N_{\pi}}{\mathcal{D}^{2}}-\frac{C_{\pi}}{\mathcal{D}}\right)^{2}+\sigma_{C_{\pi}}^{2}\left(\frac{C_{\pi} N_{\pi}^{2}}{\mathcal{D}^{2}}-\frac{N_{\pi}}{\mathcal{D}}\right)^{2}+N_{e}\left(\frac{C_{\pi} C_{e} N_{\pi}}{\mathcal{D}^{2}}\right)^{2} \\
& +\sigma_{C_{e}}^{2}\left(\frac{C_{\pi} N_{\pi} N_{e}}{\mathcal{D}^{2}}\right)^{2}+2 \operatorname{cov}\left(C_{\pi}, C_{e}\right)\left(\frac{C_{\pi} N_{\pi}^{2}}{\mathcal{D}^{2}}-\frac{N_{\pi}}{\mathcal{D}}\right)\left(\frac{C_{\pi} N_{\pi} N_{e}}{\mathcal{D}^{2}}\right),
\end{aligned}
$$

where the short-hand $\mathcal{D}$ has been used again. 
The asymmetry correction to $A_{\text {raw }}$ may be determined by extending the above procedure to the \pm helicity distributions for the uncharged and charged quasielastic coincidence preshower data, and then forming the asymmetry:

$$
A_{\pi}=\frac{\Delta_{\pi}}{\Sigma}=\frac{C_{\pi}^{+} N_{\pi}-C_{\pi}^{-} N_{\pi}}{\Sigma}
$$

where $C_{\pi}^{ \pm}$have analogous interpretations as the dilution factor case except the superscript + or - denotes the helicity. Note that $N_{\pi}$ does not require a helicity superscript; the value of $N_{\pi}$, namely the integral of the simulated pion preshower spectrum prior to scaling, gets matched to reality by the scaling factors $C_{\pi}^{ \pm}$. The uncertainties are uncorrelated; therefore, the derivative vector transposed, the diagonal elements of $\mathbf{V}$ and the variance in $A_{\pi}$ are the following:

$$
\begin{aligned}
& \mathbf{g}^{\mathbf{T}}=\left(\begin{array}{llll}
\frac{\partial A_{\pi}}{\partial C_{\pi}^{+}} & \frac{\partial A_{\pi}}{\partial C_{\pi}^{-}} & \frac{\partial A_{\pi}}{\partial N_{\pi}} & \frac{\partial A_{\pi}}{\partial \Sigma}
\end{array}\right), \\
& =\left(\begin{array}{llll}
\frac{N_{\pi}}{\Sigma} & -\frac{N_{\pi}}{\Sigma} & \frac{C_{\pi}^{+}-C_{\pi}^{-}}{\Sigma} & -\frac{C_{\pi}^{+} N_{\pi}-C_{\pi}^{-} N_{\pi}}{\Sigma^{2}}
\end{array}\right), \\
& V_{i i}=\left(\begin{array}{cccc}
\sigma_{C_{\pi}^{+}}^{2} & \sigma_{C_{\pi}^{-}}^{2} & N_{\pi} & N
\end{array}\right) \\
& \Rightarrow\left(\delta A_{\pi}\right)^{2}=\left(\sigma_{C_{\pi}^{+}}^{2}+\sigma_{C_{\pi}^{-}}^{2}\right)\left(\frac{N_{\pi}}{\Sigma}\right)^{2}+N_{\pi}\left(\frac{C_{\pi}^{+}-C_{\pi}^{-}}{\Sigma}\right)^{2} \\
& +N\left(\frac{C_{\pi}^{+} N_{\pi}-C_{\pi}^{-} N_{\pi}}{\Sigma^{2}}\right)^{2} \text {. }
\end{aligned}
$$

The results for $D_{\pi}$ and $A_{\pi}$ for the charged and uncharged quasielastic samples may be seen by Table 6.8 and Table 6.9. The simulated count sum, or $\Sigma_{\operatorname{sim}}=\left(C_{\pi}^{+}+C_{\pi}^{-}\right) N_{\pi}+$ $\left(C_{e}^{+}+C_{e}^{-}\right) N_{e}$ where $N_{\pi}$ and $N_{e}$ are the individual simulated spectra prior to scaling, agrees with the data to within one percent, i.e. $\left(\Sigma-\Sigma_{\text {sim }}\right) / \Sigma \approx 1 \%$. 


\begin{tabular}{cccl}
\hline Parameter & Neutral & Charged & Description \\
\hline$C_{\pi}$ & $0.560 \pm 0.019$ & $8.81 \pm 0.07$ & Sim. $\pi^{-}$normalization \\
$C_{e}$ & $1.022 \pm 0.002$ & $7.82 \pm 0.01$ & Sim. $e^{-}$normalization \\
$\operatorname{cov}\left(C_{\pi}, C_{e}\right)$ & $-5 \times 10^{-6}$ & $-5 \times 10^{-5}$ & Covariance b/t normalizations \\
$N_{\pi}$ & $975 \pm 31$ & $975 \pm 31$ & Sim. $\pi^{-}$count prior to scaling \\
$N_{e}$ & $1.788 \times 10^{5} \pm 400$ & $1.788 \times 10^{5} \pm 400$ & Sim. $e^{-}$count prior to scaling \\
$D_{\pi}$ & $0.997 \pm 0.001$ & $0.9940 \pm 0.0002$ & Dilution factor \\
\hline
\end{tabular}

Table 6.8: Results of the BigBite preshower pion dilution factor analysis.

\begin{tabular}{|c|c|c|c|c|}
\hline Parameter & \multicolumn{2}{|c|}{ Neutral } & \multicolumn{2}{|c|}{ Charged } \\
\hline Helicity & + & - & + & - \\
\hline$C_{\pi}$ & $0.262 \pm 0.013$ & $0.295 \pm 0.014$ & $3.90 \pm 0.05$ & $4.91 \pm 0.05$ \\
\hline$C_{e}$ & $0.481 \pm 0.002$ & $0.540 \pm 0.002$ & $3.91 \pm 0.01$ & $3.91 \pm 0.01$ \\
\hline$N_{\pi}$ & \multicolumn{2}{|c|}{$975 \pm 31$} & \multicolumn{2}{|c|}{$975 \pm 31$} \\
\hline$N_{e}$ & \multicolumn{2}{|c|}{$1.788 \times 10^{5} \pm 400$} & \multicolumn{2}{|c|}{$1.788 \times 10^{5} \pm 400$} \\
\hline$\Sigma_{\text {sim }}$ & \multicolumn{2}{|c|}{$1.831 \times 10^{5} \pm 430$} & \multicolumn{2}{|c|}{$1.406 \times 10^{6} \pm 1200$} \\
\hline$\Sigma$ & \multicolumn{2}{|c|}{$1.816 \times 10^{5} \pm 430$} & \multicolumn{2}{|c|}{$1.393 \times 10^{6} \pm 1200$} \\
\hline$A_{\pi}$ & \multicolumn{2}{|c|}{$-0.0002 \pm 0.0001$} & \multicolumn{2}{|c|}{$-0.0007 \pm \mathcal{O}\left(10^{-5}\right)$} \\
\hline
\end{tabular}

Table 6.9: Results of the BigBite preshower pion asymmetry analysis. See text for further details. 

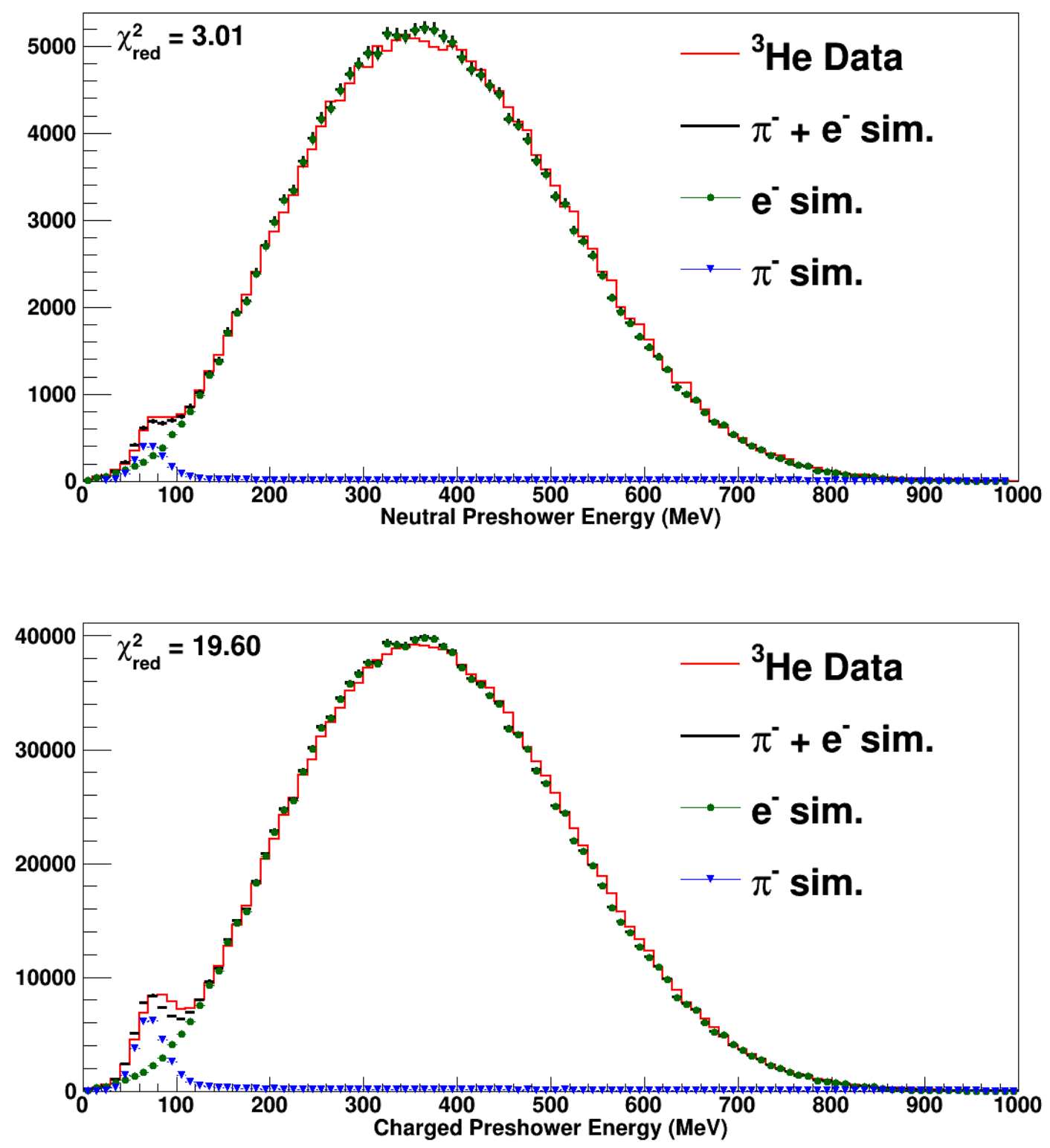

Fig. 6.5: The result of scaling the neutral and charged samples by factors determined in a $\chi^{2}$ minimization procedure; the simulation (black) is in good agreement with the data. All six data distributions and the corresponding scaled simulated spectra may be seen in a log scale in Figure 6.6. Note: The reduced $\chi^{2}$ is defined as $\chi_{\text {red }}^{2}=\frac{\chi^{2}}{\nu}=\frac{\chi^{2}}{100-2}$ where $\nu$ is the number of degrees of freedom. 

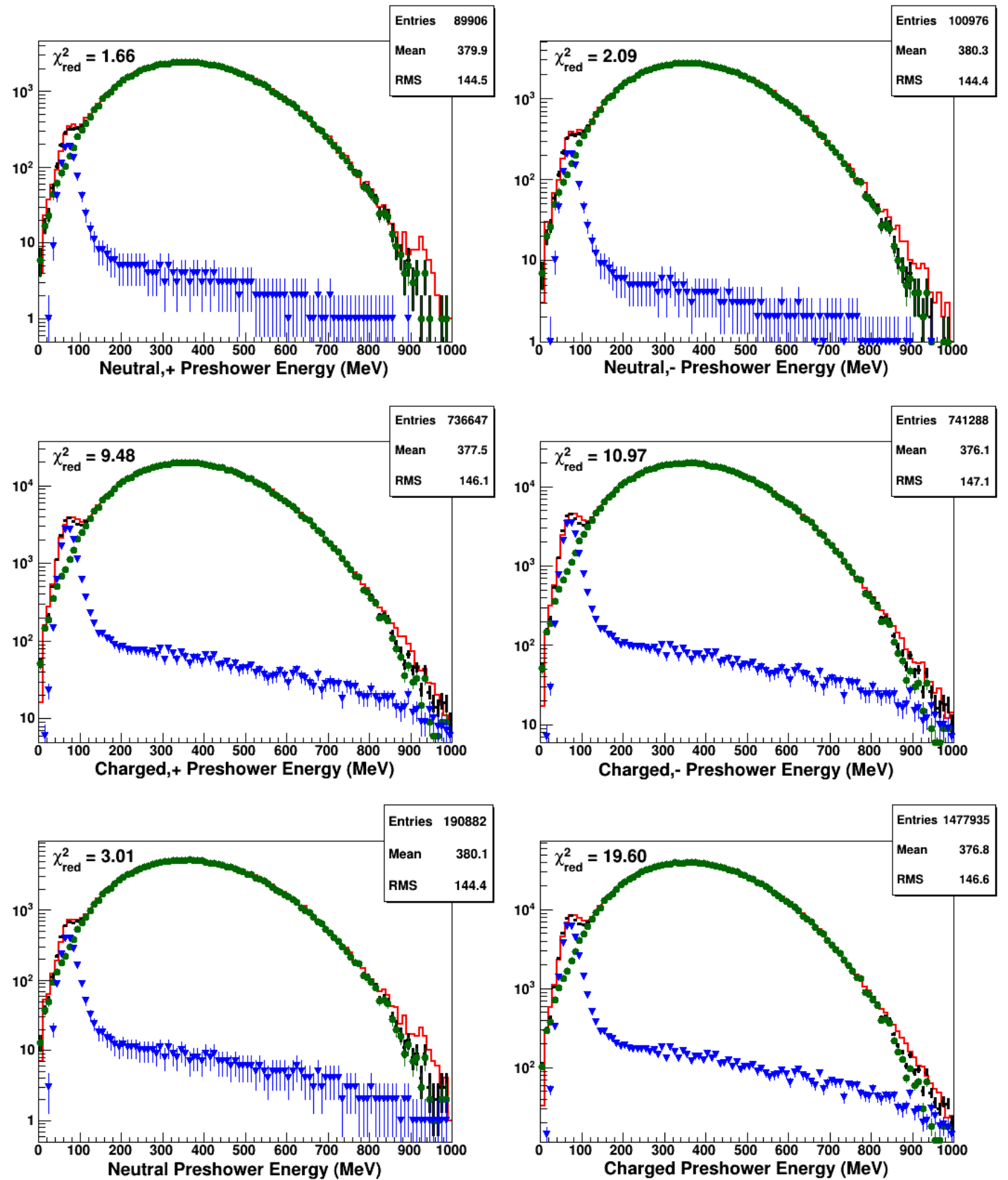

Fig. 6.6: All six distributions used in the calculation of $D_{\pi}$ and $A_{\pi}$ for the quasielastic charged and uncharged samples. Displayed are the \pm charged, \pm uncharged, and the uncharged/charged data distributions and the corresponding scaled simulated spectra. The legend is the same as Figure 6.5 . 


\subsubsection{Inelastic Contamination}

The inelastic correction formalism has been introduced in Section 5.7.7, and in summary follows a similar path as the analysis for the BigBite pion correction, namely scaling simulated data to match the observed data where the normalization factors are determined in a $\chi^{2}$ minimization procedure. However, the analysis of the simulated data must be in a more mature stage, and to be specific the following tasks are required:

1. a coincidence event, or the union of a $\mathrm{T} 1$ and a T2 trigger event;

2. nucleon cluster reconstruction, resulting in a method to calculate the missing momenta and the missing mass; and,

3. charge identification such that charged and neutral $W$ spectra may be generated.

The ability to complete the above tasks in simulation are demonstrated in Section 5.3 .6 and Section 5.4.7. For the analysis, six ${ }^{3} \mathrm{He}$ invariant mass distributions are needed just as in the BigBite pion case; however, four simulated spectra are now required, an elastic and an inelastic distribution for the charged and uncharged samples. At this stage in the analysis, the inelastic contamination after quasielastic coincidence cuts have been applied to the ${ }^{3} \mathrm{He}$ data is of particular interest. However, a presentation of the results of the $\chi^{2}$ minimization procedure with a loose cut selection, i.e. a vertex cut, a preshower cut and a coincidence cut, is a beneficial starting point for the quasielastic coincidence analysis; the spectra with the loose cut selection may be seen by Figure 6.7. The red distribution is the result of the addition of the simulated elastic and inelastic $W$ spectra after scaling by normalization factors determined in a $\chi^{2}$ minimization procedure. The 

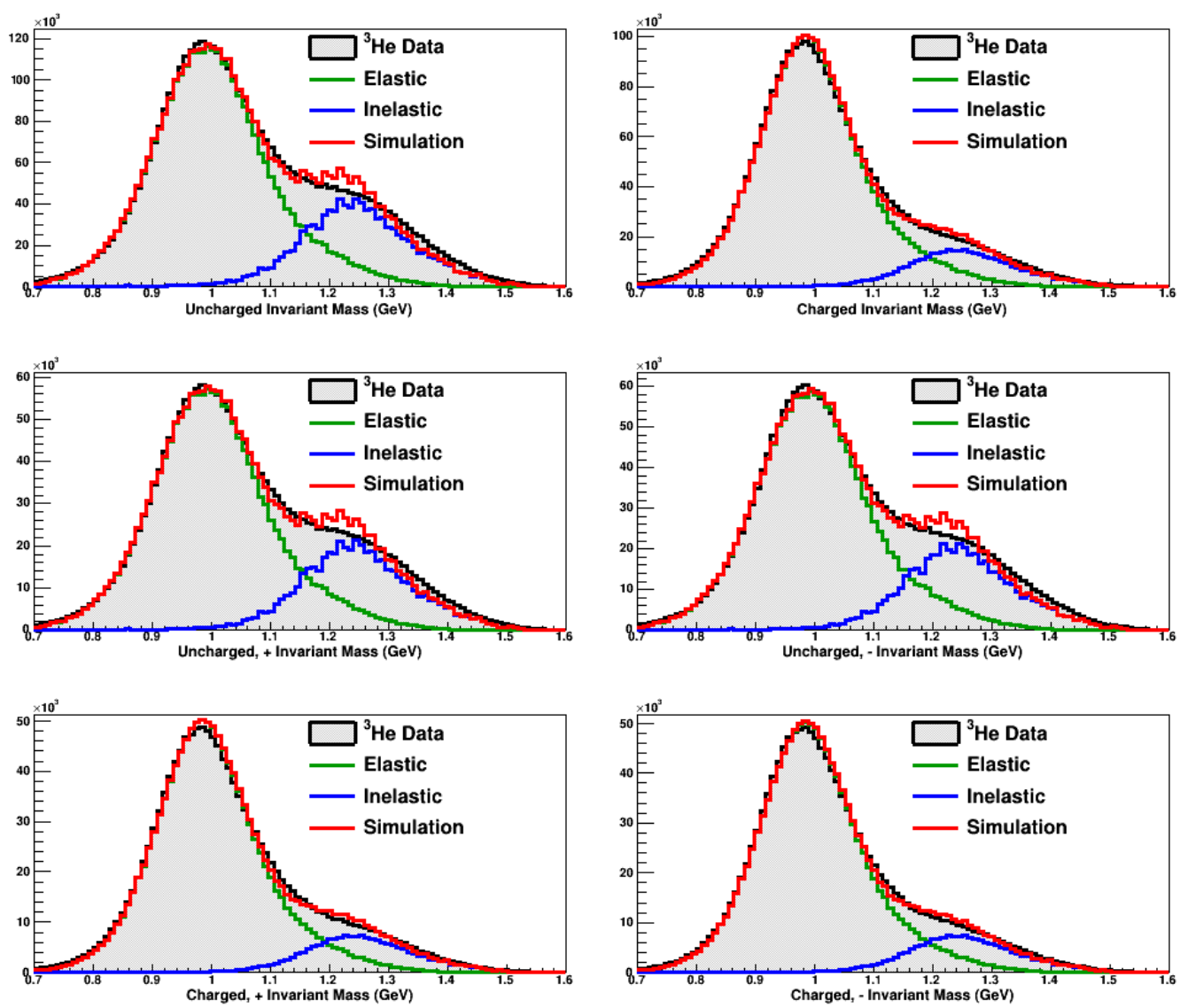

Fig. 6.7: Comparing the simulated $W$ spectra to the data with a loose cut selection.

signature of elastic $e N$ scattering is the peak at $W=M$ in the invariant mass spectrum (see Eqn 5.11), but a small positive offset is observed in the ${ }^{3} \mathrm{He}$ data relative to the elastic expectation. The most likely source of this discrepancy may be attributed to the binding energy of the ${ }^{3} \mathrm{He}$ nucleus which is not currently handled in the simulation. An ad hoc solution is to shift $E^{\prime}$ by $-7 \mathrm{MeV}$ which roughly corresponds to the binding energy of the ${ }^{3}$ He nucleus, resulting in peak alignment between simulation and data. In order to generate an apples-to-apples comparison for a contamination estimation, the simulated data must have all quasielastic cuts applied with the exception of the invari- 
ant mass cut of $0.8<W<1.15 \mathrm{GeV}$. Throughout the course of this study, two items have revealed an importance in regards to replicating the quasielastic coincidence ${ }^{3} \mathrm{He}$ $W$ spectra: elastic radiative corrections and charge identification. If elastic radiative corrections are simply ignored, then the high- $W$ shoulder of the quasielastic $W$ peak is dramatically underestimated, and as a result the inelastic contribution will be overestimated in the $\chi^{2}$ procedure. In other words, due to the mismatch between the data and the elastic simulation, the $\chi^{2}$ procedure wants to compensate by making the simulated inelastic normalization coefficient larger than necessary such that minimization occurs; the result is qualitatively poor. The other item, namely the ability to perform charge identification, alters the shape of the simulated quasielastic $W$ tail, specifically around an invariant mass of $1.1-1.3 \mathrm{GeV}$, such that less inelastic events are needed to mimic the observed spectra. A comparison of the quasielastic coincidence spectra without (with) charge identification may be seen by the top (bottom) row of Figure 6.8 the first and second column are the results for the uncharged and charged samples, respectively. Clearly charge identification alters the $W$ spectra far away from the quasielastic peak in both $W$ directions.

The six required quasielastic coincidence $W$ spectra may be seen by Figure 6.9 and in $\log$ scale by Figure 6.10. According to the simulation, the simulated elastic spectrum by itself does most of the work in regards to data replication, yielding more confidence in the naive expectation that the inelastic contamination is small for the $Q^{2}=$ $1.16 \mathrm{GeV}^{2}$ setting. Recall that in Section 5.6 in which the quasielastic cut selection is introduced, the $W$ spectrum before and after cuts motivated the initial expectation that 

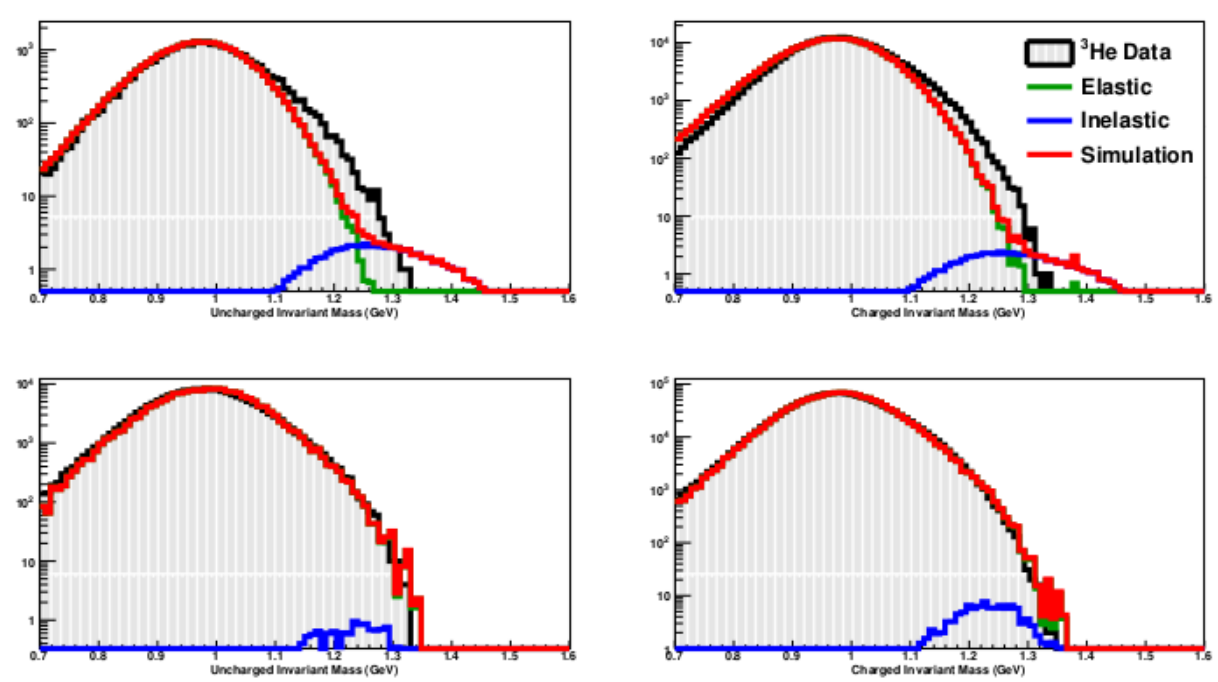

Fig. 6.8: The quasielastic coincidence spectra without (with) charge identification may be seen by the top (bottom) row.

the inelastic contamination has the potential to be small, see Figure 5.57 for example. The results of the inelastic corrections to the raw asymmetry, namely the dilution factors and asymmetry corrections to the uncharged and charged samples, could be presented in a similar manner as the BigBite pion correction; however, as the inelastic contribution is consistent with zero, a value of $D_{\text {in }}=1.000 \pm 0.050$ and $A_{\text {in }}=0.0000 \pm 0.0150$ for both the uncharged and charged samples are assigned.

\subsubsection{Nuclear Corrections}

The results of the Monte Carlo simulation written by Misak Sargsian are used to estimate the corrections to the raw asymmetry due to nuclear effects. For more information on the details of the simulation, see Section 5.7.8. The differential cross section $d^{4} \sigma /\left(d E_{e} d \Omega_{e} d p_{f} d \Omega_{f}\right)$, where the subscripts $e(f)$ represent the scattered electron (final 

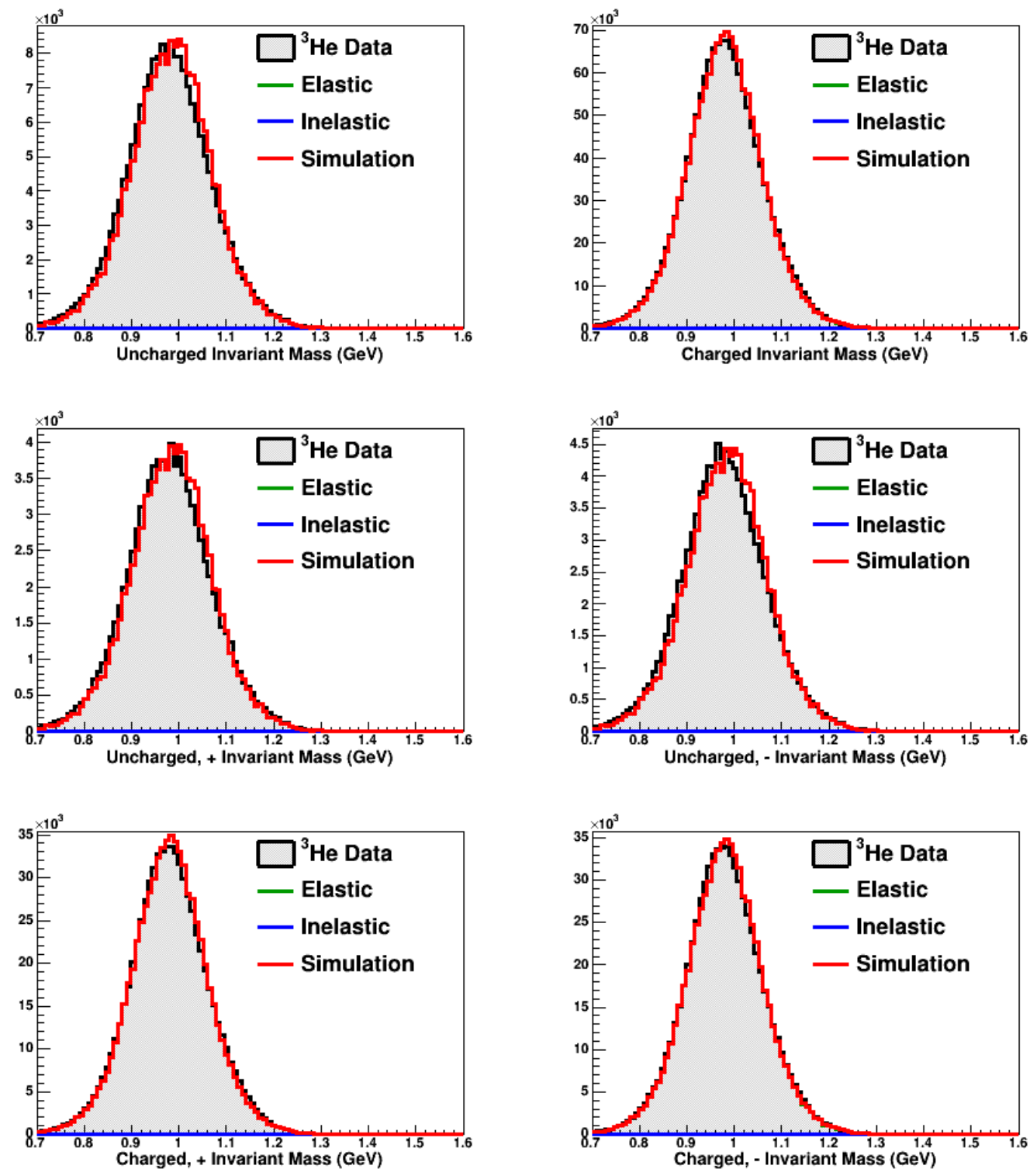

Fig. 6.9: The six $W$ spectra required for the inelastic contamination estimation. Displayed from top to bottom, left to right: uncharged, charged, \pm uncharged and \pm charged. A log scale (next page) is needed to see the negligible inelastic distributions. 

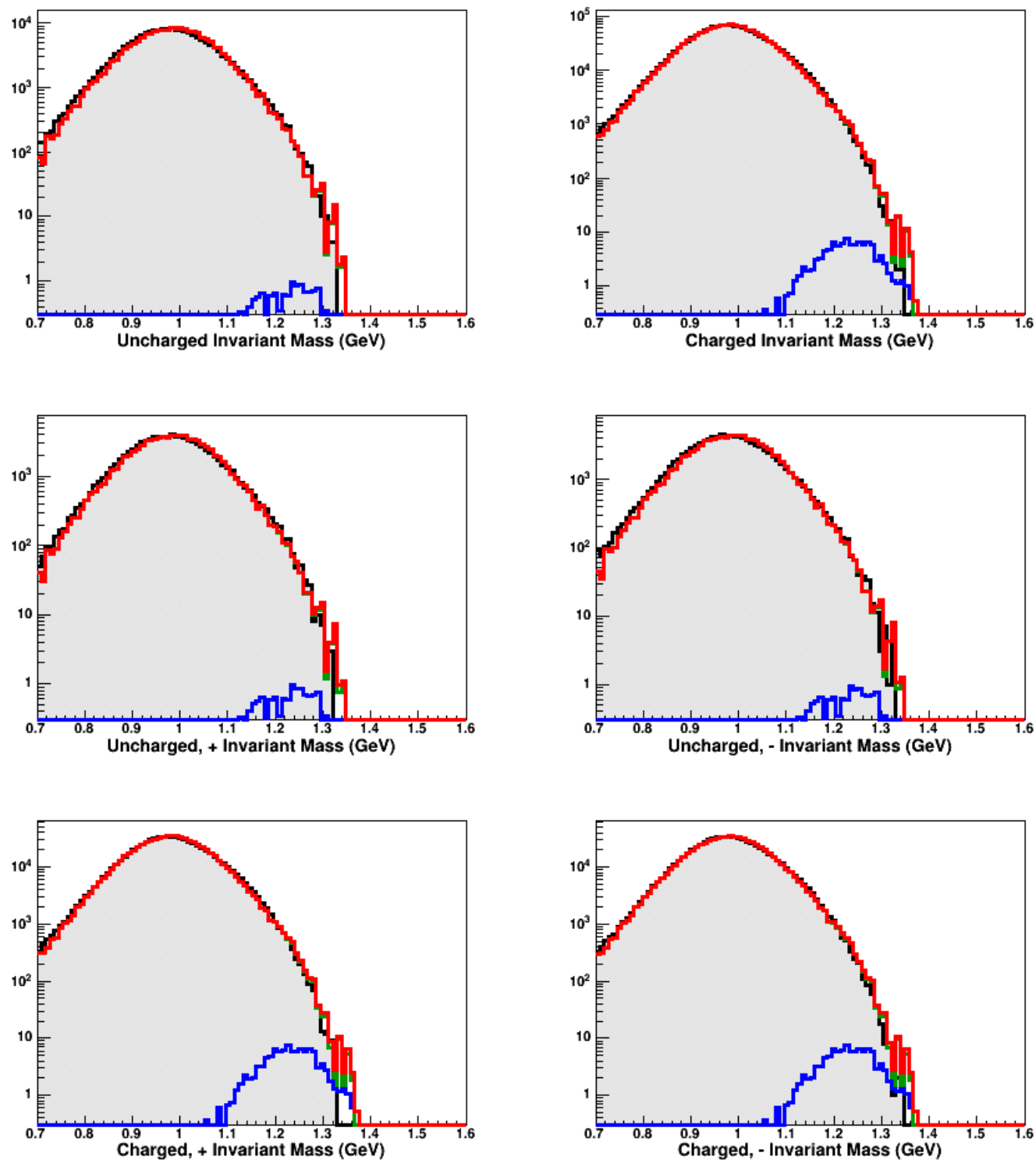

Fig. 6.10: Displaying the plots of Figure 6.9 in a log scale. According to the simulation, the inelastic contamination to the quasielastic uncharged and charged samples is negligible. 
nucleon state), is calculated for both incoming electron helicity states assuming that the generated event kinematics are within the quasielastic cut selection. The asymmetry with final state interaction processes included is denoted by $A$, and many events are combined to form an average value, or

$$
A=\frac{\sum_{i} \sigma_{i}^{+}-\sum_{i} \sigma_{i}^{-}}{\sum_{i} \sigma_{i}}
$$

where the sum runs over simulated events and $\sigma=\sigma^{+}+\sigma^{-}$. The free neutron asymmetry is calculated as a cross section weighted average as follows:

$$
A_{\text {free }}=\frac{\sum_{i} \sigma_{i} \times\left(\sum_{j=0}^{5} \bar{T}_{j} \Lambda^{j}\right)_{i}}{\sum_{i} \sigma_{i}}
$$

which includes the finite acceptance formalism discussed in Section 5.7 .2 and $\Lambda$ is calculated using the Kelly parametrization. In order to obtain the desired mapping between $A$ and $A_{\text {free }}$, the calculation is performed many times assuming a value of $\Lambda=G_{E}^{n} / G_{M}^{n}$, which may be achieved by scaling $\Lambda$ by a coefficient $f$ chosen to be in the range $[0.0,2.0]$ in increments of 0.2 . The results may be seen in Table 6.10 where each row represents a calculation in which $\Lambda$ has been scaled by factor $f$. The error in $A$ is calculated by forming the asymmetry $A$ in blocks of 100 events. The resulting distribution is fit to a Gaussian in order to calculate the standard deviation $\sigma_{\mathrm{g}}$; the uncertainty in $A$ is calculated as $\delta A=\sigma_{\mathrm{g}} / \sqrt{N / 100}$ where $N$ is the total number of events. The results of the "Gaussian-blocking" method may be seen by Figure 6.11. The mapping between $A$ and $A_{\text {free }}$ may be seen within Figure 6.12 which includes the results for the final state interaction effects in addition to the PWIA for comparison. A highly linear relationship is observed, or $A=m A_{\text {free }}+b$ where $m=0.977 \pm 0.020$ and $b=-0.0012 \pm 0.0008$. 

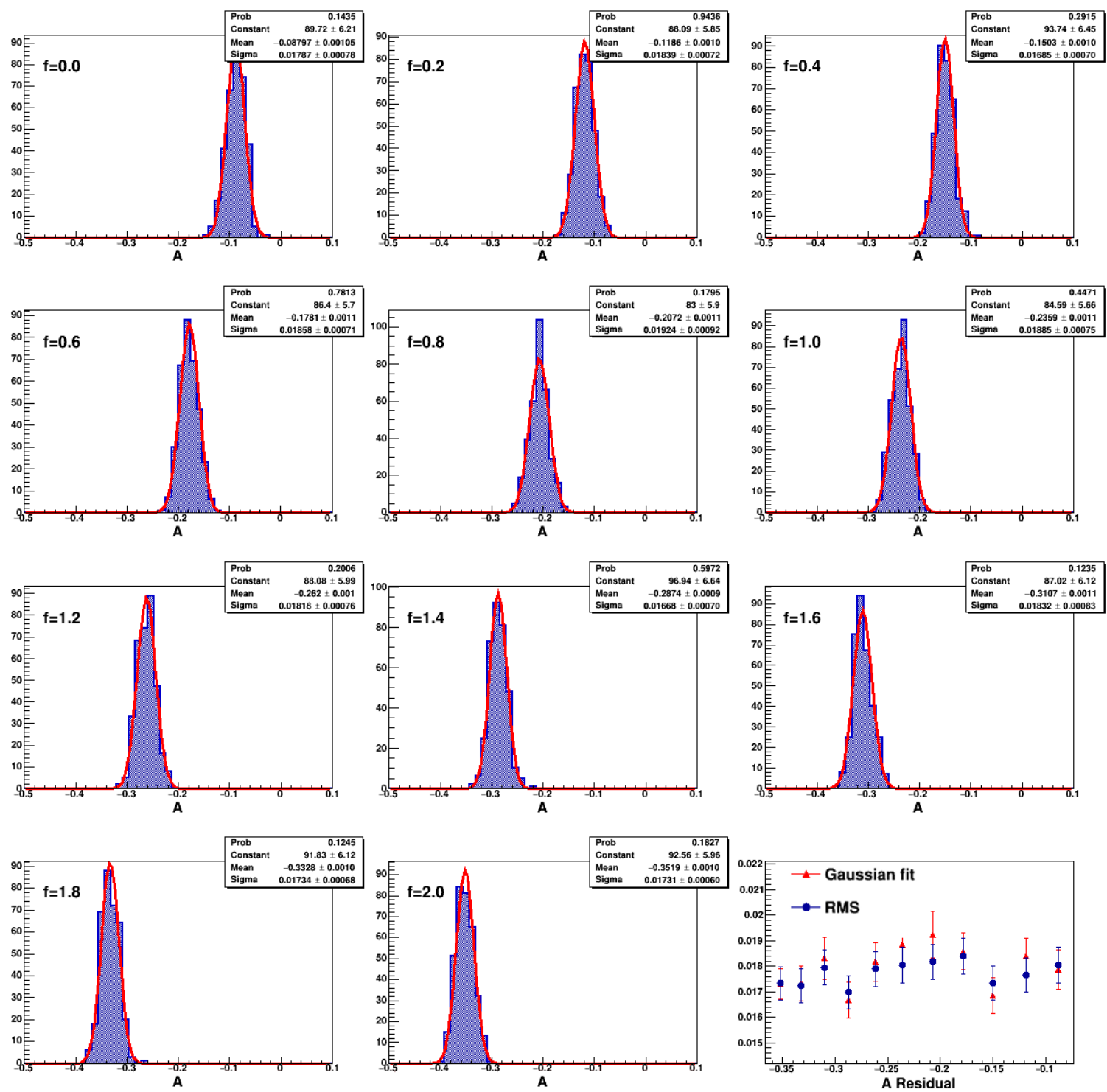

Fig. 6.11: The helicity-dependent cross sections $\sigma^{ \pm}$are summed in blocks of $N_{\text {blk }}=$ 100 events; repeat the procedure for all simulated events, and the resulting distributions are fit to a Gaussian of standard deviation $\sigma_{\mathrm{g}}$. The error in $A$ is then calculated as $\delta A=\sigma_{\mathrm{g}} / \sqrt{N / N_{\mathrm{blk}}}$. Bottom right panel: $\sigma_{\mathrm{g}}$ and the RMS in $A$ are plotted as a function of the Gaussian mean. 


\begin{tabular}{|c|c|c|c|c|}
\hline Factor $f$ & $\sum_{i} \sigma_{i}^{+}$ & $\sum_{i} \sigma_{i}^{-}$ & $\boldsymbol{A}$ & $A_{\text {free }}$ \\
\hline 0.0 & 12342.8 & 14787.7 & $-0.090 \pm 0.001$ & -0.091 \\
\hline 0.2 & 12844.9 & 16383.9 & $-0.121 \pm 0.001$ & -0.123 \\
\hline 0.4 & 11287.6 & 15319.8 & $-0.152 \pm 0.001$ & -0.154 \\
\hline 0.6 & 10767.1 & 15540.0 & $-0.181 \pm 0.001$ & -0.184 \\
\hline 0.8 & 10959.0 & 16806.2 & $-0.211 \pm 0.001$ & -0.214 \\
\hline 1.0 & 10623.7 & 17315.7 & $-0.240 \pm 0.001$ & -0.243 \\
\hline 1.2 & 10371.9 & 17902.8 & $-0.266 \pm 0.001$ & -0.270 \\
\hline 1.4 & 10061.0 & 18279.4 & $-0.290 \pm 0.001$ & -0.296 \\
\hline 1.6 & 9796.0 & 18801.9 & $-0.315 \pm 0.001$ & -0.321 \\
\hline 1.8 & 9730.7 & 19594.7 & $-0.336 \pm 0.001$ & -0.343 \\
\hline 2.0 & 9766.5 & 20601.4 & $-0.357 \pm 0.001$ & -0.365 \\
\hline
\end{tabular}

Table 6.10: The results of the FSI simulation in tabular form. Each row represents a simulation where $\Lambda$ has been scaled by $f$. See text for details on the asymmetry calculations.

The asymmetry that includes nuclear corrections originating from a Monte Carlo calculation may be compared to the results of the analysis if $A_{\text {raw }}$ has been corrected for all experimental realities excluding nuclear effects; therefore, $A_{\text {free }}=(A-b) / m$ reveals the dilution factor and the asymmetry correction to $A_{\text {raw }}$ due to nuclear effects, or $D_{\mathrm{FSI}}=m=0.977 \pm 0.020$ and $A_{\mathrm{FSI}}=b=-0.0012 \pm 0.0008$. 


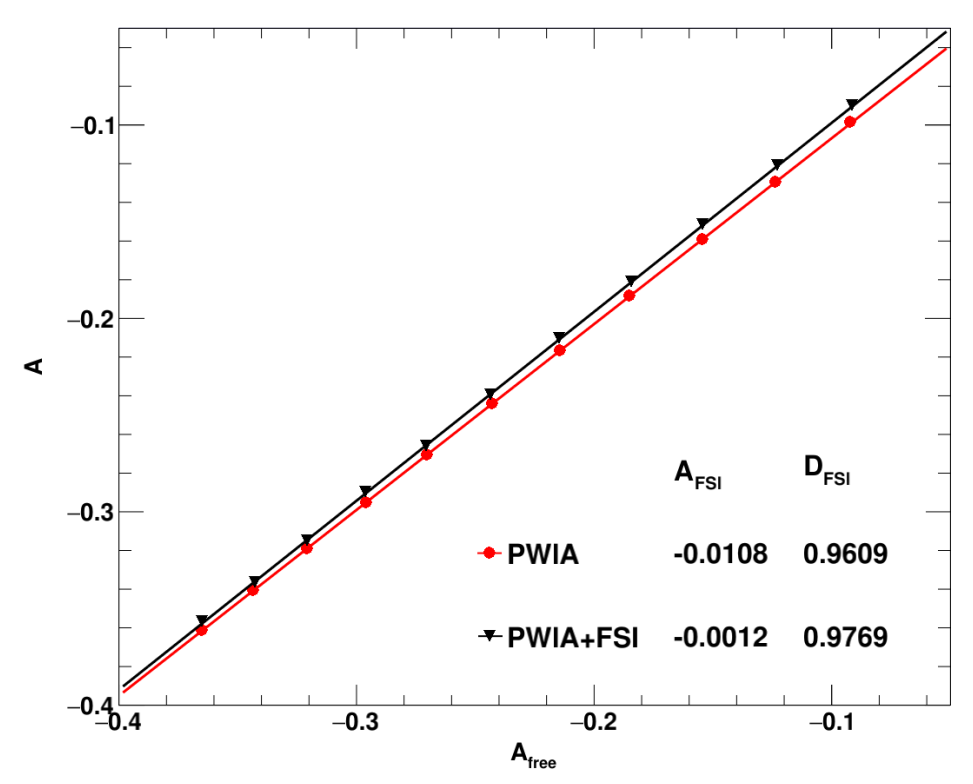

Fig. 6.12: The mapping between $A$ and $A_{\text {free }}$ in the PWIA and the full GEA (PWIA+FSI). See text for more information.

The results suggest that the effective neutron polarization within the quasielastic region exceeds $96 \%$ of $P_{3} \mathrm{He}_{\mathrm{e}}$; this is to be compared to commonly cited value of $86 \%$ [29, 30], which is for the inclusive process ${ }^{3} \mathrm{He}\left(\vec{e}, e^{\prime}\right) X$ where the integration is over the whole range of initial neutron momenta. Furthermore, the GEA ${ }^{3} \mathrm{He}\left(\vec{e}, e^{\prime} n\right) p p$ asymmetry is found to be within $2 \%$ of the asymmetry within the PWIA indicating that nuclear rescattering effects are small. Nuclear effects due to Fermi motion, depolarization, and FSI have been found to be on the $11 \%$ level with the expectation to be further reduced with an increase in $Q^{2}$. The error in these estimations is mainly due to the accuracy of the GEA, which is estimated to be $\sim 15-20 \%$ at $Q^{2} \sim 1 \mathrm{GeV}^{2}$ and improves to $\sim 5 \%$ in the high- $Q^{2}$ limit. As the overall nuclear effect is no more than $11 \%$, the GEA accuracy is estimated to be at the $2 \%$ level [219] and is taken as the systematic. 


\subsubsection{Physical Asymmetry from Data}

All dilution factors and asymmetries required to correct the raw asymmetry have been presented; these values are assumed to be constant throughout the polarized ${ }^{3} \mathrm{He}$ data set. The beam polarization is also assumed to be constant, but the target polarization is allowed to fluctuate, see Section 6.1 .2 for the values. The raw asymmetry for a particular run $i$ may change, effectively producing a set of physical asymmetries that need to be combined. Recall the final form of the physical asymmetry after all corrections have been applied is (see Eqn 5.59, Eqn 5.108, and Eqn 5.109)

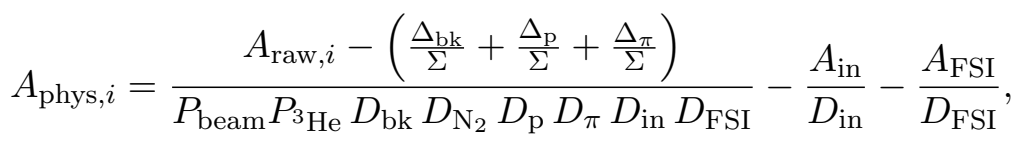

where now the index $i$ specifies the value of $A_{\text {raw }}$ and $A_{\text {phys }}$ for run $i$ of the polarized ${ }^{3}$ He data set. The statistical uncertainty in $A_{\text {phys }, i}$ is completely contained in $A_{\text {raw }, i}$ :

$$
\sigma_{\text {stat }, i}=\frac{\delta A_{\text {raw }, i}}{P_{\text {beam }} P_{3}{ }_{\mathrm{He}} D_{\mathrm{bk}} D_{\mathrm{N}_{2}} D_{\mathrm{p}} D_{\pi} D_{\mathrm{in}} D_{\mathrm{FSI}}} .
$$

Utilizing the following short-hand notation for convenience,

$$
\begin{aligned}
\mathcal{D} & \equiv P_{\text {beam }} P_{{ }^{3} \mathrm{He}} D_{\mathrm{bk}} D_{\mathrm{N}_{2}} D_{\mathrm{p}} D_{\pi} D_{\mathrm{in}} D_{\mathrm{FSI}}, \\
\mathcal{A}_{i} \equiv & \frac{A_{\mathrm{raw}, i}-\left(\frac{\Delta_{\mathrm{bk}}}{\Sigma}+\frac{\Delta_{\mathrm{p}}}{\Sigma}+\frac{\Delta_{\pi}}{\Sigma}\right)}{\mathcal{D}}
\end{aligned}
$$

the remaining terms make up the systematic contribution:

$$
\begin{aligned}
\sigma_{\mathrm{sys}, i}^{2} & =\frac{\left(\delta \frac{\Delta_{\mathrm{bk}}}{\Sigma}\right)^{2}+\left(\delta \frac{\Delta_{\mathrm{p}}}{\Sigma}\right)^{2}+\left(\delta \frac{\Delta_{\pi}}{\Sigma}\right)^{2}}{\mathcal{D}^{2}}+\left[\frac{\delta A_{\mathrm{in}}}{D_{\mathrm{in}}}\right]^{2}+\left[\frac{\delta A_{\mathrm{FSI}}}{D_{\mathrm{FSI}}}\right]^{2} \\
& +\mathcal{A}_{i}^{2}\left\{\left[\frac{\delta P_{\mathrm{beam}}}{P_{\mathrm{beam}}}\right]^{2}+\left[\frac{\delta P_{3} \mathrm{He}}{P_{3} \mathrm{He}}\right]^{2}+\left[\frac{\delta D_{\mathrm{bk}}}{D_{\mathrm{bk}}}\right]^{2}+\left[\frac{\delta D_{\mathrm{N}_{2}}}{D_{\mathrm{N}_{2}}}\right]^{2}+\left[\frac{\delta D_{\mathrm{p}}}{D_{\mathrm{p}}}\right]^{2}+\left[\frac{\delta D_{\pi}}{D_{\pi}}\right]^{2}\right\} \\
& +\left[\frac{\delta D_{\mathrm{in}}}{D_{\mathrm{in}}}\right]^{2}\left(\mathcal{A}_{i}-\frac{A_{\mathrm{in}}}{D_{\mathrm{in}}}\right)^{2}+\left[\frac{\delta D_{\mathrm{FSI}}}{D_{\mathrm{FSI}}}\right]^{2}\left(\mathcal{A}_{i}-\frac{A_{\mathrm{FSI}}}{D_{\mathrm{FSI}}}\right)^{2}
\end{aligned}
$$




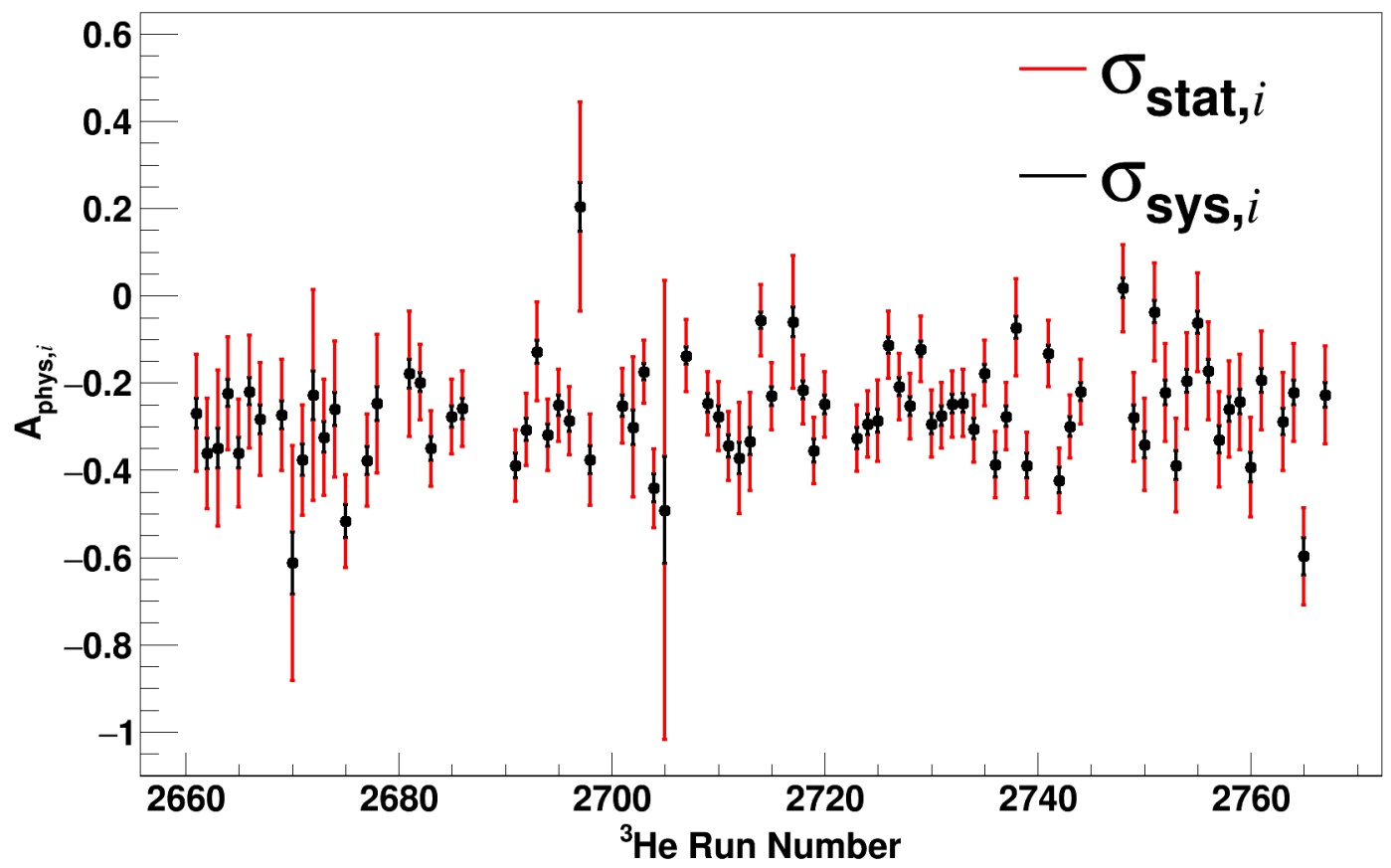

Fig. 6.13: The physical asymmetry extracted from the ${ }^{3} \mathrm{He}$ data. The statistical and systematic contributions on a per run basis are shown by the red and black lines, respectively.

where recall that the variance in the background asymmetry correction $\frac{\Delta_{\mathrm{bk}}}{\Sigma}$ has a systematic correlation that must be considered, see Eqn 6.7 for explicit details. The uncertainty in $A_{\text {phys }, i}$ may then be computed by simply adding the two contributions in quadrature, or $\delta A_{\mathrm{phys}, i}=\sqrt{\sigma_{\mathrm{stat}, i}^{2}+\sigma_{\mathrm{sys}, i}^{2}}$. The physical asymmetry extracted for ${ }^{3} \mathrm{He}$ run $i$ may be seen by Figure 6.13 where the statistical and systematic contributions are displayed by the red and black lines, respectively.

In order to compare the experimental physical asymmetry to theory, $A_{\mathrm{phys}, i}$ is combined in a statistically weighted average where the weighting variances are purely 
statistical. To be explicit, the standard expressions to compute a statistically weighted average for ${ }^{3} \mathrm{He}$ run number $i$ are

$$
A_{\text {phys }}=\frac{\sum_{i} \frac{A_{\text {phys }, i}}{\sigma_{\text {stat }, i}^{2}}}{\sum_{i} \frac{1}{\sigma_{\text {stat }, i}^{2}}} \quad \text { and } \quad \sigma_{\text {stat }}=\frac{1}{\sqrt{\sum_{i} \frac{1}{\sigma_{\text {stat }, i}^{2}}}} .
$$

The systematic contribution is assumed to affect all data points equally and may be added in quadrature at the end where $\mathcal{A}_{i}$ in Eqn 6.36 is now calculated using the statistically weighted average of $A_{\text {raw }, i}$. The results of the physical asymmetry and all relevant parameters may be seen in Table 6.11. All calculations required to extract $G_{E}^{n} / G_{M}^{n}$ have been presented.

\subsection{Neutron Form Factor Ratio}

The calculations required to correct the experimental neutral raw asymmetry for all appreciable experimental realities have been presented in the previous section. In other words, the raw asymmetry has been corrected to the point where the result most closely resembles the asymmetry of a free neutron; therefore, the data may now be compared to the theoretical physical asymmetry in order to solve for the neutron form factor ratio:

$$
A_{\mathrm{phys}}=\sum_{i=0}^{5} \bar{T}_{i} \cdot \Lambda^{i}=\bar{T}_{0}+\bar{T}_{1} \Lambda+\bar{T}_{2} \Lambda^{2}+\bar{T}_{3} \Lambda^{3}+\bar{T}_{4} \Lambda^{4}+\bar{T}_{5} \Lambda^{5},
$$

where $\Lambda=G_{E}^{n} / G_{M}^{n}$. The right-hand side is the beam-target asymmetry as derived in Section 2.3.2 and later re-expressed in Section 5.7.2. The expression has been expanded to fifth-order in $\Lambda$ where the expansion coefficients $\bar{T}_{i}$ contain the kinematics, and are meant to be averaged to handle the finite acceptance of experimental detectors. The fifth-order equation may be numerically solved for $\Lambda$ using the Newton-Raphson method, 


\begin{tabular}{|c|c|c|}
\hline Parameter & Value & Description \\
\hline$P_{\text {beam }}$ & $0.872 \pm 0.020$ & Beam polarization \\
\hline$P_{3} \mathrm{He}$ & $0.397 \pm 0.015$ & Target polarization \\
\hline$D_{\mathrm{bk}}$ & $0.949 \pm 0.029$ & Accidental background \\
\hline$D_{\mathrm{N}_{2}}$ & $0.954 \pm 0.005$ & $\mathrm{~N}_{2}$ in ${ }^{3} \mathrm{He}$ cell \\
\hline$D_{\mathrm{p}}$ & $0.812 \pm 0.017$ & Proton misidentification \\
\hline$D_{\pi}$ & $0.997 \pm 0.001$ & Preshower pion dilution \\
\hline$D_{\text {in }}$ & $1.000 \pm 0.050$ & Inelastic dilution \\
\hline$D_{\mathrm{FSI}}$ & $0.977 \pm 0.020$ & Nuclear corrections \\
\hline$\frac{\Delta_{\mathrm{bk}}}{\Sigma}$ & $-0.0003 \pm 0.0005$ & Background asymmetry correction \\
\hline$\frac{\Delta_{\mathrm{p}}}{\Sigma}$ & $-0.0008 \pm 0.0004$ & Proton asymmetry correction \\
\hline$\frac{\Delta_{\pi}}{\Sigma}$ & $-0.0002 \pm 0.0001$ & Preshower pion asymmetry \\
\hline$A_{\text {in }}$ & $0.0000 \pm 0.0150$ & Inelastic asymmetry correction \\
\hline$A_{\mathrm{FSI}}$ & $-0.0012 \pm 0.0008$ & Nuclear corrections \\
\hline$N_{\mathrm{qe}}$ & $1.816 \times 10^{5}$ & Total \# of quasielastic events \\
\hline$A_{\text {raw }}$ & $-0.0584 \pm 0.0023$ & Raw asymmetry \\
\hline$A_{\text {phys }}$ & $-0.2291 \pm 0.0094 \pm 0.0129$ & Physical asymmetry \pm stat \pm sys \\
\hline
\end{tabular}

Table 6.11: All parameters used in the calculation of $A_{\text {phys }}$. Recall that the effects of nuclear polarization are embedded within the nuclear corrections. 
i.e. an iterative root-finding algorithm solving

$$
f(\Lambda)=A_{\text {phys }}-\sum_{i=0}^{5} \bar{T}_{i} \cdot \Lambda^{i}, \quad \frac{d f}{d \Lambda}=-\sum_{i=1}^{5} i \bar{T}_{i} \cdot \Lambda^{i-1},
$$

where the step in $\Lambda$, or $\Lambda_{n+1}=\Lambda_{n}-\frac{f\left(\Lambda_{n}\right)}{f^{\prime}\left(\Lambda_{n}\right)}$, is repeated until the numerical value of $|f(\Lambda)|$ is below a convergence threshold. To ensure convergence, an initial value corresponding to the first-order solution is used, or $\Lambda_{0}=\left(A_{\text {phys }}-\bar{T}_{0}\right) / \bar{T}_{1}$, and a convergence threshold of $10^{-6}$ has been chosen which is more than sufficient.* The uncertainty in the neutron form factor ratio is

$$
\delta \Lambda=\frac{\delta A_{\mathrm{phys}}}{\left|\sum_{i=1}^{5} i \cdot \bar{T}_{i} \cdot \Lambda^{i-1}\right|},
$$

where the statistical and systematic contributions of $A_{\text {phys }}$ have been explicitly described in the previous section. The result of this thesis, namely the extraction of the form factor ratio of the neutron, may be seen by Table 6.12 where the first (second) uncertainty is the statistical (systematic) contribution. The components of the physical asymmetry, $A_{\|}$and $A_{\perp}$, may be separated by summing the even and odd $\bar{T}_{i} \Lambda^{i}$ terms, respectively, in Eqn 6.38. The electric form factor may be separated from the ratio by assuming a value of $G_{M}^{n}$ at $Q^{2}=1.16 \mathrm{GeV}^{2}$, and $G_{M}^{n}=-0.2794 \pm 0.0070$ has been chosen by a linear interpolation of $G_{M}^{n}$ data from Ref. [87]. The dominant sources to the systematic error budget may be seen by Table 6.13 where all sources are presented as fractional contributions to $G_{E}^{n}$, i.e. $\delta / G_{E}^{n}$. The systematic contribution to $G_{E}^{n}$ dominates; the largest contribution to the systematic is due to the uncertainty in target polarization measurements. A breakdown of the error budget for target cell Barbara measurements, i.e. the $\kappa_{0}$ value, EPR and NMR, density and more, may be found in Table 4.12 of

\footnotetext{
* Convergence occurs in less than five iterations using this method.
} 


\begin{tabular}{|c|c|c|}
\hline Parameter & Value & Description \\
\hline$Q^{2}\left(\mathrm{GeV}^{2}\right)$ & 1.16 & Acceptance averaged value of $Q^{2}$ \\
\hline$N_{\mathrm{qe}}$ & $1.816 \times 10^{5}$ & Total \# of quasielastic events \\
\hline $\bar{T}_{0}$ & -0.086 & $A_{\text {phys }}$ expansion coefficient, $\Lambda^{0}$ \\
\hline $\bar{T}_{1}$ & 1.198 & $A_{\text {phys }}$ expansion coefficient, $\Lambda^{1}$ \\
\hline $\bar{T}_{2}$ & 0.150 & $A_{\text {phys }}$ expansion coefficient, $\Lambda^{2}$ \\
\hline $\bar{T}_{3}$ & -2.097 & $A_{\text {phys }}$ expansion coefficient, $\Lambda^{3}$ \\
\hline $\bar{T}_{4}$ & -0.268 & $A_{\text {phys }}$ expansion coefficient, $\Lambda^{4}$ \\
\hline $\bar{T}_{5}$ & 3.743 & $A_{\text {phys }}$ expansion coefficient, $\Lambda^{5}$ \\
\hline$G_{E}^{n} / G_{M}^{n}$ & $-0.1247 \pm 0.0088 \pm 0.0121$ & Neutron form factor ratio, $\Lambda$ \\
\hline$A_{\|}$ & $-0.0836 \pm 0.0005$ & Parallel component of $A_{\text {phys }}$ \\
\hline$A_{\perp}$ & $-0.1455 \pm 0.0165$ & Perpendicular component of $A_{\text {phys }}$ \\
\hline$G_{M}^{n}$ & $-0.2794 \pm 0.0070$ & Magnetic form factor from 87 \\
\hline$G_{E}^{n}$ & $0.0348 \pm 0.0025 \pm 0.0035$ & Electric form factor \\
\hline
\end{tabular}

Table 6.12: The neutron form factor ratio extracted at $Q^{2}=1.16 \mathrm{GeV}^{2}$.

Ref. [175]. The newly extracted value of the neutron form factor ratio and $G_{E}^{n}$ may be seen amongst other double polarization data in Figure 6.14 where all fits and models are discussed, or at the very least introduced, in Section 3.3 . The remaining $Q^{2}$ points have been reanalyzed, and the details are presented in Appendix C; see Table C.28 for a summary of the results. 

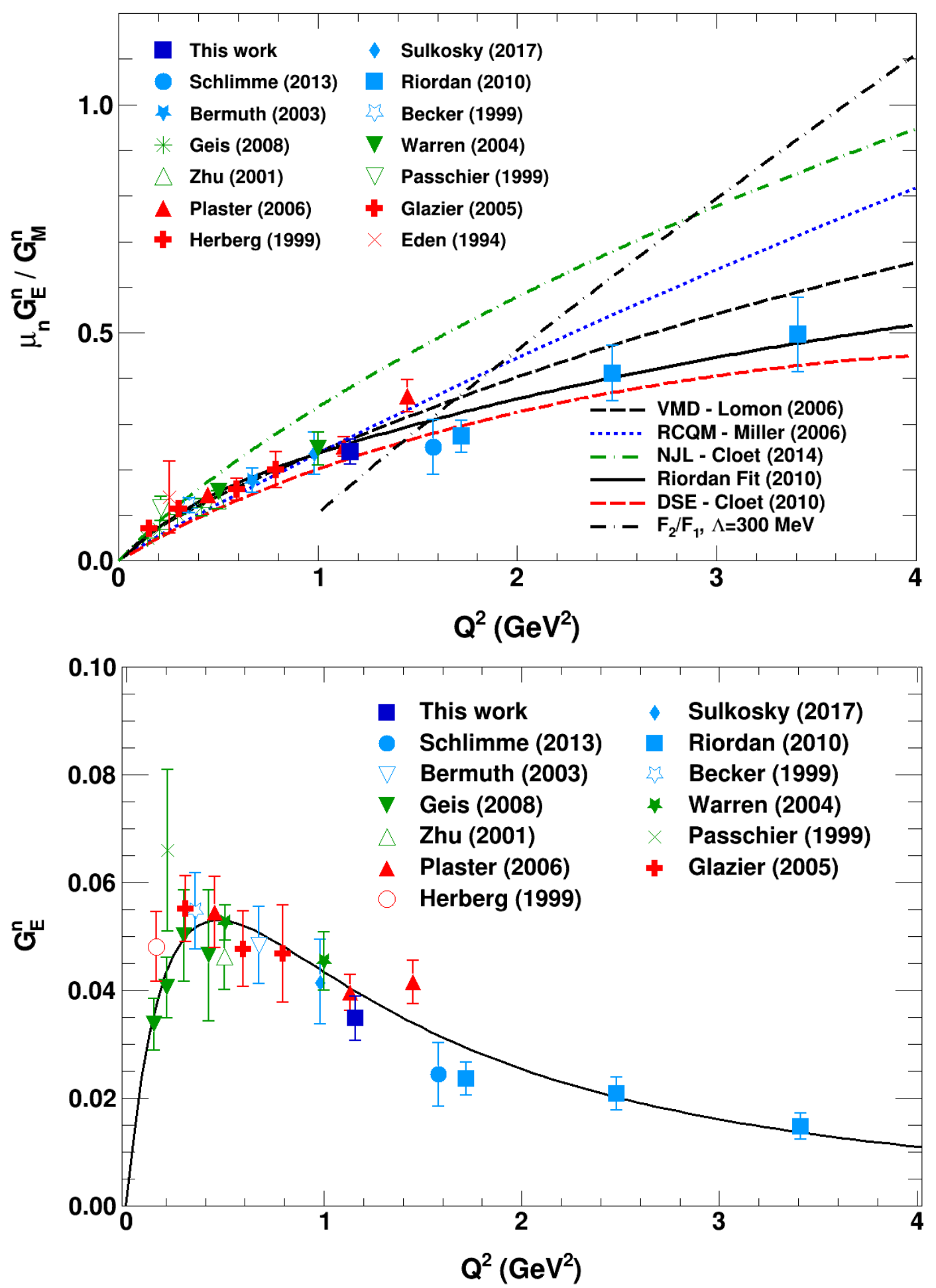

Fig. 6.14: The result of this thesis amongst double polarization neutron data. 


\begin{tabular}{lll}
\hline Source & $\boldsymbol{\delta} / \boldsymbol{G}_{\boldsymbol{E}}^{n}$ & Comments \\
\hline$\delta G_{E}^{n}$ & 0.123 & Total uncertainty contribution \\
$\delta_{\text {sys }}$ & 0.100 & Systematic \\
$\delta_{\text {stat }}$ & 0.071 & Statistical \\
\hline$\delta P_{3} \mathrm{He}$ & 0.067 & Target polarization \\
$\delta P_{\mathrm{beam}}$ & 0.040 & Beam polarization \\
$\delta D_{\mathrm{FSI}}$ & 0.035 & Nuclear corrections \\
$\delta D_{\mathrm{bk}}$ & 0.029 & Background dilution \\
$\delta D_{\mathrm{p}}$ & 0.028 & Proton dilution \\
$\delta G_{M}^{n}$ & 0.025 & Error from chosen $G_{M}^{n}$ \\
$\delta_{\text {other }}$ & 0.023 & Remaining contributions \\
\hline
\end{tabular}

Table 6.13: The systematic error budget presented as a fraction of $G_{E}^{n}$.

\subsection{Future Experiments}

There are several approved nucleon form factor experiments that will utilize Jefferson Lab's 12 GeV upgrade, see Table 6.14. Note that E12-07-108 ran in 2016 and is currently being analyzed, and E12-09-019 and E12-07-104 will extract neutron form factors via a cross section ratio measurement, i.e. $\frac{d \sigma}{d \Omega}\left[d\left(e, e^{\prime} n\right)\right] / \frac{d \sigma}{d \Omega}\left[d\left(e, e^{\prime} p\right)\right]$. Additionally, MAMI has a proposed measurement (A1-2016-1) of $G_{E}^{n} / G_{M}^{n}$ using the reaction $d\left(\vec{e}, e^{\prime} \vec{n}\right) p$ in the $Q^{2}$ range of $0.2-1.55 \mathrm{GeV}^{2}$; however, the table highlights high- $Q^{2}$ form factor experiments. Among other things, the $12 \mathrm{GeV}$ upgrade will push nucleon form factor 


\begin{tabular}{cccc}
\hline Experiment & Extraction & Reaction & Hall \\
\hline $\mathrm{E} 12-07-104[220]$ & $G_{M}^{n} / G_{M}^{p}$ & $d\left(e, e^{\prime} n\right) / d\left(e, e^{\prime} p\right)$ & $\mathrm{B}$ \\
$\mathrm{E} 12-07-108[221]$ & $G_{M}^{p}$ & $\mathrm{H}\left(e, e^{\prime} p\right)$ & $\mathrm{A}$ \\
$\mathrm{E} 12-07-109[22]$ & $G_{E}^{p} / G_{M}^{p}$ & $\mathrm{H}\left(\vec{e}, e^{\prime} \vec{p}\right)$ & $\mathrm{A}$ \\
$\mathrm{E} 12-09-016[223]$ & $G_{E}^{n} / G_{M}^{n}$ & ${ }^{3} \mathrm{He}\left(\vec{e}, e^{\prime} n\right) p p$ & $\mathrm{~A}$ \\
$\mathrm{E} 12-09-019[224]$ & $G_{M}^{n} / G_{M}^{p}$ & $d\left(e, e^{\prime} n\right) / d\left(e, e^{\prime} p\right)$ & $\mathrm{A}$ \\
$\mathrm{E} 12-11-009[225]$ & $G_{E}^{n} / G_{M}^{n}$ & $d\left(\vec{e}, e^{\prime} \vec{n}\right) p$ & $\mathrm{C}$ \\
$\mathrm{E} 12-17-004[226]$ & $G_{E}^{n} / G_{M}^{n}$ & $d\left(\vec{e}, e^{\prime} \vec{n}\right) p$ & $\mathrm{~A}$ \\
\hline
\end{tabular}

Table 6.14: JLab $12 \mathrm{GeV}$ approved experiments, focusing on high $Q^{2}$ measurements. Note that E12-07-108 ran in 2016 and is currently undergoing analysis.

measurements into a $Q^{2}$ regime where pQCD will perhaps reveal itself,* and a potential zero-crossing of $G_{E} / G_{M}$ may or may not be observed. Of particular interest with respect to the neutron is the high- $Q^{2}$ behavior of $G_{E}^{n} / G_{M}^{n}$ in which only the DSE calculations predict a zero-crossing at a $Q^{2}$ of approximately $10 \mathrm{GeV}^{2}$. Furthermore, the high- $Q^{2}$ reach using both beam-target asymmetry and recoil polarization techniques may shed light on any differing systematics, if any at all, that led to the noticeable disagreement in $G_{E}^{n}$ around $Q^{2}=1.5 \mathrm{GeV}^{2}$ between Refs. [7, 54, 107] and this work. As the neutron data is only known to $3.4 \mathrm{GeV}^{2}$ which is roughly 2.5 times lower than its nucleon counterpart, an important goal is to push neutron form factor measurements to comparable levels, which will allow for a more complete understanding of the nucleon.

\footnotetext{
* This is unexpected for elastic $e N$, though, as pQCD has been argued to only be relevant at $Q^{2} \sim 100 \mathrm{GeV}^{2}$.
} 


\section{Appendix A}

\section{Useful Results from the Dirac Equation}

\section{A.1 Dirac Representation}

Dirac matrices satisfy the anticommutation relation

$$
\left\{\gamma^{\mu}, \gamma^{\nu}\right\}=\gamma^{\mu} \gamma^{\nu}+\gamma^{\nu} \gamma^{\mu}=2 g^{\mu \nu}
$$

where $g^{\mu \nu}$ is the Minkowski metric with spatial components defined to be -1 . The chosen basis is referred to as the Dirac representation where $\gamma^{0}$ is diagonal, and requires four matrices:

$$
\gamma^{0}=\left(\begin{array}{cc}
1 & 0 \\
0 & -1
\end{array}\right), \quad \gamma^{i}=\left(\begin{array}{cc}
0 & \sigma^{i} \\
-\sigma^{i} & 0
\end{array}\right)
$$

where $\sigma^{i}$ are the $2 \times 2$ Pauli matrices:

$$
\sigma^{1}=\left(\begin{array}{cc}
0 & 1 \\
1 & 0
\end{array}\right), \quad \sigma^{2}=\left(\begin{array}{cc}
0 & -i \\
i & 0
\end{array}\right), \quad \sigma^{3}=\left(\begin{array}{cc}
1 & 0 \\
0 & -1
\end{array}\right) .
$$

It is useful to define another gamma matrix that anticommutes with $\gamma^{\mu}$, or

$$
\begin{gathered}
\gamma^{5} \equiv i \gamma^{0} \gamma^{1} \gamma^{2} \gamma^{3}, \\
0=\left\{\gamma^{5}, \gamma^{\mu}\right\}
\end{gathered}
$$




\section{A.2 Dirac Equation}

The Lagrangian density for a free Dirac field is given as

$$
\mathcal{L}=\bar{\psi}\left(i \gamma^{\mu} \partial_{\mu}-m\right) \psi
$$

and the Euler-Lagrange equation for $\bar{\psi}$ yields the familiar Dirac equation:

$$
\left(i \gamma^{\mu} \partial_{\mu}-m\right) \psi=0
$$

The particle solutions* to the Dirac equation are a linear combination of plane waves which when inserted back into the Dirac equation yields the following useful results:

$$
\begin{aligned}
0 & =(\not p-m) u(p), \\
0 & =\bar{u}(p)(\not p-m), \\
u^{(s)}(p) & =\sqrt{E+M}\left(\begin{array}{c}
\chi^{(s)} \\
\frac{\boldsymbol{\sigma} \cdot \mathbf{p}}{E+M} \chi^{(s)}
\end{array}\right) .
\end{aligned}
$$

The spinor $\chi^{(s)}$ is a spin state with two completeness relations:

$$
\begin{array}{ll}
\sum_{s} \chi^{(s)} \chi^{\dagger(s)}=1 & \text { (unpolarized) } \\
\sum_{s} \chi^{(s)} \chi^{\dagger(s)}=\frac{1}{2}(1+\boldsymbol{\sigma} \cdot \mathbf{h}) & \text { (polarized) }
\end{array}
$$

where $\mathbf{h}$ is the polarization vector and in this case treat $\boldsymbol{\sigma}$ like a vector, or $\mathbf{a} \cdot \boldsymbol{\sigma}=$ $a_{x} \sigma_{1}+a_{y} \sigma_{2}+a_{z} \sigma_{3}$. The positive Dirac spinors have the following completeness relation,

$$
\sum_{s} u^{s} \bar{u}^{s}=\not p+m
$$

\footnotetext{
* Antiparticle solutions are not needed for the derivations contained within this thesis.
} 


\section{Appendix B}

\section{Useful Trace Identities}

The trace of the product of an odd number of gamma matrices is zero.

$$
\begin{aligned}
\operatorname{Tr}\left(\gamma^{\mu}\right) & =0 \\
\operatorname{Tr}\left(\gamma^{\mu} \gamma^{\nu}\right) & =4 g^{\mu \nu} \\
\operatorname{Tr}\left(\gamma^{\mu} \gamma^{\nu} \gamma^{\lambda} \gamma^{\sigma}\right) & =4\left(g^{\mu \nu} g^{\lambda \sigma}-g^{\mu \lambda} g^{\nu \sigma}+g^{\mu \sigma} g^{\nu \lambda}\right) \\
\operatorname{Tr}\left(\gamma^{5}\right) & =0 \\
\operatorname{Tr}\left(\gamma^{5} \gamma^{\mu} \gamma^{\nu}\right) & =0 \\
\operatorname{Tr}\left(\gamma^{5} \gamma^{\mu} \gamma^{\nu} \gamma^{\lambda} \gamma^{\sigma}\right) & =4 i \epsilon^{\mu \nu \lambda \sigma}
\end{aligned}
$$




\section{Appendix C}

\section{Results for the Remaining Configurations}

A summary of the four kinematic configurations may be see by Table 4.1. The format of this section is a condensed version of Chapter 6, see Table C.28 for the summary.

C.1 Kinematic 4: $Q^{2}=1.72 \mathrm{GeV}^{2}$

\begin{tabular}{|c|c|c|c|c|c|}
\hline \multirow[t]{2}{*}{ Parameter } & \multicolumn{2}{|c|}{ Neutral } & \multicolumn{2}{|c|}{ Charged } & \multirow{2}{*}{$\begin{array}{l}\text { Description } \\
\text { Helicity }\end{array}$} \\
\hline & + & - & + & - & \\
\hline$N$ & 72,019 & 80,340 & 627,324 & 629,703 & QE count \\
\hline$N_{\mathrm{bk}}$ & 4,572 & 4,591 & 256 & 237 & Background count \\
\hline$D_{\mathrm{bk}}$ & \multicolumn{2}{|c|}{$0.970 \pm 0.017$} & \multicolumn{2}{|c|}{$0.996 \pm 0.002$} & Background dilution \\
\hline$\sigma_{\text {stat }}$ & \multicolumn{2}{|c|}{0.0003} & \multicolumn{2}{|c|}{ negligible } & Statistical \\
\hline$\sigma_{\text {sys }}$ & \multicolumn{2}{|c|}{0.0174} & \multicolumn{2}{|c|}{0.0021} & Systematic \\
\hline$A_{\text {raw }, \mathrm{bk}}$ & \multicolumn{2}{|c|}{$-0.002 \pm 0.010$} & \multicolumn{2}{|c|}{$-0.039 \pm 0.045$} & Background raw asymmetry \\
\hline$A_{\mathrm{bk}}$ & \multicolumn{2}{|c|}{$-0.0001 \pm 0.0004$} & \multicolumn{2}{|c|}{ negligible } & Background asymmetry $\frac{\Delta_{\mathrm{bk}}}{\Sigma}$ \\
\hline
\end{tabular}

Table C.1: Background corrections for the $Q^{2}=1.72 \mathrm{GeV}^{2}$ configuration. 
Nitrogen data is available for the $Q^{2}=1.72 \mathrm{GeV}^{2}$ kinematic setting; therefore, Eqn 5.87 may be used in order to calculate the nitrogen dilution factors.

\begin{tabular}{cccl}
\hline Parameter & Unit & Value & Description \\
\hline$\rho_{\mathrm{N}_{2}}\left({ }^{3} \mathrm{He}\right)$ & amg & 0.162 & $\mathrm{~N}_{2}$ density within the ${ }^{3} \mathrm{He}$ cell \\
$\rho_{\mathrm{N}_{2}}\left(\mathrm{~N}_{2}\right)$ & amg & 10.09 & $\mathrm{~N}_{2}$ density \\
$\delta\left(\rho_{\mathrm{N}_{2}}\left({ }^{3} \mathrm{He}\right)\right)_{\mathrm{rel}}$. & $\mathrm{N} / \mathrm{A}$ & 0.007 & Relative error in $\rho_{\mathrm{N}_{2}}\left({ }^{3} \mathrm{He}\right)$ \\
$\delta\left(\rho_{\mathrm{N}_{2}}\left(\mathrm{~N}_{2}\right)\right)_{\mathrm{rel} .}$ & $\mathrm{N} / \mathrm{A}$ & 0.007 & Relative error in $\rho_{\mathrm{N}_{2}}\left(\mathrm{~N}_{2}\right)$ \\
$Q\left({ }^{3} \mathrm{He}\right)$ & $\mathrm{mC}$ & 98.5 & Accum. beam charge for ${ }^{3} \mathrm{He}$ runs \\
$Q\left(\mathrm{~N}_{2}\right)$ & $\mathrm{mC}$ & 4.12 & Accum. beam charge for $\mathrm{N}_{2}$ runs \\
\hline
\end{tabular}

Table C.2: Common parameters used in the neutral/charged calculation of $D_{\mathrm{N}_{2}}$. Recall that $1 \mathrm{amg}=44.615 \mathrm{~mol} / \mathrm{m}^{3}$.

\begin{tabular}{cccl}
\hline Parameter & Neutral & Charged & Description \\
\hline$N$ & 14,129 & 119,095 & ${ }^{3} \mathrm{He}$ QE count \\
$N_{\mathrm{bk}}$ & 827 & 44 & ${ }^{3} \mathrm{He}$ QE background count \\
$N\left(\mathrm{~N}_{2}\right)$ & 1,864 & 8,662 & $\mathrm{~N}_{2}$ QE count \\
$N_{\mathrm{bk}}\left(\mathrm{N}_{2}\right)$ & 60 & 5 & $\mathrm{~N}_{2}$ QE background count \\
$D_{\mathrm{N}_{2}}$ & $0.949 \pm 0.002$ & $0.972 \pm 0.0004$ & Nitrogen dilution factor \\
\hline
\end{tabular}

Table C.3: The results of the nitrogen correction for the neutral/charged QE samples. 


\begin{tabular}{ccl}
\hline Parameter & Value & Description \\
\hline V1 Rate $(\mathrm{kHz})$ & 226 & Average V1 rate, weighted by counts \\
V2 Rate $(\mathrm{kHz})$ & 163 & Average V2 rate, weighted by counts \\
$R_{\mathrm{N}_{2}}$ & $0.209 \pm 0.001$ & Uncharged-to-charged ratio for $\mathrm{N}_{2}$ \\
$R_{\mathrm{H}_{2}}$ & $0.028 \pm 0.001$ & Uncharged-to-charged ratio for $\mathrm{H}_{2}$ \\
$R_{3} \mathrm{He}$ & $0.115 \pm 0.001$ & Uncharged-to-charged ratio for ${ }^{3} \mathrm{He}$ \\
\hline$f_{\mathrm{N}_{2}}$ & $1 / 0.9$ & Effective ratio of neutrons to protons \\
$f_{3} \mathrm{He}$ & $1 / 2.15$ & Effective ratio of neutrons to protons \\
$\sigma_{n} / \sigma_{p}$ & 0.40 & Nucleon x-section ratio, $Q^{2}=1.72 \mathrm{GeV}^{2}$ \\
$\frac{\eta_{p}^{n}}{\eta_{p}^{p}}$ & $0.028 \pm 0.001$ & Protons observed as neutrons \\
$\frac{\eta_{n}^{p}}{\eta_{p}^{p}}$ & $0.649 \pm 0.095$ & Neutrons observed as protons \\
$\frac{\eta_{n}^{n}}{\eta_{p}^{p}}$ & $0.563 \pm 0.020$ & Neutrons observed as neutrons \\
\hline$D_{\mathrm{n}, \mathrm{ch}}$ & $0.897 \pm 0.014$ & Neutron dilution factor \\
$D_{\mathrm{p}}$ & $0.779 \pm 0.012$ & Proton dilution factor, needed for $A_{\mathrm{phys}}$ \\
\hline & & \\
\hline
\end{tabular}

Table C.4: The dilution factors due to charge misidentification for $Q^{2}=1.72 \mathrm{GeV}^{2}$. 


\begin{tabular}{|c|c|c|}
\hline Parameter & Value & Description \\
\hline$Q^{2}\left(\mathrm{GeV}^{2}\right)$ & 1.72 & Acceptance averaged value of $Q^{2}$ \\
\hline$G_{E}^{p}$ & $0.073 \pm 0.024$ & Parametrization from Section 3.2 .4 \\
\hline$G_{M}^{p}$ & $0.256 \pm 0.028$ & Parametrization from Section 3.2 .4 \\
\hline$\Lambda$ & $0.286 \pm 0.100$ & Proton form factor ratio \\
\hline $\bar{T}_{0}$ & -0.065 & $A_{\text {phys }}$ expansion coefficient, $\Lambda^{0}$ \\
\hline $\bar{T}_{1}$ & 0.973 & $A_{\text {phys }}$ expansion coefficient, $\Lambda^{1}$ \\
\hline $\bar{T}_{2}$ & 0.081 & $A_{\text {phys }}$ expansion coefficient, $\Lambda^{2}$ \\
\hline $\bar{T}_{3}$ & -1.215 & $A_{\text {phys }}$ expansion coefficient, $\Lambda^{3}$ \\
\hline $\bar{T}_{4}$ & -0.104 & $A_{\text {phys }}$ expansion coefficient, $\Lambda^{4}$ \\
\hline $\bar{T}_{5}$ & 1.544 & $A_{\text {phys }}$ expansion coefficient, $\Lambda^{5}$ \\
\hline$\sum_{i=0}^{5} \bar{T}_{i} \cdot \Lambda^{i}$ & $0.195 \pm 0.077$ & Finite acceptance model, Eqn 5.76 \\
\hline$P_{\text {beam }}$ & $0.852 \pm 0.030$ & Beam polarization \\
\hline$P_{3} \mathrm{He}$ & $0.470 \pm 0.022$ & Target polarization \\
\hline$P_{\mathrm{p}}$ & $-0.050 \pm 0.025$ & Proton polarization in ${ }^{3} \mathrm{He}$ nucleus \\
\hline$D_{\mathrm{bk}}$ & $0.970 \pm 0.017$ & Background dilution \\
\hline$D_{\mathrm{N}_{2}}$ & $0.949 \pm 0.002$ & Nitrogen dilution \\
\hline$D_{\mathrm{p}}$ & $0.779 \pm 0.012$ & Proton dilution \\
\hline$\frac{\Delta_{p}}{\Sigma}$ & $-0.0008 \pm 0.0005$ & Proton asymmetry correction \\
\hline
\end{tabular}

Table C.5: The results of the proton asymmetry correction for $Q^{2}=1.72 \mathrm{GeV}^{2}$. 


\begin{tabular}{cccl}
\hline Parameter & Neutral & Charged & Description \\
\hline$C_{\pi}$ & $0.837 \pm 0.033$ & $11.2 \pm 0.1$ & Sim. $\pi^{-}$normalization \\
$C_{e}$ & $0.972 \pm 0.002$ & $7.94 \pm 0.01$ & Sim. $e^{-}$normalization \\
$\operatorname{cov}\left(C_{\pi}, C_{e}\right)$ & negligible & negligible & Covariance b/t normalizations \\
$N_{\pi}$ & $756 \pm 28$ & $756 \pm 28$ & Sim. $\pi^{-}$count prior to scaling \\
$N_{e}$ & $1.592 \times 10^{5} \pm 400$ & $1.592 \times 10^{5} \pm 400$ & Sim. $e^{-}$count prior to scaling \\
$D_{\pi}$ & $0.996 \pm 0.0002$ & $0.993 \pm 0.0002$ & Dilution factor \\
\hline
\end{tabular}

Table C.6: Results of the BigBite preshower pion dilution factor analysis.

\begin{tabular}{ccccc}
\hline Parameter & Neutral & \multicolumn{2}{c}{ Charged } \\
\hline Helicity & + & - & + & - \\
$C_{\pi}$ & $0.372 \pm 0.022$ & $0.465 \pm 0.024$ & $4.83 \pm 0.075$ & $6.33 \pm 0.083$ \\
$C_{e}$ & $0.459 \pm 0.002$ & $0.513 \pm 0.002$ & $3.96 \pm 0.01$ & $3.97 \pm 0.01$ \\
$N_{\pi}$ & & $756 \pm 28$ & $756 \pm 28$ \\
$N_{e}$ & $1.592 \times 10^{5} \pm 400$ & $1.592 \times 10^{5} \pm 400$ \\
$\Sigma_{\text {sim }}$ & $1.551 \times 10^{5} \pm 400$ & $1.271 \times 10^{6} \pm 1130$ \\
$A_{\pi}$ & $-0.0005 \pm 0.0002$ & $-0.0009 \pm \mathcal{O}\left(10^{-5}\right)$ \\
\hline
\end{tabular}

Table C.7: Results of the BigBite preshower pion asymmetry analysis where the simulated count is $\Sigma_{\text {sim }}=\left(C_{\pi}^{+}+C_{\pi}^{-}\right) N_{\pi}+\left(C_{e}^{+}+C_{e}^{-}\right) N_{e}$. 


\begin{tabular}{ccll}
\hline Parameter & Neutral & Charged & Description \\
\hline$D_{\text {in }}$ & $0.979 \pm 0.200$ & $0.991 \pm 0.040$ & Dilution factor \\
$A_{\text {in }}$ & $-0.002 \pm 0.018$ & $-0.001 \pm 0.029$ & Asymmetry correction \\
\hline
\end{tabular}

Table C.8: Results of the inelastic contamination utilizing MAID and G4SBS.

\begin{tabular}{ccccc}
\hline Factor $f$ & $\sum_{i} \sigma_{i}^{+}$ & $\sum_{i} \sigma_{i}^{-}$ & $\boldsymbol{A}$ & $\boldsymbol{A}_{\text {free }}$ \\
\hline \hline 0.0 & 6964.4 & 7824.1 & $-0.058 \pm 0.001$ & -0.064 \\
0.2 & 6692.4 & 7957.7 & $-0.086 \pm 0.001$ & -0.094 \\
0.4 & 6428.9 & 8157.6 & $-0.119 \pm 0.001$ & -0.127 \\
0.6 & 6232.1 & 8346.3 & $-0.145 \pm 0.001$ & -0.155 \\
0.8 & 6018.5 & 8579.1 & $-0.175 \pm 0.001$ & -0.185 \\
1.0 & 5727.1 & 8618.3 & $-0.202 \pm 0.001$ & -0.212 \\
1.2 & 5676.8 & 9025.0 & $-0.228 \pm 0.001$ & -0.239 \\
1.4 & 6101.9 & 10254.2 & $-0.254 \pm 0.001$ & -0.264 \\
1.6 & 5662.7 & 9980.3 & $-0.276 \pm 0.001$ & -0.289 \\
1.8 & 5413.0 & 10000.7 & $-0.298 \pm 0.001$ & -0.312 \\
2.0 & 5474.4 & 10617.1 & $-0.320 \pm 0.001$ & -0.334 \\
\hline
\end{tabular}

Table C.9: The results of the FSI simulation in tabular form: $D_{\mathrm{FSI}}=0.9721 \pm 0.0034$ and $A_{\mathrm{FSI}}=0.0047 \pm 0.0008$. Also, there is a systematic of $2 \%$ in $D_{\mathrm{FSI}}$. 
C.2 Kinematic 2: $Q^{2}=2.48 \mathrm{GeV}^{2}$

\begin{tabular}{|c|c|c|c|c|c|}
\hline \multirow[t]{2}{*}{ Parameter } & \multicolumn{2}{|c|}{ Neutral } & \multicolumn{2}{|c|}{ Charged } & \multirow{2}{*}{$\begin{array}{l}\text { Description } \\
\text { Helicity }\end{array}$} \\
\hline & + & - & + & - & \\
\hline$N$ & 20,688 & 22,829 & 221,242 & 221,532 & QE count \\
\hline$N_{\mathrm{bk}}$ & 811 & 839 & 58 & 95 & Background count \\
\hline$D_{\mathrm{bk}}$ & \multicolumn{2}{|c|}{$0.981 \pm 0.011$} & \multicolumn{2}{|c|}{$0.997 \pm 0.001$} & Background dilution \\
\hline$\sigma_{\text {stat }}$ & \multicolumn{2}{|c|}{0.0005} & \multicolumn{2}{|c|}{ negligible } & Statistical \\
\hline$\sigma_{\mathrm{sys}}$ & \multicolumn{2}{|c|}{0.011} & \multicolumn{2}{|c|}{0.001} & Systematic \\
\hline$A_{\text {raw,bk }}$ & \multicolumn{2}{|c|}{$-0.017 \pm 0.025$} & \multicolumn{2}{|c|}{$-0.242 \pm 0.078$} & Background raw asymmetry \\
\hline$A_{\mathrm{bk}}$ & \multicolumn{2}{|c|}{$-0.0006 \pm 0.0006$} & \multicolumn{2}{|c|}{ negligible } & Background asymmetry $\frac{\Delta_{\mathrm{bk}}}{\Sigma}$ \\
\hline
\end{tabular}

Table C.10: Background corrections for the $Q^{2}=2.48 \mathrm{GeV}^{2}$ configuration. 


\begin{tabular}{cccl}
\hline Parameter & Unit & Value & Description \\
\hline$\rho_{\mathrm{N}_{2}}\left({ }^{3} \mathrm{He}\right)$ & amg & 0.162 & $\mathrm{~N}_{2}$ density within the ${ }^{3} \mathrm{He}$ cell \\
$\rho_{\mathrm{N}_{2}}\left(\mathrm{~N}_{2}\right)$ & amg & 5.0 & $\mathrm{~N}_{2}$ density \\
$\delta\left(\rho_{\mathrm{N}_{2}}\left({ }^{3} \mathrm{He}\right)\right)_{\text {rel. }}$ & $\mathrm{N} / \mathrm{A}$ & 0.007 & Relative error in $\rho_{\mathrm{N}_{2}}\left({ }^{3} \mathrm{He}\right)$ \\
$\delta\left(\rho_{\mathrm{N}_{2}}\left(\mathrm{~N}_{2}\right)\right)_{\mathrm{rel} .}$ & $\mathrm{N} / \mathrm{A}$ & 0.007 & Relative error in $\rho_{\mathrm{N}_{2}}\left(\mathrm{~N}_{2}\right)$ \\
$Q\left({ }^{3} \mathrm{He}\right)$ & $\mathrm{mC}$ & 137.1 & Accum. beam charge for ${ }^{3} \mathrm{He}$ runs \\
$Q\left(\mathrm{~N}_{2}\right)$ & $\mathrm{mC}$ & 3.1 & Accum. beam charge for $\mathrm{N}_{2}$ runs \\
\hline
\end{tabular}

Table C.11: Common parameters used in the neutral/charged calculation of $D_{\mathrm{N}_{2}}$. Recall that $1 \mathrm{amg}=44.615 \mathrm{~mol} / \mathrm{m}^{3}$.

\begin{tabular}{cccl}
\hline Parameter & Neutral & Charged & Description \\
\hline$N$ & 3,137 & 33,476 & ${ }^{3} \mathrm{He}$ QE count \\
$N_{\mathrm{bk}}$ & 126 & 10 & ${ }^{3} \mathrm{He}$ QE background count \\
$N\left(\mathrm{~N}_{2}\right)$ & 966 & 5,203 & $\mathrm{~N}_{2} \mathrm{QE} \mathrm{count}$ \\
$N_{\mathrm{bk}}\left(\mathrm{N}_{2}\right)$ & 27 & 3 & $\mathrm{~N}_{2}$ QE background count \\
$D_{\mathrm{N}_{2}}$ & $0.950 \pm 0.002$ & $0.9750 \pm 0.0004$ & Nitrogen dilution factor \\
\hline
\end{tabular}

Table C.12: The results of the nitrogen correction for the neutral/charged QE samples. 


\begin{tabular}{|c|c|c|}
\hline Parameter & Value & Description \\
\hline V1 Rate $(\mathrm{kHz})$ & 322 & Average V1 rate, weighted by counts \\
\hline V2 Rate $(\mathrm{kHz})$ & 237 & Average V2 rate, weighted by counts \\
\hline$R_{\mathrm{N}_{2}}$ & $0.179 \pm 0.001$ & Uncharged-to-charged ratio for $\mathrm{N}_{2}$ \\
\hline$R_{\mathrm{H}_{2}}$ & $0.020 \pm 0.002$ & Uncharged-to-charged ratio for $\mathrm{H}_{2}$ \\
\hline$R_{3} \mathrm{He}$ & $0.094 \pm 0.001$ & Uncharged-to-charged ratio for ${ }^{3} \mathrm{He}$ \\
\hline$f_{\mathrm{N}_{2}}$ & $1 / 0.9$ & Effective ratio of neutrons to protons \\
\hline$f^{f_{3} \mathrm{He}}$ & $1 / 2.15$ & Effective ratio of neutrons to protons \\
\hline$\sigma_{n} / \sigma_{p}$ & 0.40 & Nucleon x-section ratio, $Q^{2}=2.48 \mathrm{GeV}^{2}$ \\
\hline$\frac{\eta_{p}^{n}}{\eta_{p}^{p}}$ & $0.020 \pm 0.002$ & Protons observed as neutrons \\
\hline$\frac{\eta_{n}^{p}}{\eta_{p}^{p}}$ & $0.466 \pm 0.122$ & Neutrons observed as protons \\
\hline$\frac{\eta_{n}^{n}}{\eta_{p}^{p}}$ & $0.437 \pm 0.024$ & Neutrons observed as neutrons \\
\hline$D_{\mathrm{n}, \mathrm{ch}}$ & $0.919 \pm 0.019$ & Neutron dilution factor \\
\hline$D_{\mathrm{p}}$ & $0.802 \pm 0.024$ & Proton dilution factor, needed for $A_{\text {phys }}$ \\
\hline
\end{tabular}

Table C.13: The dilution factors due to charge misidentification for $Q^{2}=2.48 \mathrm{GeV}^{2}$. 


\begin{tabular}{|c|c|c|}
\hline Parameter & Value & Description \\
\hline$Q^{2}\left(\mathrm{GeV}^{2}\right)$ & 2.48 & Acceptance averaged value of $Q^{2}$ \\
\hline$G_{E}^{p}$ & $0.038 \pm 0.019$ & Parametrization from Section 3.2.4 \\
\hline$G_{M}^{p}$ & $0.150 \pm 0.020$ & Parametrization from Section 3.2.4 \\
\hline$\Lambda$ & $0.252 \pm 0.133$ & Proton form factor ratio \\
\hline $\bar{T}_{0}$ & -0.011 & $A_{\text {phys }}$ expansion coefficient, $\Lambda^{0}$ \\
\hline $\bar{T}_{1}$ & 0.823 & $A_{\text {phys }}$ expansion coefficient, $\Lambda^{1}$ \\
\hline $\bar{T}_{2}$ & 0.010 & $A_{\text {phys }}$ expansion coefficient, $\Lambda^{2}$ \\
\hline $\bar{T}_{3}$ & -0.677 & $A_{\text {phys }}$ expansion coefficient, $\Lambda^{3}$ \\
\hline $\bar{T}_{4}$ & -0.009 & $A_{\text {phys }}$ expansion coefficient, $\Lambda^{4}$ \\
\hline $\bar{T}_{5}$ & 0.567 & $A_{\text {phys }}$ expansion coefficient, $\Lambda^{5}$ \\
\hline$\sum_{i=0}^{5} \bar{T}_{i} \cdot \Lambda^{i}$ & $0.186 \pm 0.095$ & Finite acceptance model, Eqn 5.76 \\
\hline$P_{\text {beam }}$ & $0.850 \pm 0.021$ & Beam polarization \\
\hline$P_{3} \mathrm{He}$ & $0.439 \pm 0.020$ & Target polarization \\
\hline$P_{\mathrm{p}}$ & $-0.044 \pm 0.037$ & Proton polarization in ${ }^{3} \mathrm{He}$ nucleus \\
\hline$D_{\mathrm{bk}}$ & $0.981 \pm 0.011$ & Background dilution \\
\hline$D_{\mathrm{N}_{2}}$ & $0.950 \pm 0.002$ & Nitrogen dilution \\
\hline$D_{\mathrm{p}}$ & $0.802 \pm 0.024$ & Proton dilution \\
\hline$\frac{\Delta_{\mathrm{p}}}{\Sigma}$ & $-0.0006 \pm 0.0006$ & Proton asymmetry correction \\
\hline
\end{tabular}

Table C.14: The results of the proton asymmetry correction for $Q^{2}=2.48 \mathrm{GeV}^{2}$. 


\begin{tabular}{ccll}
\hline Parameter & Neutral & Charged & Description \\
\hline$C_{\pi}$ & $0.920 \pm 0.060$ & $16.18 \pm 0.23$ & Sim. $\pi^{-}$normalization \\
$C_{e}$ & $1.010 \pm 0.005$ & $10.28 \pm 0.02$ & Sim. $e^{-}$normalization \\
$\operatorname{cov}\left(C_{\pi}, C_{e}\right)$ & $-\mathcal{O}\left(10^{-5}\right)$ & $-\mathcal{O}\left(10^{-4}\right)$ & Covariance b/t normalizations \\
$N_{\pi}$ & $231 \pm 15$ & $231 \pm 15$ & Sim. $\pi^{-}$count prior to scaling \\
$N_{e}$ & $4.30 \times 10^{4} \pm 200$ & $4.30 \times 10^{4} \pm 200$ & Sim. $e^{-}$count prior to scaling \\
$D_{\pi}$ & $0.995 \pm 0.0005$ & $0.992 \pm 0.0005$ & Dilution factor \\
\hline
\end{tabular}

Table C.15: Results of the BigBite preshower pion dilution factor analysis.

\begin{tabular}{ccccc}
\hline Parameter & \multicolumn{2}{c}{ Neutral } & \multicolumn{2}{c}{ Charged } \\
\hline Helicity & + & - & + & - \\
$C_{\pi}$ & $0.491 \pm 0.043$ & $0.428 \pm 0.042$ & $8.48 \pm 0.17$ & $7.70 \pm 0.16$ \\
$C_{e}$ & $0.479 \pm 0.003$ & $0.529 \pm 0.004$ & $5.13 \pm 0.01$ & $5.15 \pm 0.01$ \\
\hline$N_{\pi}$ & $231 \pm 15$ & $231 \pm 15$ \\
$N_{e}$ & $4.30 \times 10^{4} \pm 200$ & $4.30 \times 10^{4} \pm 200$ \\
$A_{\pi}$ & $0.0003 \pm 0.0003$ & $0.0004 \pm 0.0001$ \\
\hline
\end{tabular}

Table C.16: Results of the BigBite preshower pion asymmetry analysis. 


\begin{tabular}{cccl}
\hline Parameter & Neutral & Charged & Description \\
\hline$D_{\text {in }}$ & $0.970 \pm 0.220$ & $0.986 \pm 0.010$ & Dilution factor \\
$A_{\text {in }}$ & $-0.009 \pm 0.261$ & $-0.0006 \pm 0.0292$ & Asymmetry correction \\
\hline
\end{tabular}

Table C.17: Results of the inelastic contamination.

\begin{tabular}{|c|c|c|c|c|}
\hline Factor $f$ & $\sum_{i} \sigma_{i}^{+}$ & $\sum_{i} \sigma_{i}^{-}$ & $\boldsymbol{A}$ & $A_{\text {free }}$ \\
\hline 0.0 & 5832.4 & 5917.2 & $-0.007 \pm 0.001$ & -0.010 \\
\hline 0.2 & 5778.0 & 6237.0 & $-0.038 \pm 0.001$ & -0.041 \\
\hline 0.4 & 5469.1 & 6268.9 & $-0.068 \pm 0.001$ & -0.071 \\
\hline 0.6 & 5464.3 & 6641.5 & $-0.097 \pm 0.001$ & -0.102 \\
\hline 0.8 & 5274.2 & 6799.8 & $-0.126 \pm 0.001$ & -0.131 \\
\hline 1.0 & 4965.4 & 6803.0 & $-0.156 \pm 0.001$ & -0.160 \\
\hline 1.2 & 5064.6 & 7318.7 & $-0.182 \pm 0.001$ & -0.188 \\
\hline 1.4 & 5010.2 & 7643.0 & $-0.208 \pm 0.001$ & -0.215 \\
\hline 1.6 & 4981.8 & 8010.7 & $-0.233 \pm 0.001$ & -0.240 \\
\hline 1.8 & 4806.7 & 8125.4 & $-0.257 \pm 0.001$ & -0.265 \\
\hline 2.0 & 4759.5 & 8438.1 & $-0.279 \pm 0.001$ & -0.288 \\
\hline
\end{tabular}

Table C.18: The results of the FSI simulation in tabular form: $D_{\mathrm{FSI}}=0.9750 \pm 0.0019$ and $A_{\mathrm{FSI}}=0.0016 \pm 0.0004$. There is a systematic of $2 \%$ in $D_{\mathrm{FSI}}$. 
C.3 Kinematic 3: $Q^{2}=3.41 \mathrm{GeV}^{2}$

\begin{tabular}{|c|c|c|c|c|c|}
\hline \multirow[t]{2}{*}{ Parameter } & \multicolumn{2}{|c|}{ Neutral } & \multicolumn{2}{|c|}{ Charged } & \multirow{2}{*}{$\begin{array}{l}\text { Description } \\
\text { Helicity }\end{array}$} \\
\hline & + & - & + & - & \\
\hline$N$ & 32,564 & 35,039 & 413,035 & 414,534 & QE count \\
\hline$N_{\mathrm{bk}}$ & 1,651 & 1,688 & 273 & 245 & Background count \\
\hline$D_{\mathrm{bk}}$ & \multicolumn{2}{|c|}{$0.975 \pm 0.014$} & \multicolumn{2}{|c|}{$0.997 \pm 0.001$} & Background dilution \\
\hline$\sigma_{\text {stat }}$ & \multicolumn{2}{|c|}{0.0004} & \multicolumn{2}{|c|}{ negligible } & Statistical \\
\hline$\sigma_{\mathrm{sys}}$ & \multicolumn{2}{|c|}{0.014} & \multicolumn{2}{|c|}{0.001} & Systematic \\
\hline$A_{\text {raw,bk }}$ & \multicolumn{2}{|c|}{$-0.011 \pm 0.017$} & \multicolumn{2}{|c|}{$0.054 \pm 0.044$} & Background raw asymmetry \\
\hline$A_{\mathrm{bk}}$ & \multicolumn{2}{|c|}{$-0.0006 \pm 0.0006$} & \multicolumn{2}{|c|}{ negligible } & Background asymmetry $\frac{\Delta_{\mathrm{bk}}}{\Sigma}$ \\
\hline
\end{tabular}

Table C.19: Background corrections for the $Q^{2}=3.41 \mathrm{GeV}^{2}$ configuration. 


\begin{tabular}{cccl}
\hline Parameter & Unit & Value & Description \\
\hline$\rho_{\mathrm{N}_{2}}\left({ }^{3} \mathrm{He}\right)$ & amg & 0.162 & $\mathrm{~N}_{2}$ density within the ${ }^{3} \mathrm{He}$ cell \\
$\rho_{\mathrm{N}_{2}}\left(\mathrm{~N}_{2}\right)$ & amg & 4.405 & $\mathrm{~N}_{2}$ density \\
$\delta\left(\rho_{\mathrm{N}_{2}}\left({ }^{3} \mathrm{He}\right)\right)_{\mathrm{rel}}$. & $\mathrm{N} / \mathrm{A}$ & 0.007 & Relative error in $\rho_{\mathrm{N}_{2}}\left({ }^{3} \mathrm{He}\right)$ \\
$\delta\left(\rho_{\mathrm{N}_{2}}\left(\mathrm{~N}_{2}\right)\right)_{\mathrm{rel} .}$ & $\mathrm{N} / \mathrm{A}$ & 0.007 & Relative error in $\rho_{\mathrm{N}_{2}}\left(\mathrm{~N}_{2}\right)$ \\
$Q\left({ }^{3} \mathrm{He}\right)$ & $\mathrm{mC}$ & 113.8 & Accum. beam charge for ${ }^{3} \mathrm{He}$ runs \\
$Q\left(\mathrm{~N}_{2}\right)$ & $\mathrm{mC}$ & 23.2 & Accum. beam charge for $\mathrm{N}_{2}$ runs \\
\hline
\end{tabular}

Table C.20: Common parameters used in the neutral/charged calculation of $D_{\mathrm{N}_{2}}$. Recall that $1 \mathrm{amg}=44.615 \mathrm{~mol} / \mathrm{m}^{3}$.

\begin{tabular}{cccl}
\hline Parameter & Neutral & Charged & Description \\
\hline$N$ & 652 & 7,957 & ${ }^{3} \mathrm{He}$ QE count \\
$N_{\mathrm{bk}}$ & 43 & 2 & ${ }^{3} \mathrm{He}$ QE background count \\
$N\left(\mathrm{~N}_{2}\right)$ & 292 & 2,022 & $\mathrm{~N}_{2} \mathrm{QE}$ count \\
$N_{\mathrm{bk}}\left(\mathrm{N}_{2}\right)$ & 31 & 0 & $\mathrm{~N}_{2} \mathrm{QE}$ background count \\
$D_{\mathrm{N}_{2}}$ & $0.921 \pm 0.007$ & $0.954 \pm 0.001$ & Nitrogen dilution factor \\
\hline
\end{tabular}

Table C.21: The results of the nitrogen correction for the neutral/charged QE samples. 


\begin{tabular}{ccl}
\hline Parameter & Value & Description \\
\hline V1 Rate $(\mathrm{kHz})$ & \multicolumn{1}{c}{559} & Average V1 rate, weighted by counts \\
V2 Rate $(\mathrm{kHz})$ & \multicolumn{1}{c}{412} & Average V2 rate, weighted by counts \\
$R_{\mathrm{N}_{2}}$ & $0.128 \pm 0.001$ & Uncharged-to-charged ratio for $\mathrm{N}_{2}$ \\
$R_{\mathrm{H}_{2}}$ & $0.019 \pm 0.002$ & Uncharged-to-charged ratio for $\mathrm{H}_{2}$ \\
$R_{3} \mathrm{He}$ & $0.079 \pm 0.001$ & Uncharged-to-charged ratio for ${ }^{3} \mathrm{He}$ \\
\hline$f_{\mathrm{N}_{2}}$ & $1 / 1.07$ & Effective ratio of neutrons to protons \\
$f_{3 \mathrm{He}}$ & $1 / 2.43$ & Effective ratio of neutrons to protons \\
$\sigma_{n} / \sigma_{p}$ & 0.40 & Nucleon x-section ratio, $Q^{2}=3.41 \mathrm{GeV}^{2}$ \\
$\frac{\eta_{p}^{n}}{\eta_{p}^{p}}$ & $0.019 \pm 0.002$ & Protons observed as neutrons \\
$\frac{\eta_{n}^{p}}{\eta_{p}^{p}}$ & undetermined & Neutrons observed as protons \\
$\frac{\eta_{n}^{n}}{\eta_{p}^{p}}$ & $0.475 \pm 0.034$ & Neutrons observed as neutrons \\
\hline$D_{\mathrm{n}, \mathrm{ch}}$ & undetermined & Neutron dilution factor \\
\hline$D_{\mathrm{p}}$ & $0.806 \pm 0.029$ & Proton dilution factor, needed for $A_{\mathrm{phys}}$ \\
\hline & & \\
\hline
\end{tabular}

Table C.22: The dilution factors due to charge misidentification for $Q^{2}=3.41 \mathrm{GeV}^{2}$. 


\begin{tabular}{|c|c|c|}
\hline Parameter & Value & Description \\
\hline$Q^{2}\left(\mathrm{GeV}^{2}\right)$ & 3.41 & Acceptance averaged value of $Q^{2}$ \\
\hline$G_{E}^{p}$ & $0.019 \pm 0.015$ & Parametrization from Section 3.2.4 \\
\hline$G_{M}^{p}$ & $0.089 \pm 0.015$ & Parametrization from Section 3.2.4 \\
\hline$\Lambda$ & $0.209 \pm 0.172$ & Proton form factor ratio \\
\hline $\bar{T}_{0}$ & 0.046 & $A_{\text {phys }}$ expansion coefficient, $\Lambda^{0}$ \\
\hline $\bar{T}_{1}$ & 0.705 & $A_{\text {phys }}$ expansion coefficient, $\Lambda^{1}$ \\
\hline $\bar{T}_{2}$ & -0.025 & $A_{\text {phys }}$ expansion coefficient, $\Lambda^{2}$ \\
\hline $\bar{T}_{3}$ & -0.395 & $A_{\text {phys }}$ expansion coefficient, $\Lambda^{3}$ \\
\hline $\bar{T}_{4}$ & 0.014 & $A_{\text {phys }}$ expansion coefficient, $\Lambda^{4}$ \\
\hline $\bar{T}_{5}$ & 0.225 & $A_{\text {phys }}$ expansion coefficient, $\Lambda^{5}$ \\
\hline$\sum_{i=0}^{5} \bar{T}_{i} \cdot \Lambda^{i}$ & $0.188 \pm 0.111$ & Finite acceptance model, Eqn 5.76 \\
\hline$P_{\text {beam }}$ & $0.829 \pm 0.022$ & Beam polarization \\
\hline$P_{3} \mathrm{He}$ & $0.462 \pm 0.021$ & Target polarization \\
\hline$P_{\mathrm{p}}$ & $-0.035 \pm 0.030$ & Proton polarization in ${ }^{3} \mathrm{He}$ nucleus \\
\hline$D_{\mathrm{bk}}$ & $0.975 \pm 0.014$ & Background dilution \\
\hline$D_{\mathrm{N}_{2}}$ & $0.921 \pm 0.007$ & Nitrogen dilution \\
\hline$D_{\mathrm{p}}$ & $0.806 \pm 0.029$ & Proton dilution \\
\hline$\frac{\Delta_{p}}{\Sigma}$ & $-0.0004 \pm 0.0005$ & Proton asymmetry correction \\
\hline
\end{tabular}

Table C.23: The results of the proton asymmetry correction for $Q^{2}=3.41 \mathrm{GeV}^{2}$. 


\begin{tabular}{ccll}
\hline Parameter & Neutral & Charged & Description \\
\hline$C_{\pi}$ & $0.982 \pm 0.052$ & $24.50 \pm 0.24$ & Sim. $\pi^{-}$normalization \\
$C_{e}$ & $0.946 \pm 0.005$ & $11.51 \pm 0.02$ & Sim. $e^{-}$normalization \\
$\operatorname{cov}\left(C_{\pi}, C_{e}\right)$ & $-\mathcal{O}\left(10^{-5}\right)$ & $-\mathcal{O}\left(10^{-4}\right)$ & Covariance b/t normalizations \\
$N_{\pi}$ & $338 \pm 18$ & $338 \pm 18$ & Sim. $\pi^{-}$count prior to scaling \\
$N_{e}$ & $3.73 \times 10^{4} \pm 200$ & $3.73 \times 10^{4} \pm 200$ & Sim. $e^{-}$count prior to scaling \\
$D_{\pi}$ & $0.991 \pm 0.0007$ & $0.981 \pm 0.001$ & Dilution factor \\
\hline
\end{tabular}

Table C.24: Results of the BigBite preshower pion dilution factor analysis.

\begin{tabular}{ccccc}
\hline Parameter & \multicolumn{2}{c}{ Neutral } & \multicolumn{2}{c}{ Charged } \\
\hline Helicity & + & - & + & - \\
$C_{\pi}$ & $0.473 \pm 0.036$ & $0.485 \pm 0.037$ & $13.78 \pm 0.18$ & $10.71 \pm 0.16$ \\
$C_{e}$ & $0.453 \pm 0.004$ & $0.489 \pm 0.004$ & $5.73 \pm 0.01$ & $5.77 \pm 0.01$ \\
\hline$N_{\pi}$ & $338 \pm 18$ & $338 \pm 18$ \\
$N_{e}$ & $3.73 \times 10^{4} \pm 200$ & $3.73 \times 10^{4} \pm 200$ \\
$A_{\pi}$ & $-0.0001 \pm 0.0005$ & $0.0024 \pm 0.0002$ \\
\hline
\end{tabular}

Table C.25: Results of the BigBite preshower pion asymmetry analysis. 


\begin{tabular}{ccll}
\hline Parameter & Neutral & Charged & Description \\
\hline$D_{\text {in }}$ & $0.891 \pm 0.259$ & $0.956 \pm 0.010$ & Dilution factor \\
$A_{\text {in }}$ & $-0.0167 \pm 0.0150$ & $-0.003 \pm 0.022$ & Asymmetry correction \\
\hline
\end{tabular}

Table C.26: Results of the inelastic contamination.

\begin{tabular}{ccccc}
\hline Factor $f$ & $\sum_{i} \sigma_{i}^{+}$ & $\sum_{i} \sigma_{i}^{-}$ & $A$ & $A_{\text {free }}$ \\
\hline \hline 0.0 & 1797.0 & 1662.2 & $0.039 \pm 0.001$ & 0.045 \\
0.2 & 1691.6 & 1665.9 & $0.008 \pm 0.001$ & 0.014 \\
0.4 & 1753.6 & 1831.6 & $-0.022 \pm 0.001$ & -0.017 \\
0.6 & 1617.5 & 1786.8 & $-0.050 \pm 0.001$ & -0.046 \\
0.8 & 1610.2 & 1888.2 & $-0.079 \pm 0.001$ & -0.076 \\
1.0 & 1555.3 & 1935.7 & $-0.109 \pm 0.001$ & -0.106 \\
1.2 & 1532.4 & 2011.3 & $-0.135 \pm 0.001$ & -0.134 \\
1.4 & 1482.7 & 2053.9 & $-0.162 \pm 0.001$ & -0.161 \\
1.6 & 1537.5 & 2239.5 & $-0.186 \pm 0.001$ & -0.187 \\
1.8 & 1412.0 & 2172.0 & $-0.212 \pm 0.001$ & -0.213 \\
& & & & \\
2.0 & 1501.7 & 2415.6 & $-0.233 \pm 0.001$ & -0.237 \\
\hline
\end{tabular}

Table C.27: The results of the FSI simulation in tabular form: $D_{\mathrm{FSI}}=0.9668 \pm 0.0024$ and $A_{\mathrm{FSI}}=-0.0054 \pm 0.0003$. There is a systematic of $2 \%$ in $D_{\mathrm{FSI}}$. 


\begin{tabular}{|c|c|c|c|}
\hline Par. & Kin. 4 & Kin. 2 & Kin. 3 \\
\hline$P_{\text {beam }}$ & $0.852 \pm 0.030$ & $0.850 \pm 0.021$ & $0.829 \pm 0.022$ \\
\hline$P_{3} \mathrm{He}$ & $0.470 \pm 0.022$ & $0.439 \pm 0.020$ & $0.462 \pm 0.021$ \\
\hline$D_{\mathrm{bk}}$ & $0.970 \pm 0.017$ & $0.981 \pm 0.011$ & $0.975 \pm 0.014$ \\
\hline$D_{\mathrm{N}_{2}}$ & $0.949 \pm 0.002$ & $0.950 \pm 0.002$ & $0.921 \pm 0.007$ \\
\hline$D_{\mathrm{p}}$ & $0.779 \pm 0.012$ & $0.802 \pm 0.024$ & $0.806 \pm 0.029$ \\
\hline$D_{\pi}$ & $0.996 \pm 0.000$ & $0.995 \pm 0.001$ & $0.991 \pm 0.001$ \\
\hline$D_{\text {in }}$ & $0.979 \pm 0.200$ & $0.970 \pm 0.220$ & $0.891 \pm 0.259$ \\
\hline$D_{\mathrm{FSI}}$ & $0.972 \pm 0.020$ & $0.975 \pm 0.020$ & $0.967 \pm 0.020$ \\
\hline$A_{\mathrm{bk}}$ & $-0.0001 \pm 0.0004$ & $-0.0006 \pm 0.0006$ & $-0.0006 \pm 0.0006$ \\
\hline$A_{\mathrm{p}}$ & $-0.0008 \pm 0.0005$ & $-0.0006 \pm 0.0006$ & $-0.0004 \pm 0.0005$ \\
\hline$A_{\pi}$ & $-0.0005 \pm 0.0002$ & $0.0003 \pm 0.0003$ & $-0.0001 \pm 0.0005$ \\
\hline$A_{\text {in }}$ & $-0.0022 \pm 0.0181$ & $-0.0094 \pm 0.0261$ & $-0.0167 \pm 0.0150$ \\
\hline$A_{\mathrm{FSI}}$ & $0.0047 \pm 0.0008$ & $0.0016 \pm 0.0004$ & $-0.0054 \pm 0.0003$ \\
\hline$N_{\mathrm{qe}}$ & 157,348 & 44,043 & 36,217 \\
\hline$A_{\text {raw }}$ & $-0.054 \pm 0.003$ & $-0.048 \pm 0.005$ & $-0.036 \pm 0.005$ \\
\hline$A_{\text {phys }}$ & $-0.197 \pm 0.009 \pm 0.013$ & $-0.177 \pm 0.018 \pm 0.013$ & $-0.124 \pm 0.022 \pm 0.013$ \\
\hline$G_{E}^{n} / G_{M}^{n}$ & $-0.141 \pm 0.010 \pm 0.015$ & $-0.209 \pm 0.024 \pm 0.017$ & $-0.247 \pm 0.034 \pm 0.020$ \\
\hline$G_{M}^{n}$ & $-0.165 \pm 0.003$ & $-0.097 \pm 0.002$ & $-0.057 \pm 0.002$ \\
\hline$G_{E}^{n}$ & $0.023 \pm 0.002 \pm 0.003$ & $0.020 \pm 0.002 \pm 0.002$ & $0.014 \pm 0.002 \pm 0.001$ \\
\hline
\end{tabular}

Table C.28: A summary of the reanalysis. 


\section{References}

[1] S. Weinberg, The Discovery of Subatomic Particles (Cambridge University Press, 2003).

[2] O. Stern, Nature 132:169 (1933).

[3] L. W. Alvarez and F. Bloch, Phys. Rev. 57, 111 (1940).

[4] S. J. Brodsky and S. D. Drell, Phys. Rev. D22, 2236 (1980).

[5] D. Griffiths, Introduction to Elementary Particles (Wiley-VCH, 2008).

[6] K. A. Olive et al. (Particle Data Group), Chin. Phys. C38, 090001 (2014).

[7] S. Riordan et al., Phys. Rev. Lett. 105, 262302 (2010), arXiv:1008.1738 [nucl-ex] .

[8] M. Peskin and D. Schroeder, An Introduction to Quantum Field Theory (Westview Press, 1995).

[9] R. G. Sachs, Phys. Rev. 126, 2256 (1962).

[10] L. N. Hand, D. G. Miller, and R. Wilson, Rev. Mod. Phys. 35, 335 (1963).

[11] M. N. Rosenbluth, Phys. Rev. 79, 615 (1950).

[12] D. R. Yennie, M. M. Lévy, and D. G. Ravenhall, Rev. Mod. Phys. 29, 144 (1957).

[13] V. Punjabi et al., The European Physical Journal A 51, 79 (2015).

[14] R. Hofstadter, Rev. Mod. Phys. 28, 214 (1956).

[15] R. Hofstadter, H. R. Fechter, and J. A. McIntyre, Phys. Rev. 92, 978 (1953).

[16] B. Hahn, D. G. Ravenhall, and R. Hofstadter, Phys. Rev. 101, 1131 (1956).

[17] A. I. Akhiezer and M. Rekalo, Sov. Phys. Dokl. 13, 572 (1968), [Dokl. Akad. Nauk Ser. Fiz.180,1081(1968)].

[18] J. H. Scofield, Phys. Rev. 113, 1599 (1959).

[19] A. I. Akhiezer and M. Rekalo, Sov. J. Part. Nucl. 4, 277 (1974), [Fiz. Elem. Chast. Atom. Yadra4,662(1973)].

[20] N. Dombey, Rev. Mod. Phys. 41, 236 (1969).

[21] R. G. Arnold, C. E. Carlson, and F. Gross, Phys. Rev. C 23, 363 (1981).

[22] T. W. Donnelly and A. S. Raskin, Annals of Physics 169, 247 (1986). 
[23] A. S. Raskin and T. W. Donnelly, Annals Phys. 191, 78 (1989), [Erratum: Annals Phys.197,202(1990)].

[24] A. J. R. Puckett, Recoil Polarization Measurements of the Proton Electromagnetic Form Factor Ratio to High Momentum Transfer, Ph.D. thesis, Massachusetts Institute of Technology (2010).

[25] C. F. Perdrisat, V. Punjabi, and M. Vanderhaeghen, Prog. Part. Nucl. Phys. 59, 694 (2007), arXiv:hep-ph/0612014 [hep-ph].

[26] C. Patrignani et al. (Particle Data Group), Chin. Phys. C40, 100001 (2016),

[27] L. D. Faddeev, Mathematical Aspects of the Three-Body Problem (Daniel Davey and Co., Inc., New York, 1965).

[28] R. M. Woloshyn, Nucl. Phys. A496, 749 (1989).

[29] B. Blankleider and R. M. Woloshyn, Phys. Rev. C29, 538 (1984).

[30] J. L. Friar et al., Phys. Rev. C 42, 2310 (1990).

[31] C. Ciofi degli Atti, S. Scopetta, E. Pace, and G. Salme, Phys. Rev. C48, R968 (1993).

[32] C. Ciofi degli Atti and S. Scopetta, Phys. Lett. B404, 223 (1997)

[33] R. W. Schulze and P. U. Sauer, Phys. Rev. C56, 2293 (1997).

[34] F. R. P. Bissey, A. W. Thomas, and I. R. Afnan, Phys. Rev. C64, 024004 (2001).

[35] M. M. Sargsian, T. V. Abrahamyan, M. I. Strikman, and L. L. Frankfurt, Phys. Rev. C 71, 044614 (2005).

[36] R. J. Glauber, Phys. Rev. 100, 242 (1955).

[37] R. Hofstadter and R. W. McAllister, Phys. Rev. 98, 217 (1955).

[38] E. E. Chambers and R. Hofstadter, Phys. Rev. 103, 1454 (1956).

[39] M. R. Yearian and R. Hofstadter, Phys. Rev. 110, 552 (1958).

[40] M. K. Jones et al. (The Jefferson Lab Hall A Collaboration), Phys. Rev. Lett. 84, 1398 $(2000)$.

[41] T. Pospischil et al., The European Physical Journal A - Hadrons and Nuclei 12, 125 (2001).

[42] O. Gayou et al. (The Jefferson Lab Hall A Collaboration), Phys. Rev. C 64, 038202 (2001).

[43] O. Gayou et al. (Jefferson Lab Hall A), Phys. Rev. Lett. 88, 092301 (2002), arXiv:nuclex/0111010 [nucl-ex].

[44] S. Strauch et al., Phys. Rev. Lett. 91, 052301 (2003).

[45] V. Punjabi et al. (Jefferson Lab Hall A Collaboration), Phys. Rev. C 71, 055202 (2005).

[46] A. J. R. Puckett et al., Phys. Rev. Lett. 104, 242301 (2010).

[47] M. Paolone et al. (E03-104 Collaboration), Phys. Rev. Lett. 105, 072001 (2010).

[48] M. Meziane et al. (GEp2 $\gamma$ Collaboration), Phys. Rev. Lett. 106, 132501 (2011). 
[49] X. Zhan et al., Physics Letters B 705, 59 (2011).

[50] G. Ron et al. (The Jefferson Lab Hall A Collaboration), Phys. Rev. C 84, 055204 (2011).

[51] A. J. R. Puckett et al. (The Jefferson Lab Hall A Collaboration), Phys. Rev. C 85, 045203 $(2012)$.

[52] A. J. R. Puckett et al., Phys. Rev. C 96, 055203 (2017).

[53] B. R. Plaster, The Neutron Electric Form Factor to $Q^{2}=1.45(\mathrm{GeV} / \mathrm{c})^{2}$, Ph.D. thesis, Massachusetts Institute of Technology (2004).

[54] B. Plaster et al. (Jefferson Laboratory E93-038 Collaboration), Phys. Rev. C 73, 025205 (2006).

[55] I. A. Qattan et al., Phys. Rev. Lett. 94, 142301 (2005).

[56] W. Albrecht, H. J. Behrend, F. W. Brasse, W. Flauger, H. Hultschig, and K. G. Steffen, Phys. Rev. Lett. 17, 1192 (1966).

[57] M. Diehl and P. Kroll, The European Physical Journal C 73, 2397 (2013).

[58] C. Berger, V. Burkert, G. Knop, B. Langenbeck, and K. Rith, Physics Letters B 35, 87 (1971).

[59] L. E. Price, J. R. Dunning, M. Goitein, K. Hanson, T. Kirk, and R. Wilson, Phys. Rev. D 4, 45 (1971).

[60] W. Bartel et al., Nuclear Physics B 58, 429 (1973).

[61] F. Borkowski, G. G. Simon, V. H. Walther, and R. D. Wendling, Nuclear Physics B 93, 461 (1975).

[62] G. G. Simon, C. Schmitt, F. Borkowski, and V. H. Walther, Nuclear Physics A 333, 381 (1980).

[63] A. F. Sill et al., Phys. Rev. D 48, 29 (1993).

[64] L. Andivahis et al., Phys. Rev. D 50, 5491 (1994).

[65] R. C. Walker et al., Phys. Rev. D 49, 5671 (1994),

[66] M. E. Christy et al., Phys. Rev. C 70, 015206 (2004).

[67] J. Litt et al., Phys. Lett. 31B, 40 (1970).

[68] A. Lung et al., Phys. Rev. Lett. 70, 718 (1993).

[69] M. Gourdin, Nuovo Cimento 33, 533 (1963).

[70] S. Platchkov et al., Nuclear Physics A 510, 740 (1990).

[71] D. Benaksas, D. Drickey, and D. Frerejacque, Phys. Rev. 148, 1327 (1966).

[72] R. Schiavilla and I. Sick, Phys. Rev. C64, 041002 (2001), arXiv:nucl-ex/0107004 [nucl-ex].

[73] B. Grossetête, D. Drickey, and P. Lehmann, Phys. Rev. 141, 1425 (1966). 
[74] S. Galster, H. Klein, J. Moritz, K. H. Schmidt, D. Wegener, and J. Bleckwenn, Nucl. Phys. B32, 221 (1971).

[75] G. G. Simon et al., Nuclear Physics A 364, 285 (1981).

[76] E. Lomon and H. Feshbach, Rev. Mod. Phys. 39, 611 (1967).

[77] E. B. Hughes, T. A. Griffy, M. R. Yearian, and R. Hofstadter, Phys. Rev. 139, B458 (1965).

[78] P. Stein et al., Phys. Rev. Lett. 16, 592 (1966).

[79] K. M. Hanson et al., Phys. Rev. D 8, 753 (1973).

[80] S. Rock et al., Phys. Rev. Lett. 49, 1139 (1982).

[81] A. S. Esaulov, A. P. Rekalo, M. P. Rekalo, Yu. I. Titov, R. V. Akhmerov, and E. M. Smelov, Sov. J. Nucl. Phys. 45, 258 (1987), [Yad. Fiz.45,410(1987)].

[82] R. G. Arnold et al., Phys. Rev. Lett. 61, 806 (1988).

[83] P. Markowitz et al., Phys. Rev. C 48, R5 (1993).

[84] H. Anklin et al., Physics Letters B 336, 313 (1994),

[85] E. E. W. Bruins et al., Phys. Rev. Lett. 75, 21 (1995).

[86] G. Kubon et al., Physics Letters B 524, 26 (2002).

[87] J. Lachniet et al. (CLAS Collaboration), Phys. Rev. Lett. 102, 192001 (2009).

[88] C. E. Jones-Woodward et al., Phys. Rev. C 44, R571 (1991).

[89] A. K. Thompson et al., Phys. Rev. Lett. 68, 2901 (1992).

[90] M. Meyerhoff et al., Phys. Lett. B327, 201 (1994).

[91] T. Eden et al., Phys. Rev. C50, R1749 (1994).

[92] H. Gao et al., Phys. Rev. C 50, R546 (1994).

[93] J. Becker et al., Eur. Phys. J. A6, 329 (1999).

[94] M. Ostrick et al., Phys. Rev. Lett. 83, 276 (1999).

[95] C. Herberg et al., The European Physical Journal A - Hadrons and Nuclei 5, 131 (1999).

[96] I. Passchier et al., Phys. Rev. Lett. 82, 4988 (1999).

[97] D. Rohe et al., Phys. Rev. Lett. 83, 4257 (1999).

[98] J. Bermuth et al., Phys. Lett. B564, 199 (2003), arXiv:nucl-ex/0303015 [nucl-ex]].

[99] W. Xu et al., Phys. Rev. Lett. 85, 2900 (2000), arXiv:nucl-ex/0008003 [nucl-ex].

[100] W. Xu et al. (Jefferson Lab E95-001), Phys. Rev. C67, 012201 (2003), arXiv:nuclex/0208007 [nucl-ex].

[101] H. Zhu et al., Phys. Rev. Lett. 87, 081801 (2001).

[102] R. Madey et al. (E93-038), Phys. Rev. Lett. 91, 122002 (2003), arXiv:nucl-ex/0308007 $[$ nucl-ex]. 
[103] G. Warren et al. (Jefferson Lab E93-026 Collaboration), Phys. Rev. Lett. 92, 042301 (2004).

[104] D. I. Glazier et al., Eur. Phys. J. A24, 101 (2005), arXiv:nucl-ex/0410026 [nucl-ex].

[105] B. Anderson et al. (Jefferson Lab E95-001 Collaboration), Phys. Rev. C 75, 034003 (2007).

[106] E. Geis et al. (The BLAST Collaboration), Phys. Rev. Lett. 101, 042501 (2008).

[107] B. S. Schlimme et al., Phys. Rev. Lett. 111, 132504 (2013).

[108] V. Sulkosky et al., Phys. Rev. C96, 065206 (2017), arXiv:1704.06253 [nucl-ex].

[109] J. Golak et al., Phys. Rev. C63, 034006 (2001), arXiv:nucl-th/0008008 [nucl-th] .

[110] M. K. Jones et al. (Resonance Spin Structure), Phys. Rev. C74, 035201 (2006), arXiv:nuclex/0606015 [nucl-ex].

[111] C. B. Crawford et al., Phys. Rev. Lett. 98, 052301 (2007).

[112] L. W. Mo and Y.-S. Tsai, Rev. Mod. Phys. 41, 205 (1969).

[113] L. C. Maximon and J. A. Tjon, Phys. Rev. C 62, 054320 (2000).

[114] P. A. M. Guichon and M. Vanderhaeghen, Phys. Rev. Lett. 91, 142303 (2003).

[115] P. G. Blunden, W. Melnitchouk, and J. A. Tjon, Phys. Rev. Lett. 91, 142304 (2003).

[116] Y.-C. Chen, A. Afanasev, S. J. Brodsky, C. E. Carlson, and M. Vanderhaeghen, Phys. Rev. Lett. 93, 122301 (2004).

[117] J. Arrington, Phys. Rev. C 69, 032201 (2004).

[118] S. Kondratyuk, P. G. Blunden, W. Melnitchouk, and J. A. Tjon, Phys. Rev. Lett. 95, 172503 (2005).

[119] Y. M. Bystritskiy, E. A. Kuraev, and E. Tomasi-Gustafsson, Phys. Rev. C 75, 015207 $(2007)$.

[120] A. Afanasev et al., Prog. Part. Nucl. Phys. 95, 245 (2017), arXiv:1703.03874 [nucl-ex].

[121] D. Adikaram et al. (CLAS Collaboration), Phys. Rev. Lett. 114, 062003 (2015).

[122] I. A. Rachek et al., Phys. Rev. Lett. 114, 062005 (2015).

[123] P. G. Blunden, W. Melnitchouk, and J. A. Tjon, Phys. Rev. C 72, 034612 (2005),

[124] A. V. Afanasev, S. J. Brodsky, C. E. Carlson, Y.-C. Chen, and M. Vanderhaeghen, Phys. Rev. D 72, 013008 (2005).

[125] C. E. Carlson and M. Vanderhaeghen, Annual Review of Nuclear and Particle Science 57, 171 (2007).

[126] B. S. Henderson et al. (OLYMPUS Collaboration), Phys. Rev. Lett. 118, 092501 (2017).

[127] P. Lehmann, R. Taylor, and R. Wilson, Phys. Rev. 126, 1183 (1962).

[128] J. C. Bernauer et al. (A1 Collaboration), Phys. Rev. Lett. 105, 242001 (2010).

[129] I. Sick, Physics Letters B 576, 62 (2003). 
[130] R. J. Hill and G. Paz, Phys. Rev. D 82, 113005 (2010).

[131] I. Sick and D. Trautmann, Phys. Rev. C 89, 012201 (2014).

[132] P. J. Mohr, B. N. Taylor, and D. B. Newell, Rev. Mod. Phys. 84, 1527 (2012).

[133] R. Pohl et al., Nature 466, 213 (2010),

[134] R. Pohl et al., Annual Review of Nuclear and Particle Science 63, 175 (2013),

[135] R. Pohl et al. (CREMA), Science 353, 669 (2016).

[136] C. E. Carlson, Prog. Part. Nucl. Phys. 82, 59 (2015), arXiv:1502.05314 [hep-ph].

[137] I. T. Lorenz et al., The European Physical Journal A 48, 151 (2012).

[138] I. Sick, Progress in Particle and Nuclear Physics 55, 440 (2005).

[139] L. L. Foldy, Rev. Mod. Phys. 30, 471 (1958).

[140] S. Kopecky et al., Phys. Rev. C 56, 2229 (1997).

[141] E. Fermi and L. Marshall, Phys. Rev. 72, 1139 (1947).

[142] N. Isgur, Phys. Rev. Lett. 83, 272 (1999).

[143] F. Cardarelli and S. Simula, Physics Letters B 467, 1 (1999).

[144] D. B. Leinweber, A. W. Thomas, and R. D. Young, Phys. Rev. Lett. 86, 5011 (2001).

[145] M. A. Belushkin, H.-W. Hammer, and U.-G. Meißner, Phys. Rev. C 75, 035202 (2007).

[146] F. Halzen and D. Martin, Quarks 63 Leptons: An Introductory Course in Modern Particle Physics (John Wiley and Sons, Inc., 1984).

[147] J. J. Kelly, Phys. Rev. C70, 068202 (2004).

[148] J. Sakurai, Ann. of Physics 11 (1960).

[149] J. Sakurai, Currents and Mesons (The University of Chicago Press, 1969).

[150] F. Iachello, A. D. Jackson, and A. Lande, Phys. Lett. 43B, 191 (1973).

[151] M. F. Gari and W. Krümpelmann, Physics Letters B 274, 159 (1992).

[152] E. L. Lomon, Phys. Rev. C 64, 035204 (2001),

[153] E. L. Lomon, Phys. Rev. C 66, 045501 (2002).

[154] F. Iachello and Q. Wan, Phys. Rev. C 69, 055204 (2004).

[155] R. Bijker and F. Iachello, Phys. Rev. C69, 068201 (2004).

[156] E. L. Lomon, (2006), arXiv:nucl-th/0609020 [nucl-th].

[157] S. J. Brodsky and G. R. Farrar, Phys. Rev. Lett. 31, 1153 (1973).

[158] S. J. Brodsky and G. R. Farrar, Phys. Rev. D 11, 1309 (1975).

[159] V. A. Matveev et al., Lettere al Nuovo Cimento (1971-1985) 7, 719 (1973). 
[160] A. V. Belitsky, X. Ji, and F. Yuan, Phys. Rev. Lett. 91, 092003 (2003), arXiv:hepph/0212351 [hep-ph].

[161] X. Ji, Phys. Rev. Lett. 78, 610 (1997)

[162] M. Guidal, M. V. Polyakov, A. V. Radyushkin, and M. Vanderhaeghen, Phys. Rev. D 72, 054013 (2005).

[163] G. A. Miller, Phys. Rev. C 66, 032201 (2002).

[164] C. D. Roberts, Progress in Particle and Nuclear Physics 61, 50 (2008), quarks in Hadrons and Nuclei.

[165] I. C. Cloët et al., Few-Body Systems 46, 1 (2009).

[166] I. C. Cloët, C. D. Roberts, and A. W. Thomas, Phys. Rev. Lett. 111, 101803 (2013).

[167] J. Segovia, I. C. Cloet, C. D. Roberts, and S. M. Schmidt, Few Body Syst. 55, 1185 (2014), arXiv:1408.2919 [nucl-th] .

[168] I. C. Cloët, W. Bentz, and A. W. Thomas, Phys. Rev. C 90, 045202 (2014),

[169] I. C. Cloët and G. A. Miller, Phys. Rev. C 86, 015208 (2012).

[170] J. M. Alarcón and C. Weiss, Phys. Rev. C97, 055203 (2018), arXiv:1710.06430 [hep-ph].

[171] R. S. Sufian, G. F. de Tramond, S. J. Brodsky, A. Deur, and H. G. Dosch, Phys. Rev. D95, 014011 (2017), arXiv:1609.06688 [hep-ph].

[172] A. J. Chambers et al. (UKQCD, QCDSF, CSSM), Phys. Rev. D96, 114509 (2017), arXiv:1702.01513 [hep-lat].

[173] A. J. Chambers, Hadron Structure and the Feynman-Hellmann Theorem in Lattice Quantum Chromodynamics, Ph.D. thesis, The University of Adelaide (2018).

[174] G. D. Cates, C. W. de Jager, S. Riordan, and B. Wojtsekhowski, Phys. Rev. Lett. 106, 252003 (2011).

[175] A. M. Kelleher, A Measurement of the Neutron Electric Form Factor at Very Large Momentum Transfer Using Polarized Electrons Scattering from a Polarized Helium-3 Target, Ph.D. thesis, The College of William and Mary (2010).

[176] A. S. Kolarkar, Precision Measurements of the Neutron Electric Form Factor at High Momentum Transfers, Ph.D. thesis, University of Kentucky (2008).

[177] J. A. Miller, Measurement of the Electric Form Factor of the Neutron at High Momentum Transfer, Ph.D. thesis (2009).

[178] S. Riordan, Measurements of the Electric Form Factor of the Neutron at $Q^{2}=1.7$ and 3.5 $\mathrm{GeV}^{2}$, Ph.D. thesis, Carnegie Mellon University (2008).

[179] T. Ngo, Studies and calibration of the neutron arm for the GEN experiment at Thomas Jefferson National Laboratory, Master's thesis, California State University, Los Angeles (2007). 
[180] S. Agostinelli, J. Allison, et al., Nuclear Instruments and Methods in Physics Research Section A: Accelerators, Spectrometers, Detectors and Associated Equipment 506, 250 (2003).

[181] "Jefferson Lab: Wikiland," http://www.wikiwand.com/en/Thomas_Jefferson_ National_Accelerator_Facility, accessed: 2017-06-13.

[182] B. Craver, Technical Notes (2007).

[183] M. Steigerwald, "MeV Mott Polarimetry at Jefferson Lab," (2000).

[184] D. W. Higinbotham, AIP Conference Proceedings 1149, 751 (2009).

[185] C. W. Leemann et al., Annual Review of Nuclear and Particle Science 51, 413 (2001).

[186] C. K. Sinclair et al., Phys. Rev. ST Accel. Beams 10, 023501 (2007).

[187] C. Hernandez-Garcia, M. L. Stutzman, and P. G. O’Shea, Phys. Today 61N2, 44 (2008).

[188] J. Alcorn et al., NIM Section A 522, 294 (2004),

[189] K. B. Unser, IEEE Trans. Nucl. Sci. 28, 2344 (1981).

[190] K. B. Unser, Proceedings, 3rd Annual Workshop on Accelerator Instrumentation: Newport News, Virginia, October 28-31, 1991, AIP Conf. Proc. 252, 266 (1992).

[191] T. H. A. Collaboration, "Jefferson Lab Hall A Standard Equipment Manual," http:// hallaweb.jlab.org/github/halla-osp/ (2011).

[192] A. V. Glamazdin et al., Fizika B8, 91 (1999), arXiv:hep-ex/9912063 [hep-ex] .

[193] M. Baylac et al., Phys. Lett. B539, 8 (2002), arXiv:hep-ex/0203012 [hep-ex] .

[194] N. Falletto et al., Nuclear Instruments and Methods in Physics Research Section A: Accelerators, Spectrometers, Detectors and Associated Equipment 459, 412 (2001).

[195] T. G. Walker and W. Happer, Rev. Mod. Phys. 69, 629 (1997),

[196] T. G. Walker, Journal of Physics: Conference Series 294, 012001 (2011).

[197] A. B.-Amar Baranga et al., Phys. Rev. Lett. 80, 2801 (1998).

[198] E. Babcock et al., Phys. Rev. Lett. 91, 123003 (2003).

[199] "E02-013 GEn-style Helium-3 Target Cell Inventory," http://galileo.phys.virginia. edu/research/groups/spinphysics/gen/gencells.html.

[200] J. Singh et al., Phys. Rev. C91, 055205 (2015), arXiv:1309.4004 [physics.atom-ph].

[201] M. V. Romalis and G. D. Cates, Phys. Rev. A 58, 3004 (1998).

[202] B. Wojtsekhowski et al., Technical Notes (2008).

[203] J. de Lange et al., Nucl. Instrum. Meth. A406, 182 (1998).

[204] A. Gavalya, "Private communication," (2018).

[205] http://coda.jlab.org/. 
[206] http://hallaweb.jlab.org/root/index.html.

[207] http://root.cern.ch.

[208] http://www.aps.anl.gov/epics/index.php.

[209] "E02-013 Experimental Homepage," http://hallaweb.jlab.org/experiment/E02-013/ wiki/tiki-index.php?page=BigBite, accessed: 2017-06-06.

[210] M. Pitt and the $\mathrm{G}^{0}$ Collaboration, (2001).

[211] A. J. R. Puckett (UConn Group), "Documentation of G4SBS," https://hallaweb.jlab. org/wiki/index.php/Documentation_of_g4sbs, accessed: 2017-09-06.

[212] A. V. Gramolin et al., Journal of Physics G: Nuclear and Particle Physics 41, 115001 (2014).

[213] G. B. Franklin, Technical Notes (2006).

[214] P. E. Ulmer, "Monte Carlo for Electro-Nuclear Coincidence Experiments," http:// hallaweb.jlab.org/software/mceep/mceep.html, access: 2017-12-13.

[215] http://www.kph.uni-mainz.de/MAID/.

[216] P. E. Bosted and M. E. Christy, Phys. Rev. C 77, 065206 (2008).

[217] M. M. Sargsian, Int. J. Mod. Phys. E10, 405 (2001), arXiv:nucl-th/0110053 [nucl-th].

[218] https://www.jlab.org/fm/wx/index.html.

[219] M. M. Sargsian, (private communication).

[220] W. Brooks, G. Gilfoyle, J. Lachniet, M. Vineyard, et al., unpublished. See https://www. jlab.org/exp_prog/proposals/07/PR12-07-104.pdf (2007).

[221] J. Arrington, M. Christy, S. Gilad, V. Sulkosky, B. Wojtsekhowski, et al., unpublished. See https://www.jlab.org/exp_prog/proposals/07/PR12-07-108.pdf (2007).

[222] E. Cisbani, M. Jones, M. Khandaker, N. Liyanage, L. Pentchev, C. Perdrisat, V. Punjabi, B. Wojtsekhowski, et al., unpublished. See https://www.jlab.org/exp_prog/proposals/ 07/PR12-07-109.pdf (2008).

[223] G. Cates, S. Riordan, B. Wojtsekhowski, et al., unpublished. See https://www.jlab.org/ exp_prog/proposals/proposal_updates/PR12-09-016_pac35.pdf (2010).

[224] J. Annand, R. Gilman, B. Quinn, B. Wojtsekhowski, et al., unpublished. See https://www. jlab.org/exp_prog/proposals/09/PR12-09-019.pdf (2008).

[225] B. D. Anderson, J. Arrington, S. Kowalski, R. Madey, B. Plaster, A. Yu, et al., unpublished. See https://www.jlab.org/exp_prog/proposals/11/PR12-11-009.pdf (2011).

[226] J. Annand, V. Bellini, K. Kohl, N. Piskunov, B. Sawatzky, B. Wojtsekhowski, et al., unpublished. See https://misportal.jlab.org/pacProposals/proposals/1297/ attachments/98337/Proposal.pdf (2017). 